\title{
DIFFERENT APPROACHES TO THE SYNTHESIS AND APPLICATIONS OF BIOMEDICAL
} AND PHARMACEUTICAL SMART BIOMATERIALS

\author{
By \\ MOHAMED MAHMOUD KHATTAB
}

BSc in Chemistry (Honor), Cairo University, Egypt, 1993

MSc in Inorganic Chemistry, Cairo University, Egypt, 1996

PhD in Inorganic Chemistry, Cairo University, Egypt, 2003

\author{
A thesis \\ presented to Ryerson University \\ in partial fulfillment of the \\ requirements for the degree of \\ Doctor of Philosophy \\ in the program of \\ Chemical Engineering
}

Toronto, Ontario, Canada, 2019

(C) Mohamed M. Khattab, 2019 


\section{AUTHOR'S DECLARATION}

I hereby declare that I am the sole author of this dissertation. This is a true copy of the dissertation, including any required final revisions, as accepted by my examiners.

I authorize Ryerson University to lend this dissertation to other institutions or individuals for the purpose of scholarly research.

I further authorize Ryerson University to reproduce this dissertation by photocopying or by other means, in total or in part, at the request of other institutions or individuals for the purpose of scholarly research.

I understand that my dissertation may be made electronically available to the public. 


\title{
DIFFERENT APPROACHES TO THE SYNTHESIS AND APPLICATIONS OF BIOMEDICAL AND PHARMACEUTICAL SMART BIOMATERIALS
}

\author{
MOHAMED MAHMOUD KHATTAB \\ Doctor of Philosophy, Chemical Engineering, Ryerson University \\ Toronto, Canada, 2019
}

\begin{abstract}
In this study, two strategies were used to functionalize cellulose nanowhiskers. Firstly, by grafting its surface with $\beta$-cyclodextrin $(\beta C D)$ for developing drug-nanocarrier. Secondly, by conjugating short polymer segments to its surface for reinforcing poly( $\varepsilon$-caprolactone) (PCL), in order to develop nanocomposites with promoted properties suitable for regenerative medicine. In addition, the production and recovery of biodegradable bioplastics poly(3-hydroxybutyrate) $(\mathrm{P}(3 \mathrm{HB}))$ from agro-industrial residues of hemp hurd biomass was also examined.

In the first part, a drug-nanocarrier system based on $\beta C D$-grafted bacterial cellulose nanowhisker $(\mathrm{BCNC}-\mathrm{g}-\beta \mathrm{CD})$ was developed as a prolonged drug release nanocarrier. Antibiotic Ciprofloxacin (CIP) and anticancer drugs; Doxorubicin (DOX) and Paclitaxel (PTX) were conjugated to BCNC- $g-\beta C D$ as model drugs to form the drug-nanocarrier (BCNC-g- $\beta C D$-drug). Compared with un-grafted BCNC, the developed drug-nanocarrier showed significant increase in drug payloads from $495 \pm 4$ to $810 \pm 7 \mu \mathrm{g} / \mathrm{mg}$ along with radical improvement in the drug release profiles. Initial burst releases was reduced significantly and prolonged and sustained release for (74.5-90\%) of drug payload over 4-5.5 days were observed. In addition, an improved drug release performances were pragmatic in acidic $\mathrm{pH}$ of 6.4 that mimicked extracellular tumor cells. In vitro drug release data pointed to zero-order kinetic model with estimated zero-order release constants $\left(\mathrm{K}_{0}\right)$ of $0.68,0.74$, and $0.79 \mu \mathrm{g}$ drug/h (at $\mathrm{pH} 6.4,37^{\circ} \mathrm{C}$ ) for $\mathrm{BCNC}-\mathrm{g}-\beta \mathrm{CD}-\mathrm{CIP}$, BCNC- $g-\beta C D-D O X$ and BCNC- $g$ - $\beta C D-P T X$ nanosystems, respectively.

In the second part, the functionalized bacterial cellulose nanowhisker (BCNW-g- $\left.\beta C D-P C L_{2000}\right)$ was synthesized. Reinforcing PCL matrix with 4 wt \% of the functionalized nanowhisker resulted in bionanocomposite with promoted bulk properties. Compared to neat PCL, the obtained bionanocomposite showed 115\% and 51\% improvements in tensile strength and Young's modulus, respectively; 20\% increase in hydrophilicity; $7 \%$ increase in degradation rate; and 6\% decrease in crystallinity. Gas foaming/combined particulate leaching technique is used to
\end{abstract}


develop highly porous structures having porosity of $86-95 \%$ and interconnected macropores with mean pore diameters of $250-420 \mu \mathrm{m}$. Porous scaffolds showed compression moduli values of 5.3-9.1 MPa in the range of cancellous bones.

In the third part a dual-function PCL scaffold was fabricated. The envisioned drug-laden scaffold would provide adequate structural and mechanical supports for the newly regenerated tissues and simultaneously serve as localized drug delivery system. In this context, reinforced PCL with 4 $\mathrm{wt} \%$ of BCNW-g- $\beta C D-\mathrm{PCL}_{2000}$ and $25 \mathrm{wt} \%$ of doxorubicin anticancer drug resulted in drugladen bionanocomposite of combined promoted bulk properties. Improvements of $165 \%$ and $107 \%$ in tensile strength and Young's modulus, respectively; $31 \%$ in hydrophilicity; $10 \%$ in degradation rate; and $8^{\circ} \mathrm{C}$ increases in thermal stability. The obtained drug-laden porous scaffolds showed compressive moduli in the range of 7.2-12.3 MPa. In vitro drug releases fit the first-order release mechanism and occurred in a diffusion-controlled and sustained manner 60 days without obvious burst releases. The scaffolds will ultimately minimize systemic toxicities of drugs, lessen the number of dosing, and diminish the need for removal procedure.

The forth study described prospective trials for greener production and extraction of the biodegradable bioplastics poly(3-hydroxybutyrate) $\mathrm{P}(3 \mathrm{HB})$ from agro-industrial residues of hemp hurd biomass. Results showed that maximum hydrolysis yield of $72.4 \%$ was achieved by alkali pretreatment with $2 \% \mathrm{NaOH}$ at $135^{\circ} \mathrm{C}$ for $60 \mathrm{~min}$ along with two-step enzymatic hydrolysis and ultrasonication. Total hydrolysate sugar concentration of $53.0 \mathrm{~g} / \mathrm{L}$ was obtained. Under optimum conditions, total $\mathrm{P}(3 \mathrm{HB})$ production of $13.4 \mathrm{~g} / \mathrm{L}$ was achieved within $80 \mathrm{~h}$ of fermentation. Ultrasonic-assisted sodium dodecyl sulfate (SDS) has showed effectiveness as economic recovery method. It recovered bioplastics directly from the broth cell concentrate with $\mathrm{P}(3 \mathrm{HB})$ content of $92 \%$. Number average molecular weights $(\mathrm{Mn})$ of recovered bioplastics were in the range of 150-270 kDa with polydispersity index (Mw/Mn) of 2.1-2.4. 


\section{Acknowledgements}

This work was carried out for the fulfillment of my PhD between 2015 and 2019 in the Nanocomposites and Biomaterials Engineering Laboratory, Chemical Engineering Department, Ryerson University, Toronto, Canada.

My great appreciation goes to my supervisor Dr. Yaser Dahman for his exceptional supervision, creative guidance, and comprehensive feedback. He provided me with astonishing opportunities, flexibilities and support during my $\mathrm{PhD}$ study. He believed in me, walked me through research projects with great patience and guidance through providing valuable suggestions and comments.

I would also like to extend my deepest gratitude to Dr. Chil-Hung Cheng and Dr. Farhad EinMozaffari for their guidance and assistance as program coordinators and Dr. Simant Upreti the Department Chair for his support throughout my $\mathrm{PhD}$ degree.

I am also so grateful to have Dr. Wensheng Qin, Department of Biology at Lakehead University; Dr. Elsayed Elbeshbishy, Department of Civil Engineering at Ryerson University; Dr. Jiangning Wu, and Dr. Philip Chan, Department of Chemical Engineering at Ryerson University on the examining committee, for their valuable comments and for the time taken to carefully review my dissertation.

I would like also to acknowledge the financial support of Ryerson Graduate Scholarship, Queen Elizabeth Scholarship (QEII GSST), Shepherdess Biotech Inc., and the Centre of Green Research Technology.

In particular, I want to thank Mr. Shawn McFadden, RUAC coordinator, Department of Chemistry and Biology at Ryerson University for providing the technical support for samples characterization using FTIR, ATR, DSC, XRD, GPC and HPLC. I also want to acknowledge the help of Mr. Qiang Li, the Technical Specialist of Scanning electron microscopy (SEM) imaging, Department of Mechanical and Industrial Engineering at Ryerson University. I would also like to extend my appreciation to Dr. Dérick Rousseau for use of the Atomic Force Microscope (AFM).

Furthermore, I would like to express my appreciation to all faculty members and technical staff in the Department of Chemical Engineering at Ryerson University for their support and encouragement during the development of my learning processes at Ryerson University.

I am very thankful to my talented officemates for their motivation and collaborations. Last but not the least, I would like to thank my family for their love and support. 


\section{DEDICATION}

This thesis is dedicated to my lovely family. 


\section{TABLE OF CONTENTS}

AUTHOR'S DECLARATION

Page

ABSTRACT

ii

ACKNOWLEDGEMENTS

iii

DEDICATION

$\mathbf{v}$

LIST OF TABLES

vi

LIST OF FIGURES

xiii

LIST OF APPENDICES

XV

$\mathbf{X X}$

CHAPTER 1 - INTRODUCTION

1.1 Overview

1.2 Research Objectives

1.3 Thesis Outlines

CHAPTER 2 - LITERATURE REVIEW 9

2.1 Biocellulose: Diversity, Structure and Molecular Assembly 9

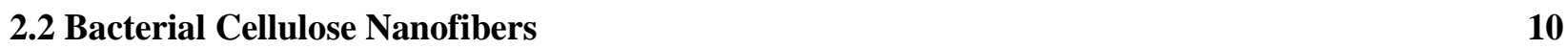

2.2.1 Source, Structure and Unique Characteristics $\quad \mathbf{1 0}$

2.2.2 Mechanical Properties of BC Nanofibers $\quad 11$

2.2.3 Bacterial Cellulose Applications in Tissue-Engineering and Drug Delivery 12

$\begin{array}{ll}2.3 \beta \text {-Cyclodextrin } & 14\end{array}$

2.3.1 Structure and Unique Properties $\quad 14$

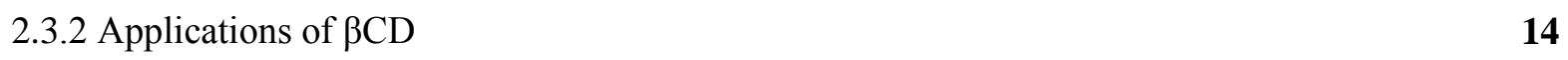

2.4 Poly(E-caprolactone) (PCL)

2.4.1 Synthesis and Physicochemical Properties of PCL $\quad 15$

2.4.2 Mechanical Properties of PCL $\quad 16$

$\begin{array}{ll}\text { 2.4.3 Biodegradation of PCL } & 17\end{array}$

2.4.4 Biocompatibility of PCL and Cell Proliferation 19

2.4.5 PCL in Tissue Engineering and Drug Delivery 19

$\begin{array}{ll}\text { 2.4.6 Challenges Facing PCL scaffolds } & \mathbf{2 3}\end{array}$

2.4.7 Developing Porosity $\quad \mathbf{2 4}$

2.4.8 Drug-laden PCL scaffolds $\quad 25$ 
2.4.9 Limb Salvage Procedure $\quad 26$

$\begin{array}{ll}2.5 \text { Poly(3-hydroxybutyrate) P(3HB) } & 28\end{array}$

2.6 Cellulose Nanocomposites: Opportunities, Challenges, and Applications 30

$\begin{array}{ll}\text { 2.6.1 Introduction } & \mathbf{3 0}\end{array}$

2.6.1.1 Different Sources of Cellulose Nanofibers

2.6.1.2 Hierarchy of Cellulose Nanofibers 32

$\begin{array}{ll}\text { 2.6.2 Nanocellulose } & \mathbf{3 4}\end{array}$

2.6.2.1 Cellulose Nanoparticles: Terminology and Production 34

2.6.2.2 Cellulose Nano Whiskers

2.6.2.3 Microfibrillated Cellulose (MFC) 39

2.6.2.4 Self-Assembly and Organization of CNC

2.6.2.5 Tensile Properties of Nanocellulose and Their Nano-reinforcement Potentials $\quad \mathbf{4 3}$

2.6.2.6 Modification of Nanocellulose

2.6.2.6.1 Non-covalent Surface Modifications $\quad \mathbf{4 4}$

2.6.2.6.2 Covalent Surface Chemical Modifications $\mathbf{4 5}$

2.6.2.6.3. Polymer Grafting $\quad \mathbf{4 6}$

$\begin{array}{ll}\text { 2.6.2.6.4. Deposition of Nanoparticles on CNC } & 47\end{array}$

2.6.3 Cellulose Based Nanocomposites $\quad 49$

2.6.3.1 Micromechanical Modeling for Predicting the Reinforcing Potential of CNC 49

2.6.3.2 Mechanical Properties of Nanocellulose Based Nanocomposites $\quad \mathbf{5 1}$

2.6.3.3 Effect of Processing Method and Interfacial Interactions on Mechanical properties $\quad 52$ of CNC-Based Nanocomposites

2.6.3.4 Thermal Properties of CNC-Based Nanocomposites $\quad \mathbf{5 3}$

2.6.3.5 Nanocellulose-Reinforced Polymer Nanocomposites: Review $\quad \mathbf{5 4}$

2.6.4 Biomedical Applications of Nanocellulose and Its Nanocomposites $\quad 55$

2.6.5 Conclusion and Future Prospects $\quad 56$

CHAPTER 3 - Functionalized Bacterial Cellulose Nanowhisker as Long Lasting Drug Nanocarrier 59 for Antibiotics and Anticancer drugs

$\begin{array}{lc}3.1 \text { Introduction } & 60\end{array}$

3.2 Material and Methods $\quad 62$

$\begin{array}{ll}\text { 3.2.1 Materials } & \mathbf{6 2}\end{array}$

3.2.2 Synthesis $\quad \mathbf{6 3}$

3.2.2.1 Production of BC Nanofibers $\quad \mathbf{6 3}$ 
$\begin{array}{ll}\text { 3.2.2.3 Grafting of Nanowhisker } & \mathbf{6 4}\end{array}$

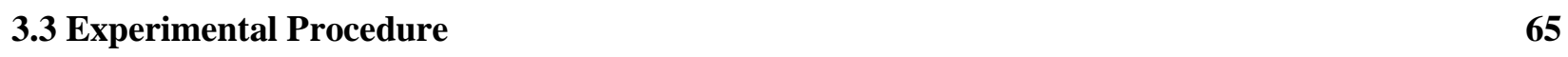

3.3.1 Determination of Grafting Ratio $\quad 65$

3.3.2 Determination of Binding Constants of Drug- $\beta C D$ Inclusion Complexes $\quad 66$

3.3.3 Effect of Drug Concentration on Loading Efficiency $\quad 66$

$\begin{array}{ll}\text { 3.3.4 In vitro Release Measurements } & 67\end{array}$

$\begin{array}{lr}\text { 3.4 Characterization Techniques } & 68\end{array}$

$\begin{array}{ll}\text { 3.4.1 SEM and AFM microscopy } & \mathbf{6 8}\end{array}$

$\begin{array}{ll}\text { 3.4.2 X-ray Diffraction } & 68\end{array}$

3.4.3 Fourier Transform Infra-Red (FTIR) $\quad \mathbf{6 8}$

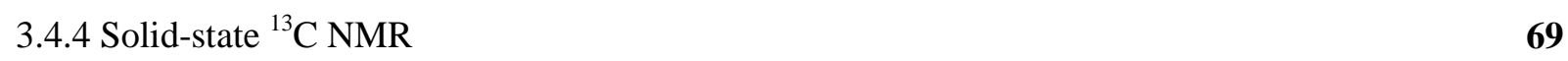

$\begin{array}{lc}3.4 .5 . H P L C & 69\end{array}$

$\begin{array}{lc}3.5 \text { Results } & 69\end{array}$

$\begin{array}{ll}\text { 3.6 Discussion } & 85\end{array}$

$\begin{array}{lr}3.7 \text { Conclusion } & 89\end{array}$

CHAPTER 4 - Synthesis and Characterization of Cellulose Nanowhisker Reinforced-Poly( $\varepsilon^{-} \quad 91$

Caprolactone) Scaffold for Tissue Engineering Applications

$\begin{array}{ll}4.1 \text { Introduction } & 92\end{array}$

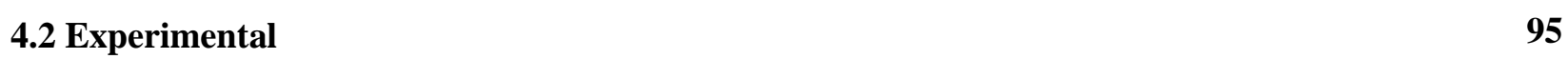

$\begin{array}{ll}\text { 4.2.1 Materials and Chemicals } & 95\end{array}$

$\begin{array}{ll}\text { 4.2.2 Preparations and Analysis } & 95\end{array}$

4.2.2.1 Preparation of BC Nanowhisker $(B C N W) \quad 95$

4.2.2.2 Preparation of $B C N W-g-\beta C D \quad 96$

4.2.2.3 Synthesis of Adamantyl End-capped $P C L_{2000}\left(P C L_{2000}-A D\right) \quad 96$

4.2.2.4 Synthesis of $B C N W-g-\beta C D-P C L_{2000}$

4.2.2.5 Dispersion of $B C N W-g-\beta C D-P C L_{2000}$ in $P C L \quad 98$

4.2.2.6 Preparation of PCL Reinforced $B C N W-g-\beta C D-P C L_{2000}$ Nanocomposites Film $\quad 98$

4.2.2.7Fabrication of PCL Porous Scaffolds $\quad 98$

4.2.2.8 Fabrication of PCL-reinforced BCNW-g- $\beta C D$-PCL $L_{2000}$ Porous Scaffolds

4.2.2.9 Porosity, Pore Volume, and Pore Size 100 
4.3.1 Fourier Transform Infra-red Spectroscopy (FTIR/ATR) $\quad 100$

4.3.2 Mechanical Properties of Nanocomposites Films and Scaffolds $\quad \mathbf{1 0 1}$

$\begin{array}{ll}\text { 4.3.3 Thermal Properties } & \mathbf{1 0 1}\end{array}$

4.3.4 Field Emission Scanning Electron Microscopy (FE-SEM) $\quad \mathbf{1 0 1}$

4.3.5 Apparent Water-in-Air Contact Angle $\quad 102$

$\begin{array}{ll}\text { 4.3.6 In vitro degradation behavior } & \mathbf{1 0 2}\end{array}$

$\begin{array}{ll}\text { 4.3.7 Statistical Analysis } & \mathbf{1 0 2}\end{array}$

4.4 Results and Discussion $\quad 102$

4.4.1 Grafting of $B C N W$ with $\beta C D$ and Assembly of $\mathrm{PCL}_{2000}-\mathrm{AD}_{2}$ on $\mathrm{BCNW}-\mathrm{g}-\beta C D$ Surface $\quad \mathbf{1 0 2}$

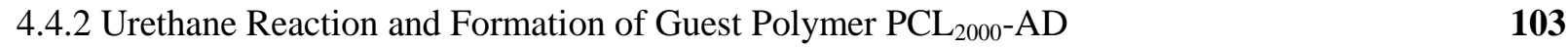

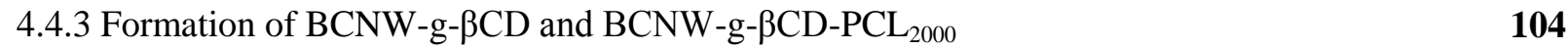

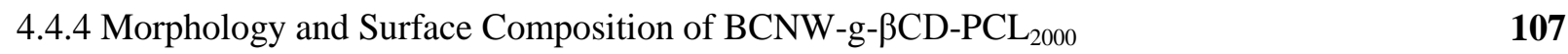

4.4.5 Mechanical Properties of BCNW-g- $\beta C D-P C L_{2000}$ Reinforced PCL Nanocomposites Films 110

4.4.6 Surface Modification Evaluated by Contact Angle $\quad \mathbf{1 1 3}$

4.4.7 Crystallization Behavior and Thermal Stability of PCL-bionanocomposite 114

4.4.8 Scaffolds Porosities and Morphologies $\quad 116$

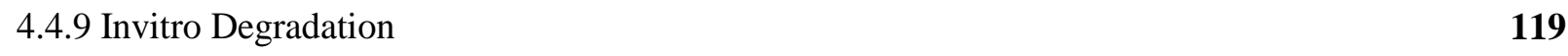

$\begin{array}{lr}4.5 \text { Conclusion } & 121\end{array}$

CHAPTER 5 - Dual-Function Poly( $\varepsilon$-Caprolactone) Scaffold for Long-Term Drug Release and 122

Tissue Engineering Applications

$\begin{array}{ll}5.1 \text { Introduction } & 123\end{array}$

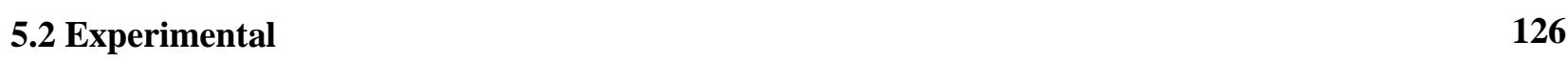

5.2.1 Materials and Chemicals $\quad 126$

5.2.2 Preparations and Analysis Procedure $\quad 126$

5.2.2.1 Preparation of BC Nanowhisker $(B C N W) \quad 126$

5.2.2.2 Synthesis of $B C N W-g-\beta C D-P C L_{2000}$

$\begin{array}{ll}\text { 5.2.2.2.1 Preparation of BCNW-g- } \beta C D & 127\end{array}$

5.2.2.2.2 Assembly of $\mathrm{PCL}_{2000}-\mathrm{AD}_{2}$ on BCNW-g- $\beta C D$ Surface $\quad 127$

5.2.2.3 Preparation of PCL Reinforced BCNW-g- $\beta C D-P C L_{2000}$ Nanocomposites 128

5.2.2.4 Fabrication of PCL-reinforced BCNW-g- $\beta C D-P C L_{2000}$ Porous Scaffolds 128

5.2.2.5 Fabrication of Drug-laden Porous Scaffold

5.2.2.6 Porosity, Pore Volume, and Pore Size 129 
5.3.1 Fourier Transform Infra-red Spectroscopy (FTIR/ATR)

5.3.2 Mechanical Properties of Nanocomposites Films and Scaffolds

5.3.3 Thermal Properties

130

5.3.4 Field Emission Scanning Electron Microscopy (FE-SEM)

131

5.3.5 Apparent Water-in-Air Contact Angle

131

5.3.6 In vitro Degradation Behavior

131

5.3.7 In vitro Drug Release Studies

132

5.3.8 Drug Release Models

132

5.4 Results and Discussion

133

5.4.1 Grafting of BCNW with $\beta C D$ and Assembly of $\mathrm{PCL}_{2000}-\mathrm{AD}_{2}$ on $\mathrm{BCNW}-\mathrm{g}-\beta C D$ Surface

133

5.4.2 Urethane Reaction and Formation of Guest Polymer PCL $\mathrm{PL}_{200}-\mathrm{AD}_{2}$

135

5.4.3 Formation of BCNW-g- $\beta C D$ and BCNW-g- $\beta C D-P C L_{2000}$

135

5.4.4 Morphology and Surface Composition of BCNW-g- $\beta C D-\mathrm{PCL}_{2000}$

137

5.4.5 Mechanical Properties of BCNW-g- $\beta C D-\mathrm{PCL}_{2000}$ Reinforced PCL Composite Films

138

5.4.6 Effect of Drug Loading on the Mechanical Properties of Bionanocomposite

140

5.4.7 Surface Modification Evaluated by Contact Angle

142

5.4.8 Crystallization Behavior and Thermal Stability of Drug-laden Bionanocomposite

143

5.4.9 Scaffolds Porosities and Morphologies

147

5.4.10 Invitro Degradation

150

5.4.11 Drug Release Profiles from Porous Scaffolds

152

5.4.12 Mathematical Model Fitting Drug Releases

155

5.5 Conclusion

157

CHAPTER 6 - Production and Recovery of Poly-3-hydroxybutyrate Bioplastics using AgroIndustrial residues of Hemp Hurd Biomass

\subsection{Introduction}

6.2.1 Materials and microbial strain

6.2.2.1 Preparation and characterization of $H H B$

162

6.2.2.2 Pre-treatment of $\mathrm{HHB}$

163

6.2.2.3 Enzymatic hydrolysis

163

6.2.2.4 Measuring sugar concentrations 
6.2.2.5 $P(3 H B)$ fermentation and production $\quad \mathbf{1 6 5}$

6.2.2.6 $P(3 H B)$ recovery $\quad \mathbf{1 6 5}$

6.2.2.7 Analytical method and characterization of $P(3 H B) \quad \mathbf{1 6 7}$

$\begin{array}{ll}6.3 \text { Results and Discussion } & 168\end{array}$

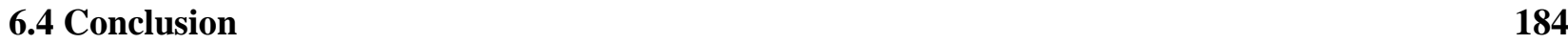

CHAPTER 7 - Original Contributions and Recommendations 186

7.1 Original Contributions to Research 186

$\begin{array}{ll}\text { 7.2 Recommendations for Future Studies } & 188\end{array}$

$\begin{array}{ll}\text { APPENDICES } & 189\end{array}$

$\begin{array}{lc}\text { REFERENCES } & 208\end{array}$ 


\section{LIST OF TABLES}

Table \#

Page\#

Table 2.1 Commercial products based on BC and/or BC based materials for biomedical applications currently available in market

Table 2.2 Bulk tensile mechanical properties of PCL reported in literature

Table 2.3 Compressive mechanical properties of PCL scaffolds manufactured through solid freeform fabrication (SFF) reported in the literature

Table 2.4 Reviews of some studies investigated the fabrication of PCL scaffold for various tissue engineering applications

Table 2.5 Different terminologies used in literature to express cellulose nanoparticles during their production and extraction processes

Table 2.6 Geometrical characteristics (length, L; diameter, D; and percolation threshold $\emptyset_{\mathrm{c}}$ of some cellulose nanocrystals

Table 2.7 Biomedical applications of nanocellulose and nanocellulose based nanocomposites

Table 3.1 Sizes, crystalinity and physicochemical properties of BCNCs samples obtained by different hydrolysis

Table 3.2 Comparison between $\beta C D$ grafting ratios upon using different linkers; loading amounts and cumulative releases of CIP, DOX and PTX drugs to and from unmodified BCNC and BCNC- $g-\beta C D$

Table 3.3 Summary of the chemical formula of the drugs used along with their binding constants as well as the modeled in vitro release kinetics

Table 4.1 Tensile properties of neat PCL, PCL/BCNW, and PCL/BCNW- $g$ - $\beta C D-$ $\mathrm{PCL}_{2000}$ nanocomposites films

Table 4.2 Thermal pentameters for neat PCL and its bionanocomposite with various nanowhisker loadings

Table 4.3 Porosity percentage, pore volume, pore size, and compression modulus for different porous scaffolds obtained from PCL/BCNW-g- $\beta C D-P_{20} L_{200}(4 \%)$

Table 5.1 The effect of BCNW- $g-\beta C D-\mathrm{PCL}_{2000}$ and drug loadings on the final mechanical properties of PCL-drug laden bionanocomposite

Table 5.2 Thermal pentameters for neat PCL, drug-loaded PCL and its bionanocomposite with various drug loadings

Table 5.3 Porosity percentage, pore volume, pore size, and compression modulus of PCL-drug laden porous scaffolds

Table 5.4 In vitro drug releases data fitting to various theoretical drug release models

Table 6.1 Chemical composition of HHB expressed as \% w/w of the pretreated DM as means of triplicates

Table 6.2 Sugar concentrations and hydrolysis yields obtained from HHB 
hydrolysates after using different pretreatments process in combination with different scenarios of enzymatic hydrolysis

Table 6.3 $\mathrm{CDW}, \mathrm{P}(3 \mathrm{HB})$ production and $\mathrm{P}(3 \mathrm{HB})$ accumulation by $R$. eutropha fed on various nitrogen sources and fixed total HHB hydrolysate sugar concentration of $53.0 \mathrm{~g} / \mathrm{L}$

Table 6.4 Investigating $\mathrm{CDW}$ and $\mathrm{P}(3 \mathrm{HB})$ production and accumulation at different $\mathrm{C}: \mathrm{N}$ ratios utilizing glucose and $\mathrm{NH}_{4} \mathrm{Cl}$ as sole carbon and nitrogen sources

Table 6.5 Comparison between $\mathrm{P}(3 \mathrm{HB})$ content, percentage of recovery, molecular 182 weights and polydispersity of $\mathrm{P}(3 \mathrm{HB})$ polymer recovered by different methods 


\section{LIST OF FIGURES}

Figure \#

Page\#

Figure 2.1 Schematic of the molecular structure of cellulose repeat units

Figure 2.2 (a) Schematic illustration of BC biosynthesis and fibril formation; (b) cellulose-producing bacteria, G. Xylinus (c) Images are showing gel-like pellicle in a three-day old culture of $G$. Xylinus. Under scanning electron microscope, the pellicle appears to be made of a nanofibrillar network of cellulose

Figure 2.3 Prospects for the various biomedical applications of BC and BC-based materials

Figure 2.4 Different examples for substitutes made from never-dried BC membranes

Figure 2.5 Chemical structure of $\alpha, \beta$ and $\gamma$ cyclodextrins (from left to right) showing their cavity dimensions

Figure 2.6 Ring opening polymerization of $\varepsilon$-caprolactone to polycaprolactone

Figure 2.7 (a)The degradation of PCL via hydrolysis intermediates 6-hydroxyl 16 caproic acid and acetyl coenzyme A, which are then eliminated from the body via the citric acid cycle. (b) Schematic visualization of how crystalline fragmentation could have taken place

Figure 2.8 Scaffold-based tissue engineering aims to promote the repair and/or regeneration of tissues through the incorporation of cells and/or biomolecules within a 3D scaffold system which can be maintained in vitro culture conditions until implantation

Figure 2.9 Structures made from PCL: nanospheres (a,b), nanofibers (c,d), foams $(\mathrm{e}, \mathrm{f}), \mathrm{knitted}$ textiles (g,h,i), selective laser sintered scaffold (j-o), and fused deposition modeled scaffolds $(\mathrm{p}-\mathrm{u})$

Figure 2.10 Developing porous Structures by gas foaming/particulate leaching techniques

Figure 2.11 (a) Doxorubicin hydrophobic anticancer drug (b) Diagram of anticancer drug-DNA complexes of two doxorubicin molecules intercalating DNA (c) Induced structural distortions due to intercalation; left: unchanged DNA strand; right: DNA strand intercalated at three locations (black areas) by Doxorubicin

Figure 2.12 Showing limb salvage procedure

Figure 2.13 Transmission electron micrograph of thin sections of R. eutropha cells containing large amounts of $\mathrm{P}(3 \mathrm{HB})$ inclusions $(90 \%$ of the dry cell weight) appear as electron-dense bodies. Bar represents $0.5 \mu \mathrm{m}$

Figure 2.14 A; Schematic representation illustrating the key aspects of heterotrophic metabolism of $R$. eutropha. The yellow circles represent the processes of central metabolism, whereas the gray circles indicate 
polyhydroxyalkanoate (PHA) storage granules. B; Cyclic metabolic nature of $\mathrm{P}(3 \mathrm{HB})$ biosynthesis and degradation in bacteria. PhaA, $\beta$ ketothiolase; PhaB, NADPH dependent acetoacetyl-CoA reductase;

PhaC, PHA synthase; PhaZ, PHA depolymerase; 1, dimer hydrolase; 2 , (R)-3-hydroxybutyrate dehydrogenase; 3, acetoacetyl-CoA synthetase; 4 , NADH-dependent acetoacetyl-CoA reductase

Figure 2.15 Comparison between average tensile properties of commonly used synthetic engineering fibers, renewable technical fibers, petroleum-based and renewable polymers, respectively. $\mathrm{BC}$ denotes bacterial cellulose

Figure 2.16 Scheme representing cellulose cell wall, bacterial cellulose production, microfibril organization and interaction between cellulose molecular chains within the crystalline region

Figure 2.17 Summarizes the main steps involved in the preparation of cellulose Nanoparticles

Figure 2.18 (Top part) shows schematics presentation for acid hydrolysis of amorphous regions and the formation of CNCs. (Bottom part) is showing AFM images of cellulose nanowhisker produced from $\mathrm{BC}$ by using (a) $\mathrm{HCl}$ hydrolysis and (b) $\mathrm{H}_{2} \mathrm{SO}_{4}$ hydrolysis

Figure 2.19 Conductometric titration curves for $\mathrm{H}_{2} \mathrm{SO}_{4}$-hydrolyzed $\mathrm{CNC}$ (open circle), $\mathrm{HCl}$-hydrolyzed $\mathrm{CNC}$ (open triangle), $\mathrm{HCl}$-desulfated CNCseventh step (solid circle), solvolytically desulfated CNC (solid triangle)

Figure 2.20 Microfibrillated cellulose (MFC) gel

Figure 2.21 (Left) aqueous $0.63 \%(\mathrm{w} / \mathrm{w}) \mathrm{CNC}$ suspension observed between crossed polarizer. Immediately after shearing, the suspension shows many iridescent birefringence patterns; after 1 week, the suspension separates into the upper isotropic and the lower anisotropic phases. (Right) schematic representation of $\mathrm{CNC}$ orientation in both isotropic and anisotropic (chiral nematic) phases

Figure 2.22 Some possible routes for chemical modification of CNC (anticlockwise from left): (A) silylation; (B) urethane linkages via isocyanates; (C) oxidation by TEMPO; (D) ester linkages via acid chlorides; (E) cationization via epoxides; (F) ester linkages via acid anhydrides

Figure 2.23 Schematic illustration of the synthetic procedure for $\mathrm{CNC} / \mathrm{CdS} @ \mathrm{ZnS}$

Figure 2.24 Schematic representation for the synthesis of CNC@Fe3O4@SiO2@ $\beta$ CD. CNC: cellulose nanocrystals; PDDA: poly (diallydimethylammonium chloride); PVP: polyvinylpyrrolidone; $\beta$-CD: $\beta$-cyclodextrin

Figure 3.1 Morphologies of BCNCs. Top; (a, $\mathbf{b}$ and $\mathbf{c})$ FE-SEM micrograph for unhydrolyzed $\mathrm{BC}$ nanofibers, $\mathrm{BCNC}$ produced by $\mathrm{H}_{2} \mathrm{SO}_{4}$ hydrolysis, and $\mathrm{BCNC}$ produced by $\mathrm{HCl}$ hydrolysis, respectively. Bottom; (d) AFM 
image of BCNC produced by enzymatic hydrolysis along with illustration for width determination, (e) SEM micrograph for BCNC- $g$ $\beta C D$

Figure 3.2 XRD diffractograms of: (a) unhydrolyzed $\mathrm{BC}$, (b) $\mathrm{H}_{2} \mathrm{SO}_{4}$ hydrolyzed

$\mathrm{BCNC}$, (c) $\mathrm{HCl}$ hydrolyzed BCNC, (d) enzymatically hydrolyzed $\mathrm{BCNC}$, and (e) BCNC- $-\beta \mathrm{CD}$

Figure 3.3 Grafting of BCNC with $\beta C D$ by using of; (a) citric acid as linker in presence of (SHP) as catalyst, (b) epichlorohydrin as a linker in basic medium, and (c) inclusion complex formation between BCNC- $g-\beta C D$ nanowhisker and conjugated drug (BCNC- $g$ - $\beta C D$-drug)

Figure 3.4 (a) ATR spectra of $\beta C D, C A-\beta C D, B C N C$ and $B C N C-g-\beta C D$; (b)

CPMAS solid-state ${ }^{13} \mathrm{C}$ NMR spectra of unmodified BCNC and BCNC$g-\beta C D$

Figure 3.5 Plot of $1 / \mathrm{A}-\mathrm{A}_{0}$ against $1 /[\beta \mathrm{CD}]$ for the interaction of $\mathrm{CIP}$ with the increased concentrations of $\beta \mathrm{CD}$ at $\mathrm{pH} 7.4$ and $37^{\circ} \mathrm{C}$. Experiments were done in triplicate, and error bars represent \pm standard deviation. Inset (a) represents schematic for inclusion complex formation. Inset (b) represents hyper-chromic shift observed for the absorption maxima of CIP due to increased $\beta C D$ concentration

Figure 3.6 Binding of CIP, DOX and PTX to $1 \mathrm{mg}$ unmodified BCNC and BCNC-g$\beta C D$ in $10 \mathrm{mM}$ PBS buffer at $\mathrm{pH} 7.4$ and $25^{\circ} \mathrm{C}$. Experiments were done in triplicate, and error bars represent \pm standard error

Figure 3.7 Comparison between the drug releases profiles of BCNC-CIP, BCNC- $g$ $\beta C D-C I P, B C N C-g-\beta C D-D O X$ and BCNC- $g-\beta C D-P T X$ at $37^{\circ} \mathrm{C}$ in PBS buffer; (a) at $\mathrm{pH} 7.4$; (b) at $\mathrm{pH}$ 6.4. Experiments were done in triplicate, and error bars represent \pm standard error

Figure 3.8 Disk diffusion of CIP from BCNC- $g$ - $\beta C D-C I P$ to prevent bacterial colonization. Left disk: unmodified BCNC; Right disk: BCNC-g- $\beta C D-$ CIP

Figure 3.9 Fitting curves of drug release data to zero-order kinetics model

Figure 4.1 Fabrication of porous scaffolds by gas foaming/combined particulate leaching technique

Figure 4.2 A: Grafting of $B C N W$ with $\beta C D$ using citric acid as linker; B: synthetic pathway of $\mathrm{PCL}_{2000}-\mathrm{AD}_{2}$ from $\mathrm{PCL}_{2000}$-diol; $\mathrm{C}$ : conceptual illustration of short polymer segments assembly on BCNW-g- $\beta C D$ surface through the formation of inclusion complexes

Figure 4.3 A: ATR spectra showing the formation of guest polymer $\left(\mathrm{PCL}_{2000}-\mathrm{AD}_{2}\right)$ from PCL 2000 -diol and AD-NCO; B: ATR spectra of BCNW after grafting with $\beta C D$ and assembly of $\mathrm{PCL}_{2000}-\mathrm{AD}_{2}$

Figure 4.4 FE-SEM micrograph (200 nm scale) showing the surface morphology of 
BCNW- $g$ - $\beta C D$ before and after $\mathrm{PCL}_{2000}-\mathrm{AD}_{2}$ assembly

Figure 4.5 EDX spectrum of A: BCNW- $g-\beta C D$ before $\mathrm{PCL}_{2000}$ assembly; $\mathrm{B}$ : spectrum after $\mathrm{PCL}_{2000}$ segments assembly

Figure 4.6 A: Stress -strain plots of neat PCL and PCL-bionanocomposite; B:

112

Tensile strength, Young's modulus of neat PCL and its nanocomposites as function of BCNW- $g-\beta C D-\mathrm{PCL}_{2000}$ loadings; $\mathrm{C}$ : Elongation at break of neat PCL and its nanocomposites as a function of BCNW- $g-\beta C D$ $\mathrm{PCL}_{2000}$ loadings

Figure 4.7 Apparent water-in-air contact angles of PCL/BCNW- $g-\beta C D-P_{2000}$ nanocomposites films containing different loadings of functionalized nanowhisker

Figure 4.8 DSC cooling and heating runs of neat PCL and its bionanocomposite

Figure 4.9 TGA curves of neat PCL, functionalized nanowhisker BCNW- $g-\beta C D-$ $\mathrm{PCL}_{2000}$, and their bionanocomposite

Figure 4.10 SEM images of surface and cross-section morphology of PCL/BCNW- $g$ $\beta C D-\mathrm{PCL}_{2000}$ scaffolds. (A, $\mathrm{B} \& \mathrm{C}$ ) are surfaces when weight $\mathrm{NH}_{4} \mathrm{HCO}_{3}$ : PCL ratios were 5:1, 7:1 and 10:1, respectively; (A1, A2) \& $(\mathrm{C} 1, \mathrm{C} 2)$ are cross-section morphology corresponding to the same series at different magnification powers; $\times 800$ and $\times 3500$

Figure 4.11 In vitro degradation behavior of neat PCL and PCL/BCNW- $g-\beta C D-$ $\mathrm{PCL}_{2000}$ bionanocomposit scaffolds in $\mathrm{PBS}$ at $\mathrm{pH} 7.4$ and $37^{\circ} \mathrm{C}$

Figure 5.1 (A) Grafting of BCNW with $\beta C D$ by using of citric acid as linker; (B) synthetic pathway of $\mathrm{PCL}_{2000}-\mathrm{AD}_{2}$ from PCL-diol; (C\&D) conceptual illustration for drug and short polymer segments assembly on $\mathrm{BCNC}-g$ $\beta C D$ surface through the formation of inclusion complexes

Figure 5.2 A: ATR spectra showing the formation of guest polymer $\left(\mathrm{PCL}_{2000}-\mathrm{AD}_{2}\right)$ from PCL 2000 -diol and AD-NCO; B: ATR spectra of BCNW after grafting with $\beta C D$ and assembly of $\mathrm{PCL}_{2000}-\mathrm{AD}_{2}$

Figure 5.3 A \& B are FE-SEM micrograph and D \&D are EDX spectrum that showing the surface morphology and composition of BCNW-g- $\beta C D$ before and after $\mathrm{PCL}_{2000}-\mathrm{AD}_{2}$ assembly

Figure 5.4 Stress-strain plots of neat PCL and PCL bionanocomposite

Figure 5.5 Apparent water-in-air contact angles for; (a) PCL/BCNW-g- $\beta C D$ $\mathrm{PCL}_{2000}$ nanocomposites films at different loadings of BCNW- $g-\beta C D$ $\mathrm{PCL}_{2000}$;(b)PCL/BCNW- $g$ - $\beta C D-\mathrm{PCL}_{2000}(4 \%)$ drug-laden nanocomposites with different DOX loadings from (10-30 \%wt)

Figure 5.6 DSC first cooling and second heating scans of neat PCL and its drugladen bionanocomposite

Figure 5.7 TGA curves of functionalized nanowhisker, neat PCL and PCLbionanocomposite 
Figure 5.8 SEM images of surface and cross-section morphology of PCL/BCNW- $g$ -

$\beta C D-\mathrm{PCL}_{2000}(4 \%) / \mathrm{DOX}(25 \%)$ scaffolds prepared by gas foaming in hot citric acid. (A, B \&C) are surfaces when weight $\mathrm{NH}_{4} \mathrm{HCO}_{3}$ : $\mathrm{PCL}$ ratios were 5:1, 7:1 and 10:1, respectively; (A1, A2) \& (C1, C2) cross-section morphology corresponding to the same series at different magnification powers; $\times 800$ and $\times 3500$

Figure 5.9 In vitro degradation behavior of neat PCL and drug-laden nanocomposites scaffolds in PBS at $\mathrm{pH} 7.4$ and $37^{\circ} \mathrm{C}$

Figure 5.10 Cumulative release of DOX from PCL+ DOX (25\%) and PCL/BCNW-g$\beta C D-\mathrm{PCL}_{2000}(4 \%)+\mathrm{DOX}(25 \%)$ at $37^{\circ} \mathrm{C}$ in $\mathrm{PBS}$ of $\mathrm{pH} 7.4$ and 6.4

Figure 5.11 A: Effect of increased porosity $(\%)$ on the cumulative release profile of DOX from nanocomposites with drug payload of $25 \% \mathrm{w} / \mathrm{w}$ in PBS at $\mathrm{pH}$ 7.4 and $37^{\circ} \mathrm{C}$; B: Effect of drug loading (\%) on the cumulative release profile of DOX from nanocomposites of $91 \%$ porosity in $\mathrm{PBS}$ at $\mathrm{pH} 7.4$ and $37^{\circ} \mathrm{C}$

Figure 6.1 Hydrolysis yield of enzymatically hydrolyzed alkali pretreated HHB samples of different particle sizes; $0.5-5 \mathrm{~mm}$ expressed as $\%$ of the total carbohydrate

Figure 6.2 Mass balance for alkali pretreated HHB based on two-step enzymatic hydrolysis and in combination with ultrasonication step

Figure 6.3 Concentration profiles showing sugars consumption (a) and PHB 154155 production (b) by $R$. eutropha feed on HHB hydrolysate in comparison with glucose and xylose control experiments. The results were presented in mean $\pm \mathrm{SD}, \mathrm{n}=3$

Figure 6.4 Accumulation of cell dry weight (a) and gradual increase in cell 176concentration (b) over fermentation time by $R$. eutropha feed on HHB hydrolysate sugars in comparison with the observed accumulation in control fermentation containing glucose and xylose simulated feedstock. The results were presented in mean $\pm \mathrm{SD}, \mathrm{n}=3$

Figure 6.5 The effect of changing $\mathrm{C} / \mathrm{N}$ ratio on cell growth and $\mathrm{P}(3 \mathrm{HB})$ accumulation by R.eutropha

Figure 6.6 Contour plot showing the effect of changing ammonium chloride and glucose concentrations on $\mathrm{P}(3 \mathrm{HB})$ accumulation

Figure 6.7 FTIR characterization of $\mathrm{P}(3 \mathrm{HB})$ produced from microbial fermentation by R. eutropha utilizing hemp sugars hydrolysate

Figure 6.8 EDX spectra (a), SEM-images (b) and photograph of extracted $\mathrm{P}(3 \mathrm{HB})$ 184 film (c) produced by $R$. eutropha feed on HHB hydrolysates 


\section{LIST OF APPENDICES}

Appendix \#

Page\#

Appendix A Main Chemical and Pharmacokinetic Data of the Drugs Used

Appendix B Activation of Bacteria strains

Appendix C Calibration/Standard Curves

Appendix D Lab Gallery Photos Showing Water-In-Air Contact Angle

Appendix E Lab Gallery Photos Showing Microbial Production and Purification of

Appendix $\mathbf{F}$ BC Nanofibers and their Conversion to Nanowhisker

PHB from Hemp Hurd Biomass

Appendix G Mechanical Testing

Appendix H Cumulative Drug Release Calculations

Appendix I Sample Calculations

Appendix J Statistical Analysis and Error Calculations

Appendix K Row data for some replicated experiments 


\section{CHAPTER 1 - INTRODUCTION}

\subsection{Overview and Background}

Sustainable biomaterials such as biocellulose nanofibrils are one of the most abundant natural biomaterial. They have been widely utilized in nanomedicine as green templates for inorganic nanoparticles, ${ }^{[1]}$ and incorporated into polymer nanocomposites scaffolds for tissue engineering (TE) applications. ${ }^{[2-4]}$ In addition to higher purity, attributes such as biocompatibility, ${ }^{[5]}$ hemocompatibility, ${ }^{[6]}$ mechanical strength ${ }^{[7]}$ biodegradability ${ }^{[8]}$ and unique surface chemistry ${ }^{[9]}$ made it attractive to wide industrial sectors. However, the large size of nanofibers limited its applications in fields where nanoscale structure is required. Nevertheless, hydrogen-bonded linear chains of $\beta$-D-glucopyranose restricted the reactivity of primary hydroxyl groups carried on their surface and often result in low grafting ratios that hindered their performance and uses. ${ }^{[10,11]}$ With adjustable and much shorter length cellulose nanocrystals (CNCs) has attracted increasing attention. It possesses rigid rod-shaped nanostructure, high surface area, good aqueous colloidal stability, favorable surface modification, and excellent mechanical strength. ${ }^{[12]}$ The later properties offer higher flexibility during its functionalization and fabrication of functional nanomaterial through bottom-up approaches. Various surface modifications for tolerating structure-function relationship of nanocellulose have been implemented. Those modifications included but not limited to esterification, oxidation, cationization, fluorescent labeling as well as polymer grafting. ${ }^{[13]}$ Such modifications enabled researchers to tune the surface energy of these nanocrystals in order to increase their compatibility with different polymer matrixes so as to explore their full potentials as green and sustainable reinforcing material for different applications. ${ }^{[13]}$ However, agglomeration and networks formation of CNCs that could be formed during their reactions are problematic during the modification processes and subsequent characterization. $^{[14]}$

By taking advantage of bacterial cellulose (BC) over plant-source cellulose, it used in the current study as main source for obtaining cellulose nanofibers. BC nanofibers are very pure, biocompatible and showed non-toxic effects toward endothelial cells. In addition, it has been approved by FDA to be used as a starting material for different biomedical applications, such as wound dressings, biomimetic scaffolds, and drug delivery devices. ${ }^{[15]}$ Bacterial cellulose nanocrystals (BCNWs) or "nanowhiskers" was obtained by acidic or enzymatic hydrolysis of BC 
nanofibers. It showed much smaller dimensions ( $150 \mathrm{~nm}$ in length; $\sim 15 \mathrm{~nm}$ in diameter; aspect ratio (length/diameter) in the range of 30 to150). ${ }^{[16]}$ Its shorter length along with higher aspect ratio maximized its surface area for promoted functionalization and drug loading applications. Moreover, BCNWs fall under the category of non-spherical nanoprticles that considered better candidates for drug targeting/delivery applications due to their enormous surface area-to-volume ratio that contributes for more interactions with targets and increased permeability. ${ }^{[17]}$ Cellular uptake of cancer drugs-conjugated to CNCs (FITC-CNC-FA) in C6 cancer cells targets was evidenced by fluorescence microscopy. ${ }^{[18]}$

$\beta$-cyclodextrins $(\beta C D)$ play significant role in drug delivery owing to their unique structure and non-toxicity. $\beta C D$ has an ideal hydrophobic central cavity dimension that can make inclusion complexes (ICs) with guest compounds such as, drug molecules ${ }^{[19]}$ and short polymers segments ${ }^{[20]}$ via non-covalent interactions. ${ }^{[21]}$ Therefore, grafting of cellulose nanofibers with $\beta C D$ is considered as promising technology for introducing new functionality to nanocellulose. Indeed, for biomedical applications, the main challenging part during production and modification of BCNWs is to make sure that either hydrolysis or chemical functionalization processes have to be done in such a way that only affect the surface of crystals, while the inherited crystalline structure, morphology, and excellent mechanical properties kept intact.

In contrast to traditional polymers, biodegradable polyesters have been the focus of extensive research for several decades due to their ease of manufacturing, desirable and tunable physical characteristics, and their hydrolytic degradation profiles throughout the unstable aliphatic-ester linkages. Later properties made them attractive candidates for utilization in various biomedical applications. ${ }^{[22]}$ Among them, poly( $\varepsilon$-caprolactone) (PCL) is one of the most widely studied biodegradable polyesters. PCL is a biocompatible, semicrystalline, slowly degrading polymer and used in several tissue engineering (TE) applications. These include bones and cartilage substitutes, sutures, fibrous meshes, adhesion barriers, temporary orthopedic fixtures and biopharmaceutical excipient for drug delivery and controlled drug releases. ${ }^{[23]}$ PCL gained popularity in the field of TE after receiving FDA approval. It also showed excellent biocompatibility, full degradation in the human body, ${ }^{[24]}$ and higher permeability for drug molecules specially hydrophobic anticancer drugs. ${ }^{[25]}$ Long-term in vivo studies have demonstrated that the PCL implants were able to maintain the physical shape after 2 years of 
implantation. ${ }^{[26]}$ Additionally, PCL was also capable to degrade slowly into low molecular weight by the end of 30 months and can be excreted completely out of the body without accumulating non-toxic degradation byproducts. ${ }^{[26]}$ Several systems have been successfully tested as vehicles for slow release of anticancer drugs at tumor sites ${ }^{[18,27]}$ and as temporal support after limb salvage procedure ${ }^{[28]}$ However, PCL showed remarkable hydrophobicity and non-osteoconductivity that stand as barrier for wider commercial use of PCL as a commodity polymer in biomedical field and led to suboptimal cell-material interactions. ${ }^{[29]}$ Nevertheless, its high degree of crystallinity and hydrophobicity also lowered its biodegradation rate.

Partial solutions for the limitations that confronting PCL were implemented by several researchers for developing the bulk properties. Those include addition for biological signaling molecules such as growth factors during scaffold fabrication, attaching cells to the scaffold surface, or seeding cells and growth factors into the scaffold before implantation. ${ }^{[26]}$ Other researchers have established to focus on the modification of the PCL by making surface decorations or blending it with other polymers until the physical and mechanical properties that required for hard and soft tissue engineering become attainable. ${ }^{[30]}$

Recently, the term tissue-engineering therapeutics has been emerged and coined TE scaffolds function as template for supporting the new tissue with additional drug delivery properties. ${ }^{[31]}$ Encapsulating drugs in nanopolymeric structures allows drugs to remain at specific sites of action in sufficiently higher concentration to exert their pharmacological effects, without causing severe systemic toxicities. Additionally, localized drug delivery also improves drug absorption and intracellular penetration, prolongs retention time, enhances drug efficacy, and reduces drug degradation. ${ }^{[32,33]}$ However, there is a significant challenge in the design and manufacture of scaffolds that possess all of the above requirements and the ability to control the release kinetics of drug over the period of treatment or tissue regeneration. ${ }^{[34]}$ For instance, TE polymeric scaffold (cell delivery) should posses the following: ${ }^{[22]}$ (i) mechanical properties that are sufficient to shield cells from tensile forces; (ii) desired volume, shape, and mechanical strength; (iii) acceptable biocompatibility; (iv) highly porous and well-interconnected open pore structure to allow cell seeding and tissue in-growth; (v) bioadsorption at predetermined time period; (vi) biocompatible chemical compositions and their degradation products, causing minimal immune or inflammatory responses; (vii) physical structure to support cell adhesion and proliferation, 
facilitating cell-cell contact and cell migration. Whereas scaffold for drug delivery should posses the following: (i) homogenous drug dispersion throughout the scaffold; (ii) ability to release the drug at a predetermined rate; (iii) low drug binding affinity that is sufficiently allow the drug released when incorporated in the scaffold; (iv) stable physical dimension and chemical structure over a prolonged period of time. ${ }^{[35]}$

Numerous therapeutic agents, including growth factors, antibiotics, and anti-inflammatories, have been successfully loaded into porous PCL scaffolds and demonstrated clinical potentials in the treatment of bone-related pathologies. ${ }^{[36]}$ However, results from PCL drug-laden 3D scaffolds such as polycaprolactone/hydroxyapatite blend (PCL/HA) showed a very slow degradation rate. ${ }^{[37]}$ In addition, PCL/anticancer drug blend of varying surface architecture used for postsurgical care of limb salvage procedure showed burst release of the loaded drug at the beginning and followed by a slow release in a sustained manner for 112 days. Nevertheless, cell attachment study demonstrated minimal potentiality of the scaffolds to provide attachment platforms for tissue regeneration. ${ }^{[28]}$

In the view of the later existing challenges and limitations that facing utilization and application of PCL polymer in biomedical applications, a novel biodegradable drug-laden bionanocomposite based on PCL and functionalized BCNW is developed. The envisioned dual-function scaffold would serve as viable alternative for metal prostheses or bone grafts. It provides adequate structural and mechanical support for newly regenerated tissues, and simultaneously serves as localized drug delivery system.

Recently, after legalizing the growth of Canadian Hemp, issues concerning the sustainable and harmless disposal of agriculture solid wastes of hemp hurd biomass (HHB) have generated interest in microbial biotechnologies. These biotechnologies are contributing to reduce the environmental footprint by converting waste materials into useful biomaterials such as poly(3hydroxybutyrate) $\mathrm{P}(3 \mathrm{HB})$. $\mathrm{P}(3 \mathrm{HB})$ is the best-known member of the polyhydroxyalkanoate family (PHA). It considered as attractive green alternative to synthetic plastics due to its biocompatibility and complete biodegradation in environment as well as within the blood and tissue of mammals. $\mathrm{P}(3 \mathrm{HB})$ is now used for absorbable suture, scaffolds, heart valves and cardiovascular tissue supports. ${ }^{[38]}$ However, its higher production cost stood as barrier and reduced the industrial sustainability of this environmentally friendly biodegradable plastic. ${ }^{\text {[39] }}$ 
Reducing production cost is one of the plausible solutions that can return this biodegradable plastic to the market to compete with petroleum-based plastics. Utilization of cheap and renewable carbon sources such as palm oil mill effluent, grass powder, rice waste biomass, waste glycerol, cassava starch hydrolysate, starch, organic waste, and fermentative effluents was reported by several researchers as a potential solution for cutting down the production cost of $\mathrm{P}(3 \mathrm{HB})$ to at least $25-40 \% .{ }^{[40]}$ Higher carbohydrate content reported for hemp fibers $(64 \% \mathrm{w} / \mathrm{w}$ of the crop dry matte) made it a promising candidate for $\mathrm{P}(3 \mathrm{HB})$ production. ${ }^{[41]}$ In addition, good selection of the microbial strains that can utilize this cheap biomass as a sole carbon source for more efficient and cost-effective production for $\mathrm{P}(3 \mathrm{HB})$ is very challenging. ${ }^{[40]}$

\subsection{Research Objectives and Research Plan}

In the present study, surface modification of BCNCs through both chemical grafting and further immobilization of short polymer segments on nanoparticle surfaces were conducted. One approach is to develop biocompatible drug-nanocarrier and the other one is to modify PCL matrix for fabricating drug-laden bionanocomposite scaffold with improved properties.

The first part of the study focuses on the biosynthesis of $\mathrm{BC}$ nanofibers as pure source for cellulose nanowhisker. Few attempts were made using controlled acidic and enzymatic hydrolysis to obtain true $\mathrm{BCNC}$ of reasonable nanosize for controlled agglomerations. Following that, surface grafting of BCNC with $\beta C D$ molecules is carried out to obtain novel functionalized BCNC-grafted- $\beta C D(B C N C-g-\beta C D)$. Tryouts involving different grafting agents were carried out in order to achieve the highest grafting ratio.

The second part focuses on harnessing the beneficial chemical and mechanical properties of the previously functionalized $\mathrm{BCNC}-\mathrm{g}-\beta \mathrm{CD}$. One time it used alone as nano-carrier for antibiotics and anticancer drug molecules via entrapping model drugs inside the hydrophobic cavities of $\beta C D$ s moieties that grafted on nanowhisker surface. Other time it used for assembling low molecular weight segments of $\mathrm{PCL}_{2000}$ on BCNC surface to produce (BCNC- $g$ - $\beta C D$ $\mathrm{PCL}_{2000}$ ) as a promising nano-reinforcing phase for PCL matrix. The later modification is not only aims to tune the surface energy of hydrophilic BCNC for better compatibility within hydrophobic PCL matrix, but also to create difference in the crystallization rates between high and low molecular weight PCL segments. This envisioned to impairing the higher crystalinity of PCL matrix and consequently improving biodegradation and drug release rates. 
The third and more challenging part of the present study aims to fabricate PCL-drug laden porous scaffold with adequate mechanical properties along with promoted cell attachment and drug loading efficiencies. Four-step process is used to implement this part. The first step is to examine the effect of different loadings of the functionalized reinforcing nanowhisker (i.e., BCNW- $g$ - $\beta C D-\mathrm{PCL}_{2000}$ ) on the mechanical, thermal, hydrophilic properties, and in-vitro degradation of the obtained PCL-bionanocomposite. The second challenging step is to reexamine the same properties to figure out the combined effect of incorporating both BCNW-g$\beta C D-\mathrm{PCL}_{2000}$ and anticancer drug on the final bulk characteristics of the obtained drug-laden bionanocomposite. More interestingly, to the best of our knowledge, there is no attempts have been made in exploiting the combined effect of introducing a reinforcing nano-phase and a drug in drug-laden porous scaffold. The third step involves the fabrication of 3D nanoconstructs of different porosity levels and have open and interconnected pores network. The forth step is to investigate the interaction between various parameters, such as nanowhisker loading, drug loadings and porosity level on the compression modulus and drug release behavior of the obtained porous drug-laden scaffold. In addition, to examine the kinetic model that best fits scaffolds' drug release data.

The last part of study aims to examine the possibility of economic production and recovery of $\mathrm{P}(3 \mathrm{HB})$ bioplastics from green sustainable and renewable agro-industrial residues of Canadian hemp hurd biomass (source of marijuana). This is a green attempt to reduce the environmental footprint to accommodate the increased disposal of the recently legalized Hemp cultivations. To date, no work has been reported on the use of Hemp hurd for $\mathrm{P}(3 \mathrm{HB})$ production.

Following a comprehensive review of the literature and the encountered challenges, the research plan of the thesis were formulated as follow:

\section{Part 1:}

1- Biosynthesis of bacterial cellulose nanofibers

2- Synthesis and characterization of bacterial cellulose nanocrystals (BCNCs)

3- Synthesis of functionalized BCNC-g- $\beta C D$ by grafting $\beta C D$ onto BCNCs using two different linkers, and investigating structure, morphology, crystallinity and grafting density by ATR, ${ }^{13}$ C NMR, TGA, DSC, XRD, FE-SEM, AFM and EDX. 


\section{Part 2:}

1- Developing drug-nanocarrier systems (BCNC-g- $\beta C D$-drug) by conjugating model drugs on BCNC-g- $\beta C D$ surface through the formation of host-guest inclusion complex, and testing their drug loading and releasing profiles

2- Synthesis of reinforcing nanowhisker (BCNC-g- $\left.\beta C D-\mathrm{PCL}_{2000}\right)$ via molecular assembly of short PCL segments $\left(\mathrm{M}_{\mathrm{n}}=2000 \mathrm{Da}\right)$ on BCNC-g- $\beta C D$, and investigating surface composition, morphology and loading density by ATR, TGA, DSC, FE-SEM, and EDX.

\section{Part 3:}

1- Reinforcing PCL $\left(\mathrm{M}_{\mathrm{n}}=45,000 \mathrm{Da}\right)$ with different loading ratios of $\mathrm{BCNC}-\mathrm{g}-\beta \mathrm{CD}$ PCL $_{2000}$, examining the optimum loading ratio, and testing mechanical, thermal, hydrophilic properties of the obtained bionanocomposite

2- Loading Doxorubicin anticancer drug to PCL-bionanocomposite, examining the optimum drug-loading ratio, and retesting the combined effect of nanowhisker and drug loading on the final mechanical, thermal, biodegradation and hydrophilic properties of the drugladen bionanocomposite

3- Fabricating 3D porous scaffold with different porosity levels

4- Studying the effect of porosity level and drug loading ratio on the compression modulus and drug release profiles of the obtained scaffolds

5- Evaluating kinetic model and drug release mechanism that fit invitro drug release data.

\section{Part 4:}

1- Examining several pretreatment process for concentrating cellulosic substrate in biomass, and carrying out composition analysis

2- Implementing several scenarios of hydrolysis for maximizing sugar concentration in hydrolysate

3- Activating producing strain, carrying out fermentation experiments and optimizing the best conditions for maximizing $\mathrm{P}(3 \mathrm{HP})$ production

4- Investigating different techniques for effective and economic extraction of product from cell concentrate

5- Analyzing composition, purity and molecular weight of the obtained bioplastics from the different extraction techniques used. 


\subsection{Thesis Outlines}

The thesis compiles seven chapters. Research results are reported in manuscript format from Chapter 3 to Chapter 6 . The scopes of every chapter is outlined as follows: Chapter 1 briefly introduces chemical/physical characteristics of $\mathrm{CNCs}, \beta C D$ and PCL and display their full potentials as promising biocompatible and nontoxic material for different TE and biomedical applications. It also spotlight on existing challenges and limitations that facing their utilizations. In addition, it draws attention to the production and important properties and uses of $\mathrm{P}(3 \mathrm{HP})$ as greener substitute for fossils-plastics. The research objectives and thesis outlines are also included in this chapter. Chapter $\mathbf{2}$ is mostly literature review. It introduces BC nanofibers (structure, synthesis, properties and medical applications); $\beta C D$ (structures, unique characteristics, and biomedical applications); PCL (synthesis, mechanical properties, biodegradation, biocompatibility, application in TE and DDS, previously developed PCLnanocomposites and the challenges confronting their uses); CNCs (terminology, structure, production, surface modification, thermal and mechanical properties, nanocomposites, processing methods, previously developed CNC-based nanocomposites, biomedical applications, and the challenges confronting their uses). Last part of chapter 2 was published as book chapter. The materials displayed in chapter 2 played key role for formulating the research ideas. Chapter 3 reports on the synthesis and characterization of BCNC- $-\beta C D$ and its capability to be used as long lasting drug-nanocarriers for antibiotics and anticancer drugs. This chapter was published as scientific article in CJCE (CJCE-18-1022). Chapter 4 reports on synthesis and characterization of $\mathrm{BCNC}-\mathrm{g}-\beta \mathrm{CD}-\mathrm{PCL}_{2000}$ and investigates its utilization as reinforcing phase for PCL for obtaining TE scaffold with improved properties. The mechanical, thermal, hydrophilic properties of the obtained scaffolds are reported. Version of this chapter was sent for publication in the Journal of Applied Polymer Science (app.20190241). Chapter 5 reports on the fabrication of dual-function PCL drug-laden scaffold for long-term drug release and TE applications. Porosities, compression moduli, drug releases profiles were studied and evaluated. Version of this chapter was sent for publication in JAPS (app.20190416). Chapter 6 demonestrates the challenge of microbial production of sustainable $\mathrm{P}(3 \mathrm{HB})$ bioplastics from hemp hurd biomass. Biomass pretreatment, fermentation, extraction and characterization are fully demonstrated and reported. This chapter was published in BPBSE (BPBSE-18-0493). Chapter 7 summarizes the key conclusions, major contributions and recommendations for future studies. 


\section{CHAPTER 2 - LITERATURE REVIEW}

\subsection{Biocellulose: Diversity, Structure and Molecular Assembly}

Biocellulose is synthesized by members of the kingdoms Planate and Animally as well as the domain Eubacteria. It considered the most abundant product on the earth and widely recognized as a sustainable raw material with lots of applicable advantages and properties. ${ }^{[42]}$ The availability of extremely heterogeneous sources of cellulose, such as wood, plants (cotton, ramie, flax, wheat straw), tunicate, algae (green, gray, red, yellow-green), fungi and bacteria have led to wide varieties of cellulosic materials, but each having unique characteristics. Although the variations they also share many common features. ${ }^{[43]}$

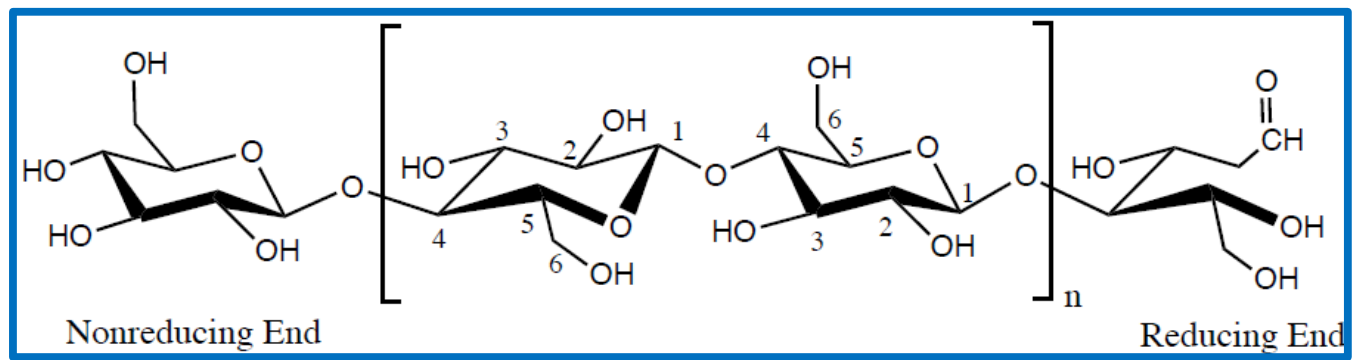

Figure 2.1 Schematic of the molecular structure of cellulose repeat units

Biochemically, cellulose is characterized as high molecular weight homopolymer with a ribbonlike conformation consisted of $\beta$-1,4-linked anhydro-D-glucose units (AGU). These repeated units bounded covalently through the oxygen atoms that join $\mathrm{C} 1$ carbon of one AGU with $\mathrm{C} 4$ carbon of the other unit (see Figure 2.1). ${ }^{[43]}$

Cellulose adopts a linear conformation, as each chair-conformed AGU is corkscrewed $180^{\circ}$ to its neighbors. The degree of polymerization of native cellulose is ranging from 10,000 to 26,000 $\mathrm{Da}$ and mainly depends on cellulose source. ${ }^{[44]}$ The terminal ends of the cellulose structure have a reducing (open-ring aldehyde) and non-reducing (closed D-glucose ring) end that create chemical symmetry. The hydroxyl groups $(-\mathrm{OH})$ are positioned in equatorial directions to the ring, while the hydrogen atoms are positioned in axial direction. ${ }^{[45]}$ The chains of poly- $\beta-(1 \rightarrow 4)$ D-glucosyl residues that formed during the biosynthesis are considered the basic structural component of cellulose. 


\subsection{Bacterial Cellulose Nanofibers}

\subsubsection{Source, Structure and Unique Characteristics}

Bacterial cellulose has the same chemical structure of plants cellulose. However, bacterial cellulose has the advantage of being very pure without lignin, pectin, hemicellulose, and other biogenic products that normally associated with plant cell wall structures. ${ }^{[46]}$

Bacterial cellulose is biosynthesized by certain bacterial strains such as Rhizobium spp., Agrobacterium spp., Acetobacter spp., and Alcaligenes spp. ${ }^{[47]}$ The resulting cellulose is termed either bacterial cellulose (BC) or microbial cellulose (MC). Acetobacter xylinum (A. xylinum) or Gluconacetobacter xylinus (G. xylinus) is the most efficient producer for BC. Typically, every single bacterium cell was able to convert up to 108 glucose molecule/h into cellulose. ${ }^{[48]}$ Cellulose molecules are naturally synthesized in the interior of bacterial cell, and then spun out of export components called "nozzles" to form protofibrils of approximately $1.5 \mathrm{~nm}$ in diameter. Immediately following synthesis, these protofibrils are crystallized into microfibrils $10-20 \mathrm{~nm}$ wide followed by their final bundling assembly into ribbons known as BC nanofibers, Figure 2.2.

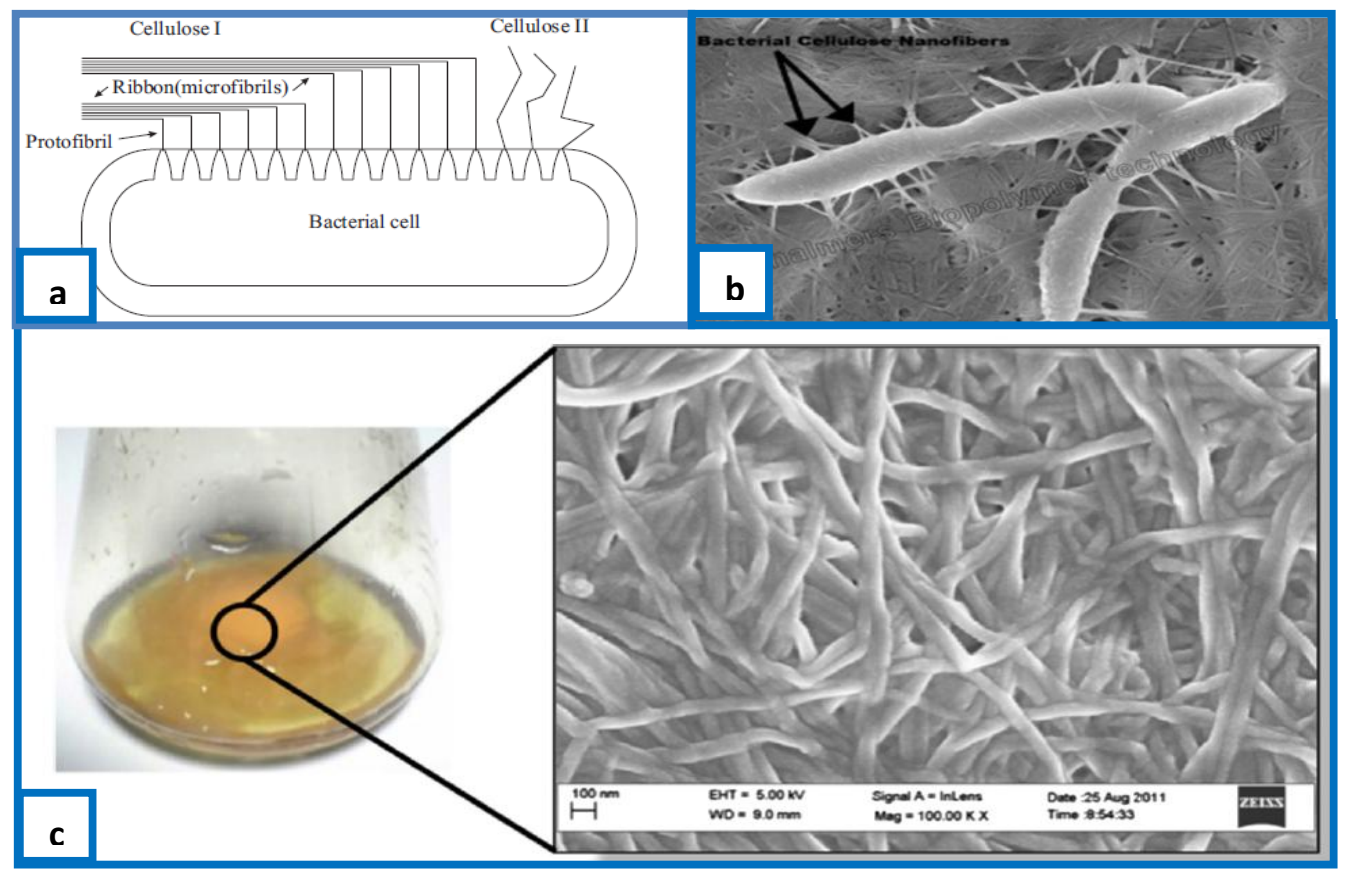

Figure 2.2 (a) Schematic illustration of BC biosynthesis and fibril formation; (b) G. Xylinus during biosynthesis of BC (c) Images showing gel-like pellicle in a three-day old culture of $G$. Xylinus; SEM of pellicle showing nanofibrillar network of BC. ${ }^{[49]}$ 
A typical BC fiber is highly crystalline and rich in cellulose $\mathrm{I}_{\alpha}$. It consists of $\sim 1000$ glucan chains having dimensions of approximately $1-9 \mu \mathrm{m} \mathrm{L} \mathrm{x} 70-80 \mathrm{~nm} \mathrm{~W} \times 3-4 \mathrm{~nm} \mathrm{H} .{ }^{[50]}$ Due to the extensive hydrogen bonding and Van der Waals forces, these ribbons bend and combine to form a stable 3D hydrogel-like membrane structure of high crystallinity ( $\sim 60 \%$ crystallinity index). Moreover, these hydrogen bonds allow $\mathrm{BC}$ to retain water in its interstitial spaces. Approximately $99.8 \%$ of $\mathrm{BC}$ matrix volume is water and $\sim 0.2 \%$ is a pure cellulose. ${ }^{[50]}$

A. xylinum strain is able to produce $\mathrm{BC}$ within a temperature range of 25 to $30{ }^{\circ} \mathrm{C}$ and a pH range of 4.5-7.5. ${ }^{[51]}$ Numerous substrates have been investigated for their suitability as potential carbon source for BC production. These include monosaccharide, disaccharide, polysaccharide, organic acid and ethylene glycol ${ }^{[47]}$ Recently, agro-industrial residues have been utilized as economic sources for BC biosynthesis as simultaneous sources of carbon and nitrogen. ${ }^{[52]}$

Beside its higher purity of $\mathrm{BC}$, attributes such as biocompatibility ${ }^{[5]}$ haemocompatibility, ${ }^{[6]}$ high aspect ratio and microporosity, ${ }^{[9]}$ mechanical strength, ${ }^{[7]}$ and biodegradability ${ }^{[8]}$ and unique surface chemistry ${ }^{[9]}$ made it an attractive raw material for wide industrial sectors. Such as food industry, biomedical applications (e.g., wound care), and tissue engineering (e.g., nanocomposites). ${ }^{[47]}$ Synthetic nanofibers, such as glass and carbon fibers, are brittle, and they are often broken into smaller fragments, whereas cellulose nanofibers is flexible and will not fracture during processing over sharp curvatures. ${ }^{[53]}$ This unique characteristic of $\mathrm{BC}$ is being widely used in producing biomedical devices of various shapes and forms. The stability of bacterial cellulose at high temperature also makes it amenable to heat sterilization. ${ }^{[54]}$ It can be purified using sodium hydroxide and yielded a form of $\mathrm{BC}$ with endotoxin values of $<20$ endotoxin units/device. As specified by FDA, the later falls within the acceptable endotoxin values range for implants used for intravenous applications. ${ }^{[55]}$

\subsubsection{Mechanical Properties of BC Nanofibers}

$\mathrm{BC}$ exhibits desirable mechanical properties, which are attributed to the high density of the interfibrilar hydrogen bonds and large contact area due to the presence of uniform reticulated structure comprising nanofibers. ${ }^{[56]}$ Variable mechanical properties have been reported for BC, which depends on its form. Mechanical properties were first studied by Yamanaka et al ${ }^{[57]}$ whom used purified BC obtained from Acetobacter aceti AJ12368 cultivated under static conditions. The Young's modulus of both air-dried and hot-pressed BC was found to be $>18 \mathrm{GPa}$, and tensile strength of air-dried BC films were found to be as high as $260 \mathrm{MPa}$, whereas that for hot- 
pressed $\mathrm{BC}$ was found equal to $216 \mathrm{MPa}$. Interestingly, the mechanical properties of $\mathrm{BC}$ were not adversely affected even after treatment with $0.1 \mathrm{M} \mathrm{NaOH}$ that used for purification. ${ }^{[58]}$ In addition, further purification with alkaline and/or oxidative solutions significantly improved the mechanical properties, where Young's modulus of the resulting sheets was $\sim 30 \mathrm{GPa}{ }^{[59]}$ Moreover, the mechanical properties of single BC nanofibers of diameters ranging from 35 to 90 $\mathrm{nm}$ have been measured using AFM nano-bending tests. In the same study, Young's modulus of the nanofibers was found to be considerably high and equal $78 \pm 17 \mathrm{GPa}^{[60]}$ In another study, elastic modulus of $114 \mathrm{GPa}$ was reported by Raman spectroscopy for single BC fiber. ${ }^{[61]}$ The outstanding mechanical properties of $\mathrm{BC}$ make it an attractive material for use in regeneration medicine of several types of tissue such as meniscus and blood vessels. Therefore, BC was produced in the form of tubes with a Young's modulus of $\sim 5 \mathrm{MPa}$ that can sustain a blood pressure of $250 \mathrm{mmHg}{ }^{[54]}$

\subsubsection{Bacterial Cellulose in Tissue-Engineering Scaffolds and Drug Delivery Applications}

The nanofibrous BC material has shown promise for tissue engineering scaffolds (TES). ${ }^{[62]}$ In this context, several researchers have already focused on designing and engineering ideal BCbased products for targeted applications. Figure 2.3 illustrates various promising biomedical applications of BC-based materials.

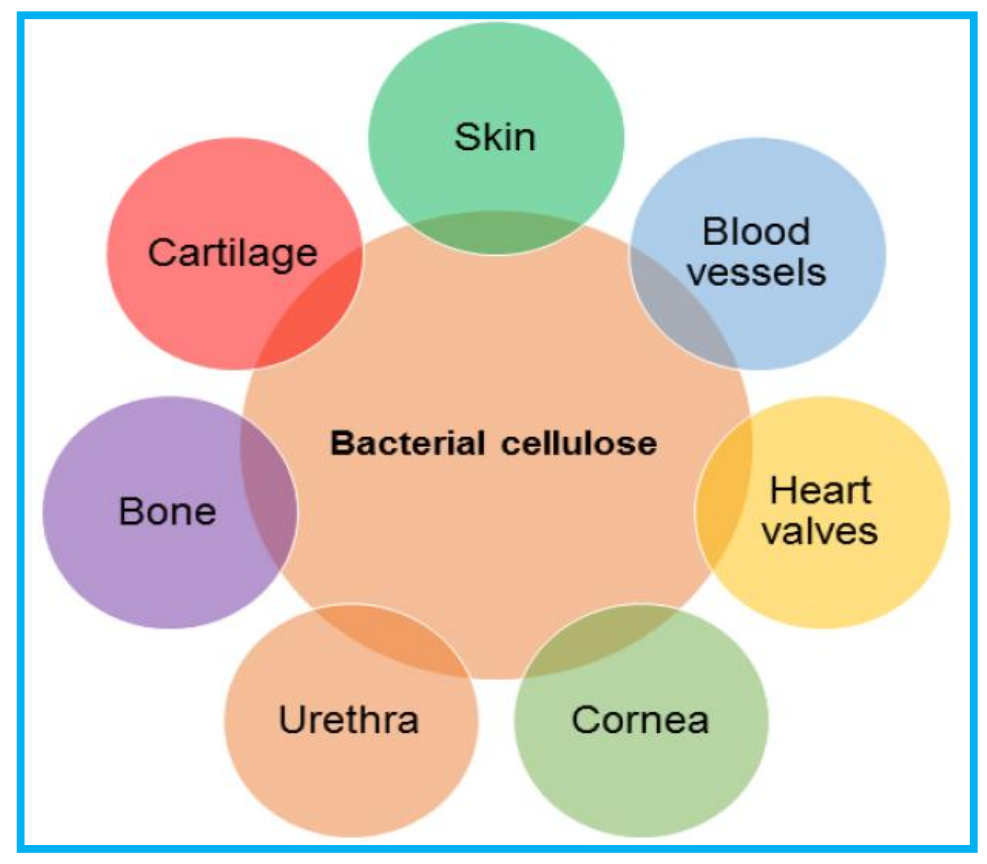

Figure 2.3 Prospects for the various biomedical applications of BC and BC-based materials. 
Due to its moist environment and bacterial barrier potential, BC is extensively used as a woundhealing device. Solway et al demonstrated the use of $\mathrm{BC}$ as an effective method for healing chronic diabetic foot ulcers. ${ }^{[63]}$ It showed a proven ability to reduce wound size, increases healing efficiency and creates less discomfort to the patient. For burn healing, it also shows remarkable conformability to various body contours and maintain moist environment that significantly reduce pain, Figure 2.4.
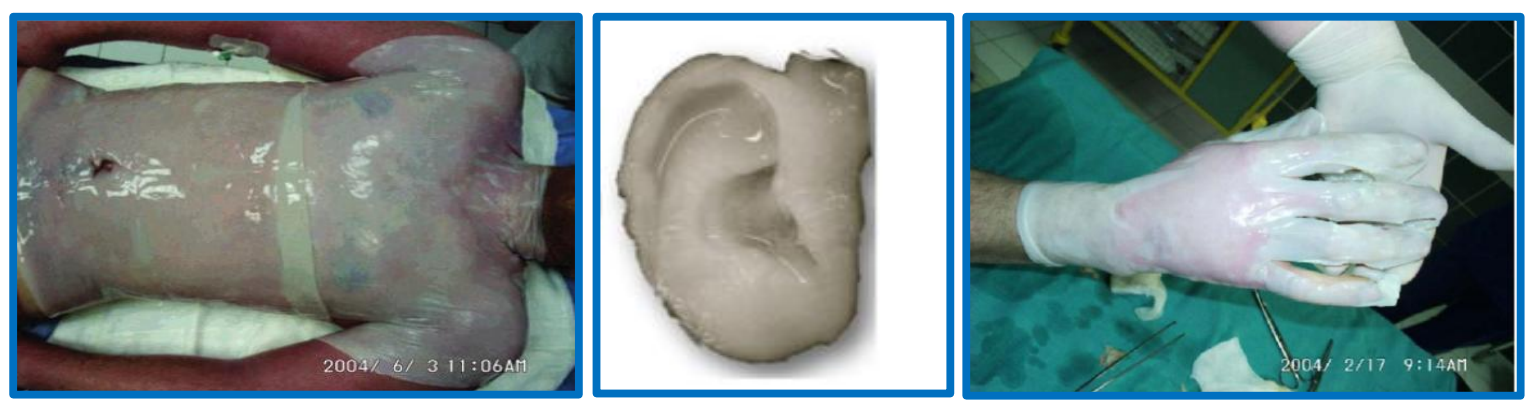

Figure 2.4 Different examples for substitutes made from never-dried BC membranes ${ }^{[64]}$

Table 2.1 compiles important biomedical applications for $\mathrm{BC}$ and/or BC-based materials that approved by FDA and already present in the market. ${ }^{\left[{ }^{[]}\right.}$

Table 2.1 Commercial products based on BC and/or BC based materials for biomedical applications currently available in market

\begin{tabular}{|l|l|l|}
\hline BC product & Medical Applications & Company/Agency \\
\hline Biofill $^{\circledR}$ & Temporary wound dressing & Robin goad \\
\hline Gengiflex $^{\circledR}$ & $\begin{array}{l}\text { Regeneration of periodontal tissues, guided } \\
\text { bone tissue regeneration }\end{array}$ & Biofill Products \\
\hline Securian & $\begin{array}{l}\text { TM } \\
\text { repair }\end{array}$ & Xylos corporation \\
\hline BASYC $^{\circledR}$ & $\begin{array}{l}\text { Artificial blood vessel, artery bypass, cuff } \\
\text { for nerve suturing }\end{array}$ & $\begin{array}{l}\text { Jenpolymer materials Ltd. \& } \\
\text { Co. }\end{array}$ \\
\hline Dermafill $^{\mathrm{TM}}$ & Wound dressing & Fibrocel \\
\hline $\begin{array}{l}\text { Mineral Trioxide } \\
\text { Aggregate }^{\circledR}\end{array}$ & Biocompatible implant & Xylos corporation \\
\hline Bioprocess $^{\mathrm{TM}}$ & Wound dressing & Biofill Produtos \\
\hline Cellulon $^{\circledR}$ & Binder, non-woven structures & CP Kelco \\
\hline
\end{tabular}




\section{$2.3 \beta$-Cyclodextrin $\beta C D$}

\subsubsection{Structure and Unique Properties}

Cyclodextrins (CDs) are a series of cyclic and non-reducing oligosaccharides composed of 6-8 $\mathrm{D}$-glucose units and named $\alpha-, \beta$-, and $\gamma-\mathrm{CD}$, respectively. The D-glucose units are covalently bonded together via $\alpha-1,4-$ glycosidic bonds to form doughnut-like structures with hydrophobic inner cavities and hydrophilic outer surfaces, ${ }^{[65]}$ (see Figure 2.5). CDs are synthesized by enzymatic degradation of starch by bacteria. In general, they considered biocompatible, do not elicit an immune response, and have low toxicities. ${ }^{[6]}$ Among them, $\beta C D$ is the most commercially attractive system due to the simplicity of synthesis, low water solubility, and availability and low cost. ${ }^{[67]}$ In addition, $\beta C D$ has ideal hydrophobic central cavity dimension that can make inclusion complexes (ICs) with different types of guest compounds such as, drugs and small organic molecules via non-covalent interactions.

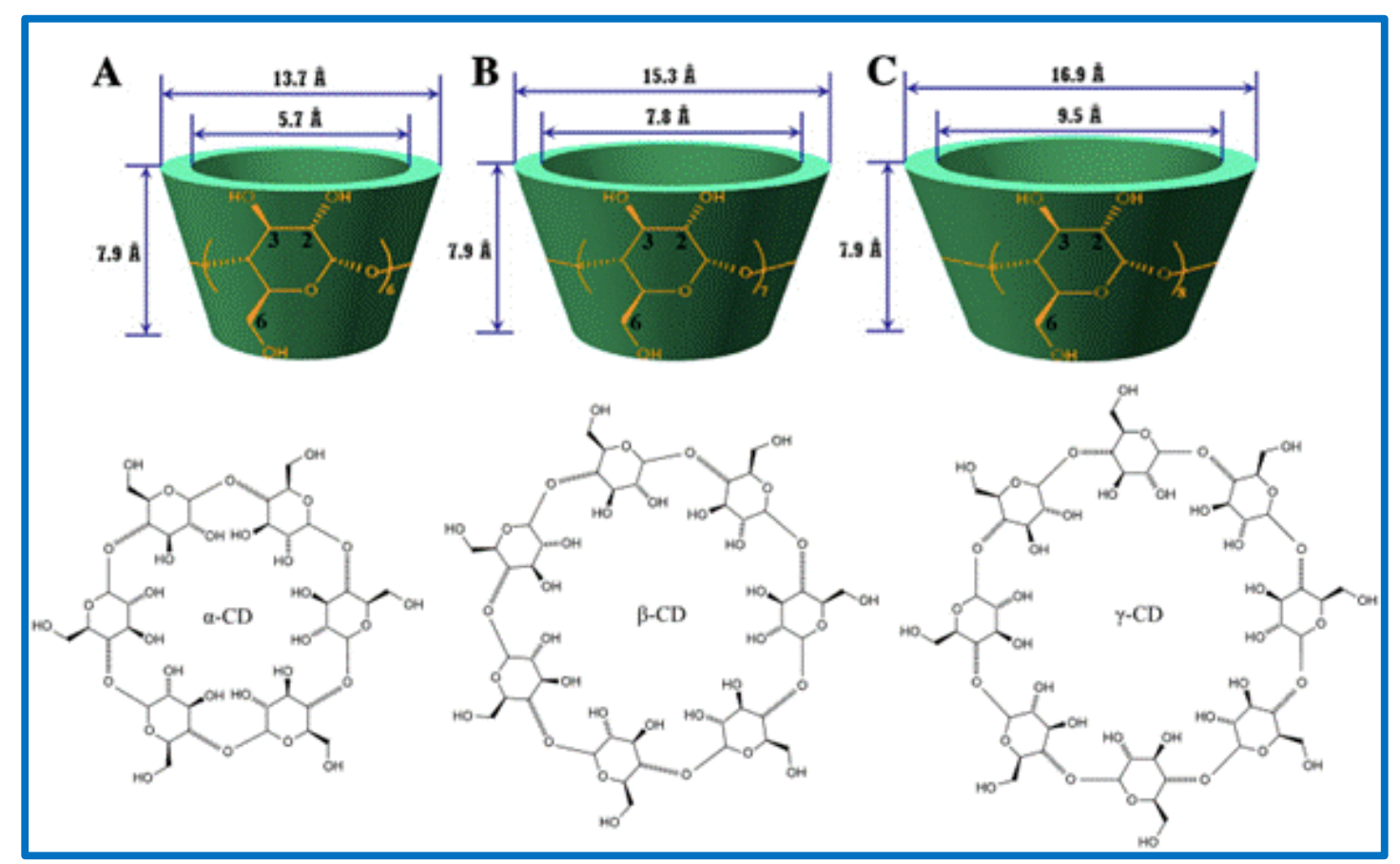

Figure 2.5 A, B and C are representing the chemical structures and cavity dimensions of $\alpha, \beta$ and $\gamma$ cyclodextrins, respectively.

\subsubsection{Applications of $\beta C D$}

Because of their interesting chemical and physical properties, $\beta C D$ have found great applications in many fields such as, pharmaceuticals, ${ }^{[68]}$ cosmetics, ${ }^{[69]}$ environmental protection ${ }^{[70]}$ and the 
food industry. ${ }^{[71]} \beta C D$ as environment-friendly auxiliaries, was grafted onto cellulose fibers to host antimicrobial agents that impart the fiber antimicrobial properties. ${ }^{[72]}$ The later became a promising technology for introducing functional agents into cellulose fibers. Where $\beta C D$ would be considered as macro container and readily form ICs with different types of guest compounds including small organic molecules as well as high and low molecular weight polymers. ${ }^{[73,74]}$ Most importantly, formation of ICs with drug molecules would improve their chemical stability, absorption, bioavailability and control their release. ${ }^{[70]}$

Inclusion complex between $\beta C D$ and adamantan is considered one of the well-known examples due to the near-perfect size match between $\beta C D$ cavity and adamantan molecule (AD). The interaction between them is extremely high with association constant of about $3 \times 10^{4} \mathrm{M}^{-1}{ }^{[75]} \mathrm{In}$ the same context, the later interaction has been employed by many researchers to form polymer networks and to assemble supramolecular polymeric compounds with various architectures. ${ }^{[74-77]}$ $\beta C D$ can be grafted onto macromolecules with crosslinkers to modify them for specific applications. A number of crosslinking agents have been utilized to graft, $\beta C D$ onto natural fibers, such as epichlorohydrin, ${ }^{[10]}$ cyanuric chloride, ${ }^{[78]} \mathrm{N}$-methylol acrylamide ${ }^{[79]}$ and polycarboxylic acids (e.g., citric acid). ${ }^{[80]}$ Although there have been several reports on the grafting of cellulose nanofibers with $\beta C D s,{ }^{[11,66,78]}$ a low grafting efficiency was achieved and hindered their uses. However, due to the larger specific surface area and unique physical properties of nano-sized cellulose, it can offer larger number of reactive surface -OH groups. ${ }^{[81]}$ The amount of $\beta C D$ s immobilized on cellulose nanofibers can reach up to $18 \%(\mathrm{w} / \mathrm{w}){ }^{[46,82]}$

\subsection{Poly( $\varepsilon$-caprolactone) PCL}

\subsubsection{Synthesis and Physicochemical Properties}

Poly( $\varepsilon$-caprolactone) (PCL) is one of the most widely studied biodegradable synthetic polyesters. PCL can be prepared by either ring-opening polymerization of the cyclic monomer $(\varepsilon-$ caprolactone) using variety of anionic, cationic and coordination catalysts or via free radical ring-opening polymerization of 2-methylene-1-3-dioxepane, ${ }^{[83]}$ (see Figure 2.6). PCL is hydrophobic, semi-crystalline polymer; its crystallinity tends to decrease with increasing molecular weight. PCL has degree of crystallization around $60 \%$, glass transition temperature $\left(\mathrm{T}_{\mathrm{g}}\right)$ around $-60{ }^{\circ} \mathrm{C}$ and melting point of about $\left(59-64^{\circ} \mathrm{C}\right) .{ }^{[30]}$ The number average molecular weight of PCL may generally vary from 3000 to 80,000 Da and can be graded according to 
molecular weight. ${ }^{[30]}$ Its homopolymeric repeating unit consists of five non-polar methylene groups and a single relatively polar ester group. This gives PCL unique properties like high miscibility with other polymers and the biodegradability that induced by the presence of hydrolytically unstable aliphatic-ester linkage. ${ }^{[84]} \mathrm{PCL}$ is soluble at room temperature in different solvents such as carbon tetrachloride, chloroform, dichloromethane, benzene, cyclohexanone and toluene. Nevertheless, it has low solubility in acetone, 2-butanone, ethyl acetate, DMF and acetonitrile, and insoluble in alcohol, petroleum ether and diethyl ether. ${ }^{[30]}$

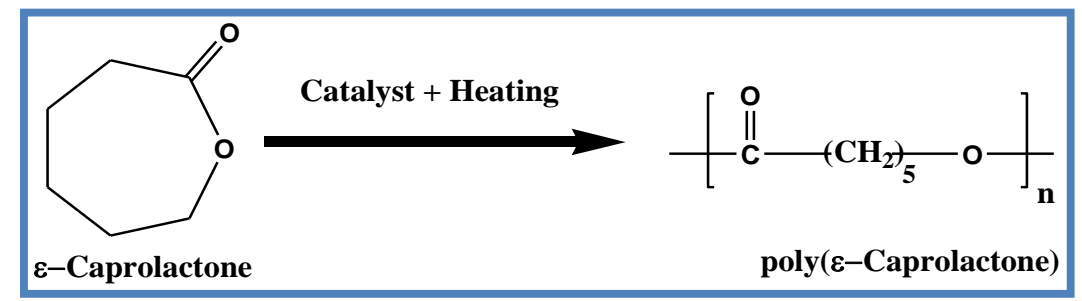

Figure 2.6 Ring opening polymerization of $\varepsilon$-caprolactone to polycaprolactone.

\subsubsection{Mechanical Properties of PCL}

Table 2.2 shows the tensile mechanical properties reported by several groups for bulk PCL processed by conventional methods. Bulk PCL shows tensile strength about 15-43 MPa and elastic modulus 250-460 MPa. ${ }^{[30]}$ However, PCL porous scaffold and fibrous scaffold showed lower value of tensile strength and elastic modulus due to porous structure. Table 2.3 lists some of the compressive properties reported in the literature of PCL scaffolds manufactured through solid freeform fabrication (SFF). ${ }^{[85]}$

Table 2.2 Bulk tensile mechanical properties that previously reported for PCL

\begin{tabular}{|l|c|c|c|c|}
\hline \multicolumn{1}{|c|}{$\begin{array}{c}\text { Manufacturing } \\
\text { Method }\end{array}$} & $\mathbf{M}_{\mathbf{n}}$ & $\mathbf{M}_{\mathbf{w}}$ & $\begin{array}{c}\text { Tensile Modulus } \\
(\mathbf{M P a})\end{array}$ & $\begin{array}{c}\text { Tensile Strength } \\
\text { (MPa) }\end{array}$ \\
\hline Injection Molding & 47,500 & 84,500 & 430 & N/A \\
\hline Melt Extruding & 50,900 & 84,500 & 264.8 & 19.3 \\
\hline Compression Molding & N/A & N/A & 340 & 21.6 \\
\hline Compression Molding & 45,000 & 50,400 & N/A & 16 \\
\hline Compression Molding & 42,500 & 72,500 & 400 & N/A \\
\hline Compression Molding & 50,500 & 101,000 & 251.9 & 16.9 \\
\hline Compression Molding & 50,000 & 80,000 & 429.1 & 27.3 \\
\hline Injection Molding & 64,000 & 124,000 & 378 & \\
\hline
\end{tabular}


Table 2.3 Compressive mechanical properties of PCL scaffolds manufactured through solid freeform fabrication (SFF)

\begin{tabular}{|l|c|c|c|c|c|}
\hline $\begin{array}{c}\text { Manufacturing } \\
\text { Method }\end{array}$ & $\begin{array}{c}\text { Compressive } \\
\text { Modulus (MPa) }\end{array}$ & $\begin{array}{c}\text { Compressive } \\
\text { Strength (MPa) }\end{array}$ & $\begin{array}{c}\text { Porosity } \\
(\mathbf{\%})\end{array}$ & $\mathbf{M}_{\mathbf{n}}$ & $\mathbf{M}_{\mathbf{w}}$ \\
\hline $\begin{array}{l}\text { Fused Deposition } \\
\text { Modeling (FDM) }\end{array}$ & 41.9 & 3.1 & 61 & 87343 & 146,739 \\
\hline FDM & $4-77$ & $0.4-3.6$ & $48-77$ & 87,000 & 147,700 \\
\hline FDM & 2.41 & 0.74 & 68 & 92,000 & 140,000 \\
\hline FDM & 4.8 & 2.2 & 65 & N/A & N/A \\
\hline $\begin{array}{l}\text { Precision Extruding } \\
\text { Deposition (PED) }\end{array}$ & $150-200$ & N/A & 53 & N/A & 44,000 \\
\hline PED & 59 & 5.3 & 65 & N/A & N/A \\
\hline PED & 109 & N/A & 0 & N/A & N/A \\
\hline PED & 21.0 & 1.3 & 70 & 65,000 & N/A \\
\hline $\begin{array}{l}\text { Selective Laser } \\
\text { Sintering (SLS) }\end{array}$ & $52-68$ & $2.0-3.2$ & $37-55$ & 50,000 & N/A \\
\hline
\end{tabular}

\subsubsection{Biodegradation of PCL}

Biodegradation (i.e. biotic degradation) is a chemical degradation of PCL provoked by the action of microorganisms that present in ecosystem such as bacteria, fungi and algae. ${ }^{[86]}$ Whereas in physiological conditions (such as in the human body), implantable PCL scaffolds are degraded by the hydrolysis of ester linkages. In particular, PCL have special interest for fabricating longterm implantable devices due to its slower degradation rate that polylactide. Long-term in vivo studies have demonstrated that the PCL implants were able to maintain the physical shape after 2 years of implantation. ${ }^{[26]}$ Additionally, PCL was also capable to degrade slowly into low molecular weight by the end of 30 months and can be excreted completely out of the body without accumulating non-toxic degradation byproducts. ${ }^{[26]}$ The most important factors that affect biodegradability are the molecular weight of polymer in implanted device and its degree of crystalinity. ${ }^{[87]}$ Where during the degradation process, the amorphous fraction of PCL degrades before the crystalline fraction because ester-linkages are easily hydrolyzed in amorphous domain. ${ }^{[8]}$ From degradation studies presented in the literature one can concluded that PCL degradation happens in a two-stage process. The first stage involves non enzymatic hydrolytic ester cleavage that autocatalyzed by carbon end groups of the polymer chains until the molecular weight is reduced to $3,000 \mathrm{~g} / \mathrm{mol}$. Whereas the second stage is enzymatic and starts with slower rate than the hydrolytic scission. ${ }^{[89]}$ The second degradation step was claimed to undergo intracellular as evidenced from the observed PCL fragments-uptake in the phagosomes of macrophages and giant cells and within fibroblasts. ${ }^{[90]}$ This in turn supports the fact that PCL is 
completely degraded and biosorbed via intracellular mechanism once the molecular weight was reduced to $3000 \mathrm{~g} / \mathrm{mol}$ or less. Figure 2.7 illustrates the mechanism by which PCL degrades hydrolytically. Hydrolysis intermediates 6-hydroxyl caproic acid and acetyl coenzyme-A are formed which in turn enter the citric acid cycle and are eliminated from the body. ${ }^{[1]}$

Researchers utilized two approaches to alter or accelerate the rate of hydrolysis of PCL; (i) by using low-molecular weight samples or (ii) by copolymerization with other lactones or glycolides/lactides. In that regard, intracellular degradation of low molecular weight PCL $\left(\mathrm{M}_{\mathrm{n}}=\right.$ $3000 \mathrm{~g} / \mathrm{mol}$; powders, 53-500 nm) was used. The authors reported rapid degradation and absorption within 13 days inside the phagosomes of macrophage and giant cells, and the sole metabolite was 6-hydroxyl caproic acid. ${ }^{[92]}$ In addition, copolymers of PCL with $\delta$-valerolactone and DL-lactide such as commercial suture (MONOCRYL, Ethicon) that shows higher degradation rates. ${ }^{[93]}$

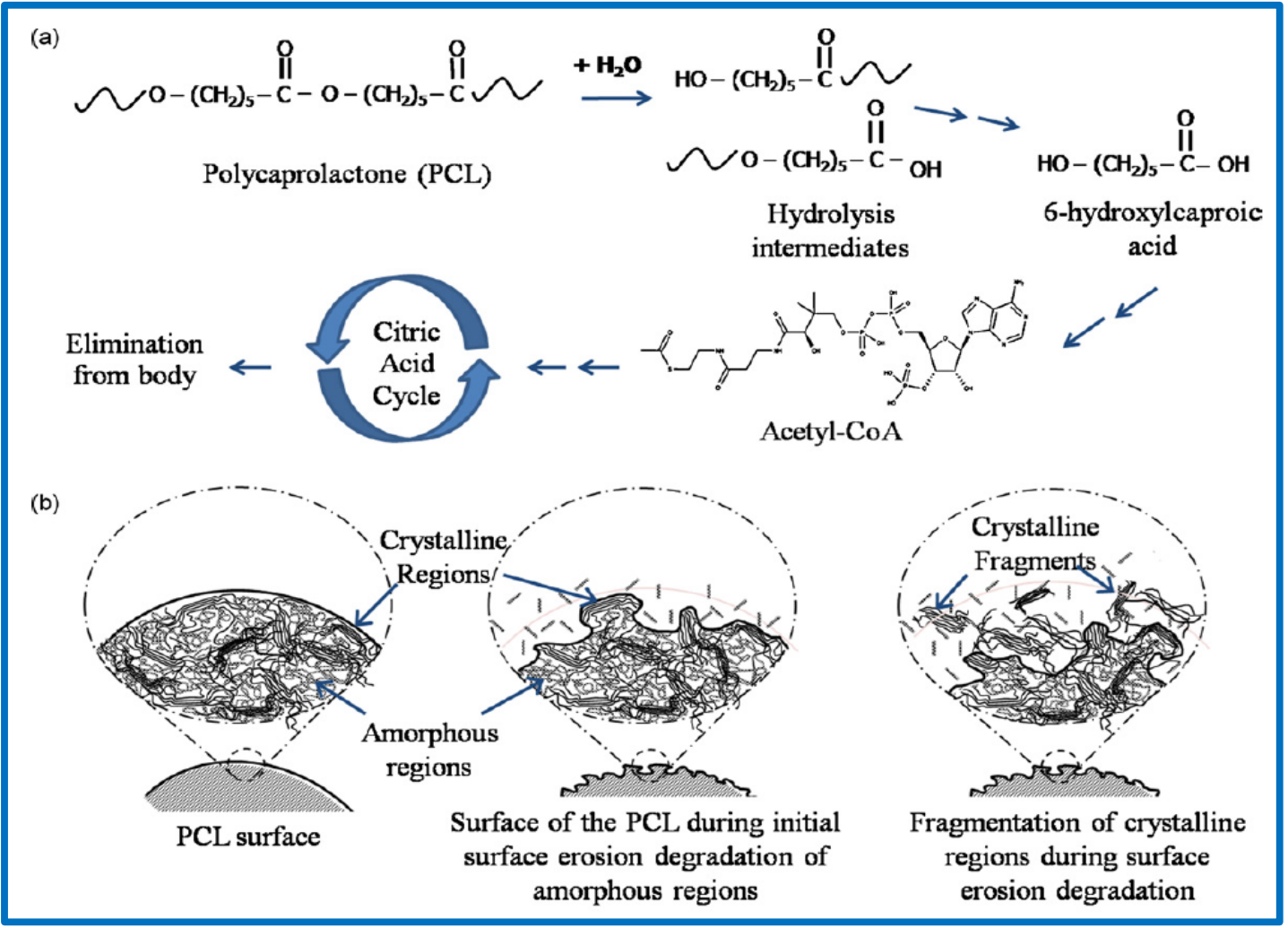

Figure 2.7 (a)The degradation of PCL via hydrolysis intermediates 6-hydroxyl caproic acid and acetyl coenzyme A, which are then eliminated from the body via the citric acid cycle. (b) Schematic visualization of how crystalline fragmentation could have taken place. ${ }^{[94]}$ 


\subsubsection{Biocompatibility of PCL and Cell Proliferation}

Biocompatibility is a factor that must be considered before the selection of biodegradable polymers to be used as permanent devices/implants or as drug-delivery systems. Originally, biocompatibility referred to the ability of a material to perform with an appropriated host response. ${ }^{[95]}$ In vitro, biocompatibility and cytotoxicity are generally evaluated through cell culture systems. In vivo histological and pathological examination of pre-implant PCL and the host responses (immunogenic, carcinogenic and thrombogenic responses) are also studied. ${ }^{[96]}$ However, most in vivo studies in the tissue-engineering field suffer from being prematurely ended in order to extract histological and/or biomechanical data before the PCL scaffold itself has been cleared from the implantation site. It is accepted that long-term in vivo data is costly to acquire. These studies compound the biocompatibility of the PCL and PCL-composites with no adverse biocompatibility effects found at short-term time points of 15 weeks up to long-term implantations of 2 years.

Tang et al ${ }^{[97]}$ proved that the different solvents used in fabricating PCL do not have an effect on the surface properties and biocompatibility (adhesion and proliferation) of the scaffolds. It was also reported that during the culture period, the growth of the cells in recombinant PCL composite scaffold were significantly higher than in the pure PCL such as in, spider silk proteinPCL-gelatin (pNSR32/PCL/Gt), ${ }^{[98]}$ PCL/silk fibroin (PCL/SF), ${ }^{[99]}$ PCL/biphasic calcium phosphate, ${ }^{[100]}$ and PCL/chitosan. ${ }^{[101]}$ In addition, endothelial cell growth has been improved on the PCL/PEG scaffold by modifying it with fibrin, fibronectin, gelatin, growth factors, and proteoglycans. ${ }^{[102]}$

\subsubsection{PCL in Tissue Engineering and Drug Delivery}

Scientific advances in biomaterials, stem cells, growth and differentiation factors, and biomimetic environments have created unique opportunities to fabricate tissues in the laboratory from combinations with engineered extracellular matrices scaffolds. However, the basic requirements needed for tissue engineering scaffold candidates should be fulfilled; (i) three dimensional and highly porous structures with an interconnected pore network for cell growth and flow transport of nutrients and metabolic waste, (ii) biocompatible and bioresorbable with a controllable degradation and resorption rate to match cell/tissue growth in vitro and/or in vivo, 
(iii) suitable surface chemistry for cell attachment, proliferation and differentiation, and (iv) mechanical properties to match those of the tissues at the site of implantation, ${ }^{[92]}$ see Figure 2.8 .

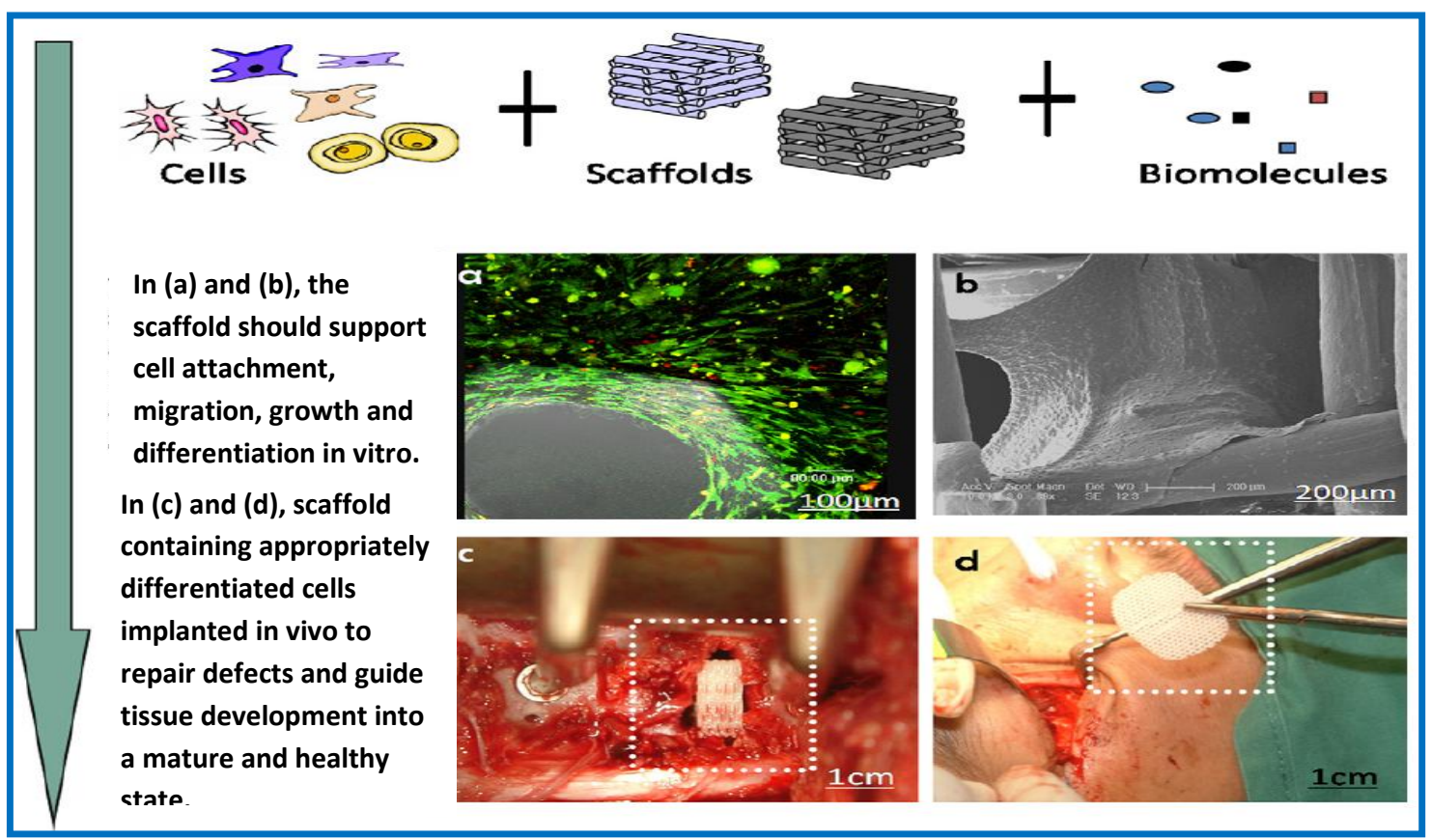

Figure 2.8 Scaffold-based tissue-engineering aims to promote the repair and/or regeneration of tissues through the incorporation of cells and/or biomolecules within a 3D scaffold system that can be maintained in vitro culture conditions until implantation. Reproduced from CRC Press"Handbook of fabrication and processing of biomaterials" (2008).

Amongst the different classes of biodegradable polymers, PCL has found widespread uses in biomedical arena, especially in the field of tissue engineering and drug delivery. Compared to PLA and PGAs, its incredible and versatile flexibility besides its superior rheological properties, bioresorbability, excellent biocompatibility, negligible tendency to generate an acidic environment during degradation made it attractive for TE scaffolds fabrication. It can be shaped by any polymer processing technology to fabricate enormous array of fibrous meshes and porous scaffolds (see Figure 2.9). Furthermore, PCL showed a slower degradation rate and higher degree of permeability toward low molecular weight drugs ( $<400 \mathrm{Da})$ rather than any other biodegradable polyesters. ${ }^{[24]}$ The later properties made it attractive candidate for fabricating long-term and diffusion-controlled drug delivery systems that remain active for more than 1 year. In fact, numerous drug-delivery devices fabricated from PCL were already approved by FDA and have CE mark registration. 


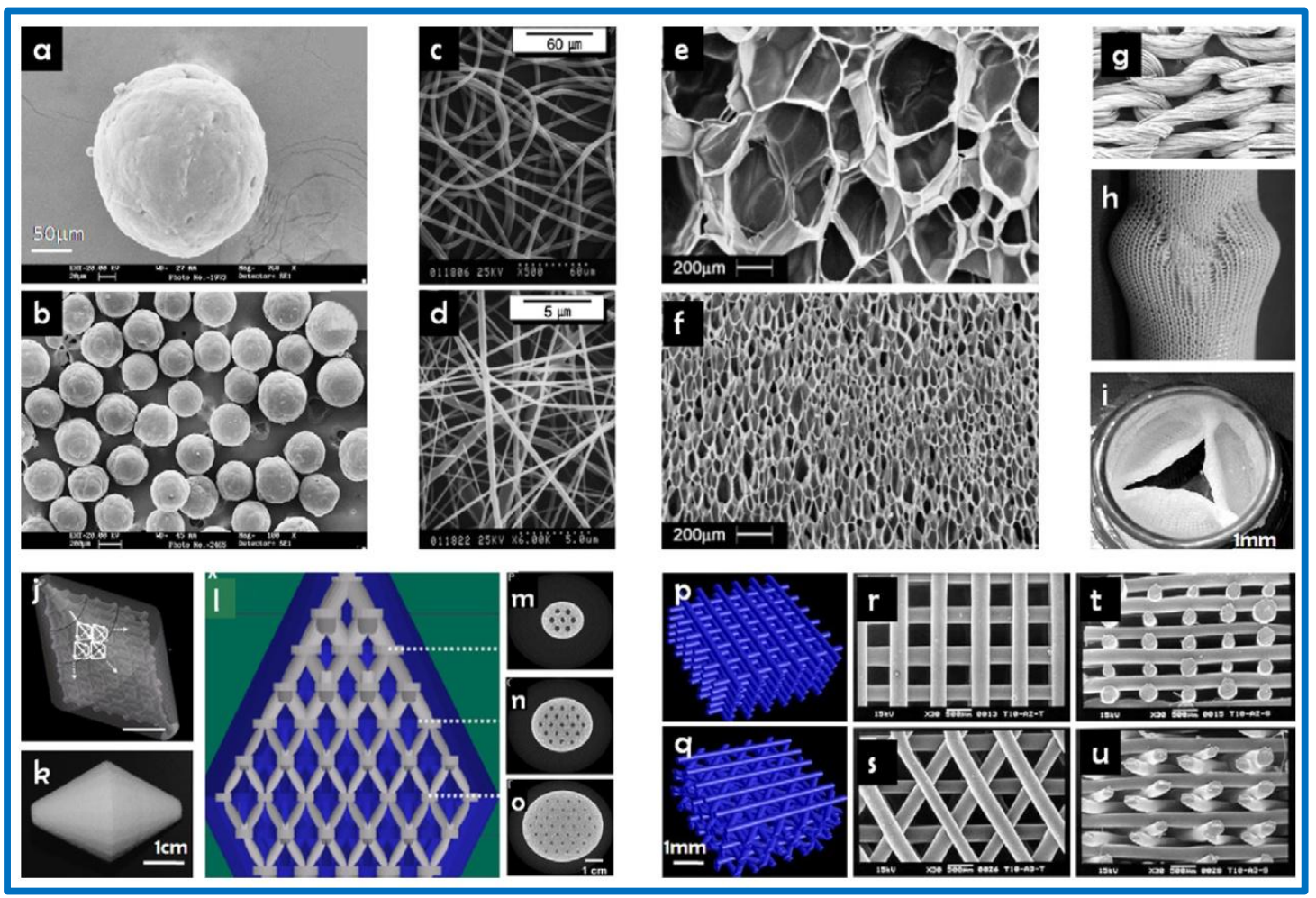

Figure 2.9 Structures made of PCL: nanospheres (a,b), nanofibers (c,d), foams (e,f), knitted textiles (g,h,i), selective laser sintered scaffold (j-o), and fused deposition molded scaffolds (pu). ${ }^{[103]}$

Due to its nontoxic nature, biodegradability and biocompatibility PCL has been gained significant popularity in the field of localized and targeted drug delivery. In the scope of the local drug delivery applications, PCL has been successfully tested as a vehicle for slow release of drugs at tumor reactions sites. ${ }^{[104]}$ Numerous drugs such as cisplatin, ${ }^{[105]}$ doxorubicin, ${ }^{[106]}$ and gentamicin sulfate, ${ }^{[107]}$ have been successfully incorporated into PCL in the form of nanoparticles. Table 2.4 lists the recent studies that investigating the fabrication and utilization of PCL scaffold for various TE and drug delivery applications. 
Table 2.4 Review of some studies investigated the fabrication of PCL scaffolds for use in various TE applications

\begin{tabular}{|c|c|}
\hline Brief method and outcomes & Ref. \\
\hline $\begin{array}{l}\text { Microporous, non-woven PCL scaffolds were made by electrospinning. Mesenchymal stem cells (MSCs) derived from the bone marrow } \\
\text { of rats were cultured, expanded and seeded on electrospun PCL scaffolds. }\end{array}$ & [108] \\
\hline $\begin{array}{l}\text { A highly porous, degradable PCL scaffold with an extracellular matrix-like topography was produced by electrospinning. Bone } \\
\text { formation from MSCs on a novel nanofibrous scaffold in a rat model was assessed. }\end{array}$ & [109] \\
\hline $\begin{array}{l}\text { A three-dimensional nanofibrous scaffold fabricated from PCL for its ability to support and maintain multiline age differentiation of } \\
\text { bone marrow-derived human mesenchymal stem cells (hMSCs) was tested. }\end{array}$ & [110] \\
\hline $\begin{array}{l}\text { To evaluate the repair potential in large osteochondral defects on high load-bearing sites, a hybrid scaffold system that comprised 3D } \\
\text { porous PCL scaffold for the cartilage component and tricalcium phosphate-reinforced PCL scaffold for the bone portion were fabricated. }\end{array}$ & [111] \\
\hline PCL/collagen nanofibers of different orientations for restoring large skeletal muscle tissue defects were produced and tested. & [112] \\
\hline $\begin{array}{l}\text { MSCs-seeded PCL nanofibrous scaffolds for cartilage repair using } 7 \mathrm{~mm} \text { full-thickness cartilage defects in a swine model were } \\
\text { evaluated. The approach showed promising and effective cartilage repair in vivo. }\end{array}$ & [113] \\
\hline $\begin{array}{l}\text { 3D microfibrous meshes of PCL as potential scaffolds for tissue engineering applications were developed. Cell culture experiments } \\
\text { employing MC3T3-E1 osteoblast like cells showed good cell viability, adhesion and collagen production on the PCL scaffolds. }\end{array}$ & [114] \\
\hline $\begin{array}{l}\text { The structural and functional properties of three-dimensional (3D) nerve guides fabricated from PCL using the air gap electrospinning } \\
\text { process was describe. }\end{array}$ & [115] \\
\hline $\begin{array}{l}\text { PCL nanofibers scaffolds were fabricated to include both } 10 \text { and } 20 \%(\mathrm{w} / \mathrm{w} \text { ) rifampicin (RIF). RIF release kinetics and bactericidal } \\
\text { efficacies of the scaffolds were evaluated and compared to RIF-free control scaffolds. }\end{array}$ & [116] \\
\hline $\begin{array}{l}\text { Porous PCL scaffolds serve as both targeted drug delivery system and attachment platform for tissue regeneration post limb salvage } \\
\text { procedure were fabricated and tested. In vitro drug release studies showed that rhodamine B was released in a slow and sustained manner } \\
\text { for } 112 \text { days. Cell attachment study demonstrated low affinity for the scaffolds to provide attachment platforms for tissue regeneration. }\end{array}$ & [117] \\
\hline $\begin{array}{l}\text { Hallow fibers were successfully manufactured from blends of PCL/PLGA by phase separation. These have adequate elongation } \\
\text { characteristics to be applied in small-caliber blood vessel regeneration. The PCL/PLGA }(85 / 15) \text { ratio yielded a miscible blend after } \\
\text { processing, whereas higher PLGA contents in the blend led to separation of the polymer phases. }\end{array}$ & [118] \\
\hline $\begin{array}{l}\text { PCL/PLA blend scaffolds are produced by novel biomanufacturing system called BioCell Printing. Scaffolds with } 0 / 90^{\circ} \text { architecture and } \\
350 \mu \mathrm{m} \text { of pore size were morphologically evaluated. Biological tests using osteosarcoma cell line G- } 63 \text { were performed and results } \\
\text { showed BioCell printing system produces scaffolds with regular and reproducible architecture, presenting no toxicity and enhancing cell } \\
\text { attachment and proliferation. }\end{array}$ & [119] \\
\hline $\begin{array}{l}\text { Porous scaffolds were produced by thermal crosslinking of PCL-diacrylate in the presence of hydroxyapatite (HA) and sodium chloride } \\
\text { porogen for bone TE. Results showed both the storage modulus (E') and Tag increased, while the loss factor (tan } \delta \text { ) decreased. In } \\
\text { addition, cytocompatibility of PCL/HA network scaffold indicated no toxicity and cells were attached and spread to the scaffold walls. }\end{array}$ & [120] \\
\hline $\begin{array}{l}\text { 3D composite scaffold composed of PCL backbone network and a poly(lactide-co-glycolide)-block-poly(ethyleneglycol)-block- } \\
\text { poly(lactide-co-glycolide) (PLGA-PEG-PLGA) thermogel surface was prepared for enhanced cartilage TE. 3D PCL/Thermogel } \\
\text { composite scaffold showed a greater number of cell retention and proliferation in comparison to the PCL platform. }\end{array}$ & [121] \\
\hline
\end{tabular}




\subsubsection{Challenges Facing PCL Scaffolds}

The wider commercial use of PCL as a commodity polymer in biomedical field is still constrained by its low melting temperature and low mechanical properties. In addition, the higher degree of crystalinity, non-osteoconductivity and hydrophobicity that decrease its biocompatibility with soft tissues and lowers its biodegradation. ${ }^{[29]}$ Nevertheless, the current fabrication techniques of drug-laden PCL nanoparticles, namely, emulsion solvent extraction/evaporation, phase separation, and spray drying, unfortunately could only achieve low drug loading efficiency and produce scaffolds with one sole function of controlled drug release. ${ }^{[122]}$

However, thermo-mechanical limitations of PCL were partially solved by researchers through the development of PCL-based nanocomposites. For instance, the addition of relatively small amounts of nanosized particles to PCL matrix lead to dramatical improvement of mechanical, thermal and heat distortion properties. Recently, efforts to increase the mechanical properties of PCL nanofibers have been reported by the addition of several synthetic nanofillers such as layered silicate (Montmorillonite) ${ }^{[123]}$ and carbon nanotubes (CNTs). ${ }^{[124]}$ Although the introduction of CNTs yielded composite with increased mechanical properties, manufacture protocols and special processing conditions made such types of composites quite costly. ${ }^{[125]}$ In addition, CNTs possess some degree of cytotoxicity toxicity in vivo applications predominately due to their sizes or their contamination with traces of transition metal catalysts that used in their production. ${ }^{[126]}$ The use of biological materials such as proteins from egg shells has also been reported as a reinforcing agent for PCL based nanofibers. ${ }^{[127]}$ Although the mechanical and interfacial properties of the composites were improved, the incorporation of soluble polymers in the PCL matrixes required special manufacturing protocols. However, due to renewability, biocompatibility and excellent thermo-mechanical properties of $\mathrm{CNCs}$, it considered as potential nanoreinforcing candidate for the preparation of bio-nanocomposites. ${ }^{[128]}$ Composites based on biodegradable PCL with both unmodified and chemically grafted CNCs derived from ramie fibers were successfully produced via the electrospinning technique. ${ }^{[129]}$ Results showed the reinforcement with $2.5 \%$ unmodified CNCs in PCL fiber webs induced minimal changes in the thermal behavior, but increased Young's modulus and the ultimate strength of $c a$. 1.5-fold compared to neat PCL webs. Results also showed that chemically grafted ramie-CNCs with short PCL chains was led to better compatibility between the hydrophobic PCL matrix and the hydrophilic CNC dispersed phases. Moreover, when short PCL chains were grafted onto the surface of CNCs, annealed febrile morphologies were obtained due to the differences in the crystallization rates 
of the high and low molecular weight PCL segments which impaired the crystalline properties of PCL matrix. ${ }^{[129]}$ Nanocomposites materials obtained from chitin whiskers and polycaprolactone were produced by evaporation, freeze drying and melt-compounding in a mini-extruder followed by hot pressing. As revealed from thermo gravimetric analysis, nanocomposites showed higher thermal stability than that of the pure PCL, the highest increase in Young's moduli was obtained for a chitin whisker content of $10 \mathrm{wt} \%$. Higher chitin whisker contents resulted in a more brittle failure behavior of the nanocomposites, which was accompanied by reduced tensile strength and elongation at break. However, meltcompounded specimens showed improved mechanical properties compared with those processed by evaporation and freeze drying techniques. ${ }^{[130]}$ In another study, PCL-BC biocomposites were prepared by melt-compounding using a mini twin-screw extruder. In this study, BC was utilized as reinforcing agent for PCL matrix in two different forms; (i) particulate $\mathrm{BC}(\mathrm{PBC})$, and fibrous $\mathrm{BC}$ (FBC). Mechanical testing reported that the addition of BC positively contributed to the Young's modulus of PCL by increasing it up to six times. However, FBC showed better homogeneous dispersion and higher composite yield stress and strain at break than PBC. Moreover, the addition of BC had a significant positive effect on the heat deflection temperature (HDT) of PCL, which was doubled with the addition of as little as 10 vol\% of BC. ${ }^{[3]}$

\subsubsection{Developing Porosity}

Cell adhesion and proliferation in scaffolds are a must and can be promoted by generating porosity within the $3 \mathrm{D}$ constructs. ${ }^{[131]}$ Porosity is induced in polymeric matrices using a variety of methods including electrospinning, freeze-drying, and solvent casting/salt leaching. ${ }^{[132]}$ However, the disadvantages of these techniques include the use of toxic organic solvent, formation of thin 2D structures, nonhomogenous and limited porosity, irregularly shaped pores, and insufficient pore interconnectivity. ${ }^{[132]}$ Gas foaming process has been widely employed to eliminate the problems associated with the use of these conventional methods for porosity generation. Porous structures of amorphous or semi-crystalline hydrophobic polymers such as poly(lactic) acid (PLA), PLGA, PCL, poly(methyl methacrylate) (PMMA) and polystyrene have been obtained using gas foaming technique. ${ }^{[133]}$ However, the formation of a non-porous external skin layer ${ }^{[134]}$ and lack of interconnectivity between pores ${ }^{[135]}$ are common issues in gas foaming technique. Gas foaming/salt leaching methods have been developed to address these issues, ${ }^{[136]}$ see Figure 2.10. For instance, Salerno et al. produced PCL foams with porosity in the range of 78-93\% and pore sizes 
between $10-90 \mu \mathrm{m}^{[136]}$ However, the gas foaming technique is not efficient for the creation of porosity in crystalline and hydrophilic polymers.

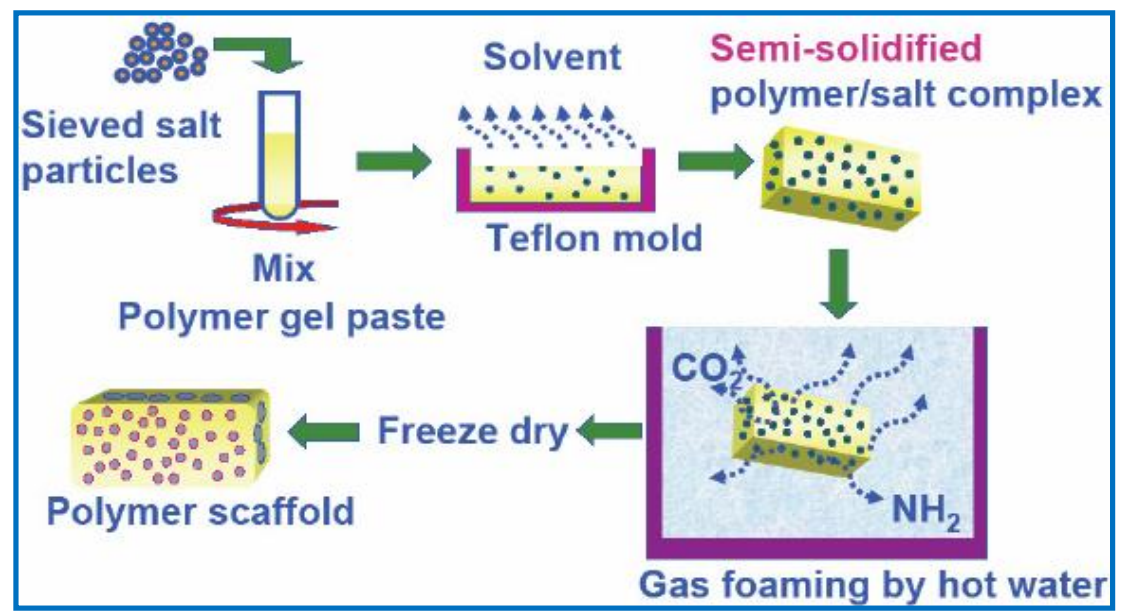

Figure 2.10 Developing porous structures by gas foaming/particulate leaching technique. ${ }^{[136]}$

\subsubsection{Drug-laden PCL Scaffolds}

The drug-laden PCL nanoparticles used in targeted drug delivery often possess no other function rather than to release drugs, while the regular 3D PCL scaffolds developed for TE usually contain no drug. The term tissue engineering therapeutics emerged to coined 3D scaffolds with additional drug delivery properties. ${ }^{[31]}$ To minimize systemic drug toxicities and to ensure adequate supply of the various therapeutic agents, targeted and controlled drug delivery systems were designed. By encapsulating drugs in micropolymeric or nanopolymeric structures, this allows drugs to remain at specific sites of action at sufficiently high concentration and to exert their pharmacological effects without causing severe systemic toxicities. Additionally, localized drug delivery also improves drug absorption and intracellular penetration, prolongs retention time, enhances drug efficacy, and reduces drug degradation. ${ }^{[33]}$

Numerous therapeutic agents, including growth factors, antibiotics, and anti-inflammatory, have been successfully loaded into three-dimensional bioactive scaffolds and demonstrated clinical potentials in the treatment and management of bone-related pathologies. ${ }^{[36]}$ Although PCL has demonstrated tremendous potentials in targeted drug delivery and tissue engineering, very limited studies were reported these two distinct combined functions. Recently, dual-function drug-laden PCL scaffolds of varying surface architecture were fabricated by microneedle arrays to serve as targeted drug delivery system and attachment platform for regenerated tissue following limb salvage procedure. However, results from in 
vitro drug release and cell attachment showed burst release at the beginning and followed by a slow drug release for about 112 days along with poor cell attachment ability. ${ }^{[28]}$

Doxorubicin is hydrophobic anticancer drug, sold under the trade names Adriamycin. It works by intercalating infected DNA strands, (see Figure 2.11). It widely used in the treatment of a wide range of cancers, including blood cancers, (like leukemia and lymphoma), many types of carcinoma (solid tumors) and soft tissue sarcomas. ${ }^{[137]}$ It is often used in combination chemotherapy as a component of various chemotherapy regimes. ${ }^{[138]}$ Doxorubicin is administered intravenously as a hydrochloride salt. Its common side effects include hair loss, nausea and vomiting, skin reactions (including hand-foot syndrome) and localized swelling and redness along the vein in which the drug is delivered. ${ }^{[139]}$
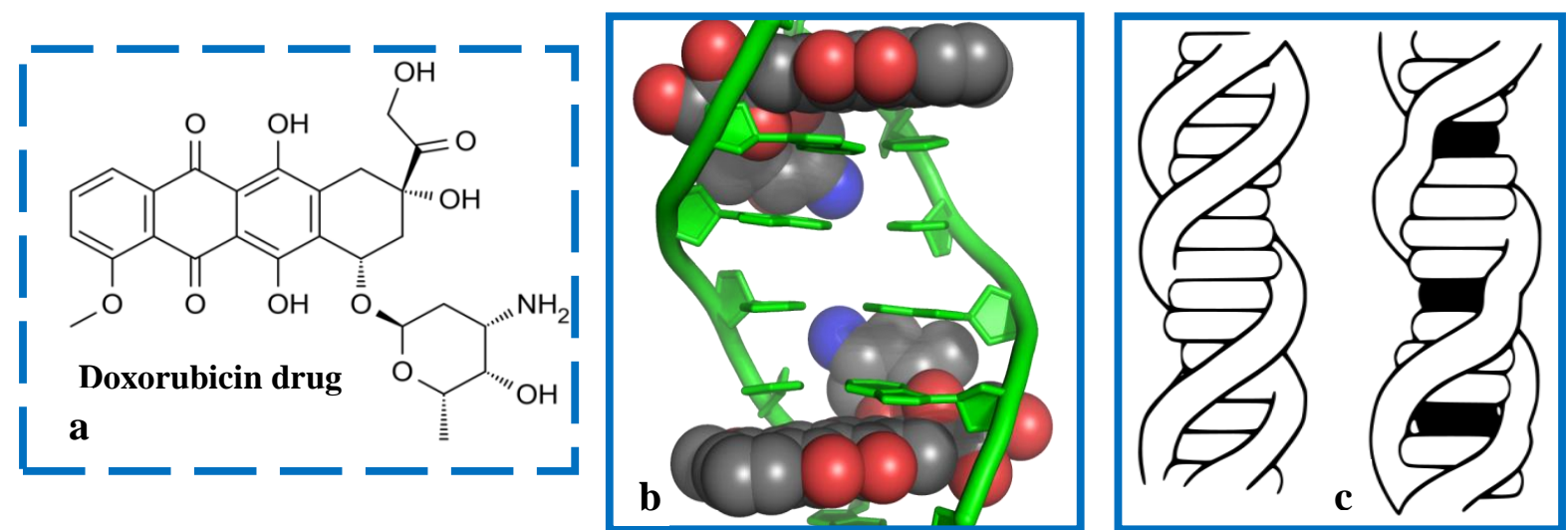

Figure 2.11 (a) Doxorubicin hydrophobic anticancer drug (b) Diagram of anticancer drugDNA complexes of two doxorubicin molecules intercalating DNA (c) Induced structural distortions due to intercalation; left: unchanged DNA strand; right: DNA strand intercalated at three locations (black areas) by Doxorubicin.

\subsubsection{Limb Salvage Procedure}

Limb salvage procedure is a type of surgery that involves local resection of bone or soft tissue cancers in order to avoid amputation. ${ }^{[140]}$ This procedure has proven to be effective in the treatment of osteosarcoma due to its superior ability to maintain the external appearances and physiological functions of the patients' limbs. ${ }^{[141]}$ Typically, the surgery involves the removal of tumors and some surrounding tissues, followed by the placement of either metal prosthesis or bone graft in the resected space to provide mechanical support and platform for new bone regeneration (see Figure 2.12). The use of metal prosthesis is constrained with numerous limitations, such as corrosion, ${ }^{[142]}$ metal hypersensitivity, ${ }^{143]}$ stress shielding, ${ }^{[144]}$ growth restriction, ${ }^{[145]}$ imaging interference, ${ }^{[146]}$ and the need for additional removal operation. ${ }^{[147]}$ The usage of bone grafts is also not ideal because allograft is often associated with nonunion, infection, disease transmission, and limited donor availability. ${ }^{[148]}$ While 
autografting can circumvent the problem of disease transmission and tissue incompatibility, limited by donor site morbidity, increased operative time, and chronic pain. ${ }^{[149]}$ Biodegradable polymer implants, therefore, emerged as viable alternatives for limb salvage procedure and have demonstrated promising results in bone regeneration of cranioplasty and bone defects. ${ }^{[150]}$

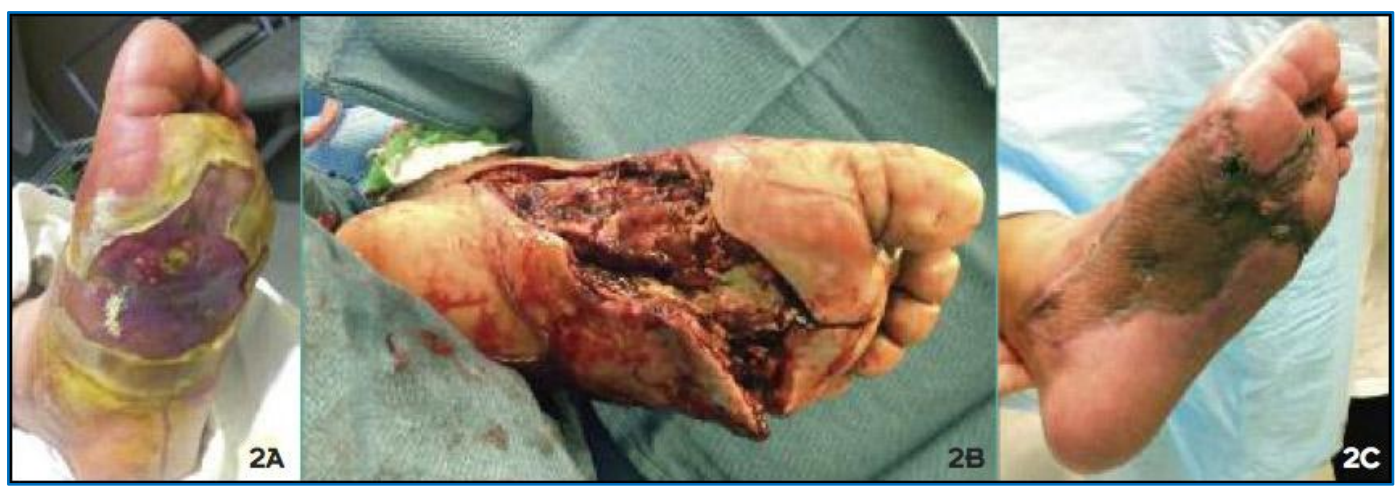

Figure 2.12 Showing limb salvage procedure. (Adapted from Photos courtesy of the Southern Arizona Limb Salvage Alliance (SALSA).

Following limb salvage procedure, chemotherapy is often prescribed to patients to destroy any lingering microscopic deposits of the malignant cells and reduce cancer recurrence. ${ }^{[151]}$ Although chemotherapy has proven to be highly effective in increasing the disease-free survival rate of localized osteosarcoma, ${ }^{[152]}$ conventional systemic chemotherapy is unfortunately highly toxic. ${ }^{[153]}$ While radiotherapy is generally considered safe and effective, it also possesses several disadvantages such as, soft tissue damage, increased pain, the need for additional orthopedic procedures, and also some bone cancer cells also do not respond well to radiotherapy. ${ }^{[154]}$ Moreover, one major complication following a limb salvage procedure is surgical wound and orthopedic device infections. ${ }^{[155]}$ Hence, oral or intravenous broad-spectrum antibiotics, opioids, mild narcotics, anti-inflammatory are regularly prescribed to control pain and to prevent or treat infections associated with limb salvage surgeries. ${ }^{[156]}$

Therefore, in view of the existing challenges and limitations confronting limb salvage surgery, a novel PCL drug-laden bionanocomposite scaffold is developed. The envisioned dual-function scaffold would serve as viable alternative of metal prostheses or bone grafts (promote bone and tissue regeneration) and simultaneously act as localized and controlled drug delivery system. Getting these two functions into one scaffold is a noble attempt to minimize undesirable systemic toxicities of chemotherapeutic drugs, ensure adequate drug supply, and reduces cancer patients' suffering from taking long-term medications. 


\subsection{Poly(3-hydroxybutyrate) P(3HB)}

$\mathrm{P}(3 \mathrm{HB})$ is the best known member of the polyhydroxyalkanoate family (PHA). It considered as energy storing material that synthesized and accumulated intracellulray by many microbial strains. ${ }^{[157]}$ It characterized by its strength, hydrophobicity, inertness, relatively high melting point, optical purity and thermoplastic process ability similar to those of polypropylene. ${ }^{[158]}$ The later properties made $\mathrm{P}(3 \mathrm{HB})$ an attractive green alternative to synthetic plastics. In addition, low molecular weight $\mathrm{P}(3 \mathrm{HB})$ and its degradation product $\mathrm{D}(-)$-3-hydroxybutyrate are common metabolite detected in humans plasma. ${ }^{[158]}$ This significant advantage of being completely biocompatible and biodegradable within the blood and tissue of mammals has further exploited the high-value of $\mathrm{P}(3 \mathrm{HB})$ for medical implants. $\mathrm{P}(3 \mathrm{HB})$ is currently used for absorbable suture, heart valves and cardiovascular tissue supports. ${ }^{[39]}$

Ralstonia eutropha ( $R$. eutropha), or Cupriavidus necator, is among the bacterial strains that have proven to assimilate sugar carbons to $\mathrm{P}(3 \mathrm{HB})$. R. eutropha has been the most commonly used strain for the industrial production of $\mathrm{P}(3 \mathrm{HB})$ from lignocellulosic biomass. It can metabolize sugar carbons obtained from the hydrolyzed lignocellulosic biomass such as glucose, xylose, and arabinose and accumulate $\mathrm{P}(3 \mathrm{HB})$ as discrete inclusions in cell cytoplasm whenever they exposed to stresses. ${ }^{[159]}$ These $\mathrm{P}(3 \mathrm{HB})$ granules, typically $0.2 \pm 0.5$ $\mu \mathrm{m}$ in diameter were visualized quite clearly with transmission electron microscopy or phase contrast light microscope due to their high refractivity, ${ }^{[160]}$ (Figure 2.13). P(3HB) accumulation to the level of approximately $90 \%$ of its dry cell weight has been reported. ${ }^{[40]}$ In $\mathrm{R}$. eutropha, two acetyl-CoA moieties are condensed to acetoacetyl-CoA by a $\beta$ ketothiolase (PhaA). The product then undergoes reduction by NADPH-dependent reductase (PhaB) which produces the (R)-isomer of 3-hydroxybutyryl-CoA, ${ }^{[161]}$ (Figure 2.14). Studies using R. eutropha, showed that the intracellular degradation of $\mathrm{P}(3 \mathrm{HB})$ inclusions is a very slow process, with rate degradation calculated to be about 10 times slower than the rate of its synthesis. ${ }^{[162]}$ Previous studies also showed the quantity of $\mathrm{P}(3 \mathrm{HB})$ accumulated increased as the carbon to nitrogen ratio increased. ${ }^{[163,164]}$ 


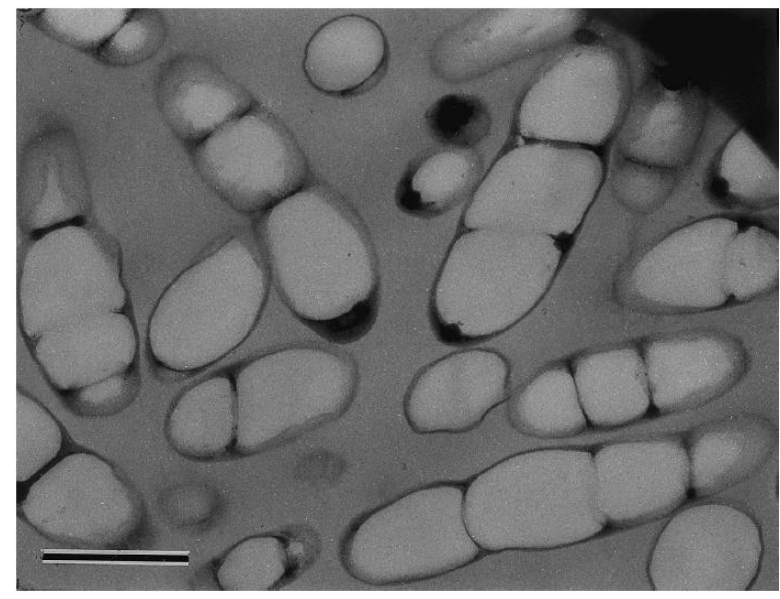

Figure 2.13 Transmission electron micrograph of thin sections of $R$. eutropha cells containing large amounts of $\mathrm{P}(3 \mathrm{HB})$ inclusions (90\% of the dry cell weight) appear as electron-dense bodies. Bar represents $0.5 \mu \mathrm{m} .^{[159]}$

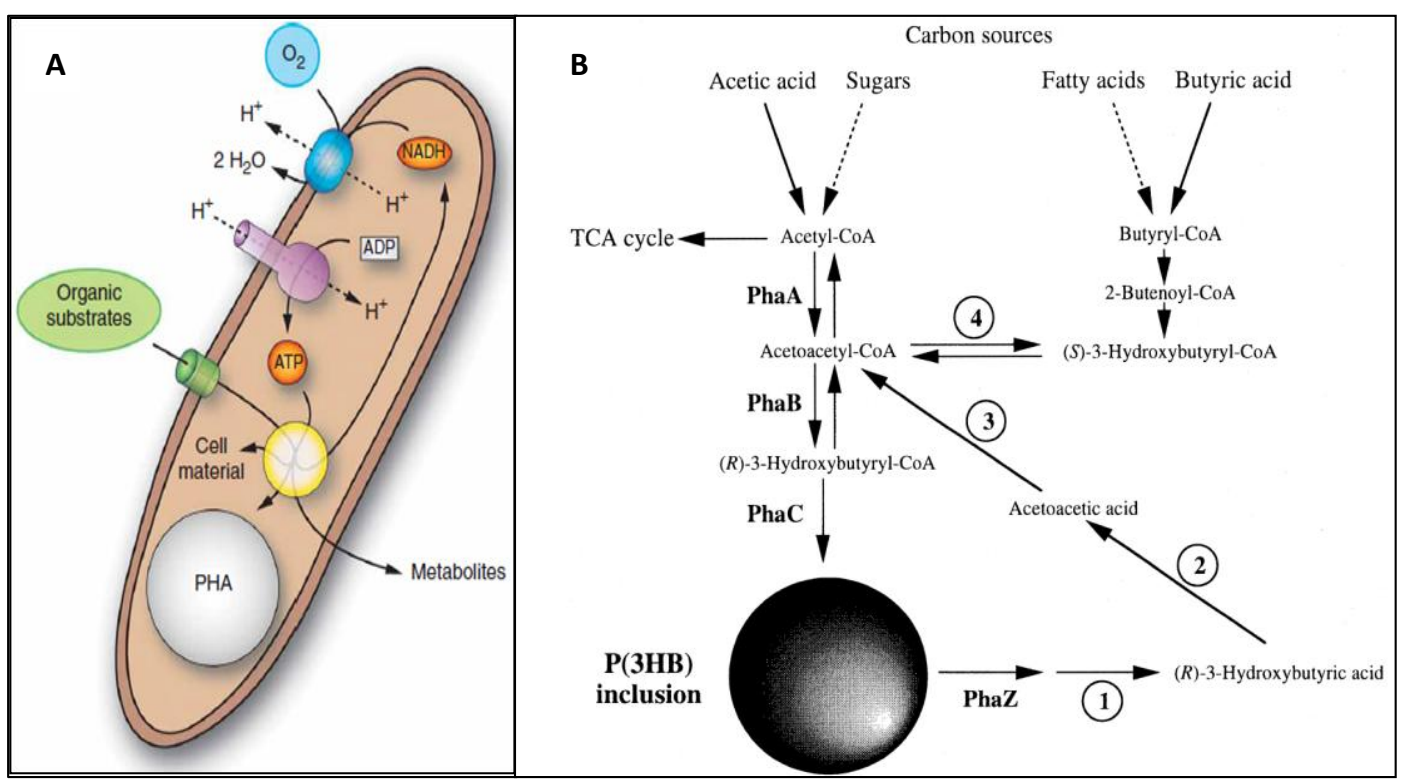

Figure $2.14 \mathrm{~A}$; Schematic representation illustrating the key aspects of heterotrophic metabolism of $R$. eutropha. The yellow circles represent the processes of central metabolism, whereas the gray circles indicate polyhydroxyalkanoate (PHA) storage granules. B; Cyclic metabolic nature of $\mathrm{P}(3 \mathrm{HB})$ biosynthesis and degradation in bacteria. PhaA, $\beta$-ketothiolase; PhaB, NADPH dependent acetoacetyl-CoA reductase; PhaC, PHA synthase; PhaZ, PHA depolymerase; 1, dimer hydrolase; 2, (R)-3-hydroxybutyrate dehydrogenase; 3, acetoacetylCoA synthetase; 4, NADH-dependent acetoacetyl-CoA reductase. ${ }^{[161]}$ 


\subsection{Cellulose Nanocomposites: Opportunities, Challenges and applications*}

Nanocellulose as renewable natural fibers has gained significant research interest and considered the prime and promising candidate for replacing synthetic fibers. It found enormous biomedical applications when combined with bio-based/renewable polymers. This chapter compiles four main sections. The first section briefly discussed the different sources and hierarchy of cellulose nanofibers, while the second section critically discussed the production of cellulosic nanoparticles, i.e. cellulose nanocrystals (CNCs) and microfibrillated cellulose (MFC), their self-assembly in solution and solid phase, their tensile and reinforcing properties, as well as surface modification done to assist their incorporation into different polymer matrices. The third section discussed the mechanical properties, the effect of processing methods on mechanical properties and displayed micromechanical modeling used for predicting their reinforcing potential in nanocomposites. In addition, this section also reviewed the up to date challenges and progresses that were accomplished by researchers to fabricate potential nanocellulose-based nanocomposites. Enormous biomedical applications of nanocellulose and its nanocomposites were listed and highlighted in the last section.

\subsubsection{Introduction}

"Back to Nature" is the present day mantra of the modern world. The current trend is to find out new materials based on natural substances. Increased environmental awareness and the current economic situations tempt the modern man to make use of natural fibers in developing new composite materials. Natural fibers are extracted from different resources, the fibers properties depend on their origin and the extraction methods used as well.

Cellulose fibers, the most abundant renewable and biodegradable polymer, are the promising feedstock owing to their applications in various industries. Annual production of natural cellulose is estimated to be of $10^{11}-10^{12}$ tons. ${ }^{[165]}$ The value of cellulose is also recognized as a versatile starting material for subsequent chemical transformation in the production of artificial cellulose based threads and films, as well as a variety of cellulose derivatives such as food, printing, cosmetic, oil well drilling, textile, pharmaceutical, domestic life and biomedical applications. ${ }^{[166]}$

\footnotetext{
*This chapter is adapted from a published book chapter "Mohamed M. Khattab, Noha A. Hady and Yaser Dahman. "Cellulose nanocomposites: opportunities, challenges, and applications." In Cellulose-Reinforced Nanofibre Composites: Production, Properties and Applications, edited by Mohammad Jawaid et al., Woodhead, Elsevier, 2017, pp.483-516. DOI:org/10.1016/B978-0-08100957-4.00021-8."
} 
Nowadays, researchers are increasingly acknowledging the importance of the use of natural cellulose nanofibers, not only because of their large surface area to volume ratio, but also due to their sustainability, biocompatibility, biodegradability, cheapness, environmental friendly nature, tendency to be recycled and their comparative mechanical properties. ${ }^{[167]}$ Moreover, special attention is given to their composites when they are mixed with natural and synthetic polymers to form nanocomposites with improved mechanical and physical properties if compared to the neat polymers. Those types of nanocomposites could address the propertyperformance gap between renewable and non-renewable petroleum based polymers (see Figure 2.15). They will likely become the next generation of novel eco-friendly nanocomposites. ${ }^{[168]}$

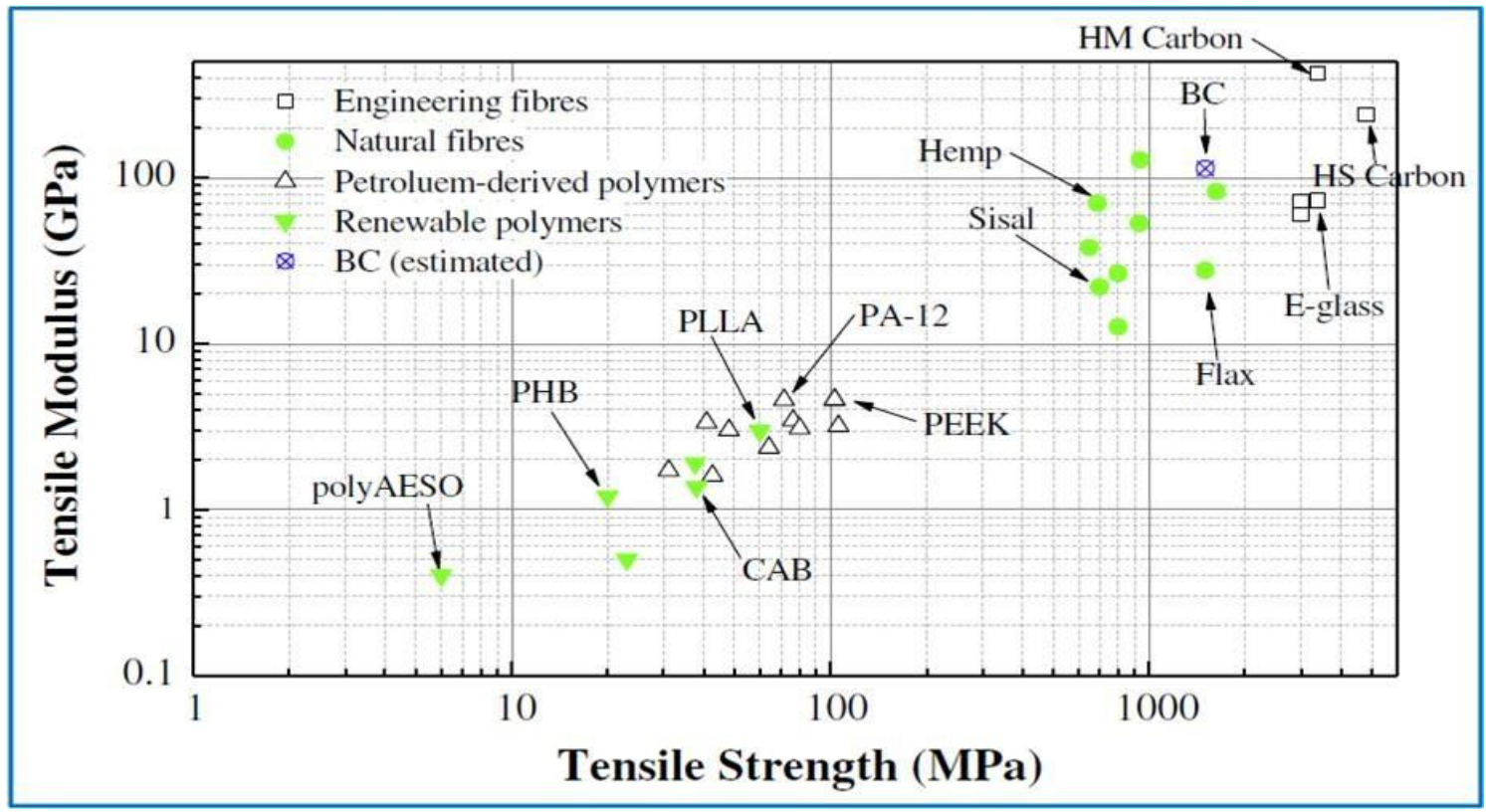

Figure 2.15 Comparison between average tensile properties of commonly used synthetic engineering fibers, renewable technical fibers, petroleum-based and renewable polymers, respectively. $\mathrm{BC}$ denotes bacterial cellulose. ${ }^{[128]}$

\subsubsection{Different Sources of Cellulose Nanofibers}

Cellulose is derived from a variety of sources, such as annual plants, animals and microbes. These include seed fiber (cotton), wood fibers (hardwoods and softwoods), bast fibers (flax, hemp, jute, ramie), grasses (bagasse, bamboo), simple marine animals, such as tunicates, which can also deposit cellulose in their cell walls, ${ }^{[169]}$ algae (Valonica ventricosa), and bacteria. The bacteria that secrete cellulose extracellulary belong to the genera Acetobacter, Agrobacterium, Alcaligenes, Pseudomonas, Rhizobium, and Sarcina. ${ }^{[170]}$ Acetobacter xylinum (A. xylinum) (or Gluconacetobacter xylinus) is the most efficient producer of bacterial 
cellulose. Typically, every single bacterium cell was able to convert up to 108 glucose molecule/h into cellulose. ${ }^{[48]}$ It is worth to mention that cellulose produced from plant serves as a structural material within the complex architecture of the plant cell walls. It is usually impure and contains hemicelluloses, and a relatively small amount of lignin. In wood, pure cellulose constitutes about 40-50\%; in leaf fibers: sisal fibers (55-73\%), in bast fibers: flax $70-75 \%$, hemp $75-80 \%$, jute $60-65 \%$, ramie $70-75 \%$, kenaf $47-57 \%$, in canes: bamboo $40-$ $55 \%$, baggase $33-45 \%$, and in cereal straw: barley $48 \%$, oat $44-53 \%$, rice $43-49 \%$, rye 50 $54 \%$, wheat $49-54 \%$, cotton seed hairs, the purest source, contain $90-99 \%$ of cellulose ${ }^{[171]}$ In comparison with plants cellulose, BC is usually produced in a relatively pure form. In addition, BC also has higher water holding capacity, higher degree of polymerization (up to $8,000 \mathrm{Da}$ ), and a finer web-like network, and therefore, no chemical treatments are needed to remove lignin and hemicelluloses, as is the case for plant cellulose. ${ }^{[172]}$

\subsubsection{Hierarchy of Cellulose Nanofibers}

Structurally, cellulose consists of a linear homo polysaccharide composed of $\beta$-Dglucopyranose units linked together by $\beta$ - $(1 \rightarrow 4)$-linkages. Each monomer unit bears three hydroxyl groups. Hence, it is obvious that these hydroxyl groups with their abilities to form hydrogen bonds are playing a very important role in directing the crystal packing structure and also controlling the physical properties of cellulose. ${ }^{[173]}$ Actually, the chains of poly- $\beta$ $(1 \rightarrow 4)$-D-glucosyl residues that formed during the biosynthesis are considered the basic structural component of cellulose. These chains are called microfibrils and have diameters ranging from 2 to $20 \mathrm{~nm} .^{[174]}$ These microfibrills ultimately aggregate to form fibrils. Fibrils are long thread-like bundles, laterally stabilized by intermolecular hydrogen bonds, ${ }^{[175]}$ as shown in Figure 2.16. Each microfibril is considered as a string of cellulose crystals linked together along the microfibril axis by disordered amorphous domains, e.g., twists and kinks. $^{[42]}$ Hydroxyl groups in equatorial positions of the glucopyranose moieties are responsible for the stabilization of structure and the increase its rigidity. As a result, an extensive intra and inter-molecular hydrogen bonding is formed and caused water insolubility. ${ }^{[176]}$ The degree of polymerization (DP) of native cellulose is approximately 10,000 in wood, 15,000 in cotton, ${ }^{[177]} 26,500$ in valonia fibers, ${ }^{[178]}$ and DP ranging from 20,000 to 14,000 in cotton fibers, depending on the plant part that analyzed. Bledzki and Gassan have reported that purification procedures usually lead to reduction in the DP, for instance, a DP of 14,000 in native cellulose can be reduced to about $2,500{ }^{[179]}$ 


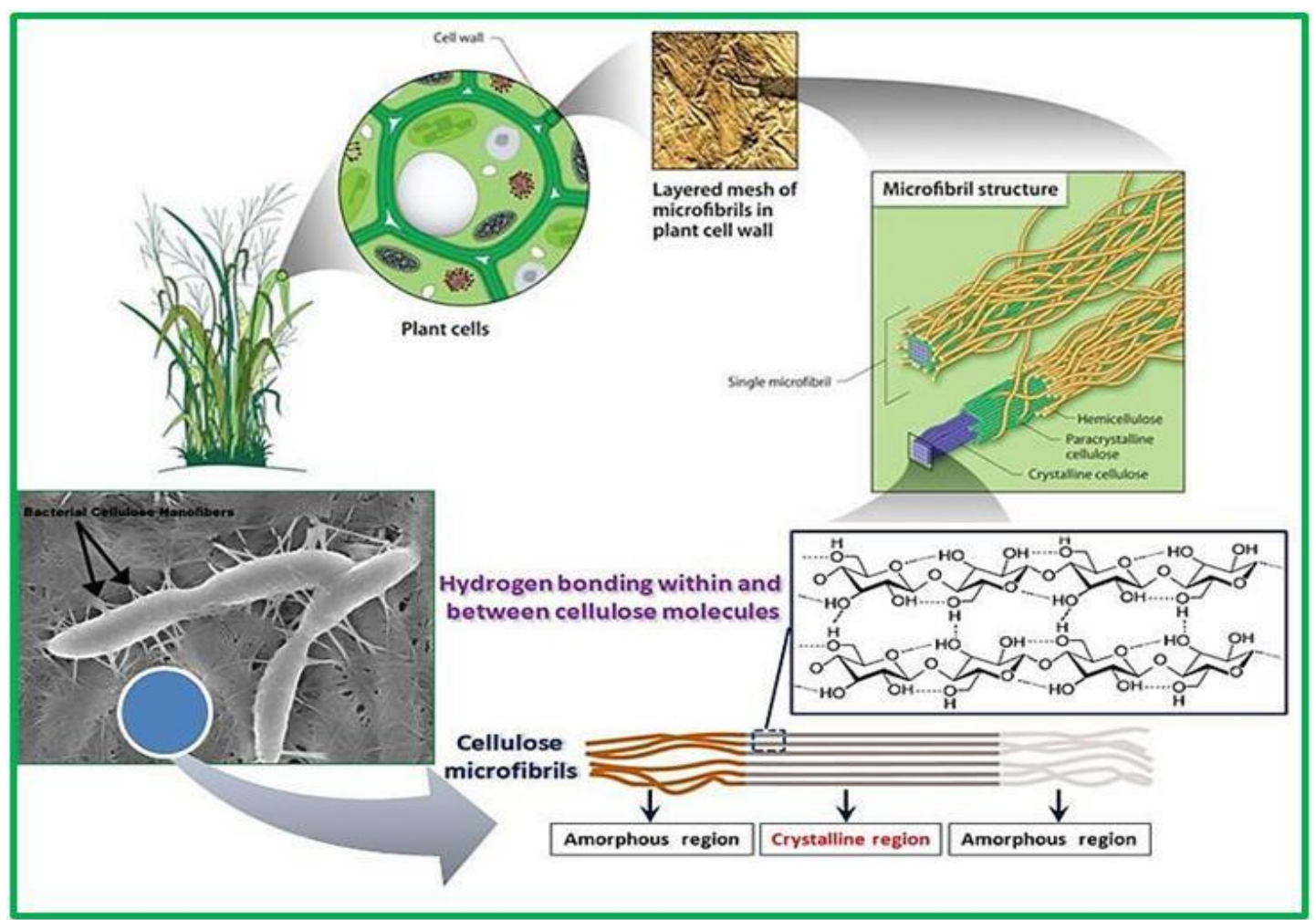

Figure 2.16 Scheme representing cellulose cell wall, bacterial cellulose production, microfibril organization and interaction between cellulose molecular chains within the crystalline region.

Two main polymorphs of cellulose are present; native cellulose or "cellulose I", and regenerated cellulose or "cellulose II". Cellulose I is crystalline and made up of parallel chains, ${ }^{[180]}$ whereas cellulose II is precipitated out of alkali solutions ${ }^{[181]}$ and has antiparallel chains. ${ }^{[182]}$ Thermodynamically, cellulose II is the most stable allomorph of cellulose due to the presence of additional hydrogen bond per glucose residue. ${ }^{[183]}$

The existence of two crystalline forms in native cellulose i.e. $\mathrm{I}_{\alpha}$ and $\mathrm{I}_{\beta}$, was first confirmed with $\mathrm{x}$-ray and NMR experiments, ${ }^{[184]}$ and then by electron diffraction and FTIR spectroscopies. ${ }^{[185]}$ They also claimed that the native cellulose is a mixture of cellulose $\mathrm{I}_{\alpha}$ and $I_{\beta}$ and some physical properties of cellulose fibers are based on the ratio of these two allomorphs. It is also reported by the same authors that the structural forms of $I_{\alpha}$ and $I_{\beta}$ are found within the same cellulose sample along a given microfibrill, and the metastable cellulose $\mathrm{I}_{\alpha}$ can be converted into the more stable $\mathrm{I}_{\beta}$ form by an annealing treatment. In these two allomorphs i.e. $I_{\alpha}$ and $I_{\beta}$, the conformation of the polysaccharide chains is similar, although the hydrogen-bonding pattern is different. ${ }^{[186]}$ The triclinic $I_{\alpha}$ allomorph is predominant in bacterial celluloses, while the monoclinic $\mathrm{I}_{\beta}$ form is the allomorph present in the plants cellulose (ramie and cotton). ${ }^{[187]}$ Nishiyama et al ${ }^{[188]}$ has reported that cellulose 
from tunicate (sea animal) consists of $\sim 90 \%$ of $\mathrm{I}_{\beta}$. On the contrary, cellulose from freshwater alga Glaucocystis sp. contains nearly $90 \%$ pure $\mathrm{I}_{\alpha}$ cellulose.

\subsubsection{Nanocellulose}

\subsubsection{Cellulose Nanoparticles: Terminology and Production}

There are two main categories of nanosized cellulosic particles. The first one is cellulose nanocrystals or nanowhisker, while the second one is called microfibrillated cellulose (MFC). Different terminologies have been used in literature to name these rod-like nanoparticles, what can lead to misunderstanding and ambiguities sometimes, e.g. microfibrils, microcrystals or microcrystallites, despite of their nanoscale dimensions. ${ }^{[174,187]}$ These terminologies, as well as raw cellulosic materials used for their production along with extraction processes, are summarized in Table 2.5. For obtaining cellulose whiskers or MFC from raw cellulosic materials, fibers are first milled and then submitted to alkaline treatments, followed by bleaching with $\mathrm{NaClO}_{2}$. During these steps, lignin and hemicelluloses were eliminated while cellulose moieties kept intact. The bleached fibers are further hydrolyzed or mechanically disintegrated at high pressure to obtain nanoparticles. Figure 2.17 presents the main steps involved in the preparation of cellulose whiskers and MFC.

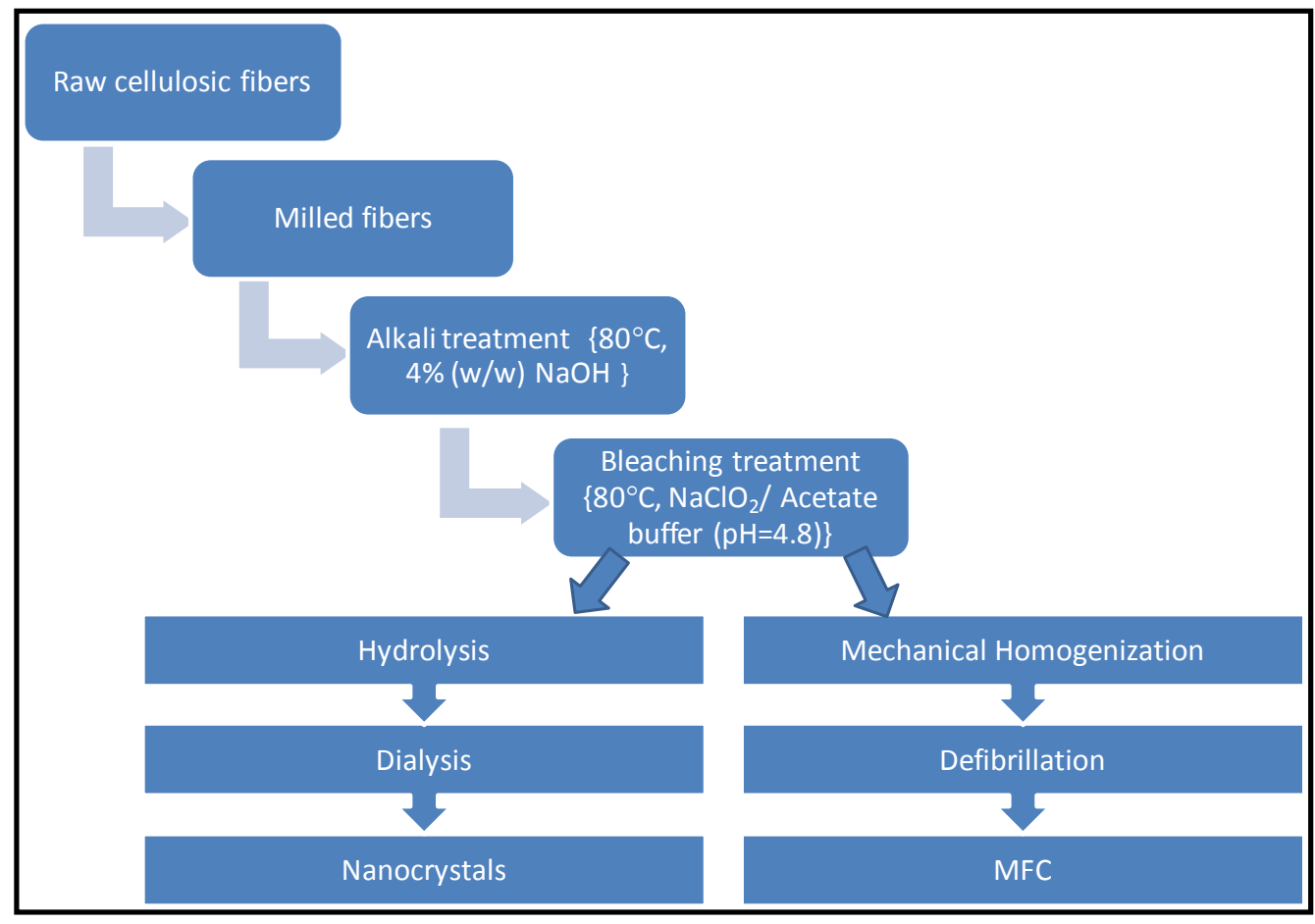

Figure 2.17 Summarizes the main steps involved in the preparation of cellulose nanoparticles. 


\subsubsection{Cellulose Nanowhisker}

The amorphous cellulose regions are randomly oriented and have lower density compared to crystalline regions. Therefore, they are more susceptible to acid attack and under controlled conditions and can be hydrolyzed, leaving behind the crystalline regions intact, Figure 2.18 (top). These resulting crystalline regions are rod-like shaped particles with dimension 5-20 $\mathrm{nm}$ in diameters and 50-500 $\mathrm{nm}$ in length and called cellulose nanocrystals (CNCs) or nanowhisker. ${ }^{[176,186]}$ Cellulose nanowhisker that produced from bacterial cellulose have larger dimension compared to those derived from plan source, since BC nanofibers have longer crystalline regions and lower fractions of amorphous region. ${ }^{[189]}$

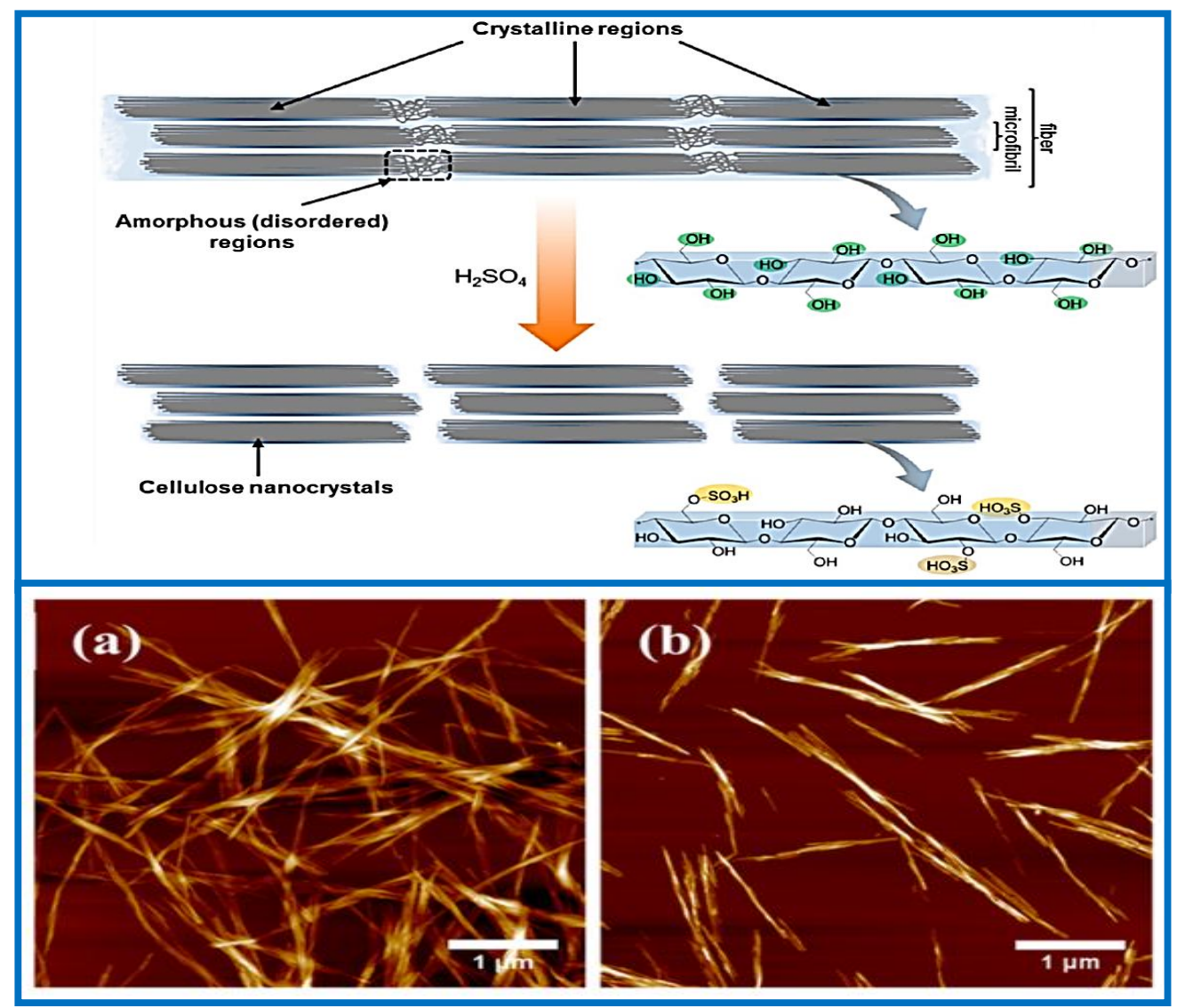

Figure 2.18 (Top) shows schematics presentation for acid hydrolysis of amorphous regions and the formation of CNCs. (Bottom) is showing AFM images of cellulose nanowhisker produced from $\mathrm{BC}$ by using (a) $\mathrm{HCl}$ hydrolysis and (b) $\mathrm{H}_{2} \mathrm{SO}_{4}$ hydrolysis. ${ }^{[190]}$

Typical methods used for the extraction of CNCs include harsh acid hydrolysis, enzymatic hydrolysis and ammonium persulfate oxidation. All methods have been researched and the obtained nanocrystals showed morphologies and properties different from native cellulose nanofibers. Atomic Force micrographs (AFM) showing cellulose nanocrystals are presented in Figure 2.18 (bottom a \& b). 
Table 2.5 Different terminologies used in literature to express cellulose nanoparticles during their production and extraction processes.

\begin{tabular}{|c|c|c|c|c|}
\hline Acronyms & Name & Source & Process & Ref. \\
\hline \multirow[t]{4}{*}{ CNW } & \multirow[t]{4}{*}{ Cellulose nanowhisker } & Ramie & $\mathrm{H}_{2} \mathrm{SO}_{4}$ hydrolysis & [191] \\
\hline & & $\mathrm{MCC}$ & $\mathrm{H}_{2} \mathrm{SO}_{4}$ hydrolysis & [192] \\
\hline & & Grass fiber & $\mathrm{H}_{2} \mathrm{SO}_{4}$ hydrolysis & [193] \\
\hline & & MCC & LiCl: DMAc & [194] \\
\hline \multirow{4}{*}{ CNXL } & \multirow{4}{*}{ Cellulose Nanocrystals } & Whatman filter paper & $\mathrm{H}_{2} \mathrm{SO}_{4}$ hydrolysis & [195] \\
\hline & & Bacterial cellulose & $\begin{array}{l}\mathrm{H}_{2} \mathrm{SO}_{4} \text { hydrolysis } \\
\mathrm{HCl} \text { hydrolysis } \\
\text { Enzymatic hydrolysis }\end{array}$ & $\begin{array}{l}{[196]} \\
{[197]} \\
{[198]} \\
\end{array}$ \\
\hline & & Cotton (cotton wool) & $\mathrm{H}_{2} \mathrm{SO}_{4}$ hydrolysis & [199] \\
\hline & & MCC & Sonication & [200] \\
\hline NF & Nanofibers & Wheat straw & $\mathrm{HCl}+$ Mechanical & [201] \\
\hline \multirow[t]{3}{*}{ MFC } & \multirow[t]{3}{*}{ Microfibrillated Cellulose } & Pulp Gaulin & Homogenizer & [202] \\
\hline & & Wood pulp & Acid hydrolysis + mechanical & [203] \\
\hline & & Wood pulp & Enzymatic + mechanical & [204] \\
\hline NFC & Nanofibrillated cellulose & Sulfite pulp & Mechanical & [205] \\
\hline MCC & Microcrystalline cellulose & $\begin{array}{l}\text { Alpha-cellulose fibers } \\
\text { Bleachead kenaf bast fiber }\end{array}$ & $\begin{array}{l}\mathrm{H}_{2} \mathrm{SO}_{4} \text { Hydrolysis } \\
\mathrm{HCl} \text { hydrolysis }\end{array}$ & $\begin{array}{l}{[206]} \\
{[207]}\end{array}$ \\
\hline \multirow[t]{2}{*}{$\mathrm{CNC}$} & \multirow[t]{2}{*}{ Cellulose nanocrystals } & \multirow[t]{2}{*}{ Cellulose fibers } & $\mathrm{H}_{3} \mathrm{PO}_{4}$ & [208] \\
\hline & & & $\mathrm{HBr}$ & [209] \\
\hline \multirow[t]{2}{*}{ NCC } & \multirow[t]{2}{*}{ Nanocrystalline cellulose } & $\mathrm{MCC}$ & $\mathrm{H}_{2} \mathrm{SO}_{4}$ hydrolysis & [210] \\
\hline & & Biomasses & Ammonium persulfate (APS) & [211] \\
\hline
\end{tabular}


Azizi Samir ${ }^{[42]}$ and Habibi ${ }^{[45]}$ described cellulose whiskers as nanofibers, which have been formed under controlled acidic hydrolysis. Beck-Candanedo ${ }^{[212]}$ studied the properties of cellulose nanocrystals obtained from soft and hard wood pulps by $\mathrm{H}_{2} \mathrm{SO}_{4}$ hydrolysis.

After studying the influence of hydrolysis time and acid-to-pulp ratio, it claimed that too long reaction times completely digest the cellulose nanofibers into its sugar molecules, whereas lower reaction times will only yield large aggregates of non-dispersed fibers. De Souza Lima described the penetration of hydronium ions throughout amorphous domains for promoting the hydrolysis of glycosidic bonds and releasing individual crystallites. ${ }^{[186]}$ Dong et al has studied the effect of hydrolysis conditions on the properties of resulting cellulose nanocrystals and reported that longer hydrolysis time leads to shorter nanocrystals with increased surface charge. ${ }^{\text {[213] }}$

Dufresne reported that the stability of nanocrystals suspensions depends on the dimensions, size, surface charge and the polydispersivity of dispersed particles. ${ }^{[187]}$ Beck-Candanedo reported that sulfuric acid-prepared nanocrystals have negative charge surface due to the esterification of surface hydroxyl groups to sulfate groups, which provide more stable aqueous suspensions due to repulsion. ${ }^{[212]}$ On the contrary, Corrêa ${ }^{[14]}$ reported that CNCs produced by hydrochloric acid hydrolysis have minimum surface charge and poor stability in solutions and tend to flocculate. However, it acquired higher crystalinity and thermal stability. Bondeson at al considered the necessity to optimize the hydrolysis condition in order to obtain large quantities of whiskers suspensions to be used as nanoreinforcement material in biopolymers. ${ }^{[214]}$ They investigated the effect of variation of sulfuric acid concentration, time and temperature of hydrolysis, as well as duration of the sonication step on microcrystalline cellulose (MCC) hydrolysis. They also reported that cellulose whiskers of length ranging between 200 and $400 \mathrm{~nm}$ were obtained by using $63.5 \mathrm{wt} \%$ sulfuric acid concentrations for approximately $2 \mathrm{~h}$ and with a yield of $30 \%$. Further studies were done by Elazzouzi-Hafraoui et al. ${ }^{[215]}$ They demonstrated the ability of forming CNCs with narrow size distribution by means of sulfuric acid hydrolysis followed by sonication. However, this sonication method usually provokes structural deformities as it generates an abundance of sheer stress on the fiber.

Moreover, it was also reported that sulfate groups might take up as much as $40 \%$ of the most reactive hydroxyl groups and make them nonreactive. ${ }^{[216]}$ The same group conducted different ways for $\mathrm{CNCs}$ desulfation. It was reported that CNCs desulfation under the condition of $2 \mathrm{M}$ $\mathrm{NaOH}$ at $65^{\circ} \mathrm{C}$ for 5 hours was effective. It was also indicated that alkaline-catalyzed desulfation 
of CNCs resulted in 3:6 and 2:3 anhydride structures. In another study for desulfation by using $\mathrm{HCl},{ }^{[217]}$ it was found that it has a drawback on the glycosidic linkages where polysaccharides are susceptible to acid hydrolysis and depolymerization may take place when treated with strong acids. Another limitation is reported by Pomin et al, ${ }^{[218]}$ where the desulfation process by using acids can be stereo-selective. Solvolytic desulfation that involves heating pyridinium salt of polysaccharide in a mixture of dimethyl sulfoxide and methanol was done by Jiang et al, ${ }^{[219]}$ (see Figure 2.19). This method resulted in a nearly complete desulfation within a single step. Liu et al was able to synthesize CNCs with little to no sulfate residues through sulfuric acid hydrolysis followed by 5 cycles of homogenization. ${ }^{[220]}$

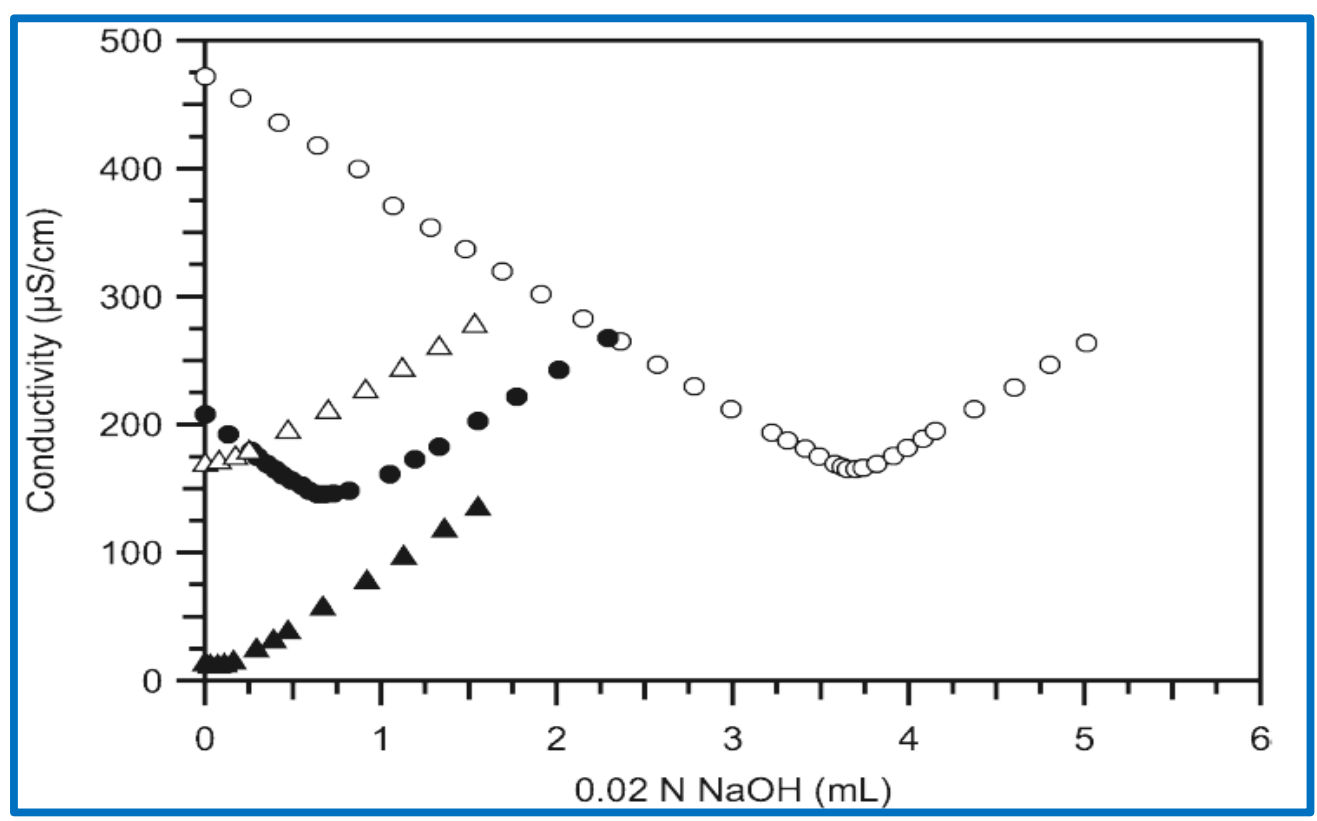

Figure 2.19 Conductometric titration curves for $\mathrm{H}_{2} \mathrm{SO}_{4}$-hydrolyzed $\mathrm{CNC}$ (open circle), $\mathrm{HCl}$ hydrolyzed CNC (open triangle), HCl-desulfated CNC-seventh step (solid circle), solvolytically desulfated CNC (solid triangle). ${ }^{[219]}$

Post-treatment of hydrochloric acid-generated CNCs by sulfuric acid has been studied by Araki et al to achieve controlled amounts of sulfate groups onto the $\mathrm{CNC}$ surface. ${ }^{[221]}$ A combination of sulfuric acid and hydrochloric acid during hydrolysis steps generates cellulose nanoparticles with spherical shapes instead of rod-like appearance, besides showing better thermal stability due to decreased amount of sulfate groups on the surface.

Hydrolysis using hydrobromic and phosphoric acids for the production of CNCs has also been reported. ${ }^{[208,209]}$ Those groups demonstrated that the resulting hydrolysis product is related to 
time, where the amorphous regions of the fiber are removed faster at the beginning of hydrolysis. They also observed that as time increases, the rate of hydrolysis decrease as the acid begins to attack the crystalline regions of the cellulose fiber. This leveling off period is highly discouraged as structural changes begin to occur. The highest yield obtained by this group was $70 \%$. Other claim was that the $30 \%$ loss could be attributed to the loss of amorphous groups during hydrolysis. It was also noticed that at a high $\mathrm{HBr}$ concentration (4M), discolouration occurred. This could be ascribed to dehydration due to harsh acid conditions.

Leung et al has prepared more homogeneous CNCs in one-step from biomasses by using ammonium persulfate (APS) as strong oxidant instead of acid hydrolysis. ${ }^{[211]}$ This versatile onepot procedure can process a variety of cellulosic biomass without the need for pretreatments to remove non-cellulosic plant contents. Compared with sulfonated CNCs that produced by using $\mathrm{H}_{2} \mathrm{SO}_{4}$, the use of APS resulted in formation of highly carboxylated CNCs. As such, highly carboxylated CNCs can be produced directly from this method without the need to undergo an additional step with (2,2,6,6-tetramethylpiperidin-1-yl)oxyl (TEMPO) oxidation that is often required to obtain highly carboxylated CNCs from that one produced by acid hydrolysis. ${ }^{[22]}$

George et al produced CNCs from BC fibers through an enzymatic process by treating it with commercially available cellulose (Trichoderma reesei ATCC26921). ${ }^{[198]}$ The resulting product had nearly two fold higher thermal stability than CNCs produced from the standard sulfuric acid hydrolysis. The obtained fibers length was in the range of 100-300 nm while its diameter was between 10-15 nm, which are typical dimensions for CNC. Compared to chemical hydrolysis routes, this enzymatic green method showed chemical and physical properties very similar to that of native cellulose fibers and displayed a more consistent production of "true" CNCs; however, further research still needed to validate this claim.

\subsubsection{Microfibrillated Cellulose (MFC)}

Herrick et al ${ }^{[223]}$ is the first group who reported the extraction of microfibrillated cellulose (MFC) from wood by mechanical disintegration process. This new type of cellulosic material was named microfibrillated cellulose (MFC) or 'nanofibrillar cellulose' (NFC). MFC can be viewed as a cellulosic material which are moderately degraded by a homogenization process and greatly expanded in surface area and volume. ${ }^{[22]}$ The isolation of MFC can be done by various mechanical techniques such as refining, grinding and high pressure homogenization. ${ }^{[225]}$ In these mechanical methods, a repeated homogenization treatment at pressure of 30,000 psi and cooling 
was used to maintain a product temperature in the range of $70-80{ }^{\circ} \mathrm{C} .{ }^{[226]}$ The final product was obtained as diluted dispersion of MFC, having a gel-like appearance, Figure 2.20.

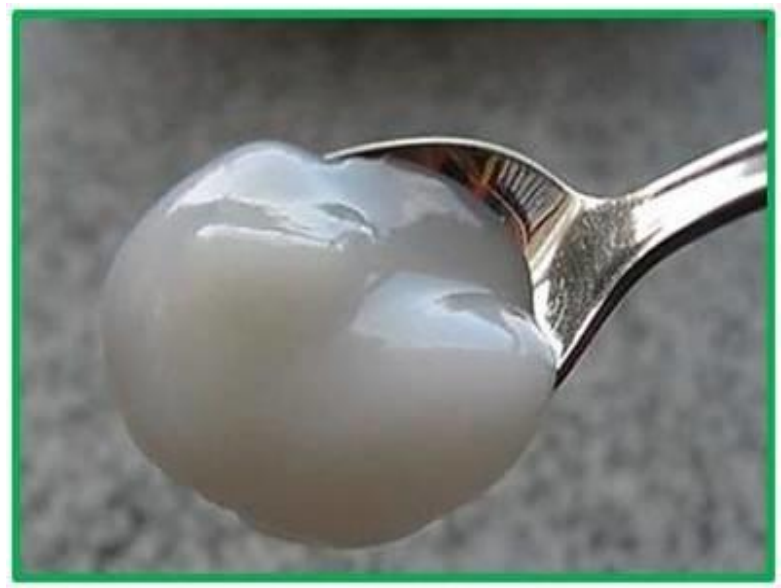

Figure 2.20 Microfibrillated cellulose (MFC) gel.

Contrary to straight cellulose whiskers, MFC is long and flexible nanoparticles and contain more or less individualized cellulose microfibrils with alternating crystalline and amorphous domains and forming web like structure. Its lateral dimensions are in the order of 10 to $100 \mathrm{~nm}$, and length is generally in the micrometer scale. ${ }^{[227]}$

High energy consumption is considered one of the main drawbacks that confront its production. Pretreatments have been developed by many researchers in order to solve the energy problem associated with the process. Zimmerman et al applied an acid hydrolysis as pre-step before pumping the pulp through the homogenizer. They claimed that sulfuric acid treatment combined with mechanical dispersion could be resulted in finer fibril structures than the ones obtained only by a mechanical treatment. They obtained fiber diameter below $50 \mathrm{~nm}$, but their lengths were still in the micrometer range. ${ }^{[203]}$

In another environment friendly treatment, a combination between enzymatic hydrolysis and mechanical shearing was done by Habibi. ${ }^{[228]}$ Results showed that MFC obtained by enzymatically pretreated pulps has better structures and higher aspect ratio than that produced by acid hydrolysis. Saito et al have proposed another process to obtain MFC based on TEMPO reaction and strong mixing where individual MFC was obtained after oxidation by TEMPO at room temperature and $500 \mathrm{rpm}$ stirring. ${ }^{[222]}$ They also claimed that optimal conditions were reached at pH 10 and giving cellulose nanofibers with 3-4 nm in width and a few microns in 
length. Ultrasonications, cryocrushing, high speed blending and steam expulsion are another new developed methods in their early stages. ${ }^{[228]}$

\subsubsection{Self-Assembly and Organization of CNC}

The presence of negatively charged sulfate groups on the surface of cellulose whiskers promotes a uniform dispersion of the whiskers in water via electrostatic repulsions. Therefore, CNC suspension is isotropic at very low concentration where particles are randomly oriented, and when the concentration increases to a critical value, the suspension becomes anisotropic with chiral nematic ordering, forming a liquid crystalline phase. ${ }^{[22]}$ With further increase in the CNC concentration, a shear birefringence phenomenon is observed, Figure 2.21. The explanation to this behavior was derived by analyzing the critical concentration of $\mathrm{CNC}$ as a function of its aspect ratio and charge density. Bordel et al reported that the application of AC electric or magnetic field on CNC suspension could introduce ordering of the nanoparticles. ${ }^{[230]}$ Habibi et al observed that application of $\mathrm{AC}$ field is resulted in alignment of CNC in suspensions. ${ }^{\text {[231] }}$ Additionally, it was observed that the film formed from aligned suspensions is more homogeneous with increasing electronic field strength. Pan et al conducted a similar study and reported the factors that control the chiral nematic property of CNC films. They found that the magnetic field did not influence the formation of chiral nematic phase, however it increased the chiral nematic pitch of the suspension. ${ }^{[232]}$

Due to the polyelectrolyte nature of CNC nanoparticles, they are highly sensitive towards electrolytes and counter ions, as well as polymers. Studies on the addition of electrolyte to CNC suspensions, and then monitoring the phase behavior have been conducted by using, for example, $\mathrm{NaCl}$ and $\mathrm{KCl}$, which found to significantly reduce the volume fraction of anisotropic phase. ${ }^{\text {[233] }}$ The types of counter ions and polymers in solution are also having influence on phase separation behavior of CNC suspension. ${ }^{[234]}$ It is also reported that addition of surfactants was improved the dispersability of CNC in non-polar solvents for applications in nanocomposites. With the addition of surfactants, a layer of surfactant coating was formed around CNC particles, but the chiral nematic phase was still observed. ${ }^{[235]}$

Sulfuric acid-hydrolyzed CNCs obtained from bacterial cellulose have been reported to spontaneously separate in a nematic phase. This phase separation event is preceded by a birefringent glass-like state that can persist for up to 7 days. However, adding a trace electrolyte 
$(<1 \mathrm{mM} \mathrm{NaCl})$ caused separation in 2 days and permitted the anisotropic phase to become chiral nematic. ${ }^{[236]}$

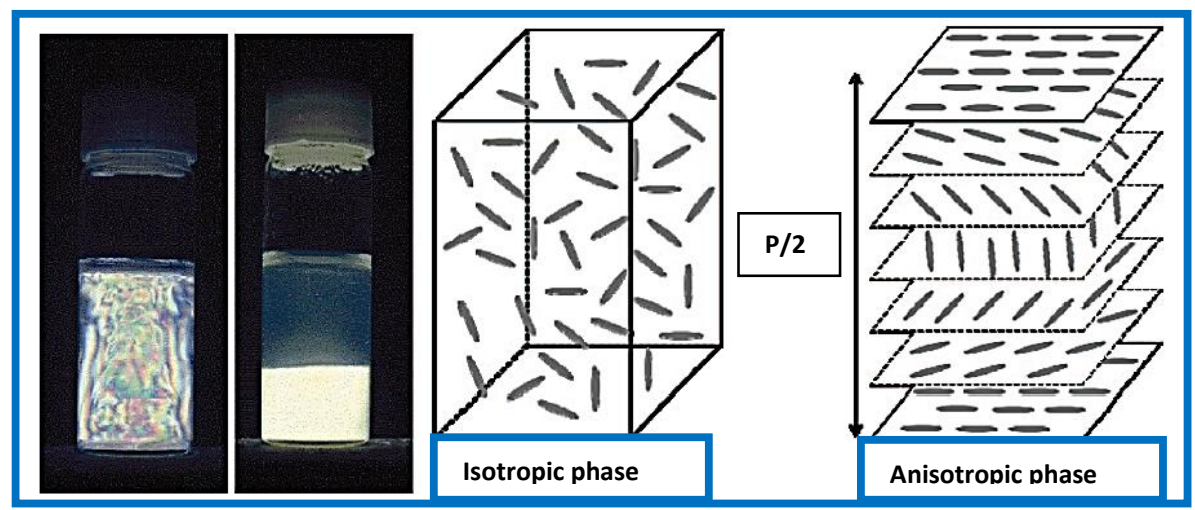

Figure 2.21 (Left) aqueous $0.63 \%$ (w/w) CNC suspension observed between crossed polarizer. Immediately after shearing, the suspension shows many iridescent birefringence patterns; after 1 week, the suspension separates into the upper isotropic and the lower anisotropic phases. (Right) schematic representation of $\mathrm{CNC}$ orientation in both isotropic and anisotropic (chiral nematic) phases. $^{[236]}$

The nature and density of the charges on the surface of CNCs have also been reported to affect the formation of the chiral nematic phase. It was reported distinctly different behaviors for using post-sulfated $\mathrm{HCl}$-hydrolyzed CNCs, which have sulfur content approximately one-third of the directly $\mathrm{H}_{2} \mathrm{SO}_{4}$-hydrolyzed CNCs. Interestingly, a high viscosity suspension of post sulfated CNCs does not yield a chiral nematic phase most likely due to its low charge content. ${ }^{[221]}$

Self-Assembly and organization of CNCs in organic medium was first investigated by Heux et $\mathrm{al},{ }^{[237]}$ and then detailed examination of the structure of this chiral nematic phase has been reported by Elazzouzi-Hafraoui et al. ${ }^{[215]}$ They studied the correlation of the aspect ratio of CNCs extracted from cotton fibers and their dispersion in nonpolar solvent such as cyclohexane. They reported that the critical concentration in which spontaneous phase separation was changed into a chiral nematic phase was higher than that in water. In addition, suspensions prepared with CNCs having high aspect ratios did not show any phase separation, but instead, produced an anisotropic gel phase at a high concentration. When $\mathrm{CNC}$ dispersions are casted on a solid support to prepare solid films the particles can maintain their alignment to form iridescent structures with chiral nematic ordering. ${ }^{[238]}$ The films reflect polarized light on a wavelength determined by the pitch 
of the liquid crystals. A recent approach reports the effect of sonication treatment to change the chiral nematic pitch in CNC suspensions and the resultant dry films. ${ }^{[239]}$

\subsubsection{Tensile Properties of Nanocellulose and Their Nano-reinforcement Potentials}

The mechanical properties of cellulose nanoparticles are characterized in terms of the properties of both ordered (crystalline) and disordered (amorphous) regions constituting the nanoparticle. The disordered regions contribute to the flexibility and plasticity of the bulk material and the ordered regions contribute to the stiffness and elasticity of the material. The modulus of different types of nanocellulose is expected to result from the mixing rule between the modulus of the crystalline domains and the amorphous fraction. Therefore, the stiffness and modulus of CNC with more crystalline regions should have higher values than pristine fibers that have both crystalline and amorphous structures. ${ }^{[240]}$

The elastic modulus of crystalline cellulose was investigated either by theoretical evaluations or by experimental measurements, e.g. wave propagation, X-ray diffraction, Raman spectroscopy, and atomic force microscopy. A broad range of values was reported, and it is generally accepted that the Young's modulus of crystalline cellulose (assimilated to the one of CNC) should be in the range 100-200 GPa. The elastic modulus of crystalline cellulose was also investigated based on nanoscale indentation and reported as $139.5 \pm 3.5 \mathrm{GPa}$ (similar to Kevlar). ${ }^{[16]}$ In another study, Dri et al computed the Young's modulus of crystalline cellulose using quantum mechanics; the predicted modulus of crystalline cellulose was found to be as high as $206 \mathrm{GPa}$ (similar to steel). ${ }^{[241]}$ A three point bending experiment using AFM tips was performed on cellulose microfibrils to calculate the elastic modulus. The dimension of cellulose microfibrils was found to significantly affect the mechanical properties, and a value of $81 \pm 12 \mathrm{GPa}$ was reported to be the longitudinal modulus. ${ }^{[242]}$ The modulus of BC nanofibers was reported as $114 \mathrm{GPa}$ by using of Raman spectroscopic technique, the measurement involved the determination of local molecular deformation of BC nanofibers via a shift in the central position of the Raman band at $1095 \mathrm{~cm}^{-1} \cdot{ }^{[243]}$ Lastly, Young's modulus of $250 \mathrm{GPa}$ were determined for cellulose whiskers by De la Motte. ${ }^{[244]}$ The later value is the closest to the modulus of the perfect crystal of native cellulose, since cellulose whiskers are devoid of chain folding and contain only a small number of defects. 
Nanocellulose showed considerable potential as reinforcing material for biobased and biodegradable polymer matrices. However, the main challenge with the incorporation of cellulose nanofibers in polymer matrix is to obtain good dispersion, which is reflected directly on the final mechanical properties of nanocomposites. ${ }^{[245]}$ Moreover, the enhancement of tensile strength and elastic modulus is usually at the expense of elongation at break. ${ }^{[239]}$ It is also very important to mention that nanocellulose-based nanocomposites can be hygroscopic in nature and any swilling that may happen can diminish the mechanical properties. ${ }^{[226]}$ Several researchers reported that adding small quantities between (0.5-5 wt $\%)$ of cellulose nanofibers will substantially improve the mechanical properties of the nanocomposites, and higher amounts of cellulose nanofibers over $5 \mathrm{wt} \%$ could lead to miscibility problems and deteriorate the mechanical properties of the nanocomposites. ${ }^{[246]}$

\subsubsection{Modification of Nanocellulose}

It is worth mentioning that, in order to use nanocellulose in biomedical applications such as wound healing, internal implant, or as drug delivery systems, a pure product is necessary. Therefore, from industrial point of view, powdered nanocellulose is much preferred. However, in dry state, the hydrophilic nature of cellulose nanofibers, supported by their high surface energy and hydrogen bonding between nanoparticles, cause irreversible agglomeration and hinder their distribution in hydrophobic polymer matrix. ${ }^{[247]}$ Although melting processing is the most practical and economical way for fabrication of CNC based bionanocomposite, it tends to cause aggregation and consequent phase separation, which finally limits the mechanical properties of nanocomposites. ${ }^{[248]}$ Lowering surface energy of nanocellulose is one of the important parameters that needs control and improvement to ensure nonfoloculated dispersion in polymer matrix and ultimately enhances the quality of melting processing. Two main strategies were used; surface physical modification by coating CNCs with surfactants, or chemical modification by grafting of CNCs surface with small molecules or polymer. The main challenge in surface modification of CNCs is to conduct the process in such a way that it only decorates the surface of CNCs, while original morphology and crystals integrity are kept intact.

\subsection{Noncovalent surface modifications}

Noncovalent surface modifications of CNCs are typically made by adsorption of surfactants on

fiber surfaces. This approach has been introduced by Heux et al, ${ }^{[237]}$ who used surfactants 
consisting of mono- and di-esters of phosphoric acid bearing alkylphenol tails. The obtained modified surfactant-coated CNCs displayed well dispersion in nonpolar solvents. Nevertheless, another study showed the surfactant molecules form a thin layer of about $15 \AA$ on the surface of the CNCs. ${ }^{[249]}$ An anionic surfactant was also used by Bondeson and Oksman to enhance the dispersion of CNCs in poly(lactic acid) (PLA). ${ }^{[250]} \mathrm{Kim}$ et $\mathrm{al}^{[251]}$ and Rojas et $\mathrm{al}^{[129]}$ used nonionic surfactants to disperse CNCs in polystyrene-based composite fibers. CNCs surface modification based on the adsorption of saccharide-amphiphilic block copolymers that mimic lignin-carbohydrate copolymers, e.g., adsorption of xyloglucan oligosaccharide-PEG-polystyrene triblock copolymer onto the surface of CNCs was done by Zhou et al. ${ }^{[252]}$ The resulting CNCs showed excellent dispersion abilities in nonpolar solvents.

\subsection{Covalent surface chemical modifications}

Depending on the desired application, chemical modifications of CNC were carried out at the hydroxyl groups of the glucose units on the crystalline backbone structure. This includes esterification, ${ }^{[253]}$ silylation, ${ }^{[254]}$ oxidation, ${ }^{[255]}$ cationization, ${ }^{[216]}$ and grafting; via acid chloride, ${ }^{[256]}$ acid anhydride, ${ }^{[257]}$ or isocyanate, ${ }^{[227]}$ (see Figure 2.22). Nevertheless, $(2,2,6,6-$ Tetramethylpiperidine-1-oxyl) or (TEMPO-mediated oxidation) of $\mathrm{CNCs}$ has been first introduced by De Nooy et al ${ }^{[258]}$ and has been used to convert the hydroxymethyl groups present on CNC surface to their carboxylic form. This oxidation reaction is highly discriminative of primary hydroxyl groups, and considered "green" and simple to be implemented. Recently, more homogeneous highly carboxylated CNCs have been prepared using ammonium persulfate oxidation. This one-pot procedure can process a variety of cellulosic biomass without the need for pretreatments to remove non-cellulosic plant contents. ${ }^{\text {[211] }}$

Moreover, an environmental friendly CNCs surface acetylation route involving a low reagent consumption and simple-to-apply procedure was reported by Yuan et al. ${ }^{[259]}$ In this method, aqueous emulsions of alkyenyl succinic anhydride (ASA) as a template were mixed with CNC suspensions followed by freeze-drying and then heating to $105^{\circ} \mathrm{C}$. The obtained solid was highly hydrophobic in character which evident from its high dispersability in solvents of different polarities. Surface trimethyl silylation of CNCs derived from BC was conducted by Roman and Winter and the resulted cellulose acetate butyrate was investigated. ${ }^{[260]}$ Finally, coupling CNCs with $\mathrm{N}$-octadecyl isocyanate, via a bulk reaction in toluene, has also been reported to enhance their dispersion in organic medium, and to improve the compatibility with polycaprolactone 
matrix. Significant improvement of the stiffness and ductility of the resultant nanocomposites was also reported.

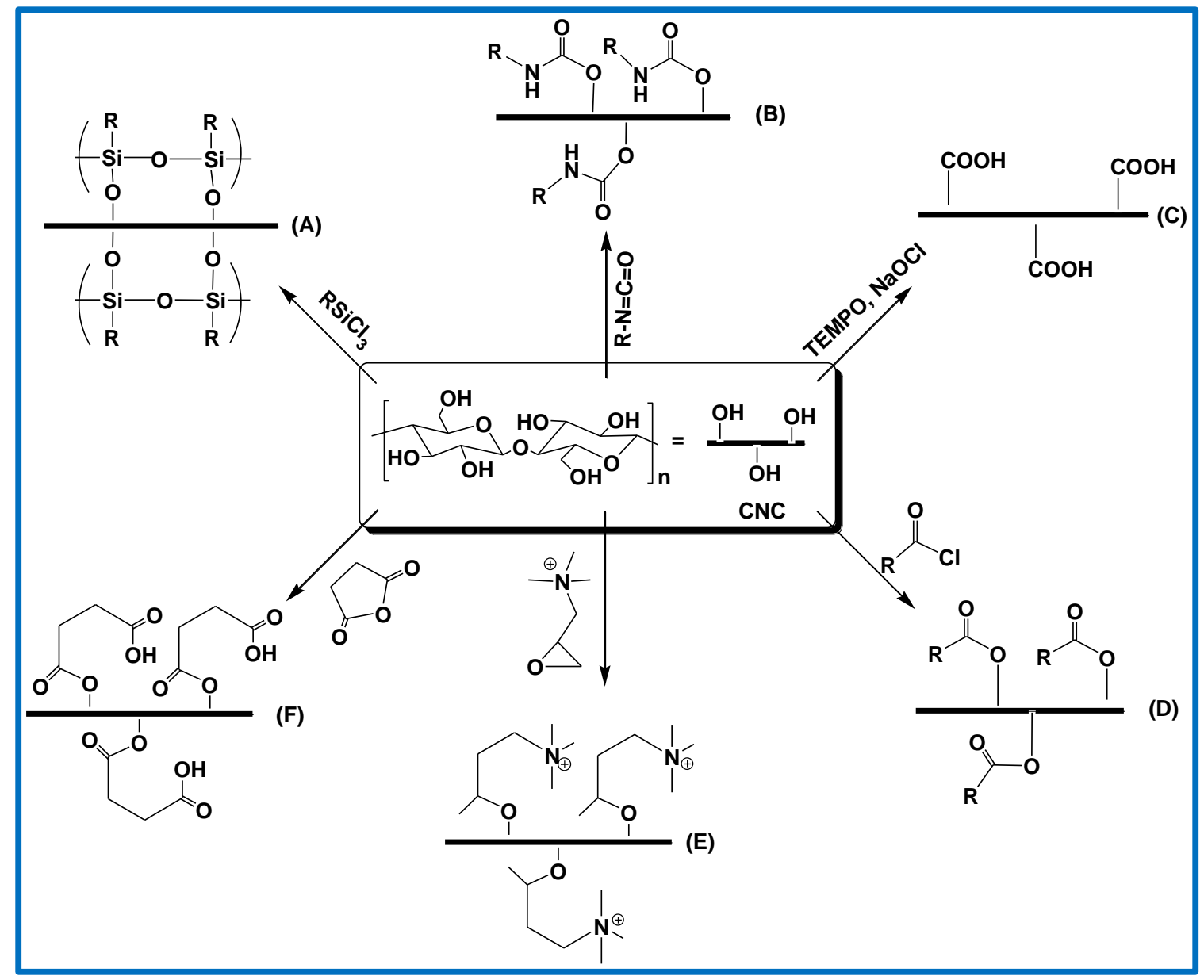

Figure 2.22 Some possible routes for chemical modification of CNC (anticlockwise from left): (A) silylation; (B) urethane linkages via isocyanates; (C) oxidation by TEMPO; (D) ester linkages via acid chlorides; (E) cationization via epoxides; (F) ester linkages via acid anhydrides.

\subsection{Polymer grafting}

Polymer grafting on the surface of CNCs was carried out using two main strategies, namely, the "grafting-onto" and "grafting-from". ${ }^{[261]}$ The "grafting onto" approach involves attachment of pre-synthesized polymer chains onto hydroxyl groups of CNCs/MFC surface by using a coupling agent. In this method, high density of grafting is not possible to achieve due to steric hindrance of polymer matrix. ${ }^{[262]}$ In the "grafting from" approach, the polymer chains are growing from the surface of $\mathrm{CNCs} / \mathrm{MFC}$ via in situ surface-initiated polymerization from immobilized initiators on 
the surface. Although this approach is difficult to control, it can achieve higher grafting density. ${ }^{[263]}$

The "grafting onto" approach was used by Ljungberg et al to graft maleated polypropylene (PPgMA) onto the surface of CNCs extracted from tunicate. ${ }^{[264]}$ The results showed that the grafted nanocrystals have very good compatibility and high adhesion in atactic polypropylene matrix. Vignon et al studied the grafting of amine terminated polymers on the surface of TEMPO oxidized CNCs by using a peptide coupling process catalyzed by carbodiimide derivatives in water. ${ }^{[265]}$ The same approach has been used by Mangalam et al who grafted DNA oligomers onto the surface of CNCs. ${ }^{[195]}$ Grafting of low molecular weight PCL fragments on the surface of CNCs has been reported by using isocyanate mediated coupling. These authors also reported the reaching of high grafting density that was enough for allowing the grafted PCL chains to crystallize at the surface of CNCs. ${ }^{[266]}$ Furthermore, they also claimed that the co-crystallization phenomenon induced the formation of a co-continuous phase between the matrix and filler, and significantly enhanced the interfacial adhesion and consequently contributed to a highly improved mechanical strength of the resulting nanocomposites.

The "grafting from" approach applied to CNCs was first reported by Habibi et al who grafted polycaprolactone onto the surface of $\mathrm{CNCs}$ via ring-opening polymerization (ROP) using stannous octoate $\left(\mathrm{Sn}(\mathrm{Oct})_{2}\right)$ as grafting agent. ${ }^{[266]}$ Likewise, Chen et al, ${ }^{[267]}$ and Lin et al ${ }^{[268]}$ conducted similar grafting reactions under microwave irradiation to enhance the grafting efficiency. In another study, surface of CNCs have been modified by grafting from PLA by ringopining polymerization to improve the compatibility of CNCs with hydrophobic polymer. ${ }^{\text {[269] }}$ The grafting of poly(3-hydroxybutyrate) onto bacterial cellulose by using of laccase was also reported and caused an improvement in mechanical strength and hydrophilicity of composite. ${ }^{[2]}$

\subsection{Deposition of Nanoparticles on CNC}

The most common method for the deposition of metallic nanoparticles on CNC surface is the treatment of metallic salts with reducing agents such as $\mathrm{NaBH}_{4}$, in presence of CNC. Modification of CNC with inorganic nanoparticles is popular because CNC acts as a green and attractive biotemplate capable of promoting nucleation of monodispersed nanoparticles and preventing agglomeration to achieve a very narrow particle size distribution. ${ }^{[270]}$ There are several reports on the use of $\mathrm{CNC}$ as a stabilizing matrix for $\mathrm{Ag}, \mathrm{Au}, \mathrm{Ni}, \mathrm{Pt}$ and $\mathrm{Pd}$ nanoparticles for use as antimicrobial wound dressing and catalysts. ${ }^{\text {[271-274] }}$ 
Recently, CNC is used as biotemplate for synthesis and growth of CNC/CdS@ZnS QDs nanohybrids for their application in the in vitro bioimaging of HeLa cells. ${ }^{[275]}$ (see Figure 2.23). In addition, hybrid inorganic-organic nanocomposites materials are of practical interest due to their multifunctionality, processability, and potential large-scale production for removal of pharmaceutical residues. ${ }^{[276]}$ For instance, CNC@ $\mathrm{Fe}_{3} \mathrm{O}_{4} @ \mathrm{SiO}_{2} @ \beta-\mathrm{CD}$ hybrids where $\mathrm{CNC}$, silica act as supporting biotemplate and coating materials respectively for iron oxide nanoparticles and grafted $\beta C D$ onto the surface of $\mathrm{CNC} @ \mathrm{Fe}_{3} \mathrm{O}_{4} @ \mathrm{SiO}_{2}$ hybrids is used for adsorption of organic contaminants, ${ }^{[277]}$ (see Figure 2.24).

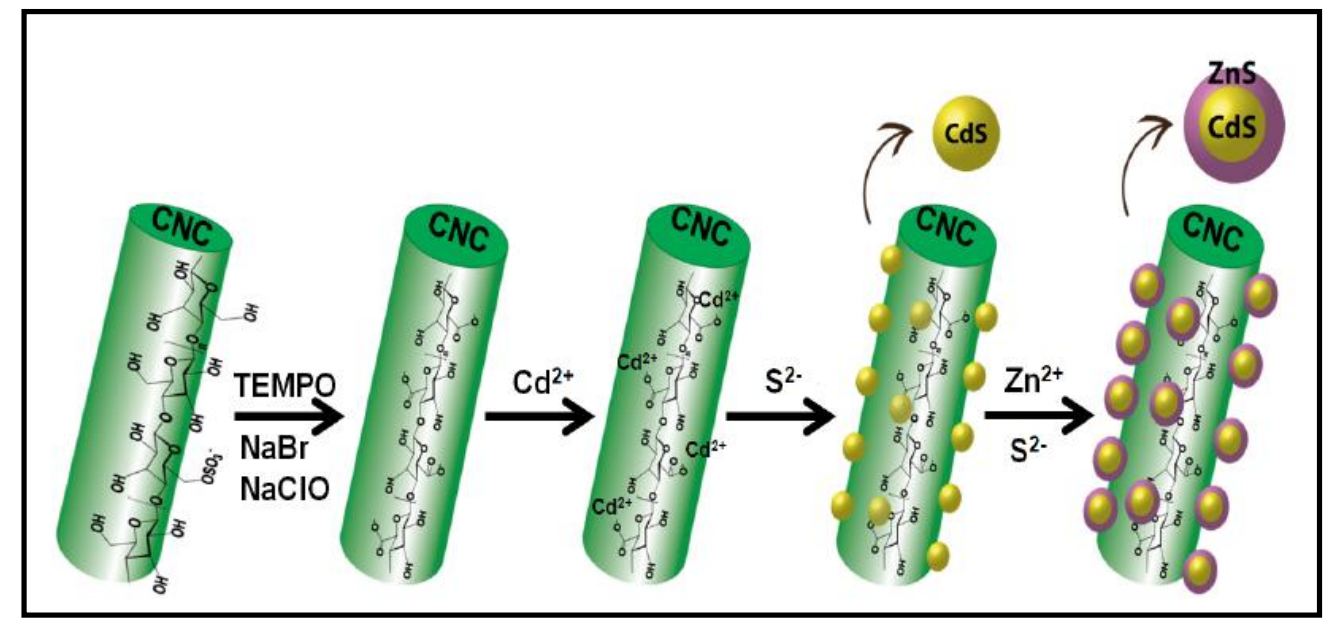

Figure 2.23 Schematic illustration of the synthetic procedure for CNC/CdS@ZnS. ${ }^{[275]}$

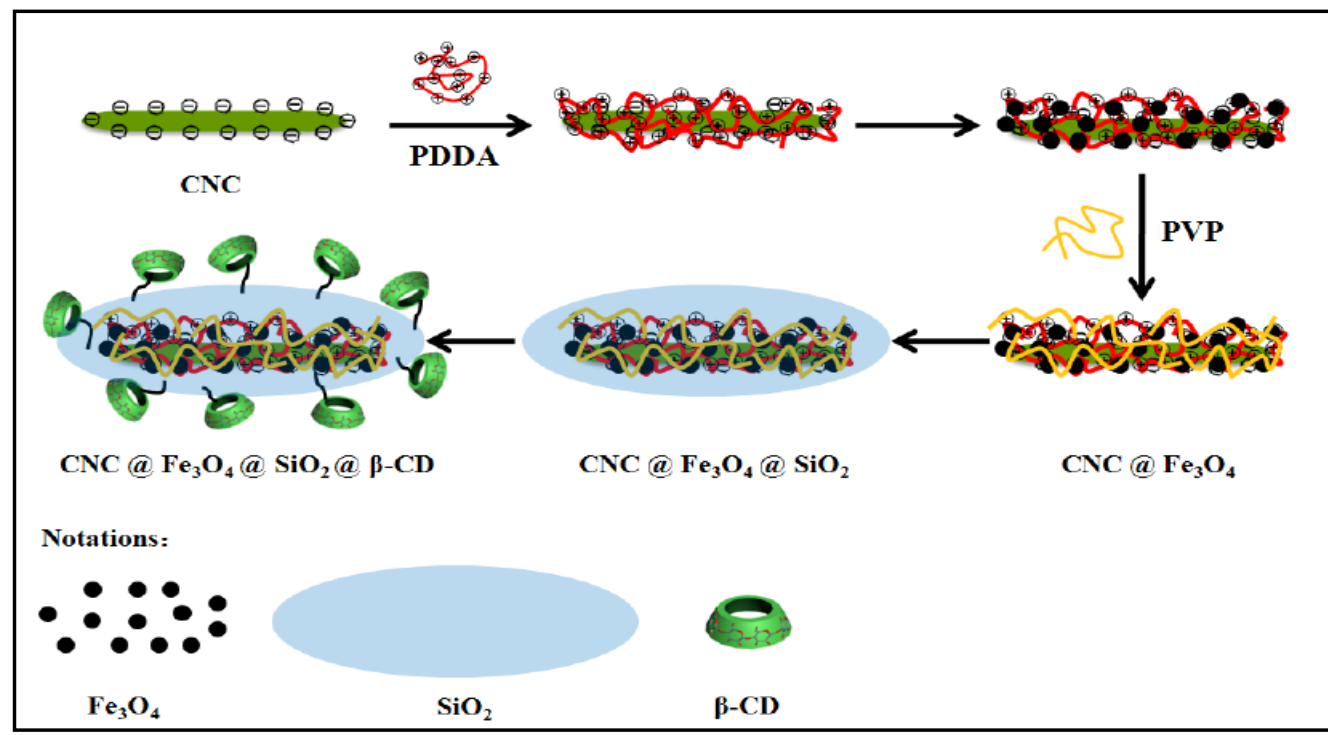

Figure 2.24 Schematic representation for the synthesis of $\mathrm{CNC} @ \mathrm{Fe}_{3} \mathrm{O}_{4} @ \mathrm{SiO} 2 @ \beta-\mathrm{CD}$. PDDA: poly (diallydimethylammonium chloride); PVP: polyvinylpyrrolidone; $\beta$-CD: $\beta$-cyclodextrin. ${ }^{[277]}$ 


\subsubsection{Cellulose Based Nanocomposites}

Structurally, nanocomposites are materials which involve nano sized filler particles (dispersed phase), matrix (dispersion phase) and interfacial region. Compared to the conventional composites, nanocomposites have unique characteristics mainly due to their large surface area to volume ratio. ${ }^{[253]}$ The extra ordinary mechanical, thermal and structural properties of nanocellulose gave it the potential to act either as matrix, or as reinforcing agent (filler). ${ }^{[278]}$ Originating from these impressive mechanical properties, nanocellulose has been potentially used as a load bearing element for various host materials provided that homogenous dispersion. Besides, strong interfacial adhesion with matrix is attained, which ultimately allows proper stress transfer from matrix to the reinforcing phase. Several new nanocomposites that incorporate nanosized cellulose have been developed within the last decade and many systems are being commercialized. ${ }^{[279]}$

On the other hand, the reinforcement applications with cellulose nanoparticles present some drawbacks. For instance, high moisture absorption, poor wettability and incompatibility with most hydrophobic polymeric matrices, and limitation of processing temperature, where lignocellulosic materials start to degrade near $220^{\circ} \mathrm{C}$, restricting the type of matrix that can be used. $^{[280]}$

\subsubsection{Micromechanical Modeling for Predicting the Reinforcing Potential of CNC}

It was found that the effect of CNCs on the nanocomposites' mechanical properties has exceeded the conventional predictions of the traditional classical model of Halpin-Kardos that applied to study filler reinforced nanocomposites. ${ }^{[281]}$ This model was used to predict the mechanical behavior of short-fibers homogeneously dispersed in a continuous matrix of semi-crystalline polymers. In this approach, the modulus and the geometry of the fibers are accounted for, but no interactions between the fibers are assumed. In the recent years, various studies have been developed with the aim of elucidating the origin of the mechanical reinforcing effect, and to explain the extraordinary high reinforcing effect observed for cellulose whiskers. Therefore, in order to explain the unusual high modulus values of cellulose whiskers reinforced nanocomposites, the following phenomena were suggested: (i) strong interactions between whiskers that cemented together by hydrogen bonds, and (ii) a mechanical percolation effect. In this regard, the model was proposed and then modified by Ouali et al to include the percolation approach which allowed better understanding for the mechanical behavior of these 
nanocomposites. ${ }^{[282]}$ Percolation is a statistical geometrical model that can be applied to any random multiphase material involving components that able to be mixed. ${ }^{[283]}$ This approach allows a transition from a disconnected set of objects to an infinite connected state. The percolation threshold is defined as the critical volume fraction that makes separations between two states. The volume threshold depends upon a number of variables, primarily the shape and size of particles, ${ }^{[248]}$ aspect ratio, ${ }^{[285]}$ orientation, ${ }^{[286]}$ and the interparticle interactions. ${ }^{[287]}$ Therefore, to study the reinforcing effect of cellulose nanoparticles, the model invoked should be involving the three different phases in a typical nanocomposites: the matrix, the filler percolating network, and the nonpercolating filler. According to Takayanagi's model, elastic tensile modulus $\mathrm{G}_{\mathrm{c}}$ of the composite can be given by Equation (2.1).

$$
\mathrm{G}_{\mathrm{c}}=\frac{\left(1-2 \varphi+\varphi \emptyset_{\mathrm{r}}\right) \mathrm{G}_{\mathrm{s}} \mathrm{G}_{\mathrm{r}}+\left(1-\emptyset_{\mathrm{r}}\right) \varphi \mathrm{G}_{\mathrm{r}}^{2}}{\left(1-\emptyset_{\mathrm{r}}\right) \mathrm{G}_{\mathrm{r}}+\left(\emptyset_{\mathrm{r}}-\varphi\right) \mathrm{G}_{\mathrm{s}}}
$$

Where $G_{s}$ and $G_{r}$ are the shear moduli of the soft (polymeric matrix) and rigid (cellulosic filler) phases, respectively. $\emptyset_{\mathrm{r}}$ corresponds to the volume fraction of the rigid phase (whiskers) and $\varphi$ is an adjustable parameter that corresponds to the volume fraction of the percolating rigid phase.

When the stiffness of the reinforcing phase is much higher than that of the matrix material (i.e., when $\mathrm{G}_{\mathrm{r}} \gg \mathrm{G}_{\mathrm{s}}$ ), Equation (1) can be reduced to $G_{c}=\varphi G_{r}$.

In the modification proposed by Ouali et al. to include the percolation approach, $\varphi$ was estimated by Equation (2.2).

$$
\begin{array}{clrl}
\varphi & =0 & \emptyset_{r}<\emptyset_{c} \\
\varphi=\emptyset_{r}\left(\frac{\emptyset_{r}-\emptyset_{c}}{1-\emptyset_{c}}\right)^{b} & \emptyset_{r} \geq \emptyset_{c}
\end{array}
$$

where $\mathrm{b}$ is the critical percolation exponent, which is equal to 0.4 for a three-dimensional system and $\emptyset_{c}$ is the percolation threshold, which varies depending on the studied material and their orientation distribution.

Dufresne $^{[288]}$ reported a good agreement between experimental and predicted values by using the modified model of Ouali et al and stated that the percolation approach is related to the formation of infinite aggregates of cellulose whiskers.

For rod-like nanoparticles, the percolation threshold was linked to the aspect ratio of the nanoparticles by Equation (2.3).

$$
\emptyset_{c}=\frac{0.7}{L / d}
$$


In Equation (2.3), L/d is the aspect ratio, assuming a cylindrical shape for the nanofiller, and $\emptyset_{c}$ is the percolation threshold. The geometrical characteristics and the corresponding percolation threshold values for some cellulose nanocrystals obtained from different systems are reported in Table 2.6.

Table 2.6 Geometrical characteristics (length, L; diameter, D; and percolation threshold $\emptyset_{\mathrm{c}}$ of some cellulose nanocrystals.

\begin{tabular}{|l|c|c|c|c|c|}
\hline \multicolumn{1}{|c|}{ Source } & $\mathbf{L ~ ( n m )}$ & $\mathbf{D}(\mathbf{n m})$ & $\mathbf{L} / \mathbf{d}$ & $\boldsymbol{\Phi}_{\boldsymbol{c}}$ & Reference \\
\hline MCC & 200 & 5 & 40 & 1.75 & {$[289]$} \\
\hline Wheat straw & 225 & 5 & 45 & 1.6 & {$[290]$} \\
\hline Cotton & 171.6 & 14.6 & 11.8 & 5.9 & {$[291]$} \\
\hline Ramie & 200 & 7 & 28.6 & 2.5 & {$[191]$} \\
\hline Sugar beet pulp & 210 & 5 & 42 & 1.7 & {$[292]$} \\
\hline Palm tree & 260 & 6.1 & 43 & 1.6 & {$[293]$} \\
\hline Tunicin & 1,000 & 15 & 67 & 1.0 & {$[294]$} \\
\hline BC & $100-1000$ & $5-50$ & 20 & 6 & {$[167,295]$} \\
\hline
\end{tabular}

\subsubsection{Mechanical Properties of Nanocellulose Based Nanocomposites}

Owing to nanocellulose's high aspect ratio, high stiffness, and high strength, a combination of nanocellulose as reinforcing materials with polymer matrix has showed enhanced mechanical properties for the resultant nanocomposites. ${ }^{[240]}$ Bondeson and Oksman reported an improvement in the elastic modulus (from $2600 \mathrm{MPa}$ to $3,100 \mathrm{MPa}$ ), tensile strength (from $35 \mathrm{MPa}$ to 52 $\mathrm{MPa}$ ), and also slight increase in elongation at break (from 1.8\% to $3.1 \%$ ) for PLA reinforced with $5 \mathrm{wt} \%$ of surfactant modified cellulose nanocrystals (sCNC). ${ }^{[250]}$ Whereas the addition of 5 $\mathrm{wt} \%$ of the pristine CNC produced nanocomposites with lower tensile strength and elastic modulus than neat PLA. A similar study for the effect of adding $5 \mathrm{wt} \%$ pristine or sCNC on the mechanical properties of PLA-PHB blend was carried out by Arrieta et al. ${ }^{[296]}$ It was reported that the pristine $\mathrm{CNC}$ has reduced elastic modulus of PLA-PHB film from $1800 \mathrm{MPa}$ to $900 \mathrm{MPa}$ and tensile strength from $40 \mathrm{MPa}$ to $27 \mathrm{MPa}$, while sCNC increased elastic modulus to 1900 $\mathrm{MPa}$ and tensile strength to $47 \mathrm{MPa}$. In the same way, PVA-chitosan blend (elastic modulus = $950 \mathrm{MPa}$, tensile strength $=60 \mathrm{Mpa}$, and toughness $=1500 \mathrm{MPa}$ ) loaded with CNCs resulted in 
nanocomposites with improved mechanical properties with an increasing amount of CNCs, reaching the highest $5 \mathrm{wt} \%$ addition (elastic modulus $=1900 \mathrm{MPa}$, tensile strength $=105 \mathrm{Mpa}$, and toughness $=2500 \mathrm{MPa}){ }^{[297]}$

\subsubsection{Effect of Processing Method and Interfacial Interactions on Mechanical Properties of CNC-Based Nanocomposites}

The processing rate and the viscosity of the system are two important factors that affect the ability of the percolating structure to be cemented or to withstand imposed stresses. Although sol-gel processing method seems to give the highest mechanical performance because of the strong percolation network that formed between CNCs contact points prior the addition of polymer solution, this method is limited only for hydrophilic polymer matrices. The castingevaporation technique also gives satisfactory results in terms of mechanical performance. During such slow processing methods, CNCs have adequate time to interconnect and to form a percolation network throughout Brownian motion, if the viscosity of the system remains low until the end of the process. In contrast, during hot pressing or extrusion processes, high viscosity of melt polymer that occurs during the process strongly limits random movement and consequently hinders the interconnection between CNCs. Possible alignments of CNCs owing to induced shear stresses during extrusion or molding may also affect the network formation. Hajji et al studied the effect of the processing method on the mechanical properties of CNC-based nanocomposites and classified them in ascending order according to their reinforcement efficiency as: extrusion < hot pressing < evaporation. ${ }^{[298]}$

Surprisingly, a very good compatibility between CNCs and the host polymer has tendency to decrease the elastic modulus of the composite, typically when nanocomposites are processed via casting method. This behavior is most likely due the restricted mobility of CNCs, which allow for strong interact with the polymer chains rather than extending to each other to interconnect and form the network. An example of this behavior has been clearly evidenced in the case of glycerol plasticized starch reinforced with CNCs extracted from cotton seed linters. ${ }^{[299]}$ On the other hand, strong matrix/CNC interactions seem to enhance the nonlinear mechanical properties, especially the ultimate strain, when CNCs are chemically modified with long-chain molecules such as surfactants, fatty acids or polymer chains. For instance, when CNCs were grafted with high molecular weight PCL and then incorporated in PCL matrix, the final nanocomposites presented a lower modulus, but significantly higher strain at break when 
compared with the control, which filled with unmodified CNCs. ${ }^{[266]}$ This behavior clearly reflected the restricted $\mathrm{CNC} / \mathrm{CNC}$ interactions that drop the modulus and the high $\mathrm{CNC} /$ matrix compatibilization resulting from the formation of a percolating network held by chain entanglements and possible co-crystallization between the grafted chains and the matrix. Similarly, Cao et al have reported that chain entanglements and co-crystallization occurred in nanocomposites based on a completely amorphous polymer, e.g., polyurethane reinforced with CNCs that have been grafted with the same polymer in a one-pot process. ${ }^{[300]}$

\subsubsection{Thermal Properties of CNC-Based Composites}

Surprisingly, most studies in this field report that the addition of CNCs into polymers matrices does not seem to affect the glass-rubber transition temperature $\left(T_{g}\right)$, regardless the nature of the host polymer, the origin of the CNCs, or the processing conditions. ${ }^{[301,302]}$ This is seems to be in conflict with the fact that CNCs have a high specific area. However, there are a few reported cases in the literature where the addition of CNCs as filler in nanocomposites materials affected the $\mathrm{T}_{\mathrm{ag}}$ especially in moisture-sensitive matrixes. ${ }^{[303]}$ This may be related to the plasticization effect of water, which causes strong interaction between CNCs and the matrix.

In the case of semicrystalline polymers, it was reported that the addition of unmodified CNCs has no influence on the melting temperature $\left(\mathrm{T}_{\mathrm{m}}\right)$ of the nanocomposites, as has been shown in the case of plasticized starch, ${ }^{[304]}$ poly(ethylene-oxide), ${ }^{[292]}$ cellulose acetate butyrate, ${ }^{[305]}$ polycaprolactone-reinforced polymers. ${ }^{[306]}$ However, when chemically modified CNCs were used in nanocomposites, a change of $\mathrm{T}_{\mathrm{m}}$ was observed. Strong interactions between chemically modified CNCs and matrices have been reported to be the origin of this $\mathrm{T}_{\mathrm{m}}$ change. Moreover, CNCs can act as nucleating agents in semicrystalline polymers, which significantly increases the crystallinity of the polymer matrix forming the nanocomposites. ${ }^{[307]}$ The later effect is mainly governed by the $\mathrm{CN}$-matrix compatibility, which depends on surface chemical considerations.

Cao et al $^{[308]}$ investigated the use of chemically modified CNCs (grafted with the same polymer as the matrix) as filler in completely amorphous polyurethane. They observed partial crystallization and transformation of the nanocomposites material from an elastomer-like to a thermoplastic-like material. This unusual behavior is related to the chain entanglements that take place between the grafted chains and those from the polymer matrix. 


\subsubsection{Nanocellulose-Reinforced Polymer Nanocomposites: A Review}

This part compiles a short review for the efforts that have been made by researches during recent years for incorporating of cellulose nanocrystals into different polymer matrix as reinforcing material. Hassan and Peppas ${ }^{[309]}$ have worked on poly(vinyl alcohol)-nanocellulose as candidate for biomedical applications; including tissue reconstruction, tissue replacement, cell entrapment, drug delivery, soft contact lens materials and burn wound covering bandages. Leitner et al prepared poly(vinyl alcohol) nanocomposites with a range of nanocellulose contents (0-90 $\mathrm{wt} \%$ ), where at cellulose content of $50 \mathrm{wt} \%$, the elastic modulus increased by a factor of 20 and tensile strength increased by a factor of 3.5 than that of native poly(vinyl alcohol). ${ }^{[310]}$ Bhatnagar and Sain ${ }^{[311]}$ reported nanofibers-reinforced composite material by a solution casting of blend containing $10 \%$ cellulose nanofibers obtained from various sources and $90 \%$ poly (vinyl alcohol). Poly(vinyl alcohol) films reinforced with regenerated MFC have been also reported. ${ }^{\text {[312] }}$ When the fibril content was $20 \mathrm{wt} \%$, Elastic modulus increased up to three times higher and up to five times higher tensile strength when compared to the native polymer.

Nakagaito and Yano ${ }^{[313]}$ impregnated MFC with a phenol formaldehyde resin to produce high strength cellulose nanocomposites. Hayashi and Shimo ${ }^{[314]}$ reported the use of MFC in phenolic resin for the preparation of car fenders. Nogi and Yano ${ }^{[315]}$ prepared a foldable and ductile transparent nanocomposites film by combining acrylic resin with $5 \mathrm{wt} \% \mathrm{BC}$. Studies by Pu et al have also shown improvements in strength properties of acrylic films using cellulose whiskers. ${ }^{[316]}$ Nogi and Yano ${ }^{[317]}$ also reported that transparent cellulose nanofibers sheets prepared from MFC and acrylic resin. Bruce at al prepared composites based on MFC with acrylic and epoxy resins. ${ }^{[318]}$ Dispersed cellulose nanofibrils in poly(styrene-co-butyl acrylate) (poly (S-co-BuA)) latex was also reported. ${ }^{[319]}$ Seydibeyoglu and Oksman ${ }^{[320]}$ prepared polyurethane-cellulose nanofibers composite by compression molding. Auad et al reported the utilization of MFC to reinforce shape memory polyurethanes (SMPs) which are capable of fixing a transient shape and recovering their original dimensions by the application of an external stimulus. ${ }^{[321]}$ Similar studies on polyurethane and waterborne polyurethane were done by Marcovich et al. ${ }^{[323]}$

Biocompatible, biodegradable, hydrophilic, and flexible bacterial cellulose/poly(ethylene-oxide)nanocomposites were studied by Brown and Laborie. ${ }^{[323]}$ They claimed that thermal and 
mechanical properties of composite could be tuned by tailoring the composition and morphology of $\mathrm{BC} / \mathrm{PEO}$ nanocomposites.

Okubo et $\mathrm{al}^{[324]}$ reported an effective technique for improving the mechanical properties of polylactic acid (PLA) by adding MFC up to $20 \mathrm{wt} \%$. However, Mathew et al ${ }^{[325]}$ reported a nonuniform distribution of $5 \mathrm{wt} \%$ cellulose nanowhisker and MFC fillers within PLA matrix when nanocomposites were prepared by twin-screw extrusion. Wang and Sain $^{[326]}$ used chemically treated cellulose nanofibers to prepare PLA and poly(hydroxybutyrate) (PHB) nanocomposites. Suryanegara et al reported that the increase in PLA crystallization leads to an increase in the strength and storage modulus, as well as Young's modulus of PLA/MFC nanocomposites without significant reduction in the strain at break. ${ }^{[327]} \mathrm{CNC}$ grafted with poly(D-lactide) for incorporation in poly(L-lactide) was studied by Habibi et al. ${ }^{[328]}$ Poly(ethylene glycol) (PEG)-grafted CNCs were successfully synthesized and incorporated into poly(lactic acid) (PLA) as a reinforcing filler by using an electrospinning technique for fabrication of nanocomposites scaffolds consisting of CNC-g-PEG and PLA to be used as potential scaffolds for bone tissue engineering. ${ }^{[329]}$ Recently, Kiziltas et al have reinforced polylactic acid with cellulose nanofibers via a polyhydroxybutyrate carrier system. ${ }^{\text {[330] }}$ Nanocellulose was successfully grafted with different molecular weights of PCL in order to improve compatibility with a large molecular weight PCL matrix. ${ }^{[331]}$ Moreover, nanocellulose has been incorporated into a wide range of polymer matrices, including poly(oxyethylene), ${ }^{[280,292]}$ cellulose acetate butyrate,${ }^{[196,332]}$ polysiloxanes, ${ }^{[333]}$ polysulfonates, ${ }^{[334]}$ poly(vinyl acetate) ${ }^{[335]}$ carboxymethyl cellulose, ${ }^{[336]}$ epoxides, ${ }^{[337]}$ poly(ethylene-vinyl acetate) (EVA), ${ }^{[338]}$ polypropylene, ${ }^{[339]}$ polyethylene ${ }^{[256]}$ and poly(vinyl chloride). ${ }^{[340]}$ The incorporation of cellulose nanofibers into biopolymers, such as starch-based polymers, ${ }^{[229]}$ chitosan, ${ }^{[341]}$ soy protein, ${ }^{[342]}$ poly(hydroxyoctanoate) ${ }^{[343]}$ regenerated cellulose,${ }^{[344]}$ polyhydroxybutyrates ${ }^{[345]}$ and Poly (vinyl pyrrolidone $)^{[346]}$ have also been reported.

\subsubsection{Biomedical Applications of Nanocellulose and Its Nanocomposites}

Nanocellulose and its bio-nanocomposites have found important rule and versatility in biomedical field due to their biodegradability and cyto/hemo-compatibility. Such applications include disease diagnostics, ${ }^{[347]}$ drug delivery, ${ }^{[348]}$ antibacterial activity and tissue engineering. ${ }^{[349]}$ Various important applications of nanocellulose and cellulose nanocomposites in biomedical arena are highlighted and listed in Table 2.7. 


\subsubsection{Conclusion and Future Prospects}

The research and development of nanocellulose-reinforced polymer composites have dramatically increased in recent years due to the possibility of exploiting their potentials in different sectors, and their abilities to address the property-performance gap between renewable and non-renewable petroleum based polymers. Owing to the good mechanical properties, renewability, biodegradability, biocompatibility, low cost production, low density, flexibility, high aspect ratio, low abrasivity, and reactive surface nanocellulose fibers serve as promising and potential candidate for preparing different kinds of bionanocomposite. Although optimizing reinforcing effect by cellulose whiskers is in progress, it was concluded that modified cellulose whiskers might overcome the dispersion problems up to certain extent. However, there are several challenges still confronting the scale-up production of cellulose-based nanocomposites.

For future objectives, it worth to notice that there are many safety concerns and debates in literature about cellulose nanowhisker specially for biomedical applications, as size allows their penetration into cells and consequently it might be accumulate in biological system if they are not resorbable. Until now, there is no sharp decision taken to categorize these nanofibers as completely safe. 
Table 2.7 Biomedical Applications of Nanocellulose and nanocellulose based Nanocomposites.

\begin{tabular}{|c|c|c|c|c|}
\hline Reinforcing material & Matrix & Modifications & Applications & References \\
\hline \multicolumn{5}{|c|}{ Disease diagnostics } \\
\hline & $\mathrm{CNCs}$ & $\begin{array}{c}\text { Covalent attachment with flourocine- } \\
5 \text {-isothiocyanate (FITC) }\end{array}$ & $\begin{array}{l}\text { Bioassay and bio- } \\
\text { imaging }\end{array}$ & {$[350]$} \\
\hline & Cellulose nanofibrils & Luminescent carbon dots & Bio-imaging & [351] \\
\hline & $\begin{array}{c}\text { Cellulose } \\
\text { nanoparticles }\end{array}$ & & $\begin{array}{l}\text { Biodegradable contrast } \\
\text { agent in biomedical } \\
\text { imaging }\end{array}$ & {$[352]$} \\
\hline \multirow[t]{2}{*}{$\begin{array}{l}\text { Ag-Pd alloy } \\
\text { nanoparticles }\end{array}$} & $\mathrm{CNCs}$ & $\begin{array}{l}\text { TEMPO mediated carboxylation of } \\
\text { CNC }\end{array}$ & $\begin{array}{l}\text { Labels for electrical } \\
\text { detection of DNA } \\
\text { hybrids }\end{array}$ & {$[353]$} \\
\hline & $\begin{array}{l}\text { Nanofibrillated } \\
\text { cellulose }\end{array}$ & $\begin{array}{c}\text { TEMPO mediated oxidation followed } \\
\text { by bovine serum albumin (BSA) and } \\
\text { ati-human IgG conjugation }\end{array}$ & $\begin{array}{l}\text { Immunoassay and } \\
\text { diagnostic }\end{array}$ & {$[354]$} \\
\hline \multicolumn{5}{|c|}{ Antibacterial applications } \\
\hline Chitin nanocrystals & Bacterial cellulose & & Antibacterial film & {$[355]$} \\
\hline Ag nanoparticles & $\begin{array}{l}\text { Microcrystalline } \\
\text { cellulose }\end{array}$ & & Antimicrobial film & {$[356]$} \\
\hline \multicolumn{5}{|c|}{ Drug delivery } \\
\hline $\begin{array}{l}\text { Titania nanoparticles } \\
\text { along with drug }\end{array}$ & Cellulose nanofibers & & $\begin{array}{l}\text { Transdermal drug } \\
\text { delivery and wound } \\
\text { dressing }\end{array}$ & {$[357]$} \\
\hline \multirow[t]{2}{*}{$\begin{array}{l}\text { Various drug } \\
\text { excipients }\end{array}$} & Nanocellulose & & Tablets coating & [27] \\
\hline & Bacterial cellulose & & $\begin{array}{l}\text { Model carrier for serum } \\
\text { albumin }\end{array}$ & {$[358]$} \\
\hline
\end{tabular}




\begin{tabular}{|l|l|l|l|l|}
\hline & CNCs & $\begin{array}{l}\text { Surface modification by Cetyl } \\
\text { trimethylammonium bromide (CTAB) } \\
\text { followed by loading with hydrophobic } \\
\text { anticancer drug docetaxel, paclitaxel, } \\
\text { and etoposide }\end{array}$ & $\begin{array}{l}\text { Anticancer controlled } \\
\text { release drug delivery } \\
\text { system. }\end{array}$ \\
\hline & Bacterial cellulose & $\begin{array}{l}\text { BC is loaded with berberine } \\
\text { hydrochloride and berberine sulfate.. }\end{array}$ & Sustained drug release \\
\hline $\begin{array}{l}\text { Bacterial cellulose } \\
\text { nanofibers }\end{array}$ & $\begin{array}{l}\text { Sodium alginate } \\
\text { hydrogel }\end{array}$ & $\begin{array}{l}\text { Hybrid hydrogel was loaded with } \\
\text { Ibuprofen }\end{array}$ & $\begin{array}{l}\text { pH and electro } \\
\text { responsive dual } \\
\text { controlled drug delivery } \\
\text { system }\end{array}$ \\
\hline Collagen & Nanocellulose fibers & Oxidation to aldehyde nanofibers & $\begin{array}{l}\text { Wound dressing and } \\
\text { tissue engineering } \\
\text { scaffold }\end{array}$ \\
\hline CNFs & Porous starch & $\begin{array}{l}\text { Cell scaffold for repair } \\
\text { and regenerate cartilage }\end{array}$ & [360] [362] \\
\hline Silk fibroin & $\begin{array}{l}\text { Bacterial cellulose } \\
\text { nanofibers }\end{array}$ & $\begin{array}{l}\text { Scaffold for tissue } \\
\text { regeneration }\end{array}$ \\
\hline nanofibers & [361] \\
\hline $\begin{array}{l}\text { Bacterial cellulose } \\
\text { nanofibers }\end{array}$ & hydroxyapatite & $\begin{array}{l}\text { Scaffold for bone tissue } \\
\text { engineering }\end{array}$ & $\begin{array}{l}\text { Template for bone grafts } \\
\text { and bone repair }\end{array}$ \\
\hline
\end{tabular}




\section{CHAPTER 3*}

\section{Functionalized Bacterial Cellulose Nanowhisker as Long Lasting Drugs Nanocarriers for Antibiotics and Anticancer drugs}

A nano drug carrier based on sustainable and biocompatible nanocellulose was developed for use in prolonged drug releases. Grafting of $\beta$-cyclodextrin $(\beta C D)$ on bacterial cellulose nanowhisker (BCNC) led to the formation of functionalized BCNC-grafted- $\beta C D$ (BCNC- $g-\beta C D$ ). Broadspectrum antibiotic Ciprofloxacin (CIP) and anticancer drugs Doxorubicin (DOX) and Paclitaxel (PTX) were used as model drugs and were conjugated to BCNC- $g-\beta C D$ to form the drugnanocarrier systems (BCNC-g- $\beta C D$-drug). Functionalized nanowhisker showed significant increase in drug payloads ranged from $495 \pm 4$ to $810 \pm 7 \mu \mathrm{g} / \mathrm{mg}$ along with radical improvement in the drug release profiles and reduced initial burst releases. The observed drug releases showed sustained and controlled manner, with cumulative releases of 75-90\% over 5-5.5 days. An improved drug release performance was observed in acidic $\mathrm{pH}$ of 6.4 that mimicked extracellular tumor cells. In vitro drug release data were fitted zero-order kinetic model with drug release constants $\left(\mathrm{K}_{0}\right)$ of $0.68,0.74$, and $0.79 \mu \mathrm{g}$ drug/h (at $\mathrm{pH} 6.4$ and $37^{\circ} \mathrm{C}$ ) for BCNC- $g$ - $\beta \mathrm{CD}-\mathrm{CIP}$, BCNC- $g$ - $\beta C D-D O X$ and BCNC- $g$ - $\beta C D-P T X$ nanosystems, respectively. The observed higher payloads along with the slow releases of drugs from the developed nanocarrier suggests its promising potential for reducing the frequent daily dosing and minimizing systemic toxicities.

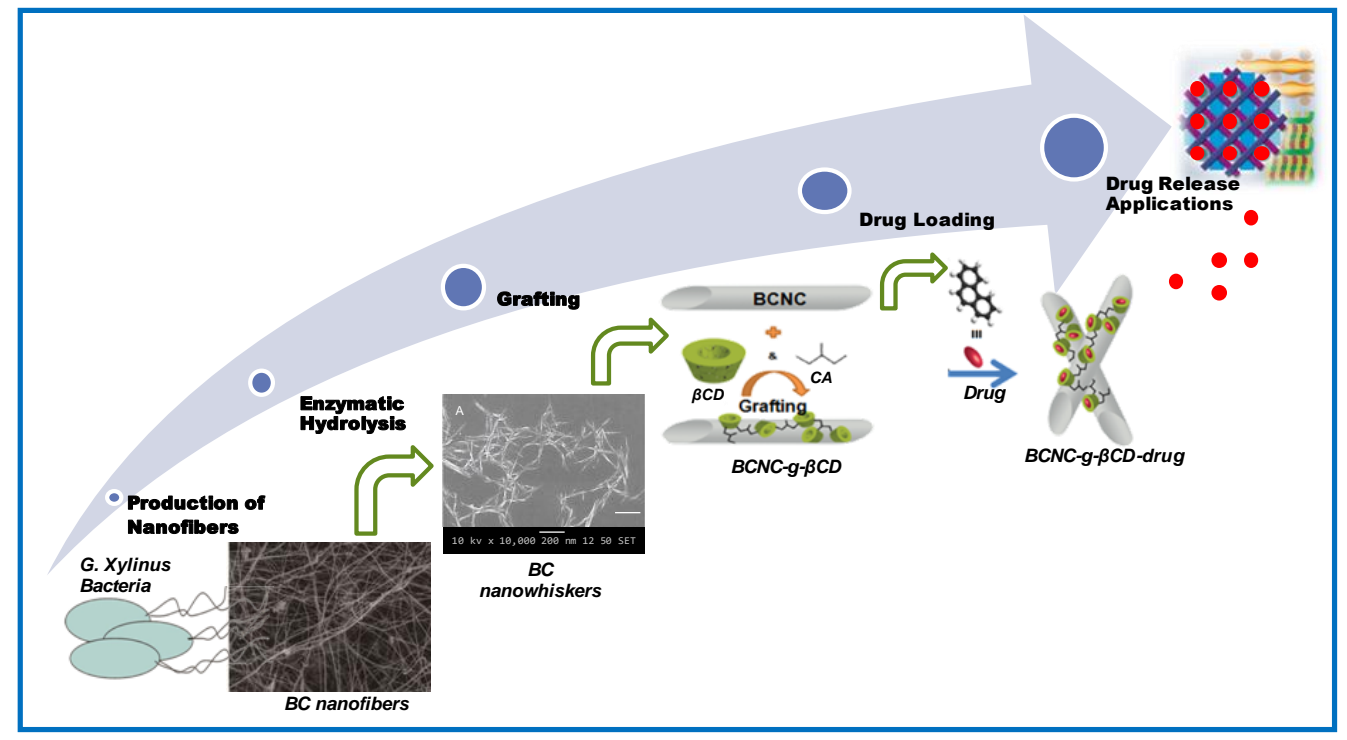

*This chapter is adapted from a manuscript accepted for publication by the Canadian Journal of Chemical Engineering (CJCE-18-1022). Mohamed M. Khattab, Yaser Dahman. Functionalized Bacterial Cellulose Nanowhisker as Long Lasting Drugs Nanocarriers for Antibiotics and Anticancer Drugs. 


\subsection{Introduction}

Nanocellulose is a natural nanoscaled material that possesses diverse of unique characteristics. These not limited to its biocompatibility and lack of toxicity, but also include surface chemical reactivity, nano-dimensions, higher crystalinity, special rheological properties, morphology, selfassembly and liquid crystalline behavior and mechanical reinforcing ability. ${ }^{[8]}$ Based on these unique properties, both "nano-enhanced" and "nano-enabled" cellulosic products have been envisioned for different uses. These ranged from bulk applications like rheological modifier, paper additive and composite reinforcement, or to high-end applications such as functional materials, tissue engineering applications and drug delivery. ${ }^{[366]}$

Cellulose nanofibers can be subjected to acidic or enzymatic hydrolysis that subsequently leads to the formation of nanocrystals. Cellulose nanocrystals (CNCs) or nanowhiskers (CNWs) are used interchangeably in literature for referring to nanocellulose. ${ }^{[367]}$ Bacterial cellulose nanowhisker (BCNC) are obtained by hydrolysis of bacterial cellulose nanofibers (BC). It was biosynthesized in highly crystalline and pure form by the most widely used bacterial strain Gluconacetobacter xylinus (G. xylinus). ${ }^{[368]}$ Contrary to CNC obtained from the hydrolysis of plant-derived cellulose, no exhaustive processing is required to remove unwanted impurities (lignin, pectin and hemicelluloses) from BC. ${ }^{[46]}$ The stability of $\mathrm{BC}$ over wide range of temperatures makes it amenable to heat sterilization. ${ }^{[54]} \mathrm{BC}$ can be easily purified by sodium hydroxide from any cell debris. This yields a form with an acceptable range of endotoxin values $(<20$ endotoxin units/device) which is potentially safe for intravenous applications (FDA, 2012) ${ }^{[369]}$ Attributed to its biosynthetic procedure BC is commonly regarded as a material possessing advantages, both biocompatibility and hemocompatibility compared to other types of cellulose ${ }^{[370]}$ Surprisingly, direct investigations on the biocompatibility of BCNC are rare. In terms of biodegradation, cellulose is considered nonbiodegradable in vivo, or at best slowly degradable in human body due to its lack of cellulolytic enzymes. However, recent reports have shown that the form of cellulose (i.e. size, crystallinity and hydration) might affect the degree of degradation and absorption. ${ }^{[64]}$ For instance, compared with crystalline cellulose $75 \%$ (w/w) of equivalent amorphous regenerated samples can be degraded and absorbed over 6-weeks. ${ }^{[371]}$ Very recently researchers attempted to improve the biodegradation of nanocellulose (from 2-4 weeks) through in vitro oxidation in a simulated body fluid by periodate oxidation. ${ }^{[372]}$ 
BCNC or "nanowhisker" are rod-like shaped particles with typical dimensions of 5-20 nm in diameter and 50-500 $\mathrm{nm}$ in length, with a high aspect ratio (length/diameter) of 2 to100. ${ }^{[16]}$ BCNC falls under the category of non-spherical nanocarriers that are considered as better candidates for drug targeting/delivery applications. This is due to their enormous surface area-tovolume ratio, which contributes to more interactions with targets and increased permeability. ${ }^{[17]}$ Cellular uptake of cancer drugs-conjugated CNCs (FITC-CNC-FA) in C6 cancer cells targets was found evident by fluorescence microscopy. ${ }^{[18]}$

The abundant of surface hydroxyl groups provides sites for surface modification and functionalization of nanocellulose by a variety of methods. These methods are esterification, oxidation, cationization, fluorescent labeling, and polymer grafting. ${ }^{[13]}$ Surface modification provides great opportunity for more control over structure-function relationship. It can also be used to modulate the loading and release of drugs that would not normally bind to nanowhisker, such as non-ionized hydrophobic cancerous drugs. Latest developments concerning the use of functionalized CNCs for emerging biomedical applications include drug delivery systems, ${ }^{[27}$, 208,357] tissue engineering scaffolds, ${ }^{[373,374]}$ and bioimaging. ${ }^{[199]}$ Additionally, BCNC is a low-cost material and can be produced from renewable and sustainable resources. Therefore, its uses provide an environmental advantage compared to other non-sustainable nanomaterials.

Cyclodextrins (CDs) are a series of cyclic and non-reducing oligosaccharides that have doughnut-like structures with hydrophobic inner cavities and hydrophilic outer surfaces. ${ }^{[65]}$ CDs do not elicit an immune response due to its biocompatibility and low toxicities. ${ }^{[66]}$ Among them, $\beta C D$ is the most commercially attractive system due to its low water solubility and availability at low cost production. ${ }^{[67]}$ Furthermore, $\beta C D$ has an ideal hydrophobic central cavity with dimensions that match the size of drugs and small organic molecules. This allows the formation of inclusion complexes (IC) with those guest compounds via non-covalent interactions. Which in tern improves the chemical stability of drugs, bioavailability, absorption, and controls their releases. ${ }^{[70]}$ The formations of IC significantly increase the solubility of the drugs in body fluids and facilitate their transportation through the lipid cell membrane. ${ }^{[375]}$ For instance, the inclusion complex produced from the reaction between the cationic $\beta$-cyclodextrin $(\beta C D)$ and anionic CNCs was used to encapsulate curcumin for controlling its release. The later nanocomplex showed an anti-proliferative effect on rectal and prostatic cancer cell lines, where the $\mathrm{IC}_{50}$ was found to be lower than using free curcumin. ${ }^{[376]}$ 
A number of crosslinking agents have been used by researchers for grafting $\beta C D$ onto cellulose nanofibers. Such as epichlorohydrin, ${ }^{[10]}$ cyanuric chloride, ${ }^{[78]} \mathrm{N}$-methylol acrylamide ${ }^{[377]}$ and polycarboxylic acids (e.g. citric acid) ${ }^{[80]}$ However, a low grafting efficiency was obtained which ultimately hindered their performance and uses. ${ }^{[10,11]}$ The maximum grafting ratio reported for immobilizing $\beta C D$ on $\mathrm{CNC}$ was about $18 \%(\mathrm{w} / \mathrm{w}){ }^{[46]}$ In this work epichlorohydrin and citric acid were used as crosslinking agents for grafting $\beta \mathrm{CD}$ onto $\mathrm{BCNC}$ to obtain functionalized $\beta C D-g$-BCNC with higher grafting ratio. This was done to produce drug nanocarrier systems based on BCNC, having a higher surface area. In addition, to have a large number of immobilized $\beta C D$ molecules on the surface that is capable for loading and releasing higher concentration of drug molecules.

The objective of this work is to develop drug-nanocarrier based on the biocompatible BCNC. The envisioned nanocarrier is able to conjugate a high payload of either ionized or hydrophobic anticancer drugs. As well as releases the conjugated drug in a sustained and controlled manner over longer time. The first part of the present study focused on the synthesis and characterization of true cellulose nanowhisker. This was followed by grafting its surface with $\beta C D$ molecules to obtain functionalized BCNC-g- $\beta C D$. The second part focused on harnessing the chemical and physical properties of the functionalized BCNC-g- $\beta C D$. This was done by examining their capabilities for loading and releasing a variety of model drugs, such as antibiotics and ionized/hydrophobic cancerous drugs. In addition, the drug release profiles and the kinetics of releases are also examined and compared.

\subsection{Material and Methods}

\subsubsection{Materials}

Bacterial strain G. xylinus (ATCC 700178) was supplied by American Type Culture Collection (ATCC), Manassas, VA 20108, USA. All materials used to prepare the different media of G. xylinus bacterial strain were as follows: Agar, Corn steep liquor (CSL), Ammonium Sulfate $\left(\mathrm{NH}_{4}\right)_{2} \mathrm{SO}_{4}, \mathrm{~L}(+)$ Albinos, D-biotin, Calcium Carbonate $\left(\mathrm{CaCO}_{3}\right)$, Calcium Chloride dihydrate $\left(\mathrm{CaCl}_{2} \cdot 2 \mathrm{H}_{2} \mathrm{O}\right)$, Copper Sulfate pentahydrate $\left(\mathrm{CuSO}_{4} \cdot 5 \mathrm{H}_{2} \mathrm{O}\right)$, Ferrous Sulfate heptahydrate (FeSO $\mathrm{S}_{4} .7 \mathrm{H}_{2} \mathrm{O}$ ), Folic Acid, Fructose, Hydroxymethyl Furfural (5-HMF), Inositol, Magnesium Sulfate heptahydrate $\left(\mathrm{MgSO}_{4} \cdot 7 \mathrm{H}_{2} \mathrm{O}\right)$, Manganese Sulfate Pentahydrate $\left(\mathrm{MnSO}_{4} \cdot 5 \mathrm{H}_{2} \mathrm{O}\right)$, Monopotassium Phosphate $\left(\mathrm{KH}_{2} \mathrm{PO}_{4}\right)$, Nicotinic Acid, D-Pantothenic Acid, Pyridoxine 
Hydrochloride, Riboflavin, Sodium Molybdenum Oxide Dihydrate $\left(\mathrm{NaMoO}_{4} \cdot 2 \mathrm{H}_{2} \mathrm{O}\right)$, Zinc Sulfate heptahydrate $\left(\mathrm{ZnSO}_{4} \cdot 7 \mathrm{H}_{2} \mathrm{O}\right)$, Thiamine Hydrochloride, and D-(+)Xylose.

For preparing BCNC, grafting reactions, and drug loading and release studies the following chemicals were used: Citric acid (CA), Epichlorohydrin (ECH, 99+\%), $\beta$-cyclodextrin $(\beta C D)$, Sodium Hypophosphite (SHP), Sodium Hydroxide, Ciprofloxacin Hydrochloride (CIP), Doxorubicin Hydrochloride (DOX), Paclitaxel (PTX), Cellulase enzyme (Trichoderma reesei ATCC26921; specific enzyme activity $700 \mathrm{U} / \mathrm{g}$ ), Phosphate buffered saline (pH 7.4 and 6.4), DMSO, Acetone, and Methanol. All chemicals were supplied by Sigma Aldrich.

\subsubsection{Synthesis}

\subsubsection{Production of BC Nanofibers}

In this study G. xylinus was used as BC producing strain. It was activated in accordance with ATCC guidelines in a sterile YGC 459 medium. Production was done according to the method of Sani and Dahman. ${ }^{[378]}$ In brief, the production was conducted in $500 \mathrm{~mL}$ flasks under static and stirring conditions. Aseptically inoculated fermentation media $(200 \mathrm{~mL}$ ) containing fructose (as carbon source) and CSL (as nitrogen source) were used for production experiments. A production of $6.0 \mathrm{~g} / \mathrm{L}$ was achieved from stirred culture, while lower yield of $4.0 \mathrm{~g} / \mathrm{L}$ were obtained from static culture.

\subsubsection{Preparation of BC Nanowhisker (BCNC)}

\subsection{Enzymatic hydrolysis}

BC microfibril paste was transformed into nanocrystals by enzymatic hydrolysis using cellulose enzyme (Trichoderma reesei ATCC26921). ${ }^{[198]} 10 \mathrm{~g}$ wet weight of BC paste was suspended in $250 \mathrm{ml}$ of acetate buffer $(0.1 \mathrm{M}, \mathrm{pH} 5.0)$ to which $1 \mathrm{ml}$ of cellulase enzyme was added. This suspension was kept in shaker at $50^{\circ} \mathrm{C}$ for $24 \mathrm{~h}$. The resulting suspension was filtered through sintered crucibles No. 1 to remove unhydrolyzed fibers. Filtrate was then centrifuged at $(4,000$ $\mathrm{rpm} \times 30 \mathrm{~min}$ ) to terminate the enzymatic reaction and to collect the cellulosic nanowhisker. Finally, BCNC suspension was re-concentrated to $0.5 \%(\mathrm{w} / \mathrm{w})$ and stored at $5^{\circ} \mathrm{C}$ for further use. 


\subsection{Acid hydrolysis}

According to Sacui et al, ${ }^{[190]}$ ground BC $(10 \mathrm{~g})$ was dispersed in hydrochloric acid (200 ml of $37 \%$ by mass) for $48 \mathrm{~h}$ at $60^{\circ} \mathrm{C}$ under stirring in oil bath. BCNC was recovered by diluting reaction mixture 10 times with deionized water followed by filtration through sintered crucibles No. 1 to remove unhydrolyzed fibers. Extra acid in the filtrate was removed by repeated filtration and re-dispersion cycles using centrifuge $(4000 \mathrm{rpm} \times 20 \mathrm{~min})$ until a neutral $\mathrm{pH}$ was obtained. Similarly, $\mathrm{H}_{2} \mathrm{SO}_{4}$-hydrolysis BCNC was obtained using the method of Habibi et al. ${ }^{[45]} \mathrm{BC}(10 \mathrm{~g})$ was dispersed in $175 \mathrm{ml}$ of $65 \%$ (w/w) $\mathrm{H}_{2} \mathrm{SO}_{4}$ solution. The hydrolysis was carried out at $55^{\circ} \mathrm{C}$ for $3 \mathrm{~h}$ under continuous stirring in oil bath. The resulting suspensions were filtered and then washed by distilled water. Finally, the suspension was dialyzed against deionized water for approximately 5 days using a dialysis bag of membranes with molecular weight cut-off 12,00014,000. In all cases BCNC suspension was re-concentrated to $0.5 \%(\mathrm{w} / \mathrm{w})$ and kept at $5^{\circ} \mathrm{C}$ for further use.

\subsubsection{Grafting of Nanowhisker}

Grafting of $\beta C D$ onto $\mathrm{BCNC}$ surface was attempted using two different methods. Citric acid (CA) was employed as green linker for grafting $\beta C D$ in the first method attempted. A two-step reaction was applied according to El-Tahlawy et al. ${ }^{[379]}$ The esterification reaction is activated by the weak acid salt catalyst sodium hypophosphite (SHP). In the first reaction $\beta$-cyclodextrin citrate (CA- $\beta C D$ ) was prepared by boiling $3.0 \mathrm{~g}$ of $\beta \mathrm{CD}, 1.1 \mathrm{~g}$ of CA, $0.29 \mathrm{~g}$ of SHP, and $1.8 \mathrm{~g}$ of distilled water at $100^{\circ} \mathrm{C}$ for $1.5 \mathrm{~h}$. The product was purified by washing with ethanol several times, followed by drying at $60^{\circ} \mathrm{C}$ for $24 \mathrm{~h}$ to obtain pure dry $\mathrm{CA}-\beta \mathrm{CD}$. In the second reaction a total of $3.0 \mathrm{~g}$ of the previously prepared CA- $\beta C D$ and $0.1 \mathrm{~g}$ of SHP were dissolved in $10 \mathrm{ml}$ of distilled water. This was followed by the addition of $20 \mathrm{ml}$ of $0.5 \%(\mathrm{w} / \mathrm{w}) \mathrm{BCNC}$ suspension preultrasonicated for $10 \mathrm{~min}$. The reaction proceeded at $160^{\circ} \mathrm{C}$ for $15 \mathrm{~min}$. After the reaction, excess solution was removed by decantation, and then the suspension was washed with acetone and distilled water through multi centrifugation cycles until the suspension became neutral.

The other grafting method of $\beta C D$ onto the surface of $\mathrm{BCNC}$ was examined using epichlorohydrin $(\mathrm{ECH})$ as a linker under alkaline conditions. ${ }^{[11]}$ First $20 \mathrm{~mL}$ of $0.5 \%(\mathrm{w} / \mathrm{w})$ BCNC suspension in $50 \mathrm{ml}$ conical flask was diluted with $20 \mathrm{ml}$ of distilled water and then ultrasonicated for 15 min. Following that, $5.0 \mathrm{~g}$ of $\mathrm{NaOH}$ was dissolved into the reaction mixture 
with frequent shaking. Then, $1.3 \mathrm{~g}$ of $\beta C D$ was added to the flasks with continuous shaking for 2 h until a homogeneous suspension was obtained. Two hours later the desired amount of ECH was added into the reaction medium, and the reaction was heated at $45^{\circ} \mathrm{C}$ for $6 \mathrm{~h}$. The $\mathrm{ECH} / \mathrm{\beta CD}$ molar ratio was controlled below 10 in order to avoid self-cross-linking of $\beta$-CD. ${ }^{[32]}$ After grafting, the suspension of (BCNC-g- $\beta \mathrm{CD})$ was re-concentrated to $0.5 \%(\mathrm{w} / \mathrm{w})$ and kept at $5^{\circ} \mathrm{C}$ for further use.

\subsection{Experimental Procedure}

Experiments carried out in triplicate and results were expressed as mean \pm standard error. Standard errors are represented as error bars in all figures.

\subsubsection{Determination of Grafting Ratio}

The grafting ratio of $\beta \mathrm{CD}$ onto the $\mathrm{BCNC}$ was determined using two different methods for comparison. These include gravimetric method ${ }^{[380]}$ and UV-vis spectrophotometry using phenolphthalein $(\mathrm{Ph} . \mathrm{Ph})$ inclusion method. ${ }^{[46]}$ In case of gravimetric method; grafting ratio is determined from the mass of nanowhisker before and after grafting reaction. For spectrophotometric determination of grafting ratio; $0.5,1,1.5,2,2.5$ and $3 \mathrm{~mL}$ of $0.02 \mathrm{M} \beta \mathrm{CD}$ stock solution were diluted to $25 \mathrm{~mL}$ by freshly prepared $0.01 \mathrm{M} \mathrm{Ph}$. $\mathrm{Ph}$ solution dissolved in $0.02 \mathrm{M} \mathrm{Na}_{2} \mathrm{CO}_{3}$ buffer. The flasks containing $\mathrm{Ph}$. $\mathrm{Ph} / \beta \mathrm{CD}$ suspensions were stirred for $30 \mathrm{~min}$ and equilibrated overnight before measuring the absorbance. Sample without $\mathrm{Ph}$. $\mathrm{Ph}$ was also prepared as blank control. Afterward, a weighed amount of BCNC- $g-\beta C D$ is ultrasonicated for $15 \mathrm{~min}$, and then diluted to $25 \mathrm{ml}$ with buffered $\mathrm{Ph}$. Ph solution and stabilized for $20 \mathrm{~h}$. The absorbance of all solutions was acquired at the characteristic wavelength of $552 \mathrm{~nm}$ (Perkin Elmer Lambda 40 dual beam UV/vis). The negative correlation standard curve was constructed between the weights of $\beta C D$ used and the corresponding $\mathrm{Ph}$. Ph absorbance values. The Grafting ratio was calculated from the weight of $\mathrm{BCNC}-g-\beta C D$ used, and the amount of grafted $\beta C D$ that determined from the calibration curve according to Equation (3.1) below;

$$
\text { Grafting Ratio }=\frac{\mathrm{m}_{(\beta C D)}}{\mathrm{m}_{(\mathrm{BCNC}-g-\beta \mathrm{CD})}} \times 100 \%
$$

where $\mathrm{m}_{(\beta \mathrm{CD})}$ and $\mathrm{m}_{(\mathrm{BCNC}-\mathrm{g}-\beta \mathrm{CD})}$ are the mass of $\beta \mathrm{CD}$ and $\mathrm{BCNC}-\mathrm{g}-\beta \mathrm{CD}$. 


\subsubsection{Determination of Binding Constants of Drug- $\beta C D$ Inclusion Complexes}

Absorption measurements are performed to determine the binding constant $(K)$ of the inclusion complexes that formed between drugs and $\beta \mathrm{CD}$, as well as assessing the spontaneity of reaction. The measured solutions were prepared as follow: $0.25 \mathrm{~mL}$ of $2 \times 10^{-3} \mathrm{M}$ stock drug solution is added to six $5 \mathrm{ml}$ volumetric flasks. All flasks are made up to the final volume using different concentrations of $\beta C D$ solutions; 0.002, 0.004, 0.006, 0.008, 0.010 and $0.015 \mathrm{M}$. Final concentration of drug in each flask was kept constant at $10^{-4} \mathrm{M}$.

The binding constant of the (1:1) inclusion complexes $\left(K_{1: 1}\right)$ was evaluated from BenesiHildebrand plot ${ }^{[381]}$ shown in Equation (3.2).

$$
\begin{gathered}
\left.(\text { Drug })_{\text {free }}+(\beta C D)_{\text {free }} \stackrel{K_{1: 1}}{\longleftrightarrow}(\text { Drug- } \beta C D)_{\text {inclusion complex }}, K_{1: 1}=\frac{[\text { Drug- } \beta C D]}{[\text { Drug }][\beta C D]}\right] \\
{[\text { Drug }]+[\beta C D] \stackrel{K_{1: 1}}{\longleftrightarrow}[\text { Drug- } \beta C D]} \\
\frac{1}{\Delta A}=\frac{1}{\Delta \varepsilon}+\frac{1}{\mathrm{~K}[\text { Drug }]_{0} \Delta \varepsilon[\beta C D]_{0}}
\end{gathered}
$$

Where $[$ Drug- $\beta C D]$ denotes to the concentration of inclusion complex formed between drug and $\beta \mathrm{CD} ; K_{1: 1}$ is the binding constant of 1:1 complex; $\Delta \mathrm{A}$ is the difference between the absorbance of drug in presence and absence of $\beta C D ; \Delta \varepsilon$ is the difference between the molar extinction coefficients of drug and drug- $\beta C D$ complex, $[\text { Drug }]_{0}$ and $[\beta C D]_{0}$ are the initial concentrations of drug and $\beta C D$. Spontaneity of inclusion reaction is proved from the Gibbs free energy change $(\Delta \mathrm{G})$ for the host-guest inclusion process. It was evaluated from the calculated binding constant values using Equation (3.3) at $310 \mathrm{~K}$.

$$
\Delta \mathrm{G}=-2.303 \mathrm{RT}(\log K)
$$

where $\Delta \mathrm{G}$ is the change in Gibbs free energy, $\mathrm{R}$ is the universal gas constant $(\mathrm{J} / \mathrm{mol} \mathrm{K}), \mathrm{T}$ is the temperature in Kelvin, and " $K$ " is the binding constant in mole ${ }^{-1}$.

\subsubsection{Effect of Drug Concentration on Loading Efficiency}

The maximum drug payload on nanowhisker was estimated spectrophotomertically as follow: $0.5 \mathrm{~mL}$ of $0.2 \% \mathrm{w} / \mathrm{w}(1 \mathrm{mg})$ of either unmodified BCNC or BCNC- $g-\beta C D$ was suspended in 10 $\mathrm{mM}$ PBS at $\mathrm{pH} 7.4$ and then ultrasonicated for $15 \mathrm{~min}$. Each of which was then mixed with increased increments of $2000 \mu \mathrm{g} / \mathrm{ml}$ drug; [Drug total]. Drug/BCNC suspensions were kept in 5 $\mathrm{mL}$ centrifuge tube and incubated at $25^{\circ} \mathrm{C}$ overnight with continuous stirring. Drug/BCNC suspensions were centrifuged at $4000 \times \mathrm{g}$ for 15 minutes to separate the nanowhisker with 
conjugated drug. The concentration of unbound drug in the supernatant [Drug unbound $_{\text {d was assayed }}$ using UV-vis spectrophotometer at wavelengths of 275, $482 \mathrm{~nm}$ for CIP and DOX and using

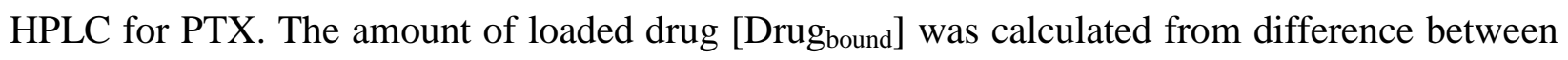
total and final drug concentrations in the supernatant. Drug loading \% (w/w) was calculated according to Equation (3.4).

$$
\text { Drug loading } \%(\mathrm{w} / \mathrm{w})=\frac{\text { weight of the drug bound to nanowhiskers }}{\text { weigh of nanowhiskers }} \times 100
$$

For drug loading and release studies calibration curves were constructed for every drug, using series of standard solutions prepared in PBS that have final concentrations ranged from 10-900 $\mu \mathrm{g} / \mathrm{mL}$. The released amount of drug at different time intervals was estimated from the calibration curve using Equation (3.5).

$$
\mathrm{q}_{\mathrm{t}}(\mu \mathrm{g} / \mathrm{mg})=\frac{\left(\mathrm{C}_{0}-\mathrm{C}_{\mathrm{t}}\right) \mathrm{V}}{\mathrm{w}}
$$

Where $\mathrm{q}_{\mathrm{t}}$ is the amount of loaded drug $(\mu \mathrm{g})$ at time $(\mathrm{t})$ on $1 \mathrm{mg}$ equivalent of oven dried nanowhisker, $\mathrm{C}_{0}(\mu \mathrm{g} / \mathrm{mL})$ is the initial drug concentration, $\mathrm{C}_{\mathrm{t}}$ is the concentration of drug at time $(\mathrm{t}), \mathrm{V}$ is the volume of the solution $(\mathrm{mL})$, and $\mathrm{W}$ is the mass of oven dried nanowhisker $(\mathrm{mg})$.

\subsubsection{In vitro Release Measurements}

The drug loaded BCNC- $g-\beta C D$ samples were suspended in $1 \mathrm{~mL}$ of PBS (pH 7.4 and 6.4) and then maintained at $37^{\circ} \mathrm{C}$ in $5 \mathrm{~mL}$ centrifuge tube under continuous stirring $(15 \mathrm{rpm})$. At specific times the suspensions were centrifuged $(4,000 \mathrm{rpm} \times 20 \mathrm{~min})$ and supernatant was removed. The concentration of CIP or DOX in the supernatant was determined by UV-vis spectroscopy, whereas PTX was determined by HPLC. After measurements samples were returned to the $5 \mathrm{ml}$ tubes where drug-BCNC- $g-\beta C D$ nanocomplexes were re-suspended. The percentage of cumulative drug release in PBS solution (i.e., at pH 6.4 and 7.4 ; and $37^{\circ} \mathrm{C}$ ) was calculated as a function of time using Equation (3.6).

$$
\text { Cumulative release }(\%)=\frac{\mathrm{C}_{\mathrm{t}} \mathrm{V}}{\mathrm{m}_{0}} \times 100
$$

Where $\mathrm{m}_{0}(\mu \mathrm{g})$ is the initial loaded amount of drug on BCNC-g- $\beta$ CD samples, $\mathrm{Ct}(\mu \mathrm{g} / \mathrm{mL})$ is the concentration of drug at any time, and $\mathrm{V}(\mathrm{mL})$ is the volume of the solution. 


\subsection{Characterization Techniques}

\subsubsection{SEM and AFM microscopy}

Surface morphology of BCNCs and BCNC- $g-\beta C D$ samples were examined using JEOL 6380LV scanning electron microscope (SEM), with an accelerating voltage of 15-20 kV. Samples made conductive by depositing a fine layer of gold using an ion sputter coater with a low deposition rate. The topography and morphology of the BCNCs were imaged using AFM (NT-MDT NTEGRA-Multimode Scanning Probe Microscope). Before imaging, a droplet of sample suspension was initially dried on mica slide and scans were obtained in air using semi-contact mode. The length of the nanocrystals was measured using image analysis software and the diameter was determined from transverse line profile of nanocrystals (i.e. the height difference between mica substrate and particle).

\subsubsection{X-ray Diffraction}

The structural analysis of the nanowhisker samples was evaluated by X-ray diffraction using a (MiniFlex600 XRD) diffractometer equipped with PDXL2 analysis software. The XRD patterns were obtained over $2 \theta$ range of $\left(5-50^{\circ}\right)$ with scan rate of $5^{\circ}$ per min. $\mathrm{Cu} \mathrm{K}_{\alpha}$ radiation source $(\lambda=$ $1.54060 \AA$ ) was operating at $40 \mathrm{kV}$ and $30 \mathrm{~mA}$. The crystal thickness of cellulose-I structure (t; $\mathrm{nm}$ ) was calculated from XRD analysis using width at half height of the diffraction peak in respect of (200) crystal plane using Equation (3.7). ${ }^{[382]}$

$$
\text { Crystal thickness }(t)=\frac{\kappa \lambda}{\beta_{1 / 2} \cos \theta}
$$

Where $\kappa$ is the correction factor and usually taken to be $0.91, \lambda$ is the radiation wavelength, $\theta$ is the diffraction angle, and $\beta_{1 / 2}$ is the corrected angular width (in radians) at half maximum intensity.

Crystallinity index (Ic) was calculated by Ic $=\left[\left(\mathrm{I}_{(002)}-\mathrm{I}_{(\mathrm{am})}\right) / \mathrm{I}_{(002)}\right] \times 100$, where, $\mathrm{I}_{(002)}$ is the intensity corresponds to the peak signal at $\left(2 \theta=19.5^{\circ}\right), \mathrm{I}_{(\mathrm{am})}$ is the intensity of the corresponding amorphous material at $\left(2 \theta=12^{\circ}\right) .^{[383]}$

\subsubsection{Fourier Transform Infra-Red (FTIR)}

The structural changes that accompanied the surface chemical modifications of BCNC were investigated by FTIR spectroscopy using Perkin Elmer Spectrum1 FTIR/ATR spectrophotometer. FTIR spectra in attenuated total reflection mode (ATR) were recorded for 
oven dried samples $\left(105^{\circ} \mathrm{C} ; 4-5 \mathrm{~h}\right)$ in the range of $4000-500 \mathrm{~cm}^{-1}$. ATR-crystal is ZnSe, the spectral resolution is $4 \mathrm{~cm}^{-1}$ and 20 scans were collected for each spectrum.

\subsubsection{Solid-state ${ }^{13}$ C NMR}

Cross-Polarization Magic Angle Spinning (CPMAS) solid-state ${ }^{13} \mathrm{C}$ NMR spectra of BCNC and BCNC-g- $\beta$ CD were obtained using Bruker II Advance-300 spectrometer (Karlsruhe, Germany). A 4.0 mm MAS spinning Bruker probe was operating at resonance frequencies of $75.47 \mathrm{MHz}$. The spinning speed was set to $5 \mathrm{kHz}$ at room temperature and time delay was $60 \mathrm{~s}$. Each spectrum is an accumulation of 50 scans. A $100 \mathrm{mg}$ dried nanocellulose sample was pressed into a $6 \mathrm{~mm} \times 7 \mathrm{~mm}$ disk, placed into a Macor rotor and spun at $3800 \pm 100 \mathrm{~Hz}$.

\subsubsection{HPLC}

HPLC analysis for drug concentration was done by using Perkin Elmer Series 200 pump and autosampler HPLC system equipped with UV-vis detector working at $232 \mathrm{~nm}$. Separation was achieved using Novapak $\mathrm{C}_{18}$ column with $20 \mu \mathrm{L}$ injections. The flow rate of mobile phase was 1 $\mathrm{mL} / \mathrm{min}$. The mobile phase composed of $58 \%$ acetonitrile, $37 \%$ distilled $\mathrm{H}_{2} \mathrm{O}$, and $5 \%$ methanol.

\subsection{Results}

Figure 3.1a-c compiles FE-SEM micrographs show the morphologies of unhydrolyzed BC nanofibers as well as BCNCs that obtained by different hydrolysis methods. According to this figure, all BCNC samples showed rod-shape morphology in the nanoscale with an average size of 200-500 nm. Similarly, AFM of enzymatically hydrolyzed BCNC shown in Figure 3.1d confirmed the size and morphology by SEM in addition to the measured width. The width was determined using the height difference between the nanocrystals and mica wafer and found equal to $4.6 \mathrm{~nm}$. SEM image of the grafted BCNC in Figure 3.1e shows similar length to unmodified ones, but with larger width of $\sim 15 \mathrm{~nm}$. 

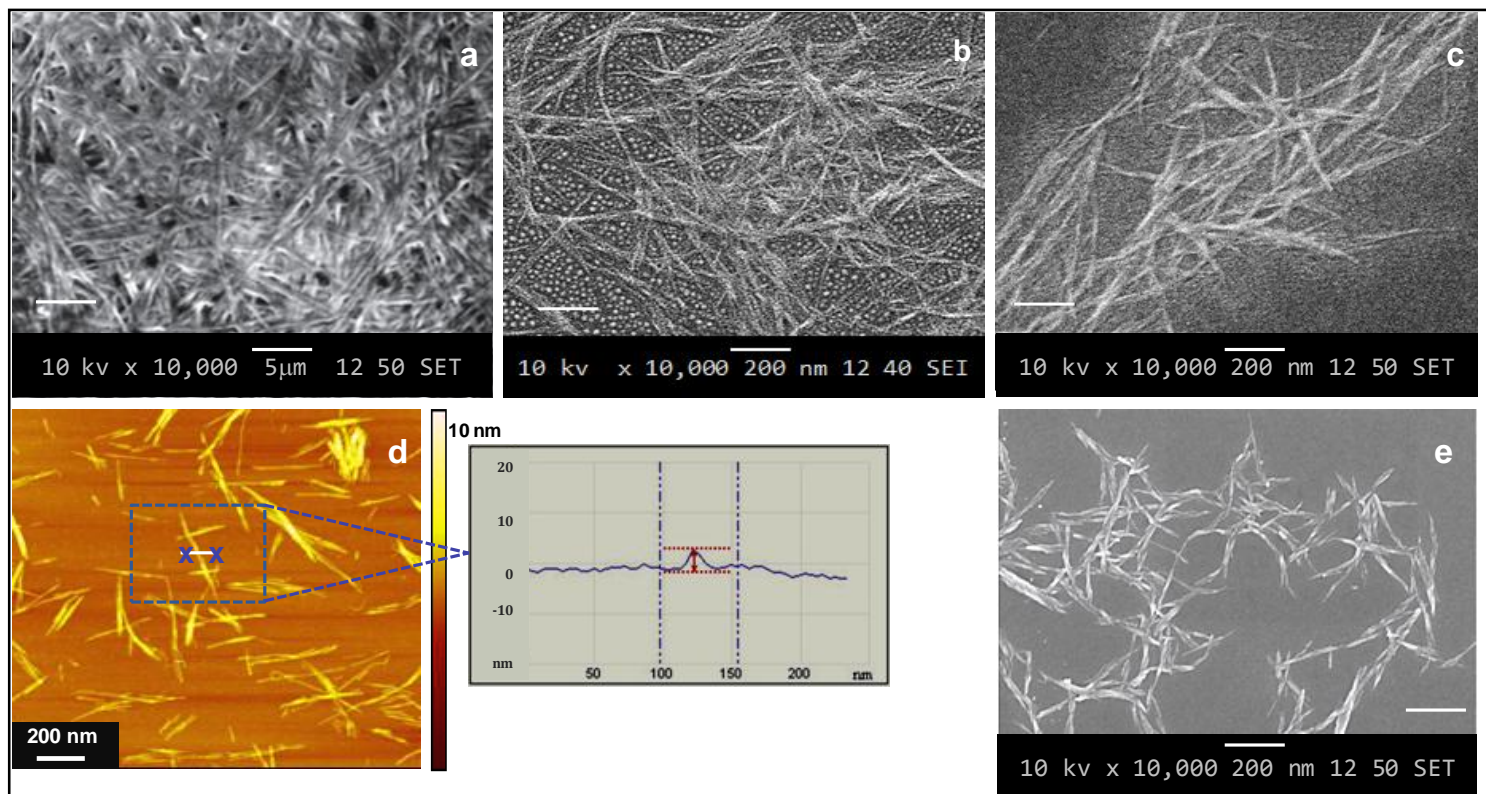

Figure 3.1 Morphologies of BCNCs. Top; (a, b and c) FE-SEM micrograph for unhydrolyzed $\mathrm{BC}$ nanofibers, $\mathrm{BCNC}$ produced by $\mathrm{H}_{2} \mathrm{SO}_{4}$ hydrolysis, and $\mathrm{BCNC}$ produced by $\mathrm{HCl}$ hydrolysis. Bottom; (d) AFM image of BCNC produced by enzymatic hydrolysis along with illustration for width determination, (e) SEM micrograph for BCNC- $g-\beta C D$.

XRD diffractograms in Figure 3.2a-d show the change in nanocellulose crystallinity up on hydrolysis and grafting reactions. Analyzing this figure shows that all BCNCs diffractograms exhibited very similar diffraction peaks around $2 \theta$ values of $12^{\circ}, 19.5^{\circ}$, and $32^{\circ}$. These diffraction peaks represent the presence of (110), (200), and (004) crystal planes typical to cellulose-I structure, respectively. The crystalinity index $\left(\mathrm{I}_{\mathrm{c}}\right)$ of BCNC samples was calculated from the corresponding diffractograms using the amorphous subtraction method. ${ }^{[383]}$ Values of $I_{c}$ are ranged from 69.3-79.5\% and listed in Table 3.1. The obtained values are found to be higher than unhydrolyzed BC nanofibers (65\%). The highest crystalinity of $79.5 \%$ was achieved by the enzymatically hydrolyzed BCNC. Moreover, the crystal thickness that estimated from the diffraction peaks at (200) crystal plane using Scherrer's equation ${ }^{[382]}$ were ranged from 6.5-7.1 nm (Table 3.1). Figure 3.2e shows XRD pattern of BCNC- $g-\beta C D$, which exhibited the same peak positions of the ungrafted BCNC, but with decreased intensity. 


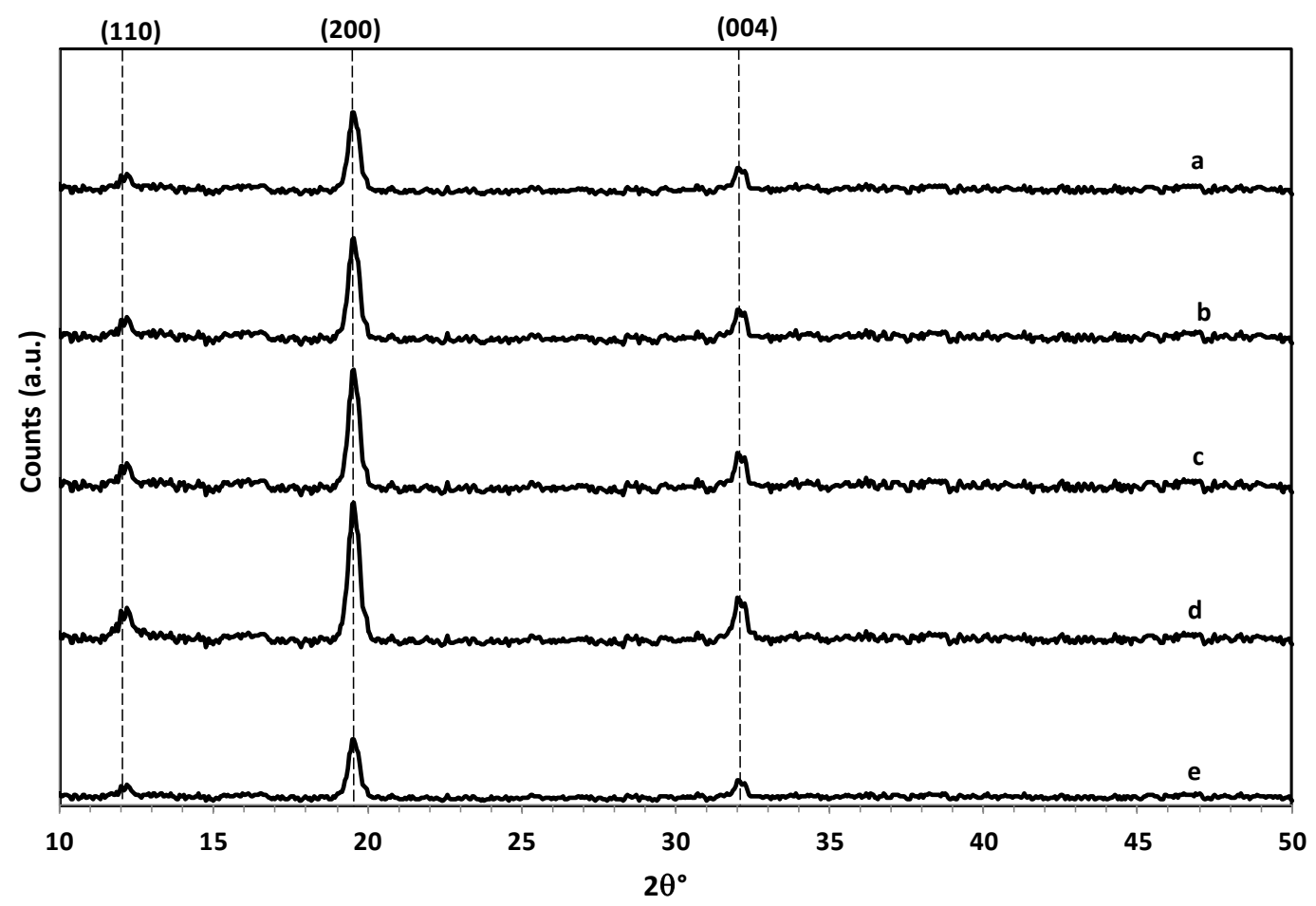

Figure 3.2 XRD diffractograms of: (a) unhydrolyzed $\mathrm{BC}$, (b) $\mathrm{H}_{2} \mathrm{SO}_{4}$ hydrolyzed $\mathrm{BCNC}$, (c) $\mathrm{HCl}$ hydrolyzed BCNC, (d) enzymatically hydrolyzed BCNC, and (e) BCNC- $g-\beta C D$.

Table 3.1 lists the observed physicochemical properties, sizes and crystallinities of the obtained BCNC by using different methods of hydrolysis. Sulfuric acid hydrolyzed BCNC showed the highest dispersion in aqueous medium, but the least chemical reactivity towards grafting reaction. Moreover, $\mathrm{BCNC}$ that was prepared by hydrochloric acid and enzymatic hydrolysis showed limited dispersion in aqueous media with greater tendency for flocculation, however, grater reactivity towards grafting reaction. 
Table 3.1 Sizes, crystalinity and physicochemical properties of BCNCs samples obtained by different hydrolysis

\begin{tabular}{|c|c|c|c|c|}
\hline Nanowhisker type & $\mathrm{I}_{\mathrm{c}}(\%)^{*}$ & $\begin{array}{c}\text { Crystal thickness } \\
(\mathrm{nm})^{* *}\end{array}$ & Advantages & Disadvantages \\
\hline Unhydrolyzed BC & 65.2 & 12.7 & NA & NA \\
\hline $\mathrm{H}_{2} \mathrm{SO}_{4}$-hydrolyzed & 69.3 & 5.6 & $\begin{array}{l}\text { - Well dispersion with } \\
\text { no agglomeration }\end{array}$ & $\begin{array}{ll}\text { - } & \text { Low thermal stability } \\
\text { - } & \text { Lowest reactivity toward } \\
\text { grafting reaction }\end{array}$ \\
\hline HCl-hydrolyzed & 73.6 & 7.1 & $\begin{array}{ll}\text { - } & \text { High thermal } \\
\text { stability } \\
\text { - } & \text { High crystalinity } \\
\end{array}$ & $\begin{array}{l}\text { Poor stability in solutions } \\
\text { and tend to flocculate }\end{array}$ \\
\hline $\begin{array}{l}\text { Enzymatically } \\
\text { hydrolyzed }\end{array}$ & 79.5 & 6.7 & $\begin{array}{l}\text { - Highest crystalinity } \\
\text { - Highest aspect ratio }\end{array}$ & $\begin{array}{l}\text { - Higher cost production } \\
\text { - High tendency for } \\
\text { agglomeration } \\
\end{array}$ \\
\hline $\mathrm{BCNC}-g-\beta \mathrm{CD}$ & 63.4 & 15 & $\begin{array}{l}\text { - Stable suspension at } \\
\text { low concentration }\end{array}$ & $\begin{array}{l}\text { - Higher tendency for } \\
\text { agglomeration }\end{array}$ \\
\hline
\end{tabular}

Nanowhisker average length is $200-500 \mathrm{~nm}$ as determined by SEM

*Crystalinity index $\left(\mathrm{I}_{\mathrm{c}}\right)$ was calculated according to amorphous subtraction method. ${ }^{\text {[383] }}$

** Calculated for cellulose-I structure in respect of (200) plane using Scherrer's equation. ${ }^{[382]}$ 
Figure 3.3a represents grafting reactions schemes of $\beta C D$ onto the surface of $B C N C$ with citric acid as a green linker. ${ }^{[384]}$ According to the reaction schemes, every two adjacent carboxylic groups in citric acid (total of three groups) dehydrate to form a reactive cyclic anhydride. This ultimately reacts with the primary hydroxyl groups of both BCNC and $\beta C D$ to form ester bonds. In the current study, a two-step reaction was employed in order to obtain a higher grafting ratio of $\beta C D$ and to reduce the side effects on cellulose nanowhisker. ${ }^{[379]}$ In addition, the optimal reaction conditions were examined and used as reported by Dong et al. ${ }^{[66]}$ Accordingly, grafting ratio was enhanced by increasing reaction temperature with an upper limit of $160^{\circ} \mathrm{C}$, reducing the heating time to $15 \mathrm{~min}$, and maintaining CA- $\beta \mathrm{CD}$ concentration at $300 \mathrm{~g} / \mathrm{L}$ for each gram of BCNC. Results of grafting reactions are listed in Table 3.2. Under optimum reaction conditions, $33.8 \%(\mathrm{w} / \mathrm{w})$ grafting ratio was achieved for enzymatically hydrolyzed BCNC compared to only $7.6 \%(\mathrm{w} / \mathrm{w})$ for unhydrolyzed BC nanofibers. It is worth to mention that for a similar grafting reactions carried out at the later reaction conditions, either $\mathrm{HCl}$-hydrolyzed $\mathrm{BCNC}$ and $\mathrm{H}_{2} \mathrm{SO}_{4}$ hydrolyzed BCNC have achieved much less grafting ratio of 17.3 and $5.6 \%$ than that obtained with enzymatically hydrolyzed BCNC.

Figure 3.3b represents scheme for grafting of $\beta C D$ onto $\mathrm{BCNC}$ using epichlorohydrin as a linker. Under optimum grafting reaction conditions, ${ }^{[80]}$ enzymatically hydrolyzed BCNC was achieved less grafting ratio of $19.7 \%(\mathrm{w} / \mathrm{w})$. Results of grafting reactions using different linkers and different types BCNCs are listed in Table 3.2 for comparison. 


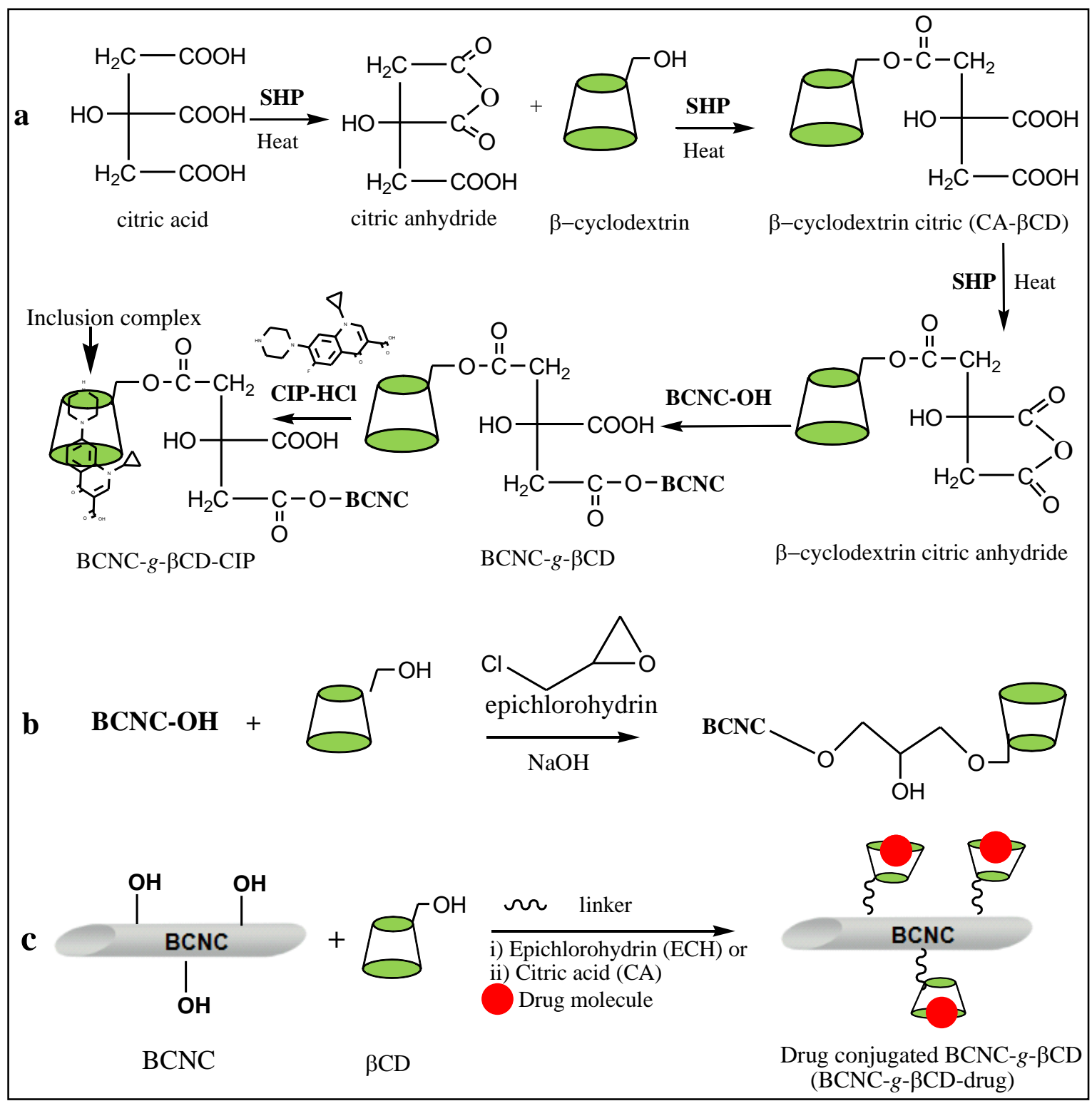

Figure 3.3 Grafting of $\mathrm{BCNC}$ with $\beta \mathrm{CD}$ by using of; (a) citric acid as linker in presence of (SHP) as catalyst, (b) epichlorohydrin as a linker in basic medium, and (c) inclusion complex formation between BCNC- $g$ - $\beta C D$ nanowhisker and conjugated drug (BCNC- $g-\beta C D$-drug). 
Table 3.2 Comparison between $\beta C D$ grafting ratios upon using different linkers; loading amounts and cumulative releases of CIP, DOX and PTX drugs to and from unmodified BCNC and BCNC- $g-\beta C D$

\begin{tabular}{|c|c|c|c|c|c|}
\hline \multirow[b]{2}{*}{ Samples } & \multicolumn{4}{|c|}{ CA as linker } & ECH as linker \\
\hline & $\begin{array}{c}\text { Grafting ratio } \\
(\%)\end{array}$ & $\begin{array}{l}\text { Loaded amount of drug } \\
\text { ( } \mu \mathrm{g} / \mathrm{mg} \text { dry nanowhisker) }\end{array}$ & $\begin{array}{c}\text { Cumulative release } \\
(\%)\end{array}$ & $\begin{array}{c}\text { Release time } \\
\text { (h) }\end{array}$ & $\begin{array}{c}\text { Grafting ratio } \\
(\%)\end{array}$ \\
\hline Native BC nanofibers & $7.6 \pm 0.3$ & $47 \pm 2(\mathrm{CIP})$ & NA & NA & $6.4 \pm 0.4$ \\
\hline $\begin{array}{c}\text { Enzymatically hydrolyzed } \\
\text { BCNC }\end{array}$ & $33.8 \pm 0.4$ & $115 \pm 3(\mathrm{CIP})$ & $97 \%$ & 10 & $17.6 \pm 0.3$ \\
\hline HCl-hydrolyzed BCNC & $17.3 \pm 0.2$ & NA & NA & NA & $14.1 \pm 0.5$ \\
\hline $\mathrm{H}_{2} \mathrm{SO}_{4}$-hydrolyzed BCNC & $5.6 \pm 0.2$ & NA & NA & NA & $5.3 \pm 0.3$ \\
\hline BCNC-g- $\beta C D-C I P$ & \multirow{3}{*}{ NA } & $810 \pm 7(\mathrm{CIP})$ & $90 \%$ & 96 & \multirow{3}{*}{ NA } \\
\hline BCNC-g- $\beta C D-D O X$ & & $690 \pm 6(\mathrm{DOX})$ & $85 \%$ & 120 & \\
\hline BCNC-g- $\beta C D-P T X$ & & $495 \pm 4(\mathrm{PTX})$ & $74 \%$ & 130 & \\
\hline
\end{tabular}


Figure 3.4a shows ATR spectra of BCNC before and after grafting with $\beta C D$. Analyzing ATR spectra of the ungrafted enzymatically hydrolyzed BCNC shows all characteristic bands reported for cellulose- ${ }^{[385]}$ as follow: band at $3180-3495 \mathrm{~cm}^{-1}$ is due to (O-H stretching) of intramolecular hydrogen bonds for cellulose-I; band at $2850-2960 \mathrm{~cm}^{-1}$ is due to (C-H stretching); broad band observed at $1635-1650 \mathrm{~cm}^{-1}$ is due to the $\left(\mathrm{O}-\mathrm{H}\right.$ bending); band at $1425-1435 \mathrm{~cm}^{-1}$ for $\left(\mathrm{CH}_{2}\right.$ scissoring); band at $1375-1385 \mathrm{~cm}^{-1}$ (C-H bending); band at $1335(\mathrm{O}-\mathrm{H}$ in plane bending); band at $1055 \mathrm{~cm}^{-1}$ (C-O-C pyranose ring stretching); band at $890-905 \mathrm{~cm}^{-1}$ is associated with (cellulosic $\beta$-glycosidic linkages); and the band at $1105 \mathrm{~cm}^{-1}$ ( $\mathrm{C}-\mathrm{O}-\mathrm{C}$ glycosidic ether band). Compared to ATR spectrum of free $\beta C D$ (Figure 3.4a), the new $\mathrm{C}=\mathrm{O}$ stretching vibration shown at $1727 \mathrm{~cm}^{-1}$ in the spectrum of CA- $\beta C D$ confirms the esterification reaction between $\beta C D$ and $C A$ and the successfulness of the first step in grafting reaction. In addition, new vibration at $1708 \mathrm{~cm}^{-1}$ that appeared in the spectrum of BCNC- $g-\beta C D$ is due to $\mathrm{C}=\mathrm{O}$ stretching vibration of ester bond formed between citric anhydride moiety of $\mathrm{CA}-\beta \mathrm{CD}$ and $\mathrm{OH}$ groups of $\mathrm{BCNC}$. This band confirms the completion of the second step of grafting reaction and the formation of BCNC- $g-\beta C D$.

Solid state ${ }^{13} \mathrm{C}$ NMR spectra of unmodified and grafted BCNC are shown in Figure 3.4b. ${ }^{13} \mathrm{C}$ chemical shifts between 55 and 110 ppm are assigned to various carbon atoms that belong to the glucose moieties in BCNC. Six peaks correspond to ${ }^{13} \mathrm{C}$-chemical shifts of cellulose carbons as follow; C-1 (106 ppm), C-4 (78 ppm), C-2/C-3/C-5 (70-80 ppm), and C-6 (66 ppm) for anhydroglucose units of cellulose. ${ }^{[386]}$ However, the new signal arose in the spectrum of BCNC$g-\beta C D$ at $174 \mathrm{ppm}$ is due to the carbonyl group of the ester bond. In addition, an observable signal at $45 \mathrm{ppm}$ was appeared due to $-\mathrm{CH}_{2}$ - group of citric acid. 

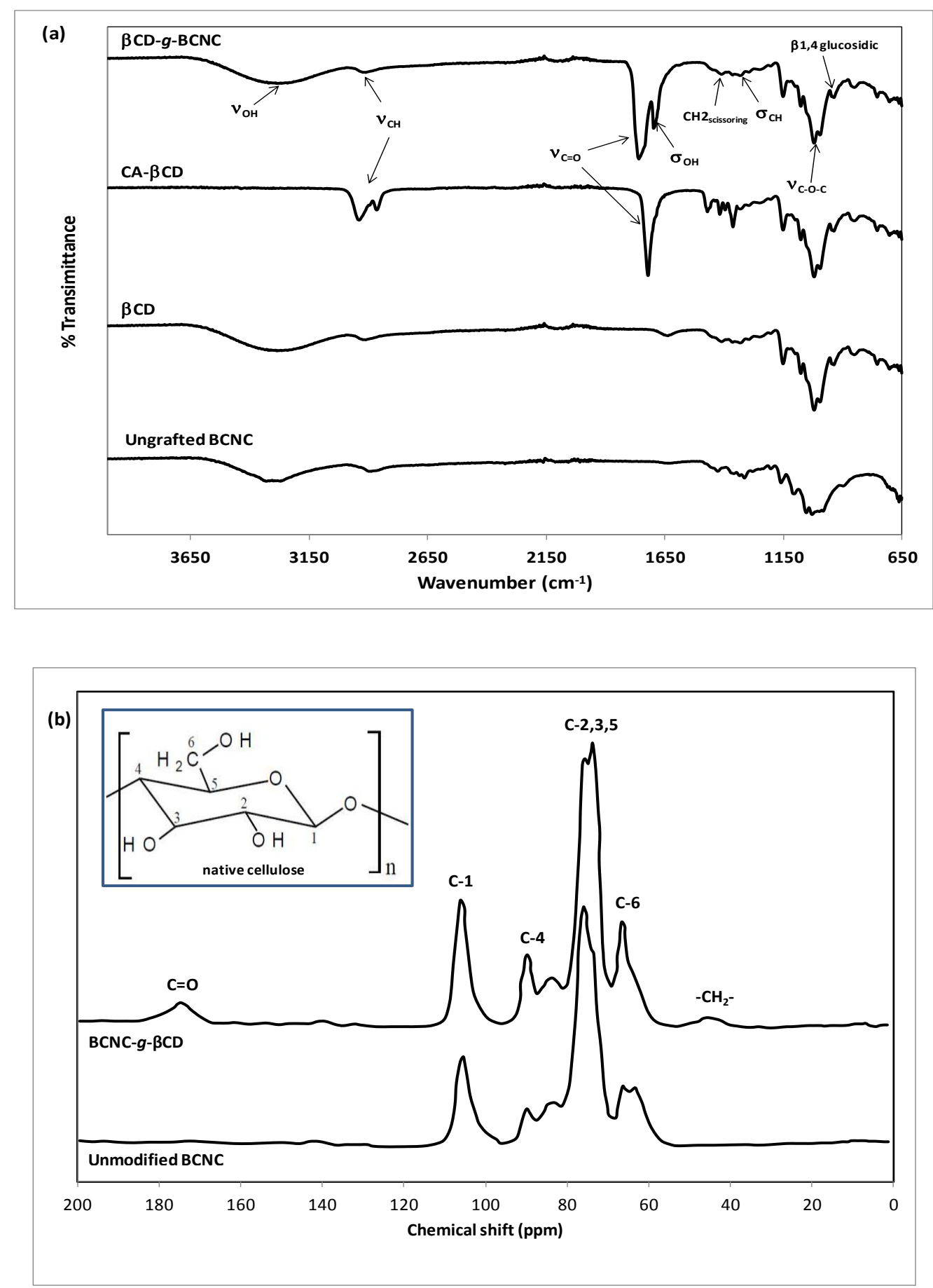

Figure 3.4 (a) ATR spectra of $\beta C D, C A-\beta C D, B C N C$ and $B C N C-g-\beta C D$; (b) CPMAS solidstate ${ }^{13} \mathrm{C}$ NMR spectra of unmodified BCNC and BCNC- $g-\beta C D$. 
Figure 3.5 presents UV-vis absorption measurements of the formation of inclusion complex between CIP model drug and non-conjugated $\beta \mathrm{CD}$ at $\mathrm{pH} 7.4$ and $37^{\circ} \mathrm{C}$. Results show that increasing $[\beta C D]$ is associated with an increased absorbance for the absorption maxima of CIP (i.e., hyper-chromic shift at $275 \mathrm{~nm}$ ). The stoichiometry of 1:1(Drug: $\beta C D$ ) IC was confirmed from the perfect linear correlation $\left(\mathrm{R}^{2}=0.992\right)$ that obtained from fitting the absorption data to the double-reciprocal Benesi-Hildebrand plots ${ }^{[381]}$ of $1 /\left(\mathrm{A}-\mathrm{A}_{0}\right)$ vs. $1 /[\beta C D]_{0}$. Binding constants of (1:1) ICs (i.e. $K_{1: 1}$ ) were evaluated from the slopes of the plots and found to equal 19.25, 27.17, and $35.12 \mathrm{M}^{-1}$ at $310 \mathrm{~K}$ for CIP, DOX, and PTX, respectively. Spontaneity of the inclusion reaction is verified from the negative Gibbs free energy change $(\Delta G)$ of the host-guest binding process. These calculated $\Delta \mathrm{G}$ values are found to equal $-7.62,-8.51$, and $-9.17 \mathrm{kJmol}^{-1}$ for CIP, DOX and PTX, respectively.

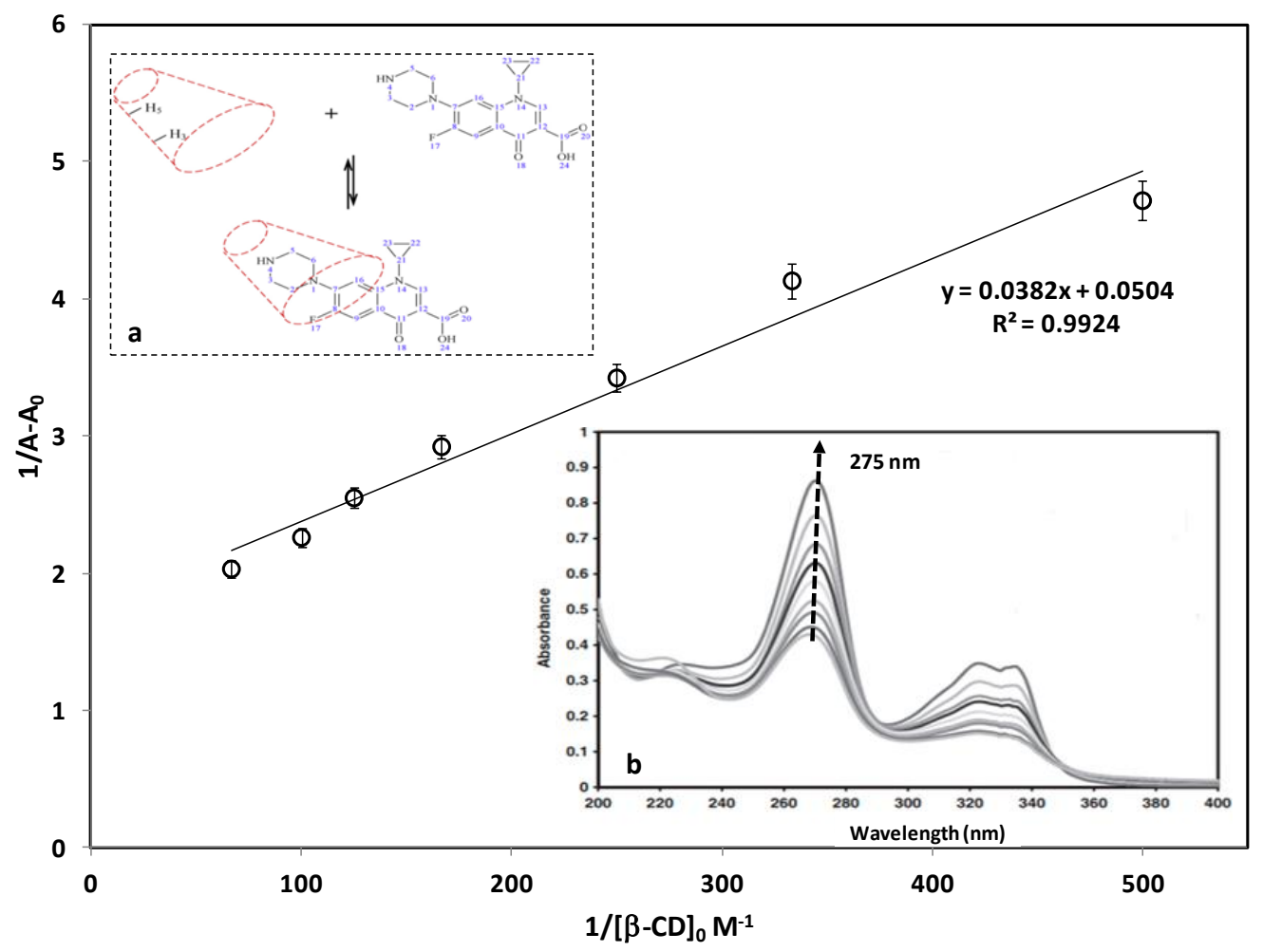

Figure 3.5 Plot of $1 / \mathrm{A}-\mathrm{A}_{0}$ against $1 /[\beta C D]$ for the interaction of CIP with the increased concentrations of $\beta \mathrm{CD}$ at $\mathrm{pH} 7.4$ and $37^{\circ} \mathrm{C}$. Experiments were done in triplicate, and error bars represent \pm standard deviation. Inset (a) represents schematic for inclusion complex formation. Inset (b) represents hyper-chromic shift observed for the absorption maxima of CIP due to increased $\beta C D$ concentration. 
Figure 3.6 shows the effect of increased drugs concentrations (i.e., CIP, DOX and PTX) on the drug loading onto unmodified BCNC and BCNC-g- $\beta \mathrm{CD}$ in $10 \mathrm{mM}$ PBS buffer at $\mathrm{pH} 7.4$ and $25^{\circ} \mathrm{C}$. Results show that the drug payloads conjugated to BCNC-g- $\beta C D$ enhanced significantly with the increased total drugs concentration. This was continued until maximum values were attained, after which the drugs binding satiated due to the saturation of binding sites on the nanowhisker surface. Analyzing the results shows the maximum equilibrium-loading amount that estimated from the calibration curve was about $810 \mu \mathrm{g}$ of $\mathrm{CIP} / \mathrm{mg}$ of BCNC-g- $\beta \mathrm{CD}$. This corresponds to $81 \%$ loading efficiency considering total CIP available initially in the solution. The corresponding maximum loading of DOX was $690 \mu \mathrm{g}$ of DOX/mg of BCNC- $g-\beta C D$. This corresponds to $69 \%$ loading efficiency. Nevertheless, less PTX drug was bound to BCNC- $g-\beta C D$ surface with maximum of $495 \mu \mathrm{g} / \mathrm{mg}$ of BCNC-g- $\beta C D$ that represents $49.5 \%$ binding efficiency.

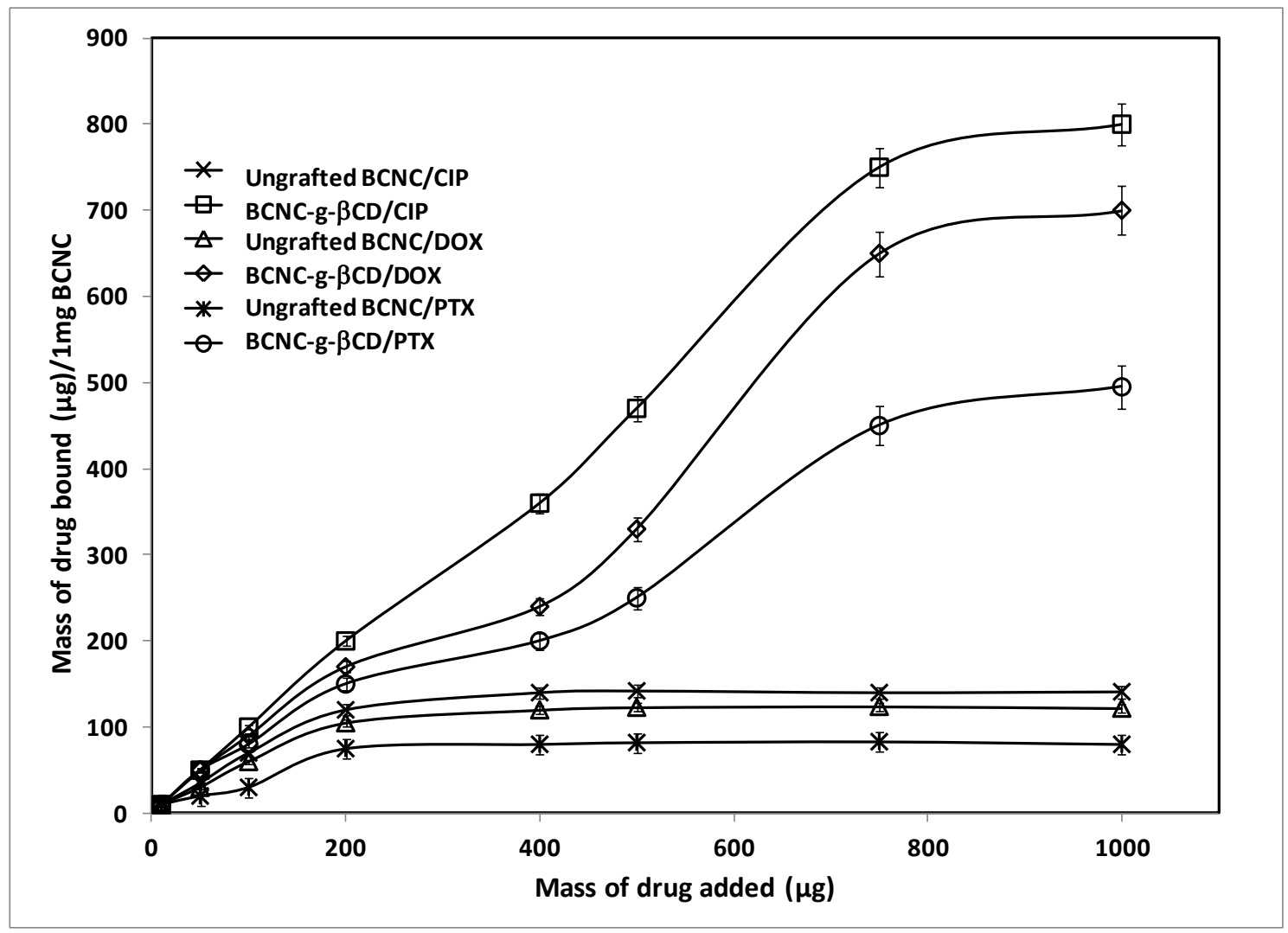

Figure 3.6 Binding of CIP, DOX and PTX to $1 \mathrm{mg}$ unmodified BCNC and BCNC- $g-\beta C D$ in 10 $\mathrm{mM}$ PBS buffer at $\mathrm{pH} 7.4$ and $25^{\circ} \mathrm{C}$. Experiments were done in triplicate, and error bars represent \pm standard error. 
Figures 3.7a, b show drug releases profiles of the three model drugs (i.e., CIP, DOX and PTX) conjugated to the grafted nanofibers in comparison to unmodified ones (CIP-BCNC). PBS solutions maintained at $\mathrm{pH}$ of 7.4 and 6.4 , at $37^{\circ} \mathrm{C}$ were used as a releasing media mimicking the physiological conditions and the slightly acidic environment of extracellular cancer cells. Results of cumulative release of CIP from the unmodified BCNC under the physiological pH show burst release of $85 \%$ of the loaded amount of drug in the first $3 \mathrm{~h}$. A maximum cumulative release of 97\% was achieved after the next $10 \mathrm{~h}$; see Figures 3.7a. However, in the case of CIP-conjugated to $\mathrm{BCNC}-g-\beta C D$ (i.e. BCNC-g- $\beta C D-C I P$ ), CIP molecules were slowly released in a controlled manner over longer time period. Cumulative release of $\sim 45 \%$ of drug payload was achieved over $24 \mathrm{~h}$. By end of the second day, the drug release increased to reach $67 \%$, while maximum cumulative release of $90 \%$ was achieved after $\sim 120$ h (5 days). A similar release profile was observed for BCNC-g- $\beta C D$-DOX nanosystem, where a rapid drug release of $\sim 35 \%$ was observed in the first 24 hour and then followed by a slower release. After the second day, $49 \%$ drug release was achieved. A maximum cumulative release of DOX had a plateau of $85 \%$ after $120 \mathrm{~h}$ ( $\sim$ days). The hydrophobic cancerous drug PTX showed slower release compared with the other two hydrophilic drugs (i.e., CIP and DOX). This resulted in $\sim 12 \%$ cumulative release of the bound PTX in the first $24 \mathrm{~h}$ (Figure 3.7a). By second day, the drug cumulative release was increased to $30 \%$ and the maximum release had a plateau at $74.5 \%$ after $122 \mathrm{~h} \mathrm{(} \sim 5$ days).

Figure 3.7b demonstrated that at a slightly acidic $\mathrm{pH}$ of 6.4 , all drug conjugated-nanocarrier systems (i.e., BCNC-g- $\beta$ CD-CIP, BCNC-g- $\beta C D-D O X$, and BCNC-g- $\beta C D-P T X$ ) have displayed higher cumulative drug releases in a sustained manner over longer times. Cumulative releases of CIP, DOX, and PTX after 130 h (5.5 days) were equal to 93, 86.5 and $79 \%$. 

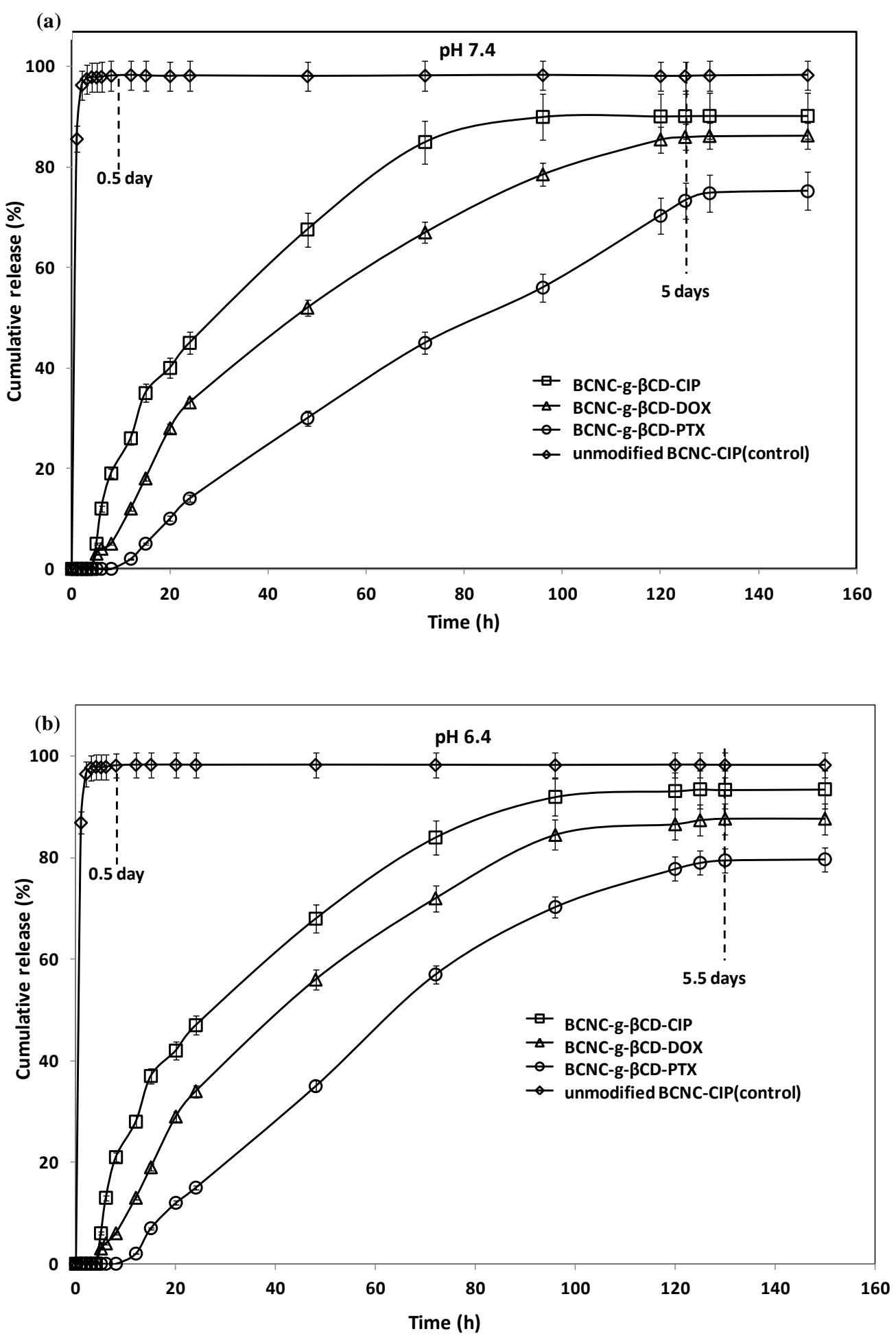

Figure 3.7 Comparison between the drug releases profiles of BCNC-CIP, BCNC- $g-\beta C D-C I P$, BCNC- $g$ - $\beta C D-D O X$ and BCNC- $g-\beta C D-P T X$ at $37^{\circ} \mathrm{C}$ in PBS buffer; (a) at $\mathrm{pH} 7.4$; (b) at pH 6.4. Experiments were done in triplicate, and error bars represent \pm standard error. 
Figure 3.8 represents the disk diffusion assay experiment, which was performed with the compressed pellet of antibiotic-loaded nanowhisker (i.e., BCNC-g- $\beta C D-C I P$ ) against $E$. coli. The antibacterial efficacy determined as visual zones of inhibition on the agar plate. An inhibition zone area with sharp edge and diameter of $1.9 \pm 0.5 \mathrm{~cm}$ was clearly observed gradually after 3 days around the desk containing BCNC- $g-\beta C D-C I P$.

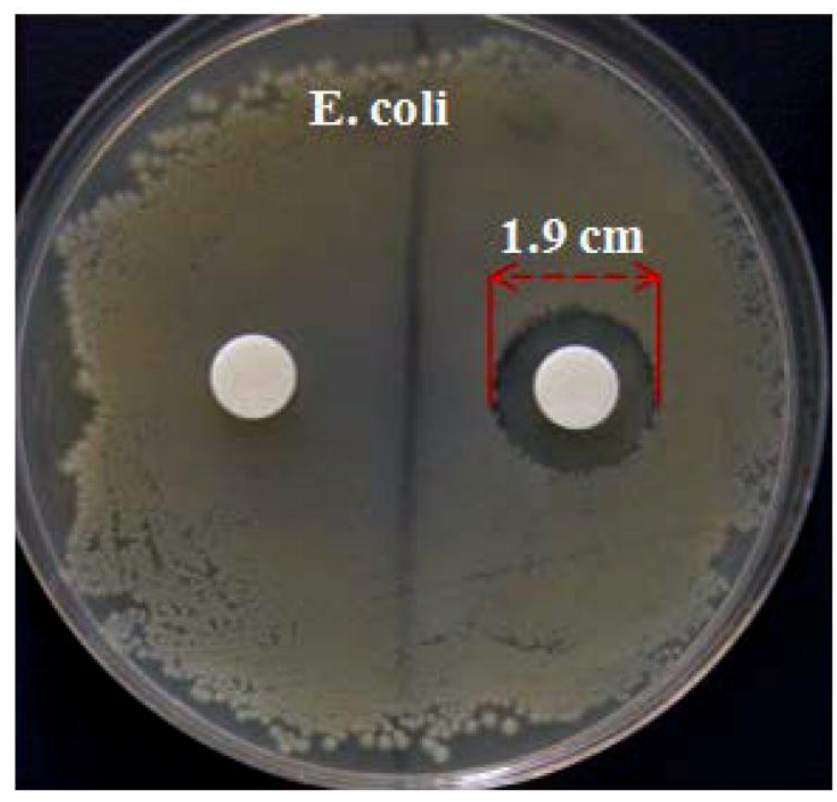

Figure 3.8 Disk diffusion of CIP from BCNC- $g$ - $\beta$ CD-CIP to prevent bacterial colonization. Left disk: unmodified BCNC; Right disk: BCNC-g- $\beta C D-C I P$.

Figure 3.9 shows the fitting of in vitro drug releases data obtained from BCNC- $g$ - $\beta C D$-drug nanocarriers systems to zero-order kinetics model. Table 3.3 summarizes the values of the fitting parameter $\left(\mathrm{R}^{2}\right)$ that obtained from data-fitting to the different examined kinetic models. According to the obtained $\mathrm{R}^{2}$ values the in vitro drug releases from BCNC- $g$ - $\beta C D$-drug nanocarriers were best represented by zero-order kinetic model. The estimated zero-order release constant values $\left(\mathrm{K}_{0}\right)$ from the linear plots at $\mathrm{pH} 7.4$ were found to equal $0.67,0.66$, and $0.61 \mu \mathrm{g} / \mathrm{h}$ for BCNC- $g$ - $\beta C D-C I P, B C N C-g-\beta C D-D O X$ and BCNC- $g$ - $\beta C D-P T X$ nanosystems, respectively. Whereas the estimated $\mathrm{K}_{0}$ values for all nanocarrier systems at $\mathrm{pH} 6.4$ were found to be higher and equal to $0.68,0.74$, and $0.79 \mu \mathrm{g} / \mathrm{h}$ for BCNC- $g-\beta C D-C I P, B C N C-g-\beta C D-D O X$, and BCNCg- $\beta$ CD-PTX nanosystems, respectively. See Table 3.3. 


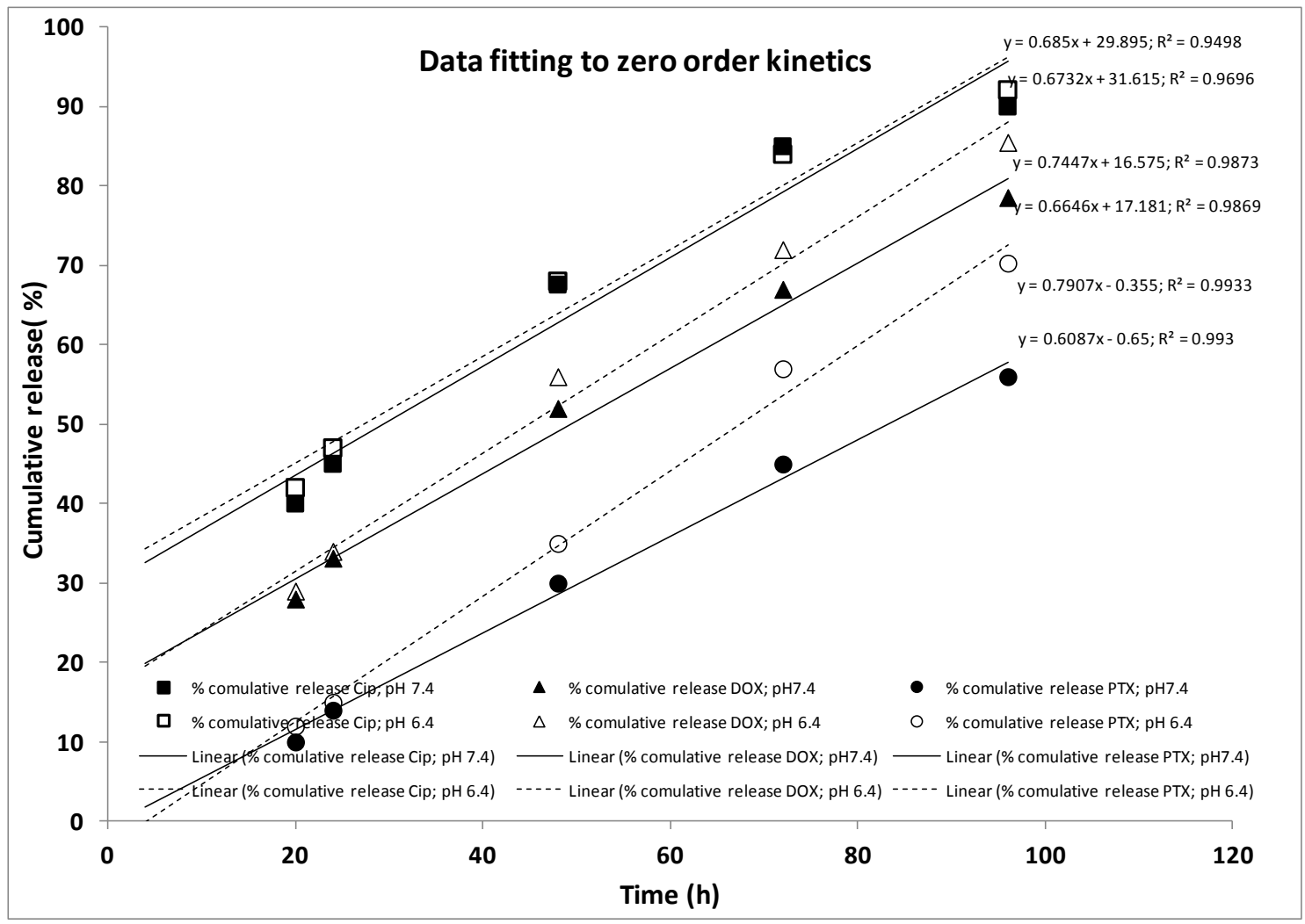

Figure 3.9 Fitting curves of drug release data to zero-order kinetics model. 
Table 3.3 Summary of the chemical formula of the drugs used along with their binding constants as well as the modeled in vitro release kinetics

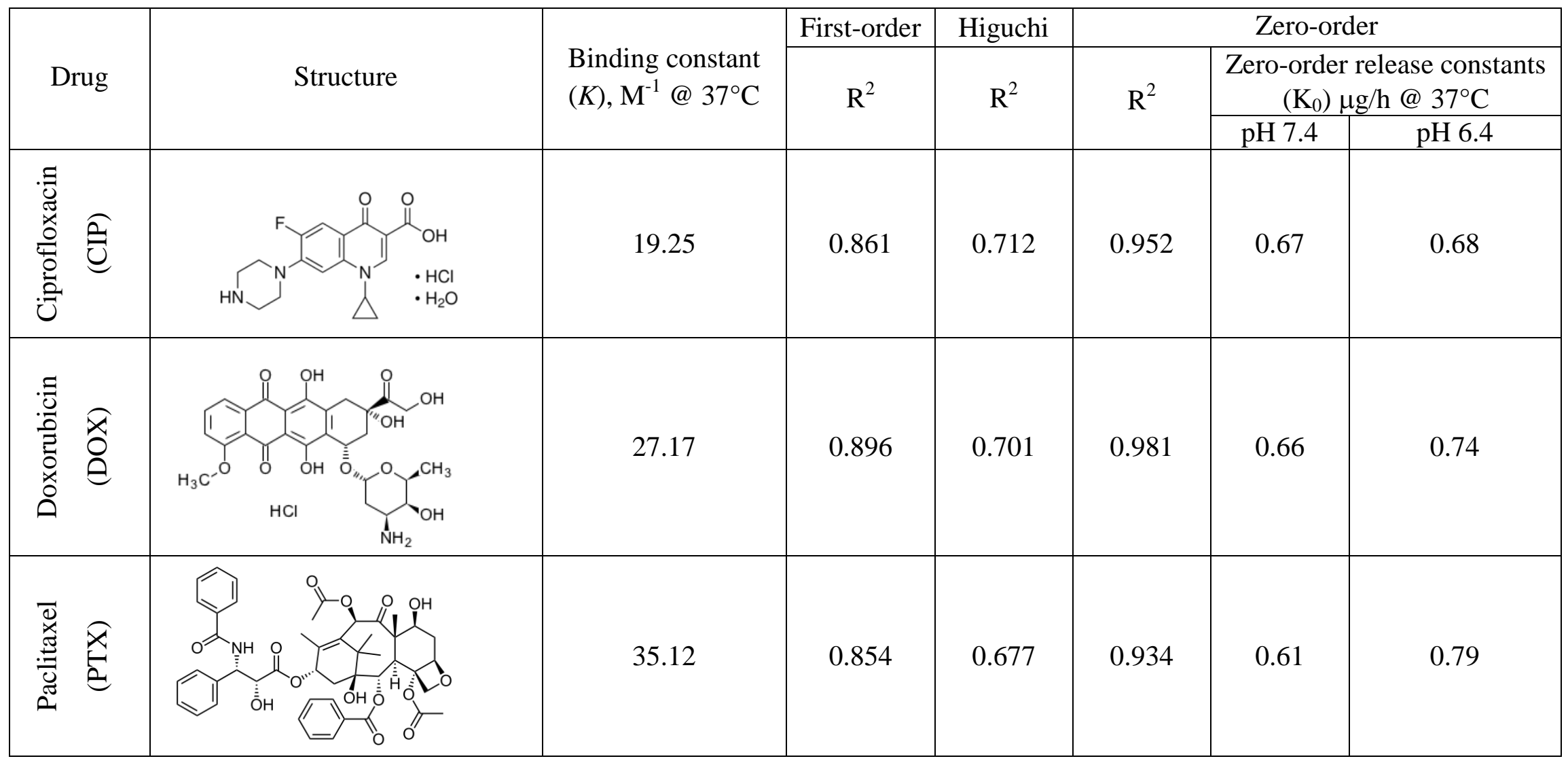




\subsection{Discussion}

SEM and AFM morphological analysis of well individualized BCNC (Figure 3.1) have evidenced rod-like shaped nanowhisker. Regardless the used method of hydrolysis, these nanowhisker showed an average length of 200-500 nm and width of $4.6 \mathrm{~nm}$.

XRD diffractograms of BCNC samples obtained with different methods of hydrolysis (Figure 3.2) confirm that the crystal integrity of cellulose-I structure is kept intact after hydrolysis. The observed increase in $\mathrm{I}_{\mathrm{c}}$ of $\mathrm{BCNC}$ (i.e., 69.3-79.5 \%) over unhydrolyzed BC nanofibers (i.e., 65.2), as revealed from Table 1, is related to the removal of amorphous cellulosic parts upon hydrolysis. The highest $I_{c}$ of $79.5 \%$ was achieved by enzymatic hydrolysis rather than acid hydrolysis methods. Therefore, changing the method of hydrolysis is solely affecting the crystallinity rather than particle sizes. Moreover, XRD pattern of grafted nanowhisker (i.e. BCNC- - - $\beta C D$ ) exhibited similar peak positions to the ungrafted BCNC (Figure 3.2e). This indicates that the crystal integrity of the native cellulose is preserved even after grafting reaction. The observed unique physical and chemical properties of BCNC samples obtained by different ways of hydrolysis are summarized in Table 3.1. As revealed from the table, enzymatically hydrolyzed nanowhisker showed limited dispersion in aqueous media with a greater tendency for agglomeration due to hydrogen bonding. When the later limitation was overcome before the reaction by carrying out a pre-ultrasonication step, enzymatically hydrolyzed nanowhisker displayed an increased reactivity towards grafting reaction with $\beta C D$. The highest grafting ratio of 33.8\% was achieved (Table 3.2). Contrary, BCNC obtained by sulfuric acid hydrolysis showed less aggregation tendency and higher dispersion in aqueous medium. This is related to the effect of repulsion forces between the negatively charged sulphate groups on their surface. However, its chemical reactivity toward grafting reaction was the least (i.e., 5.6\%). This might be related to the presence of sulfate groups, which take up as much as $40 \%$ of the reactive hydroxyl groups and make them uncreative towards grafting reaction. ${ }^{[255]}$

Hence, owing to the remarkable characteristics of the enzymatically hydrolyzed BCNC (i.e. reactivity, highest crystallinity and highest aspect ratio) it was chosen in the current study as promising potential candidate for grafting and drug release studies.

Citric acid is a well-known nontoxic and eco-friendly crosslinking agent. ${ }^{[384]}$ It was employed in this study as a linker for grafting $\beta C D$ onto the surface of BCNC. Two-step reaction was employed ${ }^{[379]}$ to achieve a higher grafting ratio along with reduced side effects on the inherited 
characteristics of cellulose nanowhisker (Figure 3.3a). This consequently increases the drug payload of nanowhisker. Table 3.2 summarizes the results obtained from different employed grafting reactions. Under optimum reaction conditions, $33.8 \%$ grafting ratio was achieved by the enzymatically hydrolyzed BCNC compared to 7.6, 5.6, and $17.3 \%$ for a similar experiment implemented on unhydrolyzed BC nanofibers, $\mathrm{H}_{2} \mathrm{SO}_{4}$-hydrolyzed $\mathrm{BCNC}$, and $\mathrm{HCl}$-hydrolyzed BCNC. The grafting ratio achieved in this study, using $\mathrm{CA}$ as a linker, is higher than the maximum reported grafting ratio for immobilizing $\beta \mathrm{CD}$ on the plant-derived $\mathrm{CNC}$ (i.e. $18 \%$ $\mathrm{W} / \mathrm{w}){ }^{[46]}$

In order to evaluate the grafting efficiency of CA as a green linker, less bulky and non-green epichlorohydrin was employed as grafting agent (Figure 3.3b). However, under optimum reaction conditions results showed that less grafting ratio of $19.7 \%$ was achieved by enzymatically hydrolyzed BCNC, see Table 3.2 for comparison. Figure 3.3c represents a schematic for the formation of host-gust inclusion complex (IC) between drug molecules and the $\beta C D$ moieties grafted on nanowhisker surface.

The change in chemistry accompanied the two-step grafting reaction of $\beta C D$ onto $B C N C$ surface was followed and confirmed by FTIR and solid state ${ }^{13} \mathrm{C}$ NMR (Figure 3.4a, b). Emerging of new $\mathrm{C}=\mathrm{O}$ stretching vibration band at $1708 \mathrm{~cm}^{-1}$ in the ATR spectra of BCNC- $g-\beta C D$ (Figure 3.4a), as well as the new ${ }^{13} \mathrm{C}$ NMR signal arose in the spectrum of BCNC- $g-\beta C D$ at $174 \mathrm{ppm}$ (Figure 3.4b) are due to the new carbonyl ester bond that formed as a result of the esterification reaction between citric anhydride moiety of $\mathrm{CA}-\beta \mathrm{CD}$ and $\mathrm{OH}$ groups of $\mathrm{BCNC}$. These previous evidences confirm the successfulness of the grafting reaction between $\beta C D$ and $B C N C$ and the formation of BCNC- $g-\beta C D$.

The spontaneity of IC formation reaction between drugs and $\beta C D$ was confirmed and assessed by UV-vis absorption measurements. The hyper-chromic shift observed for the absorption maxima of CIP spectral bands at $\lambda=275 \mathrm{~nm}$ is a great evidence for the formation of host-guest $\mathrm{IC}^{[387,388]}$ (Figure 3.5). In addition, the observed binding constant values $(\mathrm{K})$ that are considered fundamental properties for describing the strength of interaction between drugs and grafted $\beta C D$ of 1:1(Drug: $\beta C D$ ) inclusion complex at $310 \mathrm{~K}$ are ranged from $19.25-35.12 \mathrm{M}^{-1}$. These obtained values are found to be smaller than the reported $\mathrm{K}_{1: 1}$ for drugs- $\beta C D$ ICs, which have reported range from 50-2000 $\mathrm{M}^{-1}$ and mean value of $490 \mathrm{M}^{-1}$. ${ }^{[389]}$ The smaller obtained $\mathrm{K}$ values suggest the low affinity of drugs toward $\beta C D$ and the ease of their releases from $\beta C D$ cavities. In 
addition, the negative $\Delta \mathrm{G}$ values indicate the spontaneity of the binding process between drug molecules and $\beta C D$ moieties grafted on nanowhisker surface.

Effects of increasing drug concentration on the maximum drug loading efficiency for both unmodified $\mathrm{BCNC}$ and $\mathrm{BCNC}-\mathrm{g}-\beta \mathrm{CD}$ were investigated as displayed in Figure 3.6. Surprisingly, for all examined model drugs, grafting of $\beta C D$ on the surface of BCNC resulted in increased drug payloads. Where a maximum value of $\sim 500-800 \mu \mathrm{g} / \mathrm{mg}$ were achieved by the grafted nanowhisker compared to $\leq 100 \mu \mathrm{g} / \mathrm{mg}$ for unmodified nanowhisker. The later significant increase in the drug payloads confirms the effectiveness of the model as a new promising nanocarrier for drug molecules. This high drug payload is most probably related to the formation of host-guest IC between the drug molecules and the $\beta C D$ moieties grafted on BCNC surface. The increased binding efficiency of drug payload of the two ionized drugs CIP and DOX (i.e. 81, $69 \%$, respectively) over the hydrophobic PTX (49.5\%) is probably because of ionic interaction between the slightly cationic nature of drug molecules and the anionic alkalized $\mathrm{OH}$ groups that present on BCNC surface ungrafted. ${ }^{[390]}$ Even though the highly hydrophobic drug PTX bound to the surface of BCNC- $g-\beta C D$ at relatively higher weight ratio of $(\sim 33 \% \mathrm{w} / \mathrm{w})$. This is larger than the reported value of for the same drug conjugated on the surface of NCC coated with cationic surfactant CTAB (i.e. $20 \% \mathrm{w} / \mathrm{w}){ }^{[27]}$

In vitro drugs release profiles in Figure 3.7a,b showed fast and burst release of $85 \%$ of CIP payload from unmodified BCNC in the first 3h. This result supports the fact that hydrophilic antibiotic is solely physically adsorbed by weak electrostatic attraction onto the surface of BCNC. However, the presence of grafted $\beta C D$ molecules on the surface of BCNC are not only dramatically increased drug loadings but also effectively reduced their initial burst releases and prolonged the release process in a sustained manner. In this case, the drug molecules that conjugated to BCNC- $g-\beta C D$ (i.e. BCNC- $g-\beta C D$-Drug) are captured in the cavities of the grafted $\beta C D$ on $\mathrm{BCNC}$ surface. Therefore, their releases are basically controlled by the binding constant values between the drugs and the grafted $\beta C D$ moieties. Cumulative releases of $75-90 \%$ of drug payloads were achieved over $\sim 120 \mathrm{~h}$ (5 days). In the same context, higher cumulative releases of $79-93 \%$ over longer period of 5.5 days were achieved in acidic $\mathrm{pH}$ by BCNC- $g$ - $\beta \mathrm{CD}-\mathrm{CIP}$, BCNC- $g$ - $\beta C D-D O X$ and BCNC- $g$ - $\beta C D-P T X$ nanosystems. The higher cumulative release of drugs at acidic $\mathrm{pH}$ can be ascribed to the greater solubility of the drugs in aqueous medium due to the protonation of the amine groups of drug molecules. This in turn assists the diffusion 
process. Previous studies on acid-hydrolyzed CNCs bound to the water-soluble ionized drugs tetracycline and doxorubicin reported a rapid release of drugs over a period of one day. ${ }^{[17]}$ This confirms the effectiveness of the current model and promotes the validity of BCNC- $g-\beta C D$ as potential prolonged drug release carrier. Moreover, the slower drug release of PTX and DOX than CIP may be due to the higher binding constant value between these two anticancer drugs and $\beta C D$ molecules. The considerably faster release of CIP and DOX over the hydrophobic PTX is more likely due to their ionic nature and the presence of large amounts of counter ions in PBS. These counter ions interfere with the positively charged CIP and DOX binding sites and displacing them quickly from nanowhisker surface via ion exchange.

The faster-release observed for CIP may find potential applications in antibacterial wounddressing. In vitro anti-bacterial activity of antibiotic-loaded nanowhisker (i.e. BCNC- $g-\beta C D-$ CIP) against E. coli showed clearly an inhibition zone area after 3 days (Figure 3.8). E. coli is aerobic bacterium and commonly found on the skin. It is considered one of the major causes of postoperative nosocomial wound infections problem in health care facilities. The weak interaction between CIP and $\beta C D$ moieties grafted on nanowhisker surface likely assisted the sustained release of drug from the nanowhisker to bacterial cells. This prolonged and sustained release of antibiotic from BCNC- $g$ - $\beta C D-C I P$ nanocarrier can find applications such as nanospray or nanocream. This nanocarrier can inhibit $E$. coli activities over a longer period with a less frequent dosage of antibiotic.

Nevertheless, the slower release profiles for DOX and PTX can be utilized in the post operative procedures that need long-lasting drug release. For instance, the cement materials that used for filling the voids that left behind after resection of cancer tumors and implantation of new surgical devices. As for one of the treatment regimen for cancer, patients would be placed on doxorubicin $25 \mathrm{mg} / \mathrm{m}^{2} /$ day on days 1 to 3 and cisplatin $100 \mathrm{mg} / \mathrm{m}^{2}$ on day 1 , every 3 weeks for six cycles. ${ }^{[17]}$ As the developed nanocarrier in this study could potentially release from $75-93 \%$ from their total drug payload over 5-5.5 days. Therefore, it can secure sufficient quantity of the total drug required for one week from the entire postoperative period. This in turn reduces the number of daily dosing of chemotherapeutic material.

Analyzing the drug release profiles of BCNC- $g$ - $\beta C D$-drug nanocarriers (Figure 3.7a,b) shows two distinct phases for drug release. In the first phase, a relatively faster release of small amount of loaded drug of $\sim 20-25 \%$ was observed over the first $24 \mathrm{~h}$. This followed by the second phase 
where a gradual release of $\sim 75 \%$ of loaded drug over next 4 days was achieved. Different mathematical models are tested for investigating the kinetics and drug release mechanism from BCNC-g- $\beta C D$-drug nanocarrier systems. These models included zero-order model, first order model and Higuchi model. ${ }^{[391]}$ The model that best fits the drug release data (i.e., showed the highest correlation coefficient value; $\mathrm{R}^{2}$ ) will be considered the most probable drug release mechanism. In the present study, in vitro drug releases data for all BCNC-g- $\beta C D$-drug nanosystems were best fitted the purposed zero order kinetics model with $\mathrm{R}^{2}$ values ranged from 0.934-0.981, see Table 3.3.

Basically, zero-order release model represents drug dissolution from the dosages that do not disaggregate and release the drug slowly and independent on drug concentration. ${ }^{[392]}$ Such model is important for delivery of special classes of medications specifically those intended to deliver antibiotic, blood pressure maintainers, pain controllers and antidepressants. This kinetic model can be presented by $\mathrm{Q}_{\mathrm{t}}=\mathrm{Q}_{0}+\mathrm{K}_{0} \mathrm{t}$. Where, $\mathrm{Q}_{\mathrm{t}}$ is cumulative amount of drug released in time $\mathrm{t}, \mathrm{Q}_{0}$ is the initial amount of drug in the solution (most times, $\mathrm{Q}_{0}=0$ ), and $\mathrm{K}_{0}$ is zero order release constant in unit of (concentration/time). $\mathrm{K}_{0}$ values can be evaluated from the slope of the linear fitting graph shown in Figure 3.9. In acidic medium, higher zero-order release rate constants values of $0.68,0.74$ and $0.79 \mu \mathrm{g} / \mathrm{h}$ were obtained for BCNC- $g-\beta C D-C I P, B C N C-g-\beta C D-D O X$ and BCNC-g- $\beta$ CD-PTX nanosystems. The later finding is commendatory for cancer therapy due to the need of prolonged availability of drugs at the site of action compared to normal cells.

\subsection{Conclusion}

A green nanosized drug carrier system (i.e., BCNC-g- $\beta C D$ ) based on the biocompatible bacterial cellulose was developed by grafting of $\beta C D$ to the surface of bacterial cellulose nanowhisker. This developed nanocarrier would spontaneously load and release significant quantities of antibiotics and anticancer drugs. Results of the current study showed that the maximum drug payload achieved by BCNC-g- $\beta$ CD were $810 \pm 7 \mu \mathrm{g} / \mathrm{mg}, 690 \pm 6 \mu \mathrm{g} / \mathrm{mg}$, and $495 \pm 4 \mu \mathrm{g} / \mathrm{mg}$ for CIP, DOX, and PTX. Contrary, the unmodified BCNC bound smaller quantities of drug that was not exceed $100 \pm 3 \mu \mathrm{g} / \mathrm{mg}$. The drug release profiles for all drug-conjugated nanocarriers (i.e. BCNC-g- $\beta C D$-drug) showed controlled and sustained drug releases over longer time periods of 5-5.5 days, and without obvious burst releases. However, unmodified BCNC showed noticeable burst release of the loaded drug over $3 \mathrm{~h}$. Nevertheless, an improved drug release performance of 
BCNC- $g-\beta C D$-drug nanocarrier was revealed at acidic $\mathrm{pH}$ of 6.4 that mimicked the extracellular environment of tumor cells. The release profiles and the evaluated zero-order release constants for BCNC- $g-\beta C D$-drug nanocarrier revealed a promising potential for the developed nanocarriers for encapsulating and controlling the release antibiotics and anticancer drugs. Thus, this nanocarrier can find versatile medical applications as internal localized drug delivery system or in superficial application such as nanocream or nanospray. In addition, this nanocarrier could also be able to solve the well-known solubility and delivery problem of hydrophobic anticancer drugs due to its ability to bind either highly hydrophobic or hydrophilic anticancer drugs.

Lastly, the obtained promising results could pave the way for developing new green bio-based controlled release nanocarriers with higher efficiency. The model can be also expanded for dualdrug delivery applications. 


\section{CHAPTER 4*}

\section{Synthesis and Characterization of Cellulose Nanowhisker Reinforced-Poly( $\varepsilon$-Caprolactone) Scaffold for Tissue Engineering Applications}

Poly( $\varepsilon$-caprolactone) (PCL) is bioresorbable and biocompatible polymeric compound with lots of medical applications. However, its remarkable hydrophobicity and non-osteoconductivity have stood as a barrier to limit its applications. The present study aims to modify the bulk characteristics of PCL to develop polymeric scaffold with adequate structural and mechanical properties to support regenerated tissues. For this purpose, functionalized bacterial cellulose nanowhisker (BCNW-g- $\left.\beta C D-P C L_{2000}\right)$ is synthesized. Reinforcing PCL matrix with 4 wt\% of the functionalized nanowhisker resulted in bionanocomposite with promoted bulk properties. Compared to neat PCL, the obtained bionanocomposite shows improvements of $115 \%$ and $51 \%$ in tensile strength and Young's modulus; 20\% increase in hydrophilicity; 7\% increase in degradation rate; and $6 \%$ decrease in crystallinity. Gas foaming/combined particulate leaching technique is used to develop interconnected macropores structures with porosity of 86-95\% and mean pore diameters of $250-420 \mu \mathrm{m}$. Porous scaffolds showed compression modulus values of 5.3-9.1 MPa and would have promising applications in regenerative medicine.

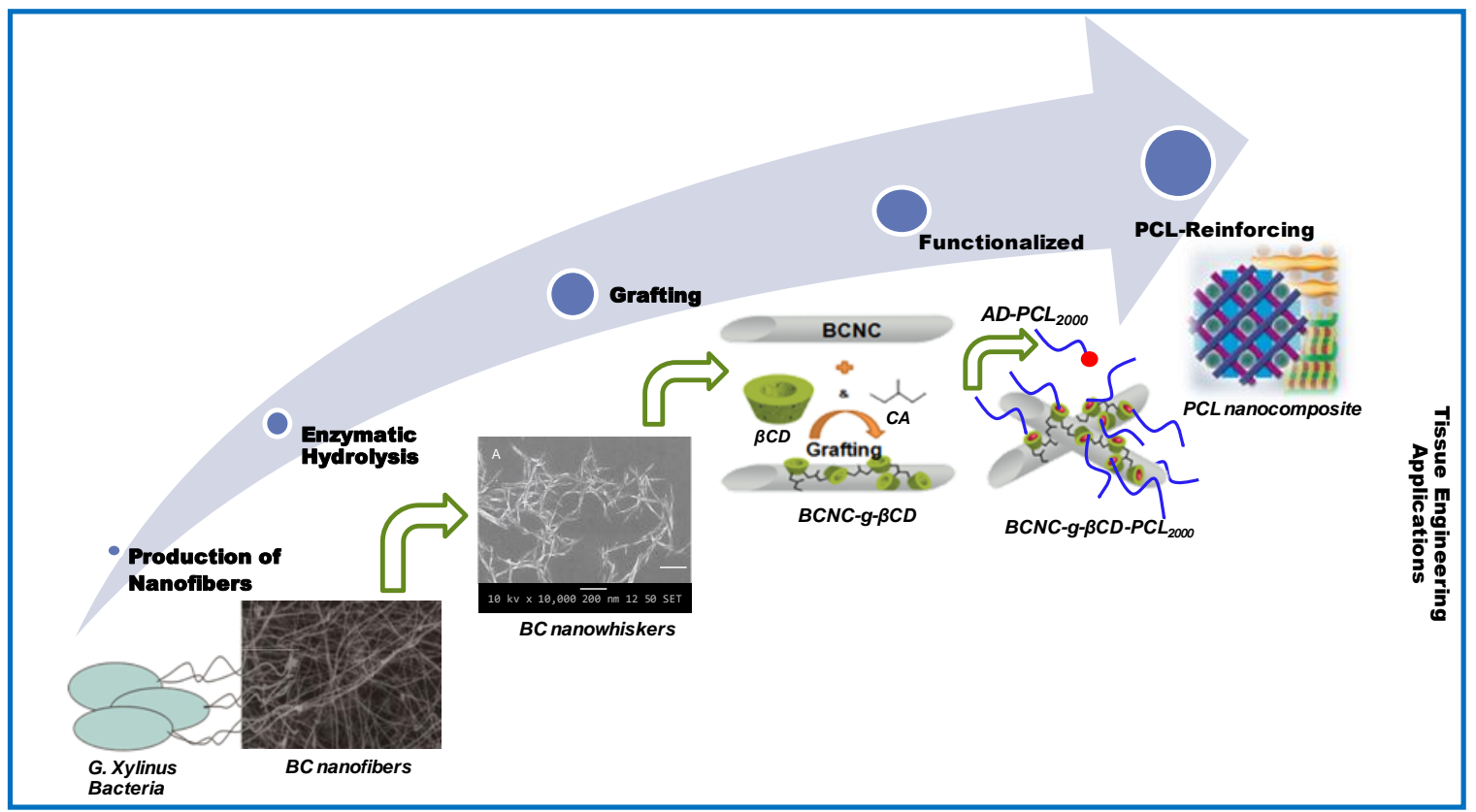

*This chapter is adapted from a submitted manuscript to the Journal of Applied Polymer Science (app.20190241). Mohamed M. Khattab, Yaser Dahman. Synthesis and Characterization of Cellulose Nanowhisker Reinforced-Poly(E-Caprolactone) Scaffold for Tissue Engineering Applications. 


\subsection{Introduction}

Limb salvage procedure is a type of surgery that involves local resection of bone or soft tissue cancers in order to avoid amputation and placement of either metal prosthesis or bone graft to provide mechanical support and platform for bone-tissue regeneration. This procedure has proven to be effective in the treatment of osteosarcoma due to its superior ability to maintain the external appearances and physiological functions of the patients' limbs. ${ }^{[141]}$

However, numerous limitations associated with metal prosthesis, such as corrosion, ${ }^{[142]}$ growth restriction, ${ }^{[145]}$ and the need for additional removal operation. ${ }^{[147]}$ As well as several drawbacks associated with bone graft; such as donor, nonunion, disease transmission, and rejection. ${ }^{[150]}$

Yet, the treatments with biodegradable polymeric scaffolds are emerged as viable alternatives procedure and considered as promising solution for bone and tissue defects. ${ }^{[393]}$ A variety of aliphatic polyesters have been used to fabricate biodegradable tissue engineering scaffolds. Polycaprolactone, polylactic acid, polyglycolic acid, and their copolymers, are most extensively used due to their excellent inherent biocompatibility, biodegradability, mechanical strength and the effortlessness processing into various 3D structures. ${ }^{[29,394]}$

PCL is a biocompatible, semicrystalline, slowly-degrading polymer used in several tissue engineering (TE) applications including bone, cartilage, sutures, adhesion barriers as well as in controlled release and targeted drug delivery. ${ }^{[395]}$ Additionally, this polymer gained popularity in the field of TE after receiving FDA approval due to its biocompatibility, ease of degradation in the human body, ${ }^{[23]}$ and higher permeability for drug molecules. ${ }^{[2]}$ Long-term in vivo studies have demonstrated that the PCL implant was able to maintain its physical shape after 2 years of implantation. In addition, PCL was also shown to be capable of being degraded slowly into low molecular weight by the end of 30 months and can be excreted completely out of the body, without accumulating non-toxic byproducts after degradation. ${ }^{[25]}$

PCL showed remarkable hydrophobicity and non-osteoconductivity that have stood as barrier and led to suboptimal cell-material interactions. ${ }^{[29]}$ However, bulk properties was modified by researchers to improve its osteoconductivity potential. This was achieved by adding growth factors during scaffold fabrication, attaching cells to the scaffold surface, or seeding cells and growth factors into the scaffold before implantation. ${ }^{[26]}$

The potential uses of biodegradable surface-engineered scaffolds as 3D templates mimicking extracellular cell matrix to encourage initial cell attachment, proliferation, and differentiation 
have been widely studied in vitro and in vivo. ${ }^{[396]}$ Basically, scaffold properties vary from tissue to tissue, as each has its unique mechanical and biochemical properties. A highly porous scaffold with an interconnected pores-network is necessary to mediate nutrient, waste diffusion and ultimately allows the growth of new load-bearing tissue at the implantation site. ${ }^{[26]}$

The pore size of TE scaffold is an important parameter that affects the quantity and characteristics of new tissue formation. Scaffold's pore size not only should be small enough to ensure mechanical integrity, but also large enough to allow the diffusion of nutrient and waste materials. An optimal pore size for TE engineering scaffolds is not well defined. However, pore sizes in the range of 100-400 $\mu \mathrm{m}$ are projected as optimum for osteoconduction, whereas pore sizes smaller than $100 \mu \mathrm{m}$ was known to be inadequate in terms of mass transport and cell migrations. ${ }^{[26]}$

A variety of techniques have been used for processing biodegradable polymeric 3D porous scaffolds. ${ }^{[396]}$ These conventional methods include freeze drying, melt-based technologies, phase inversion, thermally induced phase separation, fiber bonding, fiber felts, melt molding, solvent casting, gas foaming, particulate leaching, high-pressure processing and electrospinning. However, the obtained constructs by those methods commonly facing drawbacks due to random structures, unpredictable pore sizes, reduced pore interconnectivity, and low mechanical strength and structural stability. ${ }^{[397]}$ In modern TE, solvent casting is often used in combination with other technique to produce more efficient porous 3D structure. For instance, solvent casting-particulate leaching is probably the most convenient fabrication technique for developing porous scaffolds. ${ }^{[147]}$ This method involves casting of a polymer/salt/organic solvent mixture followed by solvent evaporation and dissolution of the salt particulates in an aqueous solution. The commonly used porogens are salt particles with different crystal sizes; such as sodium chloride, ammonium bicarbonate, glucose; low molecular weight polymers; paraffin microspheres, or emulsion particles of a defined size. ${ }^{[397,398]}$ Previous studies showed that the porogen leaching technique led to the formation of porous scaffolds with architecture properties that support integration between construct and host. ${ }^{[399]}$ However, this technique has limitations; it can only produce thin membranes with a dense surface skin layer, and might contain residual salt particles that left over from the process. In the current project a modified casting technique was introduced to fabricate 3D porous scaffolds of promoted porosities and pores-interconnectivity. This technique is based on the gas foaming/particulate leaching method, ${ }^{[400]}$ but with using two 
porogens; an effervescent ammonium bicarbonate as gas foaming agent for creating the main porosity, and the water soluble $\mathrm{PEG}_{2000}$ as porosity and pores-interconnectivity enhancer.

Cellulose nanocrystals (CNC) or nanowhisker (CNW), is highly promising green nanofibers with outstanding mechanical strength (Young's modulus of $150 \mathrm{GPa}$ ). It has highly hydrophilic surface due to the plenty of surface hydroxyl groups. ${ }^{[401]}$ It was utilized by many researchers to tune the hydrophilicity, mechanical properties, biodegradation and drug release behavior of PLA nanofibers. ${ }^{[402]}$ However, there is a big challenge confronting the dispersion and compatibility between $\mathrm{CNC}$ and polymer matrixes. For instance, it was reported that CNC content of $10 \mathrm{wt} \%$ was induced an obvious reduction in the tensile properties of PLA due to poor dispersion and incompatibility within polymer matrix. Xiang et al ${ }^{[403]}$ reported that $\mathrm{CNC}$ acts as nucleating agents and increases PLA crystallization, degradation and drug release rates. However, the increased CNC contents would induce a higher initial burst of the drug due to the strong interaction between CNC and drugs that resulted in a poor dispersion of drugs into the PLA matrix.

$\beta$-cyclodextrins $(\beta C D)$ play significant rule in drug delivery owing to their unique structure and promising features. $\beta C D$ has an ideal hydrophobic central cavity dimension that can make inclusion complexes (ICs) with different types of guest compounds such as, drugs and small organic molecules via non-covalent interactions. ${ }^{[19,21]}$ Moreover, grafting of cellulose nanofibers with $\beta C D$ is considered as promising technology for introducing another functional agents, such as short and long polymers segments. ${ }^{[20]}$ The non-covalent binding between $\beta C D$ and adamantane (AD) is selected as the model for immobilizing compounds due to the near-perfect size match between $\beta C D$ cavity and $\mathrm{AD}$. $\beta-\mathrm{CD} / \mathrm{AD}$ inclusion complexes showed extremely high binding constant of about $10^{4} \mathrm{M}^{-1}$. ${ }^{[75]}$ The later interaction is utilized by several researchers to make supramolecular assembly of different polymers. ${ }^{[74,404]}$

The present study aims to fabricate 3D porous scaffold with outstanding mechanical properties along with promoted cell attachment abilities. Two-step process is used to modify PCL matrix. The first step involves synthesis of the functionalized reinforcing nanowhisker (i.e., BCNW-g$\beta C D-\mathrm{PCL}_{2000}$ ) via grafting of $\beta C D$ molecules onto BCNW. This in turn allows for subsequent assembly of short $\mathrm{PCL}_{2000}$ segments on nanowhisker surface that consequently tuned the surface energy of nanowhisker and improved its dispersion in PCL matrix. The second challenging step is to examine the effect of nanowhisker loading on the final mechanical, thermal and hydrophilic 
properties of the obtained PCL-bionanocomposite. Finally, nanoconstructs of different porosity levels were fabricated. The combined effect of nanowhisker loading and porosity level on the compression modulus and in-vitro degradation of the porous scaffold was also investigated.

\subsection{Experimental}

\subsubsection{Materials and Chemicals}

Bacterial strain Gluconacetobacter xylinus (G. xylinus ATCC 700178) was supplied by American Type Culture Collection (ATCC), Manassas, VA 20108, USA. Citric acid (CA), $\beta$ cyclodextrin $(\beta C D)$, sodium hypophosphite (SHP), sodium hydroxide, cellulose enzyme (Trichoderma reesei ATCC26921) with specific enzyme activity of around $700 \mathrm{U} / \mathrm{g}$, ammonium bicarbonate $\left[\left(\mathrm{NH}_{4} \mathrm{HCO}_{3}\right) ; 99 \%, 100-500 \mu \mathrm{m}\right]$, polyethylene glycol (PEG; $\left.\mathrm{M}_{\mathrm{n}}=4000\right)$, Poly $(\varepsilon-$ caprolactone) diol (PCL-diol; $\left.\mathrm{M}_{\mathrm{n}}=2000\right)$, Poly( $\varepsilon$-caprolactone) (PCL; $\left.\mathrm{M}_{\mathrm{n}}=45,000\right)$, tin octoate ( $\mathrm{Sn}\left(\mathrm{Oct}_{2}\right)$ as catalyst, 1-adamantyl isocyanate (AD-NCO; 97\%), dimethylsulfoxide (DMSO), dimethyformamide (DMF), dichloromethane (DCM; 99.5\%), acetone, chloroform, methanol, diethyl ether, phosphate buffered saline (PBS; $\mathrm{pH} 7.4)$, sodium azide $\left(\mathrm{NaN}_{3}\right)$, and Lipase from porcine pancreas type II (lipase activity: 100-500 units/mg protein (using olive oil (30 min incubation), 30-90 units/mg protein (using triacetin)). All chemicals were of analytical grade and obtained from Sigma-Aldrich (Oakville, ON, Canada), and used as received without further purification.

\subsubsection{Preparations and Analysis Procedure}

\subsubsection{Preparation of BC Nanowhisker $(B C N W)$}

BC producing strain (G. xylinus) was activated according to ATCC guidelines. BC nanofibers were produced as fermentation product from fructose as C-source and corn steep liquor (CSL) as $\mathrm{N}$-source. ${ }^{[405]} \mathrm{BC}$ cellulose was further purified by boiling the pellicles in $0.2 \mathrm{M} \mathrm{NaOH}$ solution for 30 min followed by several rinses with distilled water until a neutral $\mathrm{pH}$ was attained. The pellicles were mechanically disintegrated to a cellulosic paste using a laboratory blender. The cellulose paste was suction filtered through Whatman No. 1 filter paper to remove excess water prior to hydrolysis. The obtained BC nanofibers paste was further transformed into nanocrystals by treating it with commercially available cellulose enzyme Trichoderma reesei. ${ }^{[198]} 10 \mathrm{~g}$ wet weight was suspended in $250 \mathrm{ml}$ of acetate buffer $(0.1 \mathrm{M}$; $\mathrm{pH} 5.0)$ to which $1 \mathrm{ml}$ of cellulase was 
added with gentle stirring. This suspension was kept at $50^{\circ} \mathrm{C}$ for $24 \mathrm{~h}$ in an incubator. The resulting suspension was filtered through sintered crucibles (No. 1) to remove unhydrolyzed fibers. The filtrate was centrifuged at $(4,000 \mathrm{rpm} \times 30 \mathrm{~min})$ to remove the excess enzyme and to collect the nanowhisker. BCNW was further washed with distilled water to remove the residual enzyme. Finally, BCNW suspension was re-concentrated to $0.5 \mathrm{wt} \%$ as confirmed from oven dry weight. The suspension kept at $5^{\circ} \mathrm{C}$ for further use.

\subsubsection{Preparation of $B C N W-g-\beta C D$}

Grafting reaction took place using citric acid (CA) as a linker via a two-step reaction. ${ }^{[379]}$ In the first reaction, CA- $\beta C D$ was prepared by refluxing of $3.0 \mathrm{~g}$ of $\beta \mathrm{CD}, 1.1 \mathrm{~g}$ of $\mathrm{CA}, 0.29 \mathrm{~g}$ of SHP, and $1.8 \mathrm{~g}$ of distilled water at $100^{\circ} \mathrm{C}$ for $1.5 \mathrm{~h}$. The product was purified by washing with ethanol several times, followed by drying at $60^{\circ} \mathrm{C}$ for $24 \mathrm{~h}$ to obtain pure dry CA- $\beta C D$. In the second reaction step, $3 \mathrm{~g}$ of the previously prepared CA- $\beta C D$ and $0.1 \mathrm{~g}$ of SHP were dissolved in $10 \mathrm{ml}$ of distilled water, followed by the addition of $20 \mathrm{ml}$ of $0.5 \mathrm{wt} \%$ BCNC suspension preultrasonicated for $10 \mathrm{~min}$. The reaction proceeded at $160^{\circ} \mathrm{C}$ for $15 \mathrm{~min}$ to obtain the higher grafting density ${ }^{[66]}$ After reaction, excess solution was removed by decantation, and then the suspension was washed with acetone and distilled water through multi centrifugation cycles until become neutral. The obtained suspension of $(\mathrm{BCNW}-g-\beta \mathrm{CD})$ was re-concentrated to $0.5 \mathrm{wt} \%$ and kept at $5^{\circ} \mathrm{C}$ for further use.

\subsubsection{Synthesis of Adamantyl End-capped $P C L_{2000}\left(P C L_{2000}-A D\right)$}

This reaction is carried out as described elsewhere. ${ }^{[406]} \mathrm{A}$ total of $1.5 \mathrm{~g}$ of PCL-diol was dissolved in $20 \mathrm{~mL}$ of chloroform. To this solution, 2-fold excess (mole ratio) of AD-NCO and a trace amount of $\mathrm{Sn}\left(\mathrm{Oct}_{2}\right)$ catalyst (about $10 \mu \mathrm{L}$ ) were added. The reaction was preceded at $60^{\circ} \mathrm{C}$ with stirring for $16 \mathrm{~h}$. Subsequently, the product was precipitated into $150 \mathrm{~mL}$ of diethyl ether cooled in ice water bath and kept still overnight. The final product was obtained by vacuum filtration, washed my methanol, and then dried at $45^{\circ} \mathrm{C}$.

\subsubsection{Synthesis of $B C N W-g-\beta C D-P C L_{2000}$}

Functionalized nanowhisker (BCNW-g- $\beta C D-\mathrm{PCL}_{2000}$ ) was prepared via supramolecular assembly of the guest polymer $\left(\mathrm{PCL}_{2000}-\mathrm{AD}_{2}\right)$ on $\mathrm{BCNW}-\mathrm{g}-\beta C \mathrm{C}$ using the method of Zhao et al. ${ }^{[11]}$ In brief, $40 \mathrm{mg} / \mathrm{mL}$ DMF solution of $\mathrm{PCL}_{2000}-\mathrm{AD}_{2}$ was prepared and added to an aqueous 
dispersion of BCNW-g- $\beta \mathrm{CD}$ which was solvent-exchanged from water to DMF by multi centrifugation cycles and sonicated for 15 min. Afterward, BCNW-g- $\beta C D$ suspension were mixed with the later solution and incubated in a shaker $(150 \mathrm{rpm})$ at room temperature overnight. The solution was decanted by centrifugation to remove excess DMF. After that, the mixture was put into deionized water and ultrasonicated for $15 \mathrm{~min}$ to precipitate and remove the excess free guest polymers from the suspension. The suspension was re-suspended again in DMF and washed many times by fresh DMF portions to insure the complete removal of any residue of the gust polymer. Eventually, the excess DMF was removed by centrifugation and replaced by DCM. The solution containing BCNW-g- $\beta C D-\mathrm{PCL}_{2000}$ was re-concentrated to $2 \%$ (wt $\%$ ) and kept at $5^{\circ} \mathrm{C}$ for further use.

\subsubsection{Dispersion of BCNW-g- $\beta C D-P C L_{2000}$ in $P C L$}

The less hydrophilic BCNW-g- $\beta C D-\mathrm{PCL}_{2000}$ was dispersed directly in DMF. Before adding it to PCL solutions, the dispersion was sonicated for 10 minutes in ice bath using high intensity homogenizer (Hielscher UP200Ht) equipped with $14 \mathrm{~mm}$ sonotrode. The amplitude and the output power will be kept at $26 \mathrm{kHz}$ and $160 \mathrm{~W}$. Homogenous dispersions containing BCNW-g$\beta C D-\mathrm{PCL}_{2000}$ of loadings (1-5 wt\%) in PCL were prepared as follow. A total of $1 \mathrm{~g}$ of PCL was first dissolved in DCM, and then mixed with freshly sonicated DMF solution containing the required amount of $\mathrm{BCNW}-\mathrm{g}-\beta \mathrm{CD}-\mathrm{PCL}_{2000}$. The mixture was kept under continuous stirring for 10 min until a homogenous dispersion was formed. The dispersions up $5 \mathrm{wt} \%$ of nanowhisker are colloidally stable over longer periods of time $\sim 2$ days. In case of dispersing unmodified BCNW in PCL, aqueous dispersion of unmodified BCNW was solvent-exchanged from water to acetone (three times), and then to DMF by using successive centrifugations $(4000 \mathrm{rpm} \times 15$ min). The dispersion was concentrated by evaporating excess solvent. Unmodified BCNW in DMF were sonicated before adding it to PCL solution as described before. Dispersions up to 5 wt $\%$ of BCNW were found to be colloidally stable over 3 hour period.

\subsubsection{Preparation of PCL Reinforced BCNW-g- $\beta C D-P C L_{2000}$ Nanocomposites Films}

Neat PCL, PCL reinforced BCNW and PCL reinforced BCNW-g- $\beta C D-$ PCL $_{2000}$ nanocomposites films were prepared by solvent casting method. ${ }^{[407]}$ The viscous polymer/nanowhisker solution mixture was preheated at $70^{\circ} \mathrm{C}$ and then gradually poured into hot-handmade stainless steel mold. The mold is subsequently cooled by flashed frozen in liquid nitrogen. The mold was then 
transferred to a refrigerator set at $-20^{\circ} \mathrm{C}$ and kept for $24 \mathrm{~h}$. After which, the obtained nanocomposites were placed under vacuum condition at $40^{\circ} \mathrm{C}$ for $48 \mathrm{~h}$ until complete solvent evaporation. The films were taken out from the mould and kept dry in a desiccator.

\subsubsection{Fabrication of PCL Porous Scaffolds}

Gas foaming/combined particulate leaching method ${ }^{[396]}$ was employed for fabricating 3D porous scaffold, but with some modifications. In the modified method, two progene salts are used; an effervescent salt progene ammonium bicarbonate as a gas foaming agent and $\mathrm{PEG}_{2000}$ as porosity and pores-interconnectivity enhancer, see Figure 4.1. Gel slurry composed of binary mixture of $\mathrm{PCL} \mathrm{PEG}_{2000}$ gel in chloroform and dispersed ammonium bicarbonate salt particles were casted in Teflon mold at room temperature. The semi-solidified construct is subsequently immersed in hot citric acid. The evolution of ammonia and carbon dioxide gases, along with the leaching out of ammonium bicarbonate and PEG particulates from the semi-solidified polymer matrix caused the formation of pores with high inter-connectivity. Briefly, highly viscous PCL polymer solution of concentration $30 \mathrm{wt} \%$ was first prepared by dissolving PCL pellets together with PEG $(1: 1 \mathrm{w} / \mathrm{w})$ in chloroform (3 $\mathrm{g}$ of $\mathrm{PCL}+3 \mathrm{~g}$ PEG $+4 \mathrm{~g}$ chloroform). Sieved ammonium bicarbonate particles of known crystal size ranged from 180-500 $\mu \mathrm{m}$ were used to develop the porous structure in PCL matrix. Standard sieved mesh was used to isolate the required particle size. An overnight oven dried and sieved ammonium bicarbonate particles in the range of 180300 or $300-500 \mu \mathrm{m}$ was used. $\mathrm{NH}_{4} \mathrm{HCO}_{3}$ particles was added to $4.0 \mathrm{~g}$ the binary polymer mixture where the salt: PCL mass ratios were kept at $(5: 1,7: 1$ and 10:1 w/w) in order to obtain nanocomposites of three levels of porosity. The mixtures were then stirred at $40^{\circ} \mathrm{C}$ for $30 \mathrm{~min}$.

Disc-shaped molds were constructed out of Teflon sheet of $\sim 4 \mathrm{~mm}$ thickness by drilling $15 \mathrm{~mm}$ diameter holes. Second Teflon sheet of the same thickness was used as the base of mold. The holed-sheet and base were clipped with binder clips. The mixture was casted into Teflon molds in the form of circular discs of approximately $(15 \mathrm{~mm}$ in diameter and $3 \mathrm{~mm}$ in height) by using $5 \mathrm{~mL}$ syringes with the tip cutoff. Briefly, back fill the syringe with the polymer/salt paste while putting fingers over the cutoff end of the syringe. When syringe is full, the plunger reinserted and used to press the paste against finger to compact the discs. The syringe was used to deposit the paste into the Teflon molds. The cutoff end of the syringe was used to scrape off the paste flush with the top of the mold. Afterwhich, chloroform was partially evaporated under atmospheric 
pressure, the semi-solidified constructs were immersed into an excess amount of hot citric acid solution $\left(50^{\circ} \mathrm{C}\right)$ for $30 \mathrm{~min}$ until no more gas bubbles were generated. Afterwards, the samples were placed into warm water for another $30 \mathrm{~min}$. finally the scaffolds washed three times with ethanol and then air dried and stored at $5^{\circ} \mathrm{C}$ until use. In a similar fashion, the paste mixture was casted into a cylinder-shaped mold ( $6 \mathrm{~mm}$ diameter $\times 12 \mathrm{~mm}$ height $)$ for compression tests.

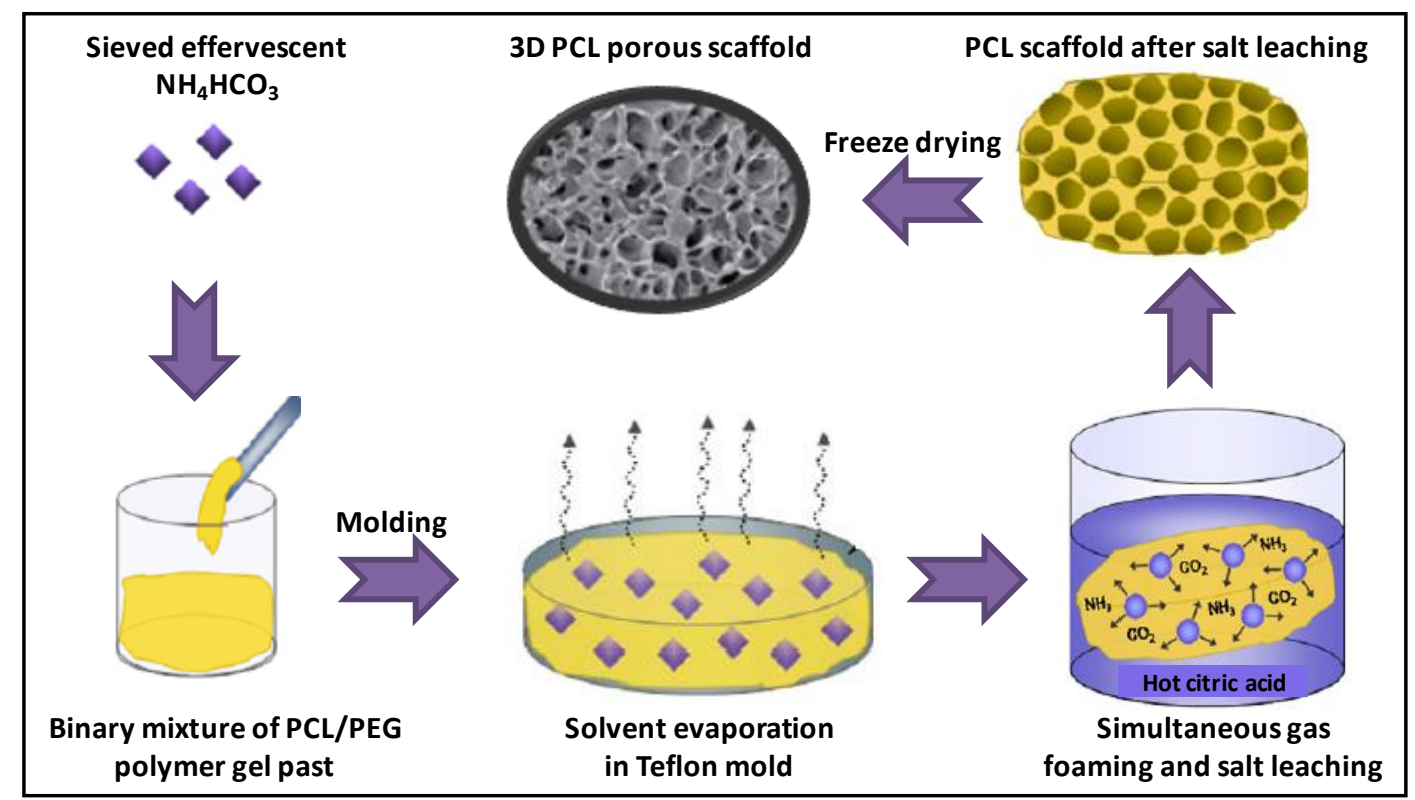

Figure 4.1 Fabrication of porous scaffolds by gas foaming/combined particulate leaching technique.

\subsubsection{Fabrication of PCL-reinforced BCNW-g- $\beta C D-P C L_{2000}$ Porous Scaffolds}

The required amounts of $\mathrm{BCNW}-g-\beta C D-\mathrm{PCL}_{2000}$ to prepare nanocomposites containing (1-5 $\mathrm{wt} \%$ ) of reinforcing nanowhisker were dispersed in DMF. Nanowhisker solutions were sonicated for 15 min before adding it to PCL/PEG polymer solution. The sonicated nanowhisker solutions were added slowly with continuous stirring to $4.0 \mathrm{~g}$ of $30 \%$ PCL/PEG polymer solution in chloroform for $30 \mathrm{~min}$ at $50^{\circ} \mathrm{C}$. Sieved ammonium bicarbonate particles of known crystal size were added to the mixture to develop the porous structure. The mixture was casted in circular Teflon molds as describer before, and then kept at $-20^{\circ} \mathrm{C}$ for $24 \mathrm{~h}$. The resultant scaffold was salt-leached and dried as before. 


\subsubsection{Porosity, Pore Volume, and Pore Size}

The porosity and pore volume of the scaffolds were estimated gravimetrically using Equations (4.1\& 4.2), ${ }^{[398]}$ where $\rho_{\text {polymer }}$ is the density of neat polymer used for scaffolds fabrication and $\rho_{\text {scaffold }}$ is the apparent density of the scaffold which determined by using Sartorius YDK 01LP density measurement kit.

$$
\begin{aligned}
& \text { Porosity }(\%)=\frac{\rho_{\text {scaffold }}}{\rho_{\text {polymer }}} \times 100 \\
& \text { Pore volume }=\left(\frac{1}{\rho_{\text {scaffold }}}-\frac{1}{\rho_{\text {polymer }}}\right) \times 100
\end{aligned}
$$

Thus, $\rho_{\text {PCL }}$ was considered to be $1.145 \mathrm{~g} / \mathrm{cm}^{3}$. Three samples were assessed for estimating both porosity and pore volume average values. The Pore size distribution and pores interconnectivity were also directly measured from the images developed by SEM.

\subsection{Characterization Techniques}

\subsubsection{Fourier Transform Infra-red Spectroscopy (FTIR/ATR)}

The structural changes accompanied the surface chemical modifications of BCNCs were followed by (FTIR/ATR) spectroscopy using (Perkin Elmer Spectrum1 FTIR/ATR spectrophotometer). ATR spectra were recorded in transmittance mode in the range of 4000-500 $\mathrm{cm}^{-1}$. ATR-crystal is ZnSe, the spectral resolution is $4 \mathrm{~cm}^{-1}$ and 20 scans were collected for each spectrum.

\subsubsection{Mechanical Properties of Nanocomposites Films and Scaffolds}

Mechanical parameters were determined for neat PCL and its bionanocomposite films. $\sigma_{\mathrm{y}}$ and $\varepsilon_{\mathrm{y}}$ are stress and strain at yield; $\sigma_{\mathrm{b}}$ and $\varepsilon_{\mathrm{b}}$ are stress and strain at break. Young's modulus (E) was calculated from slope of lines between 0.5 and $2.5 \%$ strain on stress-strain plots. Labthink's Param XLW (PC) Auto Tensile Tester equipped with a $500 \mathrm{~N}$ load cell and operating at a crosshead speed of $100 \mathrm{~mm} / \mathrm{min}$ at room temperature at a span distance of $25 \mathrm{~mm}$. The dimensions of the tested films were $\left(100 \times 10 \times 1 \mathrm{~mm}^{3}\right)$. Compression modulus of the scaffolds was evaluated at room temperature using the same load. Cross-head speed was set at $2 \mathrm{~mm} / \mathrm{min}$. Cylinder-shaped samples $(6 \mathrm{~mm}$ diameter $\times 12 \mathrm{~mm}$ height $)$ were used for compression tests according to ASTM method F451-95. 


\subsubsection{Thermal properties}

Thermogravimetric Analysis (TGA) and Differential Scanning Calorimetry (DSC) measurements of PCL and its bionanocomposites were conducted on a (Perkin Elmer Pyris Diamond) controlled with PYRIS 7 software. For TGA analysis $20 \mathrm{mg}$ samples were heated to $500^{\circ} \mathrm{C}$ in air current of $100 \mathrm{~cm}^{3} \mathrm{~min}^{-1}$ and heating rate of $10^{\circ} \mathrm{C} \mathrm{min}$. For DSC measurements sample specimens of $5-10 \mathrm{mg}$ were dried at $60^{\circ} \mathrm{C}$ for $2 \mathrm{~h}$ and sealed in $20 \mu \mathrm{L}$ aluminum pan. Samples heated from room temperature to $230^{\circ} \mathrm{C}$, held for $2 \mathrm{~min}$ to remove prior thermal history, and then cooled down to $-20^{\circ} \mathrm{C}$. The samples were then reheated to $230^{\circ} \mathrm{C}$. The heating/cooling rate was $10^{\circ} \mathrm{C} / \mathrm{min}$ under nitrogen atmosphere. The heat flow of the second heating scan was recorded for calculating the thermal parameters; cold crystallization temperature $\left(\mathrm{T}_{\mathrm{cc}}\right)$, melting temperature $\left(\mathrm{T}_{\mathrm{m}}\right)$, cold crystallization enthalpy $\left(\Delta \mathrm{H}_{\mathrm{cc}}\right)$, and melting enthalpy $\left(\Delta \mathrm{H}_{\mathrm{m}}\right)$. The degree of crystallinity $\left(\chi_{c}\right)$ was calculated using Equation (4.3). ${ }^{[408]}$

$$
\chi_{c}=\frac{\Delta \mathrm{H}_{\mathrm{m}}-\Delta \mathrm{H}_{\mathrm{cc}}}{\Delta \mathrm{H}_{0, \mathrm{~m}}\left(1-\mathrm{m}_{\mathrm{f}}\right)} \times 100
$$

where $\Delta \mathrm{H}_{\mathrm{m}}$, and $\Delta \mathrm{H}_{\mathrm{cc}}$ are enthalpies related to the second heating scan, $\Delta \mathrm{H}_{0, \mathrm{~m}}$ is melting enthalpy

for $100 \%$ crystalline PCL taken as $139.5 \mathrm{~J} / \mathrm{g},{ }^{[409]}$ and $\left(1-\mathrm{m}_{f}\right)$ is the weight fraction of PCL matrix in the sample.

\subsubsection{Field Emission Scanning Electron Microscopy (FE-SEM)}

SEM micrographs were taken at room temperature with JEOL/OE scanning electron microscope model JSM-6380 LV, equipped with Oxford energy dispersive X-ray spectroscopy (EDX). The surface morphology of unmodified and modified BCNWs was examined using microscope with an accelerating voltage of $10-20 \mathrm{kV}$ and at magnification of $\times 10,000$. Elemental analyses of nanowhisker were analyzed by EDX. Morphology and pore sizes of porous scaffolds were examined using $\left(5 \times 5 \mathrm{~mm}^{2}\right)$ samples, which were placed on an aluminum holder and sputter coated with $10 \mathrm{~nm}$ gold layer. The samples were analyzed at magnifications of $\times 200$ to $\times 3500$. Average pore diameters were measured from at least 30 pores using an image analyzer (Bum-Mi Universe Co., Ltd., Seoul, Korea). 


\subsubsection{Apparent Water-in-Air Contact Angle}

Static contact angle measurements were performed on nanocomposites films using Dataphysics OCA 15 optical contact angle measuring system using the sessile drop method. Deionised water droplets of $\sim 4 \mu \mathrm{L}$ were deposited on the samples surface via a motorized syringe. Five measurements per sample type were performed. The measurements were performed 2 min after droplet addition.

\subsubsection{In vitro degradation behavior}

Hydrolytic degradation was carried out for neat PCL and its nanocomposites in PBS solution (0.1 M PBS with $0.9 \% \mathrm{NaCl}, 0.02 \% \mathrm{NaN}_{3}$ and $\mathrm{pH}$ 7.4). Whereas enzymatic degradation was employed in the same PBS solution but in presence of Lipase enzyme from porcine pancreas at concentration level $0.1 \mathrm{mg} \mathrm{mL}^{-1}$. Samples were placed into $15 \mathrm{ml}$ vials containing $10 \mathrm{~mL}$ PBS and then incubated with shaking at $37^{\circ} \mathrm{C}$ to simulate in vivo dynamic tissue environment. The weight loss was recorded gravimetrically every 5 days using Equation (4.4). The reported weight loss was the average of triplicate.

$$
\text { Weight loss }(\%)=\left(\mathrm{W}_{0}-\mathrm{W}_{\mathrm{t}}\right) / \mathrm{W}_{0} \times 100 \%
$$

Where $\mathrm{W}_{0}$ is the initial dry weight of sample and $\mathrm{W}_{\mathrm{t}}$ is recorded dry weight after degradation time (t).

\subsubsection{Statistical Analysis}

All experiments were performed in triplicate. Results are presented as mean $\pm \mathrm{SE}$.

\subsection{Results and Discussion}

\subsubsection{Grafting of $B C N W$ with $\beta C D$ and Assembly of $P C L_{2000}-A D_{2}$ on $B C N W-g-\beta C D$ Surface}

Figure 4.2 A represents the grafting reactions of $\beta C D$ onto the surface of $\mathrm{BCNW}$ by using ecofriendly citric acid as a well-known nontoxic crosslinking agent. In order to obtain a higher grafting ratio of $\beta C D$ and to reduce the side effects on cellulose nanowhisker during the grafting process, a two-step reaction was employed. ${ }^{[379]}$ In the first step $\beta$-cyclodextrin citrate (CA- $\beta C D$ ) was obtained by condensation reaction between $\beta C D$ and $\mathrm{CA}$. The second step was grafting of $(\mathrm{CA}-\beta \mathrm{CD})$ onto $\mathrm{BCNW}$. Under the optimum reaction conditions the highest grafting ratio obtained was $33.8 \%$. 
Figure 4.2 $\mathrm{B}$ demonstrates the urethane reaction between $\left(\mathrm{PCL}_{2000}-\mathrm{diol}\right)$ and (AD-NCO) in presence of $\mathrm{Sn}\left(\mathrm{Oct}_{2}\right)$ as a catalyst to form the guest $\mathrm{PCL}_{2000}$ polymer with both ends capped with adamantane groups; abbreviated as $\left(\mathrm{PCL}_{2000}-\mathrm{AD}_{2}\right)$.

Figure 4.2 $\mathrm{C}$ represents the immobilization of $\mathrm{PCL}_{2000}-\mathrm{AD}_{2}$ onto the surface of $\mathrm{BCNW}-g-\beta C D$ to form the functionalized BCNW-g- $\beta C D-P_{2} L_{200}$. This achieved through the formation of hostguest inclusion complex between ADs moieties attached to the guest polymers and the grafted $\beta C D$ on the surface of BCNW. Because of $\beta C D$ s cavities can perfectly bind AD moieties in a polar solvent, ${ }^{[380]}$ therefore, $\mathrm{PCL}_{2000}-\mathrm{AD}_{2}$ was first assembled on the surface of $\mathrm{BCNW}-g-\beta C D$ by incubating the grafted nanowhisker in $\mathrm{DMF}$ solution of $\mathrm{PCL}_{2000}-\mathrm{AD}_{2}$, where the guest polymer molecules were captured via molecular recognition. After the assembly process, the solution mixture of $\mathrm{BCNW}-g-\beta C D$ and $\mathrm{PCL}_{2000}-\mathrm{AD}_{2}$ was transferred to an aqueous medium in order to enhance the interaction between grafted $\beta C D$ and $A D$ moieties and to precipitate non-reacted $\mathrm{PCL}_{2000}-\mathrm{AD}_{2}$ molecules as well. Moreover, it worth to mention the concentration of $\mathrm{PCL}_{2000^{-}}$ $\mathrm{AD}_{2}$ in solution was a key factor to maximize the efficiency of assembly process. The maximum weight gain by $\mathrm{BCNW}-g-\beta \mathrm{CD}$ due to $\mathrm{PCL}_{2000}-\mathrm{AD}_{2}$ assembly was archived when the concentration of $\mathrm{PCL}_{2000}-\mathrm{AD}_{2}$ in the mixture was in the range of $40 \mathrm{mg} / \mathrm{mL}$ for every $1 \mathrm{~g}$ of BCNW- $g-\beta C D .^{[11]}$

In addition, because of the guest polymers are both end-capped with $\mathrm{AD}$ groups, therefore two possibilities for their assembly on $\mathrm{BCNW}-\mathrm{g}-\beta \mathrm{CD}$ are suggested; (i) single-end immobilization and (ii) double-end immobilization; see Figure 4.2 C. A rough approximation based on obtained results indicates that either assembly fashions coexist.

\subsubsection{Urethane Reaction and Formation of Guest Polymer PCL $2000^{-A D}$}

Figure 4.3 A shows urethane reaction between $\mathrm{PCL}_{2000}$-diol and AD-NCO to form the guest polymer with both ends capped with adamantane groups (i.e., $\mathrm{PCL}_{2000}-\mathrm{AD}_{2}$ ). Analyzing ATR spectra show the main characteristic spectral bands for both free PCL-diol and AD-NCO before urethane reaction. The absorption peaks at 2945, 1726 and $1182, \mathrm{~cm}^{-1}$ were ascribed to C-H stretching of $-\mathrm{CH}_{2}$ groups, ester $\mathrm{C}=\mathrm{O}$ stretching and $\mathrm{C}-\mathrm{O}$ stretching of PCL-diol, ${ }^{[410]}$ respectively. The spectral bands at 2287 and $1485 \mathrm{~cm}^{-1}$ are characteristic peaks for the isocyanate stretching and $\mathrm{C}-\mathrm{N}$ stretching of the free AD-NCO. After urethane reaction, the characteristic bands due to $\mathrm{C}=\mathrm{O}$ and $\mathrm{C}-\mathrm{O}$ stretching of PCL-diol are retained in $\mathrm{PCL}_{2000}-\mathrm{AD}_{2}$ but with little shift to lower frequencies, while isocyanate peak of $\mathrm{AD}-\mathrm{NCO}$ was disappeared and a new secondary amide 
peak due to (-C=O-NH-) stretching was emerged at $3473 \mathrm{~cm}^{-1}$. This later evidences confirm the creation of urethane bond ${ }^{[411]}$ between $\mathrm{OH}$ groups of PCL-diol and isocyanate moieties of AD$\mathrm{NCO}$ and imply the successful formation of the gust polymer $\mathrm{PCL}_{2000}-\mathrm{AD}_{2}$.

\subsubsection{Formation of BCNW-g- $\beta C D$ and BCNW-g- $\beta C D-P C L_{2000}$}

Figure 4.3 B shows ATR spectra that monitored the change in chemistry of BCNW after grafting the surface with $\beta C D$ using $\mathrm{CA}$ as linker. Analyzing the spectral bands of $\mathrm{BCNW}-\mathrm{g}$ $\beta C D$ is showing very similar spectra to ungrafted $B C N W$ where all the characteristic bands reported for cellulose-I were present. ${ }^{[386]}$ However, a new vibration was emerged at $1708 \mathrm{~cm}^{-1}$ due to the presence of $\mathrm{C}=\mathrm{O}$ stretching vibration due to ester bond formed between citric anhydride moiety of $\mathrm{CA}-\beta \mathrm{CD}$ and $\mathrm{OH}$ groups of $\mathrm{BCNW}$. This band indicates the successfulness of the grafting reaction and confirms the formation of BCNW- $g-\beta C D$.

Figure 4.3 B depicts $\mathrm{ATR}$ spectrum of $\mathrm{BCNW}-\mathrm{g}-\beta \mathrm{CD}$ after $\mathrm{PCL}_{2000}-\mathrm{AD}_{2}$ assembly is completed. Compared to ungrafted BCNW, BCNW-g- $\beta C D-\mathrm{PCL}_{2000}$ spectra demonstrated an intense and overlapped peaks due to $\mathrm{C}=\mathrm{O}$ groups stretching in the range of $1707-1735 \mathrm{~cm}^{-1}$. This band arose from the combination of the carbonyl group stretching of both $\mathrm{PCL}_{2000}-\mathrm{AD}_{2}$ and BCNW-g- $\beta C D$. Additional peaks were also indentified at 3473, 2943 and $2866 \mathrm{~cm}^{-1}$ that ascribed to the secondary amide $-\mathrm{C}=\mathrm{O}-\mathrm{NH}$ - stretching, asymmetric and symmetric stretching of $\mathrm{CH}_{2}$ group of $\mathrm{PCL}_{2000}-\mathrm{AD}_{2}$, respectively. Therefore, all the previous evidences confirm the successful formation of the functionalized nanowhisker (BCNW-g- $\beta C D-\mathrm{PCL}_{2000}$ ). 


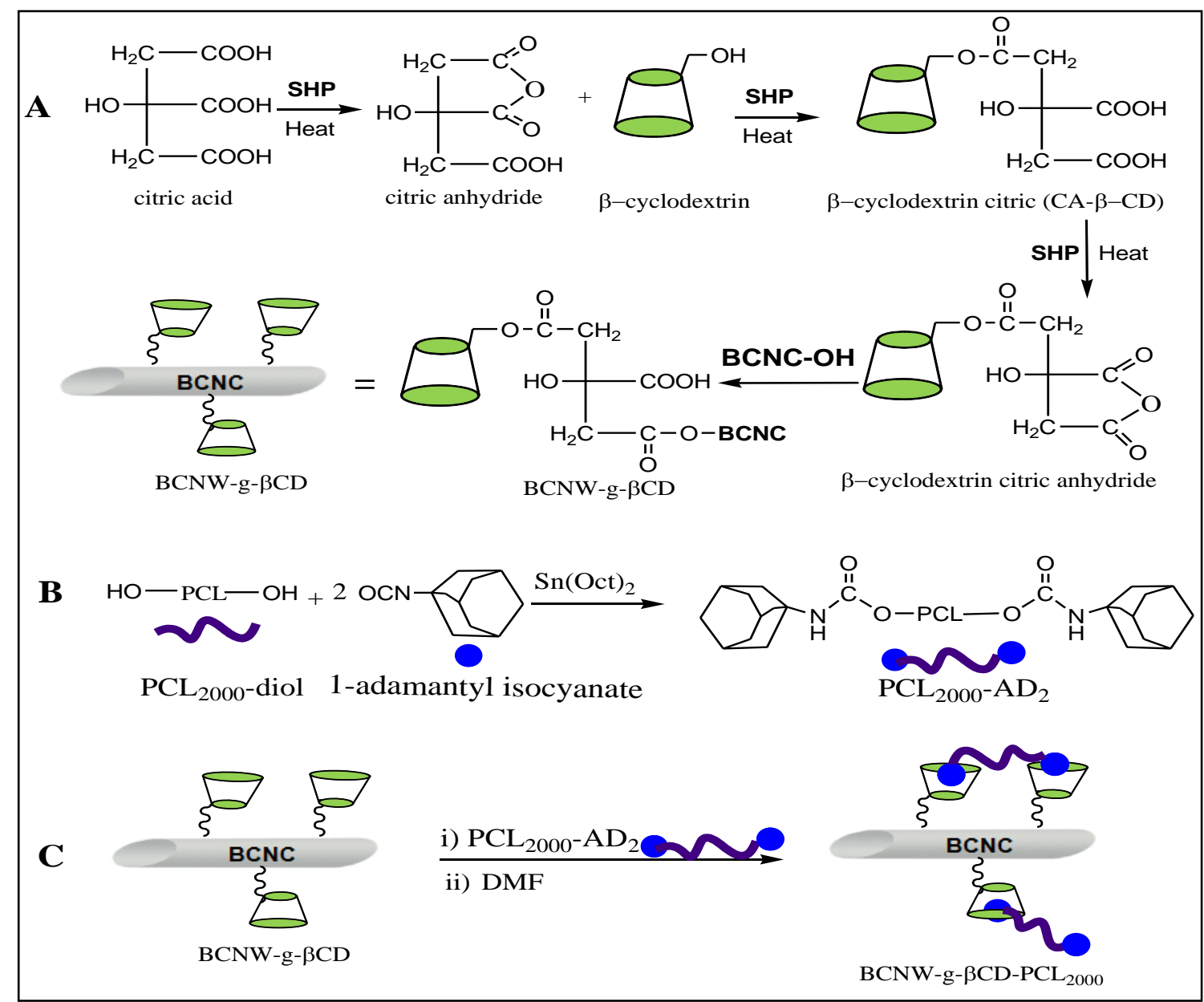

Figure 4.2 A: Grafting of $\mathrm{BCNW}$ with $\beta C D$ using citric acid as linker; B: synthetic pathway of $\mathrm{PCL}_{2000}-\mathrm{AD}_{2}$ from $\mathrm{PCL}_{2000}$-diol; $\mathrm{C}$ : conceptual illustration of short polymer segments assembly on BCNW- $g-\beta C D$ surface through the formation of inclusion complexes. 

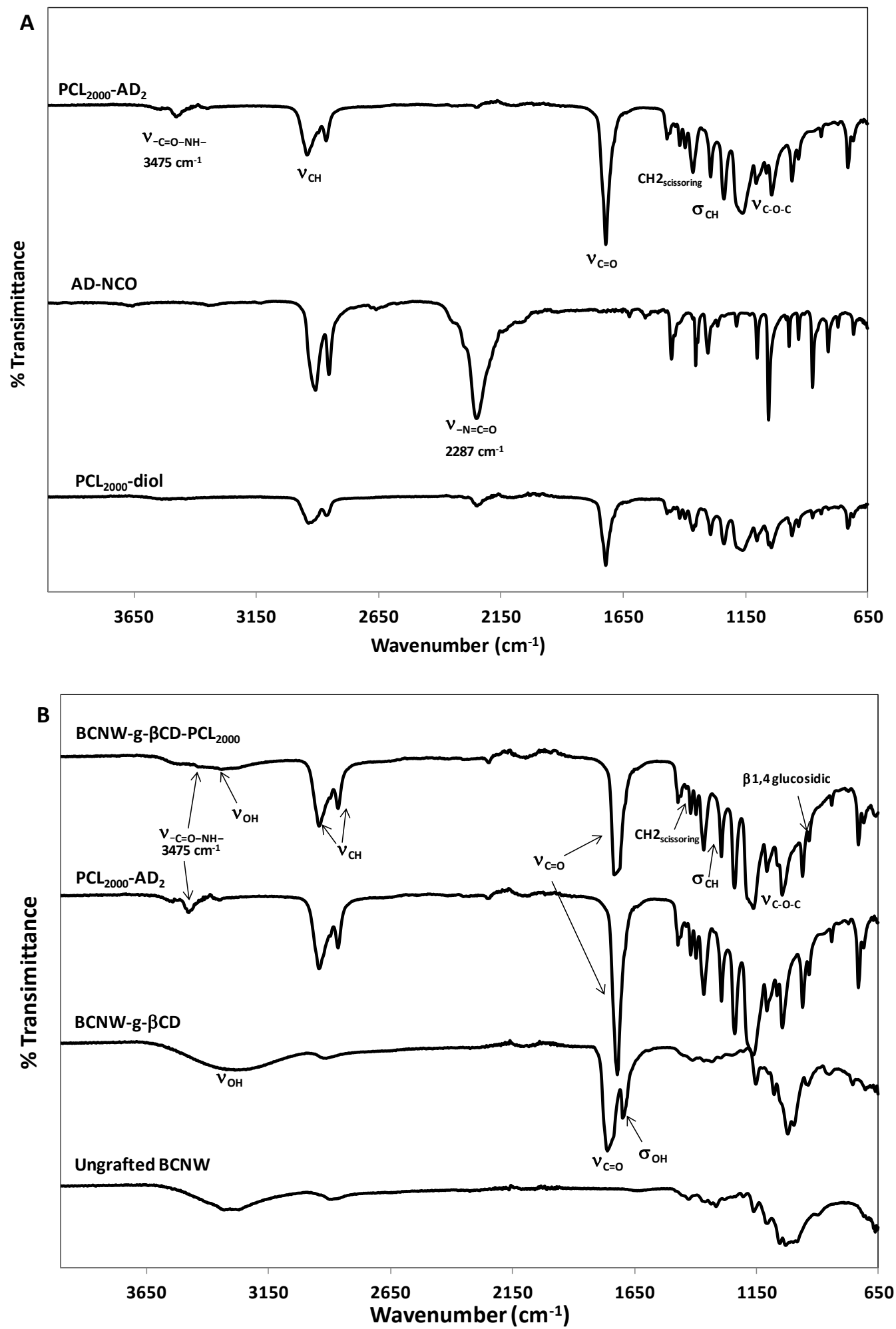

Figure 4.3 A: ATR spectra showing the formation of guest polymer $\left(\mathrm{PCL}_{2000}-\mathrm{AD}_{2}\right)$ from $\mathrm{PCL}_{2000}-$ diol and AD-NCO; B: ATR spectra of BCNW after grafting with $\beta C D$ and assembly of $\mathrm{PCL}_{2000}-\mathrm{AD}_{2}$. 


\subsubsection{Morphology and Surface Composition of BCNW-g-BCD-PCL ${ }_{2000}$}

Figure 4.4 shows FE-SEM images that describe the morphology of BCNW- $g$ - $\beta C D$ before and after $\mathrm{PCL}_{2000}-\mathrm{AD}_{2}$ assembly on the surface. Analyzing the image before $\mathrm{PCL}_{2000}-\mathrm{AD}_{2}$ assembly (Figure $4.4 \mathrm{~A}$ ) shows somewhat smoother surface for BCNW-g- $\beta C D$ similar to ungrafted BCNW. However, after polymer assembly the surface became a little rougher with a clearly observed patches; see Figure $4.4 \mathrm{~B}$. These observations were attributed to the assembled $\mathrm{PCL}_{2000}-\mathrm{AD}_{2}$ guest polymers. ${ }^{[412]}$ In addition, the few amount of small particles deposited on the surface of $\mathrm{BCNW}-\mathrm{g}-\beta C D-\mathrm{PCL}_{2000}$ may be related to the nonspecific absorption of $\mathrm{PCL}_{2000}-\mathrm{AD}_{2}$ molecules that were not completely removed during the post treatment of the modified nanowhisker.
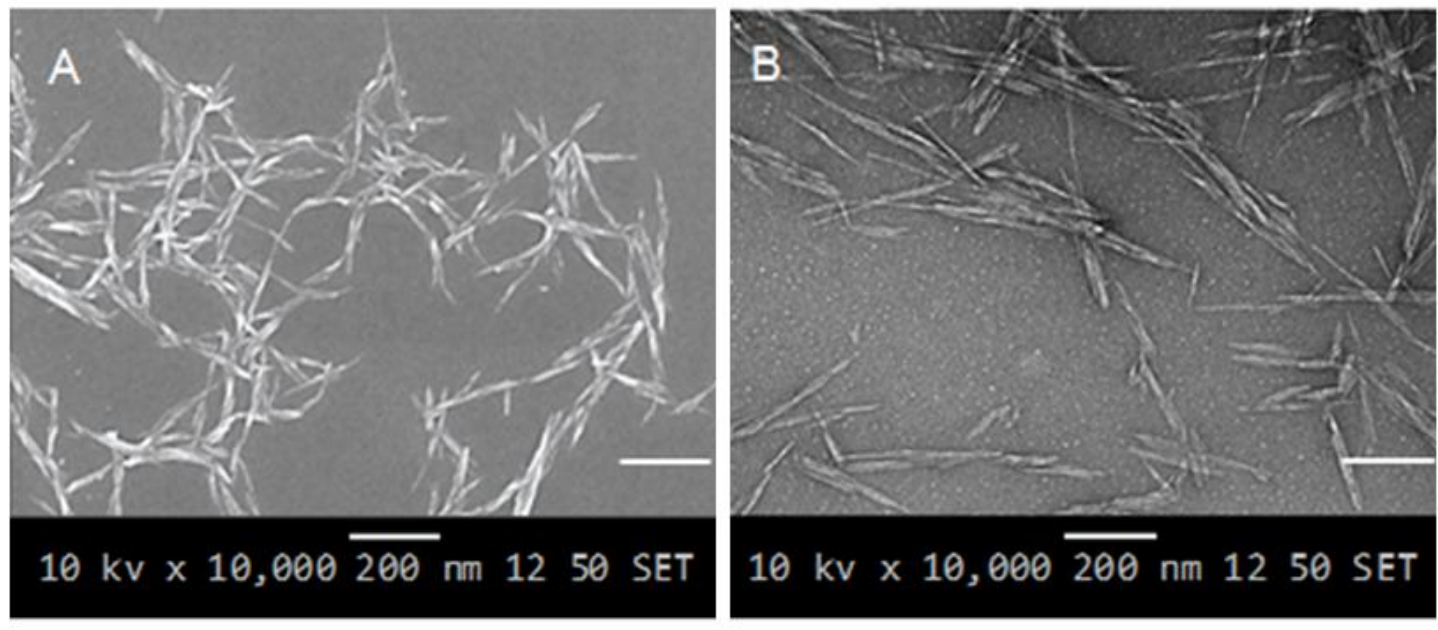

Figure 4.4 FE-SEM micrograph (200 nm scale) showing the surface morphology of BCNW- $g$ $\beta C D$ before and after $\mathrm{PCL}_{2000}-\mathrm{AD}_{2}$ assembly.

Figure 4.5 shows EDS spectra that analyze the variation in the surface chemical composition of BCNW- $g-\beta C D$ before and after $\mathrm{PCL}_{2000}-\mathrm{AD}_{2}$ assembly. Based on the fact that carbon and oxygen atoms are the main composition of BCNW-g- $\beta C D$ surface. Therefore, their corresponding atomic ratio can be quantitatively determined from the integration of Cs and Os signals. Results showed the carbon content increased from 54.1 to $65.3 \%$, where the oxygen content dropped from 49.9 to $34.7 \%$. These changes in the surface composition correspond to an increase in $\mathrm{C} / \mathrm{O}$ ratio from 1.1 to 1.8 after polymer assembly. This is due to that assembled $\mathrm{PCL}_{2000}-\mathrm{AD}_{2}$ polymer has higher carbon atomic content. Ultimately, the mole fraction of the assembled guest polymer on the surface of BCNW- $g-\beta C D$ can be estimated from Equation (4.5).

$$
\% \mathrm{C}(\mathrm{BCNW}-g-\beta \mathrm{CD}) \cdot x+\% \mathrm{C}\left(\mathrm{PCL}_{2000}-\mathrm{AD}_{2}\right) \cdot(1-x)=\% \mathrm{C} \text { (assembly) }
$$


where $x$ is the mole percentage of $\mathrm{BCNW}-g-\beta C D$, and $\% \mathrm{C}$ is the atomic carbon content of the corresponding component.

The calculated amount of assembled polymer was found to equal $24.8 \mathrm{wt} \%$. Proximate amount of $25.1 \mathrm{wt} \%$ was also estimated gravimetrically. The later finding suggests that the entire surface of BCNW-g- $\beta C D$ has not been fully covered by PCL $_{2000}$ after the assembly process. Moreover, based on the previous finding that $33.8 \mathrm{wt} \%$ grafting ratio of $\beta C D$ was achieved on the surface of $\mathrm{BCNW}$, and the fact that $\mathrm{PCL}_{2000}-\mathrm{AD}_{2}$ can only make inclusion complex with $\beta C D$ moieties grafted on nanowhisker surface. Therefore, by simple calculation one can estimate that approximately $73.4 \%$ of $\beta C D$ is occupied with $\mathrm{PCL}_{2000}$ segments, where the other $26.6 \%$ was still vacant. 

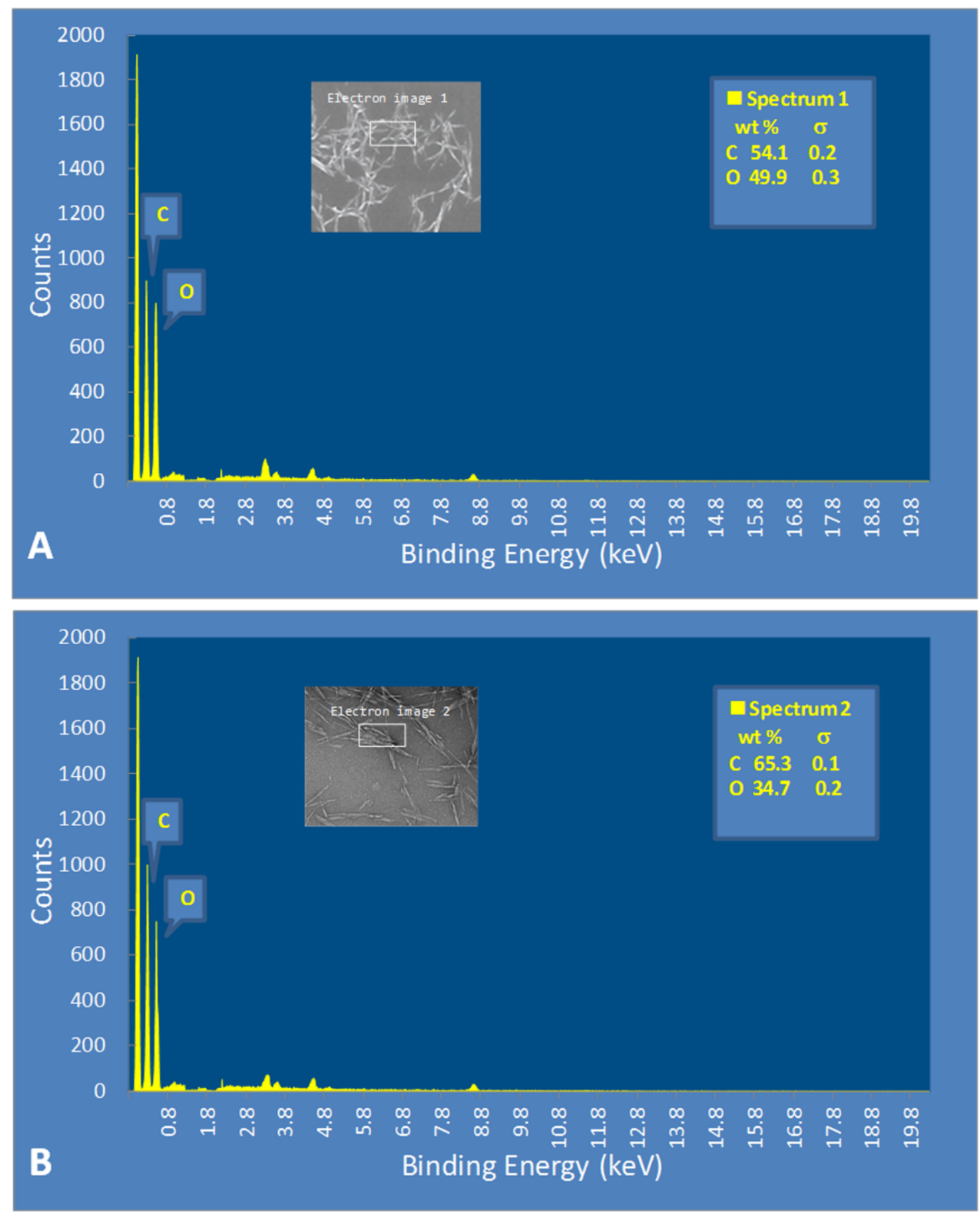

Figure 4.5 EDS spectrum of A: $B C N W-g-\beta C D$ before $\mathrm{PCL}_{2000}$ assembly; $\mathrm{B}$ : spectrum after PCL $_{2000}$ segments assembly 


\subsubsection{Mechanical Properties of BCNW-g-BCD-PCL $L_{2000}$ Reinforced PCL Composite Films}

Figure 4.6 A depicted the stress-strain curves of neat PCL and its bionanocomposite with both unmodified nanowhisker (i.e., BCNW) and functionalized nanowhisker (i.e., BCNW-g- $\beta C D-$ $\mathrm{PCL}_{2000}$ ). The mechanical testing parameters are summarized in Table 4.1. Results show that neat PCL film presents a less stiff and semi-ductile behavior where $\sigma_{\mathrm{y}}$ and $\varepsilon_{\mathrm{y}}$ values of $15.3 \mathrm{MPa}$ and $5.3 \%$ and $\sigma_{\mathrm{b}}$ and $\varepsilon_{\mathrm{b}}$ values of $14 \mathrm{MPa}$ and $10.3 \%$ were achieved. Initial addition of unmodified BCNW showed improvement in tensile strength at expenses of elasticity; see Table 4.1. An initial increase in $\sigma_{b}$ (i.e., $19.0 \pm 1.4$ ) was achieved when $1 \mathrm{wt} \%$ of unmodified BCNW was added to PCL matrix. Further increase in unmodified nanowhisker loading ratios from (2-5 $\mathrm{wt} \%)$ resulted in deterioration for all mechanical properties of PCL/BCNW nanocomposites. Hence, tensile strength, elasticity and modulus decreased monotonically with increasing nanowhisker loading ratios. This observation confirms poor wettability and incompatibility of hydrophilic nanowhisker within hydrophobic PCL matrix. In contrast, increasing of BCNW-g$\beta C D-\mathrm{PCL}_{2000}$ loadings ratio from (1-4 wt $\left.\%\right)$ in PCL matrix was associated with gradual enhancement for both strength and tensile modulus at expenses of ductility; see Figures 4.6 B and $\mathbf{C}$. The maximum increase was achieved at $4 \mathrm{wt} \%$ of BCNW- $g-\beta C D-P C L_{2000}$, where the tensile strength and Young's modulus significantly improved by $115 \%$ and $51 \%$, while elongation at break reduced by $47 \%$. This significant improvement in the stiffness and strength of $\mathrm{PCL} / \mathrm{BCNW}-\mathrm{g}-\beta \mathrm{CD}-\mathrm{PCL}_{2000}$ nanocomposites is reflecting an improved wettability and compatibility between the functionalized nanowhisker (i.e., BCNW-g- $\beta C D-P C L_{2000}$ ) and PCL matrix. This improvement can be related to the assembled short $\mathrm{PCL}_{2000}$ segments on nanowhisker surfaces, which create co-continuous phase between nanowhisker and PCL matrix. This in turn increased the interfacial adhesion between nanowhisker and matrix and ultimately allows proper stress transfer from PCL matrix to the high strength reinforcing phase network of the added BCNW- $g-\beta C D-P_{2} L_{2000}$. The enhancement of tensile strength and elastic modulus at the expense of elongation at break up on adding small quantities of cellulose nanofibers $(0.5-5$ wt\%) was previously reported. ${ }^{[240]}$ However, higher loading amounts of cellulose nanofibers over $5 \mathrm{wt} \%$ could lead to miscibility problems and deteriorate the mechanical properties of the nanocomposites. ${ }^{[246]}$ 
Table 4.1 Tensile properties of neat PCL, PCL/BCNW, and PCL/BCNW-g- $\beta C D-P C L_{2000}$ nanocomposites films

\begin{tabular}{|c|c|c|c|c|c|}
\hline Nanocomposites formulations & $\sigma_{\mathbf{y}}(\mathrm{MPa})$ & $\boldsymbol{\varepsilon}_{\mathbf{y}}(\%)$ & $\sigma_{\mathbf{b}}(\mathrm{MPa})$ & $\boldsymbol{\varepsilon}_{\mathbf{b}}(\%)$ & $\mathbf{E}(\mathrm{MPa})$ \\
\hline Neat PCL & $15.3 \pm 1.3$ & $5.3 \pm 0.2$ & $14.0 \pm 0.8$ & $10.3 \pm 0.2$ & $1560 \pm 126$ \\
\hline $\mathrm{PCL}+\mathrm{BCNW}(1 \%)$ & - & - & $19.0 \pm 1.4$ & $7.1 \pm 0.5$ & $1610 \pm 100$ \\
\hline $\mathrm{PCL}+\mathrm{BCNW}(2 \%)$ & - & - & $13.2 \pm 1.3$ & $5.8 \pm 0.3$ & $1540 \pm 115$ \\
\hline $\mathrm{PCL}+\mathrm{BCNW}(3 \%)$ & - & - & $11.1 \pm 1.2$ & $4.6 \pm 0.6$ & $1412 \pm 135$ \\
\hline $\mathrm{PCL}+\mathrm{BCNW}(4 \%)$ & - & - & $7.0 \pm 1.3$ & $3.7 \pm 0.4$ & $1350 \pm 110$ \\
\hline $\mathrm{PCL}+\mathrm{BCNW}(5 \%)$ & - & - & $5.0 \pm 1.1$ & $3.1 \pm 0.4$ & $1220 \pm 107$ \\
\hline $\mathrm{PCL}+\mathrm{BCNW}-g-\beta C D-\mathrm{PCL}_{2000}(1 \%)$ & $28.3 \pm 1.1$ & $5.9 \pm 0.3$ & $26.2 \pm 1.5$ & $9.3 \pm 0.2$ & $1870 \pm 126$ \\
\hline $\mathrm{PCL}+\mathrm{BCNW}-g-\beta C D-\mathrm{PCL}_{2000}(2 \%)$ & $29.2 \pm 1.6$ & $5.1 \pm 0.2$ & $28.3 \pm 1.3$ & $8.5 \pm 0.4$ & $1960 \pm 112$ \\
\hline $\mathrm{PCL}+\mathrm{BCNW}-g-\beta C D-\mathrm{PCL}_{2000}(3 \%)$ & $30.5 \pm 1.1$ & $4.7 \pm 0.3$ & $29.9 \pm 1.4$ & $7.4 \pm 0.2$ & $2210 \pm 130$ \\
\hline $\mathrm{PCL}+\mathrm{BCNW}-g-\beta C D-\mathrm{PCL}_{2000}(4 \%)$ & $31.4 \pm 1.2$ & $4.2 \pm 0.2$ & $30.2 \pm 1.1$ & $5.5 \pm 0.3$ & $2350 \pm 110$ \\
\hline $\mathrm{PCL}+\mathrm{BCNW}-g-\beta C D-\mathrm{PCL}_{2000}(5 \%)$ & - & - & $26.8 \pm 1.2$ & $3.6 \pm 0.3$ & $2243 \pm 102$ \\
\hline
\end{tabular}

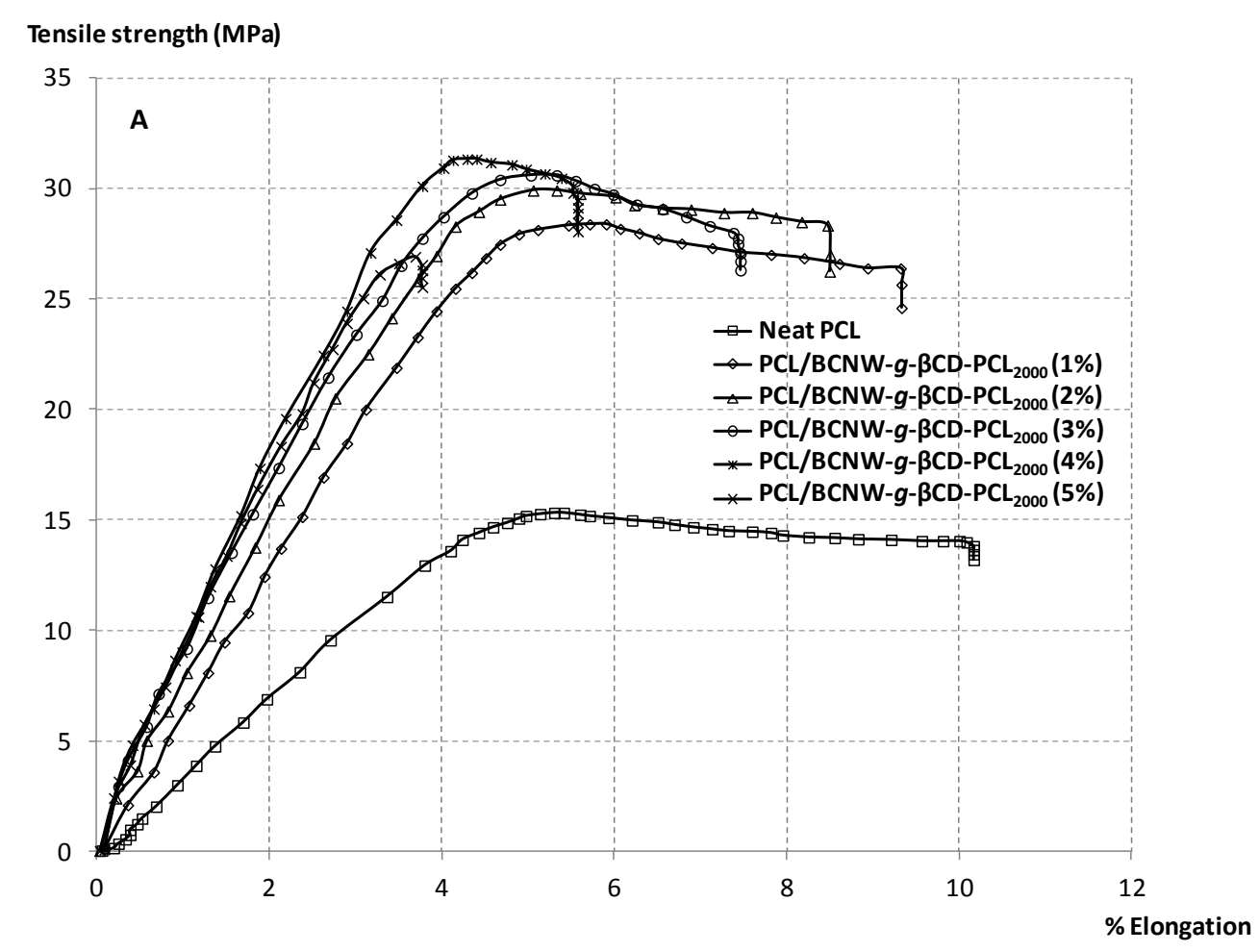

Figure 4. 6A: Stress-strain plots of neat PCL and PCL-bionanocomposite. 


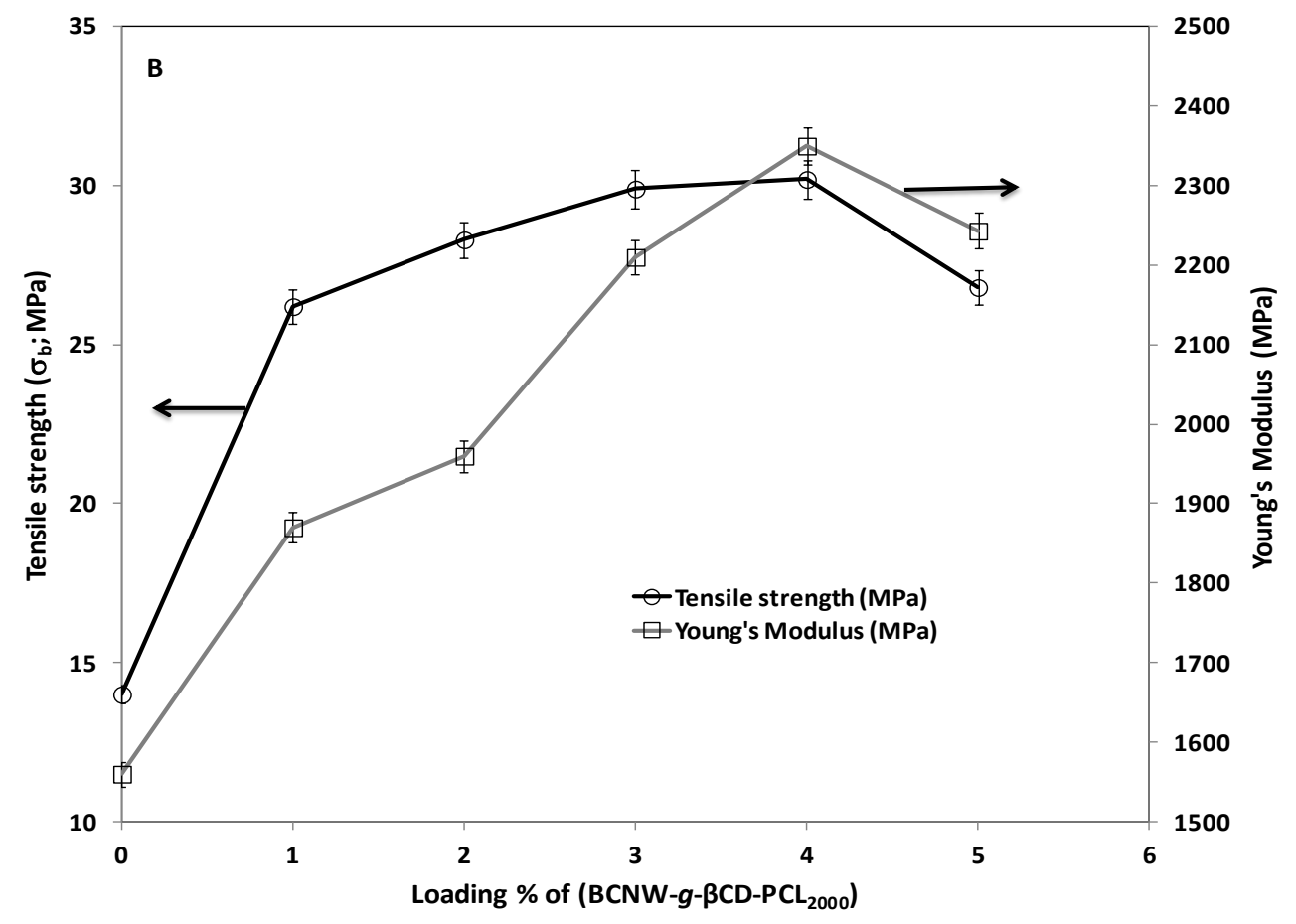

Figure 4.6B: Tensile strength, Young's modulus of neat PCL and its nanocomposites as function of BCNW-g- $\beta C D-\mathrm{PCL}_{2000}$ loadings.

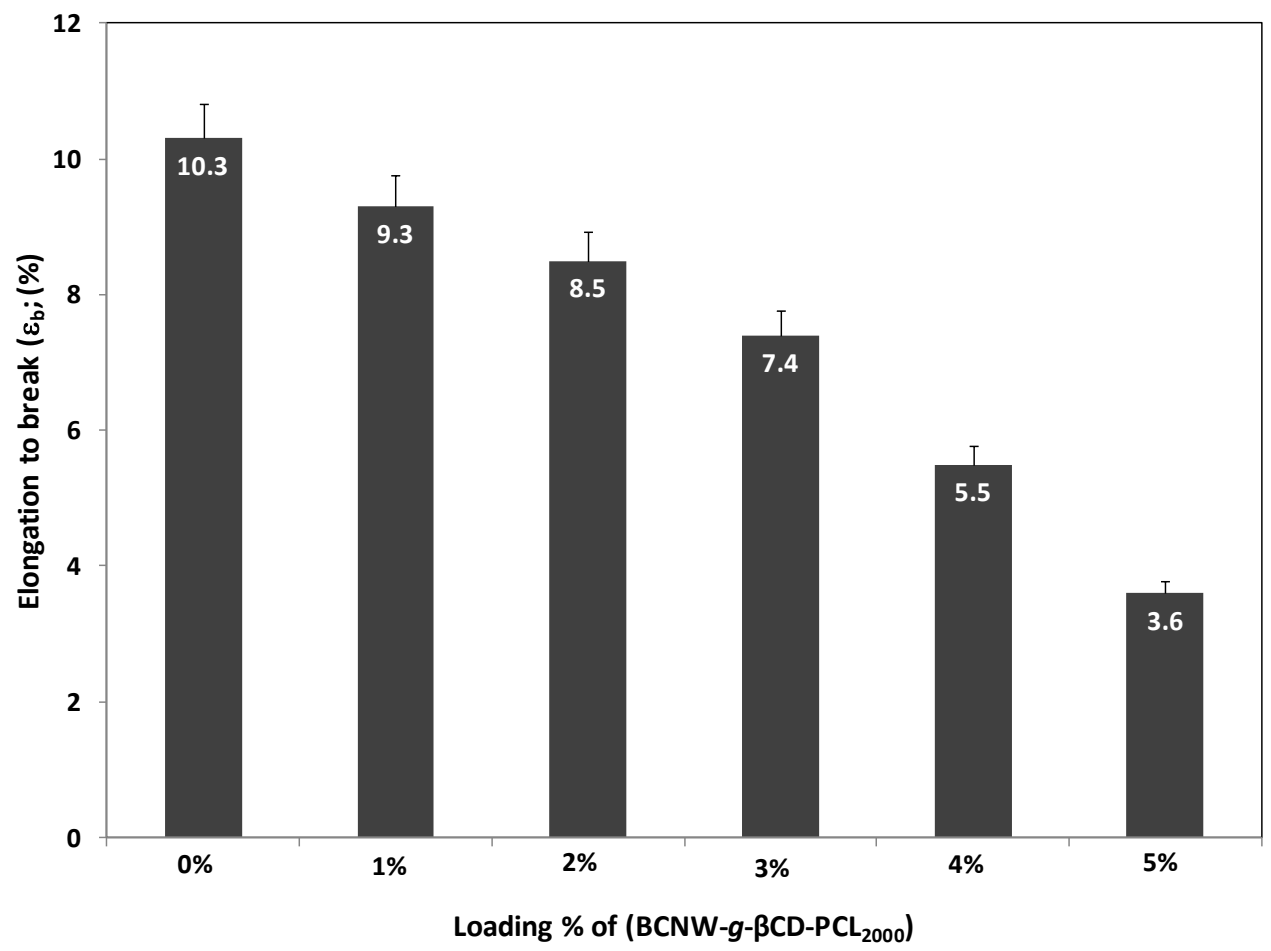

Figure 4.6C: Elongation at break of neat PCL and its nanocomposites as a function of $\mathrm{BCNW}-g$ $\beta C D-\mathrm{PCL}_{2000}$ loadings. 


\subsubsection{Surface Modification Evaluated by Contact Angle}

The bar chart in Figure 4.7 shows the evaluated apparent water-in-air contact angle of neat PCL and PCL-bionanocomposite films at different BCNW-g- $\beta C D-\mathrm{PCL}_{2000}$ loadings. In general, a contact angle value above $90^{\circ}$ corresponds to a hydrophobic surface, while a contact angle value below $90^{\circ}$ represents a hydrophilic surface. ${ }^{[413]}$ Results shows neat PCL shows contact angle values of $94^{\circ} \pm 2.5^{\circ}$. The later value was gradually decreased upon addition of (1-5 wt $\%$ ) of BCNW-g- $\beta$ CD-PCL 2000 to PCL matrix until a minimum plateau of $70^{\circ} \pm 2.9^{\circ}$ at $5 \mathrm{wt} \%$ loading was reached. However, compared to neat PCL, approximately $25.5 \%$ improvement in hydrophilicity of PCL-bionanocomposite was achieved by incorporating $5 \mathrm{wt} \%$ of BCNW-g$\beta C D-P_{2} L_{200}$ into PCL matrix. This increased hydrophilicity could be related to the inherited hydrophilic properties of $\mathrm{BCNW}$ due to the presence of free ungrafted hydroxyl groups on BCNW-g- $\beta C D-P_{2000}$ surface.

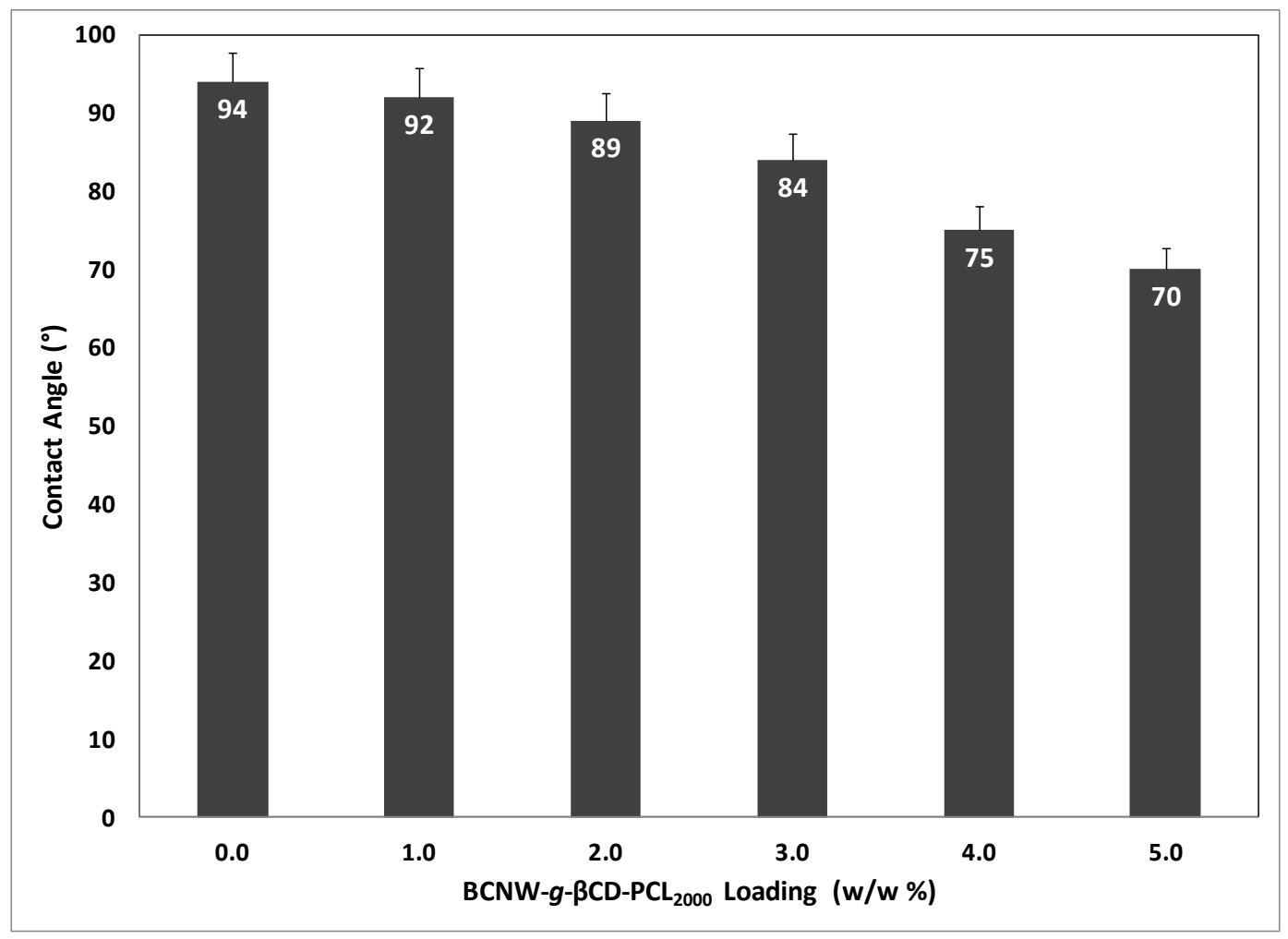

Figure 4.7 Apparent water-in-air contact angles of $\mathrm{PCL} / \mathrm{BCNW}-g-\beta C D-\mathrm{PCL}_{2000}$ nanocomposites films containing different loadings of functionalized nanowhisker. 


\subsubsection{Crystallization Behavior and Thermal Stability of PCL-bionanocomposite}

Differential scanning calorimetry was used to investigate the effect of grafted nanowhisker loadings on the crystallization and melting behaviors of PCL-bionanocomposite. Figure 4.8 displays thermal properties of the first cooling and second heating runs in the DSC thermograms of neat PCL and its bionanocomposite (i.e., PCL/BCNW-g- $\beta C D-\mathrm{PCL}_{2000}$ ). The obtained thermal properties (i.e., cold crystallization temperature; $\mathrm{T}_{\mathrm{cc}}$, melting temperature; $\mathrm{T}_{\mathrm{m}}$, cold crystallization enthalpy; $\Delta \mathrm{H}_{\mathrm{cc}}$, melting enthalpy; $\Delta \mathrm{H}_{\mathrm{m}}$ and crystallinity degree; $\chi_{\mathrm{c}}$ ) are summarized in Table 4.2. Generally, polymer crystallization behavior determined by DSC is dependent on the thermal history of sample preparation. As a result, recrystallization may occur during cooling or heating scan. Examining DSC results in Figure 4.8 shows that no obvious melt-crystallization peaks appeared during the first cooling scans. However, in the second heating scan, the cold crystallization peaks appeared for either neat PCL or its nanocomposites. Results show that neat PCL has $\mathrm{T}_{\mathrm{cc}}$ of $31^{\circ} \mathrm{C}$ and $\mathrm{T}_{\mathrm{m}}$ around $58^{\circ} \mathrm{C}$. An incorporation of $(1-4 \mathrm{wt} \%)$ of BCNW- $g-\beta C D-P_{200}$ in PCL matrix was induced obvious decrease in $\mathrm{T}_{\mathrm{cc}}$ values from 31 to $23^{\circ} \mathrm{C}$ but without significant increase in $\mathrm{T}_{\mathrm{m}}$ values $\left(58-59^{\circ} \mathrm{C}\right)$; see Table 4.2. This observed change in the crystallization behavior of nanocomposites can be attributed to the effect of lowmolecular weight $\mathrm{PCL}_{2000}$ segments conjugated on nanowhisker surface. These short polymer segments hindered the mobility of PCL chains, and consequently impaired the crystallization of PCL matrix. ${ }^{[414]}$

In addition, results in Table 4.2 show that an increase in nanowhisker loading leads to a decrease in the degree of crystallinity $\chi_{\mathrm{c}}$ of nanocomposites. The degree of crystalinity decreased from $27.5 \%$ (for neat PCL) to $21.7 \%$ up on addition of $4 \mathrm{wt} \%$ of BCNW- $g-\beta C D-\mathrm{PCL}_{2000}$. 
Table 4.2 Thermal pentameters for neat PCL and its bionanocomposite with various nanowhisker loadings

\begin{tabular}{|c|c|c|c|c|c|}
\hline Samples & $\mathbf{T}_{\mathrm{cc}}\left({ }^{\circ} \mathbf{C}\right)$ & $\Delta H_{c c}(J / g)$ & $\mathbf{T}_{\mathrm{m}}\left({ }^{\circ} \mathrm{C}\right)$ & $\Delta H_{m}(J / g)$ & $\chi_{c}(\%)^{\mathrm{a}}$ \\
\hline Neat PCL & 31 & 27.3 & 58 & 64.0 & 27.5 \\
\hline \multirow{4}{*}{$\begin{array}{l}\text { PCL+ BCNW- } g-\beta C D-P_{2000}(1 \%) \\
\text { PCL+ BCNW- } g-\beta C D-P C L_{2000}(2 \%) \\
\text { PCL+ BCNW- } g-\beta C D-P_{2000}(3 \%) \\
\text { PCL+ BCNW- } g-\beta C D-P_{2000}(4 \%)\end{array}$} & 29 & 28.1 & 58 & 63.1 & 25.9 \\
\hline & 27 & 29.5 & 58.5 & 62.0 & 23.8 \\
\hline & 25 & 32.0 & 58.5 & 61.5 & 22.0 \\
\hline & 23 & 34.0 & 59 & 60.8 & 21.7 \\
\hline
\end{tabular}

${ }^{\mathrm{a}} \chi_{c}=\left[\left(\Delta \mathrm{H}_{\mathrm{m}}-\Delta \mathrm{H}_{\mathrm{cc}}\right) /\left(\left(\Delta \mathrm{H}_{0, \mathrm{~m}}(1-(\mathrm{wt} \% \mathrm{BCNW}))\right] \times 100 ; \Delta \mathrm{H}_{0, \mathrm{~m}}\right.\right.$ of $100 \%$ crystalline PCL sample taken as $139.5 \mathrm{~J} / \mathrm{g} .{ }^{[409]}$

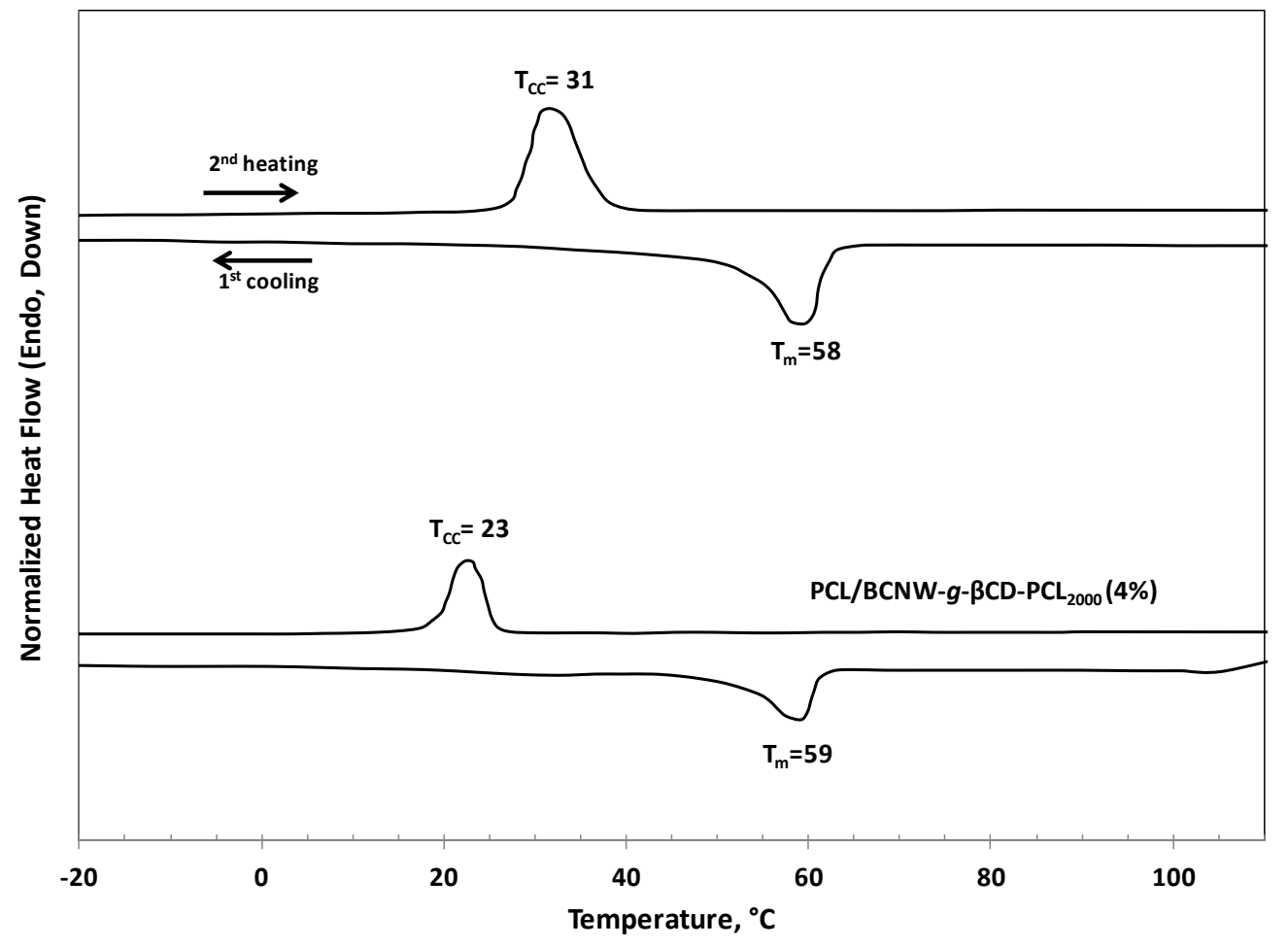

Figure 4.8 DSC cooling and heating runs of neat PCL and its bionanocomposite.

Figure 4.9 depicts TGA thermograms of neat PCL, functionalized nanowhisker BCNW- $g-\beta C D-$ $\mathrm{PCL}_{2000}$, and PCL/BCNW-g- $\beta C D-\mathrm{PCL}_{2000}(4 \%)$ nanocomposites. Investigating Figure 4.9 shows that the maximum decomposition temperature $\left(\mathrm{T}_{\max }\right)$ achieved by each thermogram was as follow; $445^{\circ} \mathrm{C}$ for neat PCL; $436^{\circ} \mathrm{C}$ for $\mathrm{BCNW}-g-\beta C D-\mathrm{PCL}_{2000}$; and $446^{\circ} \mathrm{C}$ for PCL/BCNW- $g$ $\beta C D-\mathrm{PCL}_{2000}(4 \%)$. Examining the obtained results shows that adding $4 \mathrm{wt} \%$ of functionalized 
nanowhisker to PCL matrix does not cause obvious change in $\mathrm{T}_{\max }$ value of nanocomposites if it compared with neat PCL polymer.

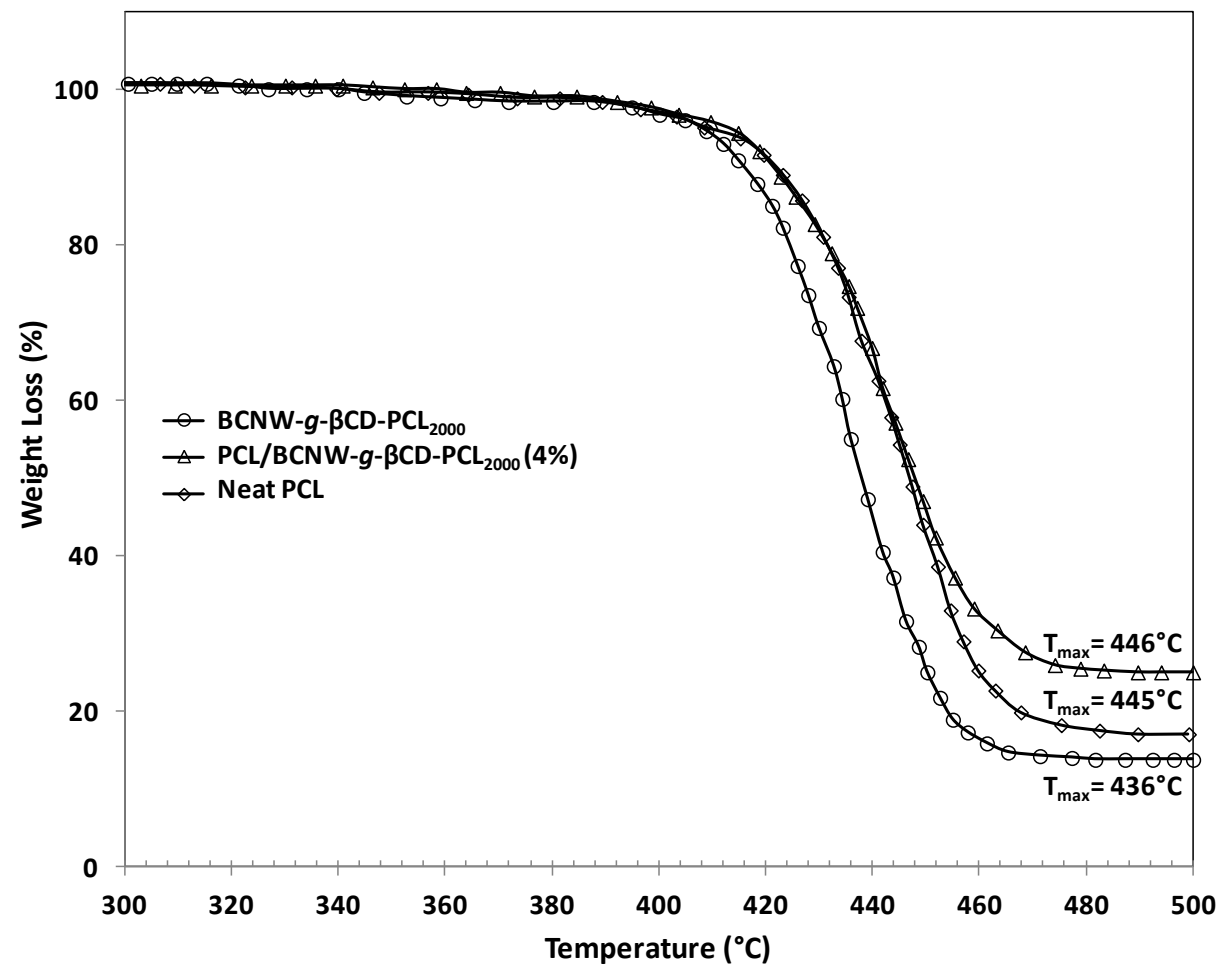

Figure 4.9 TGA curves of neat PCL, functionalized nanowhisker BCNW-g- $\beta C D-\mathrm{PCL}_{2000}$, and their bionanocomposite

\subsubsection{Scaffolds Porosities and Morphologies}

Due to the impressive mechanical and thermal properties of the nanocomposites formulation (PCL/BCNW-g- $\beta C D-P_{2000}(4 \%)$ ) it was chosen as a representative for developing nanostructures of different porosities. Gas foaming/combined particulate leaching method incorporating two progene; fixed amount of PEG, and different ratios of sieved ammonium bicarbonate salt having two different size distributions (180-300 $\mu \mathrm{m}$ and 300-500 $\mu \mathrm{m})$ were used for developing nanostructures of different porosities.

Table 4.3 summarizes percentage porosity, pore volume, and pore size of the obtained porous scaffolds. Results show that percentage porosities of the obtained scaffolds that obtained gravimetrically are in the range of $86.41-94.86 \%$ with estimated pore volumes in the range of $9.66-17.81 \mathrm{~cm}^{3} / \mathrm{g}$. At the same nanowhisker loading, the observed percentage porosity of the 
scaffold was improved from $86.41 \%$ to $94.86 \%$ with increasing salt: polymer weight ratio from 5:1 to $10: 1$.

Moreover, Table 4.3 is also depicted the obtained compression modulus values that measured for the cylinder shaped nonporous and porous scaffolds. For nonporous scaffolds, results show that PCL reinforced with 4 wt\% BCNW-g- $\beta C D-\mathrm{PCL}_{2000}$ has almost three-fold compression modulus value than neat PCL (control). However, in regard to the porous bionanocomposite scaffolds a decline in the modulus values was observed with the increase in salt: polymer ratio. For instance, the scaffold fabricated using 5:1 ammonium bicarbonate/polymer ratio showed the maximum compression modulus of $9.1 \pm 0.7 \mathrm{MPa}$, but the least porosity value of $86.41 \%$. In contrast, 10:1 salt/polymer ratio showed the highest porosity of $94.86 \%$ with the least compression modulus of $5.3 \pm 0.3 \mathrm{MPa}$. However, 7:1 salt/polymer ration has maintained good compression modulus value of $8.7 \pm 0.5 \mathrm{MPa}$ along with higher porosity of $91.28 \%$. When using the same polymer: salt ratio (i.e., 7:1), the coarser ammonium bicarbonate crystals, the bigger pore sizes are and less compression modulus (Table 4.3). Therefore, 7:1 salt to polymer was identified in this study as the most suitable ratio for developing scaffolds with higher porosity (> 90\%) along with adequate mechanical properties.

Nevertheless, in case similar experiment where porous scaffolds were fabricated from neat PCL using 7:1 salt: polymer ratio (control experiment), the observed compression modulus values are harshly decreased with increasing porosity level; see Table 4.3. The later evidence confirms the main role of BCNW-g- $\beta C D-\mathrm{PCL}_{2000}$ as reinforcing phase for keeping the integrity of the drugladen nanocomposites. This is quite seen from the potentiality of $\mathrm{BCNW}-g-\beta C D-\mathrm{PCL}_{2000}$ to compensate for the deterioration in mechanical properties due to the developed porous structures. For instance, scaffold fabricated with PCL/BCNW-g- $\beta C D-P C L_{2000}$ (4\%) using 7:1 (salt: polymer) showed almost the same level of porosity of neat PCL, but with four-fold compression modulus value (Table 4.3).

Figure 4.10 shows SEM images of the porous scaffolds that obtained by gas foaming/combined particulate leaching method. Examining the morphologies of the porous scaffolds confirms an opened macropores on the surface without sign of surface skin. These macropores are generated due to immediate gas evolution from salt particulates and subsequent rapid gas flow-out from the semi-solidified polymer mixture. In addition, the cross-section views in Figure 4.10 A1, A2, B1, B2, C1, C2 reveal the presence of uniform distribution of well-interconnected opened 
macropores from surface to surface. The average pore sizes that determined by visual estimation from SEM images were ranged from $\sim 250-420 \mu \mathrm{m}$. The estimated pore sizes appeared to be dependent on the size of the sieved ammonium bicarbonate particulates.

The adequate pore sizes, highly porous structure with an interconnected pore-network, along with an improved hydrophilicity suggest the suitability of the developed scaffolds in this study for encouraging initial cell attachment, proliferation, and differentiation. ${ }^{[396]}$ In addition, the compression modulus values range (i.e., 5.3-9.1 MPa) are located in the range of cancellous bones (i.e., 2-12 MPa). ${ }^{[415]}$ Therefore, these developed drug-laden PCL scaffolds can find application as temporal implant or bone cement.

The obtained results suggest the successfulness of gas foaming/combined particulate leaching technique that introduced in this study for developing scaffold with higher porosities. It also worth to mention the used fabrication method showed two distinctive advantages over the other examined techniques. The blend mixture (i.e., PCL/PEG/nanowhisker/ammonium bicarbonate) can be easily manipulated to make any shape and size, and also the final scaffold did not show any sign for surface skin formation.

Table 4.3 Porosity percentage, pore volume, pore size, and compression modulus for different porous scaffolds obtained from PCL/BCNW-g- $\beta C D-\mathrm{PCL}_{2000}(4 \%)$

\begin{tabular}{|c|c|c|c|c|c|c|}
\hline Scaffold Formulation & $\begin{array}{c}\mathrm{NH}_{4} \mathrm{HCO}_{3} \\
: \text { PCL } \\
(\mathrm{w} / \mathrm{w})\end{array}$ & $\begin{array}{c}\mathrm{NH}_{4} \mathrm{HCO}_{3} \\
\text { Size }(\mu \mathrm{m})\end{array}$ & $\begin{array}{l}\text { Porosity* } \\
(\%)\end{array}$ & $\begin{array}{c}\text { Pore } \\
\text { Volume* } \\
\left(\mathrm{cm}^{3} / \mathbf{g}\right)\end{array}$ & $\begin{array}{l}\text { Pore } \\
\text { Size* } \\
(\mu \mathrm{m})\end{array}$ & $\begin{array}{c}\text { Compressive } \\
\text { Modulus } \\
\text { (MPa) }\end{array}$ \\
\hline \multirow{2}{*}{ PCL (control) } & - & - & non & - & - & $5.2 \pm 0.2$ \\
\hline & $7: 1$ & $180-300$ & 90.77 & 11.71 & $264 \pm 8$ & $2.3 \pm 0.1$ \\
\hline \multirow{5}{*}{$\begin{array}{l}\text { PCL/BCNW- } g-\beta C D-P^{-} L_{2000} \\
(4 \%)\end{array}$} & - & - & non & - & - & $15.1 \pm 0.6$ \\
\hline & $5: 1$ & $180-300$ & 86.41 & 9.66 & $257 \pm 10$ & $9.1 \pm 0.7$ \\
\hline & $7: 1$ & $180-300$ & 91.28 & 12.33 & $285 \pm 16$ & $8.7 \pm 0.5$ \\
\hline & $10: 1$ & $180-300$ & 94.86 & 17.81 & $295 \pm 13$ & $5.3 \pm 0.3$ \\
\hline & $7: 1$ & $300-500$ & 93.55 & 13.32 & $418 \pm 17$ & $7.6 \pm 0.7$ \\
\hline
\end{tabular}

The significant differences for each individual feature, with $\mathrm{p}<0.05$, was determined by one-way ANOVA. $*(n=5$ for porosity and pore volume, and $n=30$ for pore size). 

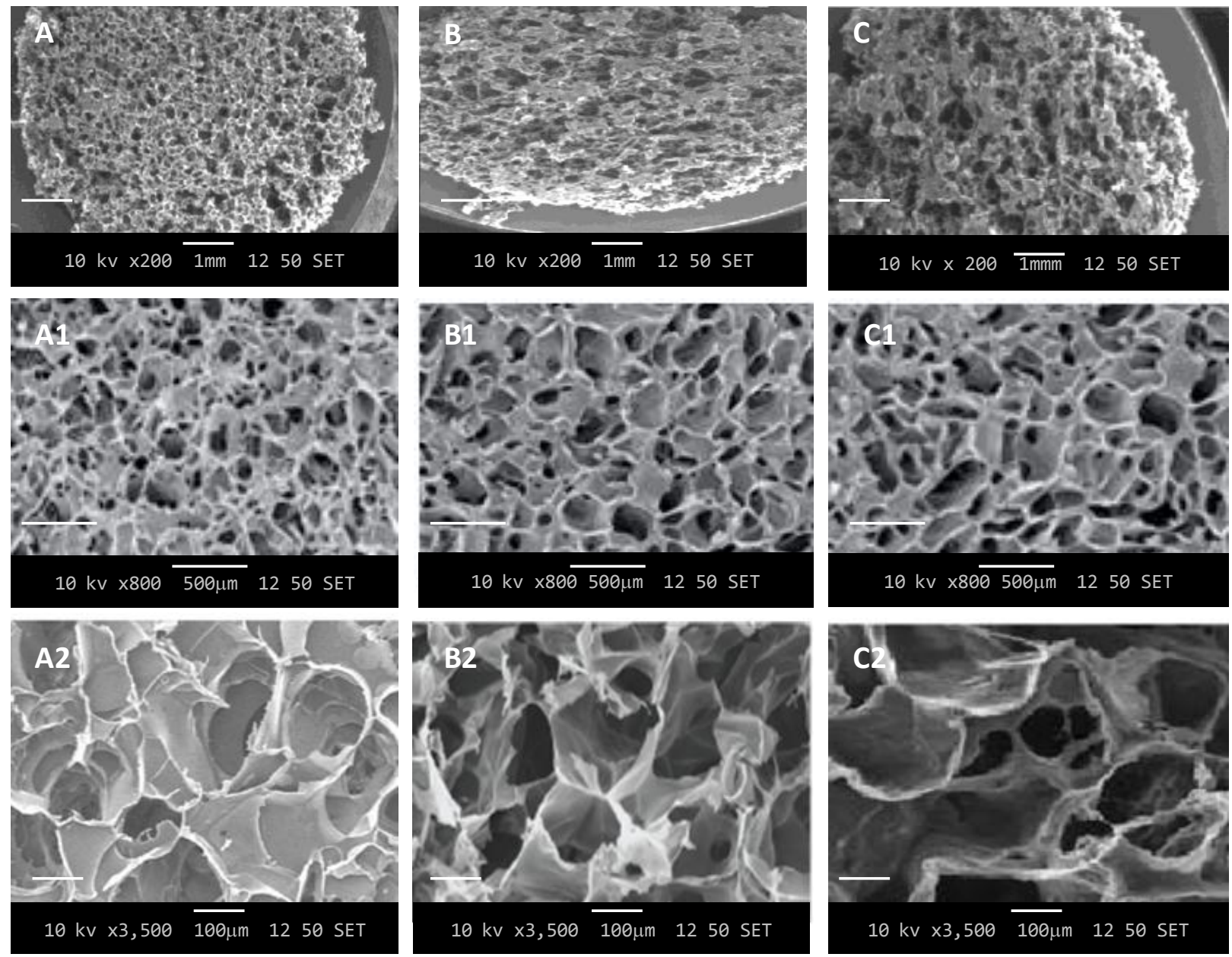

Figure 4.10 SEM images of surface and cross-section morphology of PCL/BCNW-g- $\beta C D-$ $\mathrm{PCL}_{2000}$ scaffolds. (A, B \&C) are surfaces when weight $\mathrm{NH}_{4} \mathrm{HCO}_{3}:$ PCL ratios were 5:1, 7:1 and 10:1, respectively; (A1, A2) \& (C1, C2) are cross-section morphology corresponding to the same series at different magnification powers; $\times 800$ and $\times 3500$.

\subsubsection{Invitro Degradation}

In vitro degradation behavior of neat PCL and its drug-laden scaffolds are shown in Figure 4.11. The graph shows the recorded weight loss that determined gravimetrically at different time intervals after immersing scaffolds in PBS of pH 7.4 up to $\sim 2$ month. The results show a slower degradation rate for neat PCL where only $11 \%$ weight loss was occurred after 70 days. This slow degradation rate is related to the higher crystallinity and the hydrophobic nature of PCL. ${ }^{\text {[402] }}$ In contrast, the incorporation of $4 \mathrm{wt} \%$ of BCNW-g- $\beta C D-\mathrm{PCL}_{2000}$ into PCL matrix increased its degradation rate to $18 \%$. This could be related to the increased surface hydrophilicity of 
nanocomposites that allows promoted interaction between scaffold surface and dissolution medium. The later finding is also in a good agreement with the thermal results that revealed decreased crystalinity due to the incorporation of BCNW- $g-\beta C D-P C L_{2000}$ into PCL matrix.

In addition, a comparative test for investigating the effect of enzyme on hydrolytic degradation of scaffolds was carried out in PBS at $\mathrm{pH} 7.4$ and $37^{\circ} \mathrm{C}$ in presence of porcine lipase. A substantial increase in degradation rate was observed where percentage weight loss is reached a maximum value of $26 \%$ after 70 days; see Figure 4.11. Porcine Lipase most probably accelerates the hydrolysis through attacking of ester linkages of either short and long PCL chains. ${ }^{[416]}$ This consequently promotes the degradation rate of PCL bionanocomposite scaffolds.

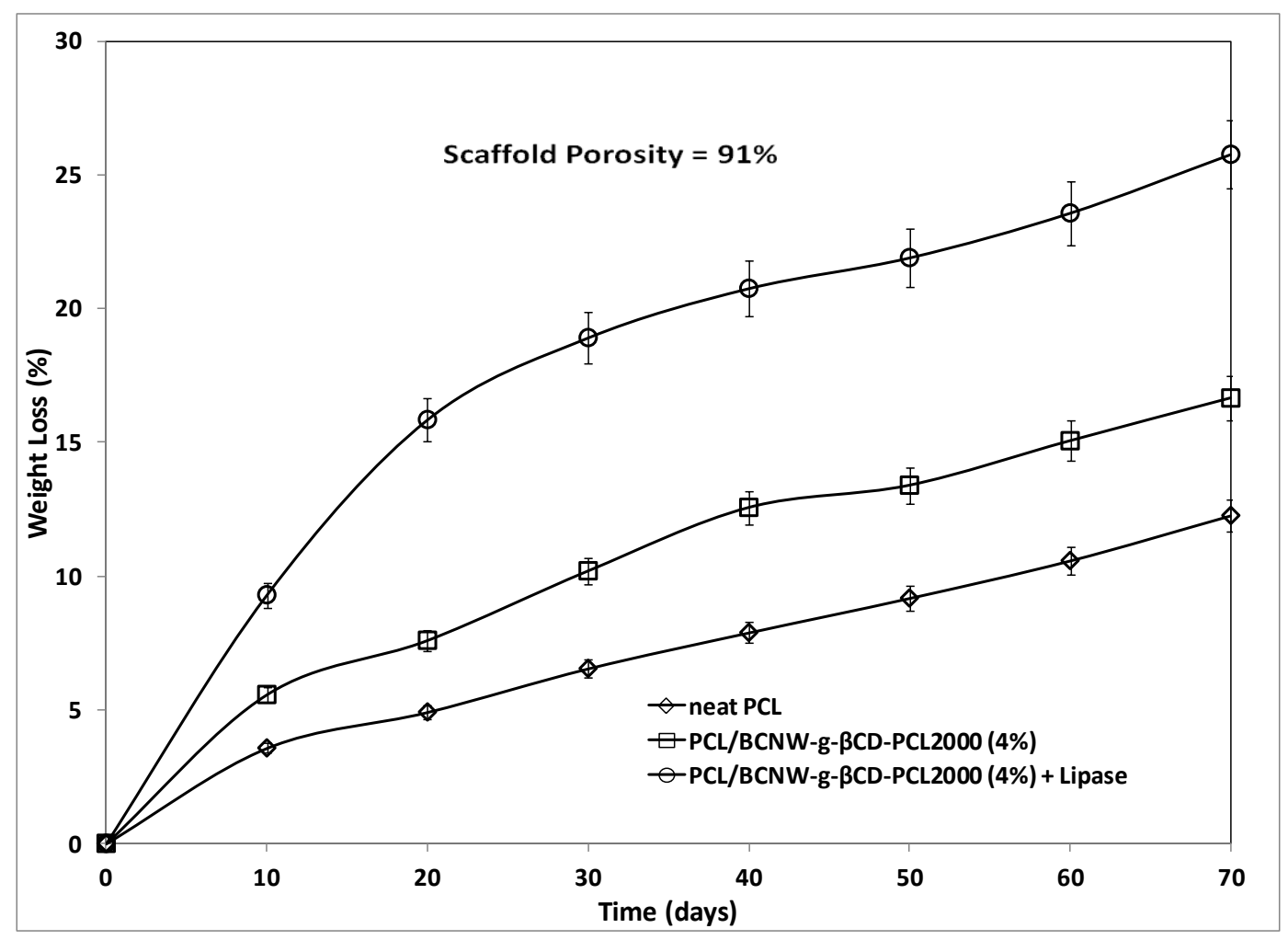

Figure 4.11 In vitro degradation behavior of neat PCL and PCL/BCNW- $g-\beta C D-P C L_{2000}$ bionanocomposit scaffolds in PBS at $\mathrm{pH} 7.4$ and $37^{\circ} \mathrm{C}$. 


\subsection{Conclusion}

In summary, nano-functionalized material (i.e., BCNW- $g-\beta C D-\mathrm{PCL}_{2000}$ ) based on the green biocompatible bacterial cellulose nanowhisker was developed by grafting nanowhisker with $\beta C D$ and conjugating low molecular weight of $\mathrm{PCL}_{2000}$ segments to its surface.

Results showed that incorporation of $4 \mathrm{wt} \%$ of BCNW- $g-\beta C D-\mathrm{PCL}_{2000}$ into PCL matrix resulted in bionanocomposite with overwhelmed changes in bulk characteristics. Compared to neat PCL, the obtained PCL-bionanocomposite showed improved mechanical and thermal properties, as well as promoted hydrophilicity and in vitro degradation. Young's modulus and tensile strength of the resulted nanocomposites were improved by $51 \%$ and $115 \%$ and hydrophilicity and degradation rate were promoted by $20 \%$ and to $7 \%$.

Porous structures having porosities of 86-95\% with mean pore diameters of 250-420 $\mu \mathrm{m}$ along with unique interconnected pore-network were developed using gas foaming/combined particulate leaching technique. The obtained scaffold characteristics could be ideal for cell seeding and proliferation. Results from mechanical testing of porous scaffolds showed compression moduli in the range of cancellous bones. Reinforcing PCL-nanocomposites with 4\% $w t \%$ of BCNW- $g-\beta C D-P_{2} L_{200}$ was not only compensated for the loss in the mechanical properties due to highly porous structure, but also enhanced the integrity of bionanocomposite.

The work done displays the ability to fabricate porous scaffolds based on the biosorbable and biocompatible PCL. The sufficient mechanical properties, higher porosity, improved hydrophilicity and bulk degradation characteristics suggest potential applications for the obtained scaffolds in biomedical field and regenerative medicine. 


\section{CHAPTER 5*}

\section{Dual-Function Poly( $\varepsilon$-Caprolactone) Scaffold for Long-Term Drug Release and Tissue Engineering Applications}

Bioresorbable and biocompatible poly(e-caprolactone) (PCL) polymer have lots of medical applications. However, remarkable hydrophobicity, non-osteoconductivity and slow degradation rate have limited its application. The present study aims to develop PCL drug-laden scaffold. The envisioned dual-function scaffold would provide adequate structural and mechanical supports for regenerated tissues, and simultaneously serve as localized drug delivery system.

Reinforcing PCL with $4 \mathrm{wt} \%$ of functionalized bacterial cellulose nanowhisker (BCNW-g- $\beta C D-$ $\mathrm{PCL}_{2000}$ ) and $25 \mathrm{wt} \%$ of doxorubicin anticancer drug resulted in drug-laden bionanocomposite of combined promoted properties. Improvements of $165 \%$ and $107 \%$ in tensile strength and Young's modulus; $31 \%$ in hydrophilicity; $10 \%$ in degradation rate; and $8^{\circ} \mathrm{C}$ increases in thermal stability are achieved. 3D porous scaffolds of porosity range $86-95 \%$ and interconnected macropores of mean pore diameters of $250-420 \mu \mathrm{m}$ were fabricated. The obtained compressive moduli of 7.2-12.3 MPa are in the range of cancellous bones. In vitro drug releases occurs in controlled and sustained manner for $\sim 60$ days without obvious burst releases, and fit first-order release mechanism and diffusion-controlled.

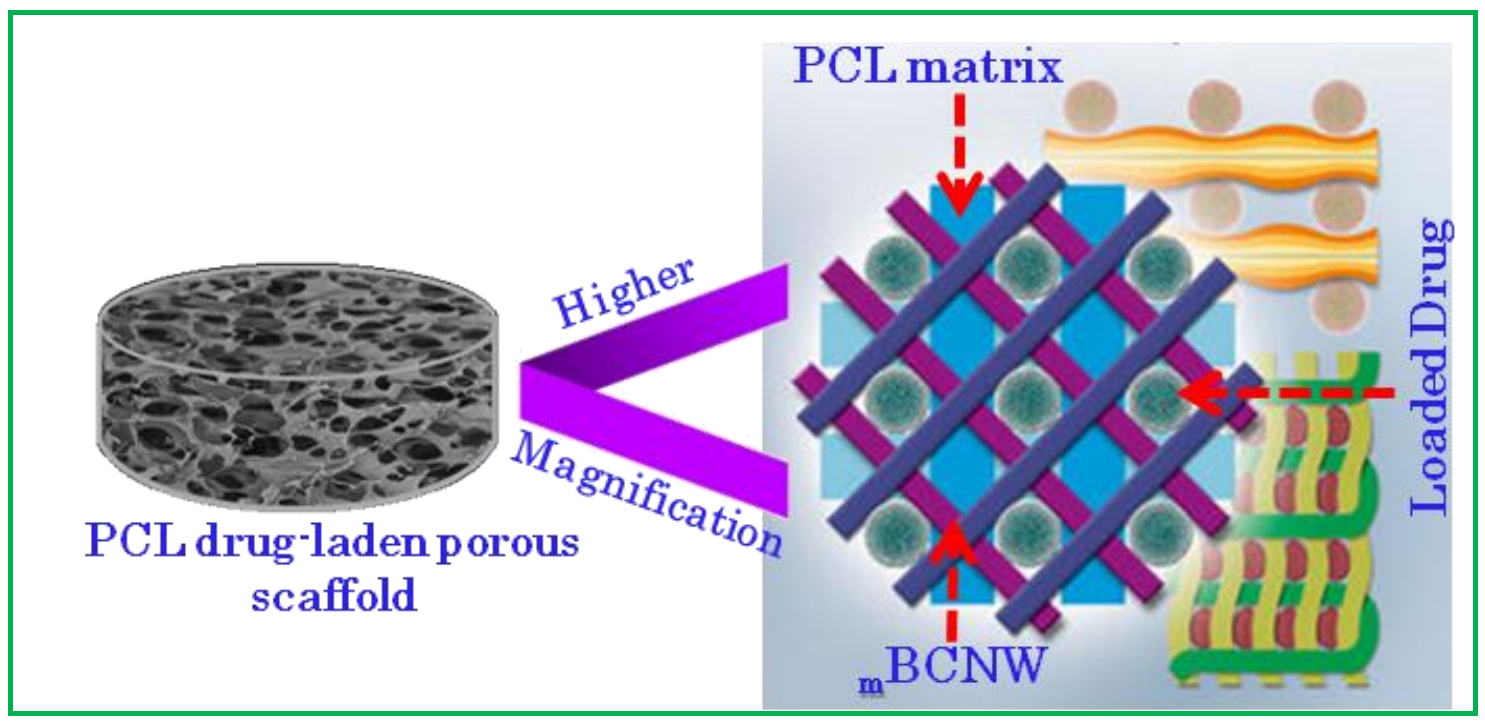

*This chapter is adapted from a submitted manuscript to the Journal of Applied Polymer Science (app.20190416). Mohamed M. Khattab, Yaser Dahman. Dual-Function Poly( $\varepsilon$-Caprolactone) Scaffold for Long-Term Drug Release and Tissue Engineering Applications. 


\subsection{Introduction}

The procedure of limb salvage has proven effectiveness in the treatment of osteosarcoma due to its superior ability to maintain the external appearances and physiological functions of the patients' limbs. ${ }^{[141]}$ The surgery involves local resection of bone or soft tissue cancers in order to avoid amputation and placement of either metal prosthesis or bone graft to provide mechanical support and platform for bone-tissue regeneration. However, numerous limitations associated with metal prosthesis, such as corrosion, ${ }^{[142]}$ growth restriction, ${ }^{[145]}$ and the need for additional removal operation. ${ }^{[147]}$ As well as several drawbacks associated with bone graft; such as donor, nonunion, disease transmission, and rejection. ${ }^{[150]}$ This is aside from the long term postoperationsuffering by patients for taking intensive doses of prescribed chemotherapeutics and antibiotics to destroy any remains of malignant cells to reduce cancer recurrence ${ }^{[156]}$ and to avoid surgical wound and orthopedic device infections. ${ }^{[394]}$

Thus, the treatments with drug-laden biodegradable polymeric scaffolds are emerged as viable alternatives procedure and considered as promising solution for bone and tissue cancer defects. ${ }^{[393]}$ Aliphatic polyesters, such as polycaprolactone (PCL), polylactic acid (PLA), polyglycolic acid (PGA), and their copolymers are most extensively used to fabricate biodegradable tissue engineering scaffolds. Due to their inherent biocompatibility, biodegradability, mechanical strength and the effortlessness processing into various 3D structures. ${ }^{[29,394]}$

PCL is a biocompatible, semicrystalline, slowly-degrading polymer and used in several tissue engineering (TE) applications. These include bones and cartilage substitutes, sutures, adhesion barriers as well as in controlling drug releases and targeting drug delivery. ${ }^{[395]}$ PCL gained popularity in the field of TE after receiving FDA approval due to excellent biocompatibility, ease of degradation in the human body, ${ }^{[23]}$ and higher permeability for drug molecules. ${ }^{[24]}$ Long-term in vivo studies have demonstrated that the PCL implants were able to maintain the physical shape after 2 years of implantation. ${ }^{[25]}$ Additionally, PCL was also capable to degrade slowly into low molecular weight by the end of 30 months and can be excreted completely out of the body without accumulating non-toxic degradation byproducts. ${ }^{[25]}$

PCL showed remarkable hydrophobicity and non-osteoconductivity that stand as barrier and led to suboptimal cell-material interactions. ${ }^{[29]}$ However, its bulk properties can be modified to improve its osteoconductivity potential by adding growth factors during scaffold fabrication, 
attaching cells to the scaffold surface, or seeding cells and growth factors into the scaffold before implantation. ${ }^{[26]}$

The potential use of biodegradable surface engineered scaffolds as 3D templates mimicking extracellular cell matrix to encourage initial cell attachment, proliferation, and differentiation are widely studied in vitro and in vivo. ${ }^{[396]}$ Basically, scaffold properties vary from tissue to tissue, as each one has its unique mechanical and biochemical properties. The pore size of TE scaffold is an important parameter that affects the quantity and characteristics of new tissue formation. Scaffold's pore size not only should be small enough to ensure mechanical integrity, but also large enough to allow the diffusion of nutrient and waste materials. An optimal pore size for TE engineering scaffolds is not well defined. However, pore sizes in the range of $100-400 \mu \mathrm{m}$ are projected as optimum for osteoconduction, whereas pore sizes smaller than $100 \mu \mathrm{m}$ was known to be inadequate in terms of mass transport and cell migrations. ${ }^{[26]}$

A variety of techniques have been used for processing biodegradable polymeric 3D porous scaffolds. ${ }^{[396]}$ These conventional methods include freeze drying, melt-based technologies, phase inversion, thermally induced phase separation, fiber bonding, fiber felts, melt molding, solvent casting, gas foaming, particulate leaching, high-pressure processing and electrospinning. However, the obtained constructs by those methods commonly facing drawbacks due to random structures, unpredictable pore sizes, reduced pore interconnectivity and low mechanical strength and structural stability. ${ }^{[397]}$ In modern TE, solvent casting is often used in combination with other technique to produce more efficient porous 3D structure. For example, solvent castingparticulate leaching is the most convenient fabrication technique that used for fabricating porous scaffolds. ${ }^{[147]}$ This method involves casting of a polymer/salt/organic solvent mixture followed by solvent evaporation and dissolution of the salt particulates in an aqueous solution. The commonly used progens are sodium chloride, ammonium bicarbonate, glucose, low molecular weight polymers, paraffin microspheres, and emulsion particles of defined sizes. ${ }^{[397,398]}$ Previous studies showed that porogen leaching technique led to creation of porous scaffolds with architecture properties which support integration between construct and host. ${ }^{[399]}$ However, this technique has limitations; it can only produce thin membranes with a dense surface skin layer, and might contain residual salt particles left from process. In this project a modified casting technique based on the gas foaming/particulate leaching method but with using two porogens salts was employed to fabricate $3 \mathrm{D}$ porous scaffolds of promoted porosities and pores- 
interconnectivity. ${ }^{[417]}$ The technique exploits the effervescent ammonium bicarbonate salt as gas foaming agent for creating the main porosity and the water soluble $\mathrm{PEG}_{2000}$ as poresinterconnectivity enhancer.

Cellulose nanocrystals $(\mathrm{CNC})$ or nanowhisker $(\mathrm{CNW})$, is highly promising green nanofibers with outstanding mechanical strength (Young's modulus of $150 \mathrm{GPa}$ ). It has highly hydrophilic surface due to the plenty of surface hydroxyl groups. ${ }^{[40]}$ It used to modify the bulk properties of PCL matrix, such as tune the hydrophilicity, mechanical properties, and biodegradation rate. It also utilized by several researchers to tune the drug release behavior of PLA nanofibers. ${ }^{[02]}$ However, there is a big challenge confronting the compatibility between CNC content, polymer properties, and the loaded amount of drugs in the composite. For instance, it was reported that higher CNC content of $10 \mathrm{wt} \%$ can induced an obvious reduction in the tensile properties of PLA due to poor dispersion and incompatibility within polymer matrix. Whereas, Xiang et al ${ }^{[403]}$ reported that the added CNC acted as a nucleating agent and increased PLA crystallization, degradation and drug release rates. However, the increased CNC contents would induce a higher initial burst of the drug due to the strong interaction between $\mathrm{CNC}$ and drugs that resulted in a poor dispersion of drugs into the PLA matrix.

Grafting of cellulose nanofibers with $\beta C D$ is considered as promising technology for introducing another functional agents, such as short and long polymers segments. ${ }^{[20]}$ The non-covalent binding between $\beta C D$ and adamantane (AD) is selected as the model for immobilizing compounds due to the near-perfect size match between $\beta \mathrm{CD}$ cavity and $\mathrm{AD}, \beta-\mathrm{CD} / \mathrm{AD}$ inclusion complexes showed extremely high binding constant of about $10^{4} \mathrm{M}^{-1}{ }^{[75]}$ In this context, the later interaction is utilized by several researchers to make supramolecular assembly of different polymers. ${ }^{[74,404]}$ Doxorubicin is a well-known anticancer drug with a broad spectrum antitumor activity to treat several types of cancer. However, treatment with doxorubicin is restricted due to several undesirable side effects like its cumulative-dose limit and cardiotoxicity. ${ }^{[418]}$

The present study aims to fabricate drug-laden 3D porous scaffold with outstanding mechanical properties along with promoted cell attachment and drug loading efficiencies. Two-step process is used to modify PCL matrix. The first step involves synthesis of the functionalized reinforcing nanowhisker (i.e., $\mathrm{BCNW}-g-\beta C D-\mathrm{PCL}_{2000}$ ) via grafting of $\beta C D$ molecules onto $\mathrm{BCNW}$ that utilized for subsequent assembly of short $\mathrm{PCL}_{2000}$ segments on nanowhisker surface. The later modifications tuned the surface energy of nanowhisker and consequently improved its dispersion 
in PCL matrix. The grafted unoccupied $\beta C D$ moieties promoted the drug release properties of the nanocomposites. The second challenging step is to examine the combined effect of incorporating different loadings of both BCNW- $g-\beta C D-\mathrm{PCL}_{2000}$ and DOX drug on the final bulk properties of the obtained drug-laden bionanocomposite. Mechanical, thermal, hydrophilicity, and in vitro degradation are significantly improved. Furthermore, the interaction between various parameters, such as nanowhisker loading, drug loadings and porosity level on the compression modulus and drug release behavior of the porous drug-laden scaffold were also investigated. Finally, drug release data of different scaffolds were fitted to various kinetic models to investigate the drug release mechanism.

\subsection{Experimental}

\subsubsection{Materials and Chemicals}

Bacterial strain Gluconacetobacter xylinus (G. xylinus ATCC 700178) was supplied by American Type Culture Collection (ATCC), Manassas, VA 20108, USA. Citric acid (CA), $\beta$ cyclodextrin $(\beta C D)$, sodium hypophosphite $(\mathrm{SHP})$, sodium hydroxide, doxorubicin hydrochloride (DOX.HCl), cellulose enzyme (Trichoderma reesei ATCC26921), with specific enzyme activity of around $700 \mathrm{U} / \mathrm{g}$, ammonium bicarbonate (100-500 $\mu \mathrm{m})$, polyethylene glycol PEG $\mathrm{M}_{\mathrm{n}}=4000$, poly $\left(\varepsilon\right.$-caprolactone) diol (PCL-diol) $\left(\mathrm{M}_{\mathrm{n}}=2000\right)$, poly ( $\varepsilon$-caprolactone) (PCL) (average $\left.\mathrm{M}_{\mathrm{n}}=45,000\right)$, tin octoate $\left(\mathrm{Sn}\left(\mathrm{Oct}_{2}\right)\right.$ as catalyst, 1-adamantyl isocyanate (AD-NCO, 97\%), dimethylsulfoxide (DMSO), dimethyformamide (DMF), dichloromethane (DCM, 99.5\%), diethyl ether, acetone, chloroform, and methanol. Phosphate buffered saline $\mathrm{pH}$ 7.4, sodium azide $\mathrm{NaN}_{3}$, sodium chloride $(\mathrm{NaCl})$ (Sigma Ultra (99.5\%) and Lipase from porcine pancreas type II (lipase activity: 100-500 units/mg protein using olive oil for 30 min incubation); 30-90 units/mg protein using triacetin). All chemicals were of analytical grade and obtained from Sigma-Aldrich (Oakville, ON, Canada), and used as received without further purification.

\subsubsection{Preparations and Analysis Procedure}

\subsubsection{Preparation of BC Nanowhisker (BCNW)}

Gluconacetobacter xylinus was used as BC producing bacteria. G. Xylinus was activated in accordance with ATCC guidelines. BC nanofibers were produced as fermentation product from fructose solution carbon source and corn steep liquor (CSL) nitrogen source. ${ }^{[405]} 10 \mathrm{~g}$ of purified 
BC nanofibers paste was further transformed into nanowhisker by treating it with commercially available cellulose enzyme Trichoderma reesei in acetate buffer (0.1 M; pH 5.0). ${ }^{[198]}$ Finally, $\mathrm{BCNW}$ suspension was re-concentrated to $0.5 \mathrm{wt} \%$ and kept at $5{ }^{\circ} \mathrm{C}$ for further use.

\subsubsection{Synthesis of $B C N W-g-\beta C D-P C L_{2000}$}

\subsection{Preparation of $B C N W-g-\beta C D$}

Grafting reaction took place using citric acid (CA) as a linker via two-step reaction. ${ }^{[379]}$ In the first reaction, CA- $\beta C D$ was prepared by refluxing of $3.0 \mathrm{~g}$ of $\beta C D, 1.1 \mathrm{~g}$ of CA, $0.29 \mathrm{~g}$ of SHP, and $1.8 \mathrm{~g}$ of distilled water at $100^{\circ} \mathrm{C}$ for $1.5 \mathrm{~h}$. In the second reaction step, $3.0 \mathrm{~g}$ of the previously prepared CA- $\beta C D$ and $0.1 \mathrm{~g}$ of SHP were dissolved in $10 \mathrm{ml}$ of distilled water. This followed by the addition of $20 \mathrm{ml}$ of ultrasonicated $0.5 \mathrm{wt} \% \mathrm{BCNW}$ and heating the reaction at $160^{\circ} \mathrm{C}$ for 15 min to obtain the higher grafting density. ${ }^{[66]}$ The obtained suspension of $(\mathrm{BCNW}-g-\beta C D)$ was reconcentrated to $0.5 \mathrm{wt} \%$ and kept at $5^{\circ} \mathrm{C}$ for further use.

Preparation of Adamantyl End-capped $P C L_{2000}\left(P C L_{2000}-A D_{2}\right)$

This reaction is carried out as described elsewhere ${ }^{[406]} \mathrm{A}$ total of $1.5 \mathrm{~g}$ of PCL-diol was dissolved in $20 \mathrm{~mL}$ of chloroform. To this solution, 2-fold excess (mole ratio) of 1-adamantyl isocyanate (AD-NCO) and a trace amount of tin octoate $\mathrm{Sn}\left(\mathrm{Oct}_{2}\right)$ as a catalyst (about $10 \mu \mathrm{L}$ ) were added. The reaction was preceded at $60^{\circ} \mathrm{C}$ with stirring for $16 \mathrm{~h}$. The product was subsequently precipitated in $150 \mathrm{~mL}$ of diethyl ether in cooled ice water bath and kept overnight. The final product was obtained by vacuum filtration, washed my methanol, and then dried at $45^{\circ} \mathrm{C}$.

\subsection{Assembly of $P C L_{2000}-A D_{2}$ on $B C N W-g-\beta C D$ Surface}

The bifunctional nanowhisker (BCNW- $g-\beta C D-\mathrm{PCL}_{2000}$ ) was prepared via supramolecular assembly of the guest polymer $\left(\mathrm{PCL}_{2000}-\mathrm{AD}_{2}\right)$ on $\mathrm{BCNW}-g-\beta C D$ using the method of Zhao et al. ${ }^{[11]}$ In brief, $40 \mathrm{mg} / \mathrm{mL}$ DMF solution of the guest polymer $\left(\mathrm{PCL}_{2000}-\mathrm{AD}_{2}\right)$ is mixed with BCNW- $g-\beta C D$ that was solvent-exchanged from water to DMF. Afterward, the mixture was then incubated in a shaker $(150 \mathrm{rpm})$ at room temperature overnight. The solution was decanted by centrifugation to remove excess DMF. An excess guest polymer was separated by adding deionized water to the mixture. The suspension was re-suspended again in DMF and washed many times by fresh DMF until complete removal of any residue of the gust polymer. Eventually, the excess DMF was removed by centrifugation and replaced by DCM. The solution 
containing the bifunctional nanowhisker BCNW-g- $\beta C D-\mathrm{PCL}_{2000}$ was re-concentrated to 2 wt\% and kept at $5^{\circ} \mathrm{C}$ for further use.

\subsubsection{Preparation of PCL Reinforced BCNW-g- $\beta C D-P C L_{2000}$ Nanocomposites Films}

BCNW-g- $\beta C D-P_{2000}$ loadings of (1-5 wt $\%$ ) were dispersed in DMF, and then sonicated for 10 minutes in ice bath using high intensity homogenizer (Hielscher UP200Ht) equipped with 14 mm sonotrode. The amplitude and the output power will be kept at $26 \mathrm{kHz}$ and $160 \mathrm{~W}$, respectively. Freshly sonicated nanowhisker solutions were mixed under continuous stirring with $1.0 \mathrm{~g}$ of PCL pre-dissolved in DCM until homogenous mixture of PCL/BCNW-g- $\beta C D-\mathrm{PCL}_{2000}$ was obtained. Nanocomposites films were prepared by solvent casting method. ${ }^{34}$ The viscous polymer/nanowhisker solution mixture was preheated at $70^{\circ} \mathrm{C}$ and then gradually poured into a hot handmade stainless steel mold. The mold is subsequently cooled by flashed frozen in liquid nitrogen. The mold was then transferred to a refrigerator set at $-20^{\circ} \mathrm{C}$ and kept for $24 \mathrm{~h}$. After which, the obtained nanocomposites were placed under vacuum condition at $40^{\circ} \mathrm{C}$ for $48 \mathrm{~h}$ until complete solvent evaporation. The films were taken out from the mould and kept dry in a desiccator.

\subsubsection{Fabrication of PCL-reinforced BCNW-g- $\beta C D-P C L_{2000}$ Porous Scaffolds}

Gas foaming/combined particulate leaching method was employed for fabricating 3D porous scaffold as published elsewhere. ${ }^{[417]}$ Two progene salts are used; an effervescent salt progene ammonium bicarbonate as a gas foaming agent and $\mathrm{PEG}_{2000}$ as porosity and poresinterconnectivity enhancer. Gel slurry composed of binary mixture of PCL/PEG 2000 gel in chloroform, nanowhisker, and dispersed ammonium bicarbonate salt particles were casted in hand-made Teflon mold at room temperature. The semi-solidified construct is subsequently immersed in hot citric acid. The evolution of ammonia and carbon dioxide gases, along with the leaching out of ammonium bicarbonate and PEG particulates from the semi-solidified polymer matrix caused the formation of pores with high inter-connectivity. Briefly, highly viscous PCL polymer solution of concentration $30 \mathrm{wt} \%$ was first prepared by dissolving PCL pellets together with PEG (1:1 w/w) in chloroform (3 g of PCL $+3 \mathrm{~g}$ PEG $+4 \mathrm{~g}$ chloroform). DMF solutions containing the required amounts $(1-5 \mathrm{wt} \%)$ of $\mathrm{BCNW}-g-\beta C D-\mathrm{PCL}_{2000}$ were sonicated for 15 min and added slowly with continuous stirring to PCL/PEG polymer solution. An overnight 
oven-dried and sieved ammonium bicarbonate particles in the range of 180-300 $\mu \mathrm{m}$ was used to develop the porous structure in PCL matrix. Standard sieved mesh was used to isolate the required particle size. $\mathrm{NH}_{4} \mathrm{HCO}_{3}$ salt was added to nanowhisker/polymer mixture where the salt: PCL mass ratios were kept at $(5: 1,7: 1$ and 10:1 w/w) in order to obtain nanocomposites of three levels of porosity. The mixtures were then stirred at $40^{\circ} \mathrm{C}$ for $30 \mathrm{~min}$.

The mixture was casted into Teflon molds in the form of circular discs of approximately (15 mm in diameter $\times 3 \mathrm{~mm}$ in height) by using $5 \mathrm{~mL}$ syringes with the tip cutoff. After chloroform was partially evaporated under atmospheric pressure, the semi-solidified samples were immersed into an excess amount of hot citric acid solution $\left(50^{\circ} \mathrm{C}\right)$ for $30 \mathrm{~min}$ until no more gas bubbles were generated. Afterwards, the samples were placed into warm water for another $30 \mathrm{~min}$. finally the scaffolds washed three times with ethanol and then air dried and stored at $5^{\circ} \mathrm{C}$ until use. The paste mixture was also casted into a cylinder-shaped mold ( $6 \mathrm{~mm}$ diameter $\times 12 \mathrm{~mm}$ height $)$ for compression testing.

\subsubsection{Fabrication of Drug-laden Porous Scaffold}

In a similar fashion, the drug-laden scaffolds were fabricated as PCL-reinforced BCNW- $g$ - $\beta C D-$ PCL $_{2000}$ porous scaffolds, but with addition of three levels of drug loadings; 10, 20 and $30 \mathrm{wt} \%$ of anticancer drug (i.e., DOX.HCl). In brief, the required mass of drug was dissolved in the least amount of chloroform, and then mixed with polymer/salt/nanowhisker mixture. The resulted scaffold was then casted, leached and dried as described before. Scaffolds without drug loading were served as control.

\subsubsection{Porosity, Pore Volume, and Pore Size}

The porosity and pore volume of the scaffolds were estimated gravimetrically as before using Equations $(4.1 \& 4.2),{ }^{[398]}$ where $\rho_{\text {polymer }}$ is the density of neat polymer used for scaffolds fabrication and $\rho_{\text {scaffold }}$ is the apparent density of the scaffold which determined by using Sartorius YDK 01LP density measurement kit.

Thus, $\rho_{\text {PCL }}$ was considered to be $1.145 \mathrm{~g} / \mathrm{cm}^{3}$. Three samples were assessed for estimating both porosity and pore volume average values. The Pore size distribution and pores interconnectivity were also directly measured from the images developed by SEM. Cross-sectional samples were vacuum dried on aluminum SEM holders and sputter coated with a $10 \mathrm{~nm}$ gold layer. The 
JEOL/OE equipment model JSM-6380 LV (Oxford Instrument, U.K. software version SEI England) was operated between 5-20 kV generating high-resolution images. The samples were analyzed between a magnification of $\times 30$ to $\times 100$ for porous structure and $\times 20 \mathrm{k}$ for nanowhisker investigations. At least 30 pores for each sample were analyzed, and average values were calculated for all of the scaffolds investigated.

\subsection{Characterization Techniques}

\subsubsection{Fourier Transform Infra-red Spectroscopy (FTIR/ATR)}

The structural changes accompanied the surface chemical modifications of BCNCs were followed by (FTIR/ATR) spectroscopy using (Perkin Elmer Spectrum1 FTIR/ATR spectrophotometer). ATR spectra were recorded in transmittance mode in the range of 4000-500 $\mathrm{cm}^{-1}$. ATR-crystal is $\mathrm{ZnSe}$, the spectral resolution is $4 \mathrm{~cm}^{-1}$ and 20 scans were collected for each spectrum.

\subsubsection{Mechanical Properties of Nanocomposites Films and Scaffolds}

Mechanical parameters were determined for neat PCL and its bionanocomposite films. Young's modulus (E) was calculated from slope of lines between 0.5 and $2.5 \%$ strain on stress-strain plots. $\sigma_{\mathrm{y}}$ and $\varepsilon_{\mathrm{y}}$ are stress and strain at yield; $\sigma_{\mathrm{b}}$ and $\varepsilon_{\mathrm{b}}$ are stress and strain at break. Labthink's Param XLW (PC) Auto Tensile Tester equipped with a $500 \mathrm{~N}$ load cell and operating at a crosshead speed of $100 \mathrm{~mm} / \mathrm{min}$ at room temperature at a span distance of $25 \mathrm{~mm}$. The dimensions of the tested films were $\left(100 \times 10 \times 1 \mathrm{~mm}^{3}\right)$. Compression modulus of the scaffolds was evaluated at room temperature using the same load and the cross-head speed was set at 2 $\mathrm{mm} / \mathrm{min}$. Cylindrical-shaped samples $(6 \mathrm{~mm}$ diameter $\times 12 \mathrm{~mm}$ height $)$ were used for compression tests according to ASTM method F451-95.

\subsubsection{Thermal Properties}

TGA and DSC measurements were employed to examine the thermal properties of PCL bionanocomposite using Perkin Elmer Diamond Differential Scanning Calorimeter controlled with PYRIS 7 software. For TGA analysis $20 \mathrm{mg}$ samples were heated to $500^{\circ} \mathrm{C}$ in air current of $100 \mathrm{~cm}^{3} \mathrm{~min}^{-1}$ and heating rate of $10^{\circ} \mathrm{C} \mathrm{min}^{-1}$. For DSC measurements sample specimens of 5-10 mg were dried at $60^{\circ} \mathrm{C}$ for $2 \mathrm{~h}$ and sealed in $20 \mu \mathrm{L}$ aluminum pan. Samples heated from room temperature to $230^{\circ} \mathrm{C}$, held for 2 min to remove prior thermal history, and then cooled down to - 
$20^{\circ} \mathrm{C}$. The samples were then reheated to $230^{\circ} \mathrm{C}$. The heating/cooling rate was $10^{\circ} \mathrm{C} / \mathrm{min}$ under nitrogen atmosphere. The heat flow of the second heating scan was recorded for calculating the thermal parameters; cold crystallization temperature $\left(\mathrm{T}_{\mathrm{cc}}\right)$, melting temperature $\left(\mathrm{T}_{\mathrm{m}}\right)$, cold crystallization enthalpy $\left(\Delta \mathrm{H}_{\mathrm{cc}}\right)$, and melting enthalpy $\left(\Delta \mathrm{H}_{\mathrm{m}}\right)$. The degree of crystallinity $\left(\chi_{\mathrm{c}}\right)$ was calculated using Equation (5.3). ${ }^{[08]}$

\subsubsection{Field Emission Scanning Electron Microscopy (FE-SEM)}

SEM micrographs were taken at room temperature with JEOL/OE scanning electron microscope model JSM-6380 LV, equipped with Oxford energy dispersive X-ray spectroscopy (EDX). The surface morphology of unmodified and modified BCNWs was examined using microscope with an accelerating voltage of $10-20 \mathrm{kV}$ and at magnification of $\times 10,000$. Elemental analyses of nanowhisker were analyzed by EDX. Morphology and pore sizes of porous scaffolds were examined using $\left(5 \times 5 \mathrm{~mm}^{2}\right)$ samples, which were placed on an aluminum holder and sputter coated with $10 \mathrm{~nm}$ gold layer. The samples were analyzed at magnifications of $\times 200$ to $\times 3500$. Average pore diameters were measured from at least 30 pores using an image analyzer (Bum-Mi Universe Co., Ltd., Seoul, Korea).

\subsubsection{Apparent Water-in-Air Contact Angle}

Static contact angle measurements were performed on nanocomposites films using Dataphysics OCA 15 optical contact angle measuring system using the sessile drop method. Deionised water droplets of $\sim 4 \mu \mathrm{L}$ were deposited on the samples surface via a motorized syringe. Five measurements per sample type were performed. The measurements were performed 2 min after droplet addition.

\subsubsection{In vitro Degradation Behavior}

Hydrolytic degradation was carried out for neat PCL and its nanocomposites in PBS solution (0.1 M PBS with $0.9 \% \mathrm{NaCl}, 0.02 \% \mathrm{NaN}_{3}$ and $\mathrm{pH}$ 7.4). Whereas enzymatic degradation was employed in the same PBS solution but in presence of Lipase enzyme from porcine pancreas at concentration level $0.1 \mathrm{mg} \mathrm{mL}^{-1}$. Samples were placed into $15 \mathrm{ml}$ vials containing $10 \mathrm{~mL}$ PBS and then incubated with shaking at $37^{\circ} \mathrm{C}$ to simulate in vivo dynamic tissue environment. The weight loss was recorded gravimetrically every 5 days using Equation (4.4). The reported weight loss was the average of triplicate. 


\subsubsection{In vitro Drug Release Studies}

First, calibration curve was constructed using different DOX standard solution; $5 \mu \mathrm{g} / \mathrm{ml}, 15 \mu \mathrm{g} / \mathrm{ml}$, $25 \mu \mathrm{g} / \mathrm{ml}$ and $50 \mu \mathrm{g} / \mathrm{ml}$ that prepared in PBS ( $\mathrm{pH} 7.4$ ) by appropriate dilutions. Absorbance of those solutions at $\lambda_{\max } 482 \mathrm{~nm}$ is measured by Uv-Vis spectroscopy. The release profile of DOX from the scaffolds was monitored by incubating a scaffold in $10.0 \mathrm{ml}$ of PBS (pH; 7.4 and 4.8) at $37^{\circ} \mathrm{C}$ for 2 months. At each time point, $1 \mathrm{ml}$ of solution was collected and replaced with $1 \mathrm{ml}$ of fresh PBS to achieve sink conditions. The concentrations of DOX released in the solutions were estimated from the calibration curve. The cumulative percentage of released is calculated using Equations (5.1-5.3). ${ }^{[419]}$

Concentration of drug $(\mu \mathrm{g} / \mathrm{ml})=($ slope $\times$ absorbance $) \pm$ intercept

Amount of drug released $(\mathrm{mg} / \mathrm{ml})=\frac{\text { Concentration }(\mu \mathrm{g} / \mathrm{ml}) \times \text { Incubation volume } \times \text { dilution factor }}{1000}$

Cumulative release $(\%)=\frac{\text { Volume of sample withdrawn }(\mathrm{ml}) \times \mathrm{P}_{(\mathrm{t}-1)}+\mathrm{P}_{\mathrm{t}}}{\text { Incubation volume }}$

Where $\mathrm{P}_{t}$ is percentage release at time $(t)$ and $\mathrm{P}_{(\mathrm{t}-1)}$ is percentage release previous to $(\mathrm{t})$.

\subsubsection{Drug Release Models}

To study the kinetics of drug releases from scaffolds, in vitro drug release data were fitted in various mathematical kinetic models. ${ }^{[117]}$ Zero-order model which states that the rate of drug release is directly proportional to time and can be used to describe the release of drugs with low solubility from a matrix tablet system; Equation (5.4). First-order model suggests that the rate of drug release is directly proportional to the amount of drug remaining in the system, and it is useful in explaining the release of drugs dispersed in porous matrix system; Equation (5.5). Higuchi's model, which was derived from the Fick's first law of diffusion. It stated that the release of drug is governed by simple diffusion; Equation (5.6). Hixon-Crowell model describes the release of drugs by dissolution associated with a change in the surface and volume of particles or tablet; Equation (5.7). In case of Peppas-Korsmeyer model; Equation (5.8), the exponent (n) gives indications about the release mechanism. ${ }^{[420]}$ Exponent values $\leq 0.45$ indicate Fickian diffusion, whereas exponent values $\geq 0.89$ indicate case II transport (typical zero-order release). However, exponent values between 0.45 and 0.89 indicate non-Fickian diffusion or anomalous release (by both diffusion and erosion). 


$$
\mathrm{A}=\mathrm{k}_{0} \mathrm{t} \quad(5.4)
$$

where $\mathrm{k}_{0}$ is zero-order rate constant and $\mathrm{A}$ is the percentage amount of drug released at time $\mathrm{t}$.

$$
\ln (100-\mathrm{A})=-\mathrm{k}_{1} \mathrm{t}
$$

where $\mathrm{k}_{1}$ is the first-order rate constant.

$$
\mathrm{A}=\mathrm{kt}^{0.5}
$$

where $\mathrm{k}$ is the Higuchi's constant.

$$
100-(100-\mathrm{A})^{1 / 3}=\mathrm{k}_{2} \mathrm{t}
$$

where $\mathrm{k}_{2}$ is the Hixon-Crowell constant

$$
\mathrm{A}_{\mathrm{t}} / \mathrm{A}_{\infty}=\mathrm{k}_{3} \mathrm{t}^{\mathrm{n}}
$$

where $\mathrm{k}_{3}$ is the Peppas-Korsmeyer constant, $\mathrm{A}_{\mathrm{t}} / \mathrm{A}_{\infty}$ is the fraction of drug released at time $\mathrm{t}$.

\subsubsection{Data Analysis}

All experiments were performed in triplicate. Results are presented as the mean value \pm SE.

\subsection{Results and Discussion}

\subsubsection{Grafting of BCNW with $\beta C D$ and Assembly of $P C L_{2000}-A D_{2}$ on BCNW-g-BCD Surface}

Figure 5.1 A represents the grafting reactions of $\beta C D$ onto the surface of $B C N W$ by using ecofriendly citric acid (CA) as a well-known nontoxic crosslinking agent. In the first step $\beta$ cyclodextrin citrate $(\mathrm{CA}-\beta \mathrm{CD})$ was obtained by condensation reaction between $\beta C D$ and $\mathrm{CA}$. The second step was grafting of $(\mathrm{CA}-\beta \mathrm{CD})$ onto $\mathrm{BCNW}$. Under the optimum reaction conditions used, the highest grafting ratio achieved was $33.8 \%$.

Figure 5.1 B demonstrates the urethane reaction between ( $\mathrm{PCL}_{2000}$-diol) and 1-adamantyl isocyanate $(\mathrm{AD}-\mathrm{NCO})$ in presence of $\mathrm{Sn}\left(\mathrm{Oct}_{2}\right)$ as a catalyst to form the guest polymer $\mathrm{PCL}_{2000}$ with both ends capped with adamantane groups, abbreviated as $\left(\mathrm{PCL}_{2000}-\mathrm{AD}_{2}\right)$.

Figure 5.1 C represents the immobilization of $\mathrm{PCL}_{2000}-\mathrm{AD}_{2}$ onto the surface of $\mathrm{BCNW}-g-\beta C D$ to form the functionalized nanowhisker ( $\mathrm{BCNW}-\mathrm{g}-\beta \mathrm{CD}-\mathrm{PCL}_{2000}$ ). This achieved by conjugating $\mathrm{PCL}_{2000}-\mathrm{AD}_{2}$ on the surface of $\mathrm{BCNW}-g-\beta C D$ through the formation of host-guest inclusion complex between $\mathrm{AD}$ moieties of the guest polymers and the grafted $\beta \mathrm{CD}$ on the surface of BCNW. Because of the guest polymers are both end-capped with AD groups, therefore two possibilities for their assembly on BCNW-g- $\beta C D$ are suggested; (i) single-end immobilization and (ii) double-end immobilization; see Figure 5.1 C. A rough approximation based on obtained results indicated that either assembly fashions coexist. 


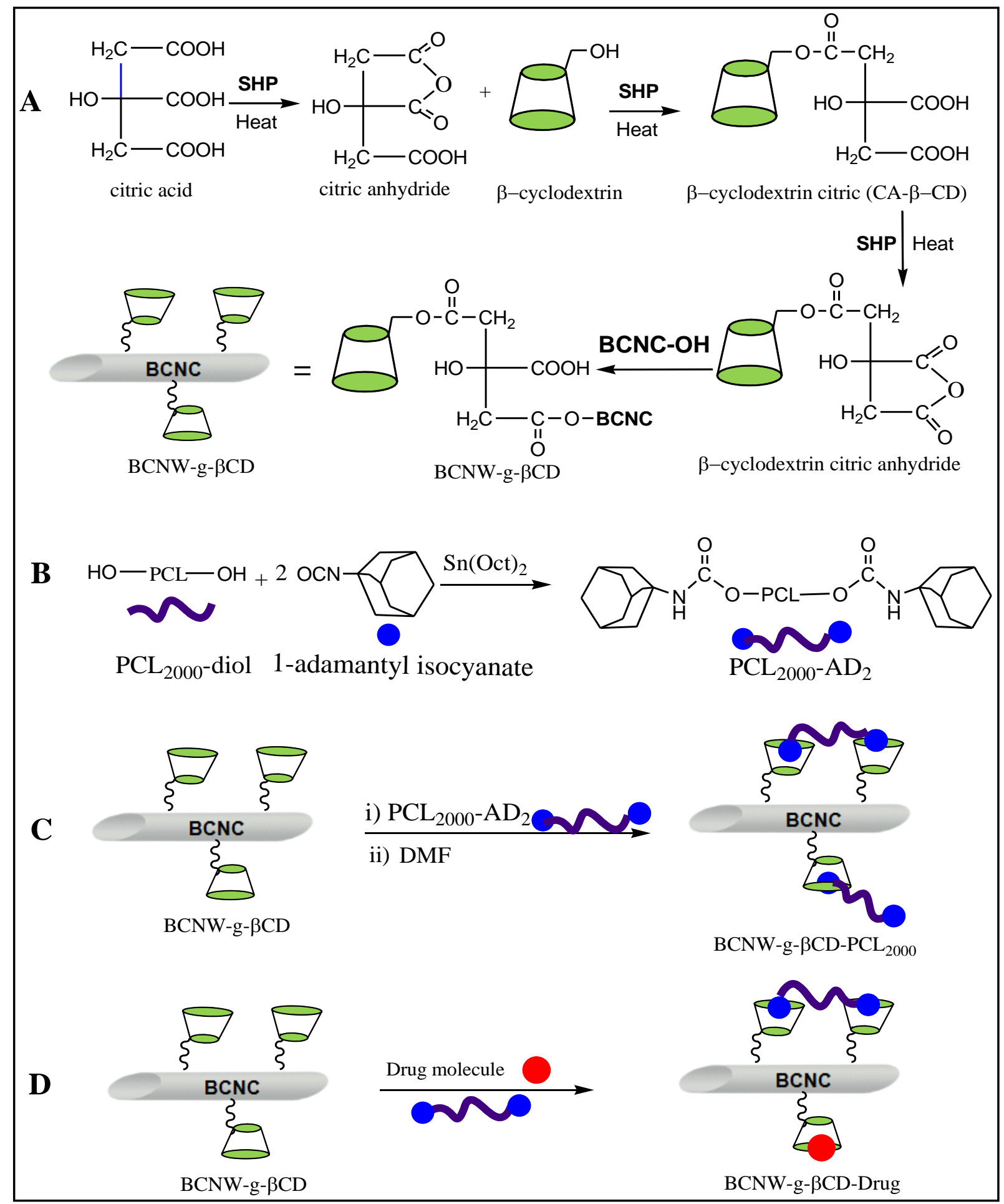

Figure 5.1 (A) Grafting of BCNW with $\beta C D$ by using of citric acid as linker; (B) synthetic pathway of $\mathrm{PCL}_{2000}-\mathrm{AD}_{2}$ from PCL-diol; (C\&D) conceptual illustration for drug and short polymer segments assembly on BCNC- $g-\beta C D$ surface through the formation of inclusion complexes. 


\subsubsection{Urethane Reaction and Formation of Guest Polymer $P C L_{2000}-A D_{2}$}

Figure 5.2 A shows urethane reaction between $\mathrm{PCL}_{2000}$-diol and $\mathrm{AD}-\mathrm{NCO}$ and formation of the guest polymer $\mathrm{PCL}_{2000}-\mathrm{AD}_{2}$. Analyzing ATR spectra confirms the successful preparation of the gust polymer $\mathrm{PCL}_{2000}-\mathrm{AD}_{2}$. In brief, $\mathrm{PCL}_{2000}-\mathrm{AD}_{2}$ show the main characteristic spectral bands due to $\mathrm{C}=\mathrm{O}$ and $\mathrm{C}-\mathrm{O}$ stretching in PCL-diol were shift to lower frequencies. ${ }^{[410]}$ Isocyanate peak of the free AD-NCO was disappeared and a new secondary amide peak due to (-C=O-NH-) stretching was emerged at $3473 \mathrm{~cm}^{-1}$. ${ }^{[411]}$

\subsubsection{Formation of $B C N W-g-\beta C D$ and $B C N W-g-\beta C D-P C L_{2000}$}

Figure 5.2 B shows ATR spectra that monitoring the change in chemistry of BCNW after grafting its surface with $\beta C D$ using $\mathrm{CA}$ as linker. Examining the spectral of BCNW-g- $\beta C D$ shows very similar bands to ungrafted $\mathrm{BCNW}$ except the new band that emerged at $1708 \mathrm{~cm}^{-1}$ due to the presence of $\mathrm{C}=\mathrm{O}$ stretching vibration of ester bond that formed between citric anhydride moiety of $\mathrm{CA}-\beta \mathrm{CD}$ and $\mathrm{OH}$ groups of $\mathrm{BCNW}$. This band indicates the successfulness of the grafting reaction between $\beta C D$ and $\mathrm{BCNW}$ and confirms the formation of $\mathrm{BCNW}-g-\beta C D .{ }^{[417]}$

In addition, Figure 5.2 B also depicts ATR spectrum of BCNW- $g-\beta C D$ after conjugating $\mathrm{PCL}_{2000}-\mathrm{AD}_{2}$ to its surface. Analyzing $\mathrm{BCNW}-\mathrm{g}-\beta \mathrm{CD}-\mathrm{PCL}_{2000}$ spectra demonstrates an intense overlapped peaks of $\mathrm{C}=\mathrm{O}$ group stretching in the range of $1707-1735 \mathrm{~cm}^{-1}$. This band arose from the combination of the carbonyl group stretching of both $\mathrm{PCL}_{2000}-\mathrm{AD}_{2}$ and $\mathrm{BCNW}-g-\beta C D$. In addition, the peaks indentified at 3473, 2943 and $2866 \mathrm{~cm}^{-1}$ were ascribed to the secondary amide $-\mathrm{C}=\mathrm{O}-\mathrm{NH}$ - stretching, asymmetric and symmetric stretching of $\mathrm{CH}_{2}$ group in $\mathrm{PCL}_{2000}-\mathrm{AD}_{2}$, respectively. Therefore, all the previous evidences confirm the successful preparation of $\mathrm{BCNW}$ g- $\beta$ CD-PCL 2000 . 

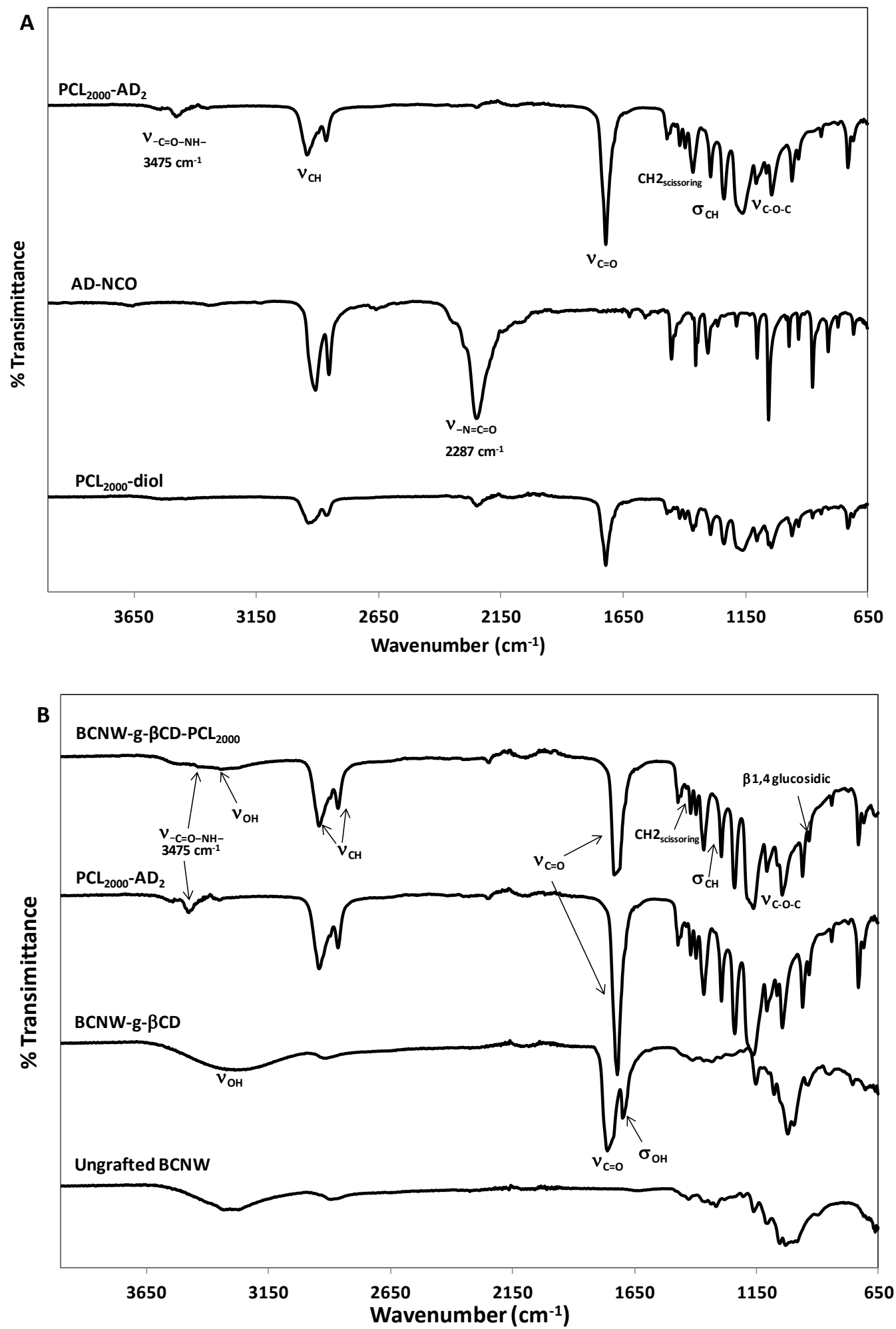

Figure 5.2 A: ATR spectra showing the formation of guest polymer $\left(\mathrm{PCL}_{2000}-\mathrm{AD}_{2}\right)$ from $\mathrm{PCL}_{2000}-$ diol and AD-NCO; B: ATR spectra of BCNW after grafting with $\beta C D$ and assembly of $\mathrm{PCL}_{2000}-\mathrm{AD}_{2}$. 


\subsubsection{Morphology and Surface Composition of BCNW-g-BCD-PCL $L_{2000}$}

Figure 5.3 A\&B show FE-SEM images that describe the morphology of BCNW- $g-\beta C D$ before and after $\mathrm{PCL}_{2000}-\mathrm{AD}_{2}$ assembly on the surface. Analyzing the image before $\mathrm{PCL}_{2000}-\mathrm{AD}_{2}$ assembly (Figure $5.3 \mathrm{~A}$ ) shows somewhat smoother surface for BCNW- $g-\beta C D$ similar to ungrafted BCNW. However, after polymer assembly the surface became a little rougher with a clearly observed patches; see Figure $5.3 \mathrm{~B}$. These observations were attributed to the assembled $\mathrm{PCL}_{2000}-\mathrm{AD}_{2}$ guest polymers. ${ }^{[412]}$ In addition, variation in the surface chemical composition of BCNW- $g-\beta C D$ before and after $\mathrm{PCL}_{2000}-\mathrm{AD}_{2}$ assembly was determined by EDX spectra (Figure $5.3 \mathrm{~B} \& \mathrm{C})$. Results showed the calculated amount of assembled polymer was found to equal 24.8 wt $\%$, where carbon content increased from 54.1 to $65.3 \%$ and oxygen content dropped from 49.9 to $34.7 \%$ due to the higher carbon atomic content of assembled $\mathrm{PCL}_{2000}-\mathrm{AD}_{2}$ polymer. ${ }^{[417]}$ The later finding suggests that the entire surface of BCNW- $-\beta C D$ is not fully covered by PCL $_{2000}$ after the assembly process. In addition, based on the previous findings that $33.8 \mathrm{wt} \%$ grafting ratio of $\beta C D$ has achieved on $\mathrm{BCNW}$ surface, and $\mathrm{PCL}_{2000}-\mathrm{AD}_{2}$ can only bind with $\beta C D$ moieties, Therefore, one can estimate that approximately $73.4 \%$ of $\beta C D$ was occupied by $\mathrm{PCL}_{2000}$ segments, where the other $26.6 \%$ is still vacant and can be ready to host drug molecules; see Figure 5.1 D. 

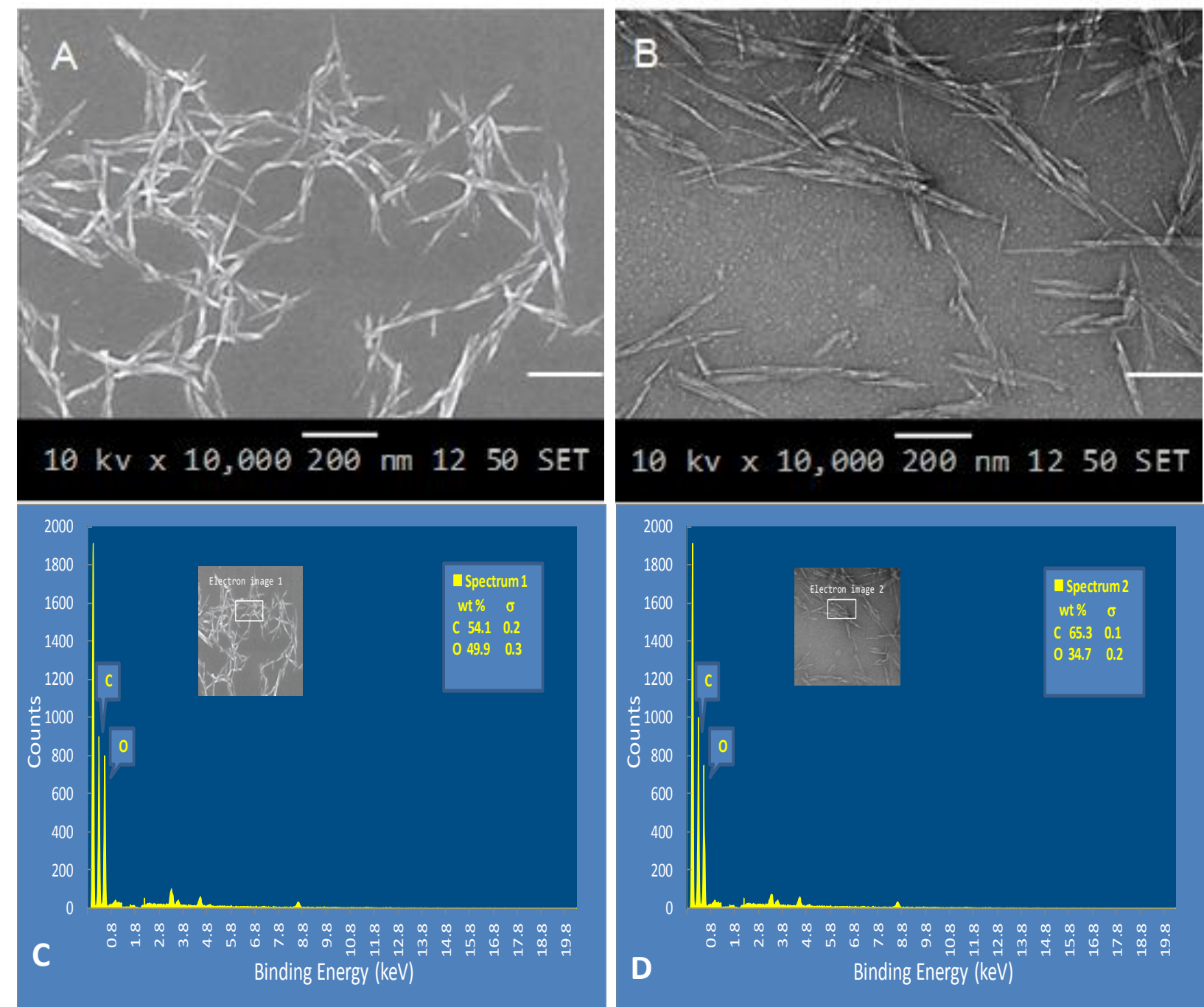

Figure 5.3 A \&B are FE-SEM micrograph and D \&D are EDX spectrum that showing the surface morphology and composition of BCNW-g- $\beta C D$ before and after $\mathrm{PCL}_{2000}-\mathrm{AD}_{2}$ assembly.

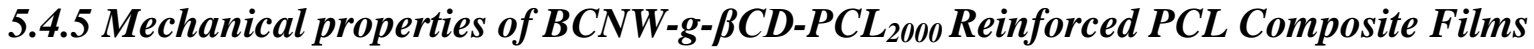

Figure 5.4 depicted the stress-strain curves of neat PCL, PCL/BCNW-g- $\beta C D-P C L_{2000}$ and drugladen bionanocomposite PCL/BCNW-g- $\beta C D-\mathrm{PCL}_{2000} / \mathrm{DOX}$. The main representative of mechanical parameters (i.e., Young's modulus (E); stress and strain at yield $\left(\sigma_{\mathrm{y}}\right.$ and $\left.\varepsilon_{\mathrm{y}}\right)$; and stress and strain at break $\left(\sigma_{\mathrm{b}}\right.$ and $\left.\left.\varepsilon_{\mathrm{b}}\right)\right)$ are summarize in Table 5.1. Results show that neat PCL film presents a less stiff and semi-ductile behavior where $\sigma_{\mathrm{y}}$ and $\varepsilon_{\mathrm{y}}$ values of $15.3 \mathrm{MPa}$ and $5.3 \%$ and $\sigma_{\mathrm{b}}$ and $\varepsilon_{\mathrm{b}}$ values of $14 \mathrm{MPa}$ and $10.3 \%$ were achieved. In addition, increasing BCNW-g$\beta C D-\mathrm{PCL}_{2000}$ loadings ratio from $1-4 \%(\mathrm{w} / \mathrm{w})$ in PCL matrix is associated with gradual enhancement for both strength and tensile modulus at expenses of elasticity; see Table 5.1. The 
maximum increase was achieved at $4 \%(\mathrm{w} / \mathrm{w})$ of $\mathrm{BCNW}-g-\beta C D-\mathrm{PCL}_{2000}$, where the tensile strength and Young's modulus significantly improved by $115 \%$ and $51 \%$, respectively over the neat PCL film, while elongation at break reduced by $47 \%$. This significant improvement in the stiffness and strength of PCL/BCNW-g- $\beta C D-\mathrm{PCL}_{2000}$ nanocomposites were related to an improved wettability and compatibility between incorporated $\mathrm{BCNW}-g-\beta C D-\mathrm{PCL}_{2000}$ and PCL matrix. The conjugated short $\mathrm{PCL}_{2000}$ segments on nanowhisker surfaces acted as co-continuous phase between modified nanowhisker and PCL matrix. This in turn increased the interfacial adhesion between nanowhisker and matrix and ultimately allows proper stress transfer from PCL matrix to the high strength reinforcing phase of the added BCNW- $-\beta C D-\mathrm{PCL}_{2000}$.

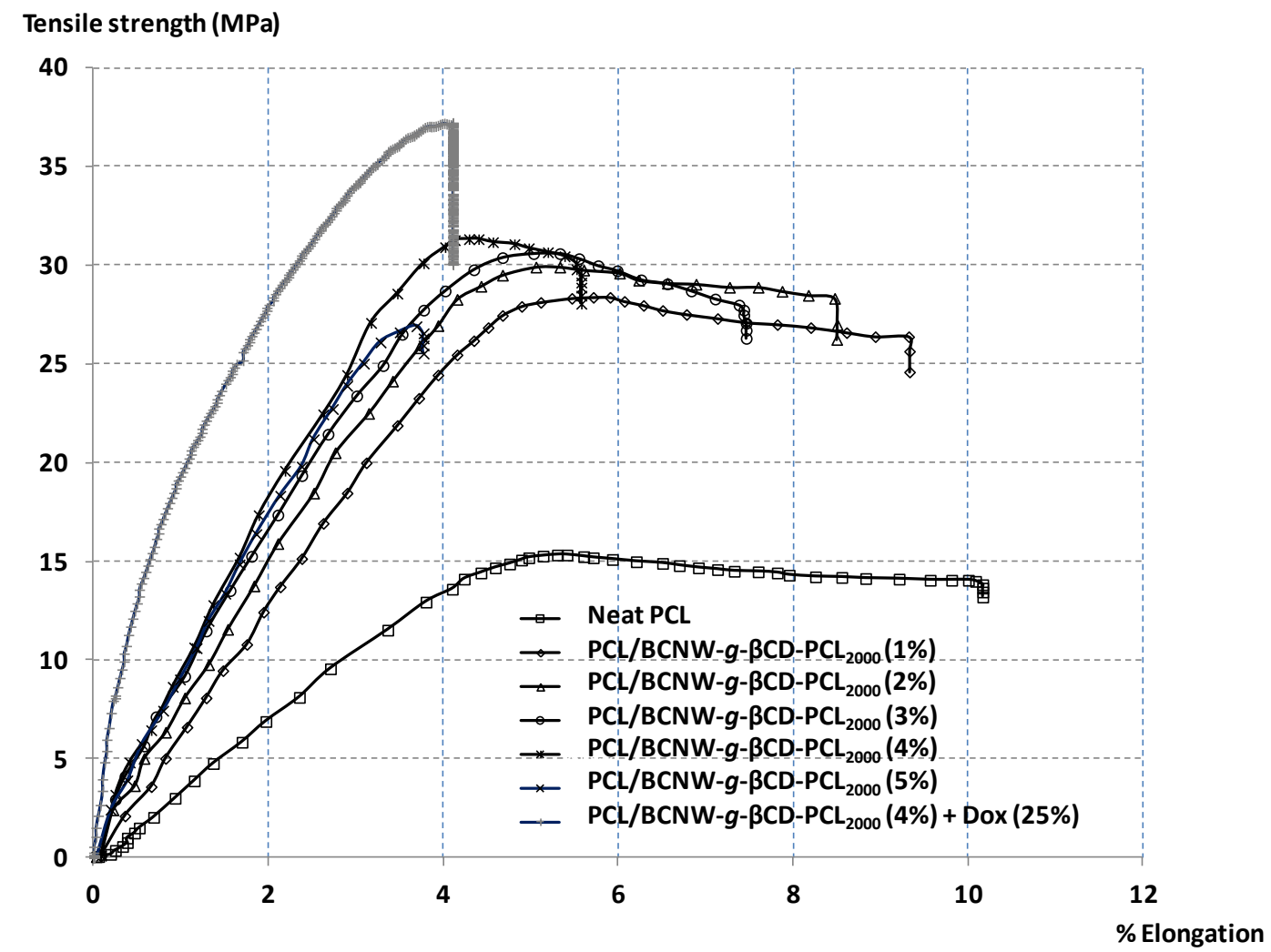

Figure 5.4 Stress-strain plots of neat PCL and PCL bionanocomposite. 


\subsubsection{Effect of Drug Loading on the Mechanical Properties of Bionanocomposite}

Due to the impressive mechanical properties of nanocomposites formulation containing $4 \mathrm{wt} \%$ of the functionalized nanowhisker ${ }^{[417]}$ (i.e., PCL/BCNW- $g-\beta C D-\mathrm{PCL}_{2000}(4 \%)$ ), it was chosen as a representative for carrying out drug loading studies. The effect of changing drug loading concentration from $(10-30 \% \mathrm{w} / \mathrm{w})$ on the final mechanical properties of drug-laden nanocomposites is also presented in Table 5.1. Surprisingly, results demonstrate that the gradual addition of DOX to bionanocomposite formulation causes further increase for either tensile strength or Young's modulus at expense of elongation at break. Maximum drug loading of 25 wt\% of DOX to PCL/BCNW- $g-\beta C D-P_{2} L_{2000}(4 \%)$ led to an improvement of 23 and $38 \%$ in tensile strength and Young's modulus, versus 27\% reduction in elongation at break. Whereas compared with neat PCL, the maximum combined improvements achieved are 165 and 107\% for tensile strength and Young's modulus, respectively, versus $61 \%$ reduction in elongation at break. Any further increase in drug loading contents more than $25 \mathrm{wt} \%$ causes decline in tensile strength and Young's modulus (Table 5.1) However, in case of a similar experiment using PCL/DOX blend at $25 \mathrm{wt} \%$ drug loading ratio but without nanowhisker loading, the obtained tensile strength and Young's modulus (i.e., 13.3 and $1490 \mathrm{MPa}$ ) are less than the corresponding values in neat polymer. This may be happened due to the reduced frictional forces between polymer chains due the presence of the low molecular weight drug molecules. Which cause an imperfect crystallization of PCL chains and could be weaken the mechanical strength of PCL/DOX blend. The obtained results revealed the strong interaction between BCNW- $g-\beta C D-$ $\mathrm{PCL}_{2000}$, DOX drug and PCL matrix and the role of the functionalized nanowhisker to keep the integrity of the drug-laden nanocomposites. Where, $\mathrm{BCNW}-\mathrm{g}-\beta \mathrm{CD}-\mathrm{PCL}_{2000}$ might act as a compatibilizer that hinders chain mobility in the non-crystalline domain of PCL matrix and thus improve their orientation. ${ }^{[421]}$ In addition to hydrogen bonding interactions that might lead to reorientation of molecules to the intermolecular chain, resulting in an increased rigidity and enhanced mechanical strength for the drug-laden nanocomposites. 


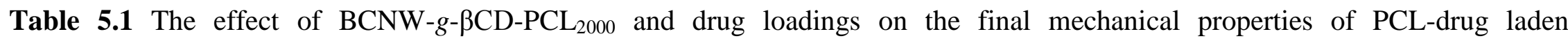
bionanocomposite

\begin{tabular}{|c|c|c|c|c|c|}
\hline Nanocomposites formulation & $\sigma_{\mathbf{y}}(\mathrm{MPa})$ & $\boldsymbol{\varepsilon}_{\mathbf{y}}(\%)$ & $\sigma_{\mathbf{b}}(\mathrm{MPa})$ & $\boldsymbol{\varepsilon}_{\mathbf{b}}(\%)$ & $\mathbf{E}(\mathrm{MPa})$ \\
\hline Neat PCL & $15.3 \pm 1.3$ & $5.3 \pm 0.2$ & $14.0 \pm 0.8$ & $10.3 \pm 0.2$ & $1560 \pm 126$ \\
\hline PCL+ BCNW-g-BCD-PCL $2000(1 \%)$ & $28.3 \pm 1.1$ & $5.9 \pm 0.3$ & $26.2 \pm 1.5$ & $9.3 \pm 0.2$ & $1870 \pm 126$ \\
\hline PCL+ BCNW- $g-\beta C D-P_{2000}(2 \%)$ & $29.2 \pm 1.6$ & $5.1 \pm 0.2$ & $28.3 \pm 1.3$ & $8.5 \pm 0.4$ & $1960 \pm 112$ \\
\hline PCL+ BCNW- $g-\beta C D-P_{2000}(3 \%)$ & $30.5 \pm 1.1$ & $4.7 \pm 0.3$ & $29.9 \pm 1.4$ & $7.4 \pm 0.2$ & $2210 \pm 130$ \\
\hline PCL+ BCNW- $g-\beta C D-P_{2000}(4 \%)$ & $31.4 \pm 1.2$ & $4.2 \pm 0.2$ & $30.2 \pm 1.1$ & $5.5 \pm 0.3$ & $2350 \pm 110$ \\
\hline PCL+ BCNW- $g-\beta C D-P C L_{2000}(5 \%)$ & - & - & $26.8 \pm 1.2$ & $3.6 \pm 0.3$ & $2243 \pm 102$ \\
\hline PCL+ DOX (10\%) & - & - & $14.6 \pm 1.4$ & $9.7 \pm 0.5$ & $1590 \pm 76$ \\
\hline PCL+ DOX (20\%) & - & - & $14.9 \pm 1.3$ & $8.5 \pm 0.3$ & $1620 \pm 95$ \\
\hline PCL+ DOX (25\%) & - & - & $13.2 \pm 1.2$ & $7.6 \pm 0.6$ & $1490 \pm 105$ \\
\hline PCL+ DOX (30\%) & - & - & $9.7 \pm 1.3$ & $7.3 \pm 0.4$ & $1405 \pm 111$ \\
\hline $\mathrm{PCL}+\mathrm{BCNW}-g-\beta \mathrm{CD}-\mathrm{PCL}_{2000}(4 \%)+\mathrm{DOX}(10 \%)$ & - & - & $34.1 \pm 1.5$ & $5.0 \pm 0.1$ & $3100 \pm 126$ \\
\hline 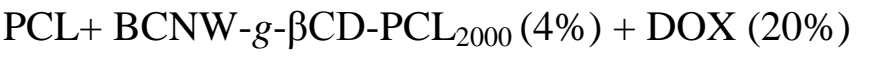 & - & - & $35.7 \pm 1.3$ & $4.4 \pm 0.4$ & $3196 \pm 117$ \\
\hline $\mathrm{PCL}+\mathrm{BCNW}-g-\beta C D-\mathrm{PCL}_{2000}(4 \%)+\mathrm{DOX}(25 \%)$ & - & - & $37.1 \pm 1.1$ & $4.0 \pm 0.3$ & $3235 \pm 110$ \\
\hline $\mathrm{PCL}+\mathrm{BCNW}-g-\beta \mathrm{CD}-\mathrm{PCL}_{2000}(4 \%)+\mathrm{DOX}(30 \%)$ & - & - & $32.8 \pm 1.2$ & $3.7 \pm 0.2$ & $2624 \pm 112$ \\
\hline
\end{tabular}




\subsubsection{Surface Modification Evaluated by Contact Angle}

The bar chart in Figure 5.5 shows the evaluated apparent water-in-air contact angle of neat PCL and PCL-bionanocomposite films at different BCNW- $-\beta C D-\mathrm{PCL}_{2000}$ and drug loadings. In general, a contact angle value above $90^{\circ}$ corresponds to a hydrophobic surface, while a contact

angle value below $90^{\circ}$ represents a hydrophilic surface. ${ }^{[413]}$ Results shows neat PCL shows contact angle values of $94^{\circ} \pm 2.5^{\circ}$. The later value was gradually decreased upon addition of (1-5 $\mathrm{wt} \%$ ) of $\mathrm{BCNW}-g-\beta C D-\mathrm{PCL}_{2000}$ to $\mathrm{PCL}$ matrix until a minimum plateau of $70^{\circ} \pm 2.9^{\circ}$ at $5 \mathrm{wt} \%$ loading was reached. In comparison to neat PCL, approximately $25.5 \%$ improvement in hydrophilicity of PCL-bionanocomposite was achieved by incorporating $5 \mathrm{wt} \%$ of BCNW-g$\beta C D-P_{2000}$ into PCL matrix. This increased hydrophilicity could be related to the inherited hydrophilic properties of $\mathrm{BCNW}$ due to the presence of free ungrafted hydroxyl groups on BCNW-g- $\beta C D-P_{2} L_{2000}$ surface. In addition, a further significant reduction in contact angle values was observed after incorporating DOX.HCl into bionanocomposite, see Figure 5.5. For instance, the addition of DOX in weight ratio of (10-30\% w/w) to PCL/BCNW- $g-\beta C D-\mathrm{PCL}_{2000}$ (4\%) led to a further drop in the observed contact angle from $75^{\circ} \pm 2.9^{\circ}$ to a stable minimum value of $63^{\circ} \pm 2.3^{\circ}$. However, compared to neat PCL, approximately $31 \%$ improvement in hydrophilicity was achieved by the drug laden bionanocomposite containing 4 wt $\%$ of PCL/BCNW- $g-\beta C D-P_{2} L_{2000}$ and loaded with $25 \mathrm{wt} \%$ of DOX. This further decrease in contact angle values of the drug-laden nanocomposites may be related due to the ionic character of DOX.HCl drug as well as the presence of hydroxyl group moieties in drug molecules. These ultimately increase the hydrophilicity of the drug-laden nanocomposites film. 


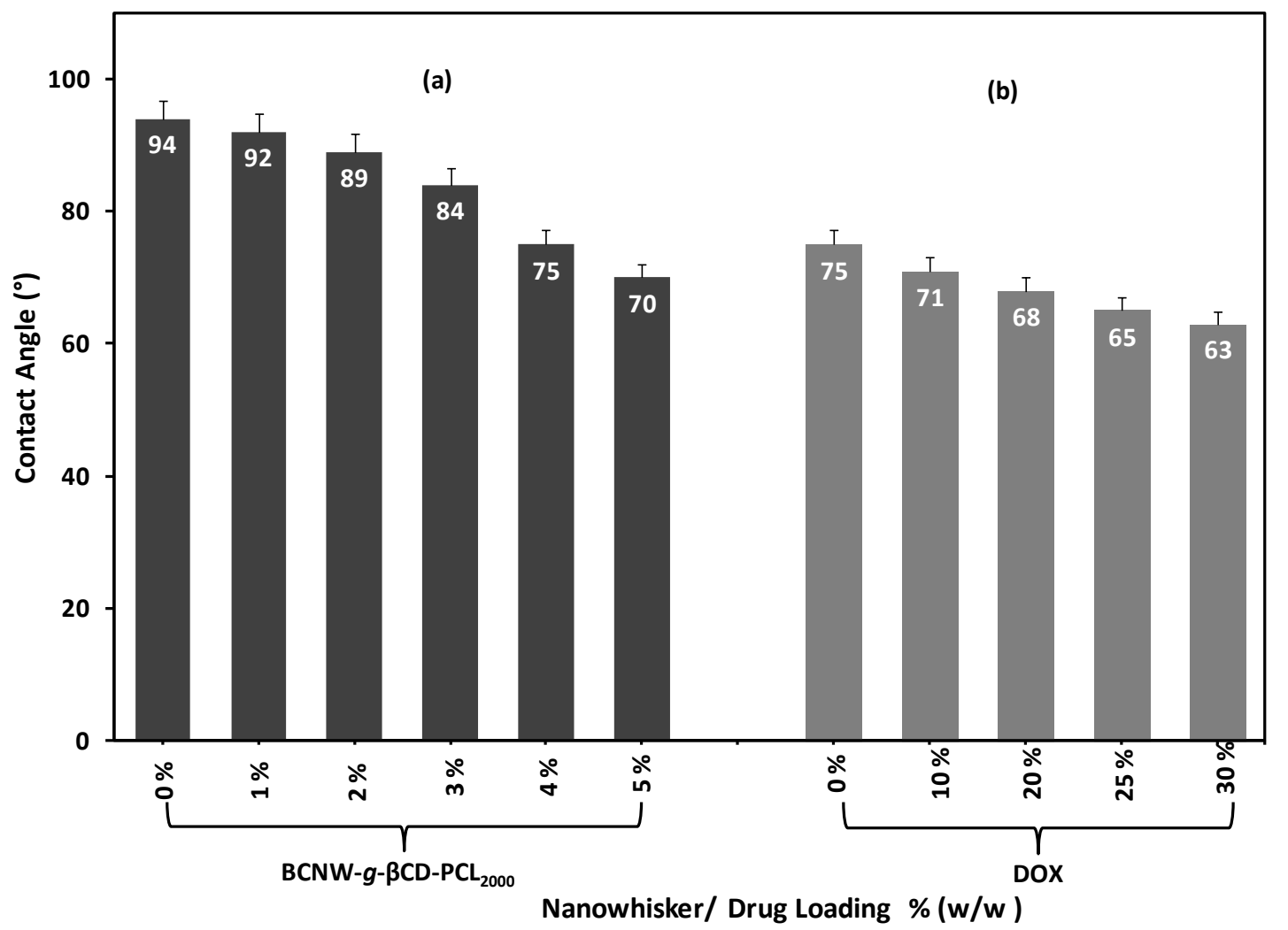

Figure 5.5 Apparent water-in-air contact angles for; (a) PCL/BCNW-g- $\beta C D-P C L_{2000}$ nanocomposites films at different loadings of BCNW-g- $\beta C D-P C L 2000$; (b) PCL/BCNW- $g-\beta C D-$ $\mathrm{PCL}_{2000}(4 \%)$ drug-laden nanocomposites with different DOX loadings from (10-30\%wt).

\subsubsection{Crystallization Behavior and Thermal Stability of Drug-laden Bionanocomposite}

Differential scanning calorimetry was used to investigate the effect of grafted nanowhisker and drug loadings on the crystallization and melting behaviors of PCL and its bionanocomposite. Figure 5.6 displays thermal properties of the first cooling and second heating runs in the DSC thermograms of neat PCL and its bionanocomposite (i.e., PCL/BCNW-g- $\beta C D-\mathrm{PCL}_{2000}$ and PCL/BCNW- $g$ - $\beta C D-\mathrm{PCL}_{2000} / \mathrm{DOX}$ ). The obtained thermal properties (i.e., cold crystallization temperature; $\mathrm{T}_{\mathrm{cc}}$, melting temperature; $\mathrm{T}_{\mathrm{m}}$, cold crystallization enthalpy; $\Delta \mathrm{H}_{\mathrm{cc}}$, melting enthalpy; $\Delta \mathrm{H}_{\mathrm{m}}$ and crystallinity degree; $\chi_{\mathrm{c}}$ ) are summarized in Table 5.2. Examining DSC results in Figure 5.6 shows that no obvious melt-crystallization peaks appeared during the first cooling scans. However, in the second heating scan, the cold crystallization peaks appeared for either neat PCL or its nanocomposites. Results in Table 5.2 show that neat PCL has $\mathrm{T}_{\mathrm{cc}}$ of $31^{\circ} \mathrm{C}, \mathrm{T}_{\mathrm{m}}$ around $58^{\circ} \mathrm{C}$ and $\chi_{\mathrm{c}}$ of $27.5 \%$. As previously reported by our group, an incorporation of $4 \mathrm{wt} \%$ of BCNW-g- 
$\beta C D-\mathrm{PCL}_{2000}$ in PCL matrix was induced obvious decrease in $\mathrm{T}_{\mathrm{cc}}$ values from 31 to $23^{\circ} \mathrm{C}$ without significant increase in $\mathrm{T}_{\mathrm{m}}$ values $\left(59^{\circ} \mathrm{C}\right)$, as well as a reduction in $\chi_{\mathrm{c}}$ value to $21.8 \%$. $^{[417]}$ These observed changes in the crystallization behavior of nanocomposites were attributed to the effect of low-molecular weight $\mathrm{PCL}_{2000}$ segments that conjugated on nanowhisker surface. Whereas these short polymer segments hindered the mobility of PCL chains, and consequently impaired the crystallization behavior of PCL matrix. ${ }^{[422]}$ The later decrease in crystallinity up on addition of $4 \mathrm{wt} \%$ of $\mathrm{BCNW}-\mathrm{g}-\beta \mathrm{CD}-\mathrm{PCL}_{2000}$ to PCL matrix was helpful in forming imperfect PCL crystals that consequently promoted the integrity of larger drug concentration in the drugladen bionanocomposite. The absence of the characteristic endothermic melting peak of

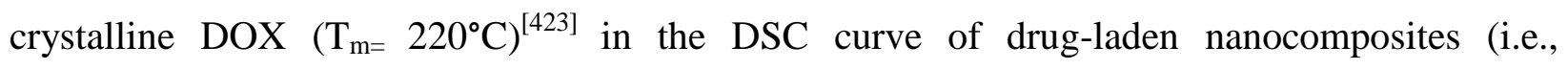
$\left.\mathrm{PCL} / \mathrm{BCNW}-\mathrm{g}-\beta \mathrm{CD}-\mathrm{PCL}_{2000} / \mathrm{DOX}\right)$ is a great evidence, where the drug is converted to amorphous phase and the entire added drug was bound to the polymer. In other words, the loading process of drug to $\mathrm{PCL} / \mathrm{BCNW}-g-\beta C D-\mathrm{PCL}_{2000}$ was appreciable. As a result, at the same BCNW-g- $\beta$ CD-PCL 2000 loading (i.e., $4 \mathrm{wt} \%$ ) the incorporation of $25 \mathrm{wt} \%$ of DOX to nanocomposites contents causes an increase in $\mathrm{T}_{\mathrm{cc}}$ and $\mathrm{T}_{\mathrm{m}}$ of the drug-laden nanocomposites from 23 to $27^{\circ} \mathrm{C}$ and from 59 to $63^{\circ} \mathrm{C}$, respectively (Figure 5.6). Moreover, an increase in $\chi_{\mathrm{c}}$ from 21.7 to $43.6 \%$ was observed upon adding of $25 \mathrm{wt} \%$ of DOX to the drug-laden nanocomposites Therefore, the addition of high concentration of low molecular weight drug (i.e., $25 \mathrm{wt} \%$ DOX) might be hindered the mobility of PCL chains in crystalline domains and decreased their packing density as well. This in turn improves its crystallization ability and increased both $T_{c c}$ and $T_{m}$ values. 
Table 5.2 Thermal pentameters for neat PCL, drug-loaded PCL and its bionanocomposite with various drug loadings

\begin{tabular}{|c|c|c|c|c|c|}
\hline Samples & $\mathrm{T}_{\mathrm{cc}}\left({ }^{\circ} \mathrm{C}\right)$ & $\Delta H_{\text {cc }}(\mathrm{J} / \mathrm{g})$ & $\mathbf{T}_{\mathrm{m}}\left({ }^{\circ} \mathrm{C}\right)$ & $\Delta H_{m}(J / g)$ & $\chi_{\mathrm{c}}(\%)^{\mathrm{a}}$ \\
\hline Neat PCL & 31 & 27.3 & 58 & 64.0 & 27.5 \\
\hline PCL+ BCNW- $g-\beta C D-P C L_{2000}(4 \%)$ & 23 & 34.0 & 59 & 60.8 & 21.7 \\
\hline \multirow{3}{*}{$\begin{array}{l}\text { PCL+ BCNW- } g-\beta C D-\mathrm{PCL}_{2000}(4 \%)+ \\
\text { DOX }(10 \%) \\
\text { PCL+ BCNW- }-\beta \text { CD-PCL } 2000(4 \%)+ \\
\text { DOX }(25 \%) \\
\text { PCL+ BCNW- } g-\beta C D-\mathrm{PCL}_{2000}(4 \%)+ \\
\text { DOX }(30 \%)\end{array}$} & 25 & 13.1 & 62 & 58.1 & 37.5 \\
\hline & 27 & 11.8 & 63 & 55.0 & 43.6 \\
\hline & 27.5 & 10.7 & 64 & 53.5 & 45.5 \\
\hline DOX & -- & -- & 220 & -- & - \\
\hline
\end{tabular}

${ }^{\mathrm{a}} \chi_{c}=\left[\frac{\Delta \mathrm{H}_{\mathrm{m}}-\Delta \mathrm{H}_{\mathrm{cc}}}{\left(\Delta \mathrm{H}_{0, \mathrm{~m}}(1-(\mathrm{wt} \% \mathrm{BCNW}+\mathrm{wt} \% \mathrm{DOX}))\right]} \times 100 ; \Delta \mathrm{H}_{0, \mathrm{~m}}\right.$ of $100 \%$ crystalline PCL sample taken as $139.5 \mathrm{~J} / \mathrm{g}^{[409]}$

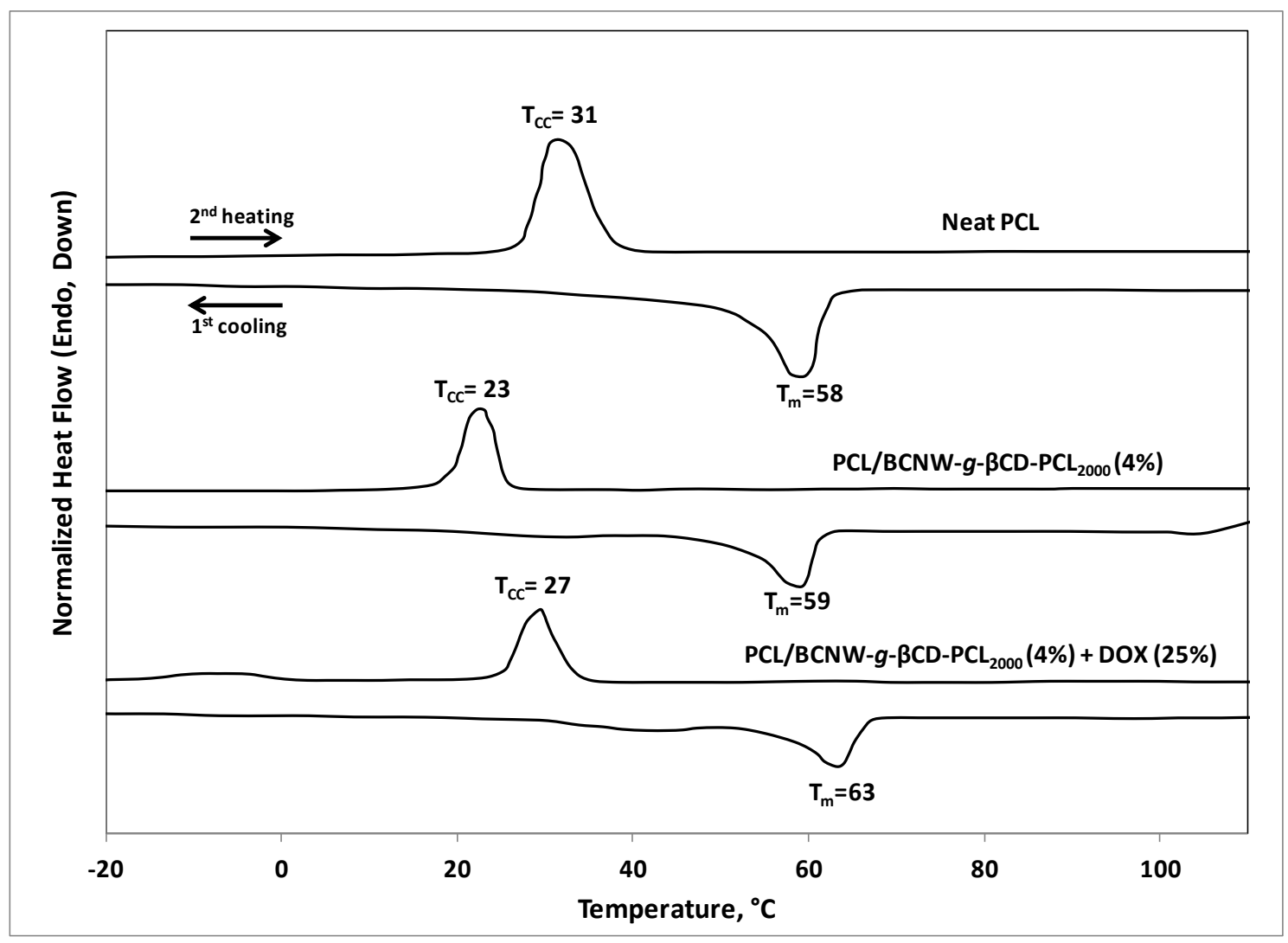

Figure 5.6 DSC first cooling and second heating scans of neat PCL and its drug-laden bionanocomposite. 
Figure 5.7 depicts TGA thermograms of neat PCL, functionalized nanowhisker BCNW- $g-\beta C D-$ $\mathrm{PCL}_{2000}$, nanocomposites PCL/BCNW-g- $\beta C D-\mathrm{PCL}_{2000}(4 \%)$ and drug laden nanocomposites PCL/BCNW- $g$ - $\beta C D-P_{2000}(4 \%) / D O X ~(25 \%)$. Investigating Figure 5.7 shows that the maximum decomposition temperature $\left(\mathrm{T}_{\max }\right)$ achieved by each thermogram was as follow; $445^{\circ} \mathrm{C}$ for neat PCL; $436^{\circ} \mathrm{C}$ for $\mathrm{BCNW}-g-\beta C D-\mathrm{PCL}_{2000} ; 447^{\circ} \mathrm{C}$ for PCL/BCNW-g- $\beta C D-\mathrm{PCL}_{2000}(4 \%)$; and $453^{\circ} \mathrm{C}$ for $\mathrm{PCL} / \mathrm{BCNW}-g-\beta C D-\mathrm{PCL}_{2000}(4 \%) / \mathrm{DOX}(25 \%)$. Examining the obtained results shows that adding $4 \% \mathrm{w} / \mathrm{w}$ of functionalized nanowhisker to PCL matrix does not cause obvious change in $\mathrm{T}_{\max }$ value of nanocomposites if it compared with neat PCL polymer. However, addition of $25 \mathrm{wt} \%$ DOX is improved $\mathrm{T}_{\max }$ value of the drug-laden nanocomposites by 8 and $7^{\circ} \mathrm{C}$ if it compared with neat PCL and its drug-unloaded nanocomposites. This increase in the thermal stability might be related to the formation of hydrogen bonds between the drug molecules, nanowhisker and polymer matrix. ${ }^{[15]}$ Moreover, the later supports the previous finding from DSC measurement where an improved crystallinity of drug-laden nanocomposites was observed.

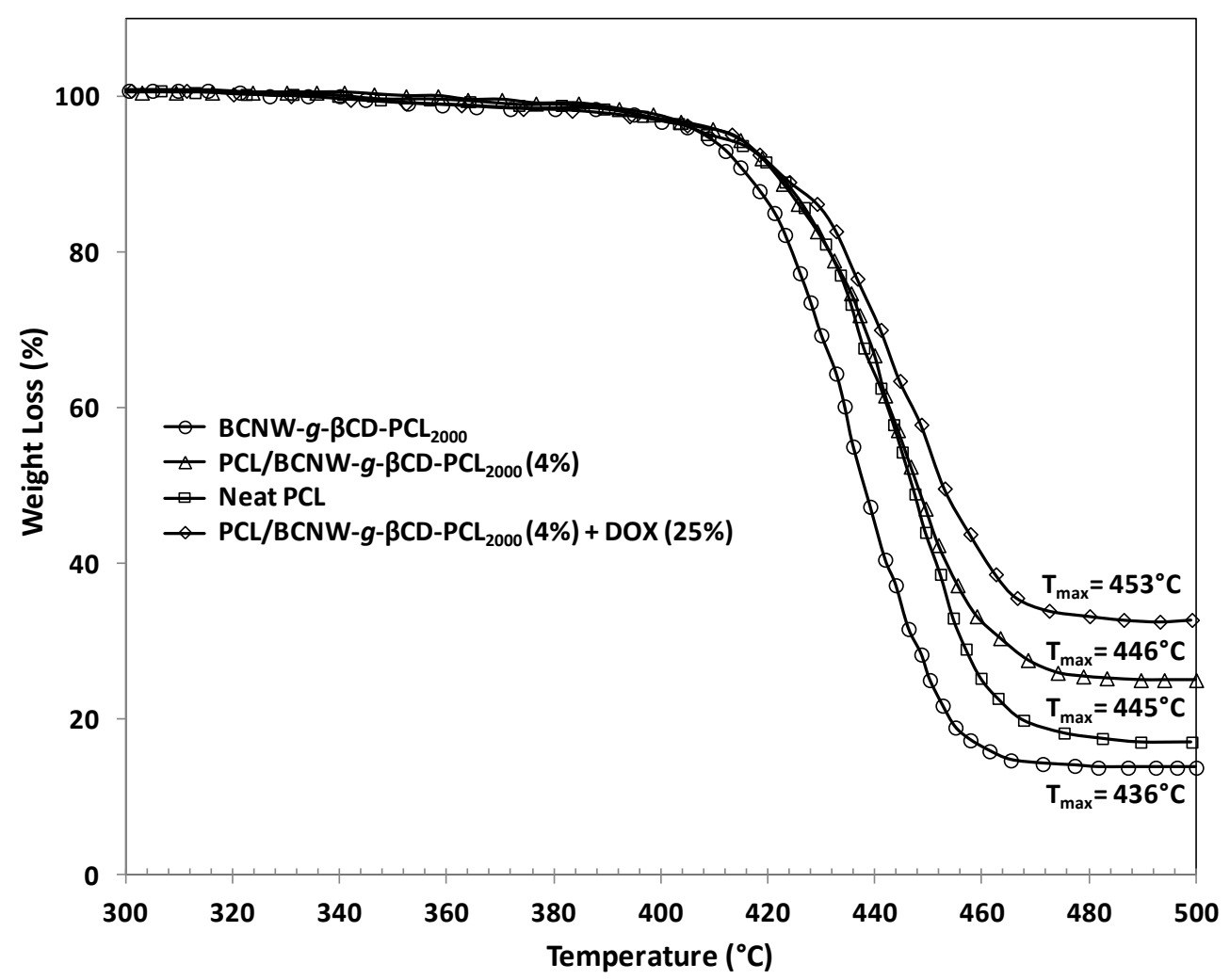

Figure 5.7 TGA curves of functionalized nanowhisker, neat PCL and PCL-bionanocomposite. 


\subsubsection{Scaffolds Porosities and Morphologies}

Due to the impressive mechanical and properties of the drug-laden nanocomposites formula involving PCL/BCNW- $\left.g-\beta C D-\mathrm{PCL}_{2000}(4 \%) / \mathrm{DOX}(25 \%)\right)$ it was chosen as a representative for developing 3D-nanostructures of different porosities. Gas foaming/combined particulate leaching method was used for developing porous structures. The method was incorporating two different progene; fixed amount of PEG, and different ratios of sieved ammonium bicarbonate salt having different size distributions (i.e., 180-300 and 300-500 $\mu \mathrm{m}$ ).

Table 5.3 summarizes percentage porosity, pore volume, and pore size of the obtained porous scaffolds. Results show that percentage porosities of the obtained scaffolds that obtained gravimetrically are in the range of $86.45-94.85 \%$ with estimated pore volumes in the range of $9.64-17.86 \mathrm{~cm}^{3} / \mathrm{g}$. The observed percentage porosities are improved from $86.45 \%$ to $94.85 \%$ with increasing salt: polymer weight ratio from 5:1 to 10:1; see Table 5.3. The average pore sizes that determined by visual estimation from SEM images were ranged from $\sim 250-340 \mu \mathrm{m}$. The estimated pore sizes appeared to be dependent on the size of the sieved ammonium bicarbonate particulates. At the same polymer: salt ratio, the coarser ammonium bicarbonate crystals used, the bigger pore sizes are.

Figure 5.8 shows SEM images of the drug-laden porous scaffolds that obtained by gas foaming/combined particulate leaching method. Examining the morphologies of the porous scaffolds confirms an opened macropores on the surface without sign of surface skin. These macropores are generated due to immediate gas evolution from salt particulates and subsequent rapid gas flow-out from the semi-solidified polymer mixture. In addition, the cross-section views in Figure 5.8 A1, A2, B1, B2, C1, C2 reveal the presence of uniform distribution of wellinterconnected opened macropores from surface to surface. The obtained results suggest the successfulness of gas foaming/combined particulate leaching technique that introduced in this study for developing scaffold with higher porosities. It also worth to mention the used fabrication method showed two distinctive advantages over the other examined techniques. The blend mixture (i.e., PCL/PEG/drug/nanowhisker/ammonium bicarbonate) can be easily manipulated to make any shape and size, and also the final scaffold did not show any sign for surface skin formation.

Moreover, Table 5.3 is also depicted the obtained compression modulus values that measured for the cylindrical shaped drug-laden porous scaffolds. Results show decline for the modulus 
with the increase in salt: polymer ratio due to the increased porosity. For instance, the scaffold prepared using 5:1 ammonium bicarbonate/polymer ratio showed the maximum compression modulus of $12.3 \pm 0.7 \mathrm{MPa}$, but the least porosity value of $86.45 \%$. In contrast, $10: 1$ salt/polymer ratio showed the highest porosity of $94.85 \%$, but the least compression modulus of $7.2 \pm 0.7$ MPa. However, 7:1 salt/polymer ration has maintained good compression modulus value of 10.6 $\pm 0.5 \mathrm{MPa}$ along with higher porosity of $91.17 \%$. Therefore, $7: 1$ salt to polymer was identified in this study as the most suitable ratio for developing drug-laden scaffolds with higher porosity (> 90\%) along with outstanding compression modulus.

Nevertheless, in case similar experiments where porous scaffolds were fabricated from neat PCL or PCL/DOX blend (control experiment) using 7:1 (salt: polymer) ratio, the observed compression modulus values are harshly decreased with increasing either drug loading or porosity level; see Table 5.3. The later evidence is not only confirming the main role of BCNW$g-\beta C D-P_{2} L_{2000}$ as reinforcing phase for keeping the integrity of the drug-laden nanocomposites, but also shows its potentiality to compensate for the deterioration in mechanical properties due to the combined effect of the increased drug loading and the developed porous structures. For instance, scaffold fabricated with PCL/BCNW-g- $\beta C D-\mathrm{PCL}_{2000}(4 \%)$ and loaded with 25 wt $\%$ DOX using 7:1 (salt: polymer) showed almost the same porosity level of control (PCL/DOX), but with three-fold compression modulus value (Table 5.3).

The adequate pore sizes, highly porous structure with an interconnected pore-network, along with an improved hydrophilicity suggest the suitability of the fabricated drug-laden scaffold that developed in this study for encouraging initial cell attachment, proliferation, and differentiation. ${ }^{[396]}$ In addition, the compression modulus values range (i.e., 7.2-12.3 MPa) are located in the range of cancellous bones (i.e., 2-12 MPa). ${ }^{[402]}$ Therefore, these developed drugladen PCL scaffolds can find application as promising temporal implant or bone cement in limb salvage procedure. ${ }^{[141]}$ 
Table 5.3 Porosity percentage, pore volume, pore size, and compression modulus of PCL-drug laden porous scaffolds

\begin{tabular}{|c|c|c|c|c|c|c|}
\hline Scaffold Formulation & $\begin{array}{c}\mathbf{N H}_{4} \mathbf{H C O}_{3}: \\
\text { PCL }(\% \text { w/w }) \\
\end{array}$ & $\begin{array}{c}\mathrm{NH}_{4} \mathbf{H C O}_{3} \\
\text { Size }(\mu \mathrm{m}) \\
\end{array}$ & $\begin{array}{c}\text { Porosity* } \\
(\%)\end{array}$ & $\begin{array}{l}\text { Pore Volume* } \\
\left(\mathrm{cm}^{3} / \mathrm{g}\right)\end{array}$ & $\begin{array}{l}\text { Pore Size* } \\
(\mu \mathrm{m})\end{array}$ & $\begin{array}{c}\text { Compressive } \\
\text { Modulus (MPa) }\end{array}$ \\
\hline Neat PCL & $7: 1$ & $180-300$ & 90.77 & 11.71 & $264 \pm 8$ & $2.3 \pm 0.1$ \\
\hline \multirow{3}{*}{ PCL+ DOX (25\%) control } & $5: 1$ & $180-300$ & 86.64 & 9.84 & $251 \pm 13$ & $4.5 \pm 0.7$ \\
\hline & $7: 1$ & $180-300$ & 90.48 & 11.92 & $274 \pm 10$ & $4.1 \pm 0.2$ \\
\hline & $10: 1$ & $180-300$ & 93.92 & 16.70 & $287 \pm 19$ & $3.6 \pm 0.2$ \\
\hline $\mathrm{PCL}+\mathrm{BCNW}-g-\beta \mathrm{CD}-\mathrm{PCL}_{2000}(4 \%)$ & $7: 1$ & $180-300$ & 91.28 & 12.33 & $285 \pm 16$ & $8.7 \pm 0.5$ \\
\hline \multirow{4}{*}{$\begin{array}{l}\text { PCL+ BCNW- } g-\beta C D-P^{-} L_{2000}(4 \%) \\
+\operatorname{DOX}(25 \%)\end{array}$} & $5: 1$ & $180-300$ & 86.45 & 9.64 & $254 \pm 11$ & $12.3 \pm 0.7$ \\
\hline & $7: 1$ & $180-300$ & 91.17 & 12.15 & $284 \pm 17$ & $10.6 \pm 0.5$ \\
\hline & $10: 1$ & $180-300$ & 94.85 & 17.86 & $292 \pm 11$ & $7.2 \pm 0.3$ \\
\hline & $7: 1$ & $300-500$ & 93.25 & 13.13 & $342 \pm 19$ & $8.7 \pm 0.7$ \\
\hline
\end{tabular}

The significant differences for each individual feature, with $\mathrm{p}<0.05$, was determined by one-way ANOVA.*(n $=5$ for porosity and pore volume, and $\mathrm{n}=30$ for pore size). 

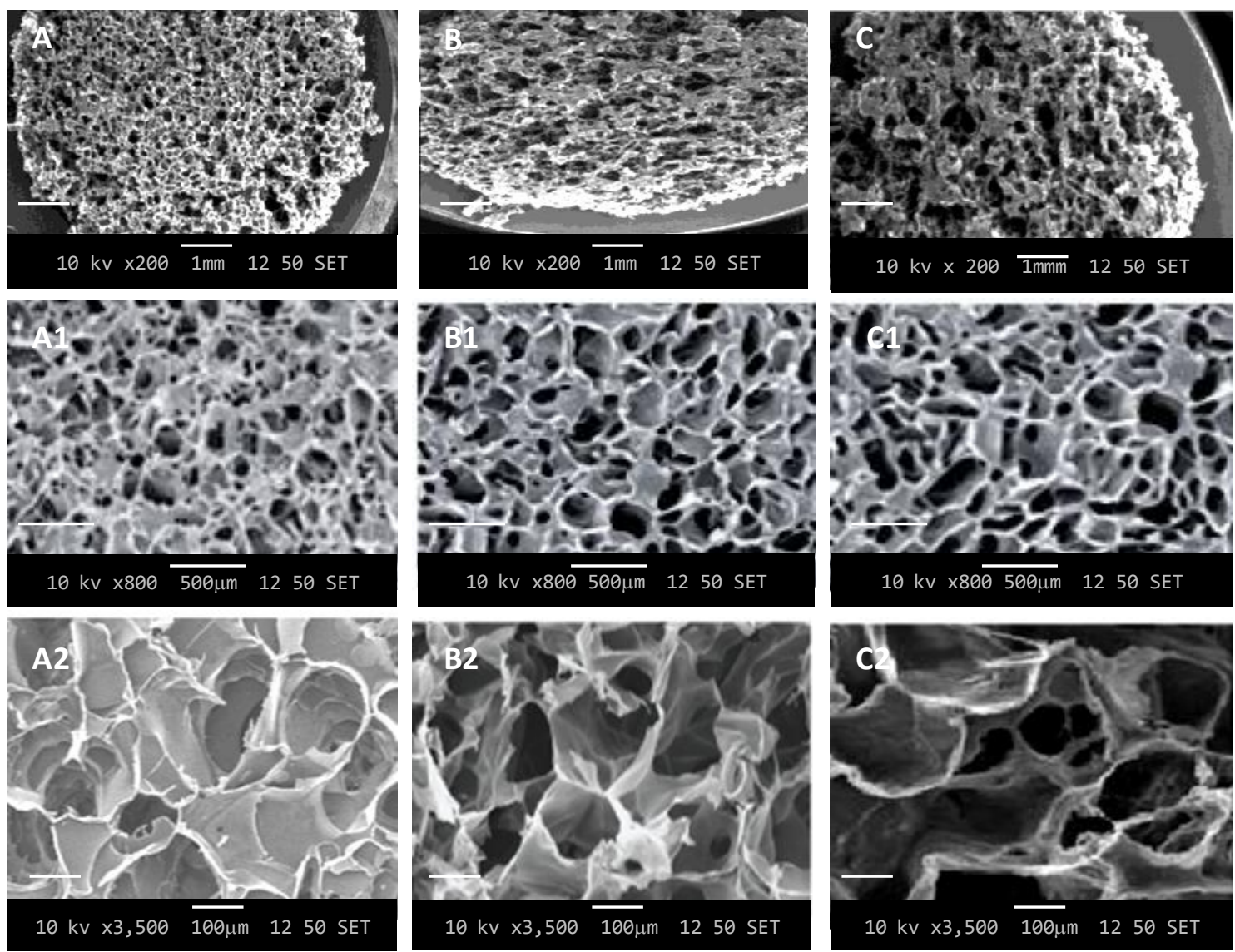

Figure 5.8 SEM images of surface and cross-section morphology of PCL/BCNW-g- $\beta C D-$ $\mathrm{PCL}_{2000}(4 \%) / \mathrm{DOX}(25 \%)$ scaffolds prepared by gas foaming in hot citric acid. (A, B \&C) are surfaces when weight $\mathrm{NH}_{4} \mathrm{HCO}_{3}$ : PCL ratios were 5:1, 7:1 and 10:1, respectively; (A1, A2) \& $(\mathrm{C} 1, \mathrm{C} 2)$ cross-section morphology corresponding to the same series at different magnification powers; $\times 800$ and $\times 3500$.

\subsubsection{Invitro Degradation}

In vitro degradation behavior of neat PCL and its drug-laden scaffolds are shown in Figure 5.9. The graph shows the recorded weight loss that determined gravimetrically at different time intervals after immersing scaffolds in PBS of pH 7.4 up to $\sim 2$ month. The results show a slower degradation rate for neat PCL where only $11 \%$ weight loss was occurred after 70 days. This slow degradation rate is related to the higher crystallinity and the hydrophobic nature of PCL. ${ }^{[416]}$ In contrast, the incorporation of $4 \mathrm{wt} \%$ of BCNW- $g-\beta C D-\mathrm{PCL}_{2000}$ into PCL matrix increased its degradation rate to $18 \%$. This improved degradation could be related to the increased surface hydrophilicity of nanocomposites, which allows promoted interaction between scaffold surface and dissolution medium. The later finding is also in a good agreement with the thermal results 
that revealed decreased crystalinity due to the incorporation of BCNW-g- $\beta C D-P C L_{2000}$ into PCL matrix. A further increase in degradation rate was observed for drug-laden nanocomposites with 25 wt $\%$ DOX, where about $21 \%$ weight loss could be achieved after 70 days; see Figure 5.9. This observation is also in a good agreement with further improvement in contact angle the previously reported with increasing drug payload. Where the ionic nature of DOX.HCl increases the interaction between scaffold surface and dissolution media. In addition, a comparative test for investigating the effect of enzyme on hydrolytic degradation of scaffolds was carried out in PBS at $\mathrm{pH} 7.4$ and $37^{\circ} \mathrm{C}$ in presence of porcine lipase. A substantial increase in degradation rate was observed where percentage weight loss is reached a maximum value of $29 \%$ after 70 days; see Figure 5.9. Porcine Lipase most probably accelerates the hydrolysis through attacking the ester linkages of either short and long PCL chains. ${ }^{[414]}$ This promoted degradation rate of PCLdrug laden bionanocomposite scaffolds makes it ideal candidate for long-term tissue regeneration.

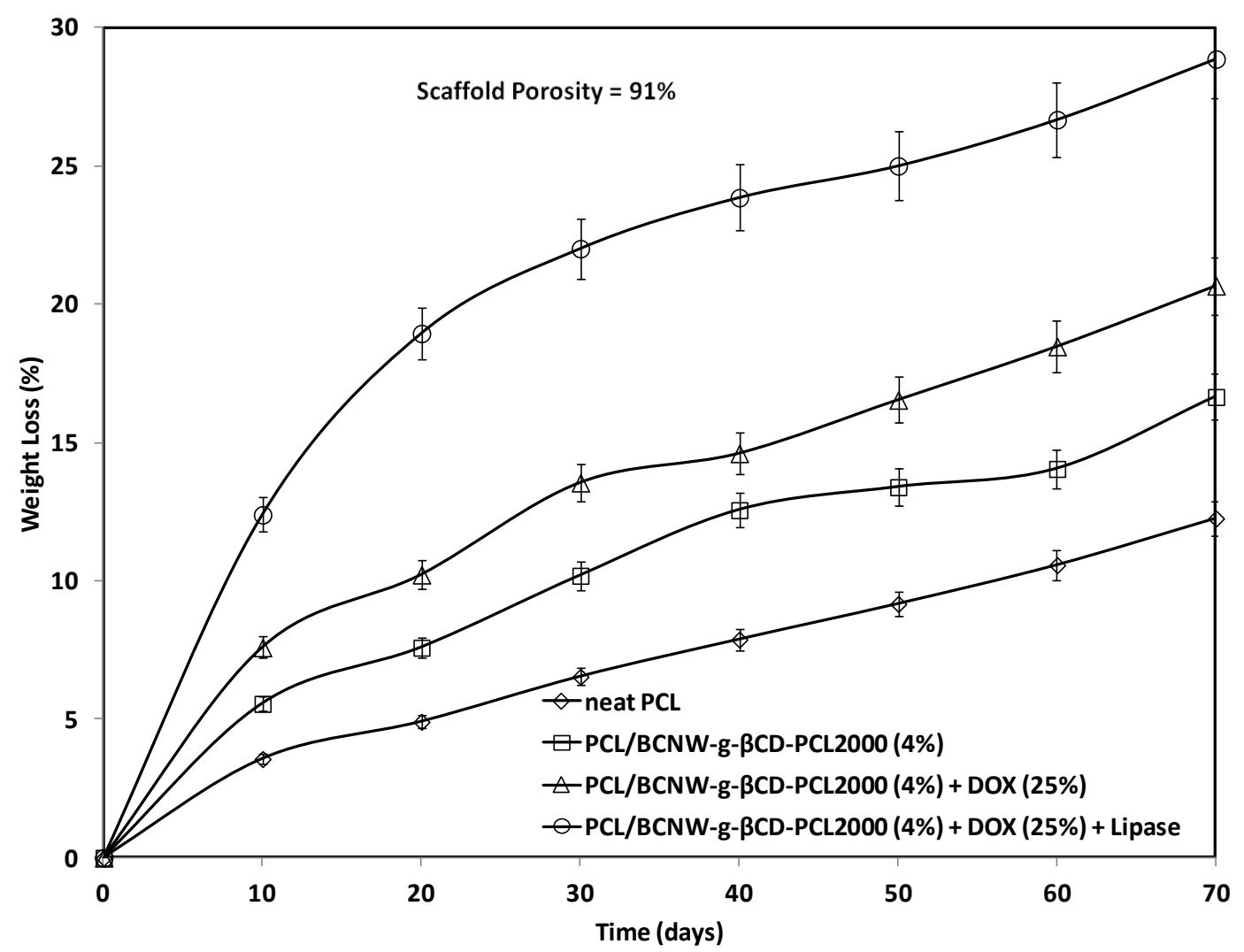

Figure 5.9 In vitro degradation behavior of neat PCL and drug-laden nanocomposites scaffolds in $\mathrm{PBS}$ at $\mathrm{pH} 7.4$ and $37^{\circ} \mathrm{C}$. 


\subsubsection{Drug Release Profiles from Porous Scaffolds}

Figure 5.10 shows cumulative release of DOX at $37^{\circ} \mathrm{C}$ from PCL/DOX blend and the drug-laden PCL/BCNW- $g-\beta C D-P_{2000}(4 \%) / D O X ~(25 \%)$ scaffolds that having the same porosity level of 91\%. PBS of two different $\mathrm{pH} ; 6.4$ and 7.4 are used as drug release media. Results indicate a minimum stained release with a larger initial burst of DOX from PCL/DOX scaffold. Analyzing these results shows a cumulative release of about $60 \%$ of the total drug payload during 2 months period. Approximately 55\% of which is released in the first 10 days, and only 5\% of drug payload is released during the subsequent period. In contrast, DOX release from nanocomposites scaffold (i.e., PCL/BCNW-g- $\beta C D-\mathrm{PCL}_{2000} / \mathrm{DOX}$ ) at $\mathrm{pH} 7.4$ is significantly slower with a controlled and sustained manner. The results indicated that more than $80 \%$ of the drug payload is released gradually during $\sim 60$ days before reaching the constant plateau. This obvious difference in the drug release behavior could be attributed to the improved bulk properties (i.e., hydrophilicity and degradation) of nanocomposites due to the presence of BCNW- $g-\beta C D-$ $\mathrm{PCL}_{2000}$. This reinforcing nanowhisker is greatly enhanced the rate of scaffold dissolution and consequently its drug release properties. In addition, a promoted drug release properties is observed in acidic $\mathrm{pH}$ of 6.4 that mimicking the physiological $\mathrm{pH}$ range of cancer tumors. The higher cumulative release of DOX in this acidic $\mathrm{pH}$ can be ascribed to the greater solubility of the drugs in aqueous acidic medium due to the protonation of the amine groups of drug molecules, which consequently assists the diffusion process. Therefore, beside the satisfactory mechanical properties showed by the developed drug-laden PCL/BCNW- $g$ - $\beta C D-$ PCL $_{2000}$ scaffolds it also demonstrates a promising potential for encapsulating higher payload of anticancer-drug and also prolongs its release. 


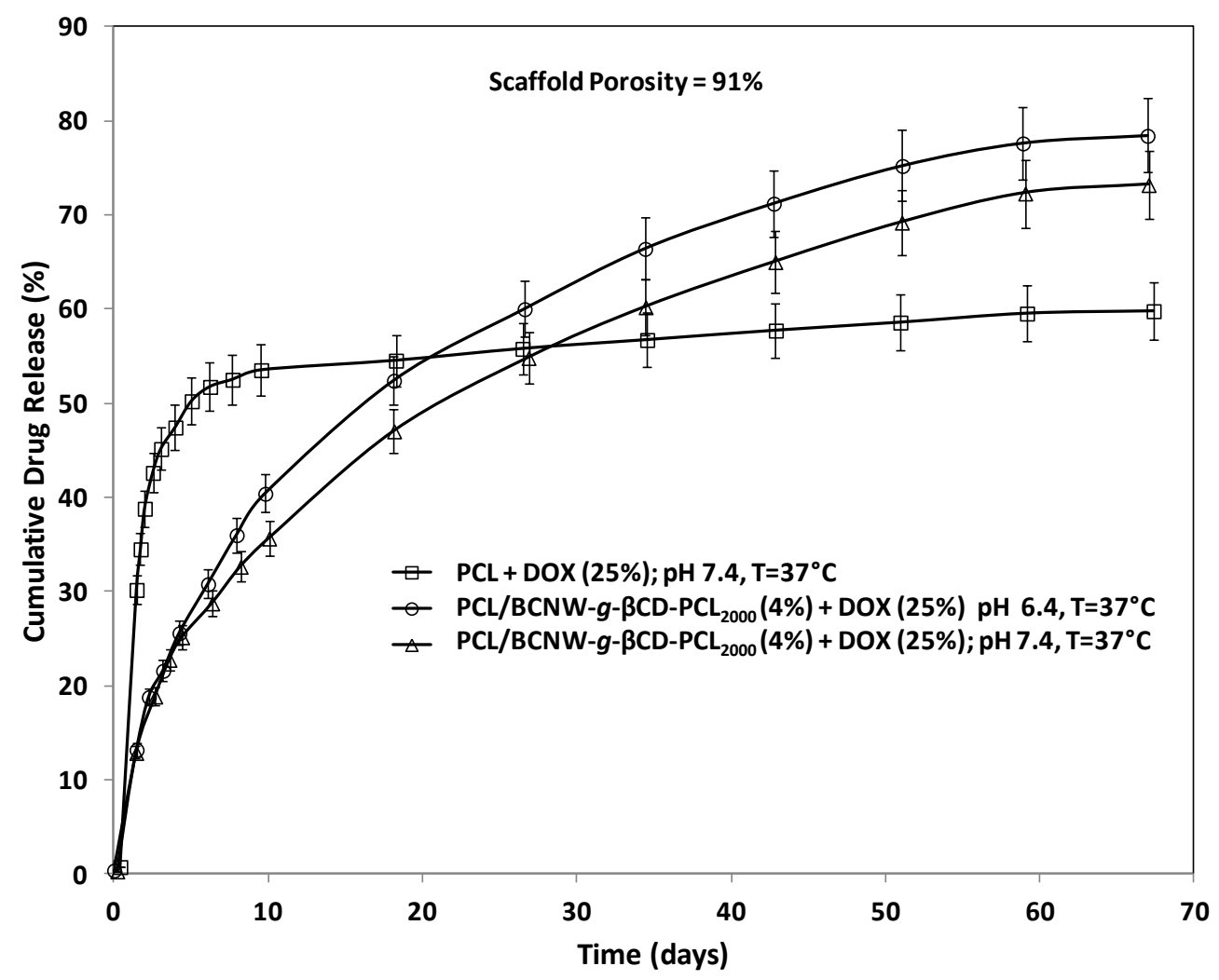

Figure 5.10 Cumulative release of DOX from PCL+ DOX (25\%) and PCL/BCNW- $g-\beta C D-$ $\mathrm{PCL}_{2000}(4 \%)+\mathrm{DOX}(25 \%)$ at $37^{\circ} \mathrm{C}$ in PBS of $\mathrm{pH} 7.4$ and 6.4 .

In order to better predict the drug-release characteristics of scaffolds, a further investigation of drug release profiles at different porosity and drug loading levels were investigated in Figure 5.11 A and B. Results show that the scaffolds loaded with the same amount of DOX (i.e., 25\%) demonstrated an improved cumulative drug release rate with increased scaffold's porosity during the first 30 days. After which, these differences become less conspicuous just before reaching the common plateaus; see Figure 5.11 A. Furthermore, Figure 5.11B shows the effect of increased drug payloads on drug release properties of scaffolds at the same porosity level. Results show that increasing drug loading concentration has a positive relationship with the cumulative releases of DOX over 60 days. Increasing drug loading concentration in the scaffold causes a steeper diffusion gradient between the scaffold and surrounding dissolution medium. This in turn leads to an increased rate of diffusion and consequently the drug release rate. As for treatment regime, most cancer patients would be placed on doxorubicin $25 \mathrm{mg} / \mathrm{m}^{2} /$ day on days 1 to 3 and cisplatin $100 \mathrm{mg} / \mathrm{m}^{2}$ on day 1 , every 3 weeks for six cycles. ${ }^{[117]}$ While the scaffolds developed in 
this study could potentially release from $50-80 \%$ from their total drug contents gradually during in the first 60 days, therefore, they could secure sufficient quantity of DOX drug that required for the entire postoperative period. In addition, the initial sustained releases of DOX from the developed drug-laden scaffolds might not make them facing the common growth-inhibition problem $^{[424]}$ of PCL/DOX blend due systemic toxicity induced by the fast initial releases of chemotherapeutic drugs during the initial phase of treatment.

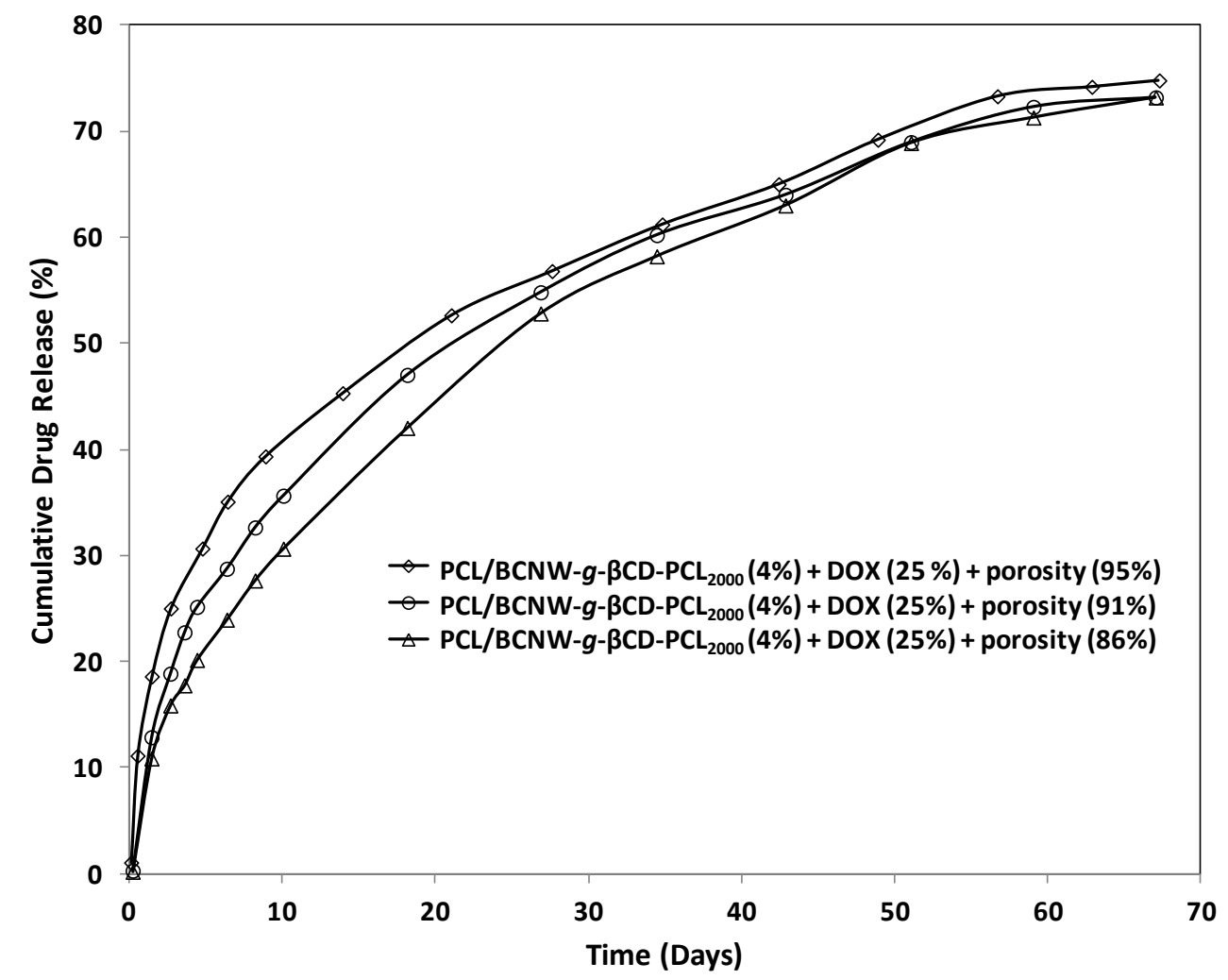

Figure 5.11 A: Effect of increased porosity (\%) on the cumulative release profile of DOX from nanocomposites with drug payload of $25 \%$ w/w in PBS at $\mathrm{pH} 7.4$ and $37^{\circ} \mathrm{C}$. 


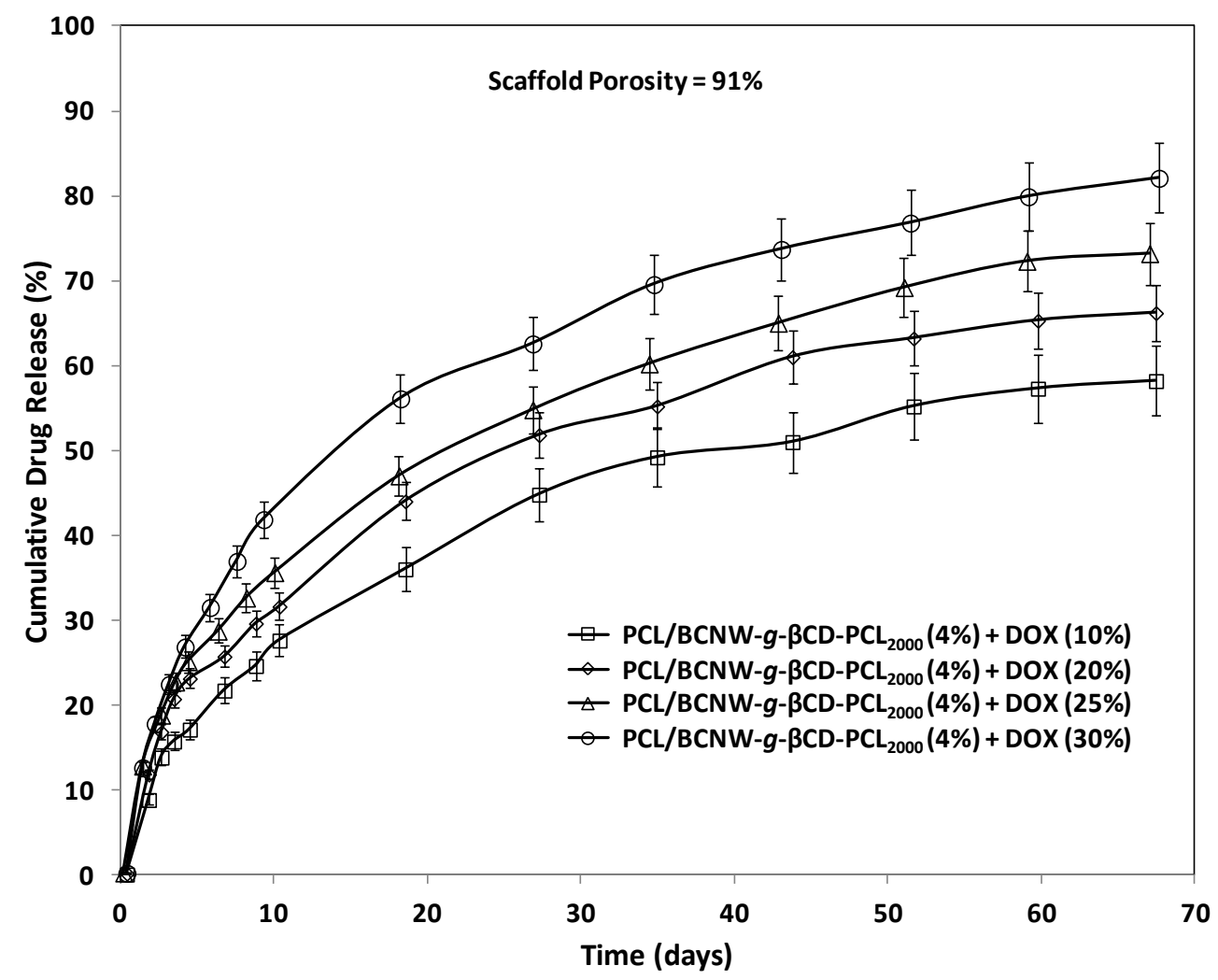

Figure 5.11 B: Effect of drug loading (\%) on the cumulative release profile of DOX from nanocomposites of $91 \%$ porosity in $\mathrm{PBS}$ at $\mathrm{pH} 7.4$ and $37^{\circ} \mathrm{C}$.

\subsubsection{Mathematical Model Fitting Drug Releases}

Table 5.4 summarizes the results of regression analysis that obtained from fitting drug release data to various theoretical models namely; zero-order, first-order, Hixson-Crowell, Higuchi and Peppas-Korsmeyer. The goodness of fit was determined by comparing the average $\mathrm{R}^{2}$ values generated from linear regression analysis. The model that best fits the data was chosen to evaluate the arbitrary release constants of drug. The release constants values were then used to compare between the rates of drug release from scaffolds having various porosities and drug loadings. In addition, the drug release data were also fitted to the Korsmeyer-Peppas model to estimate the average release exponent (n), which gives indication about the identity of release mechanism. Examining the results in Table 5.4 shows that drug releases from all scaffolds are mostly fit to first-order release pattern. In addition, the mechanism of drug releases from all scaffolds was found to be diffusion-controlled following Higuchi's model. Moreover, as revealed from the data fitting to Hixon-Crowell model, the scaffolds' erosion showed comparatively 
lesser effect on drug release. Finally, Peppas-Korsmeyer model reconfirms that the release mechanism of DOX is diffusion controlled as indicated from the exponent values (n) which varied from 0.27 to 0.42 ; see Table 5.4. The drug diffusion constants ( $\mathrm{k}$ ) that estimated from Higuchi model are also listed in Table 5.4. These diffusion constants provide a clear visual comparison between DOX release profiles from the scaffolds fabricated with different parameters. Where the higher $\mathrm{k}$ value indicates faster drug release and vice versa. Analyzing those values indicates that $\mathrm{k}$ increases as percentage porosity increases. This could be related to the total offered surface area, where rate of diffusion is directly proportional to the total exposed surface area. Hence, scaffolds with higher porosities would demonstrate a faster drug release rates. In addition, increasing drug loading concentration shows a positive effect on the cumulative release of DOX as well. This is because increasing drug payload in scaffold results in a steeper diffusion gradient between the drug concentration in scaffolds and the surrounding dissolution medium and consequently leads to an increased rate of diffusion.

Table 5.4 In vitro drug releases data fitting to various theoretical drug release models

\begin{tabular}{|c|c|c|c|c|c|c|c|}
\hline \multirow{3}{*}{ Scaffold Formulation } & \multicolumn{7}{|c|}{ Fitting Models } \\
\hline & \multirow{2}{*}{\begin{tabular}{|c|} 
Zero-order \\
$\mathrm{R}^{2}$
\end{tabular}} & \multirow{2}{*}{$\frac{\text { First-order }}{\mathrm{R}^{2}}$} & \multirow{2}{*}{$\begin{array}{c}\text { Hixon-C. } \\
\mathrm{R}^{2}\end{array}$} & \multicolumn{2}{|c|}{ Higuchi } & \multicolumn{2}{|c|}{ Peppas-Korsmeyer } \\
\hline & & & & $\mathrm{R}^{2}$ & $\mathrm{k}$ & $\mathrm{R}^{2}$ & $\mathrm{n}$ \\
\hline $\begin{array}{l}\mathrm{PCL} / \mathrm{BCNW}-g-\beta \mathrm{CD}-\mathrm{PCL}_{2000}(4 \%) \\
+\operatorname{DOX}(10 \%)+\text { porosity }(91 \%)\end{array}$ & 0.512 & 0.922 & 0.727 & 0.952 & 5.7 & 0.911 & 0.35 \\
\hline $\begin{array}{c}\mathrm{PCL} / \mathrm{BCNW}-g-\beta C D-\mathrm{PCL}_{2000}(4 \%) \\
+ \text { DOX }(20 \%)+\text { porosity }(91 \%)\end{array}$ & 0.375 & 0.915 & 0.703 & 0.981 & 6.5 & 0.901 & 0.27 \\
\hline $\begin{array}{l}\text { PCL/BCNW- } g-\beta C D-P_{C L} L_{2000}(4 \%) \\
+ \text { DOX }(25 \%)+\text { porosity }(91 \%)\end{array}$ & 0.331 & 0.910 & 0.731 & 0.955 & 6.4 & 0.900 & 0.33 \\
\hline $\begin{array}{c}\text { PCL/BCNW-g-BCD-PCL }{ }_{2000}(4 \%) \\
+ \text { DOX }(30 \%)+\text { porosity }(91 \%)\end{array}$ & 0.271 & 0.867 & 0.655 & 0.896 & 9.1 & 0.854 & 0.41 \\
\hline $\begin{array}{c}\text { PCL/BCNW- } g-\beta C D-\mathrm{PCL}_{2000}(4 \%) \\
+\operatorname{DOX}(25 \%)+\text { porosity }(86 \%)\end{array}$ & 0.443 & 0.921 & 0.654 & 0.991 & 5.5 & 0.910 & 0.32 \\
\hline $\begin{array}{l}\text { PCL/BCNW- } g-\beta C D-\mathrm{PCL}_{2000}(4 \%) \\
+\operatorname{DOX}(25 \%)+\text { porosity }(95 \%)\end{array}$ & 0.172 & 0.887 & 0.772 & 0.946 & 6.8 & 0.877 & 0.37 \\
\hline
\end{tabular}




\subsection{Conclusion}

Nano-functionalized material (i.e., BCNW-g- $\beta C D-\mathrm{PCL}_{2000}$ ) based on the green biocompatible $\mathrm{BC}$ nanowhisker was synthesized by grafting with $\beta \mathrm{CD}$ and conjugating low molecular weight segments of $\mathrm{PCL}_{2000}$ to nanowhisker surface. Incorporation of $4 \mathrm{wt} \%$ of BCNW-g- $\beta C D-P C L_{2000}$ into PCL matrix resulted in bionanocomposite with improved bulk characteristics. Compared with neat PCL, the added nanowhisker caused overwhelmed change in the bulk characteristics of PCL. Whereas Young's modulus, tensile strength, hydrophilicity and degradation rate of the resulted nanocomposites were improved by $51 \%$ and $115 \%, 20 \%$ and $7 \%$, respectively. In addition, the obtained bionanocomposite (i.e., PCL/BCNW-g- $\beta C D-P_{2} 2000(4 \%)$ ) showed high potentiality for encapsulating large drug payload. More interestingly, incorporation of $25 \mathrm{wt} \%$ of DOX drug into bionanocomposite formulation caused further improvement in Young's modulus and tensile strength to $107 \%$ and $165 \%$. The hydrophilicity and degradation rate were also promoted to $31 \%$ and $21 \%$. Additionally, $\mathrm{T}_{\mathrm{cc}}$ value of the drug-laden nanocomposites decreased by $4^{\circ} \mathrm{C}$, whereas $\mathrm{T}_{\mathrm{m}}$, and $\mathrm{T}_{\max }$ were increased by 5 and $8^{\circ} \mathrm{C}$.

Porous structures having porosities of $86-95 \%$ with mean pore diameters of $250-340 \mu \mathrm{m}$ along with unique interconnected pore-network, ideal for cell seeding and proliferation, were developed using gas foaming/combined particulate leaching technique. Results showed that reinforcing drug-laden nanocomposites with $4 \mathrm{wt} \%$ of $\mathrm{BCNW}-g-\beta C D-\mathrm{PCL}_{2000}$ is not only compensated for the loss in mechanical properties due to the higher porosity, but also enhanced the integrity and compression modulus of the drug-laden bionanocomposite. The obtained compression moduli of the drug-laden porous scaffold were in the range of cancellous bones.

In addition to the higher drug-loading efficiencies of the obtained drug-laden nanocomposites (i.e., $25 \% \mathrm{w} / \mathrm{w}$ ) it also showed long-term sustained release behavior for $80 \%$ of loaded drug within 2 months. In contrast, PCL/DOX blend released about 55\% of loaded drug within the first 10 days with burst release. In vitro drug release kinetic studies suggested first-order drug release mechanism in a diffusion-controlled manner.

The work done displays the ability to design and fabricate dual-function porous scaffolds based on the biosorbable and biocompatible PCL. The sufficient mechanical properties, higher porosity, improved hydrophilicity and bulk degradation characteristics, along with long-lasting drug release properties suggest great potential applications for the obtained scaffolds in biomedical field and regenerative medicine. 


\section{CHAPTER 6*}

\section{Production and Recovery of Poly-3-hydroxybutyrate Bioplastics using Agro-Industrial residues of Hemp Hurd Biomass}

The present study describes production and recovery of Poly(3-hydroxybutyrate) $\mathrm{P}(3 \mathrm{HB})$ from agro-industrial residues. Production was conducted using Ralstonia eutropha strain with hemp hurd biomass hydrolysates sugars as a carbon source and ammonium chloride as the nitrogen source. Results show that maximum hydrolysis yield of $72.4 \%$ was achieved with total sugar hydrolysate concentration (i.e., glucose and xylose) of $53.0 \mathrm{~g} / \mathrm{L}$. Sugar metabolism by $R$. eutropha showed preference for glucose metabolism over xylose. Under optimum conditions, cells can accumulate $\mathrm{P}(3 \mathrm{HB})$ polymer in quantity up to $56.3 \mathrm{wt} \%$ of the dry cell weight. This corresponds to total production of $13.4 \mathrm{~g} / \mathrm{L}$ (productivity of $0.167 \mathrm{~g} / \mathrm{L} . \mathrm{h}$ ). Nitrogen source showed no adverse effect on $\mathrm{P}(3 \mathrm{HB})$ biosynthesis, but rather on cell growth. Among several examined recovery techniques, ultrasonic-assisted sodium dodecyl sulfate (SDS) recovered bioplastics directly from the broth cell concentrate with $\mathrm{P}(3 \mathrm{HB})$ content of $92 \%$. Number average molecular weights $\left(\mathrm{M}_{\mathrm{n}}\right)$ of final recovered bioplastics were in the range of 150-270 kDa with polydispersity index $\left(\mathrm{M}_{\mathrm{w}} / \mathrm{M}_{\mathrm{n}}\right)$ in the range of $2.1-2.4$.

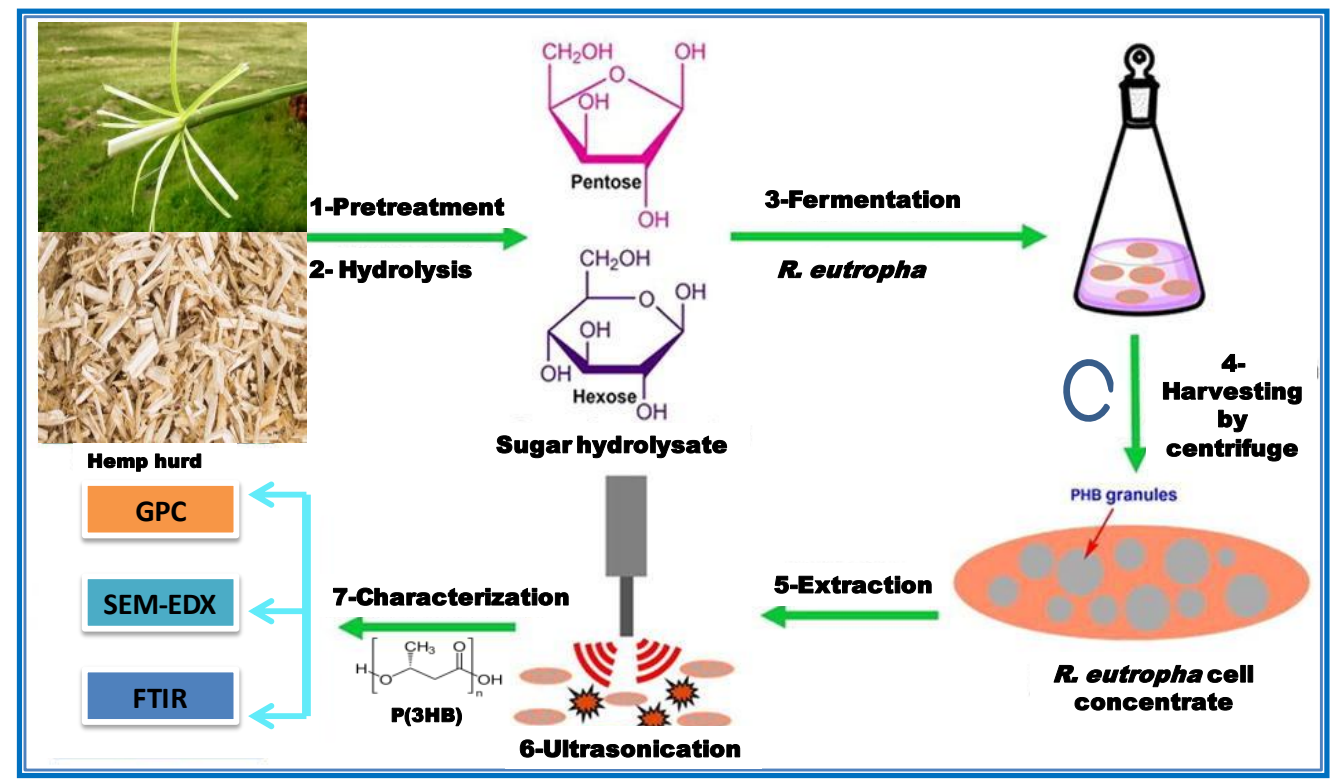

*This chapter is adapted from a manuscript accepted for publication by Bioprocess and Biosystems Engineering (BPBSE-18-0493). Mohamed M. Khattab, Yaser Dahman. Production and Recovery of Poly3-hydroxybutyrate Bioplastics using Agro-Industrial residues of Hemp Hurd Biomass. 


\subsection{Introduction}

Recent issues concerning the sustainable and harmless disposal of agrio-industerial solid wastes such as Canadian Hemp Hurd Biomass (HHB), have generated interest in microbial biotechnologies. These biotechnologies are focused on converting waste materials into useful biomaterials such as poly(3-hydroxybutyrate) $\mathrm{P}(3 \mathrm{HB})$, contributing to reduce the environmental footprint. By producing and using polyesters derived from biological processes, petrochemicalderived products can be replaced both partially and even completely. ${ }^{[425]} \mathrm{P}(3 \mathrm{HB})$, the best known member of the polyhydroxyalkanoate family (PHA), is an energy and/or carbon storing material synthesized and accumulated intracellulary by many microbial strains. It is characterized by its strength, hydrophobicity, inertness, relatively high melting point, optical purity and thermoplastic process ability similar to those of polypropylene. ${ }^{[157]}$ These properties have made $\mathrm{P}(3 \mathrm{HB})$ an attractive green alternative to synthetic plastics. In addition, low molecular weight $\mathrm{P}(3 \mathrm{HB})$ and its degradation product $\mathrm{D}(-)-3$-hydroxybutyrate are common metabolites detected in human plasma. ${ }^{[158]}$ This significant advantage of being completely biocompatible and biodegradable within the blood and tissue of mammals has further exploited the high-value of $\mathrm{P}(3 \mathrm{HB})$ for medical implants. $\mathrm{P}(3 \mathrm{HB})$ is now used for absorbable suture, scaffolds, heart valves and cardiovascular tissue supports. ${ }^{[38]}$ Enormous efforts and investigations have been undertaken by microbiologists, molecular biologists, chemical engineers, chemists, polymer experts, and medical researchers to reduce the production cost of $\mathrm{P}(3 \mathrm{HB})$ and to increase the industrial sustainability. ${ }^{[39]}$ However, much more work is still needed to reduce the productin cost of this environmentally friendly biodegradable plastic. Doing so will allow this product to be able to compete with petroleum-based plastics. Utilization of cheap and renewable carbon sources such as palm oil mill effluent, grass powder, rice waste biomass, waste glycerol, cassava starch hydrolysate, starch, organic waste, and fermentative effluents was reported by many researchers as a potential solution for cutting down the production cost of $\mathrm{P}(3 \mathrm{HB})$ to at least $25-40 \%$. $^{[40]}$ Most of the Western World banned the cultivation of Cannabis sativa (hemp fibers) in the early $20^{\text {th }}$ century because the biotypes are high in $\Delta^{9}$-tetrahydrocannabinol (THC). THC is the principal intoxicant cannabinoid and considered the source of marijuana. In 1990 dozens of countries including Canada have legalized the growth of "industrial hemp" (cultivars with quite low levels of THC) due to its potential source for oilseed crop. Since then industrial hemp has become an attractive biomass for biofuel and bioplastics production. ${ }^{[426]}$ However, this material 
is typically resistant to enzymatic hydrolysis and direct fermentation by microorganisms. ${ }^{\text {[427] }}$ This is due to the complex interactions of cellulose, lignin, hemicellulose, and pectin. ${ }^{[428]}$ Exploiting lignocellulosic materials and increasing its hydrolyzability by removal of lignin, solubilization of hemicelluloses and decrystallization of cellulose is a highly challenging process. Therefore, disassembling material and increasing accessible surface area for hydrolysis without losing the desired carbohydrates is considered the main purpose for the pretreatment process. ${ }^{\text {[29] }}$ Compared to acidic or oxidative pretreatment processes, alkaline pretreatment is the most effective pretreatment method for soft lignocelluloses material like hemp hurd. It is being processed at moderate temperatures and proven its ability to completely remove pectin and parts of lignin content rather than in hardwood. Yet, this enables better accessibility to carbohydrates by hydrolysis enzymes. ${ }^{[430]}$

Previous studies have clearly shown that the hydrolysis yields of untreated and pretreated hemp by commercial cellulases were found to increase upon adding a low dosage of pectinases and xylanase. The studies reported this was due to the synergistic actions between the three enzymes on the efficiency of hydrolysis. ${ }^{[428]}$ In addition, other studies have reported significant increase in the efficiency of hydrolysis by the commercial cellulase and cellobiase enzymes from $41.1 \%$ to $78.9 \%$. These results occurred after adding polyethylene glycol $\mathrm{M}_{\mathrm{n}}=4000\left(\mathrm{PEG}_{4000}\right)$ at $0.05 \mathrm{~g} / \mathrm{g}$ glucan (lignocellulosic substrate) during enzymatic hydrolysis. ${ }^{[428]}$

Chemical composition analysis of different agriculture biomasses, such as maize, faba bean, wheat straw and hemp fibers have shown a similarity in lignin content among them. However, considerable variation is prominent in the total content of carbohydrates. The highest carbohydrate content reported for hemp fibers was about $64 \%(\mathrm{w} / \mathrm{w})$ of the crop dry matter. ${ }^{[431]}$ Therefore, $\mathrm{HHB}$ can be considered a promising candidate for $\mathrm{P}(3 \mathrm{HB})$ production. Good selection of the microbial strains that can utilize this cheap carbohydrate biomass as a sole carbon source, can lead to more efficient and cost-effective $\mathrm{P}(3 \mathrm{HB})$ production. ${ }^{[40]}$

Ralstonia eutropha, also known as Cupriavidus necator, is among the bacterial strains that have proven to assimilate sugar carbons to $\mathrm{P}(3 \mathrm{HB})$. $R$. eutropha has been the most commonly used strain for the industrial production of $\mathrm{P}(3 \mathrm{HB})$ from the hydrolyzed lignocellulosic biomass, such as glucose, xylose, and arabinose. Thus, it accumulates $\mathrm{P}(3 \mathrm{HB})$ as discrete inclusions whenever they are exposed to stresses. ${ }^{[41]}$ These $\mathrm{P}(3 \mathrm{HB})$ granules (typically $0.2 \pm 0.5 \mu \mathrm{m}$ in diameter) are localized in the cell cytoplasm and can be visualized quite clearly with transmission electron 
microscopy. ${ }^{[159]}$ Maximum accumulation of $\mathrm{P}(3 \mathrm{HB})$ to the level of approximately $90 \%$ of its dry cell weight has been reported. ${ }^{[39]}$

In $R$. eutropha, microbial biosynthesis of $\mathrm{P}(3 \mathrm{HB})$ starts with the condensation of two molecules of acetyl-CoA to give acetoacetyl-CoA, which is subsequently reduced to hydroxybutyryl-CoA. This latter compound is then used as a monomer to polymerize $\mathrm{P}(3 \mathrm{HB}) \cdot{ }^{[159,160]}$ Studies using $R$. eutropha have shown that the intracellular degradation of $\mathrm{P}(3 \mathrm{HB})$ inclusions is a very slow process. The rate of degradation has been calculated to be about 10 times slower than the rate of its synthesis. ${ }^{[432]} \mathrm{P}(3 \mathrm{HB})$ accumulation occurs in response to nutrient limitations during the growth process. This explained the physiological role of $\mathrm{P}(3 \mathrm{HB})$ which is employed by microorganisms as a form of energy storage molecule to be metabolized when the complete range of nutrients required for cell division are not available but there is a generous supply of carbon. The biosynthesis of $\mathrm{P}(3 \mathrm{HB})$ was shown to be initiated by magnesium or sulfate deficiency. ${ }^{[162]}$ Another factor that can initiate the biosynthesis of $\mathrm{P}(3 \mathrm{HB})$ is nitrogen, phosphate ${ }^{[433]}$ and oxygen limitations. ${ }^{[434]}$ Previous studies also showed the quantity of $\mathrm{P}(3 \mathrm{HB})$ accumulated due to an increase in the carbon to nitrogen ratio. ${ }^{[164]}$

The aim of the present study is to analyze the ability of $R$. eutropha ATCC17699 to produce $\mathrm{P}(3 \mathrm{HB})$ using hemp hydrolysate sugars as a sole carbon source in separate hydrolysis and fermentation. Various pretreatment of hemp hurd raw material were examined to concentrate holocellulosic fraction in substrate as well as to open structure for subsequent enzymatic hydrolysis. Afterward, different scenarios for enzymatic hydrolysis in combination with ultrasonication were examined to maximize the total concentration of sugar yield in HHB hydrolysates. Following, the effects of different nitrogen sources and C:N ratios for maximizing $\mathrm{P}(3 \mathrm{HB})$ production from HHB hydrolysates was studied. Additionally, a comparison of the production process based on sugars mixture, mimicking composition in HHB hydrolysate was investigated. Composition analysis of hemp substrate and molecular weight determination of recovered $\mathrm{P}(3 \mathrm{HB})$ were determined using various analytical methods. 


\subsection{Materials and Methods}

\subsubsection{Materials and microbial strain}

Lignocellulosic substrate used in this study is the Canadian industrial hemp hurd biomass. It has grown in the region of Manitoba, and harvested at the end of August. The substrate is the woody inner portion of the hemp stalk, broken into pieces after separating bast fiber. $R$. eutropha (ATCC17699) was purchased from CEDARLANE, Canada. Cellulase enzyme from Trichoderma reesei (30 filter paper units FPU/g), Novozyme 188 produced by Aspergillus niger (cellobiase or $\beta$-glucosidase, $15 \beta$-glucosidase units $\mathrm{CBU} / \mathrm{g}$ ), and xylanase were all purchased from Sigma-Aldrich. D-Glucose, xylose, chloroform $\left(\mathrm{CDCl}_{3}\right)$, methanol, sodium hypochlorite $(\mathrm{NaOCl}), \mathrm{NaOH}, \mathrm{HCl}, \mathrm{H}_{2} \mathrm{SO}_{4}$, and $\mathrm{SDS}$ were procured from Sigma-Aldrich. The following nutrients for the growth and fermentation media were also produced from Sigma-Aldrich: beef extract, yeast extract, peptone, tryptone, sodium chloride, potassium dihydrogen phosphate $\left(\mathrm{KH}_{2} \mathrm{PO}_{4}\right)$, nickel chloride $\left(\mathrm{NiCl}_{2} \cdot 6 \mathrm{H}_{2} \mathrm{O}\right)$, manganese chloride $\left(\mathrm{MnCl}_{2} \cdot 4 \mathrm{H}_{2} \mathrm{O}\right)$, sodium molybdate $\left(\mathrm{NaMoO}_{4} \cdot 2 \mathrm{H}_{2} \mathrm{O}\right)$, ferric ammonium citrate (Fe(III)NH $\mathrm{NH}_{4}$-citrate), calcium chloride $\left(\mathrm{CaCl}_{2}\right)$, ammonium chloride $\left(\mathrm{NH}_{4} \mathrm{Cl}\right)$, ammonium nitrate $\left(\mathrm{NH}_{4} \mathrm{NO}_{3}\right)$, ammonium sulfate $\left(\mathrm{NH}_{4}\right)_{2} \mathrm{SO}_{4}$, urea $\left(\mathrm{N}_{2} \mathrm{H}_{4} \mathrm{CO}\right)$, boric acid $\left(\mathrm{H}_{3} \mathrm{BO}_{4}\right)$, cobalt chloride $\left(\mathrm{CoCl}_{2} \cdot 6 \mathrm{H}_{2} \mathrm{O}\right)$, cuprous chloride $\left(\mathrm{CuCl}_{2} \cdot 6 \mathrm{H}_{2} \mathrm{O}\right)$, zinc sulphate $\left(\mathrm{ZnSO}_{4} \cdot 7 \mathrm{H}_{2} \mathrm{O}\right)$, magnesium sulfate $\left(\mathrm{MgSO}_{4} \cdot 7 \mathrm{H}_{2} \mathrm{O}\right)$, and disodium hydrogen phosphate $\left(\mathrm{Na}_{2} \mathrm{HPO}_{4} \cdot 12 \mathrm{H}_{2} \mathrm{O}\right)$. Poly-3-hydroxybutyrate sample standard was used for comparison.

\subsubsection{Methods}

\subsubsection{Preparation and characterization of $\mathrm{HHB}$}

Sustainable and renewable biomass feedstock of HHB was initially washed with warm water and dried. It was then ground in a commercial blender to a maximum particle size of $1 \mathrm{~mm}$. Final stock was dried in oven at $70^{\circ} \mathrm{C}$ to constant weight, and then kept in desiccators at room temperature until use.

Composition of HHB dry matter (DM) was analyzed after drying the samples at $105^{\circ} \mathrm{C}$ until constant weight was obtained. The ash ratio was obtained from DM weight loss after pyrolysis at $550^{\circ} \mathrm{C}$ for $3 \mathrm{~h}$ using $1800 \mathrm{C}$ Quality muffin furnace. The percentage protein is estimated after measuring the elemental nitrogen content in the starting material (\% protein $=\% \mathrm{~N} \times 6.25)$, where $\% \mathrm{~N}$ was determined by Energy-dispersive X-ray spectroscopy (EDX) associated with 
(FE-SEM) microscope (FEI Quanta $200 \mathrm{~F}$, Netherlands) with accelerating voltage of 15-20 kV. Lignin and carbohydrates were analyzed in triplicate according to the methods of the National Renewable Energy Laboratory NREL-LAP protocols. ${ }^{[435,436]}$ In brief, acid hydrolysis is used to hydrolyze the biomass into monosaccharide. Acid insoluble lignin (Klason lignin) was gravimetrically determined as the residue of the hydrolysis, eventually containing some insoluble proteins. Acid soluble lignin was determined spectroscopically by measuring UV absorbance at $212 \mathrm{~nm}$ for the filtrate obtained from acid hydrolysis. Fat, wax, pectin and hemicellulose ratios were sequentially estimated by using the method of Vignon et al. ${ }^{[437]}$ The DM of hemp hurd was extracted in chloroform for $24 \mathrm{~h}$ to remove waxes and fats. The residual material was successively extracted twice for $5 \mathrm{~h}$ with boiling water, followed by $0.5 \% \mathrm{w} / \mathrm{w}$ ammonium oxalate solution $\left(100^{\circ} \mathrm{C}, 5 \mathrm{~h}\right)$, and then twice with $0.5 \%$ w/w ethylenediamine tetra acetic acid disodium salt (EDTA-2Na) at $\left(100^{\circ} \mathrm{C}, 5 \mathrm{~h}\right)$ to remove pectin. After pectin extraction, the samples were finally washed, dried, and the residual insoluble material was considered as the holocellulosic residue. The amount of holocellulosic residue was estimated from the previous measurements as shown in Equation (6.1).

Holocellulosic residue $=100 \%-\%$ (pectin + lignin + fats and waxes + ashes + proteins $)$

\subsubsection{Pre-treatment of $\mathrm{HHB}$}

Thermal pretreatment was performed in an autoclave (Sanyo MLS 3780) at $135^{\circ} \mathrm{C}$ and $0.24 \mathrm{MPa}$ for 1hour. Dry HHB samples were pretreated using different methods that include heating to $135^{\circ} \mathrm{C}$ for 60 minutes in water, in $2.0 \% \mathrm{v} / \mathrm{v}$ sulfuric acid solution, and in $2.0 \% \mathrm{w} / \mathrm{v} \mathrm{NaOH}$ solution. All pretreatments were carried out at biomass concentration of $0.1 \mathrm{~g} / \mathrm{ml}$. After pretreatments, biomass was recovered by filtration, washed with distilled water, and dried in oven at $55^{\circ} \mathrm{C}$ for $48 \mathrm{~h}$. It was then stored at $4^{\circ} \mathrm{C}$ for further enzymatic hydrolysis. The supernatant was kept at $-20^{\circ} \mathrm{C}$ until sugars concentrations analyses.

\subsubsection{Enzymatic hydrolysis}

Enzymatic hydrolysis of dry untreated (control) and pretreated HHB samples were performed at biomass concentration of $10.0 \%(\mathrm{w} / \mathrm{v})$ in $50 \mathrm{mM}$ citrate buffer $(\mathrm{pH} 4.8)$. Hydrolysis experiments were done in $1000 \mathrm{~mL}$ Erlenmeyer flask with total reaction volume of $500 \mathrm{~mL}$. For the one step hydrolysis study, the mixture was incubated for $72 \mathrm{~h}$ at $50^{\circ} \mathrm{C}$ stirred at $150 \mathrm{rpm}$ in presence of $0.005 \%(\mathrm{w} / \mathrm{v})$ sodium azide to avoid external microbial contamination. In case of two-step 
enzymatic hydrolysis, the samples were vacuum-filtered after completing the first step, and the solid fraction was re-suspended into $100 \mathrm{~mL}$ of $50 \mathrm{mM}$ citrate buffer and incubated with enzymes for another $36 \mathrm{~h}$. In all hydrolysis experiments, HHB was mixed with either two enzymes (cellulases and cellobiase) or three cellulose-degrading enzymes (cellulases, cellobiase and xylanase) in the presence and absence of $\mathrm{PEG}_{4000}$. In the case of using two-enzyme hydrolysis experiments; a mixture of cellulase and $\beta$-glucosidase was used in dosage of 3000 FPU/L and $1500 \mathrm{CBU} / \mathrm{L}$, respectively. While for the three enzymes mixture, $2 \mathrm{~g} / \mathrm{L}$ of xylanase was added to two enzymes mixture. However, in case of using of $\mathrm{PEG}_{4000}$ for improving enzymatic digestibility, mixtures were prepared by adding half of enzymes loading in addition to $5 \mathrm{~g} / \mathrm{L} \mathrm{PEG}_{4000} \cdot{ }^{[431]}$ In case of enzymatic hydrolysis that includes ultrasonication step, the enzymatic hydrolysis was achieved by pulsing samples at $(26 \mathrm{kHz}, 150 \mathrm{~W}$ for $15 \mathrm{~min})$, using Hielscher (UP200Ht) high intensity ultrasonic processor connected with $14 \mathrm{~mm}$ sonotrode, before the second enzymatic step. At the end, samples were immediately heated to $100^{\circ} \mathrm{C}$ for 15 min to deactivate enzymes, cooled, centrifuged for $15 \mathrm{~min}(4,000 \times \mathrm{g})$ and then stored at $-20^{\circ} \mathrm{C}$.

\subsubsection{Measuring sugar concentrations}

Sugar concentrations in hydrolysates were determined using a calibration curve that was constructed following 3,5-dinitrosalicylic acid (DNS) method. ${ }^{[438]}$ Calibration curves were done using UV-Vis spectrophotometry at $540 \mathrm{~nm}$ and using glucose as standard. Glucose and xylose sugar concentrations were determined by HPLC method detailed elsewhere. ${ }^{[439]}$ Perkin Elmer Series 200 pump auto sampler instrument was used with SH1011 column (Shodex) and refractive index detector. Samples were filtered through $0.2 \mu \mathrm{m}$ microfilters. The mobile phase was $10 \mathrm{mM} \mathrm{H}_{2} \mathrm{SO}_{4}$ at flow rate of $0.5 \mathrm{~mL} / \mathrm{min}$ and column oven temperature was maintained at $55^{\circ} \mathrm{C}$.

The combined single sugars that were released during the enzymatic hydrolysis are used to

calculate the hydrolysis yield according to Equation $(6.2) .{ }^{[440]}$ The factor 0.90 was used to convert polysaccharide to monosaccharide accounting for water uptake during hydrolysis.

$$
\text { Hydrolysis yield }(\%)=\frac{\text { single sugars }(\mathrm{mg}) \times 0.9 \times 100}{\text { cellulose content of substrate }(\mathrm{mg})}
$$




\subsubsection{P(3HB) fermentation and production}

For seed culture of $R$. eutropha, nutrient broth consisting of $3.0 \mathrm{~g} / \mathrm{L}$ beef extract and $5.0 \mathrm{~g} / \mathrm{L}$ peptone was used. Fermentation experiments were carried out in $250 \mathrm{~mL}$ shake flasks containing $100 \mathrm{~mL}$ of sterilized HHB hydrolysate (carbon source) mixed with mineral salt medium (MSM) with composition described elsewhere. ${ }^{[441]}$ All components of fermentation media were sterilized separately in an autoclave at $121^{\circ} \mathrm{C}$ for $30 \mathrm{~min}$. For fermentations, all flasks were aseptically inoculated with $5 \% \mathrm{v} / \mathrm{v}$ of vortexed inoculums. This inoculum was derived from $72 \mathrm{~h}$ incubated stock culture grown with MSM medium containing $20 \mathrm{~g} / \mathrm{L}$ glucose. The fermentation media were incubated at $30^{\circ} \mathrm{C}$ in a shaker at $200 \mathrm{rpm}$ for $96 \mathrm{~h}$. Production media was adjusted initially and during fermentation to $\mathrm{pH} 7$ by using $1 \mathrm{M} \mathrm{NaOH}$ and $0.5 \mathrm{M} \mathrm{HCl}$.

Several inorganic and organic nitrogen sources were examined in the present work. This includes $\mathrm{NH}_{4} \mathrm{Cl}, \mathrm{NH}_{4} \mathrm{NO}_{3},\left(\mathrm{NH}_{4}\right)_{2} \mathrm{SO}_{4}$, urea, yeast extract, beef extract, peptone and tryptone. The effect of each nitrogen source on cell growth and $\mathrm{P}(3 \mathrm{HB})$ production was investigated by testing each nitrogen source separately at fixed sugar concentration level. Furthermore, the effect of carbon to nitrogen ratio $(\mathrm{C}: \mathrm{N})$ on cell growth as well as $\mathrm{P}(3 \mathrm{HB})$ production was also examined. This was done by cultivating $R$. eutropha at various $\mathrm{NH}_{4} \mathrm{Cl}-\mathrm{N}$ contents from (3.75-18.75 g/L) at a fixed glucose level of 42.0 g/L (i.e., C:N ratio of 20:1, 20:2, 20:3, 20:4 and 20:5).

Fermentation broths were sampled periodically to monitor the change in total and single sugars concentration, $\mathrm{P}(3 \mathrm{HB})$ production, bacterial cell optical density $\left(\mathrm{OD}_{600}\right)$ and cell dry weights $(\mathrm{CDW}) . \mathrm{P}(3 \mathrm{HB})$ productions were determined gravimetrically. Cell growths were measured by quantifying optical density at $600 \mathrm{~nm}$ using UV-Vis spectrophotometer. CDW were determined gravimetrically by centrifuging $2 \mathrm{~mL}$ solutions at $(4000 \times \mathrm{g})$ for $15 \mathrm{~min}$ in pre-weighed Eppendorf tube, which were dried overnight at $75^{\circ} \mathrm{C}$ and cooled down to room temperature.

\subsubsection{6 $\mathrm{P}(3 \mathrm{HB})$ recovery}

Cell concentrates (cell and intracellular $\mathrm{P}(3 \mathrm{HB})$ product) were recovered from fermentation broth by centrifugation. It was then double washed with distilled water, and rinsed with chloroform. Following this step, four different methods were examined for recovering the intracellular $\mathrm{P}(3 \mathrm{HB})$ product. This includes solvent extraction with chloroform, ${ }^{[442]}$ digestion in sodium hypochlorite/chloroform solution, ${ }^{[443]}$ recovery by direct SDS-digestion, ${ }^{[444]}$ and disruption by ultrasonication. ${ }^{[445]}$ In the solvent extraction method, CDW of $5 \mathrm{~g}$ was dispersed in 
$200 \mathrm{ml}$ chloroform at ration of 1:40 (w/v). The solution was then sonicated for $10 \mathrm{~min}$ at a low power, and then boiled for $2 \mathrm{~h}$ at $70^{\circ} \mathrm{C}$. The cell residue was separated by filtration and $\mathrm{P}(3 \mathrm{HB})$ was then precipitated from filtrate by dropwise addition of solution into chilled methanol $(99.8 \%)$. White precipitate of the $\mathrm{P}(3 \mathrm{HB})$ polymer was collected and dried at $40^{\circ} \mathrm{C}$ in vacuum oven. In the product recovery method using digestion in sodium hypochlorite/chloroform solution, CDW of $10 \mathrm{~g}$ was dispersed in $200 \mathrm{~mL}$ of chloroform and $100 \mathrm{~mL}$ of sodium hypochlorite solution $(30 \% \mathrm{v} / \mathrm{v}, \mathrm{pH} 12.15)$. After incubation at $30^{\circ} \mathrm{C}$ for $3 \mathrm{~h}$, the dispersion was centrifuged, and the bottom chloroform phase was carefully collected. $\mathrm{P}(3 \mathrm{HB})$ was recovered by non-solvent precipitation and filtration method using $80 \%$ methanol. The resulting white precipitate was air-dried. Recovery of product by direct SDS-digestion was carried out in $100 \mathrm{ml}$ round-bottom flask with concentration ratio of $0.5(\mathrm{w} / \mathrm{w})$ of $\mathrm{SDS} / \mathrm{biomass}$ for $1 \mathrm{~h}$ at $30^{\circ} \mathrm{C}$. Then, the mixture was heated at $121^{\circ} \mathrm{C}$ for $15 \mathrm{~min}$ and then centrifuged at $4000 \times \mathrm{g}$ for $15 \mathrm{~min}$. The harvested solid sample containing $\mathrm{P}(3 \mathrm{HB})$ was washed with distilled water. The recovered $\mathrm{P}(3 \mathrm{HB})$ is dried at $60^{\circ} \mathrm{C}$ for $5 \mathrm{~h}$. Ultrasonic assisted SDS extraction was implemented as before with 10 min sonication before shaking. In ultrasonication method, $1 \mathrm{~g}$ of CDW was blended with $100 \mathrm{~mL}$ of hot chloroform in $250 \mathrm{~mL}$ round-bottom flask. After complete dispersion of cells in chloroform, the cells were disrupted by high intensity homogenizer Hielscher (UP200Ht) connected with $14 \mathrm{~mm}$ sonotrode. The amplitude and output power was kept at $26 \mathrm{kHz}$ and 160 $\mathrm{W}$, respectively for $30 \mathrm{~min}$. In order to prevent cell degradation during homogenization, the solution temperature was kept below $25^{\circ} \mathrm{C}$ using cold water jacket. After ultrasonication, the lighter density disrupted fragments was separated from $\mathrm{P}(3 \mathrm{HB})$ by centrifugation. The bioplastics polymer was obtained from chloroform solution as a film by evaporation or precipitated as white powder by using chilled methanol $(1: 9 \mathrm{v} / \mathrm{v}) . \mathrm{P}(3 \mathrm{HB})$ productions $(\mathrm{g} / \mathrm{L})$ were determined gravimetrically.

$\mathrm{P}(3 \mathrm{HB})$ accumulation was calculated as the percentage ratio of final product to initial CDW used for recovering intracellular product and evaluated by Equation (6.3).

$$
\mathrm{P}(3 \mathrm{HB}) \text { accumulation }(\%)=\frac{\text { Dry weight of extracted } \mathrm{P}(3 \mathrm{HB})(\mathrm{g} / \mathrm{L}) \times 100}{\operatorname{CDW}(\mathrm{g} / \mathrm{L})}
$$

$\mathrm{P}(3 \mathrm{HB})$ yield was expressed as the ratio of gram $\mathrm{P}(3 \mathrm{HB})$ produced per gram of total hydrolysate sugars consumed $\left(\mathrm{Y}_{\mathrm{P}(3 \mathrm{HB}) / \mathrm{S}}\right)$ or as the ratio of the ratio of gram $\mathrm{P}(3 \mathrm{HB})$ produced per gram of $\mathrm{DM}\left(\mathrm{Y}_{\mathrm{P}(3 \mathrm{HB}) / \mathrm{D}}\right)$. Productivity was expressed as $\mathrm{g}_{\mathrm{P}(3 \mathrm{HB})} / \mathrm{L} . \mathrm{h}$ and calculated by Equation (6.4). 


$$
\text { Productivity }(\mathrm{g} / \mathrm{L} \cdot \mathrm{h})=\frac{\text { Concentration of } \mathrm{P}(3 \mathrm{HB})(\mathrm{g} / \mathrm{L})}{\text { Fermentation time }(h)}
$$

\subsubsection{Analytical method and characterization of $\mathrm{P}(3 \mathrm{HB})$}

Composition, chemical structure, morphology and molecular weight of the produced $\mathrm{P}(3 \mathrm{HB})$ were confirmed using UV-Vis spectroscopy, Fourier Transform Infrared (FTIR), FE-SEM microscope and Gel Permeation Chromatography (GPC), respectively. Due to the presence of residues of other intracellular product mixed with the recovered bioplastics, ${ }^{[46]} \mathrm{P}(3 \mathrm{HB})$ content is described as the percentage of pure $\mathrm{P}(3 \mathrm{HB})$ that present among other non- $\mathrm{P}(3 \mathrm{HB})$ intracellular products. Percentage content of $\mathrm{P}(3 \mathrm{HB})$ was measured spectrophotometrically by the method of Law and Slepecky, ${ }^{[447]}$ that can only detect depolymerized $\mathrm{P}(3 \mathrm{HB})$ among other non-P(3HB) intracellular products recovered. It involves a comparison between UV absorbance of the cooled crotonic acid solutions at $235 \mathrm{~nm}$ that liberated from standard and extracted $\mathrm{P}(3 \mathrm{HB})$ after boiling each sample in concentrated sulfuric acid for $10 \mathrm{~min}$.

The functional groups and moieties present in the extracted $\mathrm{P}(3 \mathrm{HB})$ samples were determined by FTIR spectroscopy (using Perkin Elmer Spectrum1 FTIR/ATR spectrophotometer). Attenuated total reflection spectra (ATR) were recorded in the transmittance mode in the range of 4000-500 $\mathrm{cm}^{-1}$. ATR-crystal is $\mathrm{ZnSe}$, the spectral resolution is $4 \mathrm{~cm}^{-1}$ and 20 scans were collected for each spectrum.

The surface morphology and elemental composition of extracted gold-coated $\mathrm{P}(3 \mathrm{HB})$ film was examined by FE-SEM microscope with operating voltage of 15-20 kV. EDX attached with FESEM unit was used for elemental analyses.

Molecular weights of $\mathrm{P}(3 \mathrm{HB})$ using different recovery methods were determined by Gel Permeation Chromatography (GPC) using Viscotek GPC/SEC system. Molecular weight determination is accomplished through the use of Triple detector array (TDA 302) and OmniSec software. The triple detector consists of Right Angle Light Scattering (RALS), a high sensitivity Viscometer (for DP and IP), and a Refractive index detector (RI). HPLC grade chloroform was used as eluent with flow rate of $1.0 \mathrm{~mL} / \mathrm{min}$. Polymer samples were dissolved in chloroform at a concentration of $5 \mathrm{mg} / \mathrm{mL}$, and then filtered through $0.45 \mu \mathrm{m}$ filter. The internal column temperatures were kept at $25^{\circ} \mathrm{C}$. Weight and number average molecular weights of the recovered polymers $\left(\mathrm{M}_{\mathrm{w}}\right.$ and $\left.\mathrm{M}_{\mathrm{n}}\right)$ were determined after column calibration using polystyrene reference samples (GPC/SEC calibration kit; VWR) having narrow molecular weight distributions 


\subsection{Results and Discussion}

Results in Table 6.1 represent the chemical composition of unpretreated HHB (control) (i.e., holocellulose residue, pectins, lignin, proteins, ash, waxes and fats), as well as HHB samples after implementing different pretreatment processes. According to these results, untreated total solid of HHB contains 87\% DM fraction and 13\% water content. Approximately, 8\% of DM constitute waxes, fats, proteins and ash, $6 \%$ pectin, $18 \%$ lignin, and the remainder $67 \%$ of the $\mathrm{DM}$ is holocellulose residue (cellulose + hemicelluloses). The solid fractions obtained after different pretreatment processes are varied in holocellulosic content. They are also further utilized for enzymatic saccharification as potential source for fermentable sugars. Results in Table 6.1 show that alkaline pretreatment (i.e., $2 \% \mathrm{NaOH}$ ) at $135^{\circ} \mathrm{C}$ resulted in higher loss in biomass content reached $26 \%$ due to near complete removal of pectin and notable decrease in lignin content. This consequently concentrates holocellulose fraction in the raw material to $22 \%$ over untreated DM, $12.4 \%$ over acid pretreated DM and $18.4 \%$ over hydrothermal pretreated DM. In addition, the amount of wasted sugar observed in the supernatant of alkaline pretreated samples did not exceed $2 \%$ of the carbohydrate total mass. Although, the acid pretreated samples showed comparable weight loss of $19 \%$, it mostly related to the solubility of hemicelluloses where the wasted sugars observed in supernatant reached 17\%. This consequently causes decrease in the carbohydrate content of DM rather than delignification. Several researches have reported that the pretreatment with $1 \% \mathrm{NaOH}$ significantly increased the cellulose content of wheat straw in addition to high removal of lignin. ${ }^{[448]}$ However, Korte et al ${ }^{[449]}$ reported that pectin in hemp is strongly bound to lignin and forming disorganized matrix with cellulose, hemicelluloses and protein. Only strong pretreatment methods such as steam explosion and hot alkali treatment should be used to open structure. Therefore, the hot alkali pretreatment method proved its effectiveness for lignin and pectin removal in addition to keeping cellulose and hemicelluloses solid fraction intact and ready for the subsequent enzymatic hydrolysis. Figure 6.1 displays the effect of reducing substrate particle size on hydrolysis yield. Results showed that the amount of liberated sugars significantly increases when HHB particle size decreases. A hydrolysis yield of $\sim 61 \%$ was achieved after $72 \mathrm{~h}$, when particle sizes reduced to $1 \mathrm{~mm}$ range. Further decrease in particles size showed no significant effect on hydrolysis yield. In addition, the observed rate of hydrolysis is comparatively higher at the first $24 \mathrm{~h}$, and started to decrease after that. 
Table 6.1 Chemical composition of HHB expressed as \% w/w of the pretreated DM as means of triplicates

\begin{tabular}{|l|c|c|c|c|c|c|}
\hline \multirow{2}{*}{ Pretreatment methods } & Recovery & \multicolumn{4}{|c|}{ Components mass fraction (\%) $^{\mathbf{b}}$} \\
\cline { 3 - 6 } & $(\%)^{\mathbf{b}}$ & Holocellulose residue & Pectins & Lignin & Proteins & Ash \\
\hline Un-pretreated $\mathrm{HHB}$ (control) & 100 & $67.4 \pm 1.3$ & $6.1 \pm 0.6$ & $18.5 \pm 0.3$ & $3.1 \pm 0.2$ & $4 \pm 0.1$ \\
\hline $2 \% \mathrm{NaOH}\left(135^{\circ} \mathrm{C}, 1 \mathrm{~h}\right)$ & 74 & $89.0 \pm 1.5$ & $0.2 \pm 0.2$ & $4.0 \pm 0.4$ & $3.0 \pm 0.3$ & $3 \pm 0.1$ \\
\hline $2 \% \mathrm{H}_{2} \mathrm{SO}_{4}\left(135^{\circ} \mathrm{C}, 1 \mathrm{~h}\right)$ & 85 & $76.6 \pm 1.5$ & $3.0 \pm 0.1$ & $15.0 \pm 0.5$ & $3.0 \pm 0.0$ & $2 \pm 0.1$ \\
\hline Hydrothermal $\left(135^{\circ} \mathrm{C}, 1 \mathrm{~h}\right)$ & 94 & $70.6 \pm 1.2$ & $5.9 \pm 0.2$ & $16.5 \pm 0.5$ & $3.0 \pm 0.3$ & $3 \pm 0.1$ \\
\hline
\end{tabular}

${ }^{\mathrm{a}}$ Hemp hurd DM of $10 \mathrm{~g}$ used for carrying out pretreatment

${ }^{\mathrm{b}} \%$ Recovery and components mass fraction based on the $10 \mathrm{~g}$ of DM used

For all pretreatment methods, the combined waxes and fats mass fraction does not exceed $1 \pm 0.1 \%$. 


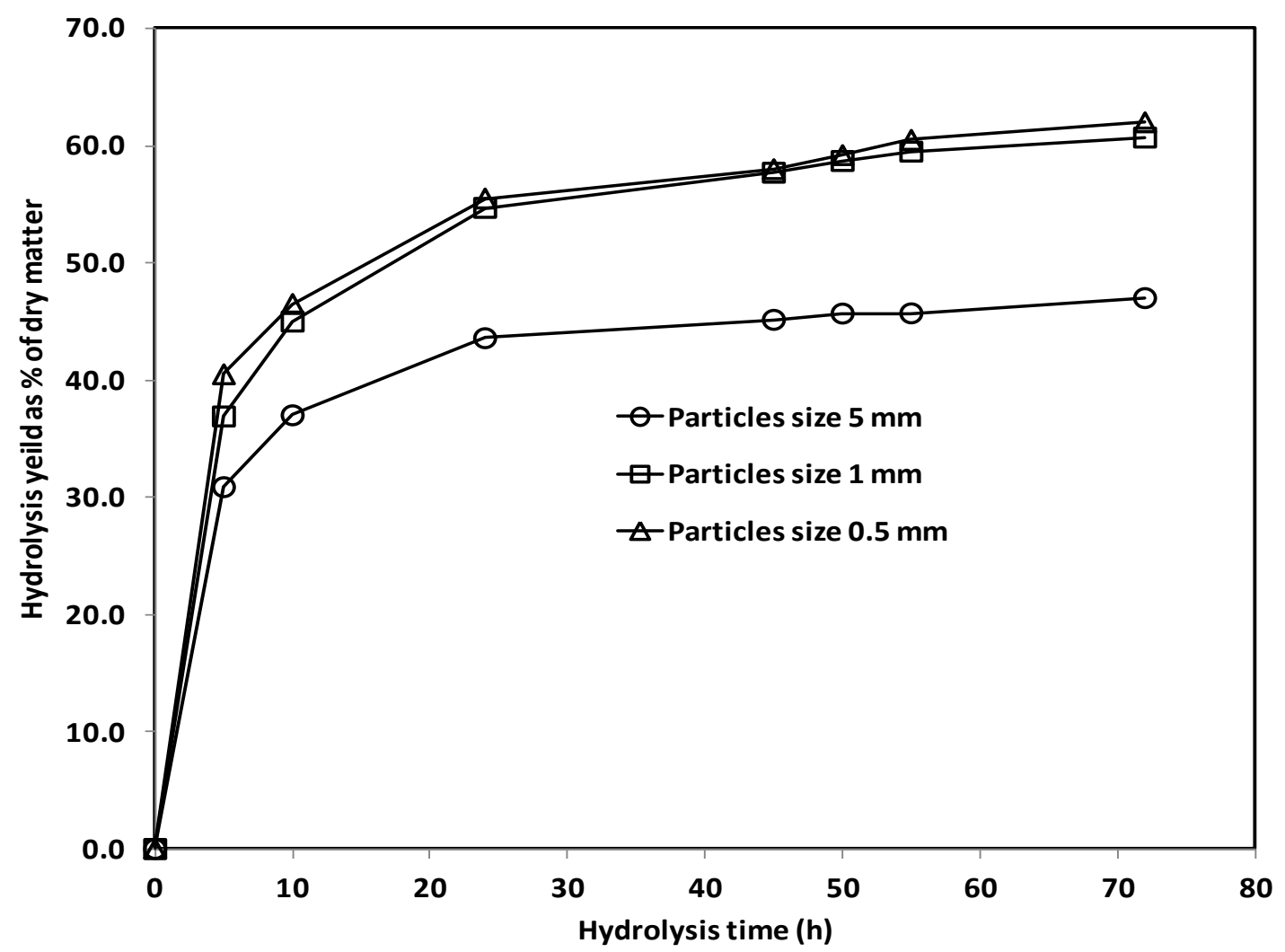

Figure 6.1 Hydrolysis yield of enzymatically hydrolyzed alkali pretreated HHB samples of different particle sizes; $0.5-5 \mathrm{~mm}$ expressed as \% of the total carbohydrate

Table 6.2 summarizes sugar concentrations and hydrolysis yields as obtained from the different pretreated HHB samples with different enzymatic hydrolysis methods. Results show that hydrolysis yield and total librated sugar concentration achieved by alkali pretreated HHB using one-step enzymatic hydrolysis with two-enzyme mixture were $43.7 \%$ and $32.0 \mathrm{~g} / \mathrm{L}$, respectively. This hydrolysis yield is $\sim 7$ times greater than untreated HHB (i.e., 6.1\%). Moreover, one-step enzymatic hydrolysis using the three enzymes showed hydrolysis yield equal to $54.7 \%$ with total sugar produced of $40.0 \mathrm{~g} / \mathrm{L}$. These values are higher than those obtained above using one-step enzymatic hydrolysis with the two-enzyme mixture. This is correlated to the efficiency of synergistic action of the major glucane and xylane enzymes (i.e., endoglucanases, cellobiohydrolases, $\beta$-glucosidase and xylanase) for hydrolyzing holocellulosic material. ${ }^{[428,450]}$ Results in Table 6.2 also demonstrates that hydrolysis yield of $47.8 \%$ with total sugar concentration of $35 \mathrm{~g} / \mathrm{L}$ can be achieved with half load of the tri-enzyme mixture together with 
0.05g $\mathrm{PEG}_{4000} / \mathrm{g}$ glucane. This may be related to the ability of PEG to make hydrophobic interaction and hydrogen bonding with lignin, which in turn could reduce the unproductive binding of enzymes. Though, the previous method provides an opportunity for using a lower amount of enzymes.

It was observed that the accumulation of higher sugar concentration released during the one-step enzymatic hydrolysis using tri-enzyme mixture causes feedback inhibition for enzymatic activity. Accordingly, a two-step enzymatic hydrolysis is proposed to uncouple enzymatic hydrolysis and liberated fermentable sugars. Up on using this method, the observed hydrolysis yield and total released sugars were increased and reached $62.8 \%$ and $46.0 \mathrm{~g} / \mathrm{L}$, respectively. This is corresponding to an increase in total sugar concentration over than one-step hydrolysis with the tri-enzyme mixture (i.e., $40 \mathrm{~g} / \mathrm{L}$ ); see Table 6.2. Moreover, a combination of an ultrasonication step in-between the two enzymatic hydrolysis steps were also performed. This has improved the hydrolysis yield and the total sugar concentration to $72.4 \%$ and $53.0 \mathrm{~g} / \mathrm{L}$, respectively. The physico-chemical effect of ultrasound and cavitations for enhancing the delignification process was previously reported, where the subsequent rate of the enzymatic hydrolysis can be increased by approximately $200 \% .^{[451]}$ Although the mode of action is not clearly investigated, it presumably due to the breakdown of hydrogen bonds of the crystalline cellulose structure by the enough energy generated from ultrasonication. The maximum total sugar concentration obtained (i.e., $53.0 \mathrm{~g} / \mathrm{L}$ ) is equivalent to sugar yield of $530 \mathrm{mg} / \mathrm{g}$ of dry HHB. This corresponds to $42.0 \mathrm{~g} / \mathrm{L}$ glucose and $11.5 \mathrm{~g} / \mathrm{L}$ xylose, respectively (from HPLC analysis). Figure 6.2 depicts the mass balance for alkali pretreated HHB based on two-step enzymatic hydrolysis and in combination with ultrasonication step In previous investigation, Zhang et al ${ }^{[452]}$ obtained $48.3 \mathrm{~g} / \mathrm{L}$ of glucose and $29.8 \mathrm{~g} / \mathrm{L}$ of xylose from alkaline pretreated oil palm empty fruit bunch hydrolysate with overall sugar concentration of $78.1 \mathrm{~g} / \mathrm{L}$ and corresponding to a sugar yield of $550 \mathrm{mg} / \mathrm{g}$. Annamalaia et al ${ }^{[453]}$ obtained overall sugar concentration of $62.9 \mathrm{~g} / \mathrm{L}$ from wheat bran corresponding to sugar yield of $629.1 \mathrm{mg} / \mathrm{g}$, and the sugars released were mainly composed of $48.4 \mathrm{~g} / \mathrm{L}$ glucose and $14.6 \mathrm{~g} / \mathrm{L}$ xylose. Contrarily, 12.6 $\mathrm{g} / \mathrm{L}$ of glucose and $5.0 \mathrm{~g} / \mathrm{L}$ of xylose was obtained by Cao et al ${ }^{[454]}$ from alkali pretreated sweet sorghum bagasse. 


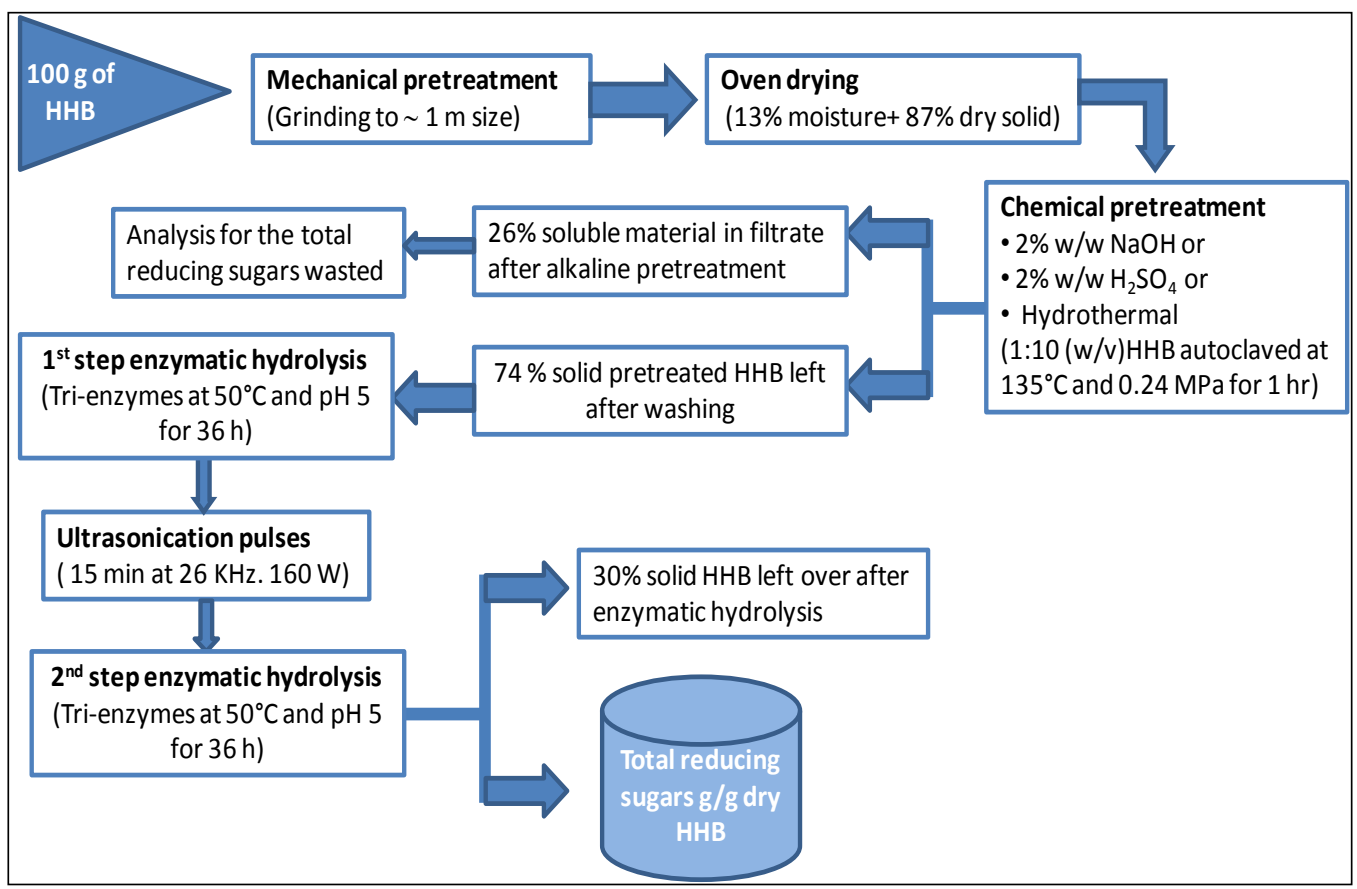

Figure 6.2 Mass balance for alkali pretreated HHB based on two-step enzymatic hydrolysis and in combination with ultrasonication step.

It was observed that for all applied glucose concentrations, growth was significantly delayed for $\sim 72$ h. Hence, $R$. eutropha is rapidly adapted to utilize glucose as growth substrate, by simply incubating the microorganism with high levels of glucose (i.e., $20 \mathrm{~g} / \mathrm{L}$ ) for a period of $\sim 72 \mathrm{~h}$ before starting fermentation. This initial incubation in the applied glucose concentration allows reducing the estimated lag-phase time span upon which glucose-utilizing strain will arise. ${ }^{\text {[455] }}$ Therefore, in this study, the onset of growth was correlated with the initial glucose concentration after the previously mentioned time span.

Figure 6.3a shows concentration profiles of sugar consumption in HHB hydrolysate and that present in glucose and xylose control experiments. Figure 6.3a demonstrates that consumption profiles of glucose were similar in the two cultures. This indicates that glucose was consumed at the same rate when it was used as single sugar feedstock or was a part of HHB hydrolysate sugars. In the presence of the two-sugar mixture of HHB hydrolysate (i.e., glucose and xylose), $R$. eutropha showed selectivity for glucose which it can assimilate first, and then xylose in a clearly distinct two-phase production steps. During the first phase, $\mathrm{P}(3 \mathrm{HB})$ production increased steadily until full consumption of glucose where an amount of $(11.7 \mathrm{~g} / \mathrm{L})$ was achieved after 72 h. In the second phase, $\mathrm{P}(3 \mathrm{HB})$ production continued to increase slightly slower and reached a 
maximum value of $13.4 \mathrm{~g} / \mathrm{L}$ after 80 hours of fermentation at full consumption of xylose; see Figures 6.3a,b. Xylose sugar appeared as an important nutrient as glucose, since its consumption was also complete and associated with more $\mathrm{P}(3 \mathrm{HB})$ production; (Figures 6.3a,b). Hence, the increased total sugar concentration that was obtained from HHB hydrolysate (i.e., 53.0 g/L) supported the higher $\mathrm{P}(3 \mathrm{HB})$ accumulation of $56.3 \%$. This corresponds to yield of about 0.134 $\mathrm{g}_{\mathrm{P}(3 \mathrm{HB})} / \mathrm{g}_{\mathrm{DM}}$ or $0.253 \mathrm{~g}_{\mathrm{P}(3 \mathrm{HB})} / \mathrm{g}_{\text {sugar }}$ and productivity of $0.167 \mathrm{~g}_{\mathrm{P}(3 \mathrm{HB}) / L}$.h. It worth to mention that after $80 \mathrm{~h}$ fermentation time, $\mathrm{P}(3 \mathrm{HB})$ production started to decrease and reached $13.1 \mathrm{~g} / \mathrm{L}$ after 110 h; see Figure 6.3b. This can be related to the existence of the intracellular PHA depolymerases in $R$. eutropha, which may be activated as a result of complete consumption of the carbon source. ${ }^{[456]}$

The maximum $\mathrm{P}(3 \mathrm{HB})$ production obtained in the present study (i.e., $13.4 \mathrm{~g} / \mathrm{L}$ ) is higher than those reported for $R$. eutropha ATCC17699 that were fed on different feedstock. P(3HB) production of $10.0 \mathrm{~g} / \mathrm{L}$ was reported with feedstock of wheat straw hydrolysate, ${ }^{[457]} 9.9 \mathrm{~g} / \mathrm{L}$ with rice paddy straw hydrolysate, ${ }^{[458]} 7.8 \mathrm{~g} / \mathrm{L}$ with emulsified palm oil, ${ }^{[459]}$ and $3.9 \mathrm{~g} / \mathrm{L}$ with bagasse hydrolysate. ${ }^{[460]} R$. eutropha MTCC 1472 fed on paddy straw hydrolysate achieved P(3HB) production of $5.2 \mathrm{~g} / \mathrm{L} \cdot{ }^{[461]}$ However, this is less than the production reported with mutant strain R. eutropha NCIMB 11599 fed on wheat bran hydrolysate (i.e., $14.8 \mathrm{~g} / \mathrm{L}){ }^{[462]}$

Production profiles for the simulated sugar culture in Figure 6.3b show that glucose culture produced $\mathrm{P}(3 \mathrm{HB})$ of $12.3 \mathrm{~g} / \mathrm{L}$ with calculated yield of $0.29 \mathrm{~g} \mathrm{P}(3 \mathrm{HB}) / \mathrm{g}$ glucose, whereas xylose

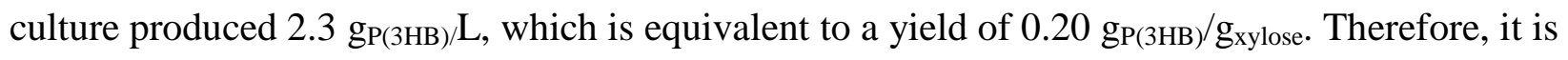
obvious that the combined total $\mathrm{P}(3 \mathrm{HB})$ produced by $R$. eutropha from the simulated synthetic individual sugars was $\sim 1.3 \mathrm{~g} / \mathrm{L}$ larger than that obtained using HHB hydrolysate sugars. This decreased production in case of hydrolysate mixed sugars substrate can be related to the induced degradation of some accumulated $\mathrm{P}(3 \mathrm{HB})$ after complete exhaustion of glucose, during the time taken by $R$. eutropha to resume the growth when switching from glucose to xylose toward $\mathrm{P}(3 \mathrm{HB})$ production. ${ }^{[455]}$ 
Table 6.2 Sugar concentrations and hydrolysis yields obtained from HHB hydrolysates after using different pretreatments process in combination with different scenarios of enzymatic hydrolysis

\begin{tabular}{|c|c|c|c|c|c|c|}
\hline \multirow{2}{*}{$\begin{array}{l}\text { Pretreatment and hydrolysis } \\
\text { methods }^{\mathrm{a}}\end{array}$} & \multirow{2}{*}{$\begin{array}{c}\text { Recovered } \\
\text { biomass }(\mathbf{g})^{\mathbf{b}}\end{array}$} & \multicolumn{2}{|c|}{ Total sugars } & \multirow{2}{*}{$\begin{array}{l}\text { Hydrolysis } \\
\text { yield }(\%)^{d}\end{array}$} & \multicolumn{2}{|c|}{ Single sugars $(\mathrm{g} / \mathrm{L})^{\mathrm{e}}$} \\
\hline & & $\mathbf{Y}(\mathrm{mg} / \mathrm{g})^{\mathrm{c}}$ & $(\mathrm{g} / \mathrm{L})$ & & Glucose & Xylose \\
\hline $\begin{array}{l}\text { Untreated HHB (control) + one step } \\
\text { enzymatic hydrolysis (two enzymes) }\end{array}$ & $92.5 \pm 1$ & 64 & 6.4 & 6.1 & 4.0 & 1.1 \\
\hline $\begin{array}{l}\text { Hydrothermal }\left(135^{\circ} \mathrm{C}, 1 \mathrm{~h}\right)+\text { one step } \\
\text { enzymatic hydrolysis (two enzymes) }\end{array}$ & $83.2 \pm 2$ & 110 & 11 & 22.4 & 7.0 & 2.0 \\
\hline $\begin{array}{l}2 \% \mathrm{H}_{2} \mathrm{SO}_{4}\left(135^{\circ} \mathrm{C}, 1 \mathrm{~h}\right)+\text { one step } \\
\text { enzymatic hydrolysis (two enzymes) }\end{array}$ & $68.8 \pm 0$ & 165 & 16.5 & 22.8 & 12.0 & 4.0 \\
\hline $\begin{array}{l}2 \% \mathrm{NaOH}\left(135^{\circ} \mathrm{C}, 1 \mathrm{~h}\right)+\text { one step } \\
\text { enzymatic hydrolysis (two enzymes) }\end{array}$ & $40.7 \pm 2$ & 320 & 32 & 43.7 & 22.0 & 8.0 \\
\hline $\begin{array}{l}2 \% \mathrm{NaOH}\left(135^{\circ} \mathrm{C}, 1 \mathrm{~h}\right)+\text { one step } \\
\text { enzymatic hydrolysis (tri-enzymes) }\end{array}$ & $32.3 \pm 1$ & 400 & 40 & 54.7 & 23.0 & 15.0 \\
\hline $\begin{array}{l}2 \% \mathrm{NaOH}\left(135^{\circ} \mathrm{C}, 1 \mathrm{~h}\right)+\text { one step } \\
\text { enzymatic hydrolysis (tri-enzymes) } \\
+\mathrm{PEG}_{4000}\end{array}$ & $36.6 \pm 2$ & 350 & 35 & 47.8 & 21.0 & 14.0 \\
\hline $\begin{array}{l}2 \% \mathrm{NaOH}\left(135^{\circ} \mathrm{C}, 1 \mathrm{~h}\right)+\text { ultrasonic } \\
\text { pulses }+ \text { one step enzymatic } \\
\text { hydrolysis (tri-enzymes) }\end{array}$ & $29.5 \pm 2$ & 430 & 43 & 58.8 & 33.0 & 10.0 \\
\hline $\begin{array}{l}2 \% \mathrm{NaOH}\left(135^{\circ} \mathrm{C}, 1 \mathrm{~h}\right)+\text { two step } \\
\text { enzymatic hydrolysis (tri-enzymes) }\end{array}$ & $17.6 \pm 2$ & 460 & 46 & 62.8 & 36.0 & 9.0 \\
\hline $\begin{array}{l}2 \% \mathrm{NaOH}\left(135^{\circ} \mathrm{C}, 1 \mathrm{~h}\right)+\text { two steps } \\
\text { enzymatic hydrolysis (tri-enzymes) } \\
+ \text { ultrasonic pulses }\end{array}$ & $19.8 \pm 2$ & 530 & 53 & 72.4 & 42.0 & 11.5 \\
\hline
\end{tabular}



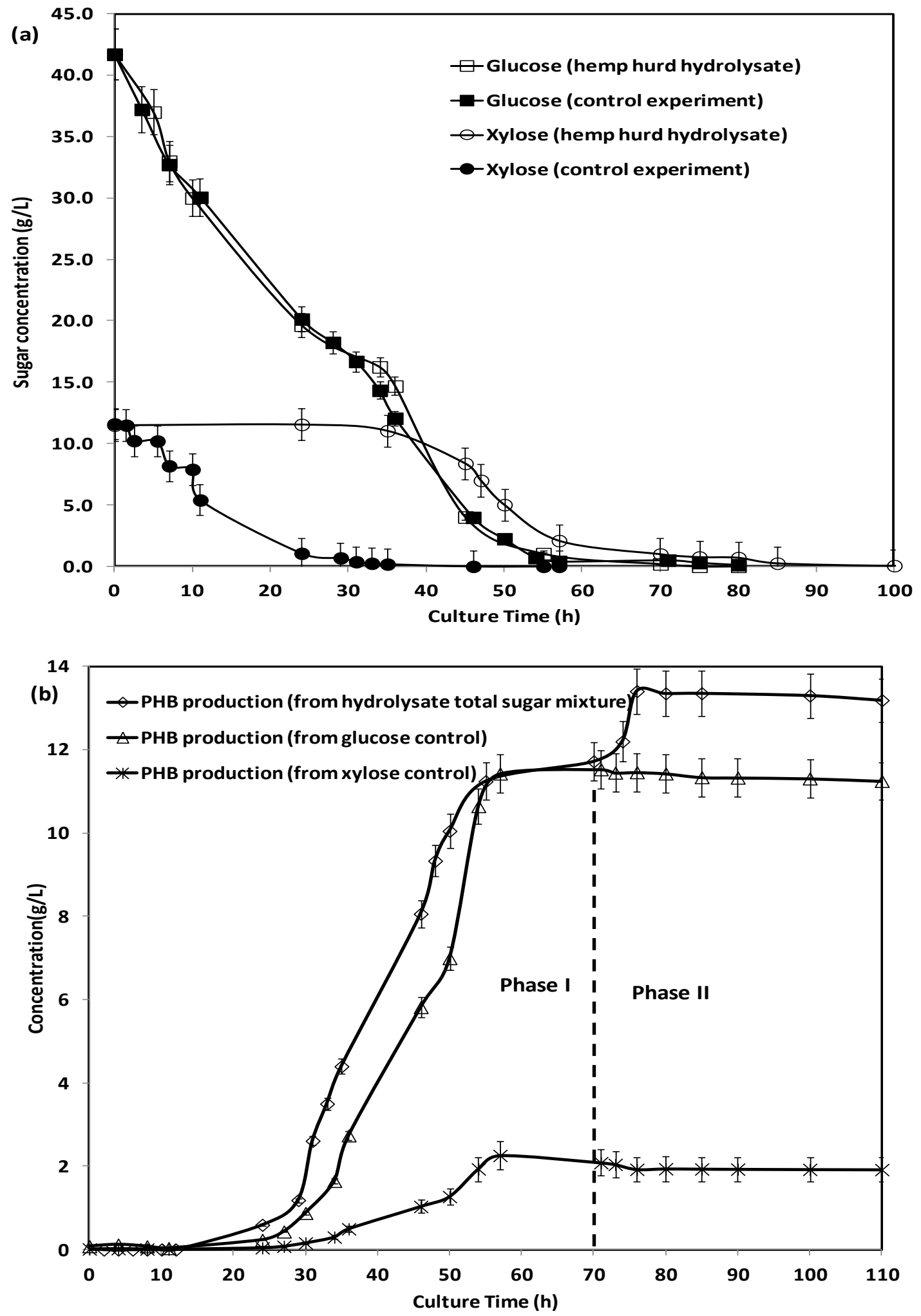

Figure 6.3 Concentration profiles showing sugars consumption (a) and PHB production (b) by $R$. eutropha feed on HHB hydrolysate in comparison with glucose and xylose control experiments. The results were presented in mean $\pm \mathrm{SE}, \mathrm{n}=3$ 
Figure 6.3a and $\mathrm{b}$ show the growth profiles of $R$. eutropha represented by the change in cell concentration $(\mathrm{CDW} ; \mathrm{g} / \mathrm{L})$ and cell density $\left(\mathrm{OD}_{600}\right)$ over fermentation time. Results in Figure 6.4 shows that both $\mathrm{CDW}$ and $\mathrm{OD}_{600}$ increased gradually over fermentation time. Maximum CDW reached was $23.8 \pm 0.3 \mathrm{~g} / \mathrm{L}$ while largest $\mathrm{OD}_{600}$ value obtained was 4.1 after $80 \mathrm{~h}$ of fermentation time. Furthermore, total CDW obtained from the combined glucose and xylose-controlled cultures was higher than that obtained from HHB hydrolysate. This is consistent with the increase in $\mathrm{P}(3 \mathrm{HB})$ production that was obtained from the combined glucose and xylosecontrolled cultures. The higher growth values achieved by $R$. eutropha with HHB hydrolysate without any detoxification demonstrate less fermentation inhibitors and water-soluble lignin were present in hydrolysate. This reflects the importance of the HHB as green feedstock that releases high concentration of fermentable sugars and less potential inhibitory effect on cell metabolism.

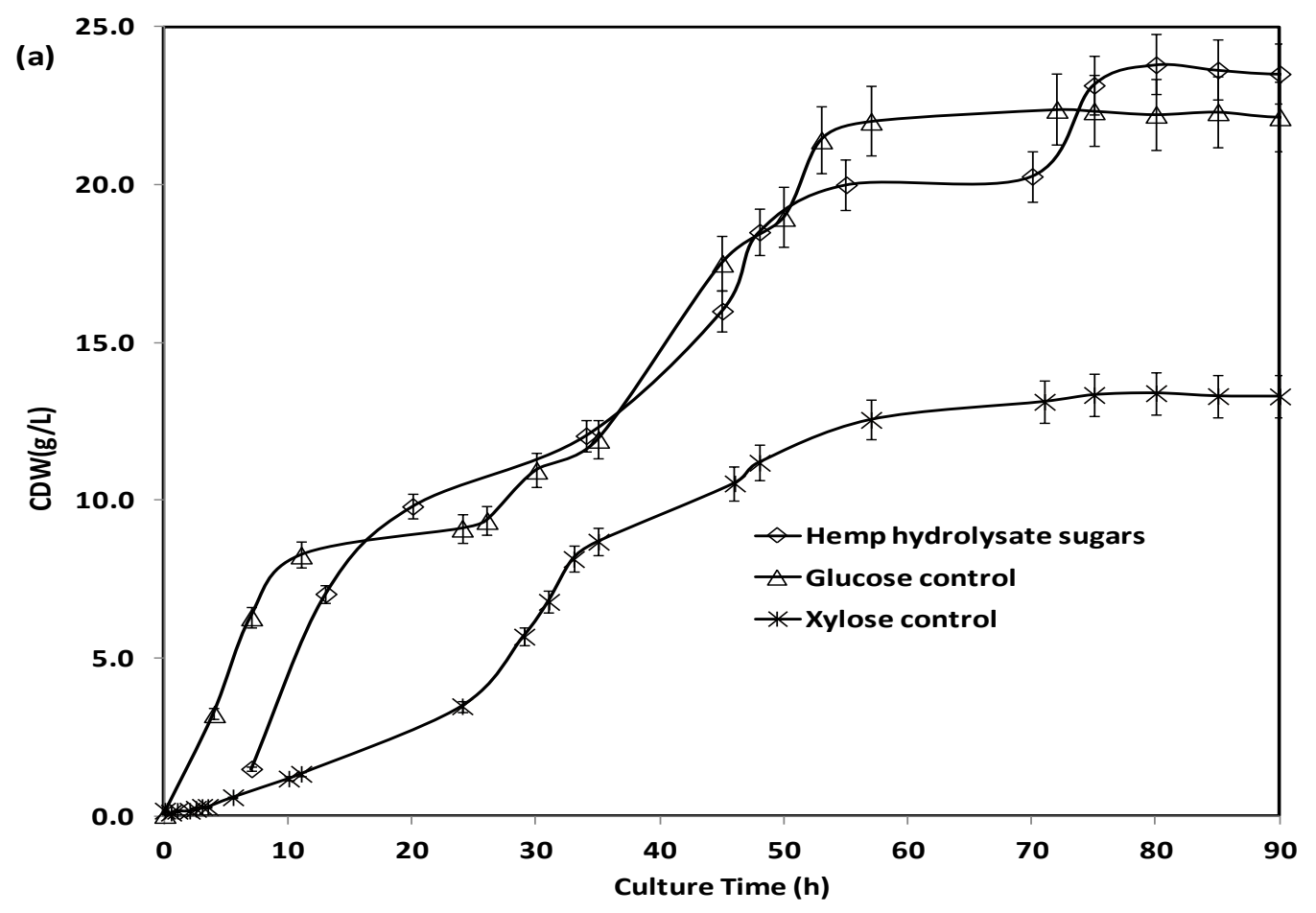




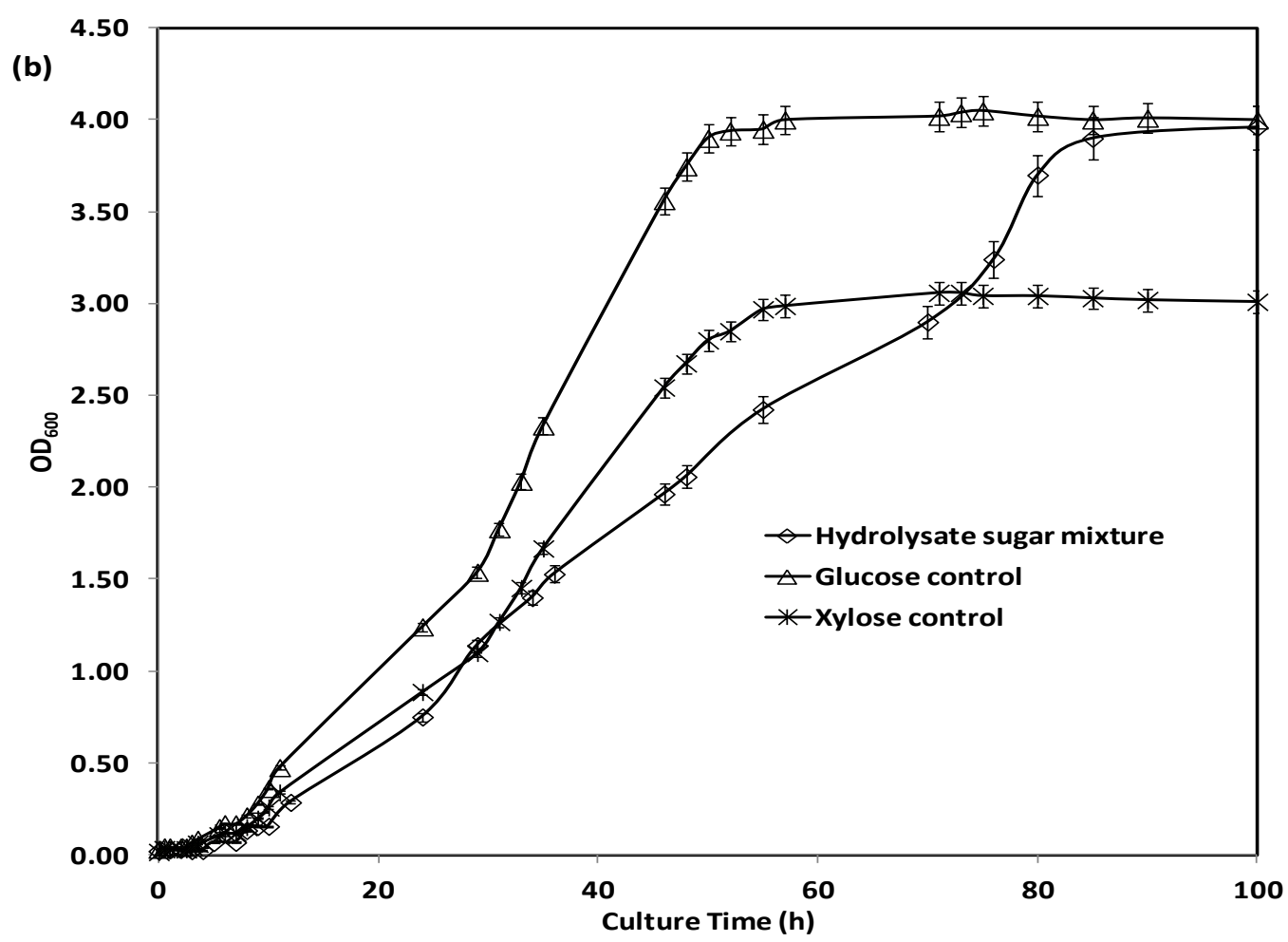

Figure 6.4 Accumulation of cell dry weight (a) and gradual increase in cell concentration (b) over fermentation time by $R$. eutropha feed on HHB hydrolysate sugars in comparison with the observed accumulation in control fermentation containing glucose and xylose simulated feedstock. The results were presented in mean $\pm \mathrm{SE}, \mathrm{n}=3$

Table 6.3 shows the effect of using various inorganic and organic nitrogen sources on cellgrowth, $\mathrm{P}(3 \mathrm{HB})$ production and yield of $R$. eutropha at same sugar level. Results in Table 6.3 demonstrate that the largest CDW obtained was 25.3 and $23.8 \mathrm{~g} / \mathrm{L}$ when using ammonium sulfate and ammonium chloride as nitrogen sources, respectively. Ammonium sulfate showed the largest $\mathrm{CDW}$, but the highest production, yield and accumulation of $\mathrm{P}(3 \mathrm{HB})$ (i.e., $13.4 \mathrm{~g} / \mathrm{L}, 0.253$ $\mathrm{g}_{\mathrm{P}(3 \mathrm{HB})} / \mathrm{g}_{\text {sugar }}$ and $56.3 \%$, respectively) were observed with ammonium chloride (see Table 6.3). This confirms that limitation of ammonium sulfate as sole nitrogen source supports cell growth rather than bioplastics accumulation. Accordingly, ammonium chloride is considered an appropriate choice as nitrogen source for fermentation and $\mathrm{P}(3 \mathrm{HB})$ production in the current study. This is in agreement with earlier reports where ammonium chloride was identified as the most suitable nitrogen source for bacterial growth and $\mathrm{P}(3 \mathrm{HB})$ production. ${ }^{[441]}$ However, other reports identified ammonium sulphate, ${ }^{[163]}$ ammonium nitrate ${ }^{[463]}$ and tryptone ${ }^{[450]}$ as the most suitable nitrogen sources. 
Table 6.3 CDW, $\mathrm{P}(3 \mathrm{HB})$ production and $\mathrm{P}(3 \mathrm{HB})$ accumulation by $R$. eutropha fed on various nitrogen sources and fixed total HHB hydrolysate sugar concentration of $53.0 \mathrm{~g} / \mathrm{L}$

\begin{tabular}{|l|c|c|c|c|}
\hline $\begin{array}{l}\text { Nitrogen } \\
\text { Sources }\end{array}$ & CDW (g/L) & $\begin{array}{c}\mathbf{P}(\mathbf{3 H B}) \text { production } \\
(\mathbf{g} / \mathbf{L})\end{array}$ & $\begin{array}{c}\mathbf{P}(3 \mathbf{3 H}) \\
\text { accumulation }(\%)\end{array}$ & $\begin{array}{c}\mathbf{P}(\mathbf{3 H B}) \text { yield } \\
\left(\mathbf{Y}_{\mathbf{P}(\mathbf{3 H}) / \mathbf{S}}\right)^{*}\end{array}$ \\
\hline $\mathrm{NH}_{4} \mathrm{Cl}$ & $23.8 \pm 0.3$ & $13.4 \pm 0.3$ & $56.3 \pm 1.4$ & $0.253 \pm 0.02$ \\
\hline $\mathrm{NH}_{4} \mathrm{NO}_{3}$ & $20.6 \pm 0.3$ & $10.0 \pm 0.3$ & $48.8 \pm 1.5$ & $0.189 \pm 0.01$ \\
\hline$\left(\mathrm{NH}_{4}\right)_{2} \mathrm{SO}_{4}$ & $25.3 \pm 0.2$ & $11.6 \pm 0.4$ & $45.8 \pm 1.5$ & $0.219 \pm 0.02$ \\
\hline Urea & $20.0 \pm 0.2$ & $9.4 \pm 0.2$ & $47.2 \pm 1.2$ & $0.177 \pm 0.03$ \\
\hline Yeast Extract & $19.3 \pm 0.1$ & $8.5 \pm 0.3$ & $44.1 \pm 1.4$ & $0.160 \pm 0.03$ \\
\hline Beef Extract & $18.6 \pm 0.1$ & $8.3 \pm 0.3$ & $44.5 \pm 1.1$ & $0.157 \pm 0.04$ \\
\hline Peptone & $20.0 \pm 0.3$ & $9.1 \pm 0.3$ & $45.5 \pm 1.2$ & $0.172 \pm 0.02$ \\
\hline Tryptone & $20.8 \pm 0.2$ & $9.6 \pm 0.3$ & $47.9 \pm 1.7$ & $0.181 \pm 0.01$ \\
\hline
\end{tabular}

P $(3 \mathrm{HB})$ yield $\left(\mathrm{Y}_{\mathrm{P}(3 \mathrm{HB}) / \mathrm{S}}\right)$ is gram of $\mathrm{P}(3 \mathrm{HB})$ produced from gram of total sugar.

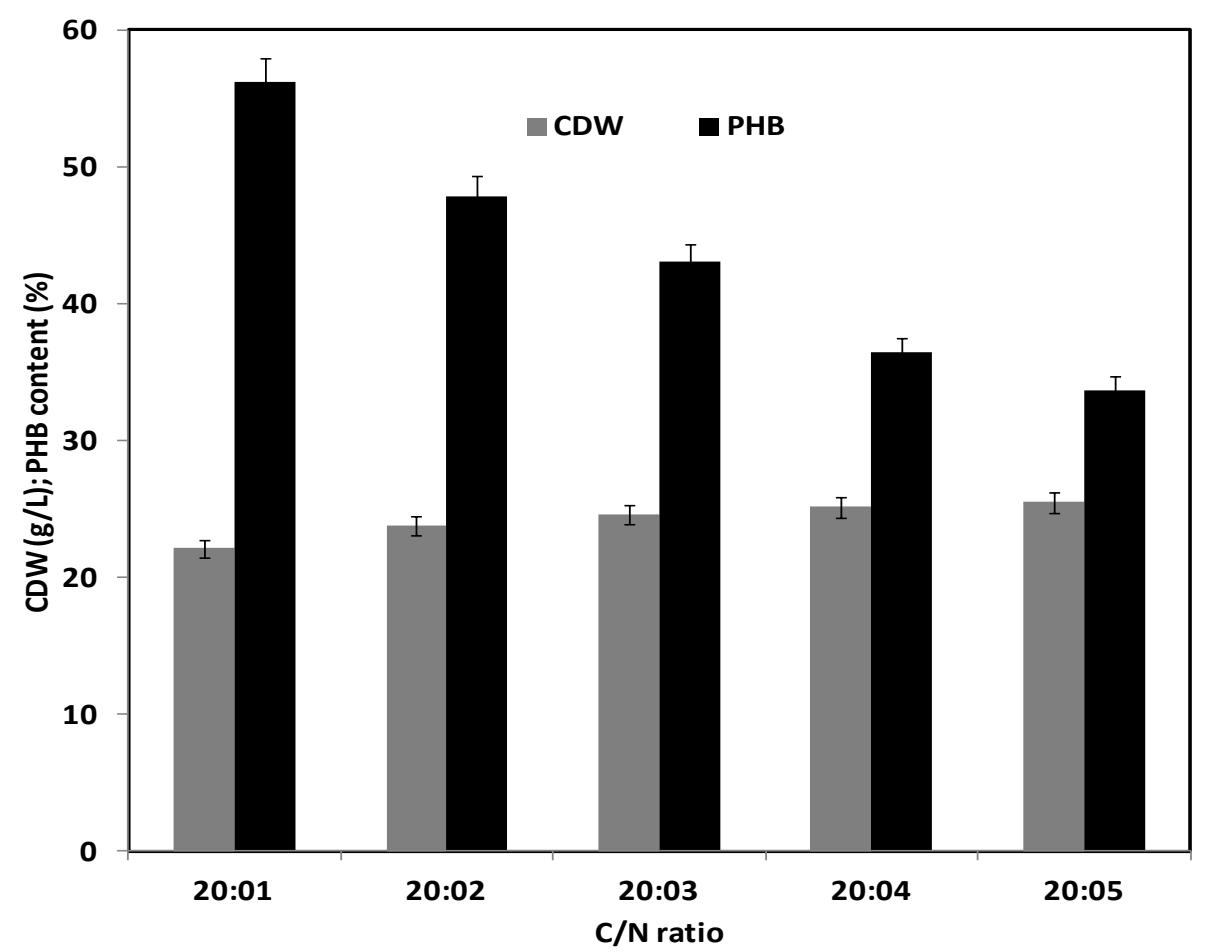

Figure 6.5 The effect of changing $\mathrm{C} / \mathrm{N}$ ratio on cell growth and $\mathrm{P}(3 \mathrm{HB})$ accumulation by R.eutropha 
In addition, several reports suggested that $\mathrm{C}: \mathrm{N}$ ratio plays an important role in $\mathrm{P}(3 \mathrm{HB})$ production but has minimal effect on cell growth. ${ }^{[435]}$ Table 6.4 shows results for the effect of $\mathrm{C}: \mathrm{N}$ ratio on $\mathrm{P}(3 \mathrm{HB})$ production. The investigation was carried out by varying $\mathrm{NH}_{4} \mathrm{Cl}-\mathrm{N}$ concentrations from $(3.75-18.75 \mathrm{~g} / \mathrm{L})$ at fixed glucose concentration of $42.0 \mathrm{~g} / \mathrm{L}$. This corresponds to C:N ratio range from 20:1 to 20:5. Results in Figure 6.4 present that when C:N ratio increased to 20:1, CDW was found to be minimum (i.e., $23.8 \mathrm{~g} / \mathrm{L}$ ) whereas $\mathrm{P}(3 \mathrm{HB})$ accumulation was found to be maximum (i.e., $56.3 \%$ ). Moreover, when $\mathrm{C}: \mathrm{N}$ ratio decreased to 20:5, P(3HB) accumulation reached a minimum value of $33.7 \%$, however, CDW reached a maximum value of $25.5 \mathrm{~g} / \mathrm{L}$. The previous results provide clear evidence that nitrogen limitation does not support cell growth, but it mostly favors $\mathrm{P}(3 \mathrm{HB})$ accumulation. Accordingly, C:N of (20:1) was used in this study as the optimal ratio for maximizing $\mathrm{P}(3 \mathrm{HB})$ production.

Table 6.4 Investigating $\mathrm{CDW}$ and $\mathrm{P}(3 \mathrm{HB})$ production and accumulation at different $\mathrm{C}: \mathrm{N}$ ratios utilizing glucose and $\mathrm{NH}_{4} \mathrm{Cl}$ as sole carbon and nitrogen sources

\begin{tabular}{|c|c|c|c|c|c|}
\hline $\begin{array}{c}\text { Glucose } \\
(\mathbf{g} / \mathbf{L})\end{array}$ & $\begin{array}{c}\mathbf{N H}_{\mathbf{4}} \mathbf{C l} \\
(\mathbf{g} / \mathbf{L})\end{array}$ & $\mathbf{C : N \text { ratio }}$ & $\mathbf{C D W}(\mathbf{g} / \mathbf{L})$ & $\begin{array}{c}\mathbf{P}(\mathbf{3 H B}) \\
\text { production }(\mathbf{g} / \mathbf{L})^{*}\end{array}$ & $\begin{array}{c}\mathbf{P}(\mathbf{3 H B}) \\
\text { accumulation }(\%)\end{array}$ \\
\hline 42 & 3.75 & $20: 1$ & $23.8 \pm 0.3$ & $13.4 \pm 0.2$ & $56.3 \pm 3$ \\
\hline 42 & 7.50 & $20: 2$ & $24.1 \pm 0.7$ & $11.4 \pm 0.5$ & $47.3 \pm 2$ \\
\hline 42 & 11.25 & $20: 3$ & $24.6 \pm 0.4$ & $10.6 \pm 0.2$ & $43.1 \pm 2$ \\
\hline 42 & 15.00 & $20: 4$ & $25.1 \pm 0.2$ & $9.2 \pm 1.0$ & $36.4 \pm 1$ \\
\hline 42 & 18.75 & $20: 5$ & $25.5 \pm 0.3$ & $8.6 \pm 0.5$ & $33.7 \pm 3$ \\
\hline 52 & 3.75 & $25: 1$ & $25.9 \pm 0.4$ & $12.6 \pm 0.6$ & $48.6 \pm 3$ \\
\hline
\end{tabular}

In all cases solvent extraction by chloroform was used as recovery technique

Figure 6.6 gives a comprehensive overview of changing ammonium chloride and glucose concentrations on $\mathrm{P}(3 \mathrm{HB})$ accumulation. At fixed ammonium chloride concentration of $3.75 \mathrm{~g} / \mathrm{L}$, an increase of glucose concentration to $52.0 \mathrm{~g} / \mathrm{L}$ (i.e., C:N ratio of 25:1) promoted biomass growth without significant effect on $\mathrm{P}(3 \mathrm{HB})$ production. This may be related to the effect of substrate inhibition at high initial concentrations on cell growth. ${ }^{[464]}$ 


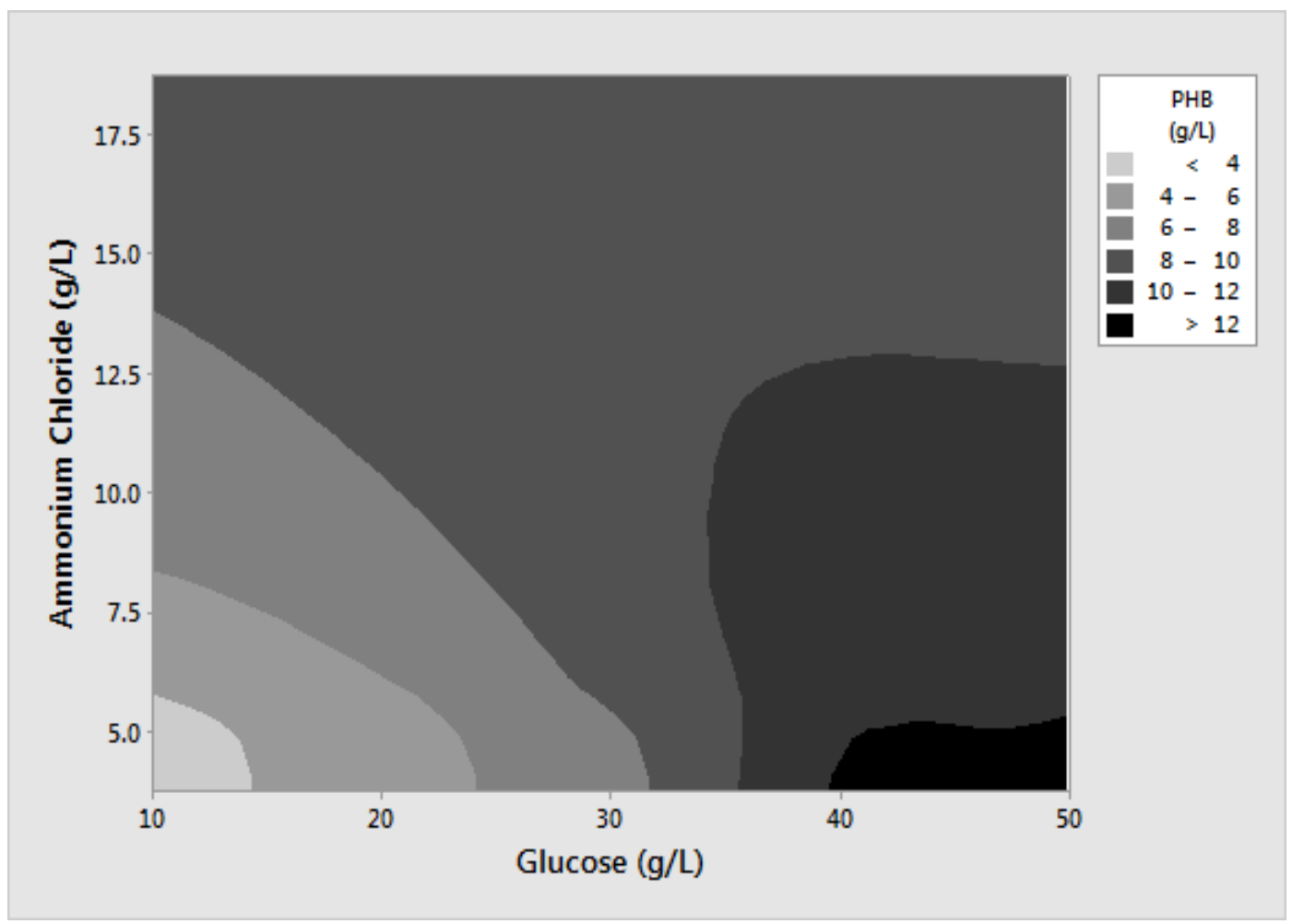

Figure 6.6 Contour plot showing the effect of changing ammonium chloride and glucose concentrations on $\mathrm{P}(3 \mathrm{HB})$ accumulation

Table 6.5 shows results for percentages contents and percentages recovery of $\mathrm{P}(3 \mathrm{HB})$ in bioplastics obtained by different recovery methods. Generally, all the recovery methods used in the current study showed near complete recovery of $\mathrm{P}(3 \mathrm{HB})$ from DCW (i.e., 92-97\%). Considering that highest recovery and degree of purity of $\mathrm{P}(3 \mathrm{HB})$ without polymer degradation are usually obtained by solvent extraction with chloroform. ${ }^{[442]}$ The recovery efficiency was evaluated with respect to the total polymer recovered by this method in the present study. Recovery method with digestion by sodium hypochlorite in chloroform achieved 91\% of polymer recovery with a higher $\mathrm{P}(3 \mathrm{HB})$ content of $95 \%$. Ultrasound-assisted extraction is another recovery method achieved the least percentage recovery of $89 \%$ along with $\mathrm{P}(3 \mathrm{HB})$ content of $93 \%$.

Furthermore, digestion of cells by the anionic surfactant SDS showed a recovery percentage of $90 \%$ with $\mathrm{P}(3 \mathrm{HB})$ content of $94 \%$. Compared to the previous method, ultrasonic-assisted SDS technique uses a lower surfactant dose of $3 \mathrm{wt} \%$ and showed higher recovery percentage of $94 \%$ but with lower $\mathrm{P}(3 \mathrm{HB})$ content of $92 \%$. 
In summary, solvent extraction method showed the highest recovery and $\mathrm{P}(3 \mathrm{HB})$ content. It does not cause obvious polymer degradation during the recovery, as revealed from the highest obtained polymer molecular weight (i.e., $\mathrm{M}_{\mathrm{n}}=271 \mathrm{kDa}$ ); see Table 6.5. This method has also proven ability to eliminate the endotoxin found in gram negative bacteria. ${ }^{[465]}$ Owing to this fact, $\mathrm{P}(3 \mathrm{HB})$ polymer obtained by this method can be useful for some medical applications. However, recovering $\mathrm{P}(3 \mathrm{HB})$ from cell debris in solution containing more than $5 \%(\mathrm{w} / \mathrm{v})$ can be difficult due to the high viscosity. Accordingly, about 20 parts of solvent is required to extract 1 part of polymer. ${ }^{[466]}$ This requires further solvent recovery and purification steps when applied on industrial scale. Hence, ultrasonic-assisted SDS technique is considered as reasonable alternative for solvent extraction method on the industrial scale as it achieves higher $\mathrm{P}(3 \mathrm{HB})$ content along with large molecular weight.

Table 6.5 also summarizes results for the polymer molecular weight and polydispersity index of $\mathrm{P}(3 \mathrm{HB})$ recovered by different techniques. Results show that $\mathrm{M}_{\mathrm{n}}$ of recovered $\mathrm{P}(3 \mathrm{HB})$ using solvent extraction method was the highest (i.e., $379 \mathrm{kDa}$ ) when glucose is used as sole carbon source. This is in good agreement with $\mathrm{M}_{\mathrm{n}}$ value of recovered $\mathrm{P}(3 \mathrm{HB})$ produced from glucose by R. eutropha (i.e., $400 \mathrm{kDa}$ ), where glucose is reported to promote polymer synthesis by $R$. eutropha [54]. However, $\mathrm{M}_{\mathrm{n}}$ value of recovered $\mathrm{P}(3 \mathrm{HB})$ produced from the metabolism of $\mathrm{HHB}$ hydrolysate sugars was equal to $271 \mathrm{kDa}$. The inclusion of another supplementary carbon sources (i.e., xylose in this study) during fermentation resulted in a significant decrease in the $M_{n}$ of the polymer produced (Table 6.5). The is in good agreement with the reported effect of supplementary carbon sources on reducing $\mathrm{M}_{\mathrm{n}}$ more $50 \%$ of that for $\mathrm{P}(3 \mathrm{HB})$ produced from glucose as sole carbon source. ${ }^{[467]}$ Nevertheless, $\mathrm{M}_{\mathrm{n}}$ values of recovered $\mathrm{P}(3 \mathrm{HP})$ polymer from $R$. eutropha grown on HHB hydrolysate sugars were in the range of 153-271 kDa, with polydispersity index $\left(\mathrm{M}_{\mathrm{w}} / \mathrm{M}_{\mathrm{n}}\right)$ in the range of 2.1-2.4. According to results in Table $6.5, \mathrm{M}_{\mathrm{n}}$ value of polymers recovered using solvent extraction method was the highest (i.e., $271 \mathrm{kDa})$. The decrease in $M_{n}$ values of the bioplastics obtained by the other recovery methods could be related to the polymer chain session that occurred as a result of the harsh chemicals or the physical method used during the recovery process. ${ }^{[468]}$ This was clearly observed with polymer samples recovered by $\mathrm{NaOCl} /$ chloroform digestion and disruption by ultrasonication method, see Table 6.5. Utilizing these two methods led to lower $M_{n}$ values of 153 and $155 \mathrm{kDa}$, respectively. This 
implies that solvent extraction with chloroform and ultrasonic-assisted SDS digestion methods are recommended when higher molecule weight is required.

Table 6.5 Comparison between $\mathrm{P}(3 \mathrm{HB})$ content, percentage of recovery, molecular weights and polydispersity of $\mathrm{P}(3 \mathrm{HB})$ polymer recovered by different methods

\begin{tabular}{|c|c|c|c|c|c|}
\hline \multirow{2}{*}{ Recovery Technique } & \multirow{2}{*}{$\begin{array}{c}\mathbf{P}(3 \mathrm{HB}) \\
\text { content }(\%)\end{array}$} & \multirow{2}{*}{$\begin{array}{l}\text { Recovery } \\
(\%)^{\mathrm{c}}\end{array}$} & \multicolumn{2}{|c|}{$\begin{array}{c}\text { Average Molar Mass } \\
(\mathbf{k D a})\end{array}$} & \multirow{2}{*}{$\begin{array}{c}\text { Polydispersity } \\
\left(\mathbf{M}_{w} / M_{n}\right)\end{array}$} \\
\hline & & & $\mathbf{M}_{\mathbf{w}}$ & $\mathbf{M}_{\mathbf{n}}$ & \\
\hline Solvent extraction $^{\mathrm{a}}$ & ND & ND & 910 & 379 & 2.4 \\
\hline Solvent extraction ${ }^{\mathrm{b}}$ & ND & ND & 469 & 213 & 2.2 \\
\hline Solvent extraction & 97 & 100 & 569 & 271 & 2.1 \\
\hline $\begin{array}{l}\mathrm{NaOCl} / \text { chloroform } \\
\text { digestion }\end{array}$ & 95 & 91 & 352 & 153 & 2.3 \\
\hline Direct digestion with SDS & 94 & 90 & 531 & 241 & 2.2 \\
\hline $\begin{array}{l}\text { Disruption by } \\
\text { ultrasonication }\end{array}$ & 93 & 89 & 372 & 155 & 2.4 \\
\hline $\begin{array}{l}\text { Ultrasonic-assisted SDS } \\
\text { digestion }\end{array}$ & 92 & 94 & 561 & 244 & 2.3 \\
\hline
\end{tabular}

All recovery experiments were done on $\mathrm{P}(3 \mathrm{HP})$ obtained from $R$. eutropha utilized HHB hydrolysates sugars. Except; ${ }^{a}$ Glucose was used as sole carbon source; ${ }^{b}$ Xylose was used as sole carbon source.

${ }^{\mathrm{c}}$ Determined based on assuming $100 \%$ recovery for solvent extraction with chloroform ND: not determined.

FTIR spectra of $\mathrm{P}(3 \mathrm{HB})$ obtained by SHF from microbial fermentation is shown in Figure 6.7. The characteristic band at 2850 to $2965 \mathrm{~cm}^{-1}$ shows the presence of antisymmetric and symmetric stretching of $-\mathrm{CH}$ (alkanes) bonding in $-\mathrm{CH}_{3}$ and $-\mathrm{CH}_{2}$ groups. The band at 1718 $\mathrm{cm}^{-1}$ represents the $\mathrm{C}=\mathrm{O}$ (carbonyl) of $-\mathrm{COO}$ (ester) group. The band found at $1452 \mathrm{~cm}^{-1}$ corresponds to the asymmetrical deformation of the $\mathrm{C}-\mathrm{H}$ bond in $-\mathrm{CH}_{2}$ groups, while the one found at $1379 \mathrm{~cm}^{-1}$ is the equivalent for $-\mathrm{CH}_{3}$ groups. The band observed at 1053 to $1259 \mathrm{~cm}^{-1}$ corresponds to the $\mathrm{C}-\mathrm{O}$ bonding. Comparison between the band assignments of various peaks in FTIR spectra for the obtained $\mathrm{P}(3 \mathrm{HB})$ samples by microbial fermentation and the standard $\mathrm{P}(3 \mathrm{HB})$ confirmed identical chemical structure of and the bioplastics produced (Figure 6.7). The bands of the above PHB samples closely match with the results of standard PHB. Moreover, all 
these bands are in full agreement with those reported by Getachew et al. ${ }^{[469]}$ Thus, the FTIR results showed not only closely match with the results of standard $\mathrm{P}(3 \mathrm{HB})$ but also substantial degree of purity in terms of peaks assignment for $\mathrm{P}(3 \mathrm{HB})$ obtained after extraction.

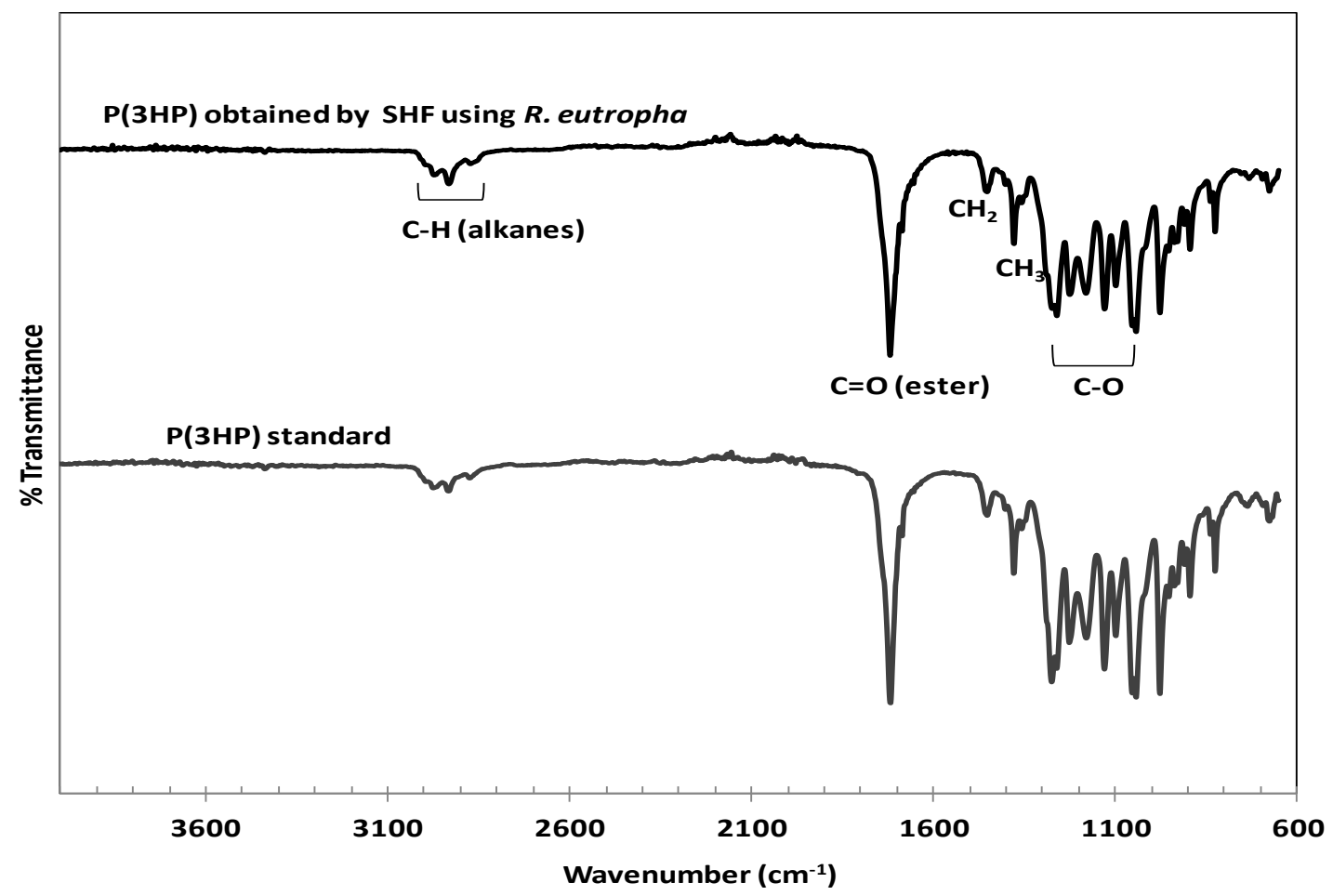

Figure 6.7 FTIR characterization of $\mathrm{P}(3 \mathrm{HB})$ produced from microbial fermentation by $\mathrm{R}$. eutropha utilizing hemp sugars hydrolysate

Figure 6.8 shows elemental composition and surface morphology of solvent-extracted $\mathrm{P}(3 \mathrm{HB})$ analyzed by FE-SEM. SEM image in Figure 6.8b shows the granular structure surface morphology of extracted bioplastics film. Moreover, EDX spectrum in Figure 6.8a shows that the weight percentage of carbon and oxygen content in the extracted $\mathrm{P}(3 \mathrm{HB})$ film were equal to $61.4 \%$ and $38.15 \%$, respectively. These results are in agreement with values theoretically calculated from the chemical formula of the polymer for both carbon and oxygen contents (i.e., $62.92 \%$ and $36.73 \%$, respectively). This confirms the composition of produced and recovered $\mathrm{P}(3 \mathrm{HB})$ polymer and promotes its higher purity as well. 
(a)

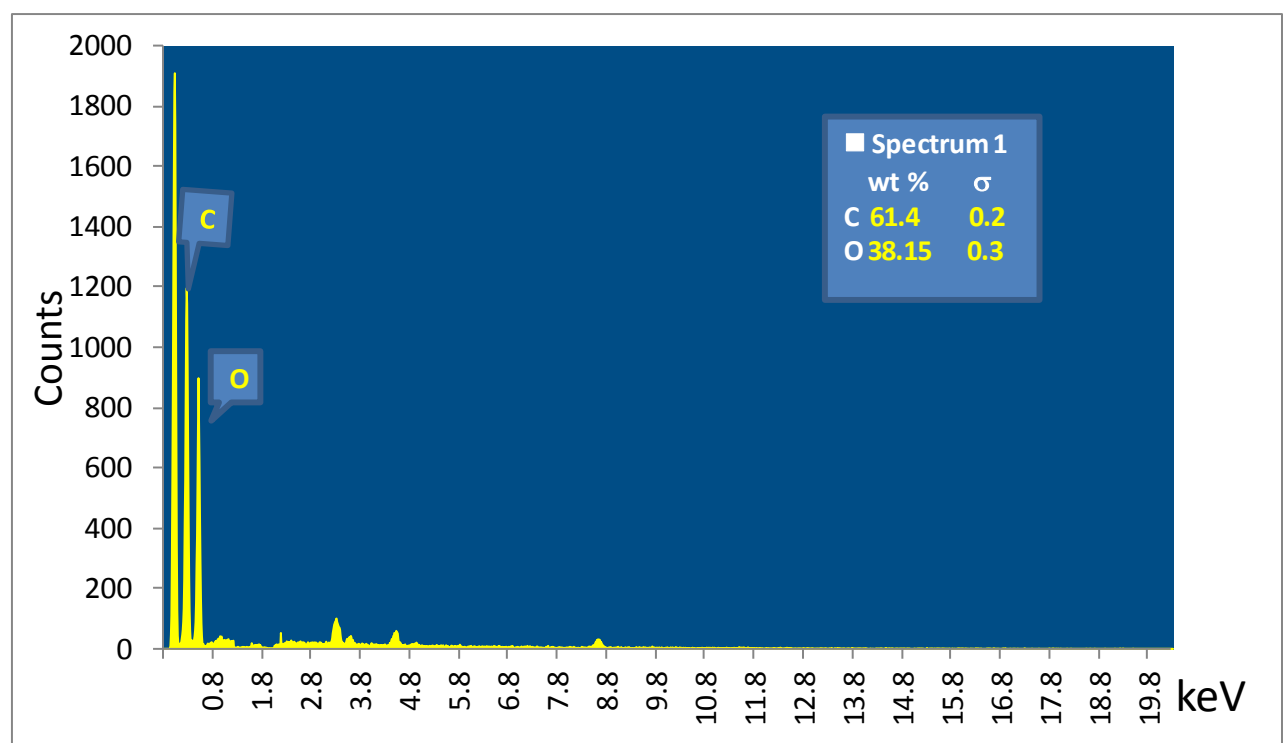

(b)

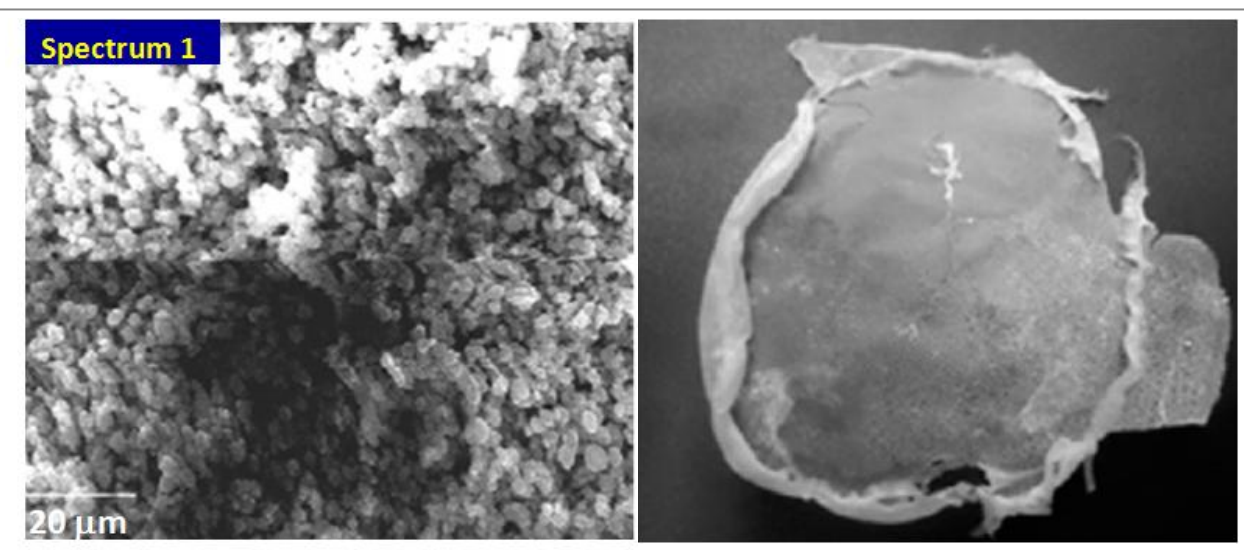

(c)

Figure 6.8 EDX spectra (a), SEM-images (b) and photograph of extracted $\mathrm{P}(3 \mathrm{HB})$ film (c) produced by R. eutropha feed on HHB hydrolysates

\subsection{Conclusion}

Poly(3-hydroxybutyrate) $\mathrm{P}(3 \mathrm{HB})$ is a biodegradable, biocompatible, and non-toxic polymer, which stands out as replacement for fossils-derived plastics. It is postulated that availability of substrate, operation and bioreactor control and the downstream processing make this polymer commercially competitive against fossils-derived plastics. In the present study, production of $\mathrm{P}(3 \mathrm{HB})$ using renewable and sustainable resources of green hemp, as an interesting alternative for reducing the production cost, was examined. Results for the different scenarios of pretreatment and hydrolysis of raw biomass showed that hot alkali pretreatment of biomass in combination with ultrasonic assisted two-step enzymatic hydrolysis released the highest sugar concentration from biomass with sugar yield of $530 \mathrm{mg} / \mathrm{g}$. 
Under optimum carbon and $\mathrm{NH}_{4} \mathrm{Cl}-\mathrm{N}$ concentration of 20:1 ratio, the highest $\mathrm{P}(3 \mathrm{HB})$ production of $13.4 \mathrm{~g} / \mathrm{L}$ was achieved. Ultrasonic assisted SDS-digestion method has proven its effectiveness as competent economic recovery method for bioplastics. It could recover $94 \%$ of $\mathrm{P}(3 \mathrm{HB})$ directly from broth cell concentrate with bioplastics content of $92 \%$. Compared to other literature values, the current study demonstrated a potential process for cost-effective microbial synthesis of $\mathrm{P}(3 \mathrm{HB})$ and can be easily scaled up for industrial production. A hyper production of PHB by using genetically modified bacterial strain is under progress by our research group. 


\section{CHAPTER 7}

\section{Original contributions and recommendations}

\subsection{Original contributions to research}

The objective of this thesis largely involved the development of novel functional materials based on BCNW by considering its attractive sustainability, biocompatibility and excellent thermal and mechanical properties. The goal is to utilize the synergistic properties of BCNW for obtaining functional nanomaterial that could assist in biomedical applications; one time as drug nanocarrier and other time as reinforcing nanophase for developing the bulk characteristics of PCL matrix. The thesis includes the synthesis and characterization of two types of functionalized nanocellulose-based materials: (i) BCNW- $g-\beta C D-D r u g ; ~(i i) ~ B C N W-g-\beta C D-P C L 2000$ and preparation techniques and characterization of two different bionanocomposite scaffolds based on PCL: (i) PCL/BCNW-g- $\beta C D-\mathrm{PCL}_{2000}$; (ii) PCL/BCNW- $g-\beta C D-\mathrm{PCL}_{2000} / \mathrm{DOX}$. In addition, different methodologies were also investigated for the preparation and recovery of $\mathrm{P}(3 \mathrm{HP})$ bioplastics from sustainable feedstock of agro-industrial residue of hemp hurd biomass. The following sections summarize the main findings of each part:

\subsubsection{Development of BCNW-g-BCD-Drug as a drug nanocarrier}

A green nanosized and long lasting drug nanocarrier systems based on the biocompatible BCNW was developed by grafting of $\beta C D$ to the surface of nanowhisker. This developed nanocarrier able to spontaneously load and release significant quantities of antibiotics and anticancer drugs without significant burst releases. Different scenarios for controlled acidic and enzymatic hydrolysis were examined to resolve the aggregation problem of BCNW for better functionalization process. In addition, different linkers and reaction conditions were examined to achieve the highest grafting ration for $\beta C D$ on nanowhisker surface. The later resulted in functionalized nanomaterial with unique properties and enhanced performance in the fields of drug delivery.

\subsubsection{Development of reinforcing nanowhisker $B C N W-g-\beta C D-P C L_{2000}$}

Sustainable and biocompatible reinforcing nanophase based on BCNW (i.e., BCNW-g- $\beta C D-$ $\mathrm{PCL}_{2000}$ ) was developed by grafting nanowhisker with $\beta C D$ and then conjugating low molecular weight segments of $\mathrm{PCL}_{2000}$ to its surface. The assembly of hydrophobic polymer segments on 
nanowhisker surface is a smart solution for tuning its surface energy and enhancing the dispersion of the hydrophilic cellulose nanowhisker in hydrophobic polymer matrix.

\subsubsection{Development of PCL/BCNW-g- $\beta C D-P C L_{2000}$ bionanocomposite for TE applications}

Incorporation of $4 \mathrm{wt} \%$ of $\mathrm{BCNW}-g-\beta C D-\mathrm{PCL}_{2000}$ into PCL matrix resulted in bionanocomposite with improved bulk characteristics. Compared to neat PCL, the obtained PCL-bionanocomposite showed improved mechanical and thermal properties, as well as promoted hydrophilicity and in vitro degradation rate. In addition, porous structures having porosities of $86-95 \%$ with mean pore diameters of $250-420 \mu \mathrm{m}$ along with unique interconnected pore-network were developed using gas foaming/combined particulate leaching technique. The obtained scaffolds showed compression moduli values in the range of cancellous bones and could be ideal for cell seeding and proliferation. The obtained scaffolds' characteristics suggest their suitability for regenerative medicine.

\subsubsection{Development of drug-laden porous scaffolds $P C L / B C N W-g-\beta C D-P C L_{2000} / D O X$}

Dual-function drug-laden porous scaffolds based on the biosorbable and biocompatible PCL was developed. The drug-laden scaffold showed high potentiality for encapsulating large drug payload of $(\sim 25 \% \mathrm{w} / \mathrm{w})$. The drug released from scaffold in a sustained and a controlled manner without obvious burst releases. The sufficient mechanical properties, higher porosity, improved hydrophilicity and bulk degradation characteristics, along with long-lasting drug release properties suggest future potential applications for the obtained scaffolds in the field of cancer therapy and regenerative medicine.

\subsubsection{Development of P(3HP) biodegradable bioplastics}

The production of biodegradable, biocompatible, and non-toxic $\mathrm{P}(3 \mathrm{HB})$ bioplastics from sustainable hemp hurd biomass was established. Different scenarios of retreating and hydrolyzing the raw biomass were implemented to concentrate cellulosic substrate and increase sugar yield in hydrolysate. In addition, different techniques for downstream extraction and purification processes were examined for commercial and competitive production. Microbial production of $13.4 \mathrm{~g} / \mathrm{L}$ was achieved with bioplastics content of $92 \%$. 


\subsection{Recommendations for future studies}

Based on the obtained results from the different studies conducted in this thesis, the following recommendations are proposed for future research. In chapter 3, the model can be also expanded to include dual or multiple drug delivery applications. Moreover, finding more effective method for increasing the grafting density of $\beta \mathrm{CD}$ on $\mathrm{BC}$ nanowhisker could pave the way for developing new green bio-based controlled release nanocarriers with higher efficiency. In addition, however the successfulness of the invitro studies, the developed nanocarrier has to be tested in vivo. In chapter $\mathbf{4}$, applying the same scenarios with other biodegradable hydrophobic polymers, such as PLA and PHB could lead to similar or better development for the bulk properties of those biodegradable polymers, and consequently expanding their application in biomedical arena. In addition after polymer degradation, until now there is no sharp decision in literature was taken to categorize nanocelluloses as completely safe. As the size allows their penetration into cells and they might be accumulated in biological system if they are not resorbable. Therefore, in this regard, in vivo safety concerns have to be studied. In chapter $\mathbf{5}$, the model can be expanded to develop dual-function drug-laden scaffolds multiple drug delivery properties. Nevertheless, the developed drug-laden scaffold has to be tested in vivo to figure out if any cytotoxicity for drug payloads. In chapter $\mathbf{6}$, for scaled-up production with a competitive cost, a hyper production of PHB should be examined using other bacterial strains, genetically modified strains and co-carbon sources. Moreover, different greener recovery and extraction techniques have to be tried out. 


\section{APENDICES}

Appendix A: Main Chemical and Pharmacokinetic Data of the Drugs Used

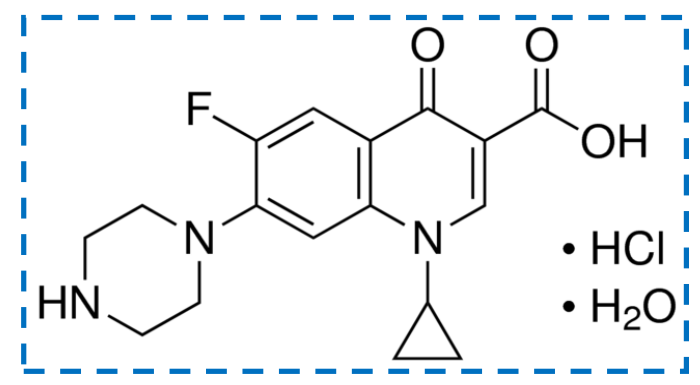

Figure A1. Ciprofloxacine Hydrochloride antibiotic

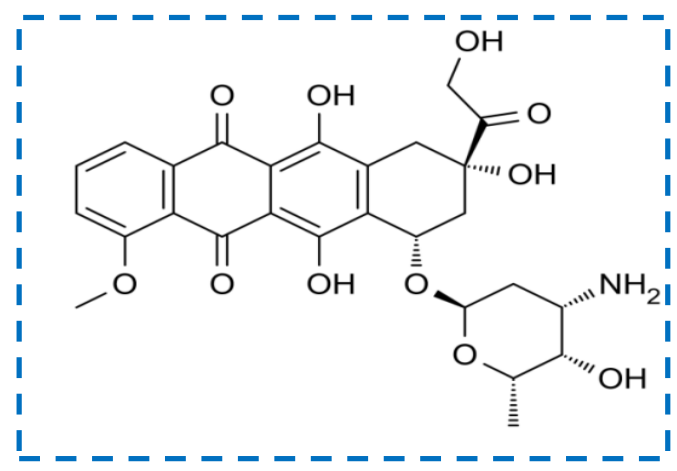

Figure A2. Doxorubicin Cancer Drug

\begin{tabular}{|c|c|c|c|}
\hline $\begin{array}{l}\text { Common Brand } \\
\text { name }\end{array}$ & Cipro & $\begin{array}{l}\text { Common Brand } \\
\text { name }\end{array}$ & Dox \\
\hline IUPAC name & $\begin{array}{l}\text { 1-cyclopropyl-6-fluoro-4- } \\
\text { oxo-7-(piperazin-1-yl)- } \\
\text { quinoline-3-carboxylic acid }\end{array}$ & \multirow[t]{2}{*}{ IUPAC name } & \multirow{2}{*}{$\begin{array}{l}(7 S, 9 S)-7-[(2 R, 4 S, 5 S, 6 S)-4- \\
\text { amino-5-hydroxy-6-methyloxan- } \\
\text { 2-yl]oxy-6,9,11-trihydroxy-9-(2- } \\
\text { hydroxyacetyl)-4-methoxy-8,10- } \\
\text { dihydro-7H-tetracene-5,12-dione }\end{array}$} \\
\hline \multirow[t]{2}{*}{ Usage } & \multirow{2}{*}{$\begin{array}{l}\text { Treatment of different types } \\
\text { of bacterial infections }\end{array}$} & & \\
\hline & & \multirow[t]{3}{*}{ Usage } & \multirow{3}{*}{$\begin{array}{l}\text { Treatment of some leukemias, as } \\
\text { well as, cancers of the bladder, } \\
\text { breast, stomach, lung, ovaries, } \\
\text { thyroid and soft tissue sarcoma }\end{array}$} \\
\hline Formula & $\mathrm{C}_{17} \mathrm{H}_{18} \mathrm{FN}_{3} \mathrm{O}_{3} \cdot \mathrm{HCl} \cdot \mathrm{H}_{2} \mathrm{O}$ & & \\
\hline Molar mass & $385.82 \mathrm{~g} / \mathrm{mol}$ & & \\
\hline Bioavailability & $69 \%$ & Formula & $\mathrm{C}_{27} \mathrm{H}_{29} \mathrm{NO}_{11}$ \\
\hline Metabolism & \multirow{2}{*}{ Hepatic } & Molar mass & $543.52 \mathrm{~g} / \mathrm{mol}$ \\
\hline \multirow{3}{*}{ Biological half-life } & & Bioavailability & $5 \%$ (Oral) \\
\hline & \multirow{2}{*}{4 hours } & Metabolism & Hepatic \\
\hline & & Biological half-life & $1-3$ hours \\
\hline
\end{tabular}




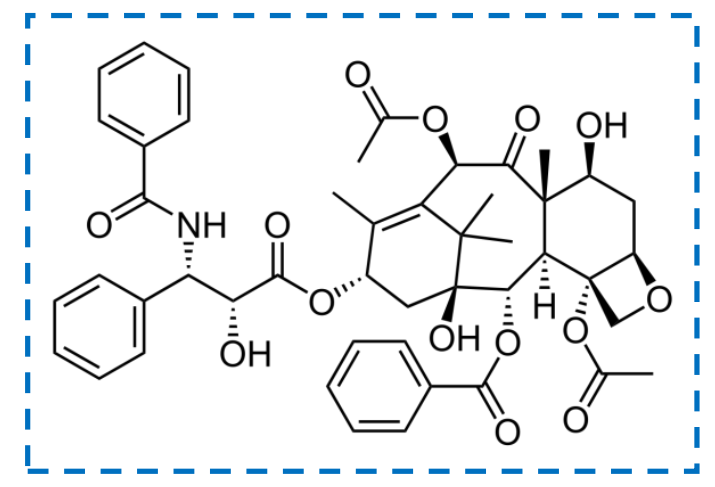

Figure A3. Paclitaxel Cancer Drug.

\begin{tabular}{|c|c|}
\hline $\begin{array}{l}\text { Common Brand } \\
\text { name }\end{array}$ & PTX \\
\hline IUPAC name & $\begin{array}{l}2 \alpha, 4 \alpha, 5 \beta, 7 \beta, 10 \beta, 13 \alpha)-4,10-\text { Bis } \\
\text { (acetyloxy)-13-\{[(2R,3S)-3- } \\
\text { (benzoylamino)-2-hydroxy-3- } \\
\text { phenylpropanoyl]oxy\}-1,7- } \\
\text { dihydroxy-9-oxo-5,20-epoxytax- } \\
\text { 11-en-2-yl benzoate }\end{array}$ \\
\hline Usage & $\begin{array}{l}\text { used to treat a number of types } \\
\text { of cancer. This includes ovarian } \\
\text { cancer, breast cancer, lung } \\
\text { cancer, Kaposi sarcoma, cervical } \\
\text { cancer, and pancreatic cancer }\end{array}$ \\
\hline Formula & $\mathrm{C}_{47} \mathrm{H}_{51} \mathrm{NO}_{14}$ \\
\hline Molar mass & $853.906 \mathrm{~g} / \mathrm{mol}$ \\
\hline Bioavailability & $6.5 \%$ (Oral) \\
\hline Metabolism & Hepatic \\
\hline Elimination half-life & 5.8 hours \\
\hline
\end{tabular}


Appendix B. Activation of Bacteria strains

\section{B1. Gluconacetobacter xylinus (ATCC® $700178^{\mathrm{TM}}$ )}

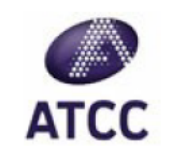

Product Sheet

\section{Gluconacetobacter xylinus (ATCC $^{\circledR} 700178^{\mathrm{TM}}$ )}

\section{Please read this FIRST}

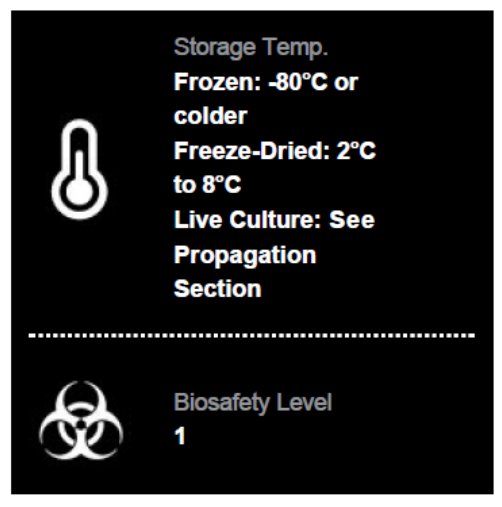

Intended Use

This product is intended for research use only. It is not intended for any animal or human therapeutic or diagnostic use.

\section{Citation of Strain}

If use of this culture results in a scientific publication, it should be cited in that manuscript in the following manner. Gluconacetobacter xylinus (ATCC ${ }^{\circledR} 700178^{\mathrm{TM}}$ )
Description

Designation: JCM 9730 [BPR2001, FERM-BP 4545, LMG 18788]

Deposited Name: Acetobacter xylinus subsp. sucrofermentans Toyosaki et al.

Product Description: Deposited as and referred to as the type strain of Acetobacter xylinus subsp. sucrofermentans. Produces large amounts of cellulose.

Pedium
ATCC ${ }^{\oplus}$ Medium $459: \mathrm{YGC}$ medium
Growth Conditions
Temperature: $26^{\circ} \mathrm{C}$
Atmosphere: Aerobic
Propagation Procedure
1. Open vial according to enclosed instructions.
2. Using a single tube of \#459 broth $(5$ to $6 \mathrm{~mL})$, withdraw approximately 0.5 to $1.0 \mathrm{~mL}$ with a Pasteur or
$1.0 \mathrm{~mL}$ pipette. Rehydrate the entire pellet.
3. Aseptically transfer this aliquot back into the broth tube. Mix well.
4. Use several drops of the suspension to inoculate a \#459 agar slant and/or plate.
5. Incubate the tubes and plate at $26^{\circ} \mathrm{C}$ for 72 hours.
Notes

Colonies on \#459 agar are small, slightly irregular, smooth, entire, pulvinate, and opaque. Undisturbed broth tube will form a thick pellicle at the surface.

Additional information on this culture is available on the ATCC ${ }^{\circledR}$ web site at www.atcc.org.

References

References and other information relating to this product are available online at www. atcc. org.

\section{Biosafety Level: 1}

Appropriate safety procedures should always be used with this material. Laboratory safety is discussed in the current publication of the Biosafety in Microbiological and Biomedical Laboratories from the U.S. Department of Health and Human Services Centers for Disease Control and Prevention and National Institutes for Health.

\section{ATCC Warranty}

ATCC $^{\oplus}$ products are warranted for 30 days from the date of shipment, and this warranty is valid only if the product is stored and handled according to the information included on this product information sheet. If the ATCC ${ }^{\circledR}$ product is a living cell or microorganism, ATCC lists the media formulation that has been found to be effective for this product. While other, unspecified media may also produce satisfactory results, a change in media or the absence of an additive from the ATCC recommended media may affect recovery, growth and/or function of this product. If an alternative medium formulation is used, the ATCC warranty for viability is no longer valid.

\section{Disclaimers}

This product is intended for laboratory research purposes only. It is not intended for use in humans While ATCC uses reasonable efforts to include accurate and up-to-date information on this product sheet, ATCC makes no warranties or representations as to its accuracy. Citations from scientific literature and patents are provided for informational purposes only. ATCC does not warrant that such information has been confirmed to be accurate.

This product is sent with the condition that you are responsible for its safe storage, handling, and use. ATCC is not liable for any damages or injuries arising from receipt and/or use of this product. While reasonable effort is made to insure authenticity and reliability of materials on deposit, ATCC is not liable for damages arising from the misidentification or misrepresentation of such materials.

Please see the enclosed Material Transfer Agreement (MTA) for further details regarding the use of this 
B2. Cupriavidus necator (ATCC® 17699ТМ)

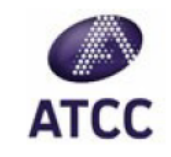

Product Sheet

\section{Cupriavidus necator (ATCC $^{\circledR}$ 17699 $^{\mathrm{TM}}$ )}

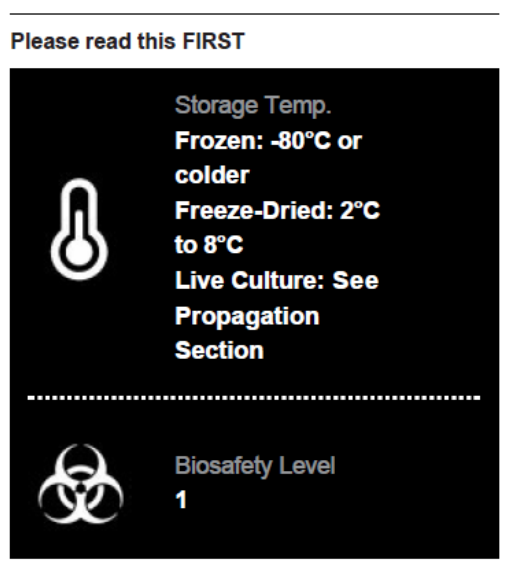

Intended Use

This product is intended for research use only. It is not intended for any animal or human therapeutic or diagnostic use.

\section{Citation of Strain}

If use of this culture results in a scientific publication, it should be cited in that manuscript in the following manner: Cupriavidus necator (ATCC ${ }^{\circledR} 17699^{\mathrm{TM}}$ ) a Description

Designation: 337 [ATCC 23440, H16, NCIB 10442, S-10-1]

Deposited Name: Ralstonia eutropha Yabuuchi et al Product Description: Formerly Wautersia eutropha.

Propagation

Medium

ATCC $^{\oplus}$ Medium 3: Nutrient agar or nutrient broth

Growth Conditions

Temperature: $26^{\circ} \mathrm{C}$

Atmosphere: Aerobic

Propagation Procedure

1. Open vial according to enclosed instructions.

2. Using a single tube of \#3 broth ( 5 to $6 \mathrm{~mL}$ ), withdraw approximately 0.5 to $1.0 \mathrm{~mL}$ with a Pasteur or $1.0 \mathrm{~mL}$ pipette. Rehydrate the entire pellet.

3. Aseptically transfer this aliquot back into the broth tube. Mix well.

4. Use several drops of the suspension to inoculate a \#3 agar slant and/or plate.

5. Incubate the tubes and plate at $26^{\circ} \mathrm{C}$ for 48 hours.

\section{Notes}

Additional information on this culture is available on the ATCC ${ }^{\oplus}$ web site at www.atcc.org.

References

References and other information relating to this product are available online at www. atcc.org

Biosafety Level: 1

Appropriate safety procedures should always be used with this material. Laboratory safety is discussed in the current publication of the Biosafety in Microbiological and Biomedical Laboratories from the U.S. Department of Health and Human Services Centers for Disease Control and Prevention and National Institutes for Health.

\section{ATCC Warranty}

ATCC ${ }^{\oplus}$ products are warranted for 30 days from the date of shipment, and this warranty is valid only if the product is stored and handled according to the information included on this product information sheet. If the ATCC ${ }^{\circledast}$ product is a living cell or microorganism, ATCC lists the media formulation that has been found to be effective for this product. While other, unspecified media may also produce satisfactory results, a change in media or the absence of an additive from the ATCC recommended media may affect recovery, growth and/or function of this product. If an alternative medium formulation is used, the ATCC warranty for viability is no longer valid.

\section{Disclaimers}

This product is intended for laboratory research purposes only. It is not intended for use in humans While ATCC uses reasonable efforts to include accurate and up-to-date information on this product sheet, ATCC makes no warranties or representations as to its accuracy. Citations from scientific literature and patents are provided for informational purposes only. ATCC does not warrant that such information has been confirmed to be accurate.

This product is sent with the condition that you are responsible for its safe storage, handling, and use. ATCC is not liable for any damages or injuries arising from receipt and/or use of this product. While reasonable effort is made to insure authenticity and reliability of materials on deposit, ATCC is not liable for damages arising from the misidentification or misrepresentation of such materials.

Please see the enclosed Material Transfer Agreement (MTA) for further details regarding the use of this product. The MTA is also available on our Web site at www. atcc.org

Additional information on this culture is available on the ATCC web site at www.atcc.org. 
Appendix C: Calibration/Standard Curves

C1: Calibration curve Used to Determine Grafting Density of $\beta C D$ on $B C N W$

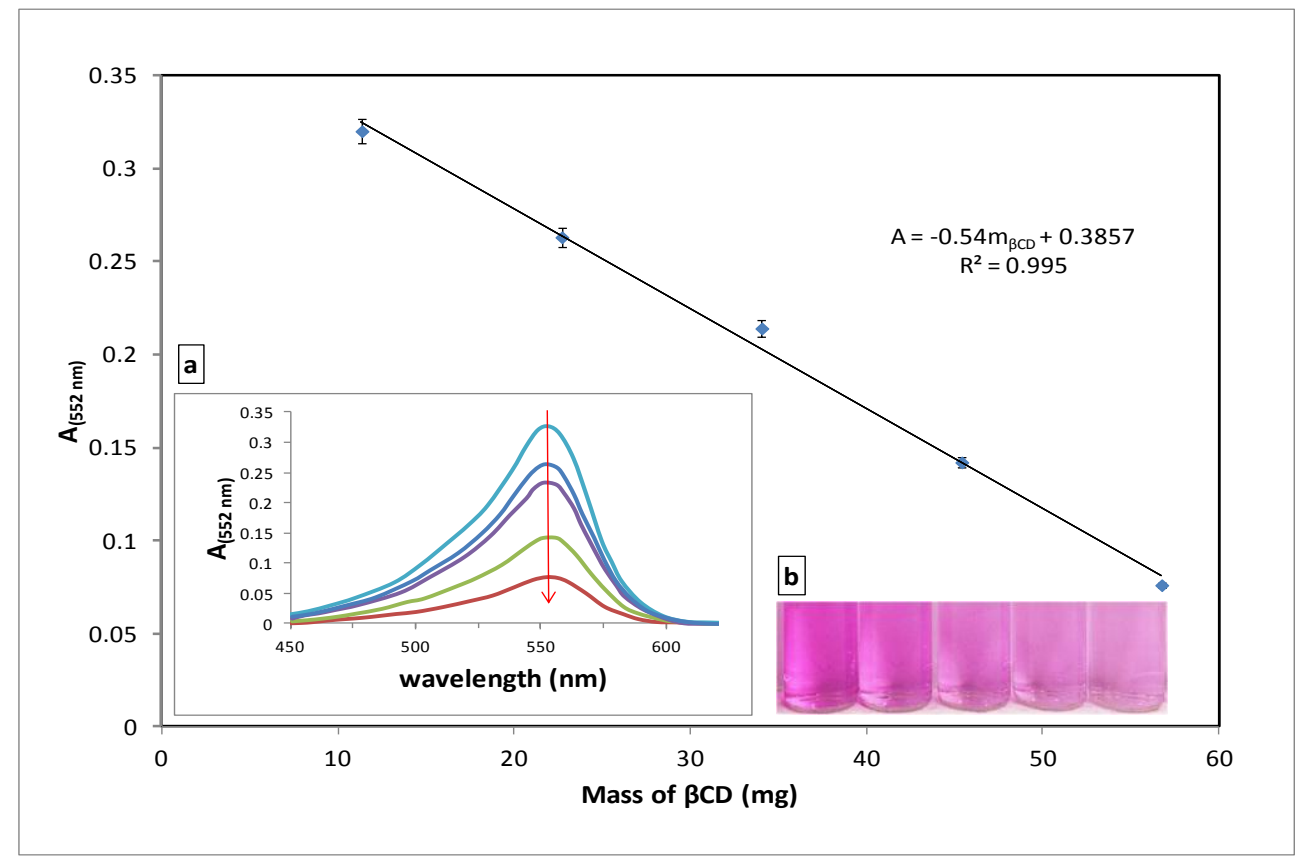

Figure C1. (A) Negative correlation standard curve of absorbance at $\lambda=552 \mathrm{~nm}$ vs. $\beta C D$ concentration (mg); (B) UV-vis absorbance of $\mathrm{Ph}$. $\mathrm{Ph} / \beta$-CD solutions with increasing $\beta C D$ concentrations; $(\mathrm{C}) \mathrm{Ph}$.Ph solutions with increasing $\beta C D$ concentration, where color faded from dark pink to faint pink. 
C2: The Standard Curves Used to Estimate the Concentration of Loaded and Relaesed Ciprofloxacin Drug

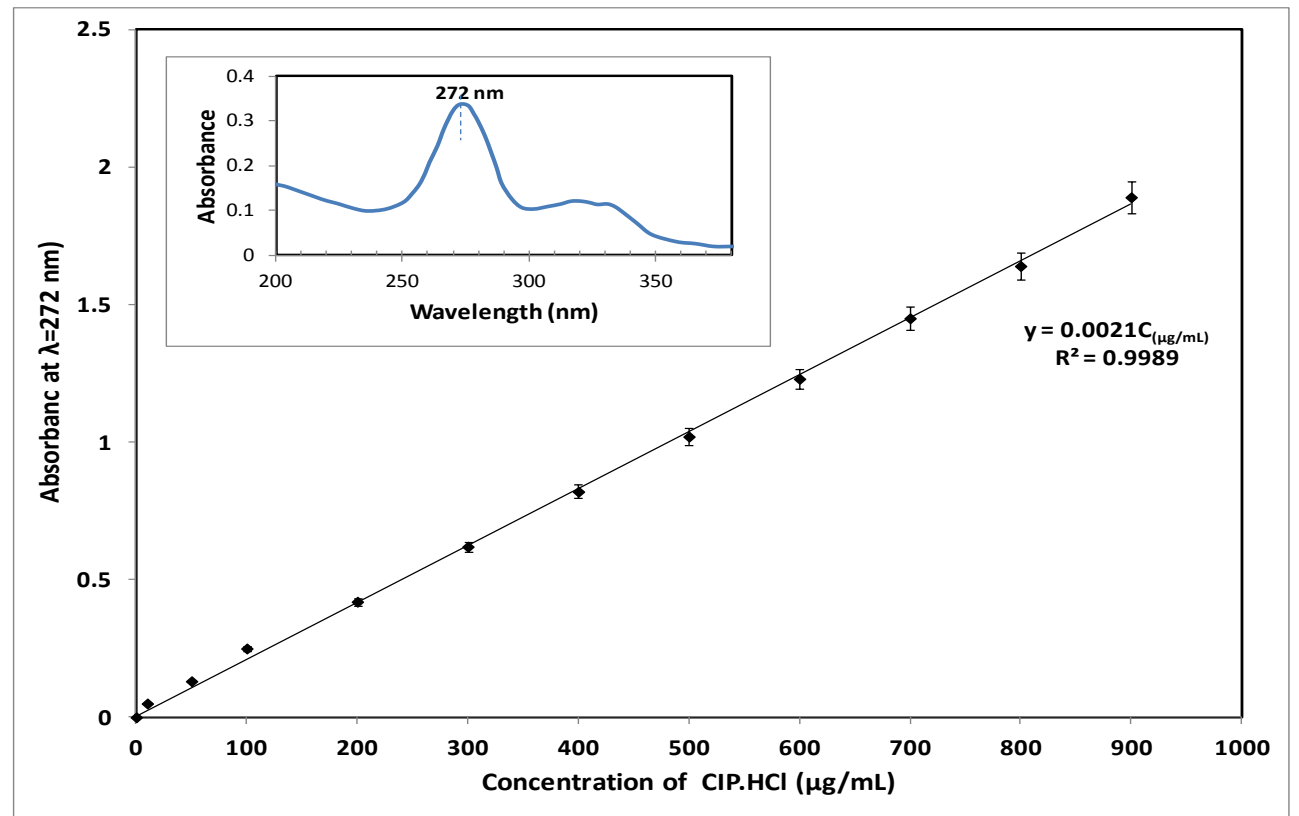

Figure C2. (A) Standard curve constructed at $272 \mathrm{~nm}$ for studying loading and release concentration of Cip-HCl; (B) Absorption spectra of ciprofloxacin hydrochloride in aqueous solution.

C3: Polystyrene calibration curve used to estimate Mn of P(3HP) using GPC

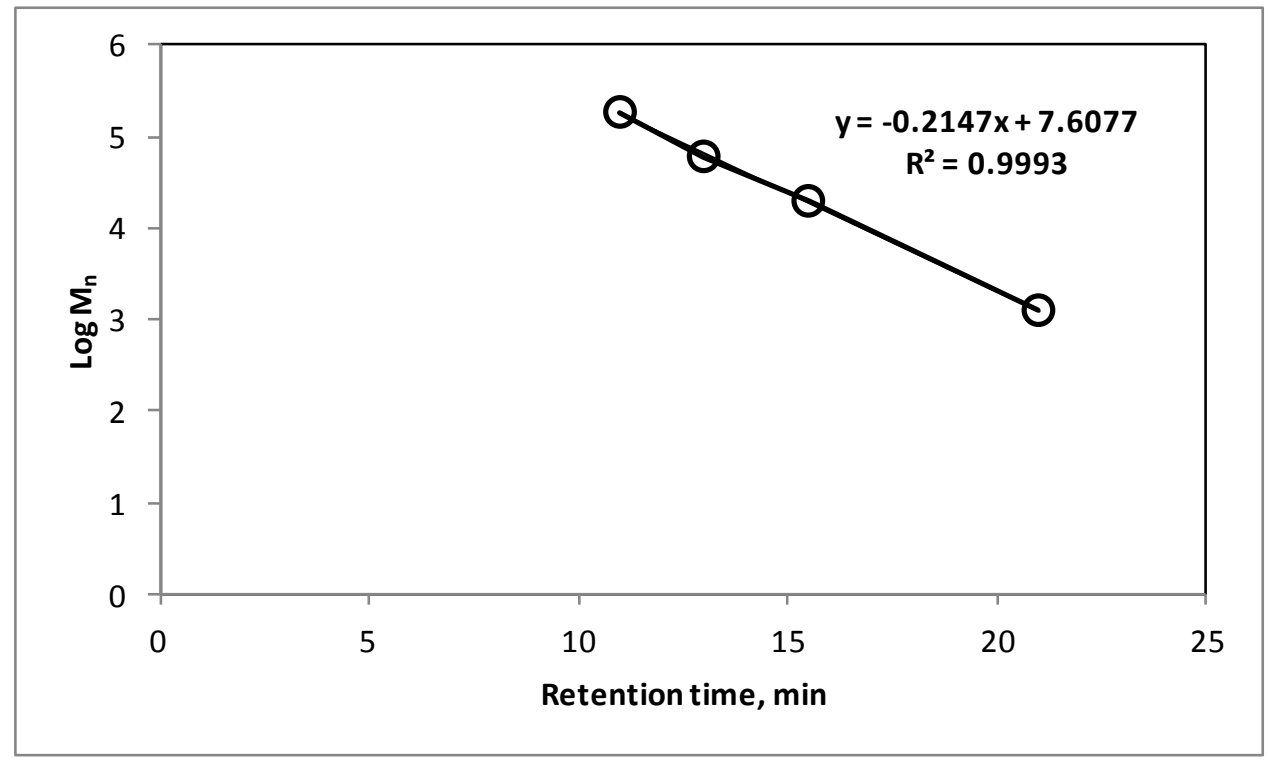

Figure C3. Polystyrene calibration generated by EasiVials for determination of number-average molecular weight of $\mathrm{P}(3 \mathrm{HB})$ 
C4. HPLC Standard Calibration Curves for Glucose Determination

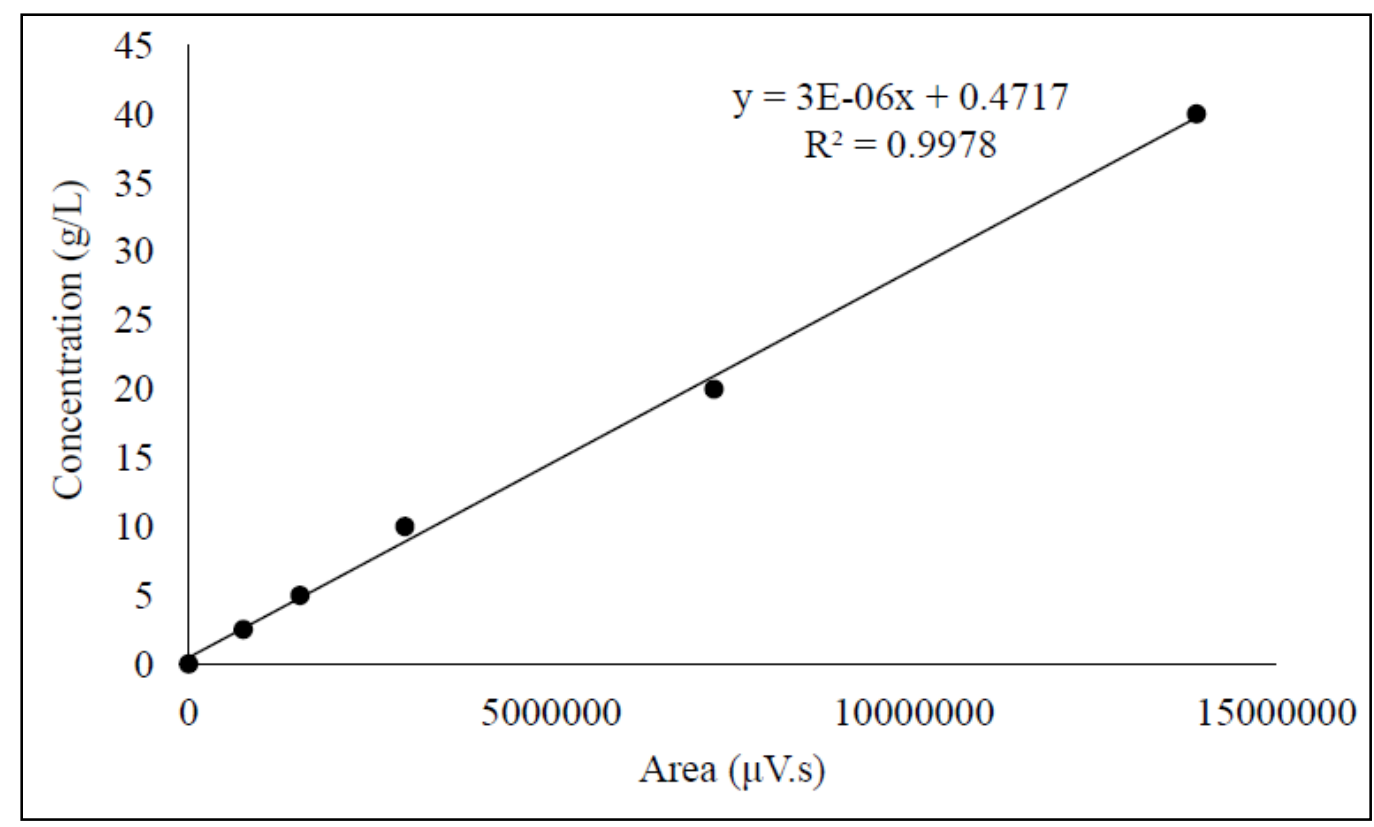

Figure C4. HPLC calibration curve for glucose (retention time $12.85 \mathrm{~min}$ ).

C5. HPLC Standard Calibration Curves for Xylose Determination

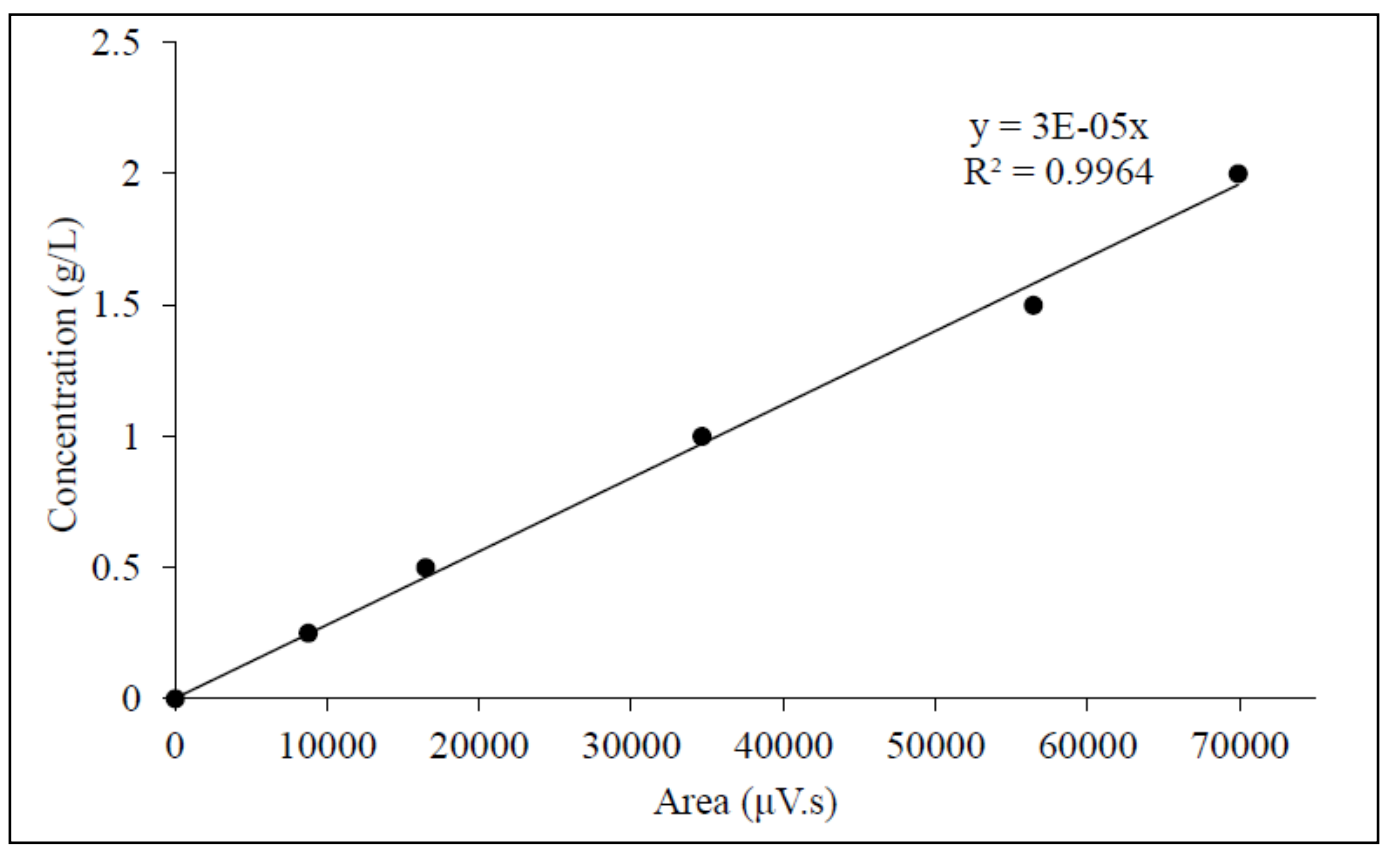

Figure C4. HPLC calibration curve for xylose (retention time $14.03 \mathrm{~min}$ ). 
Appendix D: : Lab Gallery Photos Showing Water-In-Air Contact Angle Measurements of PCL nanocomposites

D1.

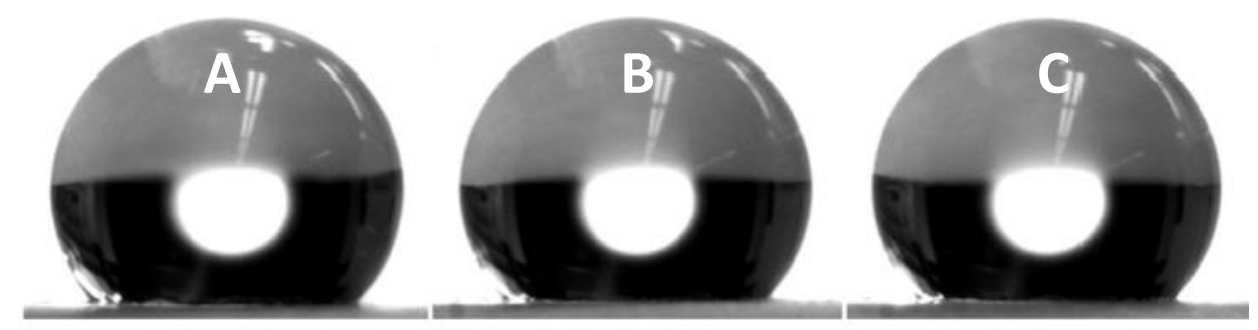

D2.

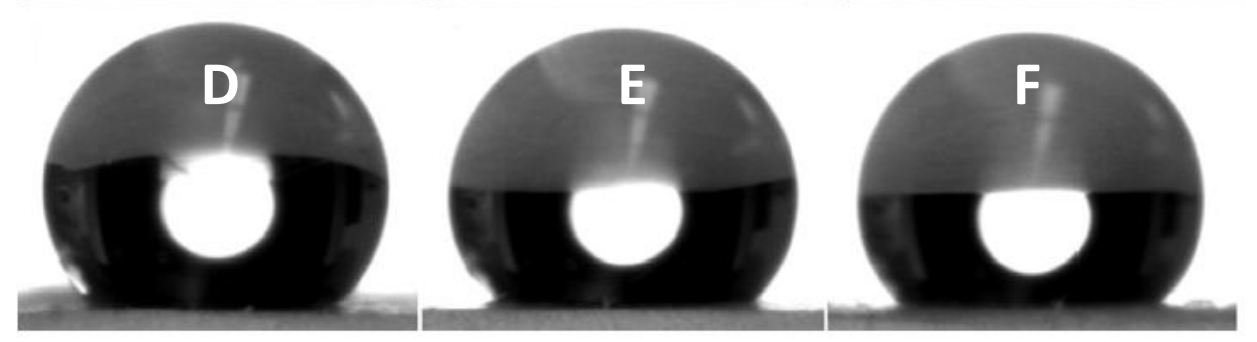

D1. PCL/BCNW- $g$ - $\beta C D-\mathrm{PCL}_{2000}$ nanocomposites films at different BCNW- $g-\beta C D-\mathrm{PCL}_{2000}$ loadings; (A) $1 \% \mathrm{w} / \mathrm{w}$, (B) $3 \% \mathrm{w} / \mathrm{w}$, (C) $4 \% \mathrm{w} / \mathrm{w}$.

D2. PCL/BCNW-g- $\beta C D-\mathrm{PCL}_{2000}(4 \%)$ drug-laden nanocomposites with different DOX loadings; (A) $10 \% \mathrm{w} / \mathrm{w}$, (B) $25 \% \mathrm{w} / \mathrm{w}$, (C) $30 \% \mathrm{w} / \mathrm{w}$. 
Appendix E: Lab Gallery Photos Showing Microbial Production and Purification of BC

Nanofibers and their Conversion to Nanowhisker

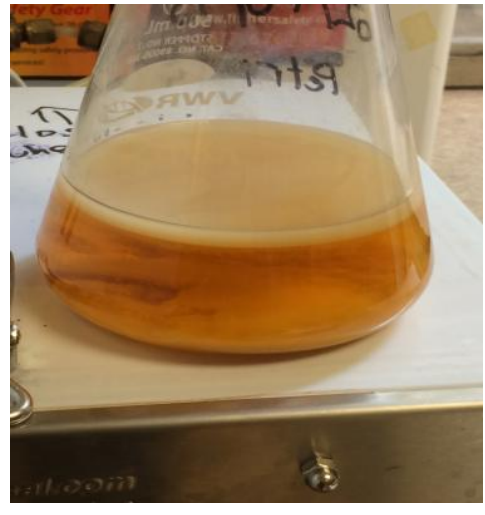

E1. BC disk from static culture

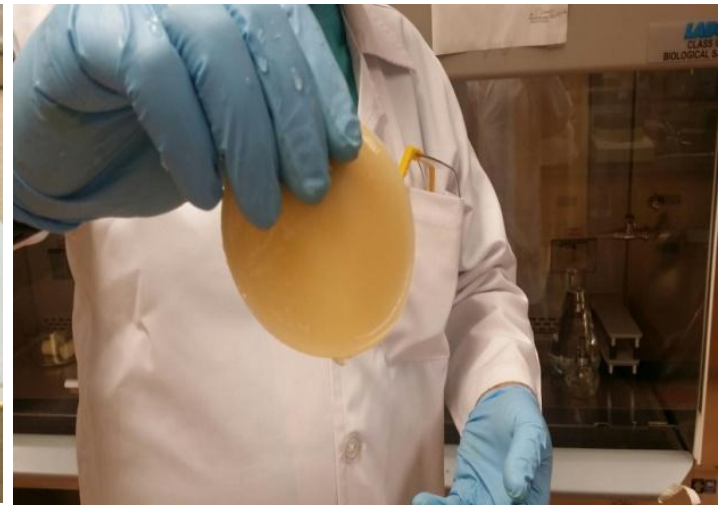

E2. Harvested unpurified BC

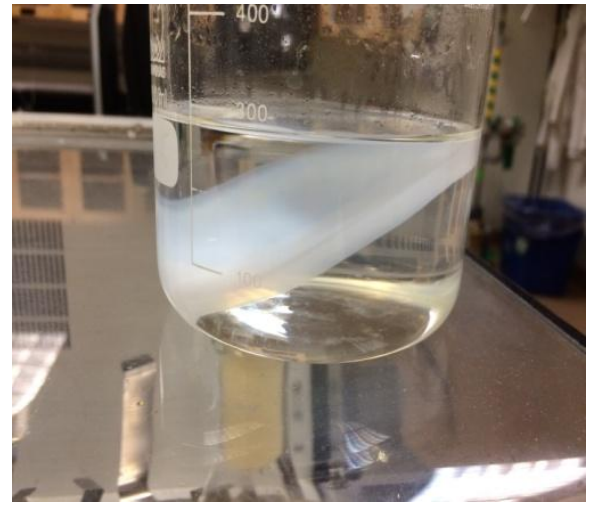

E3. BC disk after purification

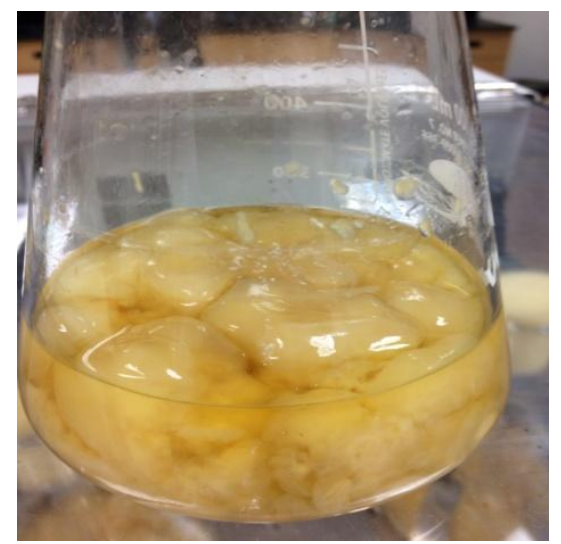

E4. $B C$ pellicle from shake culture

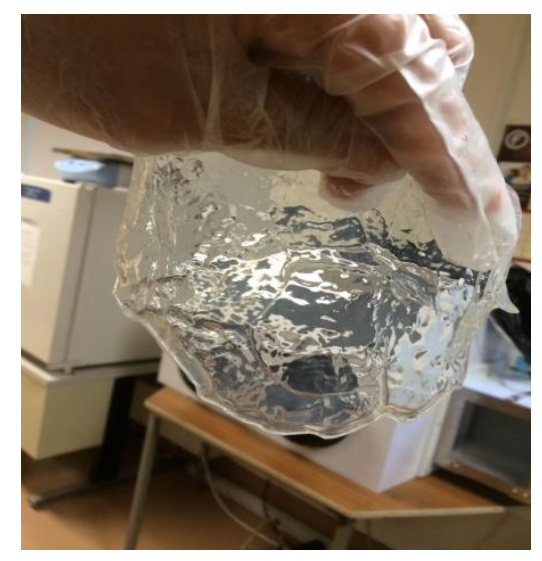

E5. BC pellicle purified

\section{Electron image 1}

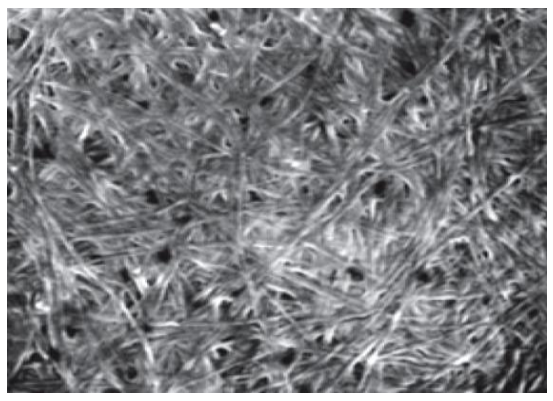

E7. SEM of nanofibers before hvdrolvsis

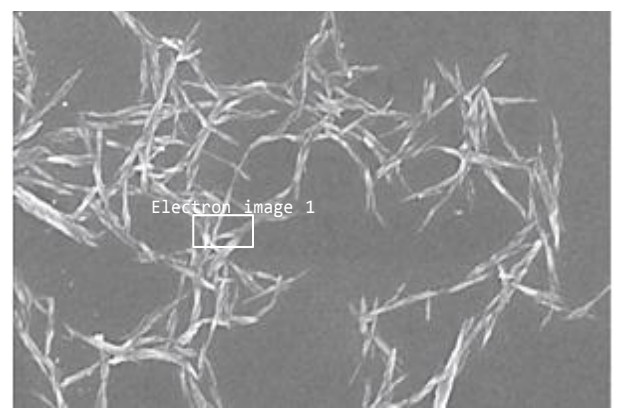

E8. SEM of nanowhiskers

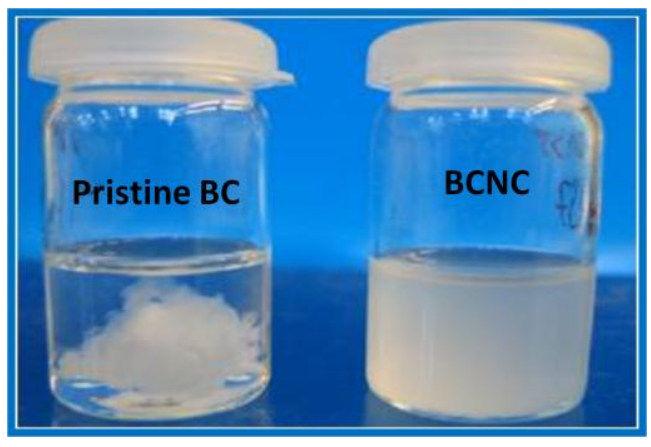

E6. $B C$ nanofibers before and after hydrolysis

Electron image 2

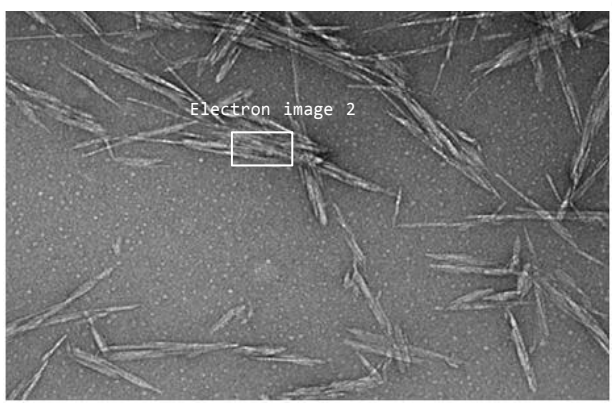

E9. SEM of BC nanowhiskers after grafting 
Appendix F: Lab Gallery Photos Showing Microbial Production and Extraction of PHB from Hemp Hurd Biomass

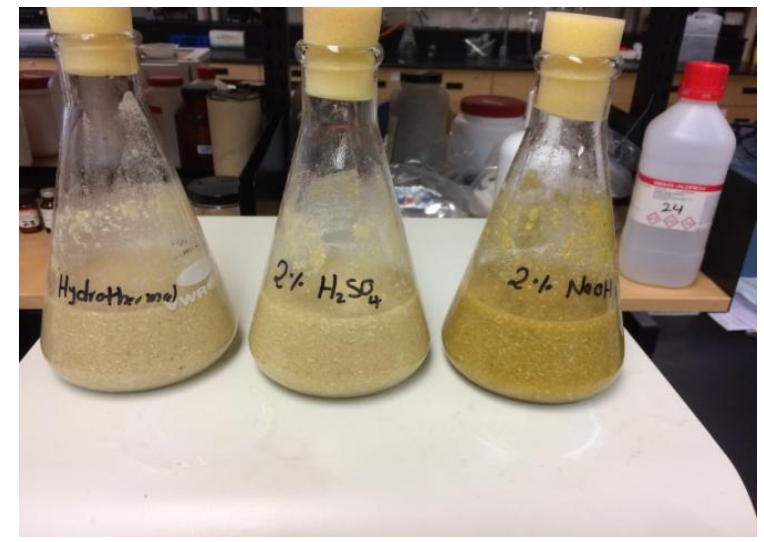

F1. Hemp Hurd biomass pretreatments

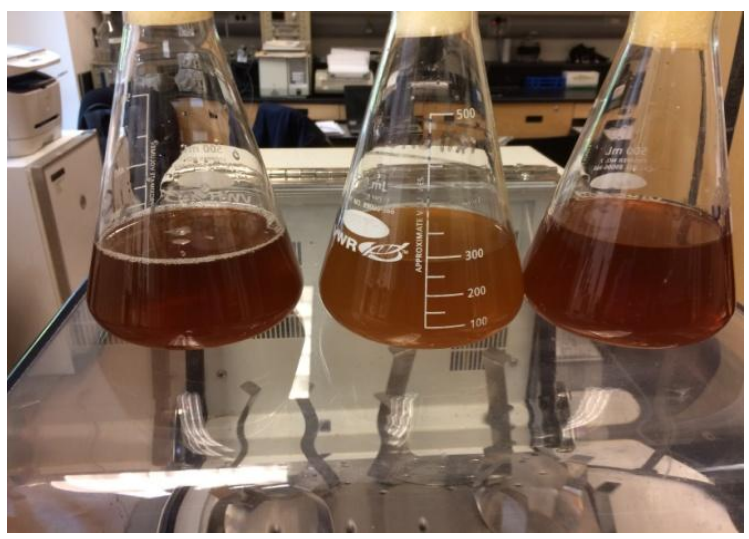

F2. Hemp Hurd hydrolysates

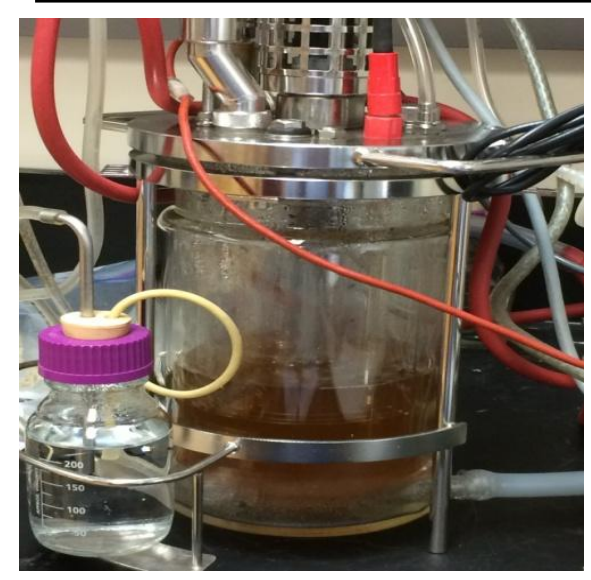

F3. Bioreactor used for fermentation

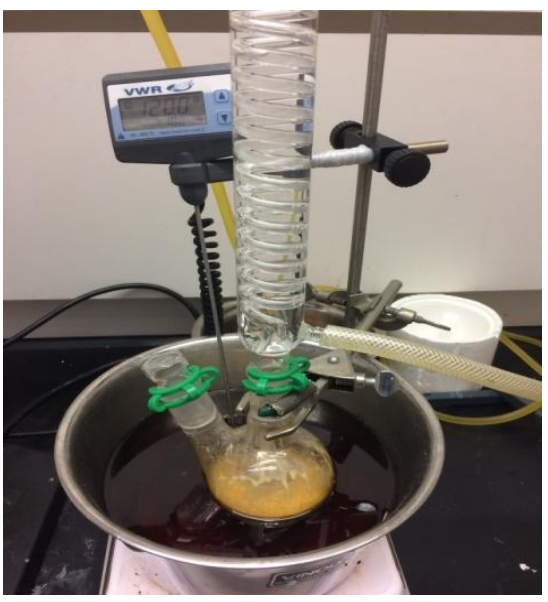

F6. Extraction PHB bioplastics

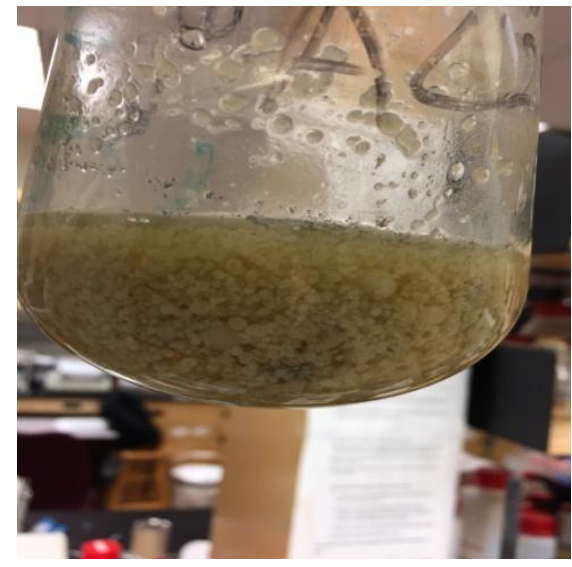

F4. PHB pellicle after fermentation

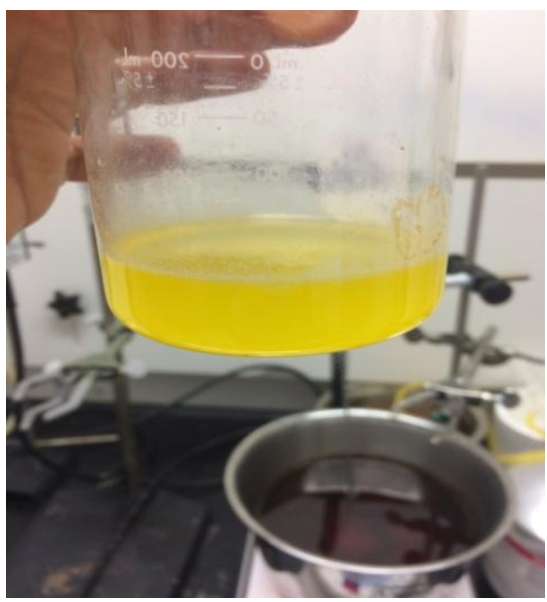

F7. PHB bioplastics purification

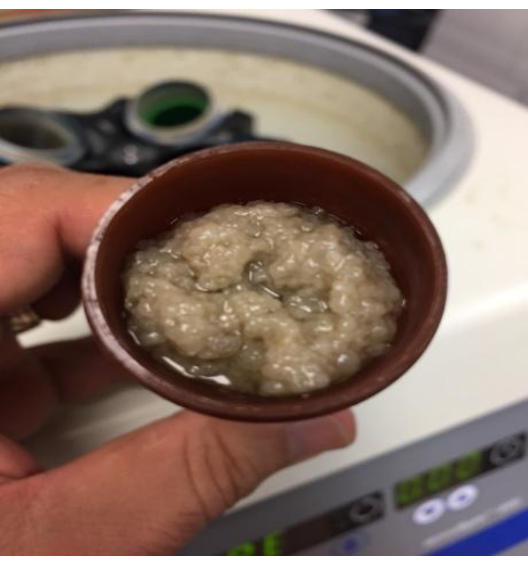

F5. PHB pellicles harvested

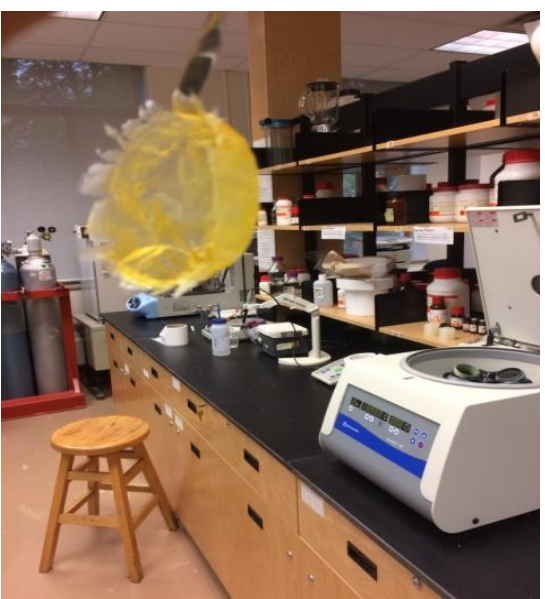

F8. PHB bioplastics film 


\section{Appendix G. Mechanical Testing}

\section{Tensile Strength}

Tensile strength is calculated by dividing the load at break by the original minimum crosssectional area. The result is expressed in megapascals (MPa) and reported to three significant figures.

Tensile strength $=($ load at break $) /($ original width $)($ original thickness $)$

\section{Percent Elongation}

Percent elongation is calculated by dividing the elongation at the moment of rupture by the initial gauge length and multiplying by 100 . When gauge marks or extensometers are used to define a specific test section, only this length is used in the calculation, otherwise the distance between the grips is used as the initial gauge length. The result is expressed in percent and reported to two significant figures.

Percent elongation $=($ elongation at rupture $) \times 100 /($ initial gage length $)$

\section{Young's Modulus}

Young's modulus is calculated by drawing a tangent to the initial linear portion of the stressstrain curve, selecting any point on this tangent, and dividing the tensile stress by the corresponding strain. For purposes of this calculation, the tensile stress shall be calculated by dividing the load by the average original cross section of the test specimen. The result is expressed in gigapascals (GPa) and reported to three significant figures.

$$
\text { Young's modulus }=\frac{\text { load at point on tangent } /(\text { original width })(\text { original thickness })}{\text { elongation at point of tangent }) /(\text { initial gage length })}
$$

\section{Toe Compensation}

In a typical stress-strain curve (see below), there is a toe region, $\mathrm{AC}$, which does not represent a property of the material. It is an artifact caused by a take-up of slack, and alignment or seating of the specimen. In order to obtain correct values of such parameters as modulus, strain, and yield point, this artifact must be compensated for to give the corrected zero point on the strain or extension axis. In the case of a material exhibiting a region of Hookean (linear) behavior as shown below, a continuation of the linear (CD) region of the curve is constructed through the zero-stress axis. The intersection (B) is the corrected zero-strain point from which all extensions 
or strains must be measured, including the yield point, if applicable. The elastic modulus can be determined by dividing the stress at any point along line $\mathrm{CD}$ (or its extension) by the strain at the same point (measured from point B, defined as zero-strain).

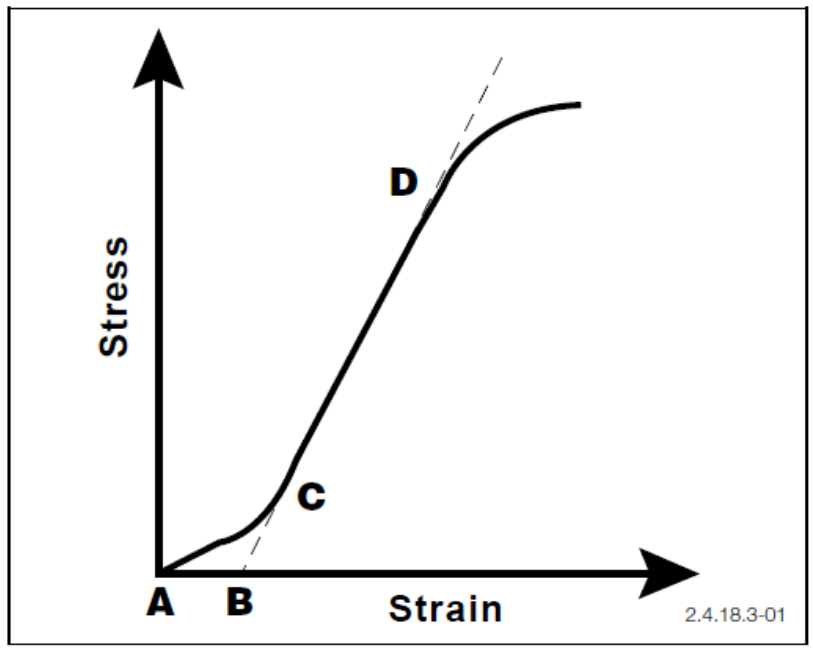

Figure G1. A typical stress-strain curve.

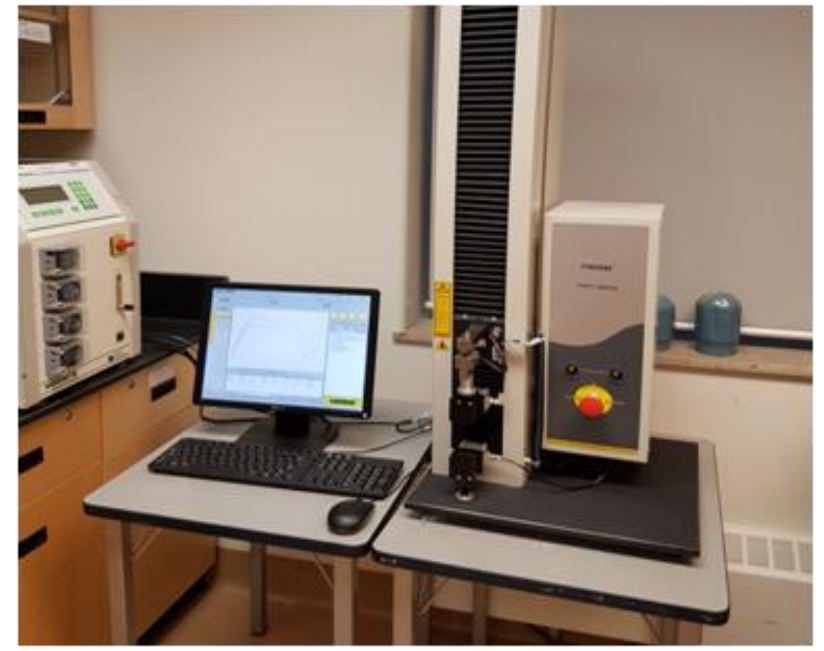

Figure G2. Labthink's Param XLW (PC) Auto Tensile Tester. 


\section{Appendix H: Cumulative Drug Release Calculations}

Before doing a cumulative release plot, the standard curve that represents the response vs. drug concentration has to be created first. For example, $\mathrm{y}=\mathrm{ax}+\mathrm{b}, \mathrm{R}^{2}>0.99$, where $\mathrm{x}$ is the concentration of the solution containing the drugused, $y$ is the corresponding UV absorbance, the constants $\mathrm{a}$ and $\mathrm{b}$ are the slope and intercept of the straight line.

For example, assume the drug loading capacity of the sample is $100 \mathrm{mg} / \mathrm{g}$, the drug-loaded sample (200 mg) was immersed into $10 \mathrm{~mL}$ PBS, and the drug-release medium $(0.9 \mathrm{~mL})$ was withdraw for UV-vis analysis at given time intervals and replaced the same volume of fresh PBS solution (to keep sink conditions).

The cumulative release was calculated as shown in the following Table. In addition, when the experimental absorbance date is going up and down several parallel drug-release experiments were carried out. Differences between the groups with $\mathrm{p}<0.05$ were considered to be statistically significant, where the average error in every set of data presented was less than $5 \%$. The mean value and standard deviation were evaluated for accuracy.

\begin{tabular}{|c|c|c|c|c|}
\hline Time/h & Absorbance & $\begin{array}{c}\text { The content of drug } \\
\text { in the drug-release } \\
\text { medium } / \mathrm{mg}\end{array}$ & The cumulative release/mg & $\begin{array}{c}\text { The cumulative } \\
\text { release percentage } \\
(\%)\end{array}$ \\
\hline 1 & 0.1478 & $10 *(0.1478-\mathrm{b}) / \mathrm{a}$ & $\mathrm{A}=10 *(0.1478-\mathrm{b}) / \mathrm{a}$ & $100 * \mathrm{~A} / 2$ \\
\hline 2 & 0.2073 & $10 *(0.2073-\mathrm{b}) / \mathrm{a}$ & $\mathrm{B}=10 *(0.2073-\mathrm{b}) / \mathrm{a}+0.9 * \mathrm{~A}$ & $100 * \mathrm{~B} / 2$ \\
\hline 3 & 0.3123 & $10 *(0.3123-\mathrm{b}) / \mathrm{a}$ & $\mathrm{C}=10 *(0.3123-\mathrm{b}) / \mathrm{a}+0.9 * \mathrm{~B}$ & $100 * \mathrm{C} / 2$ \\
\hline 4 & $\ldots$ & $\ldots$ & $\mathrm{D}=\ldots+0.9 * \mathrm{C}$ & $\ldots$ \\
\hline
\end{tabular}


Appendix I: Sample Calculations

\section{I1: Crystallite thickness}

A more useful form of Scherrer Equation can be written as

Crystallite thickness $(\mathrm{t})=0.9 \lambda /(\beta 1 / 2 \cos \theta)$

e.g., at $\beta=1.1^{\circ}, \lambda=0.154 \mathrm{~nm}, 2 \theta=27^{\circ}$

$\theta=13.5^{\circ} ; \cos (13.5)=0.972$

$360^{\circ}=2 \times 3.142$

$1.1=1.1 \times 2 \times 3.142 /(360)=0.0189$

$\mathrm{t}=7.1 \mathrm{~nm}$

\section{I2: Porosity and Pore Volume}

The porosity and pore volume of the scaffolds were estimated gravimetrically using Equations (4.1\& 4.2), where $\rho_{\text {polymer }}$ is the density of neat polymer used for scaffolds fabrication and $\rho_{\text {scaffold }}$ is the apparent density of the scaffold which determined by using Sartorius YDK 01LP density measurement kit. $\rho_{\text {PCL }}$ was is considered to be $1.145 \mathrm{~g} / \mathrm{cm}^{3}$.

$$
\begin{aligned}
& \text { Porosity }(\%)=\frac{\rho_{\text {scaffold }}}{\rho_{\text {polymer }}} \times 100 \\
& \text { Pore volume }=\left(\frac{1}{\rho_{\text {scaffold }}}-\frac{1}{\rho_{\text {polymer }}}\right) \times 100
\end{aligned}
$$

e.g., for $\rho_{\text {scaffold }}$ equal to $0.989 \mathrm{~g} / \mathrm{cm}^{3}$

$$
\begin{gathered}
\text { Porosity }(\%)=\frac{0.989}{1.145} \times 100=86.45 \% \\
\text { Pore volumewere equal to }=\left(\frac{1}{0.989}-\frac{1}{1.145}\right) \times 100=9.64 \mathrm{~cm}^{3} / \mathrm{g}
\end{gathered}
$$

\section{I3: The degree of crystalinity $\left(\chi_{c}\right)$}

The degree of crystallinity $\left(\chi_{c}\right)$ was calculated using Equation (4.3).

$$
\chi_{c}=\frac{\Delta \mathrm{H}_{\mathrm{m}}-\Delta \mathrm{H}_{\mathrm{cc}}}{\Delta \mathrm{H}_{0, \mathrm{~m}}\left(1-\mathrm{m}_{\mathrm{f}}\right)} \times 100
$$

where $\Delta \mathrm{H}_{\mathrm{m}}$, and $\Delta \mathrm{H}_{\mathrm{cc}}$ are enthalpies related to the second heating scan, $\Delta \mathrm{H}_{0, \mathrm{~m}}$ is melting enthalpy for $100 \%$ crystalline PCL taken as $139.5 \mathrm{~J} / \mathrm{g}$, and $\left(1-\mathrm{m}_{f}\right)$ is the weight fraction of PCL matrix in the sample.

e.g., for PCL+ BCNW-g- $\beta C D-\mathrm{PCL}_{2000}(4 \%) / \mathrm{DOX}(10 \%)$ scaffold $\Delta \mathrm{H}_{\mathrm{m}}$, and $\Delta \mathrm{H}_{\mathrm{cc}}$ were equal to 58.1 and $13.1 \mathrm{~J} / \mathrm{g}$, and $\left(1-\mathrm{m}_{f}\right)$ equal to 0.86 . Therefore, the corresponding $\chi_{\mathrm{c}}$ is equal

$$
\chi_{c}=\frac{58.1-13.1}{139.5(0.86)} \times 100=37.5
$$




\section{Appendix J. Statistical Analysis and Error Calculations}

Most experimental procedure was done in triplicates. Each date point in table or graph is expressed as mean for $n=3$. The mean is presented in table as an interval, in which the true value is likely to be found, and expressed mathematically as $\bar{x} \pm(\mathrm{SE})$. While in graph, the (SE) is represented by error bar on every data point. The statistical analysis of the data was conducted using one-way ANOVA. Differences between the groups with $\mathrm{p}<0.05$ were considered to be statistically significant, where the average error in every set of data presented was less than $5 \%$. Appendix $\mathrm{J}$ listed raw data that obtained from replicated experiments.

It is worth to mention that for all repeated experiments standard errors did not exceed $\pm 5 \%$ and $p$ values $<0.05$. This in turn reflects the significance of the obtained results.

Equations J1and $\mathbf{J} 2$ were used to calculate standard deviation (SD) and standard error (SE), respectively.

$$
\begin{aligned}
& S D=\sqrt{\frac{\sum(X-\bar{X})^{2}}{(n-1)}} \\
& S E_{\bar{X}}=\frac{S D}{\sqrt{n}}
\end{aligned}
$$

Where, $\bar{x}=$ sample mean value $; \mathrm{x}=$ data point $\mathrm{n}=$ sample size 
Appendix K. Row data for some replicated experiments

K1: Cumulative release of DOX from PCL/BCNW-g- $\beta C D-P C L_{2000}(4 \%) / D O X(25 \%)$ scaffold at $37^{\circ} \mathrm{C}$ in $\mathrm{PBS}$ of $\mathrm{pH} 7.4$ and 6.4, Figure 5.10

\begin{tabular}{|c|c|c|c|c|c|c|c|c|}
\hline \multicolumn{5}{|c|}{ Cumulative realesae $(\%), \mathrm{pH} 6.4$} & \multicolumn{4}{|c|}{ Cumulative realesae $(\%), \mathrm{pH} 7.4$} \\
\hline Time, day & $\mathbf{T 1}$ & $\mathbf{T} 2$ & T3 & $\bar{x} \pm(\mathrm{SE})$ & T1 & $\mathbf{T} 2$ & T3 & $\bar{x} \pm(\mathrm{SE})$ \\
\hline 0.3 & 0.40 & 0.32 & 0.37 & $0.36 \pm 0.02$ & 0.30 & 0.32 & 0.29 & $0.30 \pm 0.01$ \\
\hline 1.5 & 13.21 & 12.28 & 13.10 & $12.86 \pm 0.29$ & 12.19 & 12.21 & 11.71 & $12.04 \pm 0.16$ \\
\hline 2.3 & 18.81 & 17.81 & 16.88 & $17.83 \pm 0.55$ & 18.88 & 17.81 & 18.77 & $18.49 \pm 0.34$ \\
\hline 3.3 & 21.61 & 20.63 & 20.27 & $20.83 \pm 0.40$ & 22.78 & 21.63 & 20.77 & $21.72 \pm 0.58$ \\
\hline 4.3 & 25.61 & 24.65 & 24.50 & $24.92 \pm 0.35$ & 25.17 & 24.65 & 25.04 & $24.95 \pm 0.16$ \\
\hline 6.0 & 30.82 & 29.81 & 29.83 & $30.15 \pm 0.33$ & 28.77 & 27.81 & 29.10 & $28.56 \pm 0.39$ \\
\hline 8.0 & 36.02 & 35.21 & 35.62 & $35.62 \pm 0.23$ & 32.67 & 31.21 & 30.62 & $31.50 \pm 0.61$ \\
\hline 10.0 & 40.42 & 41.44 & 41.47 & $41.11 \pm 0.34$ & 35.66 & 34.44 & 33.66 & $34.59 \pm 0.58$ \\
\hline 18.0 & 52.43 & 53.47 & 53.54 & $53.15 \pm 0.35$ & 47.05 & 45.90 & 46.36 & $46.44 \pm 0.34$ \\
\hline 26.0 & 60.04 & 61.03 & 61.77 & $60.95 \pm 0.50$ & 54.84 & 55.17 & 54.77 & $54.93 \pm 0.12$ \\
\hline 34.0 & 66.44 & 66.89 & 66.85 & $66.73 \pm 0.14$ & 60.24 & 60.89 & 60.85 & $60.66 \pm 0.21$ \\
\hline 43.0 & 71.24 & 72.42 & 72.17 & $71.94 \pm 0.35$ & 65.03 & 61.42 & 64.17 & $63.54 \pm 1.09$ \\
\hline 51.0 & 75.24 & 76.22 & 76.87 & $76.11 \pm 0.47$ & 69.23 & 67.22 & 68.87 & $68.44 \pm 0.62$ \\
\hline 59.0 & 77.65 & 78.45 & 78.23 & $78.11 \pm 0.24$ & 72.33 & 71.45 & 70.23 & $71.34 \pm 0.61$ \\
\hline 67.0 & 78.45 & 81.04 & 81.00 & $80.16 \pm 0.85$ & 73.22 & 71.83 & 71.00 & $72.02 \pm 0.65$ \\
\hline
\end{tabular}

Anova: Single Factor

SUMMARY

\begin{tabular}{lrrrr}
\hline \multicolumn{1}{c}{ Groups } & \multicolumn{1}{c}{ Count } & \multicolumn{1}{c}{ Sum } & Average & Variance \\
\hline T1 & 15 & 668.3924 & 44.5595 & 674.0675 \\
T2 & 15 & 671.6769 & 44.77846 & 721.8297 \\
T3 & 15 & 672.457 & 44.83047 & 724.9039 \\
\hline
\end{tabular}

ANOVA

\begin{tabular}{lcccccc}
\hline $\begin{array}{l}\text { Source of } \\
\text { Variation }\end{array}$ & SS & $d f$ & MS & $F$ & P-value & Fcrit \\
\hline $\begin{array}{l}\text { Between } \\
\text { Groups }\end{array}$ & 0.620382 & 2 & 0.310191 & 0.000439 & 0.999561 & 3.219942 \\
Within & 29691.22 & 42 & 706.9337 & & & \\
Groups & & & & & & \\
Total & 29691.84 & 44 & & & & \\
\hline
\end{tabular}


K2: Porosity percentage of PCL/BCNW- $g-\beta C D-\mathrm{PCL}_{2000}(4 \%) / \mathrm{DOX}(25 \%)$ scaffold at $\mathrm{NH}_{4} \mathrm{HCO}_{3}$ to PCL 7:1 (\% w/w), Table 4.3

\begin{tabular}{|c|c|c|c|}
\hline \multicolumn{4}{|c|}{ Porosity (\%) } \\
\hline Trials & Sample 1 & Sample 2 & Sample 3 \\
\hline T1 & 91.17 & 92.02 & 91.66 \\
\hline T2 & 90.12 & 91.42 & 90.18 \\
\hline T3 & 88.87 & 91.87 & 93.01 \\
\hline T4 & 92.13 & 90.13 & 91.17 \\
\hline T5 & 91.02 & 91.42 & 90.08 \\
\hline
\end{tabular}

\section{Anova: Single Factor}

SUMMARY

\begin{tabular}{lrrrr}
\hline \multicolumn{1}{c}{ Groups } & \multicolumn{1}{c}{ Count } & \multicolumn{1}{c}{ Sum } & \multicolumn{1}{c}{ Average } & Variance \\
\hline Sample 1 & 5 & 453.31 & 90.662 & 1.51157 \\
Sample 2 & 5 & 456.86 & 91.372 & 0.55377 \\
Sample 3 & 5 & 456.1 & 91.22 & 1.44535 \\
\hline
\end{tabular}

\begin{tabular}{|c|c|c|c|c|c|c|}
\hline $\begin{array}{l}\text { Source of } \\
\text { Variation }\end{array}$ & SS & $d f$ & $M S$ & $F$ & P-value & F crit \\
\hline $\begin{array}{l}\text { Between } \\
\text { Groups }\end{array}$ & 1.397613 & 2 & 0.698807 & 0.597153 & 0.565937 & 3.885294 \\
\hline $\begin{array}{l}\text { Within } \\
\text { Groups }\end{array}$ & 14.04276 & 12 & 1.17023 & & & \\
\hline Total & 15.44037 & 14 & & & & \\
\hline
\end{tabular}


K3: Tensile properties of PCL/BCNW-g- $\beta C D-\mathrm{PCL}_{2000}$ nanocomposites films of different nanowhisker loadings, Table 4.1

\begin{tabular}{|c|c|c|c|c|c|c|c|c|c|c|c|c|c|}
\hline & & \multicolumn{4}{|c|}{ Tensile Strength, $\sigma_{b}(\mathrm{MPa})$} & \multicolumn{4}{|c|}{$\%$ Elongation at Break, $\boldsymbol{\varepsilon}_{\mathbf{b}}(\%)$} & \multicolumn{4}{|c|}{ Young Modulus, E (MPa) } \\
\hline Lot name & $\begin{array}{c}\text { Sample } \\
\#\end{array}$ & $\mathbf{T 1}$ & $\mathbf{T 2}$ & $\mathbf{T 3}$ & $\bar{x} \pm(\mathbf{S E})$ & T1 & $\mathbf{T 2}$ & $\mathbf{T 3}$ & $\bar{x} \pm(\mathbf{S E})$ & T1 & $\mathbf{T} 2$ & T3 & $\bar{x} \pm(\mathbf{S E})$ \\
\hline \multirow{3}{*}{$\begin{array}{l}\text { PCL+ BCNW- } g- \\
\beta C D-P_{2000}(1 \%)\end{array}$} & S1 & 27.6 & 27.0 & 28.2 & $27.6 \pm 1.7$ & 9.0 & 8.8 & 9.2 & $9.0 \pm 0.2$ & 1810 & 1900 & 1780 & $1830 \pm 128$ \\
\hline & S2 & 26.3 & 27.5 & 25.1 & $26.3 \pm 2.0$ & 9.3 & 9.8 & 11.2 & $10.1 \pm 0.3$ & 1819 & 1916 & 1875 & $1870 \pm 100$ \\
\hline & S3 & 25.8 & 27.8 & 24.9 & $25.5 \pm 0.8$ & 8.5 & 9.3 & 8.7 & $8.8 \pm 0.1$ & 1911 & 1919 & 1870 & $1900 \pm 150$ \\
\hline \multirow{2}{*}{$\begin{array}{l}\text { PCL+ BCNW-g- } \\
\beta C D-P_{2000}(2 \%)\end{array}$} & S1 & 28.9 & 26.7 & 27.8 & $27.8 \pm 1.8$ & 8.1 & 8.6 & 8.8 & $8.6 \pm 0.6$ & 1888 & 2011 & 1840 & $1901 \pm 106$ \\
\hline & S3 & 30.1 & 28.5 & 28.4 & $29.0 \pm 0.9$ & 8.5 & 8.1 & 8.8 & $8.4 \pm 0.2$ & 1886 & 1898 & 2150 & $1978 \pm 119$ \\
\hline \multirow{3}{*}{$\begin{array}{l}\mathrm{PCL}+\mathrm{BCNW}-g- \\
\beta C D-\mathrm{PCL}_{2000}(3 \%)\end{array}$} & S1 & 30.3 & 29.0 & 27.2 & $28.8 \pm 1.4$ & 7.4 & 7.1 & 6.8 & $7.1 \pm 0.2$ & 2315 & 2359 & 2382 & $2352 \pm 130$ \\
\hline & S2 & 30.3 & 29.5 & 32.3 & $30.7 \pm 1.8$ & 7.4 & 7.2 & 7.0 & $7.2 \pm 0.1$ & 2320 & 2205 & 2144 & $2223 \pm 36$ \\
\hline & S3 & 29.8 & 31.1 & 29.7 & $30.2 \pm 1.0$ & 7.3 & 7.8 & 7.9 & $7.7 \pm 0.3$ & 2175 & 2019 & 1971 & $2055 \pm 46$ \\
\hline \multirow{3}{*}{$\begin{array}{c}\mathrm{PCL}+\mathrm{BCNW}-g- \\
\beta C D-\mathrm{PCL}_{2000}(5 \%)\end{array}$} & S1 & 26.5 & 27.4 & 29.2 & $27.7 \pm 0.8$ & 3.4 & 3.3 & 3.8 & $3.5 \pm 0.3$ & 2302 & 2229 & 2291 & $2274 \pm 102$ \\
\hline & S2 & 28.5 & 26.1 & 26.7 & $27.1 \pm 1.2$ & 3.3 & 3.0 & 3.3 & $3.2 \pm 0.4$ & 2139 & 2133 & 2328 & $2200 \pm 66$ \\
\hline & S3 & 26.1 & 25.3 & 25.4 & $25.6 \pm 1.6$ & 4.0 & 3.9 & 4.4 & $4.1 \pm 0.2$ & 2304 & 2217 & 2244 & $2255 \pm 56$ \\
\hline
\end{tabular}


ANOVA Single Factor of the Mechanical Parameters of PCL+ BCNW-g- $\beta C D-P C L 2000$ (4\%) System as a Representative

Anova: Single Factor

for $\sigma_{b}$

SUMMARY

\begin{tabular}{lrrrr}
\hline \multicolumn{1}{c}{ Groups } & \multicolumn{1}{c}{ Count } & Sum & Average & Variance \\
\hline S1 & 3 & 91.5 & 30.5 & 2.73 \\
S2 & 3 & 88.6 & 29.53333 & 1.343333 \\
S3 & 3 & 92.1 & 30.7 & 2.41 \\
\hline
\end{tabular}

Anova: Single Factor

for $\boldsymbol{\varepsilon}_{\mathbf{b}}(\%)$

SUMMARY

\begin{tabular}{lrrrr}
\hline \multicolumn{1}{c}{ Groups } & Count & \multicolumn{1}{c}{ Sum } & Average & Variance \\
\hline S1 & 3 & 16.2 & 5.4 & 0.07 \\
S2 & 3 & 16.8 & 5.6 & 0.04 \\
S3 & 3 & 16.2 & 5.4 & 0.09 \\
\hline
\end{tabular}

Anova: Single Factor

for $\mathbf{E}$

SUMMARY

\begin{tabular}{lrrrr}
\hline \multicolumn{1}{c}{ Groups } & Count & \multicolumn{1}{c}{ Sum } & Average & Variance \\
\hline S1 & 3 & 6849 & 2283 & 2803 \\
S2 & 3 & 7293 & 2431 & 301 \\
S3 & 3 & 6954 & 2318 & 10471 \\
\hline
\end{tabular}

\begin{tabular}{|c|c|c|c|c|c|c|}
\hline $\begin{array}{l}\text { Source of } \\
\text { Variation }\end{array}$ & SS & $d f$ & $M S$ & $F$ & P-value & F crit \\
\hline $\begin{array}{l}\text { Between } \\
\text { Groups }\end{array}$ & 2.335556 & 2 & 1.167778 & 0.54036 & 0.608445 & 5.143253 \\
\hline $\begin{array}{l}\text { Within } \\
\text { Groups }\end{array}$ & 12.96667 & 6 & 2.161111 & & & \\
\hline Total & 15.30222 & 8 & & & & \\
\hline ANOVA & & & & & & \\
\hline $\begin{array}{l}\text { Source of } \\
\text { Variation }\end{array}$ & SS & $d f$ & $M S$ & $F$ & P-value & F crit \\
\hline $\begin{array}{l}\text { Between } \\
\text { Groups }\end{array}$ & 0.08 & 2 & 0.04 & 0.6 & 0.578704 & 5.143253 \\
\hline $\begin{array}{l}\text { Within } \\
\text { Groups }\end{array}$ & 0.4 & 6 & 0.066667 & & & \\
\hline Total & 0.48 & 8 & & & & \\
\hline
\end{tabular}

ANOVA

\begin{tabular}{lcccccc}
\hline $\begin{array}{l}\text { Source of } \\
\text { Variation }\end{array}$ & $S S$ & $d f$ & $M S$ & $F$ & P-value & F crit \\
\hline $\begin{array}{l}\text { Between } \\
\text { Groups }\end{array}$ & 35898 & 2 & 17949 & 3.96663 & 0.079854 & 5.143253 \\
Within & 27150 & 6 & 4525 & & & \\
Groups & & & & & & \\
Total & 63048 & 8 & & & & \\
\hline
\end{tabular}




\section{REFERENCES}

[1] Darder, M.; Aranda, P.; Ruiz-Hitzky, E. Bionanocomposite: a new concept of ecological, bioinspired, and functional hybrid materials. Adv. Mater. 2007, 19 (10), 1309-1319.

[2] Iqbal MH, Kyazze G, Tron T, Keshavarz T. Laccase-assisted grafting of poly(3hydroxybutyrate) onto the bacterial cellulose as backbone polymer: development and characterisation. Carbohydr. Polym. 2014; 113: 131-137.

[3] Saharman G, Christophe RT, Nima R, Nattakan S, Ton P. Biodegradable Composites Based on Poly(e-Caprolactone) and Bacterial Cellulose as a Reinforcing Agent, J. Biobased Mater. Bioenergy 4, 384-390, 2010

[4] Shah N, Ul-Islam M, Khattak WA, Park JK. Overview of bacterial cellulose composites: A multipurpose advanced material. Carbohydrate Polymers 2013; 98(2): 1585-1598.

[5] Petersen N, Gatenholm P. Bacterial cellulose-based materials and medical devices: current state and perspectives. Appl Microbiol Biotechnol. 2011; 91: 1277-1286.

[6] Leitão AF, Gupta S, Pedro J, Reviakine I, Gama M Hemocompatibility study of a bacterial cellulose / polyvinyl alcohol nanocomposites. Colloids Surf B: Biointerfaces. 2013; 111: 493-502.

[7] Kim J, Cai Z, Lee HS, Choi GS, Lee DH, Jo C. Preparation and characterization of a bacterial cellulose/chitosan composite for potential biomedical application. J Polym Res. 2010; 18:73944.

[8] Rajwade JM, Paknikar KM, Kumbhar JV. Applications of bacterial cellulose and its composites in biomedicine. Appl Microbiol Biotechnol. 2015; 99: 2491-2511.

[9] Hu W, Chen S, Yang J, Li Z, Wang H. Functionalized bacterial cellulose derivatives and nanocomposites. Carbohydr Polym. 2014; 101: 1043-1060.

[10] Xia YY, Wan JM. Preparation and adsorption of novel cellulosic fibers modified by $\beta$ cyclodextrin. Polym. Adv. Technol. 2008; 19(4): 270-275.

[11] Zhao Q, Wang SF, Cheng XJ, Yam RCM, Kong D, Li RKY. Surface modification of cellulose fiber via supramolecular assembly of biodegradable polyesters by the aid of host-guest inclusion complexation. Biomacromolecules. 2010; 11(5): 1364-1369.

[12] Lin, N.; Huang, J.; Dufresne, A. Preparation, properties and applications of polysaccharide nanocrystals in advanced functional nanomaterials: a review. Nanoscale 2012, 4 (11), 3274-3294.

[13] S. Eyley, W. Thielemans, Nanoscale 2014, 6, 7764.

[14] Corrêa AC, Teixeira EM, Pessan LA, Mattoso LHC. Cellulose nanofibers from curaua fibers. Cellulose. 2010; 17(6): 1183-1192.

[15] Jorfi M, Foster EJ. Recent advances in nanocellulose for biomedical applications. A Review. Journal of Applied Polymer Science. 2015; 132(14): 41719. 
[16] Wu X, Moon RJ, Martini A. Crystalline cellulose elastic modulus predicted by atomistic models of uniform deformation and nanoscale indentation. Cellulose. 2013; 20: 43-55.

[17] Simone EA, Dziubla TD, Muzykantov VR. Polymeric carriers: role of geometry in drug delivery. Expert Opin Drug Deliv. 2008; 5: 1283-1300.

[18] S. Dong, H. J. Cho, Y. W. Lee, M. Roman, Biomacromolecules 2014, 15, 1560.

[19] Loftsson, T.; Brewster, M.E. J. Pharm. Sci. 2012, 101, 3019-3032.

[20] Shuai, X.; Porbeni, F.E.; Wei, M.; Bullions, T.; Tonelli, A.E. Macromolecules. 2002, 35, 3778-3780

[21] Manakker, F.V.d.; Vermonden,T.; Nostrum, C.F.V.; Hennink, W.E. Biomacromolecules. 2009, 10, 3157-3175.

[22] Ulery BD, Nair LS, Laurencin CT. Biomedical Applications of Biodegradable Polymers. J Polym Sci B Polym Phys. 2011; 49(12): 832-864.

[23] Bikiaris, D.N.; Papageorgiou, G.Z.; Achilias, D.S.; Pavlidou, E.; Stergiou, A. Eur. Polym. J.2007, 43(6), 2491-2503.

[24] Murthy, R.S. In Biodegradable Polymers; Jain, N. K.; Eds.; CBS Publisher: New Dehli, 1997; pp 27-51.

[25] Sun, H.F.; Mei, L.; Song, C.X.; Cui, X.M.; Wang, P.Y. Biomaterials. 2006; 27(9), $1735-1740$.

[26] Roosa, S.; Moffitt, E. Journal of Biomedical Materials Research Part A. 2010, 92(1), 359-368.

[27] Jackson JK. Letchford K, Wasserman BZ. The use of nanocrystalline cellulose for the binding and L controlled release of drugs. Inter J Nanomed. 2011; 6:321-330.

[28] Bin WS, Swee-Hin T, Lifeng K. Polycaprolactone scaffold as targeted drug delivery system and cell attachment scaffold for postsurgical care of limb salvage. Drug Deliv. and Transl. Res. $2012 ; 2: 272-283$.

[29] Woesz, A. Springer. 2008, 9, 171-205.

[30] Abedalwafa M, Wang F, Wang L, Li C. Biodegradable poly-epsilon-caprolactone (PCL) for tissue engineering applications: A review. Rev. Adv. Mater. Sci. 2013; 34:123-140.

[31] Baroli B. From natural bone grafts to tissue engineering therapeutics: brainstorming on pharmaceutical formulativ requirements and challenges. J Pharm Sci. 2009; 98(4):1317-1375.

[32] Kumari A, Single R, Guliani A, Walia S, Acharya A, Yadav KS (2016). Nanoscale materials in targeted drug delivery. In: Nanoscale Materials in Targeted Drug Delivery, Theragnosis and Tissue Regeneration. S K Yadav, (eds)., (ISBN: 9811008183, 9789811008184), Springe Sciencer, Singapore, pp. 116-120.

[33] Kumari A, Yadav SK, Yadav SC. Biodegradable polymeric nanoparticles based drug delivery systems. Colloids Surf B Biointerfaces. 2010; 75(1): 1-18. 
[34] Papkov MS, Agashi K, Olaye A, shakesheff K, Domb AJ. Polymer carriers for drug delivery in tissue engineering. Adv Drug Deliv Rev. 2007;59:187-206.

[35] Tarun Garg, Onkar Singh, Saahil Arora, R.S.R. Murthy. Scaffold: A Novel Carrier for Cell and Drug Delivery. Critical Reviews ${ }^{\mathrm{TM}}$ in Therapeutic Drug Carrier Systems, 29(1), 1-63 (2012)

[36] Mouriño V, Boccaccini AR. Bone tissue engineering therapeutics: controlled drug delivery in three-dimensional scaffolds. J R Soc Interface. 2010 ;7(43): 209-227.

[37] Park SA, Lee SH, Kim WD. Fabrication of porous polycaprolactone/hydroxyapatite (PCL/HA) blend scaffolds using a 3D plotting system for bone tissue engineering. Bioprocess Biosyst Eng. 2011; 34 (4): 505-513.

[38] Martin DP, Williams SF (2003) Biochem Eng J 16:97-105

[39] Radhika D, Murugesan AG (2012) Bioresour Technol 121:83-92.

[40] Poomipuk N, Reungsang A, PlangklangP (2014) Int J Biol Macromol 65:51-64

[41] Pakarinen A, Maijala P, Stoddard FL, Santanen A, Tuomainen P, Kymalainen M, Viikari L (2011) Biomass and bioenergy 35:3071-78

[42] Azizi Samir MAS, Alloin F, Dufresne A. Review of recent research into cellulosic whiskers, their properties and their application in nanocomposite field. Biomacromolecules. 2005a; 6: 612-626.

[43] Moon RJ, Martini A, Nairn J, Simonsen J, Youngblood J. Cellulose nanomaterials review: structure, properties and nanocomposites. Chem Soc Rev. 2011; 40(7): 39413994.

[44] Nishiyama Y. Structure and properties of the cellulose microfibril. J Wood Sci. 2009; 55: 241-49.

[45] Habibi Y, Lucia LA, Rojas OJ. Cellulose nanocrystals: Chemistry, self-assembly, and applications. Chem. Rev. 2010; 110: 3479-3500.

[46] Lin N, Dufresne A. Supramolecular hydrogels from in situ host-guest inclusion between chemically modified cellulose nanocrystals and cyclodextrin. Biomacromolecules. 2013; 14(3): 871-880.

[47] Iqbal HMN, Kyazze G, Locke IC, Tron T, Keshavarz T. Development of biocomposites with novel characteristics: Evaluation of phenolinduced antibacterial, biocompatible and biodegradable behaviours. Carbohydrate Polymers. 2015; 131: 197-207.

[48] Iguchi M, Yamanaka S, Budhiono A. Bacterial cellulose - a masterpiece of nature's arts. J Mater Sci. 2000; 35: 261-270.

[49] Lee KY, Tammelin T, Schulfter K, Kiiskinen H, Samela J, Bismarck A. High performance Cellulose nanocomposites: comparing the reinforcing ability of bacterial cellulose and nanofibrillated cellulose. ACS Appl Mater Interfaces 2012;4(8):407886. 
[50] Bielecki S, Krystoynowicz A, Turkiewicz M, Kalinowska H. Bacterial cellulose. In: Vandamme EJ, De Baets S, Steinb A, editors. Biopolymers: Vol 5, Polysaccharides I, Polysaccharides from Prokaryotes. Weinham: Wiley; 2001. p. 37-46.

[51] Son HJ, Heo MS, Kim YG, Lee SJ. Optimization of fermentation conditions for the production of bacterial cellulose by a newly isolated Acetobacter. Biotechnology and Applied Biochemistry. 2001; 33(1): 1-5.

[52] Castro C, Zuluaga R, Putaux JL, Caro G, Mondragon I, Ganan P. Structural characterization of bacterial cellulose produced by Gluconacetobacter swingsii sp. from Colombian agro-industrial wastes. Carbohydr Polym. 2011; 84: 96-102.

[53] Iqbal HMN, Kyazze G, Keshavarz T. Advances in the valorization of lignocellulosic materials by biotechnology: An overview. BioResources. 2013;8(2): 3157- 3176.

[54] Bodin A, Bäckdahl H, Fink H, Gustafsson L, Risberg B, Gatenholm P. Influence of cultivation conditions on mechanical and morphological properties of bacterial cellulose tubes. Biotechnol Bioeng. 2007; 97: 425-434.

[55] Rockville MD: FDA, 2012.U.S. Department of Health and Human Services. Pyrogen and endotoxins testing: Questions and answers. In: Guidance for industry.

[56] Retegi A, Gabilondo N, Peña C, Zuluaga R, Castro C, Gañan P, de la Caba K, Mondragon I. Bacterial cellulose films with controlled microstructure-mechanical property relationships. Cellulose 2010; 17:661-669.

[57] Yamanaka DS, Watanabe K, Kitamura N, Iguchi M, Mitsuhashi S, Nishi Y, Uryu M. The structure and mechanical properties of sheets prepared from bacterial cellulose. $J$ Mater Sci. 1989; 24: 3141-3145.

[58] McKenna BA, Mikkelsen D, Wehr JB, Gidley MJ, Menzies NW. Mechanical and structural properties of native and alkali-treated bacterial cellulose produced by Gluconacetobacter xylinus strain ATCC 53524. Cellulose. 2009; 16: 1047-1055.

[59] Nishi Y, Uryu M, Yamanaka S, Watanabe K, Kitamura N, Iguchi M, Mitsuhashi S. The structure and mechanical properties of sheets prepared from bacterial cellulose. J Mater Sci. 1990; 25: 2997-3001.

[60] Guhados G, Wan W, Hutter JL. Measurement of the elastic modulus of single bacterial cellulose fibers using atomic force microscopy. Langmuir. 2005; 21: 6642-6646.

[61] Hsieh Y-C, Yano H, Nogi M, Eichhorn SJ. An estimation of the Young's modulus of bacterial cellulose filaments. Cellulose. 2008; 15: 507-513.

[62] Jozala AF, de Lencastre-Novaes LC, Lopes AM, de Carvalho Santos-Ebinuma V, Mazzola PG, Pessoa-Jr A, et al. Bacterial nanocellulose production and application: a 10-year overview. Applied Microbiology and Biotechnology. 2016; 100(5): 20632072.

[63] Solway DR, Clark WA, Levinson DJ. A parallel open-label trial to evaluate microbial cellulose wound dressing in the treatment of diabetic foot ulcers. Int Wound J. 2011; 8(1): 69-73. 
[64] Czaja WK, Young DJ, Kawecki M, Brown RM Jr. The future prospects of microbial cellulose in biomedical applications. Biomacromolecules, 2007; 8(1): 1-12.

[65] Szejtli J. Introduction and general overview of cyclodextrin chemistry. Chem Rev. 1998; 98(5):1743-1753.

[66] Dong C, Li C, XiaoH, He B, Qian L. $\beta$-Cyclodextrin Grafted Cellulose and Cationic Starch for Antibacterial Paper Products: A Comparative Study. BioResources. 2014; 9(2): 3580-3590.

[67] Landy, D., Mallard, I., Ponchel, A., and Monflier, E. Remediation technologies using cyclodextrins: An overview. Chem. Lett. 2012; 10(3):225-237.

[68] Peila R, Migliavacca G, Aimone F, Ferri A, Sicardi S. A comparison of analytical methods for the quantification of a reactive $\beta$-cyclodextrin fixed onto cotton yarns. Cellulose. 2012; 19(4): 1097-1105.

[69] Astray G, Gonzalez-Barreiro C, Mejuto JC, Rial-Otero R, Simal-Gandara J. A review on the use of cyclodextrins in foods. Food Hydrocoll. 2009; 23(7):1631-1640.

[70] Kurkov SV, Ukhatskaya EV, Loftsson T. Drug/cyclodextrin:beyond inclusion complexation. J Incl Phenom Macrocycl Chem. 2011; 69(3-4): 297-301.

[71] Singh M, Sharma R, Banerjee UC. Biotechnological applications of cyclodextrins. Biotechnol Adv. 2002; 20(5): 341-359.

[72] Gonil P, Sajomsang W, Ruktanonchai U R, Pimpha N, Sramala I, Nuchuchua O, Puttipipatkhachorn, S. Novel quaternized chitosan containing $\beta$-cyclodextrin moiety: Synthesis, characterization and antimicrobial activity. Carbohyd. Polym. 2011; 83(2): 905-913.

[73] Dong T, He Y, Shin K, Inoue Y. Formation and Characterization of Inclusion Complexes of Poly(butylene succinate) with $\alpha$ - and $\gamma$-Cyclodextrins. Macromol. Biosci. 2004; 4: 1084-1091.

[74] Hasegawa Y, Miyauchi M, Takashima Y, Yamaguchi H, Harada A. Supramolecular Polymers Formed from -Cyclodextrins Dimer Linked by Poly(ethylene glycol) and Guest Dimers. Macromolecules. 2005; 38: 3724-3730.

[75] Eftink MR, Andy ML, Bystrom K, Perlmutter HD, Kristol DSJ. Cyclodextrin inclusion complexes: Studies of the variation in the size of alicyclic guests. J. Am. Chem. Soc. 1989; 111: 6765-6772.

[76] Zhang ZX, Liu X, Xu FJ, Loh XJ, Kang ET, Neoh KG, Li J. Pseudo-block copolymer based on star-shaped poly ( $\mathrm{N}$-isopropylacrylamide) with a $\beta$-cyclodextrin core and guest-bearing PEG: controlling thermoresponsivity through supramolecular selfassembly. Macromolecules. 2008, 41, 5967-5970.

[77] Koopmans, C.; Ritter, H. Formation of Physical Hydrogels via Host-Guest Interactions of $\beta$-Cyclodextrin Polymers and Copolymers Bearing Adamantyl Groups. Macromolecules. 2008; 41: 7418-7422. 
[78] Denter U, Schollmeyer E (1997). Surface modification of synthetic and natural fibres by fixation of cyclodextrin derivatives. In: Proceedings of the 8th international symposium on cyclodextrins, pp 559-564.

[79] Lee MH, Yoon KJ, Ko S. Grafting onto cotton fiber with acrylamidomethylated $\beta$ cyclodextrin and its application. J Appl Polym Sci. 2000; 78(11):1986-1991.

[80] Gawish SM, Ramadan AM, Abo El-Ola SM, Abou El-Kheir AA. Citric acid as a cross-linking agent for grafting bcyclodextrin onto wool fabric. Polym Plast Technol Eng. 2009; 48(7):701-710.

[81] Qing Y, Sabo R, Wu Y, Zhu JY, Cai Z. Self-assembled optically transparent cellulose nanofibril films: Effect of nanofibril morphology and drying procedure. Cellulose. 2015; 22(2): 1091-1102.

[82] Chen CY, Chen CC, Chung Y C. Removal of phthalate esters by alpha-cyclodextrinlinked chitosan bead. Bioresour. Technol. 2007;98(13): 2578-2583.

[83] Labet M, Thielemans W. Synthesis of polycaprolactone: a review. Chemical Society Reviews, Vol 38, 2009, pp. 3484-3504

[84] Kalfogu NK, J. Appl. Polym. Sci. 1983: 28, 255.

[85] Eshraghi S, Das S. Mechanical and Microstructural Properties of Polycaprolactone Scaffolds with 1-D, 2-D, and 3-D Orthogonally Oriented Porous Architectures Produced by Selective Laser Sintering. Acta Biomater. 2010 ; 6(7): 2467-2476.

[86] Gautam R., Bassi A.S., Yanful E.K., A Review of Biodegradation of Synthetic Plastic and Foams. Appl Biochem Biotechnol. pp. 141, 2007.

[87] Gunatillake PA, Adhikari A. "Biodegradable synthetic polymers for tissue engineering". European Cells and Materials, Vol 5, 2003, pp. 1-16.

[88] Jayasekara R., Harding I., Bowater I., Lonergan G. Biodegradability of Selected Range of Polymers and Polymer Blends and Standard Methods for Assessment of Biodegradation. J Polymer Environ. 2005;13:231.

[89] Rutkowska M., Heimowska A., Krasowska K., Janik H. Biodegradation of PCL in sea water. Reactive\&Functional Polymers. 1998;38; 27.

[90] Woodward C S. The intracellular degradation of poly( $\varepsilon$-caprolactone). Journal of biomedical materials research, Vol 19, 1985, pp. 437-44.

[91] Albertsson AC, Karlsson S. Controlled degradation by artificial and biological processes. In: Hatada K, Kitayama T, Vogl O, editors. Macromolecular design of polymeric materials. New York/Basel/Hong Kong: Marcel Dekker Inc.; 1997. p. 739_ 80.

[92] Hutmacher DW. Scaffold design and fabrication technologies for engineering tissuesstate of the art and future perspectives. J Biomater Sci Polym Ed 2001;12:107-24.

[93] Gunatillake PA, Biotechnol Annu Rev 2006, 301.

[94] Lam CXF, Savalani MM, Teoh SH, Hutmacher DW. Dynamics of in vitro polymer degradation of polycaprolactone-based scaffolds: accelerated versus simulated physiological conditions. Biomed Mater 2008;3:1-15. 
[95] Williams DF. Revisiting the definition of biocompatibility. Med Dev Technol 2003;14(8):10-3.

[96] Williams DF. On the mechanisms of biocompatibility. Biomaterials. 2008;29:294153.

[97] Tang ZG, Black RA, Curran JM, Hunt JA, Rhodes NP, Williams DF. Biomaterials. 2004; 25, 4741.

[98] Xiang P, Li M, Zhang C-Y, Chen D-L, Zhou Z-H. Int. J. Biol. Macromol. 2011; 49:281.

[99] Chen G, Zhou P, Mei N, Chen X, Shao Z, Pan L, Wu C. J. Mater. Sci-Mater. Med. 2004; 15, 671.

[100] Bao TQ, Franco RA and Lee PT. Biochem. Eng. J. 2012; 64, 76.

[101] Savarino L, Baldini N, Greco M, Capitani O, Pinna S, Valentini S, Lombardo B, Esposito MT, et al. Biomaterials. 2007; 28, 3101.

[102] Pankajakshan D, Philipose LP, Palakkal M, Krishnan K, Krishnan LK . J. Biomed. Mater. Res. B Appl. Biomater. 2008; 87B, 570.

[103] Woodruff MA, Hutmacher DW. The return of a forgotten polymer Polycaprolactone in the $21^{\text {st }}$ century. Prog Polym Sci (2010), doi:10.1016/j.progpolymsci.2010.04.002

[104] Báez JE, Fernández AM, Martínez-Richa A, Macromolecules 2005, 38, 1599.

[105] Li X, Li R, Qian X, Ding Y, Tu Y, Guo R, et al. Superior antitumor efficiency of cisplatin-loaded nanoparticles by intratumoral delivery with decreased tumor metabolism rate. Eur J Pharm Biopharm. 2008;70(3):726-34.

[106] Gou M, Zheng X, Men K, Zhang J, Zheng L, Wang X. Poly( $\varepsilon$-caprolactone) /poly(ethylene glycol)/poly( $\varepsilon$-caprolactone) nanoparticles: preparation, characterization, and application in doxorubicin delivery. J Phys Chem B. 2009;113 (39):12928-33.

[107] Teo EY, Ong SY, Chong MS, Zhang Z, Lu J, Moochhala S, et al. Polycaprolactonebased fused deposition modeled mesh for delivery of antibacterial agents to infected wounds. Biomaterials. 2011;32(1):279-87.

[108] Yoshimoto H, Shina YM, Teraia H, Vacanti J P. "A biodegradable nanofiber scaffold by electrospinning and its potential for bone tissue engineering". Biomaterials. 2003; 24:2077-82.

[109] Shin M, Yoshimoto $\mathrm{H}$ and Vacanti J P. "In vivo bone tissue engineering using mesenchymal stem cells on a novel electrospun nanofibrous scaffold". Tissue Eng Part A. 2004; 10: 33-41.

[110] Lia W J, Tuli R, Huang X, Laquerriere P, Tuan R S. "Multilineage differentiation of human mesenchymal stem cells in a three-dimensional nanofibrous scaffold". Biomaterials, Vol 26, 2005, PP. 5158-5166.

[111] Shao X, Goh J C, Hutmacher D W, Lee E H, Zigang G., "Repair of large articular osteochondral defects using hybrid scaffolds and bone marrow-derived mesenchymal stem cells in a rabbit model". Tissue Eng Part A. 2006; 12:1539-51. 
[112] Choi J S, Lee S G, Christ G J, Atala A, Yoo J J. "The influence of electrospun aligned poly (epsilon-caprolactone)/collagen nanofiber meshes on the formation of selfaligned skeletal muscle myotubes". Biomaterials, Vol 29, 2008, pp. 2899-906.

[113] Li W J, Chiang H, Kuo T F, Lee H S, Jiang C C, Tuan R S. "Evaluation of articular cartilage repair using biodegradable nanofibrous scaffolds in a swine model: a pilot study". J. Tissue Eng. Regener. Med. 2009: 2;1-10.

[114] Puppi D, Detta N, Piras A M, Chiellini F, Clarke D A, Reilly G C, Chiellini E. "Development of Electrospun Three-arm Star Poly( $\varepsilon$-caprolactone) Meshes for Tissue Engineering Applications". Macromol. Biosci. Vol 10, 2010, pp. 887-897.

[115] Jha B S, Colello R J, Bowman J R, Sell S A, Lee K D, Bigbee J W, Bowlin G L, Chow W N, Mathern B E, Simpson D G. "Two pole air gap electrospinning: fabrication of highly aligned, three-dimensional scaffolds for nerve reconstruction". Acta Biomaterialia, 2011; 7:203-215.

[116] Ruckh T T, Oldinski R A, Carroll D A, Mikhova K, Bryers J D, Popat K C. "Antimicrobial effects of nanofiber poly (caprolactone) tissue scaffolds releasing rifampicin". J Mater Sci: Mater Med. 2012; 23:1411-1420.

[117] Wong B.S, Teoh S, Kang L. Polycaprolactone scaffold as targeted drug delivery system and cell attachment scaffold for postsurgical care of limb salvage. Drug Deliv. and Transl. Res. (2012) 2: 272. doi:10.1007/s13346-012-0096-9

[118] Diban N, Haimi S,Bolhuis-Versteeg L, Teixeira S, Miettinen S, Poot A, Grijpma D, Stamatialis D. "Hollow fibers of poly(lactide-co-glycolide) and poly(e-caprolactone) blends for vascular tissue engineering applications". Acta Biomaterialia, Vol 9, 2013, 6450-6458.

[119] Patrício T, Domingos M, Gloria A, Bártolo P. Characterization of PCL and PCL/PLA Scaffolds for Tissue Engineering. Procedia CIRP. 2013; 5:110-114.

[120] Koupaei N, Karkhaneh A. Porous crosslinked polycaprolactone hydroxyapatite networks for bone tissue engineering. Tissue Eng Regen Med (2015) 13: 251. doi:10.1007/s13770-016-9061-x

[121] Wang S-J, Zhang Z-Z, Jiang D, Qi Y-S, Wang H-J, Zhang J-Y, Ding J-X, Yu J-K. Thermogel-Coated Poly("-Caprolactone) Composite Scaffold for Enhanced Cartilage Tissue Engineering. Polymers. 2016, 8, 200; doi:10.3390/polym8050200.

[122] Takada K. Microfabrication-derived DDS: from batch to individual production. Drug Discov Ther. 2008; 2(3): 140-155.

[123] Chen B, Evans RG. Macromol. 2006; 39: 747.

[124] Saeed, K.; Park, S.-Y.; Lee, H.-J.; Baek, J.-B.; Huh, W.-S. Polymer 2006, 47, 80198025.

[125] Barron AR, Khan MR. Adv. Mater. Processes 2008, 166, $41-43$.

[126] Smart SK, Cassady A I, Lu GQ, Martin DJ. Carbon 2006, 44, 1034-1047.

[127] GeunHyung K, Taijin M, Su AP, Wan DK, Young HK. Biomed. Mater. 2007; 2: 250256. 
[128] Lee K-Y, Aitomäki Y, Berglund AL, Oksman K, Bismarck A. On the use of nanocellulose as reinforcement in polymer matrix composites. Composites Science and Technology. 2014; 105: 15-27.

[129] Rojas OJ, Montero GA, Habibi Y. Electrospun nanocomposites from polystyrene loaded with cellulose nanowhiskers. J Appl Polymer Sci. 2009; 113: 927-935.

[130] Wu X, Torres FJ, Vilaseca F, Peijs T. J. Biobased Mater. Bioenergy. 2007; 1:341.

[131] S.-M. Lien, L.-Y. Ko, T.-J. Huang, Effect of pore size on ECM secretion and cell growth in gelatin scaffold for articular cartilage tissue engineering, Acta Biomaterialia 5 (2) (2009) 670-679.

[132] N. Annabi, J.W. Nichol, X. Zhong, C. Ji, S. Koshy, A. Khademhosseini, F. Dehghani, Controlling the porosity and microarchitecture of hydrogels for tissue engineering, Tissue Engineering: Part B 16 (4) (2010) 371-380.

[133] J.J.A. Barry, M.M.C.G. Silva, S.H. Cartmell, R.E. Guldberg, C.A. Scotchford, S.M. Howdle, Porous methacrylate tissue engineering scaffolds: using carbon dioxide to control porosity and interconnectivity, J. Materials Science 41 (13) (2006) 4197-4204.

[134] D.J. Mooney, D.F. Baldwin, N.P. Suh, J.P. Vacanti, R. Langer, Novel approach to fabricate porous sponges of poly(d,1-lactic-co-glycolic acid) without the use of organic solvents, Biomaterials 17 (14) (1996) 1417-1422.

[135] D. Hutmacher, Scaffolds in tissue engineering bone and cartilage, Biomaterials 21 (2000) 2529-2543.

[136] A. Salerno, S. Iannace, P.A. Netti, Open-pore biodegradable foams prepared via gas foaming and microparticulate templating, Macromolecular Biosciences 8 (2008) 655664.

[137] Tacar, O; Sriamornsak, P; Dass, CR. "Doxorubicin: an update on anticancer molecular action, toxicity and novel drug delivery systems.". The Journal of Pharmacy and Pharmacology 2013:65 (2): 157-70. doi:10.1111/j.2042-7158.2012.01567.x. PMID 23278683.

[138] Doxorubicin: MedlinePlus Drug Information". www.nlm.nih.gov. Retrieved 2016-0201

[139] Rossi, S, ed. (2013). Australian Medicines Handbook (2013 ed.,). Adelaide: The Australian Medicines Handbook Unit Trust. ISBN 978-0-9805790-9-3.

[140] Lindell EB, Carroll NC. Limb salvage tumor surgery in children. Iowa Orthop J. 1993;13:124-35.

[141] Carty CP, Dickinson IC, Watts MC, Crawford RW, Steadman P. Impairment and disability following limb salvage procedures for bone sarcoma. Knee. 2009; 16(5): 405-408.

[142] Agins HJ, Alcock NW, Bansal M, Salvati EA, Wilson Jr PD, Pellicci PM, et al. Metallic wear in failed titanium-alloy total hip replacements. A histological and quantitative analysis. J Bone Joint Surg Am. 1988;70(3):347-56. 
[143] Lalor PA, Revell PA, Gray AB, Wright S, Railton GT, Freeman MA. Sensitivity to titanium. A cause of implant failure, J Bone Joint Surg Br. 1991;73(1):25-8.

[144] Uhthoff HK, Finnegan M. The effects of metal plates on posttraumatic remodelling and bone mass. J Bone Joint Surg Br. 1983;65(1):66-71.

[145] Yaremchuk MJ, Fiala TG, Barker F, Ragland R. The effects of rigid fixation on craniofacial growth of rhesus monkeys. Plast Reconstr Surg. 1994; 93(1): 1-10.

[146] Sullivan PK, Smith JF, Rozzelle AA. Cranio-orbital reconstruction: safety and image quality of metallic implants on CT and MRI scanning. Plast Reconstr Surg. 1994;94(5):589-96.

[147] Dhillon MS, Prabhakar S, Prasanna C. Preliminary experience with biodegradable implants for fracture fixation. Indian J Orthop. 2008; 42(3): 319-322.

[148] Moore WR, Graves SE, Bain GI. Synthetic bone graft substitutes. ANZ J Surg. 2001;71(6):354-61.

[149] Kurz LT, Garfin SR, Booth Jr RE. Harvesting autogenous iliac bone grafts. A review of complications and techniques. 1989;14(12):1324-31.

[150] Schuckert KH, Jopp S, Teoh SH. Mandibular defect reconstruction using threedimensional polycaprolactone scaffold in combination with platelet-rich plasma and recombinant human bone morphogenetic protein-2: de novo synthesis of bone in a single case. Tissue Eng Part A. 2009; 15(3):493-499.

[151] Rosen G, Caparros B, Huvos AG, Kosloff C, Nirenberg A, Cacavio A, et al. Preoperative chemotherapy for osteogenic-sarcoma: selection of postoperative adjuvant chemotherapy based on the response of the primary tumor to preoperative chemotherapy. Cancer. 1982;49 (6):1221-30.

[152] Susa M, Iyer AK, Ryu K, Hornicek FJ, Mankin H, Amiji MM, et al. Doxorubicin loaded polymeric nanoparticulate delivery system to overcome drug resistance in osteosarcoma. BMC Cancer. 2009;9:399.

[153] Hogendoorn PC, Athanasou N, Bielack S, De Alava E, Dei Tos AP, Ferrari S, et al. Bone sarcomas: ESMO clinical practice guidelines for diagnosis, treatment and follow-up. Ann Oncol. 2010;21 Suppl 5:v204-13.

[154] Matsumine A, Takegami K, Asanuma K, Matsubara T, Nakamura T, Uchida A, et al. A novel hyperthermia treatment for bone metastases using magnetic materials. Int $\mathbf{J}$ Clin Oncol. 2011;16 (2):101-8

[155] Gaur AH, Liu T, Knapp KM, Daw NC, Rao BN, Neel MD, et al. Infections in children and young adults with bone malignancies undergoing limb-sparing surgery. Cancer. 2005; 104(3): 602-10.

[156] Goff BJ, Castillo R, Raja SN. Painful sequelae following limb salvage: etiology and management. J Am Acad Orthop Surg. 2011; 19 Suppl 1:S23-7.

[157] Jeon JM, Brigham CL, Kim YH, Kim HJ, Yi DD, Kim H (2014) Appl Microbiol Biotechnol. 98 (12):5461-5469

[158] Reusch RN, Sparrow AW, Gardiner J (1992) Biochim Biophys Acta 1123:33-40 
[159] Khanna S, Srivastava AK (2005) Process Biochem 40:2173-2182

[160] K. Sudesh, H. Abe, Y. Doi, Synthesis, structure and properties of polyhydroxyalkanoates (2000) Prog Polym Sci 25:1503-1555

[161] Anderson AJ, Dawes EA. Occurence, metabolism, metabolic rate, and industrial uses of bacterial polyhydroxyalkanoates. Microbiol Rev 1990;54(4):450-72.

[162] Doi Y, Kawaguchi Y, Koyama N, Nakamura S, Hiramitsu M, Kimura H. Synthesis and degradation of polyhydroxyalkanoates in Alcaligenes eutrophus. FEMS Microbiol Rev 1992;103:103-8.

[163] Sreekanth, M.S., Vijayendra, S.V.N., Joshi, G.J., Shamala, T.R., 2013. Effect of carbonand nitrogen sources on simultaneous production of amylase and green food packaging polymer by Bacillus sp. J. Food Sci. Technol. 50 (2), 404-408.

[164] Johnson, K., Kleerebezem, R., van Loosdrecht, M.C., 2010. Influence of the C/N ratio on the performance of polyhydroxybutyrate $(\mathrm{P}(3 \mathrm{HB}))$ producing sequencing batch reactors at short SRTs. Water Res. 44, 2141-2152.

[165] Varshney VK, Naithani S. Chemical Functionalization of Cellulose Derived from Nonconventional Sources. In: Kalia S, Kaith BS, Kaur I, editrs. Cellulose Fibers Bioand Nano-Polymer Composites. London New York: Springer Heidelberg Dordrecht; 2011. p. 45.

[166] Kumari A, Single R, Guliani A, Walia S, Acharya A, Yadav KS. Nanoscale materials in targeted drug delivery. In: Yadav S K, editors. Nanoscale Materials in Targeted Drug Delivery, Theragnosis and Tissue Regeneration. Singapore: Springe Sciencer; 2016. p. 116-20.

[167] Kalia S, Dufresne A, Cherian MB, Kaith SB, Avérous L, Njuguna J, Nassiopoulos E. Cellulose-Based Bio- and Nanocomposites: A Review. International Journal of Polymer Science. 2011; 875, 35.

[168] Dufresne A. Cellulose-based composites and nanocomposites. In: Gandini A, Belgacem NM, editors, $1^{\text {st }}$ ed. Monomers, Polymers and Composites from Renewable Resources. Oxford, UK: Elsevier; 2008. p. 401-18.

[169] Coffey DG, Bell DA, Henderson A. Cellulose and cellulose derivatives. In: Stephen M, Collar C, Martinez JC, Rosell CM, editors. A Food Polysaccharides and Their Applications. New York: Marcel Dekke; 1995. p. 123-53.

[170] El-Saied H, Basta AH, Gobran RH. Research progress in friendly environmental technology for the production of cellulose products (bacterial cellulose and its application). Polym Plast Technol Eng. 2004; 43:797-820.

[171] Hon DNS. Chemical modification of lignocellulosic material. New York Inada: Marcel Dekker; 1996.

[172] Barud HS, Barrios C, Regiani T. Self-supported silver nanoparticles containing bacterial cellulose membranes. Mater Sci Eng C-Biomim Supramol Syst. 2008; 28: $515-18$. 
[173] John MJ, Thomas S. Biofibres and biocomposites. Carbohydr. Polym. 2008; 71: 34364.

[174] Lu J, Askeland P, Drzal LT. Surface modification of microfibrillated cellulose for epoxy composite applications. Polymer. 2008; 49: 1285-96.

[175] Stenstad P, Andresen M, Tanem BS, Stenius P. Chemical surface modifications of microfibrillated cellulose. Cellulose. 2008; 15: 35-45.

[176] Thielemans W, Warbey CR, Walsh DA. Permselective nanostructured membranes based on cellulose nanowhiskers. Green Chem. 2009; 11: 531-37.

[177] Sjöström E. Wood Chemistry Fundamentals and Applications. New York, USA: Academic Press; 1981.

[178] Daniel JR Cellulose structure and properties. In: Kroschwitz JI, editor. Encyclopedia of Polymer Science and Engineering. New York, USA: Wiley-Interscience Publication John Wiley \& Sons; Volume 3, 1985. p. 86-123.

[179] Bledzki AK, Gassan J. Composites reinforced with cellulose based fibres. Prog. Polym. Sci. 1999; 24: 221-74.

[180] Sugiyama J, Persson J, Chanzi H. Combined infrared and electron diffraction study of polymorphism of native cellulose. Macromolecules. 1991; 24: 2461-66.

[181] Dinand E, Vignon M, Chanzy H, Heux L. Mercerization of primary wall cellulose and its implication for the conversion of cellulose I $\rightarrow$ cellulose II. Cellulose. 2002; 9: 7-18.

[182] French AD, Bertoniere NR, Brown RM, Chanzy H, Gray D, Hattori K, et al. In: Kroschwitz JI, editor. Cellulose in Encyclopedia of Polymler Science and Technology. New Jersey, USA: Wiley Interscience Publication John Wiley \& Sons; Volume 5, 2003. p. 473-507.

[183] Saxena IM, Brown RMJ. Cellulose Biosynthesis: Current views and evolving Concepts. Ann. Bot. 2005; 96: 9-21.

[184] Atalla RH, VanderHart DL, David L. Studies of microstructure in native celluloses using solid-state carbon-13 NMR. Macromoles. 1984; 17: 1465-72.

[185] Sugiyama J, Vuong R, Chanzi H. Electron diffraction study on the two crystalline phases occurring in native cellulose from an algal cell wall. Macromolecules. 1991; 24: 4168-75.

[186] De Souza Lima MM, Borsali R. Rodlike cellulose microcrystals: Structure, properties and applications. Macromol. Rapid Commun. 2004; 25: 771-87.

[187] Dufresne A. Polymer nanocomposites from Biological Sources. In: Nalwa HS, editor. Encyclopedia of Nanoscience and Nanotechnology, 2nd ed. American Scientific Publisher: CA, USA: Valencia; 2003.

[188] Nishiyama Y, Sugiyama J, Chanzy H, Langan P. Crystal structure and hydrogen bonding system in cellulose 1(alpha), from synchrotron X-ray and neutron fiber diffraction. J. Am. Chem. Soc. 2003; 125:14300-306. 
[189] Heux L, Bonini C. Microfibrillated and/or Micrcroystalline Dispersion, in Particular of Cellulose, in an Organic Solvent. International Patent WO. 2000/077088,

[190] Sacui IA, Nieuwendaal RC, Burnett DJ, Stranick SJ, Jorfi M, Weder C, et al. Comparison of the Properties of Cellulose Nanocrystals and Cellulose Nanofibrils Isolated from Bacteria, Tunicate, and Wood Processed Using Acid, Enzymatic, Mechanical, and Oxidative Methods. ACS Appl. Mater. Interfaces. 2014; 6: 6127-138.

[191] Habibi Y, Dufresne A. Highly filled bionanocomposites from functionalized polysaccharide nanocrystals. Biomacromolecules. 2008; 9: 1974-80.

[192] Petersson L, Kvien I, Oksman K. Structure and thermal properties of poly(lactic acid)/cellulose whiskers nanocomposites materials. Compos. Sci. Technol. 2007; 67: 2535-44.

[193] Pandey JK, Chu WS, Kim CS, Lee CS, Ahn SH. Bio-nano reinforcement of environmentally degradable polymer matrix by cellulose whiskers from grass. Composites, Part. 2009; 40: 676-80.

[194] Oksman K, Mathew AP, Bondeson D, Kvien I. Manufacturing process of cellulose whiskers/polylactic acid nanocomposites. Compos. Sci. Technol. 2006; 66: 2776-84.

[195] Mangalam AP, Simonsen J, Benight A. Cellulose/DNA hybrid nanomaterials. Biomacromolecules. 2009; 10: 497-504.

[196] Grunert M, Winter WT. Nanocomposites of cellulose acetate butyrate reinforced with cellulose nanocrystals. J. Polym. Envir. 2002; 10:27-30.

[197] Blaker JJ, Lee K-Y, Walters M, Drouet M, Bismarck A. Aligned unidirectional PLA/bacterial cellulose nanocomposite fibre reinforced PDLLA composites. Reactive and Functional Polymers. 2014; 85; 185-92.

[198] George J, Ramana KV, Bawa AS. Siddaramaiah. Bacterial cellulose nanocrystals exhibiting high thermal stability and their polymer nanocomposites. Int $\mathrm{J}$ Biol Macromol. 2011; 48(1): 50-57.

[199] Morandi G, Heath L, Thielemans W. Cellulose nanocrystals grafted with polystyrene chains through surface-initiated atom transfer radical polymerization (SI-ATRP). Langmuir. 2009; 25: 8280-86.

[200] Filson PB, Dawson-Andoh BE. Sono-chemical preparation of cellulose nanocrystals from lignocellulose derived materials. Biores. Technol. 2009; 100: 2259-64.

[201] Alemdar A, Sain M. Biocomposites from wheat straw nanofibers: Morphology, thermal and mechanical properties. Comp. Sci. Technol. 2008; 68: 557-65.

[202] Andresen M, Stenius P. Water-in-oil emulsions stabilized by hydrophobized microfibrillated cellulose. J. Disp. Sci. Technol. 2007; 28: 837-44.

[203] Zimmermann T, Pohler E, Geiger T. Cellulose fibrils for polymer reinforcement. Adv. Eng. Mat. 2004; 6: 754-61.

[204] Ankerfors MP, Kosonen H, Nyknen A, Ahola S, Sterberg M, Roukolainen J, et al. Enzymatic hydrolysis combined with mechanical shearing and high-pressure 
homogenization for nanoscale cellulose fibrils and strong gels. Biomacromolecules. 2007; 8: 1934-41.

[205] Chinga-Carrasco G, Syverud K. Computer-assisted quantification of the multi-scale structure of films made of nanofibrillated cellulose. J. Nanopart. Res. 2010; 12: 84151.

[206] Battista OA, Hill D, Smith PA. Microcrystalline cellulose. Ind. Eng. Chem. 1962; 20 : 54-59.

[207] Aprilia NS, Davoudpour Y, Zulqarnain W, Khalil HA, Hazwan CC, Hossain MS, et al. Physicochemical Characterization of Microcrystalline Cellulose Extracted from Kenaf Bast. BioResources. 2016; 11(2): 3875-89.

[208] Filpponon I. The Synthetic Strategies for Unique Properties in Cellulose Nanocrystal Materials. North Carolina State University, 2009.

[209] Sadeghifar H, Filpponen I, Clarke SP, Brougham DF, Argyropoulos DS. Production of cellulose nanocrystals using hydrobromic acid and click reactions on their surface. Journal of Materials Science. 2011; 46(22): 344-55.

[210] Bai W, Holbery J, Li K. A technique for production of nanocrystalline cellulose with a narrow size distribution. Cellulose. 2009; 16:455-65.

[211] Leung AC, Hrapovic S, Lam E, Liu Y, Male KB, Mahmoud KA, Luong JH. Characteristics and properties of carboxylated cellulose nanocrystals prepared from a novel one-step procedure. Small. 2011; 7: 302-5.

[212] Beck-Candanedo S, Roman M, Gray D. Effect of conditions on the properties behavior of wood cellulose nanocrystals suspensions. Biomacromolecules. 2005; 6:1048-54.

[213] Dong XM, Revol JF, Gray D. Effect of microcrystallite preparation conditions on the formation of colloid crystals of cellulose. Cellulose. 1998; 5:19-32.

[214] Bondeson D, Mathew A, Oksman K. Optimization of the isolation of nanocrystals from microcrystalline cellulose by acid hydrolysis. Cellulose. 2006; 13:171-80.

[215] Elazzouzi-Hafraoui S, Nishiyama S, Putaux J-L, Heux L, Dubreuil F, Rochas C. The shape and size distribution of crystalline nanoparticles prepared by acid hydrolysis of native cellulose. Biomacromolecules. 2008; 9(1): 57-65.

[216] Hasani M, Cranston ED, Westman G, Gray DG. Cationic surface functionalization of cellulose nanocrystals. Soft Matter. 2008; 4: 2238-44.

[217] Viana A, Noseda M, Duarte M, Cerezo A. Alkali modification of carrageenans. Part V. The iota-new hybrid carrageenan from Eucheuma denticulatum and its cyclization to iota-carrageenan. Carbohydr. Polym. 2004; 58: 455-60.

[218] Pomin VH, Valente AP, Pereira MS, Mourão PAS. Mild acid hydrolysis of sulfated fucans: a selective 2-desulfation reaction and an alternative approach for preparing tailored sulfated oligosaccharides. Glycobiology. 2005; 15: 1376-85.

[219] Jiang F, Esker AR, Roman M. Acid-catalyzed and solvolytic desulfation of $\mathrm{H}_{2} \mathrm{SO}_{4}$ hydrolyzed cellulose nanocrystals. Langmuir. 2010; 26(23):17919-25. 
[220] Liu H, Liu D, Yao F, Wu Q. Fabrication and properties of transparent polymethylmethacrylate/cellulose nanocrystals composites. Bioresource Technology. 2010; 101(14): 5685-92.

[221] Araki J, Wada M, Kuga S, Okano T. Birefringent Glassy Phase of a Cellulose Microcrystal Suspension. Langmuir. 2000; 16: 2413-15.

[222] Saito T, Kimura S, Nishiyama Y, Isogai A. Cellulose nanofibers prepared by TEMPO-mediated oxidation of native cellulose. Biomacromolecules. 2007; 8: 248591.

[223] Herrick FW, Casebier RL, Hamilton JK, Aandberg KR. Microfibrillated Cellulose: Morphology and Accessibility. J. Aool. Poly. Sci. App. Polym. Symp. 1983; 37(9),797-813.

[224] Lavoine N, Desloges I, Sillard C. Controlled release and long-term antibacterial activity of chlorhexidine digluconate through the nanoporous network of microfibrillated cellulose. Cellulose. 2014; 21: 4429-42.

[225] Jonoobi M, Oladi R, Davoudpour Y, Oksman K, Dufresne A, Hamzeh Y, Davoodi R. Different preparation methods and properties of nanostructured cellulose from various natural resources and residues: a review. Cellulose, 2015, 22: 935-69.

[226] Miao C, Hamad WY. Cellulose reinforced polymer composites and nanocomposites: a critical review. Cellulose. 2013; 20: 2221-62.

[227] Siqueira G, Bras J, Dufresne A. cellulose nanowhiskers vs. microfibrils. Biomacromolecules. 2009; 10: 425-32.

[228] Habibi Y. Key advances in the chemical modification of nanocelluloses. Chem. Soc. Rev. 2014; 43, 1519-42.

[229] Revol JF, Bradford H, Giasson J, Marchessault RH, Gray DG. Helicoidal selfordering of cellulose microfibrils in aqueous suspension. Int. J. Biol. Macromol. 1992; 14: 170-72.

[230] Bordel D, Putaux JL, Heux L. Orientation of native cellulose in an electric field. Langmuir. 2006; 22: 4899-4901.

[231] Habibi Y, Heim T, Douillard R. AC electric field-assisted assembly and alignment of cellulose nanocrystals. J. Polym. Sci. Part B Polym. Phys. 2008; 46: 1430-6.

[232] Pan J, Hamad W, Straus SK. Parameters affecting the chiral nematic phase of nanocrystalline cellulose films. Macromolecules. 2010; 43: 3851-8.

[233] Dong XM, Kimura T, Revol JF, Gray DG. Effects of Ionic Strength on the Isotropic Chiral Nematic Phase Transition of Suspensions of Cellulose Crystallites. Langmuir. 1996; 12:2076-82.

[234] Dong XM, Gray DG. Effect of counter ions on ordered phase formation in suspensions of charged rod like cellulose crystallites. Langmuir. 1997; 13:2404-09.

[235] Elazzouzi-Hafraoui S, Putaux JL, Heux L. Self-assembling and chiral nematic properties of organophilic cellulose nanocrystals. The Journal of Physical Chemistry B. 2009; 113: 11069-75. 
[236] Araki J, Wada M, Kuga S. Steric stabilization of a cellulose microcrystal suspension by poly(ethylene glycol) grafting. Langmuir. 2001; 17:21-27.

[237] Heux L, Chauve G, Bonini C. Nonflocculatingand chiral-nematic self-ordering of cellulose microcrystals suspensions in nonpolar solvents. Langmuir. 2000; 16:821012.

[238] Liu D, Chen X, Yue Y, Chen M,Mu Q. Structure and rheology of nanocrystalline cellulose. Carbohydr. Polym. 2011; 84:316-22.

[239] Beck S, Bouchard J, Berry R. Controlling the reflection wavelength of iridescent solid films of nanocrystalline cellulose. Biomacromolecules 2011; 12:167-72.

[240] Khan, R. A.; Beck, S.; Dussault, D.; Salmieri, S.; Bouchard, J.; Lacroix, M. J. Appl. Polym. Sci. 2013, 129, 3038-3046.

[241] Dri FL, Hector JLG, Moon RJ, Zavattieri PD. Anisotropy of the elastic properties of crystalline cellulose $\mathrm{Ib}$ from first principles density functional theory with Van der Waals interactions. Cellulose. 2013; 20: 2703-18.

[242] Cheng Q, Wang S, Harper DP. Effects of process and source on elastic modulus of single cellulose fibrils evaluated by atomic force microscopy. Compos Part A. 2009; 40: 583-8.

[243] Hsieh Y-C, Yano H, Nogi M, Eichhorn SJ. An estimation of the Young's modulus of bacterial cellulose filaments. Cellulose. 2008; 15: 507-13.

[244] De la Motte H, Hasani M, Brelid H, Westman G. Molecular characterization of hydrolyzed cationized nanocrystalline cellulose, cotton cellulose and softwood kraft pulp using high resolution 1D and 2D NMR. Carbohydr. Polym. 2011; 85:738-46.

[245] López SH, Santiago EV, Mora MM, Mancilla JRF, Contreras EAZ. Cellulose-Based Polymer Composite with Carbon Black for Tetrahydrofuran Sensing. International Journal of Polymer Science. 2013. doi.org/10.1155/2013/381653.

[246] Ojijo V, Ray SS, Sadiku R. Toughening of biodegradable polylactide/poly(butylene succinate-co-adipate) blends via in situ reactive compatibilization. ACS Appl Mater Interfaces 2013; 5:4266-76.

[247] Mariano M, El Kissi N, Dufresne A. Cellulose nanocrystals and related nanocomposites: Review of some properties and challenges. J. Polym. Sci. Part B: Polym. Phys. 2014; 52:791-806.

[248] Spinella S, Lo Re G, Liu B, Dorgan J, Habibi Y, Leclère P, Raquez JM, Dubois P, Gross RA. Polylactide/Cellulose Nanocrystal Nanocomposites: Efficient Routes for Nanofiber Modification and Effects of Nanofiber Chemistry on PLA Reinforcement. Polymer. 2015; 65:9-17.

[249] Bonini C, Heux L, Cavaille J-Y, Lindner P, Dewhurst C, Terech P. Rodlike Cellulose Whiskers Coated with Surfactant: A Small-Angle Neutron Scattering Characterization. Langmuir. 2002; 18:3311-14.

[250] Bondeson D, Oksman K. Dispersion and characteristics of surfactant modified cellulose whiskers nanocomposites. Compos. Interface. 2007; 14:617-30. 
[251] Kim J, Montero G, Habibi Y, Hinestroza JP, Genzer J, Argyropoulos DS, Rojas OJ. Dispersion of cellulose crystallites by nonionic surfactants in hydrophobic polymer matrix. Polym. Eng. Sci. 2009; 49: 2054-61.

[252] Zhou Q, Brumer H, Teeri TT. Self-organization of cellulose nanocrystals observed with xyloglucan oligosaccharide-poly(ethyleneglycol)-polystyrene triblock copolymer. Macromolecules. 2009; 42:5430-32.

[253] Spinella S, Maiorana A, Qian† Q, Dawson NJ, Hepworth V, McCallum SA., et al. Concurrent Cellulose Hydrolysis and Esterification to Prepare a Surface-Modified Cellulose Nanocrystal Decorated with Carboxylic Acid Moieties. ACS Sustainable Chem. Eng. 2016; 4 (3): 1538-50.

[254] Gousse C, Chanzy H, Excoffier G, Soubeyrand L, Fleury E. Stable suspensions of partially silylated cellulose whiskers dispersed in organic solvents. Polymer. 2002; 43:2645-651.

[255] Habibi Y, Chanzy H, Vignon MR. TEMPO-Mediated surface oxidation of cellulose whiskers. Cellulose. 2006; 13: 679-87.

[256] Menezes AJ, Siqueira G, Curvelo ASA, Dufresne A. Extrusion and characterization of functionalized cellulose whiskers reinforced polyethylene nanocomposites. Polymer. 2009; 50: 4552-63.

[257] Pandey JK, Kim SC, Chu CS, Lee CS, Jang DJ, Ahn SH. Evaluation of morphological architecture of cellulose chains in grass during conversion from macro to nano dimensions. e-Polymer. 2009; 102:1-15.

[258] De Nooy AEJ, BesemerAC, van Bekkum H. Highly selective tempo mediated oxidation of primary alcohol groups in polysaccharides. Recueil des Travaux Chimiques des Pays-Bas. 1994; 113:165-66.

[259] Yuan H, Nishiyama Y, Wada M, Kuga S. Surface acylation of cellulose whiskers by drying aqueous emulsion. Biomacromolecules. 2006; 7:696-700.

[260] Roman M, Winter WT. In: Oksman K, Sain M, editors. Cellulose Nanocomposites: Processing, Characterization, and Properties. ACS Symposium Series 938; Washington, DC: American Chemical Society; 2006

[261] Zhao B, Brittain WJ. Polymer Brushes: Surface-Immobilized Macromolecules. Prog. Polym. Sci. 2000; 25: 677-710.

[262] Missoum K, Belgacem MN, Bras J. Nanofibrillated Cellulose Surface Modification: A Review. Materials. 2013; 6:1745-66.

[263] Islam MR, Beg MDH, Gupta A. Laccase-treated kenaf/PP. BioResources. 2013; 8(3): 3753-70.

[264] Liungberg N, Bonini C, Bortolussi F, Boisson C, aHeux L, Cavalle JY. New nonocompositre material reinforced with cellulose nanowhiskers in acetic polypropylene. Biomacromolecule. 2005; 6: 2732-39.

[265] Vignon M, Montanari S, Habibi Y. (Centre National de la Recherche Scientifique CNRS, Fr.) FR 2003/5195, 2004. 
[266] Habibi Y, Goffin AL, Schiltz N, Duquesne E, Dubois P, Dufresne A. Bionanocomposites based on poly(epsilon-caprolactone)-grafted cellulose nanocrystals by ring-opening polymerization. J. Mater. Chem. 2008; 18: 5002-10.

[267] Chen G, Dufresne A, Huang J, Chang PR. A novel thermoformable bionanocomposite based on cellulose nanocrystal-graft-poly (e-caprolactone). Macromol. Mater. Eng. 2009; 294: 59-67.

[268] Lin N, Chen GJ, Huang J, Dufresne A, Chang PR. Effects of Polymer-Grafted Natural Nanocrystal on Structure and Mechanical Properties of Poly(lactic acid): A Case of Cellulose Whisker-graft-Polycaprolactone. Journal of Applied Polymer Science. 2009; 113(5): 3417-25.

[269] Peltzer M, Pei A, Zhou Q, Berglund L, Jim'enez A. Surface modification of cellulose nanocrystals by grafting with poly(lacticacid). PolymInt. 2014; 63: 1056-62.

[270] Huang J, GuY. Self-assembly of various guest substrates in natural cellulose substances to functional nanostructured materials. Curr Opin Colloid Interface Sci. 2011; 16: 470-481.

[271] Shin Y, Bae I, Arey BW, Exarhos GJ. Facile stabilization of gold-silver alloy nanoparticles on cellulose nanocrystal. J. Phys. Chem. 2008;112:4844-4848.

[272] Cai J, Kimura S, Wada M, Kuga S. Nanoporous cellulose as metal nanoparticles support. Biomacromolecules. 2009; 10: 87-94.

[273] Maneerung T, Tokura S, Rujiravanit R. Impregnation of silver nanoparticles into bacterial cellulose for antimicrobial wound dressing. Carbohydr. Polym. 2008; 72: 43-51.

[274] Lam E, Hrapovic S, Majid E, Chong JH, Luong JH. Catalysis using gold nanoparticles decorated on nanocrystalline cellulose. Nanoscale. 2012; 4: 997-1002.

[275] Wang, X. F.; Zhou, Y.; Xu, J. J.; Chen, H. Y. Signal-on electrochemiluminescence biosensors based on CdS-carbon nanotube nanocomposite for the sensitive detection of choline and acetylcholine. Adv. Funct. Mater. 2009, 19, 1444-1450.

[276] Gass, J.; Poddar, P.; Almand, J.; Srinath, S.; Srikanth, H. Superparamagnetic polymer nanocomposites with uniform $\mathrm{Fe} 3 \mathrm{O} 4$ nanoparticle dispersions. Adv. Funct. Mater. 2006, 16, 71-75.

[277] L. Chen, R. M. Berry and K. C. Tam. Synthesis of $\beta$-cyclodextrin-modified cellulose nanocrystals (CNCs)@Fe3O4@SiO2 superparamagnetic nanorods. ACS Sustainable Chem. Eng., 2014, 2, 951-958".

[278] Siqueira G, Bras J, Dufresne A. Cellulosic Bionanocomposites: A Review of Preparation, Properties and Applications. Polymers. 2010; 2(4): 728-65.

[279] Samir A, Alloin F, Dufresne A. Review of recent research into cellulosic whiskers, their properties and their application in nanocomposite field. Biomacromolecules 2005; 6(2):612-26. 
[280] Azizi Samir MAS, Alloin F, Sanchez JY, Dufresne A. Nanocomposite polymer electrolytes based on poly(oxyethylene) and cellulose whiskers. Polymers: Cie^ncia e Tecnologia. 2005; 15:109-13.

[281] Halpin JC, Kardos JL. Moduli of crystalline polymers emploing composites theory. J. Appl. Phys. 1972; 43: 2235-41.

[282] Ouali N, Cavaille JY, Perez J. Elastic, viscoelastic and plastic behavior of multiphase polymer blends. Plast. Rubber Comp. Process. Appl. 1991; 16:55-60.

[283] Stauffer D, Aharony A. Introduction to Percolation Theory. London: Taylor \& Francis; 1992.

[284] Surve M, Pryamitsyn V, Ganesan V. Universality in structure and elasticity of polymer-nanoparticle gels. Phys. ReV. Lett. 2006; 96: 177805.

[285] Garboczi EJ, Snyder KA, Douglas JF. Geometrical percolation threshold of overlapping ellipsoids. Phys. ReV. E: Stat. Phys. Plasmas, Fluids, Relat. Interdiscip. Top. 1995; 52:819-28.

[286] Surve M, Pryamitsyn V, Ganesan V. Polymer-bridged gels of nanoparticles in solutions of adsorbing polymers. J. Chem. Phys. 2006; 125: 064903.

[287] Prasad V, Trappe V, Dinsmore AD, Segre PN, Cipellettie L, Weitz DA. Rideal Lecture, Universal features of the fluid to solid transition for attractive colloidal particles. Faraday Discuss. 2003; 123:1-12.

[288] Dufresne A. Comparing the mechanical properties of high performances polymer nanocomposites from biological sources. J. Nanosci. Nanotechnol. 2006; 6: 322-30.

[289] Kvien I, Oksman K. Orientation of cellulose nanowhiskers in polyvinyl alcohol. Appl. Phys. A-Mat. Sci. Process. 2007; 87: 641-43.

[290] Helbert W, Cavaille JY, Dufresne A. Thermoplastic nanocomposites filled with wheat straw cellulose whiskers. Part I. Processing and mechanical behavior. Polym. Compos. 1996; 17:604-11.

[291] Roohani M, Habibi Y, Belgacem NM, Ebrahim G, KarimiAN, Dufresne A. Cellulose whiskers reinforced polyvinyl alcohol copolymers nanocomposites. Europ. Polym. J. 2008; 44: 2489-98.

[292] Azizi Samir MAS, Mateos AM , Alloin F, Sanchez JY, Dufresne A. Plasticized nanocomposites polymer electrolytes based on poly(oxyethylene) and cellulose whiskers. Electrochim Acta. 2004; 49:4667-77.

[293] Bendahou A, Habibi Y, Kaddami H, Dufresne A. Physico-chemical characterization of palm from Phoenix Dactylifera-L, preparation of cellulose whiskers and natural rubber-based nanocomposites. J. Biobased Mat. Bioenerg. 2009; 3: 81-90.

[294] Favier V, Chanzy H, Cavaille JY. Polymer nanocomposites reinforced by cellulose whiskers. Macromolecules. 1995; 28: 6365-67.

[295] Blaker JJ, Lee K-Y, Walters M, Drouet M, Bismarck A. Aligned unidirectional PLA/bacterial cellulose nanocomposite fibre reinforced PDLLA composites. Reactive and Functional Polymers. 2014;85; 185-92. 
[296] Arrieta MP, Castro-López Mde M, Rayón E, Barral-Losada LF, López-Vilariño JM, López J, et al. Plasticized poly(lactic acid)-poly(hydroxybutyrate) (PLA-PHB) blends incorporated with catechin intended for active food-packaging applications. J Agric Food Chem. 2014; 62(41):10170-180.

[297] El Miri N, Abdelouahdi K, Zahouily M, Fihri A, Barakat A, Solhy A, et al. Bionanocomposite films based on cellulose nanocrystals filled polyvinylalcohol/chitosan polymer blend. Inc. J. Appl. Polym. Sci. 2015, 132, 42004.

[298] Hajji P, Cavaille JY, Favier V, Gauthier C, Vigier G. Tensile behavior of nanocomposites from latex and cellulose whiskers. Polym. Compos. 1996; 17:61219.

[299] Lu Y, Weng L, Cao X. Biocomposites of plasticized starch reinforced with cellulose crystallites from cottonseed linter. Macromol. Biosci. 2005; 5:1101-07.

[300] Cao X, Habibi Y, Lucia LAJ. One-pot polymerization, surface grafting, and processing of waterborne polyurethane-cellulose nanocrystal nanocomposites. J Mater Chem. 2009;19:7137-45.

[301] Azizi Samir MAS, Chazeau L, Alloin F, Cavaillé JY, Dufresne A, Sanchez JY. POEbased nanocomposite polymer electrolytes reinforced with cellulose whiskers. Electrochim Acta. 200; 50:3897-3903.

[302] Dufresne A. Polysaccharide nanocrystal reinforced nanocomposites. Can J Chem. 2008; 86: 484-494.

[303] Roohani M, Habibi Y, Belgacem NM, Ebrahim G, KarimiAN, Dufresne A. Cellulose whiskers reinforced polyvinyl alcohol copolymers nanocomposites. Europ. Polym. J. 2008; 44: 2489-2498

[304] Mathew AP, Dufresne A. Morphological investigation of nanocomposites from sorbitol plasticized starch and tunicin whiskers. Biomacromolecules. 2002; 3: 609617.

[305] Li Q, Zhou J, Zhang L. Structure and properties of the nanocomposite films of chitosan reinforced with cellulose whiskers. J Polym Sci Part B: Polym Phys. 2009; 47:1069-1077.

[306] Habibi Y, Goffin AL, Schiltz N, Duquesne E, Dubois P, Dufresne A. Bionanocomposites based on poly(epsilon-caprolactone)-grafted cellulose nanocrystals by ring-opening polymerization. J. Mater. Chem. 2008; 18: 5002-5010.

[307] Ljungberg N, Cavaille' JY, Heux L. Nanocomposites of isotactic polypropylene reinforced with rod-like cellulose whiskers. Polymer. 2006; 47: 6285-6292.

[308] Cao X, Habibi Y, Lucia LAJ. One-pot polymerization, surface grafting, and processing of waterborne polyurethane-cellulose nanocrystal nanocomposites. J Mater Chem. 2009;19:7137-45.

[309] Hassan CM, Peppas NA. Structure and applications of poly(vinyl alcohol) hydrogels produced by conventional crosslinking or by freezing/thawing methods. Biopolym/PVA Hydrogels/Anionic Polym Nanocompos. 2000; 153: 37-65. 
[310] Leitner J, Hinterstoisser B, Wastyn M, Keckes J, Gindl W. Sugar beet cellulose nanofibril-reinforced composites. Cellulose. 2007; 14: 419-25.

[311] Bhatnagar A, SainM. Processing of cellulose nanofibre-reinforced composites. J Reinf Plast Compos 2005;24:1259-68.

[312] Wang B, Sain M. Dispersion of soybean stock-based nanofibre in a plastic matrix. Polym Int. 2007; 56: 538-46.

[313] Nakagaito AN, Yano H. The effect of morphological changes from pulp fibre towards nanoscale fibrillated cellulose on the mechanical properties of high-strength plant fibre based composites. Appl Phys A. 2004; 78:547-52.

[314] Hayashi H, Shimo T (2006). Automobile outside plates with good surface smoothness from cellulose fibre prepregs. Jpn Kokai Tokkyo Koho 2005-136053:9.

[315] Nogi M, Yano H. Transparent nanocomposites based on cellulose produced by bacteria offer potential innovation in the electronics device industry. Adv Mater. 2008; 20:1849-52.

[316] Pu Y, Zhang J, Elder T, Deng Y, Gatenholm P, Ragauskas A. Investigation into acrylic films reinforced with nanocellulosics versus acacia. Composite. Eng. 2007; 38B (3):360-66.

[317] Nogi M, Yano H. Optically transparent nanofibre sheets by deposition of transparent materials: a concept for roll- to-roll processing. Appl Phys Lett. 2009; 94:1-3.

[318] Bruce DM, Hobson RN, Farrent JW, Hepworth DG. High-performance composites from low-cost plant primary cell walls. Compos Part A Appl Sci Manuf . 2005; 36:1486-93.

[319] Paillet M, Dufresne A. Chitin whisker reinforced thermoplastic nanocomposites. Macromolecules. 2001; 34: 6527-30.

[320] Seydibeyoglu MO, Oksman K. Novel nanocomposites based on polyurethane and microfibrillated cellulose. Compos Sci Technol. 2008; 68:908-14.

[321] Auad ML, Contos VS, Nutt S, Aranguren MI, Marcovich NE. Characterization of nanocellulose-reinforced shape memory polyurethanes. Polym Int. 2008; 57:651-59.

[322] Marcovich NE, Auad ML, Bellesi NE, Nutt SR, Aranguren MI. Cellulose micro/nanocrystals reinforced polyurethane. J Mater Res. 2006; 21:870-81.

[323] Brown EE, Laborie MPG. Bioengineering bacterial cellulose/poly(ethylene oxide) nanocomposites. Biomacromolecules. 2007; 8:3074-81.

[324] Okubo K, Fujii T, Yamashita N. Improvement of interfacial adhesion in bamboo polymer composite enhanced with micro-fibrillated cellulose. JSME Int J Series ASolid Mech Mater Eng. 2005; 48:199-204.

[325] Mathew AP, Chakraborty A, Oksman K. In: Oksman K, Sain M, editors. Cellulose nanocomposites: processing, characterization, and properties The structure and mechanical properties of cellulose nanocomposites prepared by twin screw extrusion. Washington, DC: American Chemical Society; 2006. 
[326] Wang B, Sain M. The effect of chemically coated nanofibre reinforcement on biopolymer based nanocomposites. Bioresources. 2007; 2:371-88.

[327] Suryanegara L, Nakagaito AN, Yano $\mathrm{H}$. The effect of crystallization of PLA on the thermal and mechanical properties of microfibrillated cellulose-reinforced PLA composites. Compos Sci Technol. 2009; 69: 1187-92.

[328] Habibi Y, Aouadi S, Raquez J-M, Dubois P. Effects of interfacial stereocomplexation in cellulose nanocrystal-filled polylactide nanocomposites. Cellulose 2013; 20: 287785.

[329] Zhang C, Salick MR, Cordie TM, Ellingham T, Dan Y, Turng LS. Incorporation of poly(ethylene glycol) grafted cellulose nanocrystals in poly(lactic acid) electrospun nanocomposite fibers as potential scaffolds for bone tissue engineering. Mater Sci and Eng: C. 2015; 49:463-71.

[330] Kiziltas A, Nazari B, Erbas Kiziltas E, Gardner DJ, Han Y, Rushing TS. Method to reinforce polylactic acid with cellulose nanofibers via a polyhydroxybutyrate carrier system. Carbohydrate Polymers. 2016; 140: 393-99.

[331] Lonnberg H, Fogelstrom L, Malstrom E. Microfibrillated cellulose films grafted with

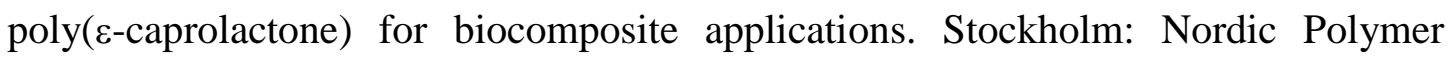
Days, (June 11-13), 2008.

[332] Petersson L, Mathew AP, Oksman K. Dispersion and properties of cellulose nanowhiskers and layered silicates in cellulose acetate butyrate nanocomposites. J Appl Polym Sci. 2009; 112: 2001-9.

[333] Grunnert M, WinterWT. Progress in the development of cellulose reinforced nanocomposites. Polym Mater Sci Eng. 2000; 82:232-38.

[334] Noorani S, Simonsen J, Atre S. In: Oksman K, Sain M, editors. Cellulose nanocomposites: processing, characterization and properties. Washington, DC: ACS symposium series; vol 938; American Chemical Society, 2006.

[335] Garcia de Rodriguez NL, Thielemans W, Dufresne A. Sisal cellulose whiskers reinforced polyvinyl acetate nanocomposites. Cellulose. 2006; 13:261-70.

[336] Choi Y, Simonsen J. Cellulose nanocrystal-filled carboxymethyl cellulose nanocomposites. J Nanosci Nanotechnol. 2006; 6: 633-39.

[337] Ruiz MM, Cavaille JY, Dufresne A, Gérard JF, Graillat C. Processing and characterization of new thermoset nanocomposites based on cellulose whiskers. Compos Interface. 2000; 7:117-31.

[338] Chauve G, Heux L, Arouini R, Mazeau K. Cellulose poly(ethylene-co-vinyl acetate) nanocomposites studied by molecular modeling and mechanical spectroscopy. Biomacromolecules. 2005; 6: 2025-31.

[339] Cheng Q, Wang SQ, Rials TG, Lee SH. Physical and mechanical properties of polyvinyl alcohol and polypropylene composite materials reinforced with fibril aggregates isolated from regenerated cellulose fibres. Cellulose. 2007; 14:593-602. 
[340] Chazeau L, Cavaille JY, Perez J. Plasticized PVC reinforced with cellulose whiskers II. Plastic behavior. J Polym Sci Part B: Polym Phys. 2000; 38: 383-92.

[341] Li Q, Zhou J, Zhang L. Structure and properties of the nanocomposite films of chitosan reinforced with cellulose whiskers. J Polym Sci Part B: Polym Phys. 2009; 47:1069-77.

[342] Wang Y, Cao X, Zhang L. Effects of cellulose whiskers on properties of soy protein thermoplastics. Macromol Biosci. 2006; 6:524-31.

[343] Dufresne A. Dynamic mechanical analysis of the interphase in bacterial polyester/cellulose whiskers natural composites. Compos Interface. 2000; 7:53-67.

[344] Qi H, Cai J, Zhang L, Kuga S. Properties of films composed of cellulose nanowhiskers and a cellulose matrix regenerated from alkali/urea solution. Biomacromolecules. 2009;10:1597-1602.

[345] Jiang L, Morelius E, Zhang JW, Wolcott M, Holbery J. Study of the poly(3hydroxybutyrate-co-3-hydroxyvalerate)/cellulose nanowhisker composites prepared by solution casting and melt processing. J Compos Mater. 2008; 42: 2629-45.

[346] Huang S, Zhou L, Li M-C, Wu Q, Kojima Y, Zhou D. Preparation and Properties of Electrospun Poly (Vinyl Pyrrolidone)/Cellulose Nanocrystal/Silver Nanoparticle Composite Fibers. Materials. 2016; 9:523.

[347] Pelton R. Bioactive paper provides a low-cost platform for diagnostics. Trac-Trends Anal Chem. 2009; 28: 925-42.

[348] Trovatti E, Silva NH, Duarte IF, Rosado CF, Almeida IF, Costa P, et al. Biocellulose membranes as supports for dermal release of lidocaine. Biomacromolecules. 2011; 12:4162-68.

[349] Elisseeff JH, Lee A, Kleinman HK, Yamada Y. Biological response of chondrocytes to hydrogels. Ann N Y Acad Sci. 2002; 961:118-22.

[350] Dong S, Roman M. Fluorescently labeled cellulose nanocrystals for bioimaging applications. J.am.Chem. Soc. 2007; 129: 13810-11.

[351] Junka K, Guo J, Filpponen I, Laine J, Rojas OJ. Modification of cellulose nanofibrils with luminescent carbon dots. Biomacromolecules. 2014; 15: 876-88.

[352] Jokerst JV, Van de Sompel D, Bohndiek SE, Gambhir S S. Cellulose nanoparticles are a biodegradable photoacoustic contrast agent for use in living mice. Photoacoustic. 2014; 2:119-27.

[353] Liu A, Walther A, Ikkala O, Belova L, Berglund LA. Clay nanopaper with tough cellulose nanofiber matrix for fire retardancy and gas barrier functions. Biomacromolecules. 2011;12:633-41.

[354] Orelma H, Filpponen I, Johansson LS, Osterberg M, Rojas OJ, Laine J. Surface functionalized nanofibrillar cellulose (NFC) film as a platform for immunoassays and diagnostics. Biointerphases. 2012; 61(7):1-4.

[355] Butchosa N, Brown C, Larsson PT, Berglund LA, Bulone V, Zhou Q. Nanocomposites of bacterial cellulose nanofibers and chitin nanocrystals: 
Fabrication, characterization and bactericidal activity. Green Chemistry. 2013; 15(12): 3404-13.

[356] Vivekanandhan S, Christensen L Misra M, Amar Kumar Mohanty. Green process for impregnation of silver nanoparticles into microcrystalline cellulose and their antimicrobial bionanocomposite films. J. Biomater Nanobiotechnol. 2012; 3: 371-76.

[357] Galkina OL, Ivanov VK, AgafonovAV. Cellulose nanofiber-titania nanocomposites as potential drug delivery systems for dermal applications. J.Mater Chem. 2015; 3:1688-98.

[358] Muller A, Ni Z, Hessler N, Wesarg F, Müller FA, Kralisch D, Fischer D. The biopolymer bacterial nanocellulose as drug delivery system: investigation of drug loading and release by using the model protein albumin. J Pharm Sci. 2013; 102: 57992.

[359] Huang L, Chen X, Nguyen TX, Tang H, Zhang L, Yang G. Nano-cellulose 3Dnetworks as controlled-release drug carriers. J. Mater Chem. 2013; 1:2976-84.

[360] Shi X, Zheng Y, Wang G, Lina Q, Fana J. pH-and electro-response characteristics of bacterial cellulose nanofiber/sodium alginate hybrid hydrogels for dual controlled drug delivery. RSC Adv. 2014; 4:47056-65.

[361] Lu T, Li Q, Chen w, Yu H. Composite aerogels based on dialdehyde nanocellulose and collagen for potential applications as wound dressing and tissue engineering scaffold. Compos Sci Technol. 2014; 94: 132-38.

[362] Nasri-Nasrabadi B, Mehrasa M, Rafienia M, Bonakdar S, Behzad T, Gavanji S. Porous starch/cellulose nanofibers composite prepared by salt leaching technique for tissue engineering. Carbohydr Polym. 2014;108:232-38.

[363] Barud HS, Vial WR, Nunes ES, Ribeiro SJL, Jafellici M, Nalin M, Marques RFC. Biocellulose-based flexible magnetic paper, Journal of Applied Physics. 2015; 117:17B734.

[364] Saska S, Teixeira LN, de Oliveira PT, Gaspar AMM, Ribeiro SJL, Messaddeq Y. Bacterial cellulose-collagen nanocomposite for bone tissue engineering. J Mater Chem. 2012; 22(41): 22102-12.

[365] Hutchens SA, Benson RS, Evans BR, O'Neill HM, Rawn CJ. Biomimetic synthesis of calcium-deficient hydroxyapatite in a natural hydrogel. Biomaterials. 2006; 27(26):4661-70.

[366] M. Österberg, E. D. Cranston, Nord Pulp Pap Res J 2014, 29, 1.

[367] R. Rusli, S. J. Eichhorn, Nanotechnology 2011, 22, 1.

[368] Y. Dahman, Journal of Nanoscience and Nanotechnology 2009, 9, 5105.

[369] Department of Health and Human Services "Pyrogen and endotoxins testing: Questions and answers", In: Guidance for industry. Rockville, MD: FDA. U.S., 2012.

[370] G. Helenius, H. Bäckdah, A. Bodin, U. Nannmark, P. Gatenholm, B. Risberg, $J$ Biomed Mater Res A 2006, 76, 431. 
[371] T. Miyamoto, S. Takahashi, H. Ito, H. Inagaki, Y. Noishiki, J Biomed Mater Res 1989, 23, 125.

[372] H. Luo, G. Xiong, D. Hu, K. Ren, F. Yao, Mater Chem Phys 2013, 143, 373.

[373] A. A. Homaei, R. Sariri, F. Vianello, J Chem Biol 2013, 6, 185.

[374] R. M. A. Domingues, M. E. Gomes, R. L. Reis, Biomacromolecules 2014, 15, 2327.

[375] T. Loftsson, M. Brewster, Journal of Pharmaceutical Sciences 1996, 85, 1017.

[376] G. M. A. Ntoutoume, R. Granet, J. P. Mbakidi, Bioorg. Med. Chem. Lett 2016, 26, 941.

[377] M. H. Lee, K. J. Yoon, S. Ko, J Appl Polym Sci 2000, 78, 1986.

[378] A. Sani, Y. Dahman, J. Chem. Technol. Biotechnol 2010, 85, 151.

[379] K. El-Tahlawy, M. A. Gaffar, S. El-Rafie, Carbohydr Polym 2006, 63, 385.

[380] J. H. Wang, Z. Cai, Carbohyd. Polym 2008, 72, 695.

[381] H. A. Benesi, J. H. Hildebrand, Am J. Chem. Soc 1949, 71, 2703.

[382] A. Patterson, Phys. Rev 1939, 56, 978.

[383] J. I. Morán, V. A. Alvarez, V. P. Cyras, A. Vazquez. Cellulose 2008, 15(1), 149.

[384] W. Ruland, Acta Cryst 1961, 14, 1180.

[385] M. P. Bajgai, S. Aryal, S. R. Bhattarai, K. C. Bahadur, K. W. Kim, H. Y. Kim, Journal of applied polymer science 2008, 108, 1447.

[386] P. Garside, P. Wyeth, Stud. Conser 2003, 48, 269.

[387] N. Sathitsuksanoh, Z. Zhu, S. Wi, Y. H. P. Zhang, Biotechnol. Bioeng 2011, 108, 521.

[388] S. Gibuad, S. Ben Zirar, P. Mutzenhardt, I. Fries, A. Astier, Int. J. Pharm 2005, 306, 107.

[389] S. Dash, P. N. Murthy, L. Nath, P. Chowdhury, Acta Pol Pharm 2010, 67, 217.

[390] K. A. Connors, Chem. Rev 1997, 197, 1325.

[391] A. Antony, M. Prabhu, R. K. Sankaranarayanan, G. Venkatesh, N. Rajendiran, J. Phys. Chem. B 2012, 166, 9061.

[392] A. M. Khan, S. S. Shah, J Dispers Sci Technol 2009, 30, 1247.

[393] Malikmammadov, E.; Tanir, T.E.; Kiziltay, A.; Hasirci, V.; Hasirci, N. Journal of Biomaterials Science, Polymer Edition. 2018, 29(7-9),1-55.

[394] Hyun, J.C.; Tae, G.P. Adv Drug Deliv Rev, 2007, 59, 249-262.

[395] Azimi, B.; Nourpanah, P.; Rabiee, M.; Arbab, S. Journal of engineered fibers and fabrics. 2014, 9(1):47-66.

[396] Chung, H.J.; Park, T.G. Advanced Drug Delivery Reviews. 2007, 59, 249-262.

[397] Carletti, E.; Motta, A.; Migliaresi, C. Methods in Mol Biol. 2011, 695, 17-39.

[398] Thadavirul, N.; Pavasant, P.; Supaphol, P. J Biomed Mater Res Part A. 2014, 102A, 3379-3392.

[399] Ekaputra, A.K.; Prestwich, G.D.; Cool, S.M.; Hutmacher, D.W. Biomacromolecules. 2008, 9, 2097-2103.

[400] Nam, Y.S.; Yoon, J.J.; Park, T.G. J. Biomed. Mater. Res: Appl. Biomater. 2000, $53,1-7$. 
[401] Abdalkarim, S.Y.H.; Yu, H.Y.; Wang, D.; Yao, J. Cellulose. 2017, 24, 2925-2938.

[402] Shi, Q.; Zhou, C.; Yue, Y.; Guo, W.; Wu, Y.; Wu, Q. Carbohydr Polym. 2012, 90, 301-308.

[403] Xiang, C.; Taylor, A.G.; Hinestroza, J.P.; Frey, M.W. J Appl Polym Sci. 2013, 127, 79-86.

[404] Kretschmann, O.; Choi, S.W.; Miyauchi, M.; Tomatsu, I.; Harada, A.; Ritter, H. Angew. Chem., Int. Ed. 2006, 45, 4361-4365.

[405] Al-Abdallah, W.; Dahman, Y. Bioprocess. Biosyst. Eng. 2013, 36(11), 1735-1743.

[406] Folmer, B.J.B.; Sijbesma, R.P.; Versteegen, R.M.; Van der Rijt, J.A.J.; Meijer, E.W. Adv. Mater. 2000, 12, 874-78.

[407] Zoppe, J.O.; Peresin, M.S.; Habibi, Y.; Venditti, R.A.; Rojas, O.J. Applied materials and interfaces. 2009, 1(9), 1996-2004.

[408] Cacciotti, I.; Fortunati, E.; Puglia, D.; Kenny, J.M.; Nanni, F. Carbohydr Polym. 2014, 103,22-31

[409] Wang, Y.; Rodriguez-Perez, M.A.; Reis, R.L.; Mano, J.F. Macromol. Mater. Eng. 2005, 290, 792-801.

[410] Lang, M.; Bei, J.; Wang, S. J. Biomater. Sci. Polymer Edn. 1999, 10: 501-512.

[411] Ali, F.B.; Kang, D.J.; Kim, M.P.; Cho, C-H.; Kim, B.J. Polym Int. 2014, 63, 16201626.

[412] Valter, C.; Marco, G.; Simone, G.; Giulia, M. Biomacromolecules. 2007, 8, 498-508.

[413] Wang, W.; Caetano, G.F.; Chiang, W.H.; Braz, A.L.; Blaker, J.J.; Frade, M.A.; Bartolo, P.J. Int. J. Bioprint. 2016, 2, 95-104.

[414] Yu, H.Y.; Wang, C.; Abdalkarim, S.Y.H. Cellulose. 2017, 24, 4461-4477.

[415] Velasco, M.A.; Narváez-Tovar, C.A.; Garzón-Alvarado, D.A. Biomed Res Int. 2015, 2015, 1-21.

[416] Fukuzaki, H.; Yoshida, M.; Kumakura, M.; Mashimo, T.; Yuasa, H.; Imai, K.; Hidetoshi, Y. Polymer, 1990, 31(10), 2006-2014.

[417] [reserved] Khattab, M.M.; Dahman, Y. Manuscript submitted for publication in Journal of Applied Polymer Science. 2019, (app.20190241).

[418] Takemura, G.; Fujiwara, H. Prog Cardiovasc Dis. 2007, 49(5), 330-352.

[419] Chandrasekaran, A.R.; Jia, C.Y.; Theng, C.S.; Muniandy, T.S.; Muralidharan, S.A. Journal of Applied Pharmaceutical Science. 2011,5, 214-217.

[420] Czarnobaj, K. Drug Deliv. 2008, 15, 485-492.

[421] Haroosh, H.J.; Dong, Y. 2013. Electrospun nanofibrous composites to control drug release and interaction between hydrophilic drug and hydrophobic blended polymer matrix, in Hoa, S.V. and Hubert, P. (ed), Proceedings of The $19^{\text {th }}$ International Conference on Composite Materials (ICCM19), Jul 28-Aug 2 2013, pp. 6300-6307. Montreal, Canada: Canadian Association for Composite Structures and Materials

[422] Missirlis, D.; Kawamura, R.; Tirelli, N. Eur J Pharm Sci. 2006, 29, 120-129.

[423] Zhang, H.; Yu, H.Y.; Wang, C.; Yao, J. Carbohydr Polym. 2017, 173,7-16 
[424] Salerno, M.; Cenni, E.; Fotia, C.; Avnet, S.; Granchi, D.; Castelli, F. Current Cancer Drug Targets. 2010, 10(7), 649-659.

[425] Akaraonye E, Keshavarz T, Roy I (2010) J Chem Technol Biotechnol 85:732-743

[426] Cherney JH, Small E (2016) Agronomy 6:58 doi:10.3390/agronomy6040058

[427] Binod P, Sindhu R, Singhania RR, Vikram S, Devi S, Nagalakshmi S (2010) Bioresour Technol 101:4767-4774

[428] Pakarinen A, Zhang J, Brock T, Maijala P, Viikari L (2012) Bioresour Technol 107:275-281

[429] Yoo J, Alavi S, Vadlani P, Amanor-Boadu V (2011) Bioresour Technol 102:75837590

[430] Alvira P, Tomás-Pejó E, Ballesteros M, Negro MJ (2010) Bioresour Technol 101:4851-61

[431] Ouyang J, Dong Z, Song X, Lee X, Chen M, Yong Q (2010) Bioresour Technol 101(17): 6685-91

[432] Uchino K, Saito T, Gebauer B, Jendrossek D (2007) Journal of Bacteriology 189: 8250-8256

[433] Repaske R, Repaske AC (1976) Environ Microbiol 32:585-91

[434] Ward AC, Rowley BI, Dawes EA (1977) J Gen Microbiol 102:61-68

[435] Sluiter A, Hames B, Hyman D, Payne C, Ruiz R, Scarlata (2008) Laboratory Analytical Procedure, NREL/TP-510-42621. Golden, CO: National Renewable Energy Laboratory, Golden, CO. 1

[436] Sluite A, Hames B, Ruiz R, Scarlata C, Sluite J, Templeton D (2010) Golden, Colorado: National Renewable Energy Laboratory 17. Report No. TP-510-42618

[437] Vignon MR, Garcia-Jaldon C, Dupeyre DM (1995) Int J Biol Macromol 17(6):395404

[438] Yu S, Olsen CE, Marcussen J (1998) Carbohydr Res 305(1):73-82

[439] Templeton DW, Quinn M, Wychen VS (2012) J Chromatogr A 1270: 225-234

[440] Ouyang J, Li Z, Li X, Ying H, Yong Q (2009) Bioresources 4:1586-1599

[441] Wang Y, Chen R, Cai J, Liu Z, Zheng Y (2013) PLOS ONE 8(4):60318

[442] Ramsay JA, Berger E, Voyer R, Chavarie C, Ramsay BA (1994) Biotechnology Techniques 8:589-594.

[443] Hahn SK, Chang YK, Kim BS, Chang HN (1994) Biotechnology and Bioengineering 44(2):256-261

[444] Kim M, Cho KS, Ryu HW, Lee EG, Chang YK (2003) Biotechnol Lett 25:55-59

[445] Hwang KJ, You SF, Don TM J (2006) Chin Inst Chem Eng 37:209-216

[446] Zakaria MR, Ariffin H, Johar NAM, Abd-Aziz S, Nishida H, Shirai Y, Hassan MA (2010), Polym. Degrad. Stabil. 95:1382-1386

[447] Slepecky RA, Law JH (1960) Anal Chem 32:1697-1699

[448] Steffien D, Aubel I, Bertau M J (2014) Mol Catal B Enzyme 103:29-35

[449] Korte S, Staiger MP (2008) Fibers Polym 9:593-603 
[450] Batcha AFM, Prasad DMR, Khan MR, Abdullah H (2014) Bioprocess Biosyst Eng 37: 943-951

[451] Imai M, Ikari K, Suzuki I (2004) Biochem Eng J 17:79-83

[452] Zhang Y, Sun W, Wang H, Geng A (2013) Bioresour Technol 147:307-314

[453] Annamalaia N, Sivakumarb N (2016) Journal of Biotechnology 237:13-17

[454] Cao W, Sun C, Liu R, Yin R, Wu X (2012) Bioresour Technol 111:215-221

[455] Franz A, Rehner R, Kienle A, Grammel H (2011) Letters in Applied Microbiology $54: 45-51$

[456] Saito T, Kobayashi T (2002). Intracellular degradation of PHAs, p. 23-40. In Doi Y and Steinbuchel A(ed.), Biopolymetrs, vol 3b. Polyester II. Willey-VCH, WEINHEIM, Germany.

[457] Dahman Y, CU Ugwu (2014) Bioprocess Biosyst Eng 37: 1561-1568

[458] Saratale GD, Oh MK (2015) International Journal of Biological Macromolecules 80: $627-635$

[459] Budde CF, Riede SL, Hübner F, Risch S, Popovic MK, Rha CK, Sinskey AJ (2011) Appl Microbiol Biotechnol 89:1611-1619

[460] Yu J, Stahl H (2008) Bioresour Technol 99: 8042-8048

[461] Sandhya M, Aravind J, Kanmani P (2013) Int J Environ Sci Technol 10:10:47-54

[462] Gouda MK, Swellam AE, Omar SH (2001) Microbiol Res 156:201-207

[463] Lee SY, Choi J, Han K, Song JY (1999) Applied and Environmental Micropiology. 65:2762-2764

[464] Wang J, Yu HQ (2007) Appl Microbiol Biotechnol 75:871-878

[465] Ramsay AJ, Berger E, Ramsay BA, Chavari C (1990) Biotechnol Technol 4:221-226

[466] Byrom D (1994) in: D.P. Mobley (Ed.), Plastics from Microbes: Microbial Synthesis of Polymers and Polymer Precursors, Hanser, Munich.

[467] Madden LA, Anderson AJ, Shah DT, Asrar J (1999) International Journal of Biological Macromolecules 25: 43-53

[468] Mohammadi M, Hassan MA, Phang L-Y, Ariffin H (2012) Biotechnol Lett 34:253259.

[469] Getachew A, Woldesenbet F (2016) BMC Res Notes 9(1):509. 